THIAGO CELSO STRANO PASQUAL

UM ESTUDO SOBRE A AÇÃO DO VENTO NAS ESTRUTURAS DE MEMBRANA

SÃO PAULO

2011 


\section{UM ESTUDO SOBRE A AÇÃO DO VENTO NAS ESTRUTURAS DE MEMBRANA}

Dissertação apresentada à Escola Politécnica da Universidade de São Paulo para obtenção do Grau de Mestre em Engenharia Civil.

Área de concentração:

Engenharia de Estruturas

Orientador:

Prof. Livre Docente Ruy Marcelo de Oliveira Pauletti

\section{Ed. Revisada}


Este exemplar foi revisado e alterado em relação à versão Original sob responsabilidade única do autor com a anuência de seu orientador.

São Paulo, 12 de Dezembro de 2011.

Autor: Thiago Celso Strano Pasqual

Orientador: Prof. Livre Docente Ruy Marcelo de Oliveira Pauletti

FICHA CATALOGRÁFICA

\section{Pasqual, Thiago Celso Strano}

Um estudo sobre a ação do vento nas estruturas de mem-

bra / T.C.S. Pasqual. -- ed.rev. -- São Paulo, 2011.

p. 109

Dissertação (Mestrado) - Escola Politécnica da Universidade de São Paulo. Departamento de Engenharia de Estruturas e Geotécnica.

1. Estruturas de membranas 2. Ação do vento nas estruturas 3. Tensoestruturas 4. Vento I. Universidade de São Paulo. Escola Politécnica. Departamento de Engenharia de Estruturas e Geotécnica II. t. 


\section{AGRADECIMENTOS}

Aos meus pais e irmão pelo apoio em todos momentos, pelo amor e companheirismo nas horas difíceis e também por compartilharem as alegrias durante estes três anos de trabalho árduo.

À minha esposa pela compreensão e carinho em todas noites passadas em claro, conciliando atividades profissionais e acadêmicas. Pelo seu apoio moral e incentivo a todo momento.

Aos meus amigos por estarem ao meu lado e entenderem um distanciamento nas periódicas viagens e fins de semana de estudo, a fim de concluir o curso.

Ao Prof. Ruy Pauletti, por todo conhecimento e experiência transmitidos ao longo de meses, sempre confiando e incentivando o aprimoramento técnico e a abrangência do trabalho. 


\section{RESUMO}

Atualmente, problemas de interação fluido-estrutura representam um grande desafio em diferentes áreas de engenharia e ciências aplicadas. Dentro das aplicações de engenharia civil, a ação do vento sobre estruturas tem grande relevância principalmente na avaliação de instabilidades elásticas de estruturas muito flexíveis, como pontes e estruturas de membrana.

Este trabalho introduz os conceitos principais das estruturas de membrana e da ação do vento em estruturas. A ação do vento em estruturas de membrana será apresentada em capítulo à parte, através de aplicações computacionais para problemas de fluidodinâmica.

A ação do vento em estruturas é tratada de forma consistente com as prescrições da norma técnica da Associação Brasileira de Normas Técnicas (ABNT) NBR 6123 Forças devidas ao Vento em Edificações, a qual está vigente desde Junho de 1988. Ainda, foi apresentado breve resumo sobre análise dinâmica do vento através de modelos reduzidos em túneis de vento.

Devido às estruturas de membrana possuírem formas incomuns e comportamento estrutural diferenciado com relação às estruturas convencionais, as forças devido à ação do vento nestas estruturas não fazem parte do escopo da NBR 6123. Utilizando-se dos conceitos básicos e benchmarks da norma supracitada, a análise fluido-dinâmica das aplicações será tratada computacionalmente. As leis físicas e equações gerais para solução deste tipo de problema são apresentadas em concordância com o software utilizado.

Neste trabalho a ação do vento em estruturas de membrana foi solucionada com acoplamento parcial em algumas aplicações tridimensionais, fazendo-se uso do software Ansys versão 11. Aqui entende-se por acoplamento parcial a tratativa do vento e da estrutura em domínios separados. Em primeira análise, foram obtidas as pressões de vento sobre uma malha de elementos infinitamente rígidos e indeslocáveis com a forma geométrica da membrana. As pressões foram então aplicadas sobre a estrutura, obtendo-se deslocamentos e tensões estáticas. Atualizando-se o domínio do vento para a nova geometria, novos processamentos foram realizados sucessivamente até que a variação de tensões ou das pressões na estrutura fosse desprezível. 


\begin{abstract}
Currently, problems of fluid-structure interaction represent a major challenge in different areas of engineering and applied sciences. Within the civil engineering applications, the action of wind on structures has great importance particularly in the evaluation of elastic instabilities of very flexible structures such as bridges and tension membrane structures

This work introduces the main concepts of tension membrane structures and wind acting on structures. The fluid-dynamic analysis are presented in a separate chapter, focused on computational applications.

The wind acting on structures is treated consistently with the prescriptions of the national code ABNT (Associação Brasileira de Normas Técnics "Brazilian Technical Standards Association”) NBR 6123 - Wind Forces on Buildings, issued on June 1988. Still, a brief summary was presented on the dynamic analysis of wind using wind tunnels.

As tension membrane structures having unusual shapes and different structural behavior compared to conventional structures, the forces due to wind action on membranes are not part of the scope of NBR 6123. Using the basic concepts and benchmarks available, the fluid-dynamics for the applications are treated computationally. The general physical laws and equations for solving this problem are presented in accordance with the software.

The wind acting on membrane structures was solved with partial coupling in some three-dimensional applications, using Ansys v.11 software. Here, partial coupling means solving wind problem and structure problem separately. In the first analysis, the wind problem is solved obtaining pressures over a rigid mesh with the geometric shape of the membrane structure. These pressures are then applied on the membrane, resulting on static displacements and stresses. Updating the domains to the new geometry, other analysis are run until the change over the structural tensions is negligible.
\end{abstract}




\section{LISTA DE FIGURAS}

Figura 1 - Parabolóide Hiperbólico...............................................................................2

Figura 2 - Superfície Esférica ................................................................................. 26

Figura 3 - Geometria do Elemento Finito Ansys SHELL 41 …………………………....... 35

Figura 4 - Isopletas de Velocidade Básica do Vento $\left(\mathrm{V}_{0}\right)$..................................................4 40

Figura 5 - Fator Topográfico S1 (z) .......................................................................... 42

Figura 6 - Mapa mundi de ameaças de tornado ………………………………………...... 48

Figura 7 - Isopletas Ampliadas na Região de São Martinho da Serra / RS ......................... 49

Figura 8 - Um dos 40 modelos para ensaio em túnel de vento do Burj Khalifa...................62 62

Figura 9 - Domínio recomendado para ação do vento na estrutura ........................................65

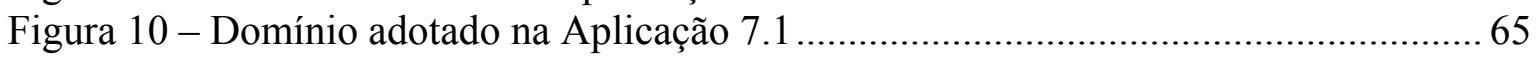

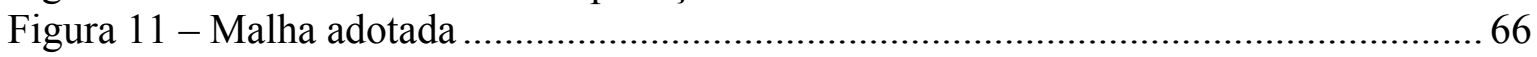

Figura 12 - Coeficientes de pressão obtidos no Ansys …………………………………..... 67

Figura 13 - Velocidade do vento no entorno do anteparo circular $(\mathrm{m} / \mathrm{s})$..............................6 68

Figura 14 - Geometria do modelo para vento em Perfil I.....................................................69 69

Figura 15 - Malha adotada para vento em Perfil I ..............................................................69 69

Figura 16 - Malha próxima ao Perfil I …………………………………………….... 70

Figura 17 - Coeficientes de pressão esperados .............................................................. 70

Figura 18 - Resultado dos coeficientes de pressão .......................................................... 71

Figura 19 - Resultado de velocidades do vento $(\mathrm{m} / \mathrm{s})$........................................................ 72

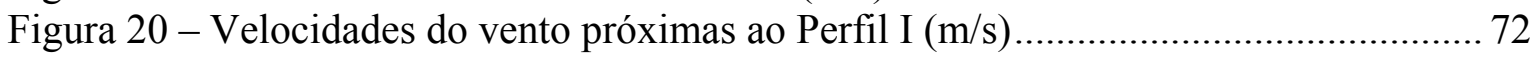

Figura 21 - Geometria do Modelo ………………………………………………..... 73

Figura 22 - Malha de Elementos Finitos......................................................................... 74

Figura 23 - Refinamento da malha em torno do muro...................................................... 74

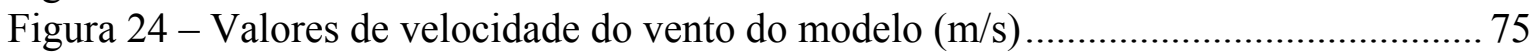

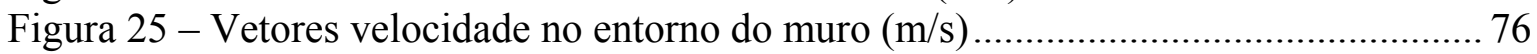

Figura 26 - Valores de pressão do vento $\left(\mathrm{N} / \mathrm{m}^{2}\right)$.......................................................... 77

Figura 27 - Coeficientes de pressão do vento ………………………………………...... 78

Figura 28 - Geometria da edificação modelada ………………………………………... 79

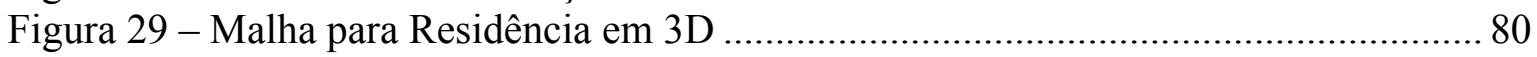

Figura 30 - Coeficientes de Pressão em Plano próximo ao Piso ………………………….... 81

Figura 31 - Valores de Velocidade do Vento próximo ao nível do Piso $(\mathrm{m} / \mathrm{s})$.................... 82

Figura 32 - KeyPoints Básicos para Projeto do Parabolóide ……………………………........ 83

Figura 33 - Superfície Inicial gerada pelo Software …………………………………..... 84

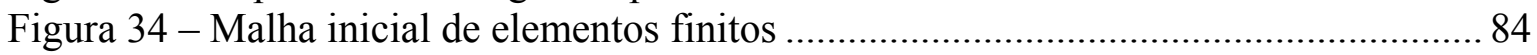

Figura 35 - Deslocamentos provocados pela condição inicial de retesamento (m) ............. 85

Figura 36 - Coeficientes de pressão sobre a membrana em $1^{\mathrm{a}} \mathrm{FSI}$.......................................... 86

Figura 37 - Velocidades máximas de vento no entorno da membrana $(\mathrm{m} / \mathrm{s})$...................... 87

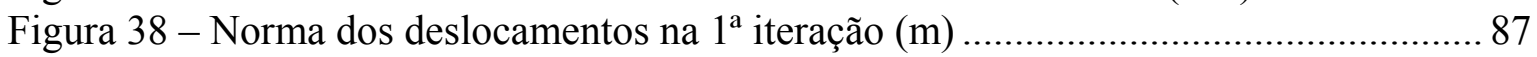

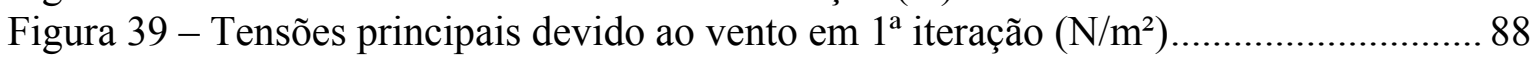

Figura 40 - Coeficientes de pressão sobre a membrana na $2^{\mathrm{a}}$ interação .................................. 89

Figura 41 - Norma dos deslocamentos da membrana na $2^{\mathrm{a}}$ interação (m)............................ 89

Figura 42 - Tensões principais da membrana na $2^{\mathrm{a}}$ interação $\left(\mathrm{N} / \mathrm{m}^{2}\right)$................................. 90

Figura 43 - Coeficientes de pressão sobre a membrana na $3^{\mathrm{a}}$ interação .............................. 91

Figura 44 - Norma dos deslocamentos da membrana na $3^{\mathrm{a}}$ interação (m)........................... 91

Figura 45 - Tensões principais da membrana na $3^{\mathrm{a}}$ interação $\left(\mathrm{N} / \mathrm{m}^{2}\right)$................................. 92

Figura 46 - Geometria inicial adotada para o conóide ....................................................... 94

Figura 47 - Malha de elementos finitos do conóide......................................................... 95 
Figura 48 - Norma dos deslocamentos pela condição inicial de retesamento $(\mathrm{m})$

Figura 49 - Norma dos deslocamentos provocados no $2^{\circ}$ processamento da busca da forma

(m)

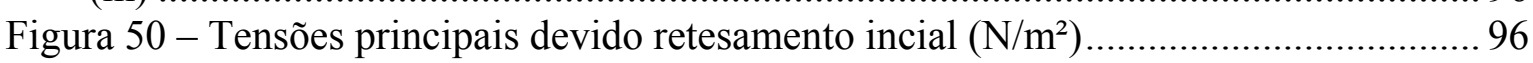

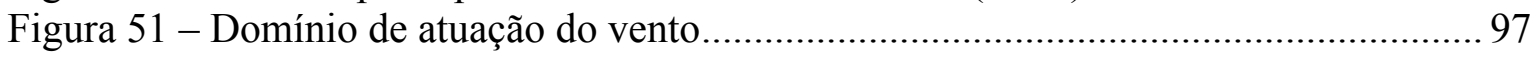

Figura 52 - Coeficientes de pressão sobre a membrana em $1^{\mathrm{a}}$ interação ............................. 98

Figura 53 - Velocidades máximas de vento no entorno da membrana $(\mathrm{m} / \mathrm{s})$...................... 98

Figura 54 - Pressão do vento na $1^{\mathrm{a}}$ interação $\left(\mathrm{N} / \mathrm{m}^{2}\right)$.......................................................... 99

Figura 55 - Deslocamentos em $\mathrm{z}$ devido ao vento na $1^{\mathrm{a}}$ interação $(\mathrm{m})$............................. 100

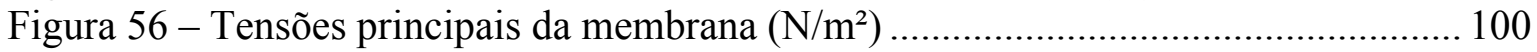

Figura 57 - Coeficientes de pressão na $2^{\mathrm{a}}$ interação ......................................................... 101

Figura 58 - Velocidades máximas no entorno do conóide na $2^{\mathrm{a}}$ interação $(\mathrm{m} / \mathrm{s}) \ldots \ldots \ldots \ldots \ldots 101$

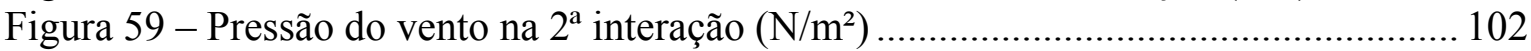

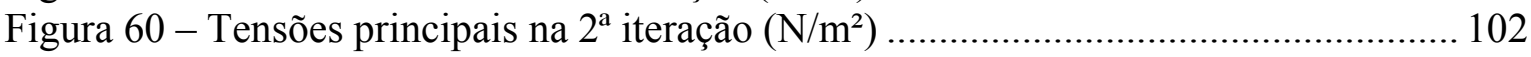

Figura 61 - Coeficientes de pressão na $3^{\mathrm{a}}$ interação ....................................................... 103

Figura 62 - Velocidades máximas no entorno do conóide na $3^{\mathrm{a}}$ interação $(\mathrm{m} / \mathrm{s}) \ldots \ldots \ldots \ldots \ldots . . .103$

Figura 63 - Pressão do vento na $3^{\mathrm{a}}$ interação $\left(\mathrm{N} / \mathrm{m}^{2}\right)$..................................................... 104 


\section{LISTA DE TABELAS}

Tabela 1 - Parâmetros para cálculo do Fator de Rugosidade $\left(\mathrm{S}_{2}\right)$

Tabela 2 - Ocorrência de Ventos em São Martinho da Serra de 2005 a 2010

Tabela 3 - Estações consideradas no código nacional de ação do vento em estruturas...... 50

Tabela 4 - Distribuição das pressões externas em edificações cilíndricas..... 


\section{SUMÁRIO}

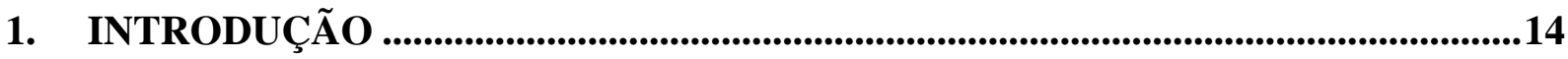

2. OBJETIVOS .........................................................................................................16

3. ESTRUTURAÇÃO E METODOLOGIA DO TRABALHO ........................................17

3.1. Modelagem ..........................................................................................................

3.2. Parâmetros estruturais........................................................................................................17

4. ESTRUTURAS DE MEMBRANA ................................................................................19

4.1. Busca da Forma ..........................................................................................................21

4.2. Padronagem ..........................................................................................................28

4.3. Carregamentos...............................................................................................................30

4.4. Materiais............................................................................................................32

4.5. Comportamento estrutural............................................................................................35

5. AÇÃO DO VENTO EM ESTRUTURAS.......................................................................37

5.1. Caracterização do Vento ...........................................................................................37

5.2. Ação do Vento Segundo a NBR 6123 ....................................................................39

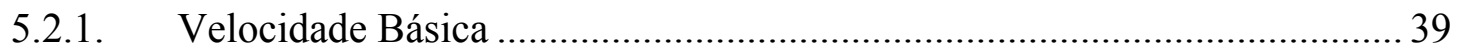

5.2.2. Velocidade Característica e Velocidade de Projeto......................................... 41

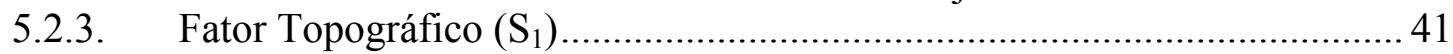

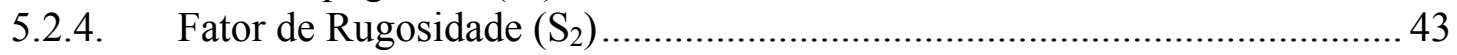

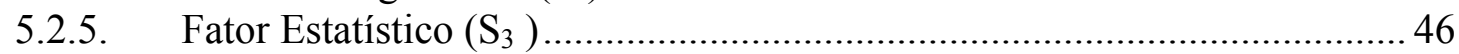

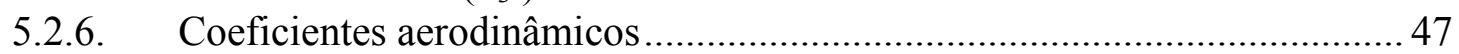

5.3. Pesquisa sobre velocidades básicas do Vento............................................................48

6. FLUIDO-DINÂMICA .........................................................................................51

6.1. Análise computacional ..........................................................................................51

6.1.1. Lei da Conservação da Massa …………………………………………... 51

6.1.2. Equação da Continuidade ........................................................................... 52

6.1.3. Lei da Conservação dos Momentos............................................................ 53

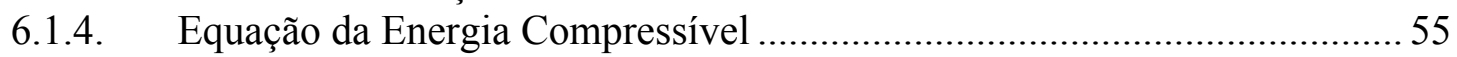

6.1.5. Equação da Energia Incompressível............................................................... 57

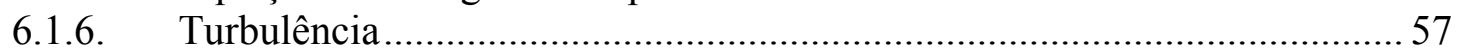

6.2. Ensaios em Túnel de Vento......................................................................................60

7. APLICAÇÕES ....................................................................................................................64

7.1. Fluido sobre Anteparo Circular 2D .............................................................................65 


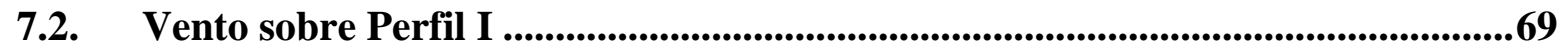

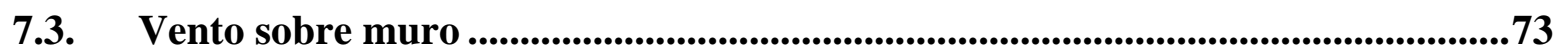

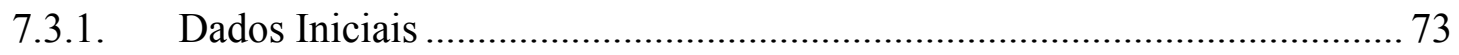

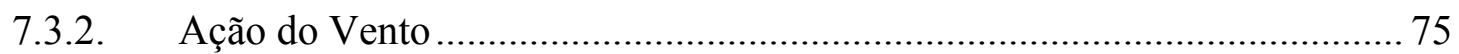

7.4. Vento sobre Edificação em 3D...............................................................................79

7.5. Parabolóide Hiperbólico .................................................................................................83

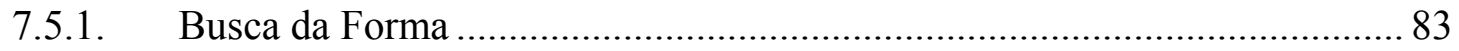

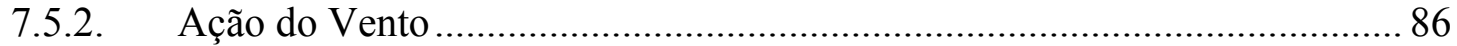

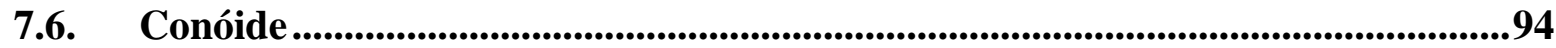

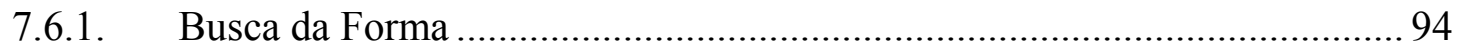

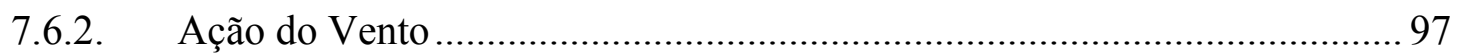

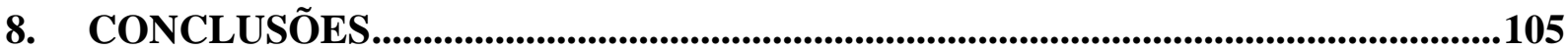

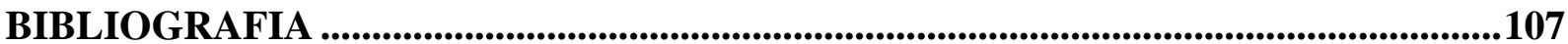




\section{NOMENCLATURAS EM LETRAS LATINAS}

Letras Latinas

$A \quad$ área

$A_{i} \quad$ área efetiva i-gésima parte da estrutura

$A_{r} \quad$ área do elemento de membrana

$A_{s} \quad$ área da seção transversal do cabo

b parâmetro meteorológico

b carregamento externo

c coeficiente de arrasto superficial

$C_{A} \quad$ coeficiente de arrasto

$C_{A a} \quad$ coeficiente de arrasto de atrito

$C_{A f} \quad$ coeficiente de arrasto de forma

$C_{\rho} \quad$ coeficiente aerodinâmico de pressão

$C_{S} \quad$ coeficiente de sustentação

$\hat{D} \quad$ matriz constitutiva

E módulo de elasticidade

$f \quad$ freqüência do vento

$F_{A} \quad$ força de arrasto do vento

$F_{S} \quad$ força de sustentação do vento

$F_{r} \quad$ fator de rajada

$F(t) \quad$ carregamento dinâmico 
$g \quad$ aceleração da gravidade

I momento de inércia

$\mathrm{k}_{\mathrm{e}} \quad$ matriz de rigidez tangente elástica

$\mathrm{k}_{\mathrm{n}} \quad$ matriz de rigidez tangente natural

$\mathrm{k}_{\mathrm{t}} \quad$ matriz tangente do elemento de membrana

K constante de Karmán, de valor 0,4

$\mathbf{N} \quad$ tensão superficial à superfície

p carregamento ortogonal à superfície de membrana

$P_{m} \quad$ probabilidade de ocorrência

$q$ pressão dinâmica do vento

Re número de Reynolds

$S_{1} \quad$ fator topográfico

$S_{2} \quad$ fator de rugosidade da superfície

$S_{3} \quad$ fator estatístico

$t \quad$ tempo

$\mathbf{u}_{\mathrm{P}} \quad$ vetor deslocamentos nodais

$V \quad$ velocidade de escoamento do vento

Vbarravalor médio da componente longitudinal do vento

$V_{k} \quad$ velocidade característica do vento

$V_{p} \quad$ velocidade de projeto do vento

$V_{r} \quad$ volume do elemento de membrana 
$V_{z} \quad$ velocidade média na altura $z$

$V_{0} \quad$ velocidade básica do vento

$V_{10} \quad$ velocidade igual à de projeto

$x \quad$ deslocamento na direção do eixo $\mathrm{x}$

$y$ deslocamento na direção do eixo y

w peso específico

$z \quad$ deslocamento na direção do eixo z

$z \quad$ altura sobre o terreno

$z_{0} \quad$ comprimento de rugosidade

Observação: a nomenclatura em letras gregas está indicada no decorrer do texto quando da sua primeira aparição 


\section{INTRODUÇÃO}

Em engenharia civil exige-se que as estruturas sejam obrigatoriamente funcionais e seguras. Além disto, existe uma constante busca por otimização de materiais e arquiteturas harmoniosas. Neste contexto, as estruturas leves como as estruturas de membrana ganharam bastante espaço, tendo aplicações das mais diversas.

Atualmente, devido ao seu baixo peso próprio e funcionamento estrutural, as estruturas de membrana são amplamente utilizadas como elementos de cobertura de grandes áreas. Entretanto, devido a sua resistência à flexão ser desprezível estas estruturas apresentam grandes deformações e mudança de forma importantes quando submetidas a carregamentos elevados.

Levando isto em conta, a ação do vento sobre as membranas tem grande importância, havendo necessidade de estudos da interação entre o meio fluido (vento) e a estrutura (membrana).

Historicamente, problemas de engenharia envolvendo interação de fluidos e estrutura sempre foram de substancial importância para o homem, desde as situações mais simples como o transporte de água e a cobertura de suas edificações (sob ações do vento) até outras mais complexas como os meios de transporte aéreo e marítimo.

Sob o ponto de vista estrutural, o objetivo principal nos problemas de engenharia é determinar a configuração equilibrada do sistema carregado, satisfazendo as condições de compatibilidade das deformações, relações constitutivas dos materiais e equilíbrio de forças.

Em breve resumo, os fluidos têm como principal característica a capacidade de se deformar continuamente quando submetidos a uma tensão de cisalhamento. Por outro lado e comparativamente, as estruturas sólidas apresentam deslocamentos menores e mais ordenados quando submetidos às solicitações externas.

Devido a estas complexidades, a maioria dos problemas envolvendo interação fluido-estrutura foi resolvida primeiramente de forma empírica ou experimental. Somente no último século, com a evolução das teorias de materiais e de estruturas, o homem produziu um conhecimento científico mais adequado sobre estas aplicações. 
Entretanto, a complexidade de tais teorias exigiria muito tempo do engenheiro civil para solução manual tanto de problemas de membrana quanto de interação fluidoestrutura. Por este motivo, sistemas computacionais estão em constante avanço e são amplamente utilizados pelos profissionais da área.

No contexto de aplicação prática, é interessante observar as limitações da mente do ser humano. Ele dificilmente consegue assimilar o comportamento do seu ambiente e criações de forma única. Por este motivo, na solução de problemas estruturais é comum o procedimento de subdivisão do sistema em parcelas elementares (discretização), cujo comportamento é prontamente compreendido, para uma posterior reconstrução do todo.

Recentemente observou-se um incessante crescimento no estudo e implementação computacional de métodos numéricos discretos para resolução de problemas de estruturas, como o Método dos Elementos Finitos (MEF), Método dos Volumes Finitos e Método dos Elementos Livres (sem malha) de Galerkin (EFG).

Tratando-se da interação fluido-estrutura, devido à complexidade dos modelos computacionais e à história muito recente de pesquisas numéricas, o método mais usual para solução destes problemas ainda é o experimental, através de ensaios em túnel de vento. 


\section{OBJETIVOS}

O objeto de pesquisa terá como foco a análise não-linear dinâmica de estruturas de membrana, sob a ação de fortes ventos através da modelagem em softwares baseados em elementos finitos.

A análise da estrutura de membrana será feita em uma configuração espacial (bi ou tridimensional) com acoplamento parcial à dinâmica do fluido, ou seja, será gerado um modelo em que o vento atua sobre um domínio em que a estrutura de membrana é apresentada como um obstáculo. As forças de pressão atuantes neste obstáculo são então aplicadas na estrutura de membrana. Com os deslocamentos provocados por esta pressão, atualiza-se a malha de elementos finitos do fluido e rodam-se novas interações.

De forma a atestar o método computacional, primeiramente serão apresentadas algumas aplicações em concordância com as condições de vento recomendadas pela Norma da Associação Brasileira de Normas Técnicas (ABNT) NBR 6123/1988, utilizando os coeficientes de pressão para algumas formas geométricas como benchmarks. Feito isto, serão apresentadas aplicações em estruturas de membranas em condições de escoamentos mais rápidos e turbulentos, compatíveis com o ciclone tropical Catarina e alguns ventos mais fortes observados no Sul do País desde 2003.

Entre as saídas deste trabalho serão apresentadas análises comparativas entre:

- análise computacional da ação do vento sobre estrutura indeformável;

- análise computacional da ação do vento sobre estrutura deformando-se em acoplamento parcial;

- benchmarks quando disponíveis. 


\section{ESTRUTURAÇÃO E METODOLOGIA DO TRABALHO}

Este trabalho foi divido em oito capítulos. Nos capítulos 4, 5 e 6 foi apresentado um breve resumo com formulações sobre estruturas de membrana, ação do vento em estruturas e fluido-dinâmica. No capítulo 7 foram apresentadas aplicações numéricas com os respectivos resultados. Finalmente, no oitavo capítulo são elaborados os comentários e conclusões do trabalho.

\subsection{Modelagem}

Foi utilizado o software Ansys versão V.11 para a modelagem das estruturas apresentadas neste trabalho.

O fluido será modelado através de uma descrição puramente euleriana, ou seja, as propriedades do escoamento são apresentadas num ponto fixo e determinado do espaço (nó fixo).

Os parâmetros característicos de vento serão obtidos conforme prescrições da norma ABNT NBR 6123/1988. Além disso, observando as crescentes notícias de catástrofes climáticas, principalmente as chuvas acompanhadas de ventos fortes no Sul do país, será realizado um estudo frente a condições mais adversas, como o ciclone tropical Catarina, modelando a turbulência pelo método $\mathbf{k}-\boldsymbol{\varepsilon}$.

Em contrapartida, as membranas serão modeladas através de uma malha de elementos finitos em descrição puramente lagrangeana, ou seja, as partículas do material estão fixas em uma malha que se deforma e desloca. $\mathrm{O}$ acoplamento entre as modelagens será feito parcialmente através do software mencionado.

\subsection{Parâmetros estruturais}

Os parâmetros estruturais considerados são condizentes com os materiais utilizados recentemente para execução de membranas de PVC no Brasil e na maioria dos países desenvolvidos.

Todos os materiais utilizados na modelagem foram considerados isotrópicos. 
O sistema de coordenadas adotado em todas análises realizadas neste trabalho foi um sistema cartesiano estacionário. 


\section{ESTRUTURAS DE MEMBRANA}

Há milhares de anos o homem convive e utiliza tecidos e cabos tracionados para construção de abrigos, instrumentos musicais e meios de transporte ou de transposição de grandes alturas, mares, lagos ou rios.

Entretanto, a sua utilização sempre foi movida pela intuição e conclusões obtidas por tentativas e erros. Somente no último século o conhecimento técnico evoluiu o bastante nas áreas de materiais, computadores e métodos numéricos para o desenvolvimento de uma teoria sobre essas estruturas, conhecida como tensoestruturas ou estruturas retesadas.

Segundo Pauletti (2003), o termo estruturas retesadas é uma alusão ao estado em que se encontram as cordas dos arcos ou as cordas dos instrumentos musicais que, para funcionarem a contento, precisam estar retesadas e não frouxas. Portanto, um estado de retesamento em cabos e membranas é essencial para que as estruturas retesadas trabalhem adequadamente.

Atualmente, o projeto e construção de estruturas retesadas como tendas, sistemas de roldanas, pontes pênseis, e coberturas exige uma teoria sofisticada, além de técnicas de construção e materiais modernos que permitem seu uso de forma mais segura, durável e barata.

As principais características das estruturas retesadas são o seu baixo peso próprio, permitindo sua utilização em grandes vãos e o seu comportamento estrutural flexível, cujo estado de retesamento é essencial para o funcionamento a contento. Adicionalmente, são estruturas que agridem menos o meio ambiente por serem feitas com materiais recicláveis. Atualmente é amplamente utilizada em coberturas de estádios, igrejas, aeroportos e outros.

As maiores vantagens em comparação com estruturas convencionais são:

- $\quad$ o peso de um teto de tecido é $1 / 30$ do peso de um teto de estrutura metálica;

- $\quad$ tempo menor de construção;

- $\quad$ possibilidade de reciclagem, facilidade de desmontagem e remontagem;

- iluminação natural;

- $\quad$ menor custo de energia; 
- $\quad$ menos manutenção;

- $\quad$ espaço coberto ilimitado.

Por outro lado, nem sempre a tensoestrutura é a solução mais viável, pois:

- o custo de uma cobertura em tecido é mais elevado do que o de uma cobertura convencional;

- a durabilidade da estrutura não é muito boa quando comparada com estruturas metálicas e de concreto armado, e é variável de acordo com o material empregado;

- mau isolamento térmico;

- $\quad$ problemas acústicos;

- restrição quanto a ventos fortes.

Quanto ao material da membrana existem vários tipos, mas os mais utilizados atualmente são três: o tecido de poliéster revestido com cloreto polivinílico (PVC), tecido de fibra de vidro revestido com politetrafluoretileno (PTFE) e filme de etileno fluoroetileno (ETFE).

Os tecidos com PVC são menor custo quando comparado aos demais e têm durabilidade de 10 a 15 anos. Os tecidos com PTFE têm maior durabilidade (mais de 25 anos) e resistência, porém são os mais caros. Já o filme de ETFE tem durabilidade de 20 anos e é muito utilizado em obras que requerem grande transparência por ter aproximadamente $90 \%$ de translucidez.

O projeto de uma estrutura de membrana, de acordo com TABARROK (1992) e PAULETTI (2003), envolve então três etapas fundamentais:

- busca da forma: determinação de uma forma geométrica inicial que equilibra o estado de solicitações devido às forças de retesamento, de modo a satisfazer os requisitos arquitetônicos; 
- padronagem ou padrões de corte: determinação aproximada de um conjunto de peças planas feitas de tecido e que, no espaço, serão unidas para formar a superfície determinada no processo de busca da forma, ou seja, a membrana;

- resposta aos carregamentos: análise do comportamento da estrutura (já definida no processo de busca da forma) devido aos vários carregamentos de serviço possíveis, como aqueles devidos ao vento, por exemplo, de modo a garantir a segurança do sistema.

Vistos os objetivos deste trabalho citado no capítulo 2, este trabalho apresentará de forma resumida questões relacionadas ao projeto de membrana. Nas aplicações (vide capítulo 7) serão apresentadas ilustrações relacionadas a busca da forma e resposta aos carregamentos, mas não serão apresentados padrões de corte.

\subsection{Busca da Forma}

A busca da forma pode ser feita mediante modelos físicos ou numéricos. Os modelos físicos são aqueles em que estrutura é construída em escala, fazendo uso desde filmes de sabão até membranas de PVC. Já os modelos numéricos tomam partido de métodos numéricos e da computação gráfica para gerar a estrutura virtualmente.

O uso de modelos físicos começou com Frei Otto, cujos livros oferecem uma descrição detalhada das principais técnicas empregadas. Parte da popularidade da modelagem física reside na possibilidade de também se empregar o modelo, subseqüentemente, para a determinação de padrões de corte.

Tradicionalmente, o processo de busca de forma se realizava com filmes de sabão, com espessura de 0,1 a 1 mícron. Por serem muito instáveis e de difícil visualização (dada sua transparência), os modelos de filme de sabão foram substituídos por outros feitos de membranas finas de borracha ou materiais de costura, como o filó. Atualmente a preferência é dada a modelos construídos com membranas de PVC, cuja superfície pode ser pintada após a deformação (usualmente de branco), de modo que possa ser marcada. 
Diferentes arranjos de películas translúcidas, marcadas com um reticulado de linhas equiespaçadas são então experimentados sobre a superfície, até que um arranjo satisfatório de padrões de corte seja obtido. O reticulado é usado então para auxiliar a determinação da geometria planificada das peças.

Além da visualização arquitetônica, os modelos físicos podem dar indicações valiosas quanto ao comportamento estrutural. Eles não provêem, no entanto, nenhuma informação específica quanto ao nível de tensão a que os materiais ficarão submetidos. Tampouco permitem que se imponham a priori condições sobre o estado de retesamento. Assim, embora não haja dúvida de que os modelos físicos das estruturas retesadas continuarão sendo feitos também parece claro que o projeto assistido por computador, combinando simulação estrutural e computação gráfica terá uso cada vez mais generalizado. Além da determinação e visualização da distribuição acurada das tensões em toda a estrutura, o computador permite uma investigação mais completa da forma e uma rápida percepção dos efeitos de alterações geométricas, retesamento, materiais, entre outros.

Em se tratando de modelagem numérica, existem alguns procedimentos para a busca da forma como, por exemplo, aqueles baseados:

- nas superfícies minimais;

- nas tensões não-uniformes;

- nos deslocamentos incrementais;

- $\quad$ nas densidade de forças;

- na relaxação dinâmica.

É importante deixar claro que, nesta etapa, existem inúmeras configurações geométricas possíveis tais que o equilíbrio seja verificado, e é o engenheiro civil quem deverá optar por aquela que melhor se adapte às condições arquitetônicas, ambientais e econômicas.

Cabe lembrar que em estruturas de membrana existe uma constância de espessura, exceto em determinadas regiões onde é necessário o reforço. Portanto, um projeto em que 
as tensões sejam tenham grande amplitude de variação em regiões distintas acarretará em uma estrutura muito cara.

Entre os procedimentos citados acima, uma das mais relevantes é a representada pelas superfícies minimais, que se caracterizam, do ponto de vista matemático, por apresentarem a menor área para um dado contorno (TABARROK, 1992).

Do ponto de vista mecânico, elas também apresentam a menor energia de deformação, para o contorno especificado, bem como campos uniformes e isotrópicos de tensões, características que podem ser tomadas como base para definições alternativas do conceito de superfícies minimais.

As características descritas acima são as mesmas existentes em filmes de sabão e, por este motivo, modelos físicos de tais filmes eram utilizados como técnica para a determinação da forma das estruturas de membrana.

Com o avanço da ciência e dos métodos computacionais esta técnica caiu em desuso.

Porquanto sejam ideais do ponto de vista do aproveitamento do material, as configurações baseadas em superfícies minimais podem não atender a alguns requisitos de projeto. Uma característica indesejável é que estas superfícies tendem a ser demasiadamente abatidas, uma vez que a curvatura média de uma superfície minimal é zero. Como a capacidade portante de uma estrutura retesada cresce com sua curvatura, fica claro que as superfícies minimais nem sempre serão desejáveis (PAULETTI, 2003).

Pequenas curvaturas podem se mostrar inadequadas também do ponto de vista funcional, prejudicando o escoamento da água da chuva para uma membrana de cobertura.

Em geral, portanto, membranas de superfícies não-minimais associadas a campos de tensão não uniformes provêem maior flexibilidade para o projetista (TABARROK, 1992). Nesta abordagem, admite-se baixo módulo de elasticidade para a membrana, mas tensões iniciais especificadas anisotrópicas e não-uniformes, levando a formas de equilíbrio distintas das minimais.

Em geral são necessárias várias tentativas de campo inicial de tensões, ajustandose a razão entre as tensões em duas direções ortogonais até que se encontre uma configuração adequada. 
Quanto à geometria espacial, existem basicamente três grandes grupos de superfícies:

- as planas;

- as desenvolvíveis;

- não-desenvolvíveis.

As superfícies planas são aquelas em que ambos os raios de curvatura principais são infinitos em todos os pontos do seu domínio.

As desenvolvíveis são aquelas cujos pontos de seu domínio possuem um dos raios de curvatura principais nulo e o outro não. Exemplos deste tipo são os cilindros e os cones.

Já as superfícies não-desenvolvíveis apresentam ambos os raios de curvatura principais não-nulos e finitos. Como os raios de curvatura podem ser positivos ou negativos, este grupo é subdividido em dois outros: o das superfícies anticlásticas e o das superfícies sinclásticas.

Uma superfície é dita anticlástica se, em qualquer ponto de seu domínio, os raios de curvatura máximo e mínimo possuírem sinais contrários, como o parabolóide hiperbólico. 


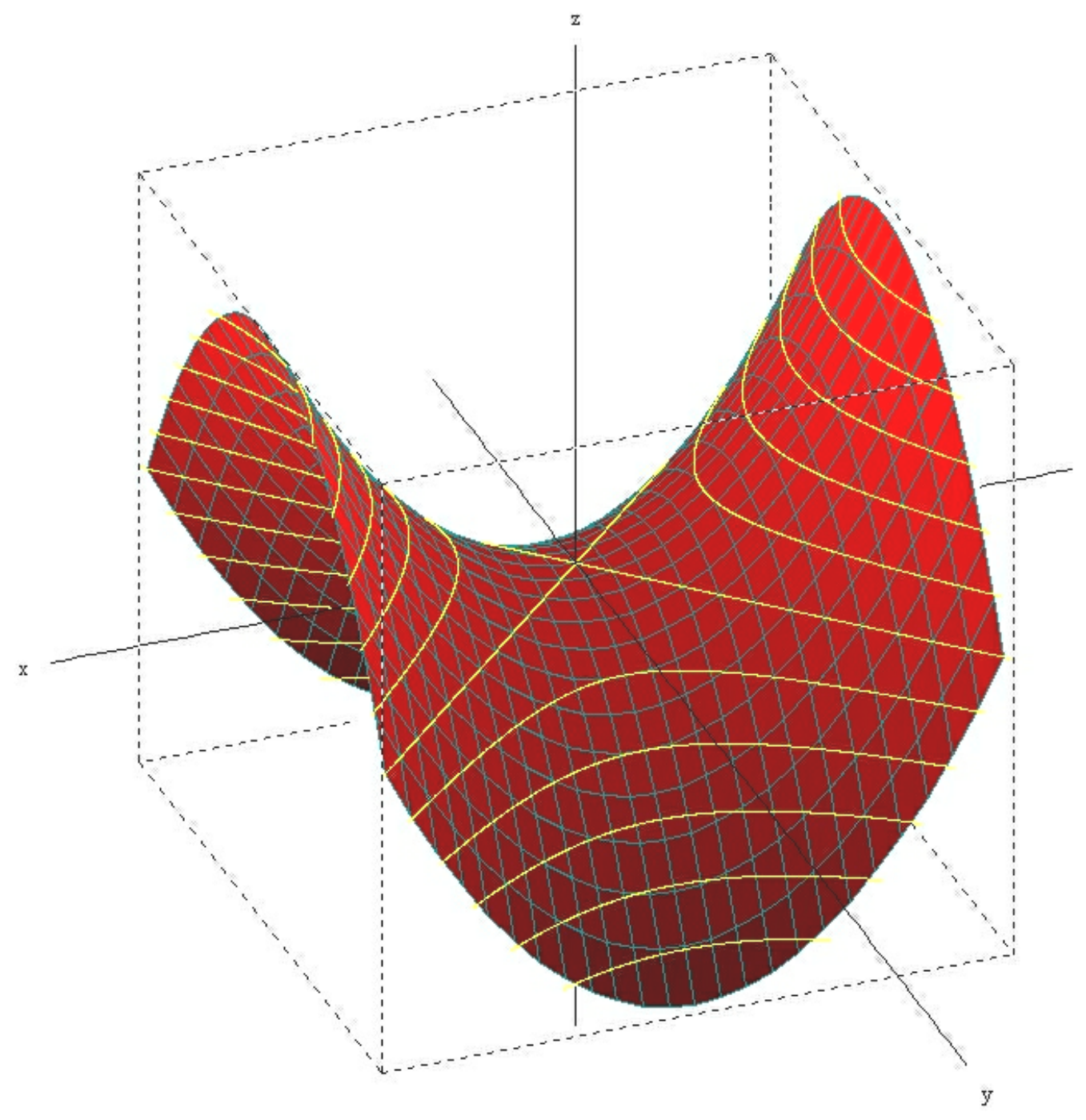

Figura 1 - Parabolóide Hiperbólico

Já as superfícies esféricas e elipsoidais, por exemplo, apresentam o mesmo sinal para os raios de curvatura máximo e mínimo em qualquer ponto de seu domínio e, portanto, são ditas sinclásticas. 


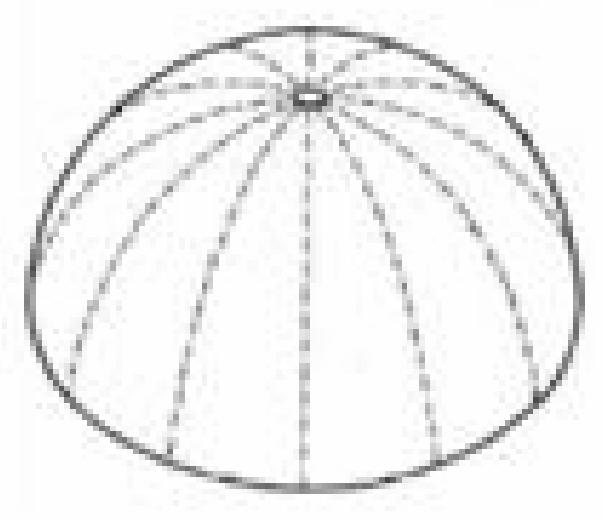

Figura 2 - Superfície Esférica

Devido ao comportamento estrutural, interessante observar que toda superfície minimal é anticlástica ou plana e membrana sinclástica é inflada.

Para auxiliar no entendimento do descrito acima, abaixo são apresentadas equações de equilíbrio de membranas considerando direções principais de superfície e estado homogêneo e isótropo de tensões:

$$
\begin{array}{ll}
\frac{\partial N_{11}}{\partial s_{1}}+\frac{\partial N_{12}}{\partial s_{2}}+b_{1}=0 & \\
\frac{\partial N_{12}}{\partial s_{1}}+\frac{\partial N_{22}}{\partial s_{2}}+b_{2}=0 & \\
\frac{N_{11}}{\rho_{1}}+\frac{N_{22}}{\rho_{2}}-2 \varphi N_{12}=p &
\end{array}
$$

Por definição, a superfície minimal tem estado de tensões homogêneo e isótropo e não existe uma componente do carregamento externo $\mathbf{b}$ que seja ortogonal à superfície, isto é, $\mathbf{p}=0$. Com isto, para satisfazer a última equação apresentada, $\rho_{\text {máx }}$ e $\rho_{\text {mín }}$ devem ter sinais contrários, determinando assim uma superfície anticlástica.

Da mesma forma, quando $\rho_{\text {máx }}$ e $\rho_{\text {mín }}$ tem mesmo sinal (superfície sinclástica), a equação acima somente estará satisfeita se $\mathbf{p}$ (carregamento ortogonal a superfície) for proporcional a $\mathbf{N}$ (tensão superficial). 
Como as membranas não possuem resistência à compressão, o valor de $\mathbf{N}$ deve ser obrigatoriamente positivo e não nulo. Conseqüentemente, neste caso, o carregamento também deverá ser positivo e não nulo, condição esta caracterizada por superfícies infladas.

Conforme já mencionado, neste trabalho as equações de equilíbrio e busca da forma foram resolvidas em aplicações através do auxílio do Software Ansys, V.11. Basicamente a metodologia para busca da forma adotada foi de:

- criar superfície plana;

- aplicar as condições de contorno, como pontos de apoio;

- criar efeito de retesamento inicial da membrana. Neste caso, dependendo da limitação do software em uso pode ser necessário utilizar artifícios como efeitos de temperatura;

- resolver o problema com condição inicial de retesamento;

- após solução do problema, atualizar condições de contorno e refazer processamentos. 


\subsection{Padronagem}

Uma vez consolidada a configuração inicial da estrutura, por meio da busca da forma, deve-se proceder à determinação dos padrões de corte, processo que compreende a identificação de linhas de corte sobre a superfície de equilíbrio inicial, desdobrando a superfície total e gerando um conjunto de peças de dupla curvatura, e o subseqüente desenvolvimento deste conjunto em peças planas.

O processo deve levar em conta uma série de restrições:

- o tecido deve ser cortado a partir de rolos de largura relativamente pequena;

- por razões econômicas, é desejável que cada peça maximize o uso da largura disponível, sendo vantajoso o emprego de peças com lados retos;

- os comprimentos do contorno de peças adjacentes devem ser iguais;

- a distorção do tecido nas bordas da estrutura deve ser evitada;

- os tecidos usualmente empregados para a confecção de membranas são incapazes de suportar carregamentos prolongados de cisalhamento e o arranjo das peças deve ser tal que as direções das tensões principais coincidam na maior extensão possível com as direções da fiação.

Além disso, as linhas de emenda são também condicionadas por fatores estéticos. Peças adjacentes devem ter uma variação gradual de tamanho. Deve-se também procurar evitar a junção de quatro peças de tecido em um canto, devido à sobre-espessura decorrente (PAULETTI, 2003 apud HERZOG, 1979; BARNES, 1988; MONCRIEFF, 1990; KNUDSON, 1991; GRUNDIG, 1993B).

Assim como o estabelecimento da sua configuração inicial, a determinação dos padrões de corte evoluiu da modelagem física para, quase que exclusivamente, processos computadorizados. No entanto, os modelos físicos continuam populares por serem 
procedimentos intuitivos capazes de fornecer estimativas iniciais para os procedimentos numéricos.

As técnicas de determinação dos padrões de corte por meio de modelos físicos são discutidas em detalhe em OTTO (1958) e OTTO (1982), as quais apresentam-se em breve resumo abaixo.

Em primeiro lugar determina-se o arranjo geral das peças sobre a superfície da maquete. Tiras de papel, tecido ou plástico são então assentadas sobre a maquete, obtendose uma descrição das peças planas que aproximam a geometria da membrana. Obviamente, o processo ressente-se de precisão, tornando-se inadequado para o caso de tecidos com altos módulos de elasticidade como aqueles de fibra de vidro ou kevlar. Nestes tecidos, pequenos erros dimensionais podem induzir a campos de tensão e conseqüentemente a formas bastante diferentes daquelas pretendidas. Abre-se ainda a possibilidade de surgirem zonas de enrugamento no caso de perda do retesamento ou rasgos. Por esta razão, os modelos físicos têm sido complementados ou substituídos por modelos numéricos.

Grande parte do sucesso na determinação dos padrões de corte está relacionada a identificação de linhas de corte, de preferência sendo dada às linhas geodésicas, isto é, linhas de curvatura geodésica nula.

Quando a superfície puder ser desenvolvida sobre um plano, a linha geodésica torna-se uma reta. Peças com as bordas definidas conforme linhas geodésicas, na configuração de equilíbrio, tornam-se retângulos, quando planificadas, possibilitando um melhor aproveitamento de material. Como, porém, as superfícies das membranas retesadas geralmente não são desenvolvíveis, as bordas das peças planificadas apresentam por via de regra algum arqueamento, e os ângulos alguma discrepância em relação ao ângulo reto [MONCRIEFF, 1990].

A partir da identificação das linhas de corte e conseqüentemente das peças de tecido, estas devem ser planificadas. Quando a superfície for desenvolvível, a geometria dos painéis individuais de tecido pode ser obtida por um procedimento essencialmente geométrico, rebatendo-se em um plano os diversos elementos da malha (em geral 
triangulares), e corrigindo-se os comprimentos dos lados para levar em conta as deformações (PAULETTI, 2003 apud MONCRIEFF, 1990; BARNES, 1994).

Quando, por outro lado, a superfície não puder ser desenvolvida, é possível forçar sua planificação empregando-se uma análise estrutural. Para cada peça definida por meio do estudo de linhas geodésicas, pode-se gerar um submodelo de elementos finitos, extraindo da malha da estrutura os elementos correspondentes à peça, e retendo as configurações e os estados de deformação obtidos no processo de busca de forma. Em seguida, impõe-se a cada submodelo deslocamentos tais que anulem as coordenadas verticais dos nós, convergindo-se para a forma planificada, seja pelo método de NewtonRaphson, seja por relaxação dinâmica. Obviamente, é importante verificar ao fim do processo de planificação que os comprimentos resultantes para os contornos de cada peça resultem compatíveis (MONCRIEFF, 1990).

\subsection{Carregamentos}

Os carregamentos típicos de estruturas retesadas no Brasil incluem as cargas concentradas, isto é, distribuídas sobre uma região muito pequena, o peso-próprio e demais as demais cargas permanentes, a sobre ou sub-pressão uniforme (no caso de estruturas pneumáticas), as cargas de vento e temperatura.

Enquanto variações de temperatura induzem a perda ou acréscimo de protensão e as cargas concentradas e distribuídas permanentes (peso próprio) usualmente são muito baixas, são os carregamentos devidos ao vento que se mostram particularmente problemáticos para as estruturas retesadas, seja pela sua severidade como pela complexidade dos fenômenos envolvidos, como aqueles decorrentes da interação ventoestrutura. Como neste trabalho, naturalmente, o vento constitui um dos focos das pesquisas experimentais e teóricas acerca dos carregamentos em estruturas retesadas (PAULETTI, 2003 apud BUCHHOLDT, 1985; GANTES, 1993; KRAUTAHAMMER, 1987; STEFANOU, 1992; TABARROK,1992).

Conforme já mencionado, a determinação das cargas devidas ao vento é um problema complexo. As pressões de vento em uma superfície arbitrária são em geral dadas por expressões do tipo $\mathrm{P}=1 / 2 \mathrm{C}_{\rho} \rho \mathrm{V}^{2}$, onde $\rho$ é a densidade, $\mathrm{V}$ é a velocidade do vento $\mathrm{e}$ 
$\mathrm{C}_{\rho}$ é um coeficiente de pressão, que depende da direção de incidência do vento e da geometria atual da superfície.

Os coeficientes $\mathrm{C}_{\rho}$ são tradicionalmente determinados para cada modelo estrutural, por meio de ensaios em túneis de vento ou, modelos numéricos de hidrodinâmica. Alternativamente, Tabarrok propõe um modelo simplificado de carregamento, em que a força exercida pelo vento em cada elemento de membrana é computada escalando-se uma magnitude especificada para a pressão de vento pelo cosseno do ângulo entre a direção do vento (também especificada) e a normal à superfície corrente do elemento. Este modelo dá pressão zero nos elementos de superfície horizontal e sucção nas superfícies posteriores. A aplicação deste modelo elimina a necessidade de medir-se o coeficiente $\mathrm{C}_{\rho}$ experimentalmente e simplifica os dados de entrada. No entanto, TABARROK enfatiza que se trata de uma aproximação relativamente grosseira. Segundo PAULETTI (2003), um tratamento mais aprofundado do problema, no contexto das estruturas retesadas, é dado por BUCHHOLDT, 1999.

A resposta de uma estrutura retesada aos carregamentos de projeto se dá em torno de uma configuração de equilíbrio inicial, em que a membrana (ou rede) se encontra basicamente enrijecida pelo efeito dos carregamentos permanentes e a protensão. Estes carregamentos induzem um campo de tensões iniciais na membrana, em relação ao qual as tensões variam, para mais ou para menos, em função da ação dos demais carregamentos de projeto, como o vento, o acúmulo de neve ou água da chuva, e as variações de temperatura. O peso próprio é um carregamento intrínseco à própria existência do material, mas sua contribuição para o campo de tensões iniciais é em geral desprezível. Por outro lado, a carga inicial de retesamento da membrana, é o carregamento essencial de uma estrutura retesada, conferindo-lhe forma e rigidez.

É ainda importante observar que, analogamente ao que acontece nos cabos, para os quais a rigidez geométrica não é função direta da tensão atuante no cabo, mas sim da força normal resultante, também no caso das membranas não é a intensidade das tensões de membrana a responsável direta pela rigidez geométrica obtida, mas sim as distribuições lineares de força, decorrentes da integração das tensões ao longo da espessura. Para os materiais atualmente disponíveis para a confecção de membranas e considerando as espessuras tipicamente disponíveis pode-se conseguir um nível adequado de rigidez para a 
membrana solicitando o material com tensões da ordem de $5 \%$ da tensão de ruptura (PAULETTI, 2003 apud LEWIS, 1998), de sorte que a imposição de um nível adequado de protensão (com as devidas folgas) não encontra grandes empecilhos, no que diz respeito ao nível de tensão.

Por outro lado, o vento pode induzir tensões consideravelmente mais altas (oito a dez vezes mais altas, conforme PAULETTI (2003 apud LEWIS, 1998), concentradas principalmente nos pontos de ancoragem da membrana. Esta característica é por vezes apontada como razão para o uso de altos fatores de segurança minorando a tensão de ruptura do material.

As razões para se trabalhar com altos coeficientes de segurança são diversas. Em primeiro lugar, além de ser o principal fator de indução de tensões na membrana, o vento é um carregamento aleatório, cujas características somente são conhecidas de modo estatístico, com grande margem de incerteza. Soma-se a isso o fato de as tensões de ruptura serem em geral determinadas por meio de ensaios uniaxiais de tiras de tecido novo, limpo e seco, enquanto que em testes biaxiais o mesmo material pode exibir tensões de ruptura até duas vezes menores (LEWIS, 1998). Ora, além de não se saber com precisão a efetiva distribuição e intensidade dos carregamentos vento e, portanto, das tensões dele decorrentes dificilmente se conhece, no estágio das análises de tensão, o efetivo arranjo das peças de tecido que compõe a membrana e muito menos a orientação da fiação em cada peça de tecido. Todas estas incertezas tornam contraproducentes os esforços para se trabalhar com menores fatores de segurança, por conta de custos de projeto crescentes.

\subsection{Materiais}

As estruturas de membrana possuem uma ampla gama de materiais construtivos, acerca dos quais apresenta-se aqui uma breve discussão, eminentemente qualitativa. Informações quantitativas devem, evidentemente, ser buscadas junto aos fabricantes e fornecedores específicos. 
As notas seguintes foram baseadas principalmente em PAULETTI (2003) apud BULSON (1973); HERZOG (1977); IRVINE (1981); OTTO (1982); FIRT (1983); LEONARD (1988); SALVADORI (1990); HAPPOLD (1994); MEEK (1994).

Há cinqüenta anos atrás, a pequena resistência e má durabilidade dos tecidos naturais limitavam o uso de membranas como estruturas. Foi somente com a invenção dos tecidos sintéticos que as membranas puderam florescer (PAULETTI, 2003 apud SALVADORI, 1990).

A aplicabilidade de um material é limitada por uma série de fatores entre eles:

- resistência mecânica;

- flexibilidade;

- trabalhabilidade;

- durabilidade;

- custo.

Dados os recentes avanços tecnológicos, atualmente a escolha do material está limitada à poucos tipos de tecido, entre eles:

- fibras de vidro ou poliéster recobertas com PVC;

- fibras de vidro ou carbono (Kevlar) recobertas com Teflon (PTFE);

- fibra de vidro ou carbono recobertas com silicone.

$\mathrm{Na}$ realidade brasileira, devido ao baixo custo, o tecido mais comumente utilizado é de fibras recobertas com PVC. Como grande desvantagem, este material deteriora-se sob a ação dos raios ultravioletas.

No Japão, as estruturas pneumáticas são classificadas em (PAULETTI, 2003 apud HAPPOLD, 1994):

- classe I: estruturas permanentes e semi-permanentes feitas de fibra de vidro com Teflon;

- classe II: estruturas manufaturadas de fibra de vidro com PVC; 
- classe III: estruturas de pequenos vãos, não permanentes, feitas com poliéster recoberto com PVC.

A fiação dos tecidos que constituem as membranas é geralmente lançada segundo duas direções: o urdume (termo amplamente utilizado em inglês warp) e a trama. Durante a fiação, os fios do urdume são mantidos retos, retesados, e os fios da trama são passados alternadamente por cima e por baixo de cada fio do urdume.

Quando o tecido é solicitado, a interação entre urdume e trama é acentuado, com a trama tendendo a retificar-se e o urdume a ondular-se. Deste comportamento resultam propriedades mecânicas anisotrópicas altamente não-lineares para a membrana.

Por este motivo, para evitar-se distorções excessivas da membrana, recomenda-se alinhar as direções da fiação com as tensões principais.

Atualmente, existe um esforço muito grande para o desenvolvimento de modelos numéricos capazes de reproduzir este comportamento das membranas. Simplificadamente, neste trabalho os materiais considerados nas aplicações foram adotados como isotrópicos. 


\subsection{Comportamento estrutural}

Segundo PAULETTI (2003), o comportamento das estruturas retesadas é sempre geometricamente não-linear. A importância da não-linearidade depende do tipo de estruturas e da fase de análise. Em geral, a busca da forma de estruturas de membrana será um procedimento não-linear.

Também a análise para o carregamento de projeto pode envolver a consideração da não-linearidade geométrica. Devido à baixa massa deste sistema estrutural, qualquer pequena mudança na carga, como a de vento e neve tem um largo impacto no tamanho dos elementos estruturais necessários e nos desvios experimentados. Conseqüentemente, a carga de estruturas de membrana deve ser cuidadosamente considerada. Por isso mais tempo e esforço devem ser gastos para definir casos de cargas.

Neste trabalho, serão utilizados elementos finitos disponíveis no software Ansys, mais especificamente o elemento SHELL41 para as aplicações em três dimensões. A formulação e características completas do elemento podem ser encontradas no guia do software.

Na Figura 3 apresenta-se a geometria do elemento SHELL 41:

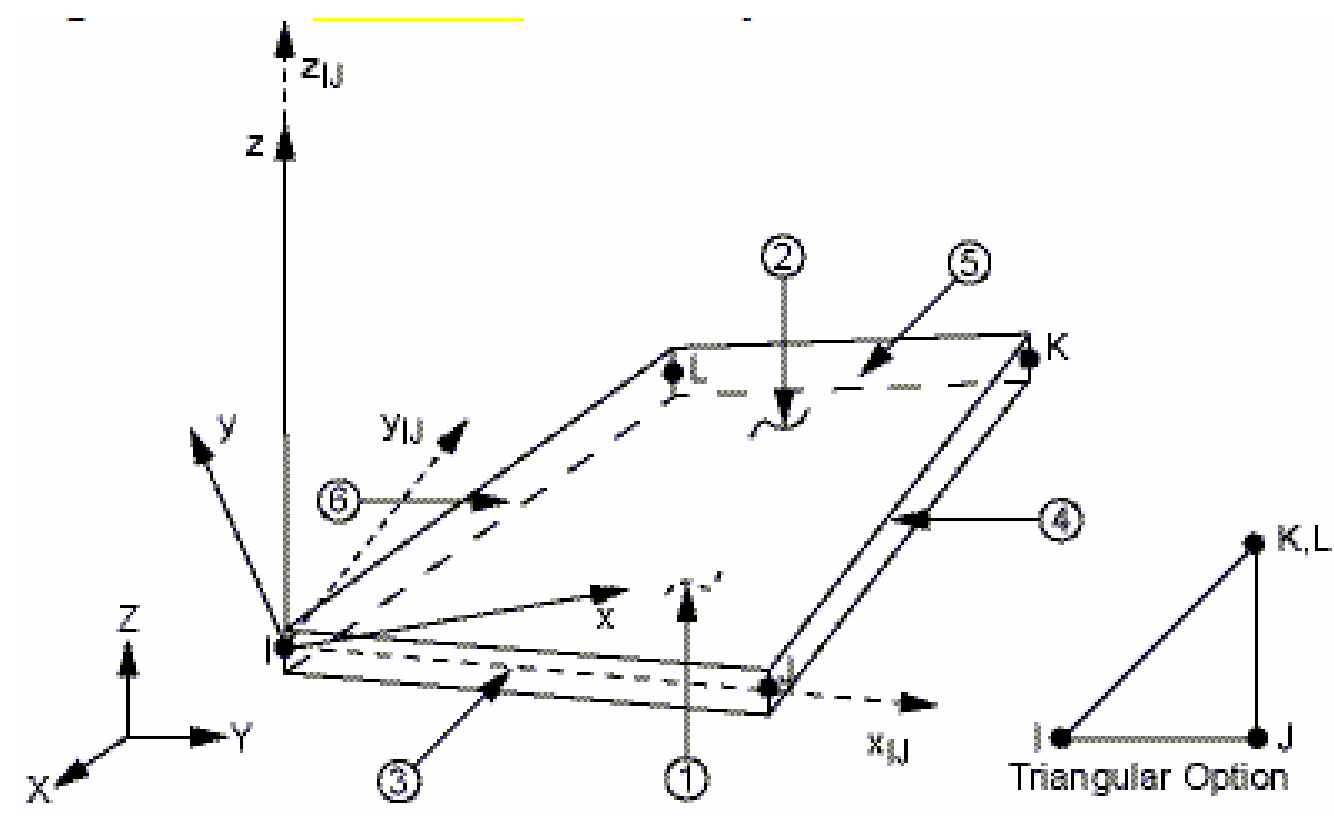

Figura 3 - Geometria do Elemento Finito Ansys SHELL 41 
O elemento é definido por quatro nós, quatro espessuras, um ângulo de direção do material e propriedades ortotrópicas do material. Admite-se que a espessura varia homogeneamente sobre a superfície do elemento.

As seguintes hipóteses e restrições foram considerados neste elemento:

- elementos de área nula não são permitidos;

- o elemento não pode ter espessura nula em nenhum nó;

- elementos triangulares têm nós K e L com mesmo número. 


\section{AÇÃO DO VENTO EM ESTRUTURAS}

A norma brasileira NBR 6123 (ABNT,1988), Forças Devidas ao Vento em Edificações, permite que as ações dinâmicas do vento que atuam em edifícios sejam consideradas como ações estáticas, bastando determinar a freqüência e conseqüentemente o período fundamental da edificação, dispensando a análise dinâmica propriamente dita se o mesmo for inferior a um segundo.

A análise estática prescrita na norma brasileira é realizada através da especificação de coeficientes aerodinâmicos para edifícios de formas geométricas variadas, porém simples como galpões, prismas retangulares, cúpulas, etc. A análise estática de edificações com forma geométrica mais complexa e a análise dinâmica de estruturas usualmente é realizadas através de ensaios com modelos reduzidos em túneis de vento.

A resposta dinâmica da estrutura à ação do vento depende não só de sua forma externa, mas também dos materiais empregados, do amortecimento e da rigidez da estrutura.

Edificações convencionais, determinadas aqui como aquelas estruturadas em concreto, aço ou alvenaria, em grande parte tem período fundamental menor que um segundo. Adicionalmente, tem rigidez elevada o bastante para serem consideradas, quase sempre, como estruturas de pequenos deslocamentos.

Em contrapartida, estruturas retesadas apresentam grandes deslocamentos quando submetidas a carregamentos, mudando de forma e, portanto, de rigidez. Neste sentido é evidente que a análise estática é pouco fiel ao comportamento real da estrutura.

\subsection{Caracterização do Vento}

A superfície terrestre, em contato com as massas de ar que se movimentam, provoca um efeito retardador sobre as camadas inferiores do escoamento atmosférico gerando uma variação vertical de velocidades do vento. A partir da altura onde o efeito do solo é desprezível, a velocidade média do vento permanece invariante, denominadas altura e velocidade gradiente. A altura gradiente varia conforme diferentes autores desde 300 até 
mais de $2000 \mathrm{~m}$ sobre o nível do solo. A região do ar compreendida entre a superfície terrestre e a altura gradiente denomina-se camada limite atmosférica.

$\mathrm{O}$ vento no interior da camada limite atmosférica constitui um escoamento turbulento cuja descrição realiza-se com as ferramentas da teoria de processos aleatórios. As cargas provocadas sobre as estruturas são relevantes quando há altas velocidades de vento. A agitação originada pela fricção superficial é superior em relação à provocada por gradientes térmicos atmosféricos, destruindo desta maneira o processo de convecção. As condições atmosféricas nos ventos fortes são de gradiente térmico vertical adiabático e estabilidade neutra. Sob estas condições, a turbulência contida no escoamento médio do ar é causada pelo movimento da massa de ar sobre rugosidades superficiais (turbulência mecânica) e sua característica depende da forma, dimensão e distribuição de obstáculos superficiais naturais e artificiais.

O modelo físico do vento de alta velocidade proposto pelos códigos de vento é composto pelo escoamento médio de direção horizontal provocado por um sistema de pressão em grande escala ao qual se sobrepõem flutuações geradas pela rugosidade superficial local. É de esperar que se a superfície rugosa permanecesse razoavelmente constante por alguns quilômetros a barlavento da estação de medição, produzir-se-ia um equilíbrio dinâmico entre o aumento uniforme da velocidade com a altura até a velocidade gradiente, com o déficit de energia cinética representado pela soma da energia cinética transferida do escoamento médio às flutuações de velocidade e o trabalho realizado pelo escoamento ao movimentar-se sobre as rugosidades superficiais.

O equilíbrio dinâmico entre as massas de ar produz perfis verticais de velocidades que permitem caracterizar os escoamentos médios com parâmetros que dependem das características da rugosidade superficial e descrever também o comportamento espacial da turbulência.

Embora exista abundante bibliografia sobre a estrutura do escoamento médio e flutuante do vento atmosférico, definem-se hipóteses e conceitos básicos utilizados na Engenharia do Vento que se utilizam neste trabalho.

BLESSMANN (1998) denomina tormenta os sistemas meteorológicos que provocam ventos em alta velocidade (fortes), independente de qual seja o mecanismo de formação. Em caso de tormentas de origem extra-tropicais ou simplesmente tormentas EPS 
("extended mature pressure systems") e com ventos de alta velocidade, a rugosidade superficial gera turbulências de tal intensidade que a mistura do ar entre as camadas adjacentes impede o processo de convecção. O gradiente térmico vertical é considerado adiabático e a estabilidade neutra.

Desta forma, as flutuações são originadas pela agitação mecânica do ar, formando redemoinhos de dimensões diversas. Os ciclones extra-tropicais, considerados na Engenharia do Vento como ventos bem comportados, geram ventos fortes em equilíbrio dinâmico com a rugosidade superficial. Estes são os ventos mais estudados e servem como base aos códigos de vento.

Em outras tormentas as flutuações de vento são principalmente de origem térmica (tormentas elétricas ou TS, tornados entre outras). Isto impede a aplicação do conceito de vento em equilíbrio dinâmico com a rugosidade da superfície terrestre, devido à reduzida extensão superficial horizontal que abarca estes fenômenos meteorológicos.

\subsection{Ação do Vento Segundo a NBR 6123}

\subsubsection{Velocidade Básica}

A velocidade básica do vento $\mathrm{V}_{0}$, adequada ao local onde a estrutura se localiza é determinada através das isopletas de velocidades básica, as quais foram elaboradas a partir dos registros de diversas estações meteorológicas: 


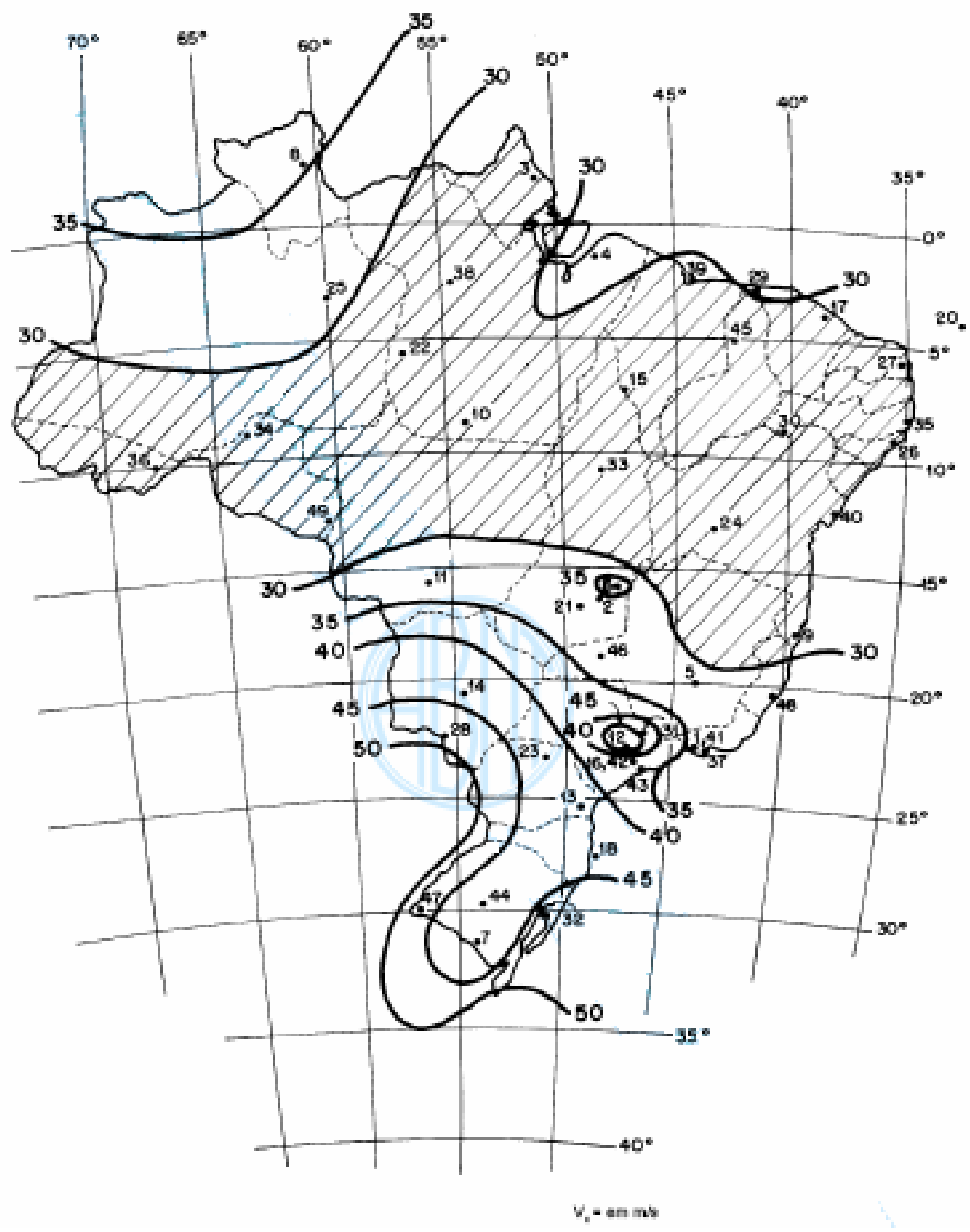

Figura 4 - Isopletas de Velocidade Básica do Vento $\left(\mathrm{V}_{0}\right)$

Fonte: digitalização da norma NBR 6123:1988 
Estas velocidades são definidas como a velocidade de uma rajada de 3 segundos, exercida em média uma vez em 50 anos, a 10 metros acima do solo em campo aberto e plano. Como regra geral é admitido que o vento básico possa soprar de qualquer direção horizontal.

\subsubsection{Velocidade Característica e Velocidade de Projeto}

A velocidade característica do vento $V_{k}$ é a utilizada no cálculo da pressão dinâmica nas estruturas, obtida a partir da velocidade básica corrigida por fatores de ajuste, segundo a apresentada pela NBR-6123 (1988):

$$
\mathrm{V}_{\mathrm{k}}=\mathrm{V}_{0} \mathrm{~S}_{1} \mathrm{~S}_{2} \mathrm{~S}_{3}
$$

A velocidade de projeto $\mathrm{V}_{\mathrm{p}}$, corresponde à velocidade média em $10 \mathrm{~min}$ a $10 \mathrm{~m}$ de altura sobre o solo, em terreno aberto e em nível ou aproximadamente em nível, com poucos obstáculos isolados, tais como árvores e edificações baixas:

$$
\mathrm{V}_{\mathrm{p}}=0,69 \mathrm{~V}_{0} \mathrm{~S}_{1} \mathrm{~S}_{3}
$$

onde $V_{0}$ é a velocidade básica do vento e $S_{1}, S_{2}$ e $S_{3}$ os coeficientes de ajuste da velocidade básica, os quais serão nos itens 5.2.3 a 5.2.5. O fator multiplicador 0,69 é uma adequação do tempo de exposição da velocidade básica para 10 minutos.

Como citado anteriormente, os valores de velocidade básica são obtidos das isopletas da NBR-6123 (1988) e os coeficientes de ajuste têm como finalidade adequar a velocidade básica às particularidades do local da edificação, suas dimensões e grau de segurança desejado.

\subsubsection{Fator Topográfico $\left(S_{1}\right)$}

$O$ fator $S_{1}$ leva em consideração a influência da topografia local na variação da velocidade do vento. Como primeira aproximação, a NBR-6123 (1988) sugere os valores indicados no seu item 5.2, ressaltando que estes devem ser usados com precaução. Caso seja necessário um conhecimento mais preciso da influência do relevo ou se a complexidade deste tornar difícil a aplicação dos valores sugeridos, deve-se proceder a 
ensaios de modelos topográficos em túnel de vento ou a medidas anemométricas no próprio terreno.

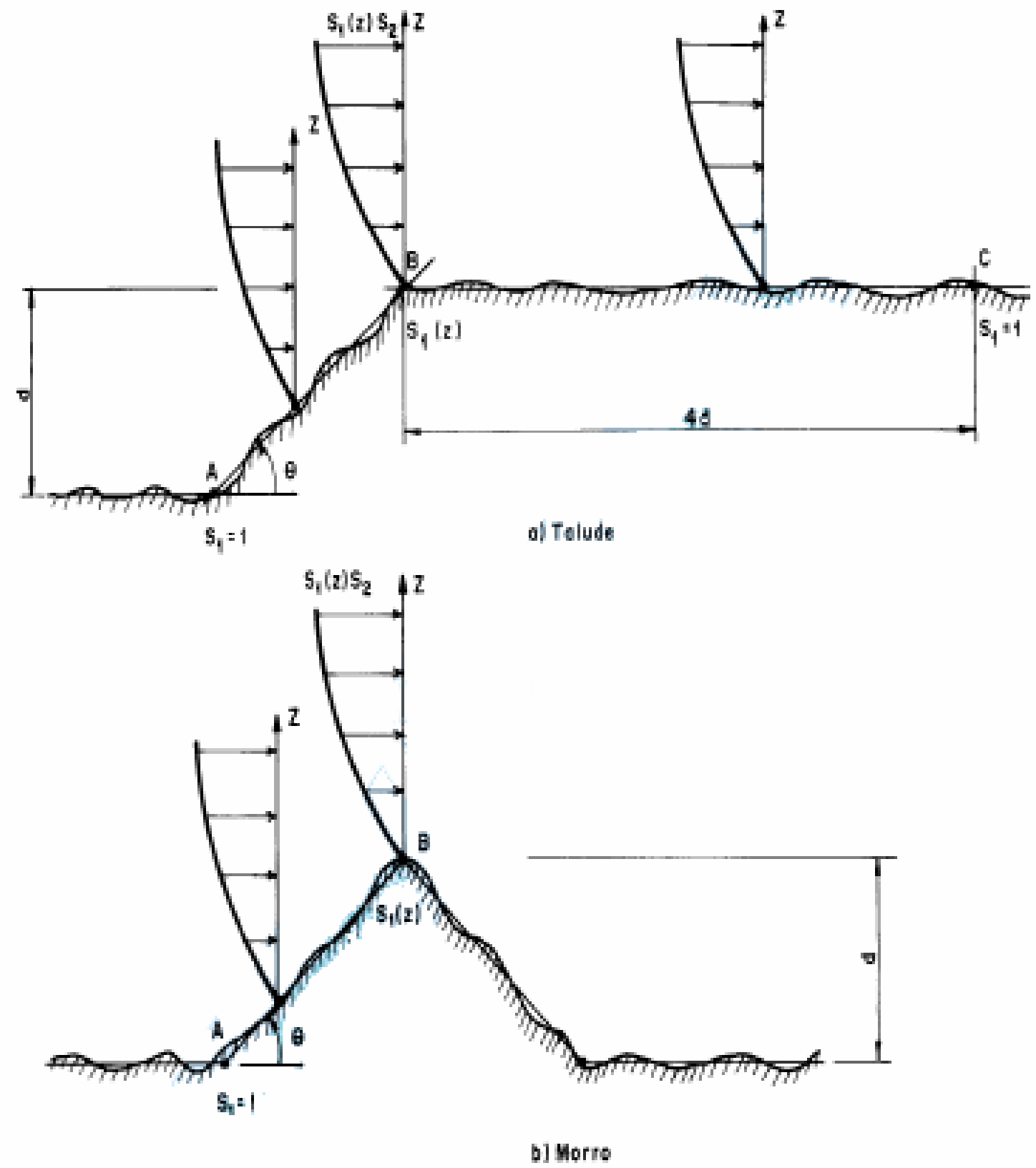

Figura 5 - Fator Topográfico S1 (z)

Fonte: digitalização da norma NBR 6123:1988 


\subsubsection{Fator de Rugosidade $\left(S_{2}\right)$}

De acordo com a NBR-6123 (1988), o fator $\mathrm{S}_{2}$ considera o efeito combinado da rugosidade do terreno, da variação da velocidade do vento com a altura acima do terreno e das dimensões da edificação ou parte desta. A altura considerada é tomada a partir do nível do terreno ao ponto desejado. Entretanto, pode-se dividir a altura da edificação em trechos, determinando-se $\mathrm{S}_{2}$ com base na altura medida do terreno à cota do topo de cada trecho.

A rugosidade do terreno é classificada em cinco categorias e quanto às dimensões da edificação a norma divide as edificações e suas partes em três classes, com intervalos de tempo para o cálculo da velocidade média de 3,5 e 10 segundos e dimensões máximas, vertical ou horizontal, de 20,50 e $80 \mathrm{~m}$. Nas edificações em que a maior dimensão ultrapasse $80 \mathrm{~m}$, o intervalo de tempo é obtido das instruções fornecidas no Anexo A da referida norma. A norma considera ainda, no seu item 5.5, os casos de transição de rugosidade:

- Categoria I

Superfícies lisas de grandes dimensões, com mais $5 \mathrm{~km}$ de extensão, medida na direção e sentido do vento incidente:

- mar calmo;

- lagos e rios;

- pântanos sem vegetação.

- Categoria II

Terrenos abertos em nível ou aproximadamente em nível, com poucos obstáculos isolados, tais como árvores e edificações baixas:

- zonas costeiras planas;

- pântanos com vegetação rala;

- campos de aviação;

- pradarias e charnecas; 
- fazendas sem sebes ou muros.

- Categoria III

Terrenos planos ou ondulados com obstáculos, tais como sebes e muros, poucos quebra-ventos de árvores, edificações baixas e esparsas.

- granjas e casas de campo, com exceção das partes com matos;

- fazenda com sebes e/ou muros;

- subúrbios a considerável distância do centro, com casas e esparsas.

- Categoria IV

Terrenos cobertos por obstáculos numerosos e pouco espaçados, em zona florestal, industrial ou urbanizada.

- zonas de parques e bosques com muitas árvores;

- cidades pequenas e seus arredores;

- subúrbios densamente construídos de grandes cidades.

- Categoria V

Terrenos cobertos por obstáculos numerosos, grandes, altos e pouco espaçados.

- florestas com árvores altas, de copas isoladas;

- centros de grandes cidades;

- complexos industriais bem desenvolvidos.

- Classe A

Todas as unidades de vedação, seus elementos de fixação e peças individuais de estruturas sem vedação. Toda edificação na qual a maior dimensão horizontal ou vertical não exceda $20 \mathrm{~m}$. 
- Classe B

Toda edificação ou parte de edificação para a qual a maior dimensão horizontal ou vertical da superfície frontal esteja entre 20 e $50 \mathrm{~m}$.

- Classe C

Toda edificação ou parte de edificação para a qual a maior dimensão horizontal ou vertical da superfície frontal exceda $50 \mathrm{~m}$.

$\mathrm{O}$ fator $\mathrm{S}_{2}$ pode ser obtido a partir da equação5.3, que é aplicável até a altura $\mathrm{z}_{\mathrm{g}}$, a qual define o contorno superior da camada atmosférica. Os valores de S2 para as diversas categorias de rugosidade do terreno e classe de dimensões das edificações são encontrados na Tabela 5.4 da NBR-6123 (1988), sendo permitida a interpolação linear entre os valores apresentados.

$$
S_{2}=b F_{r}\left(\frac{z}{10}\right)^{p}
$$

Sendo b o parâmetro meteorológico, $\mathrm{p}$ o expoente da lei potencial de variação de $\mathrm{S}_{2}, \mathrm{~F}_{\mathrm{r}} \mathrm{o}$ fator de rajada, o qual sempre corresponde à categoria II e $\mathrm{z}$ a altura acima do nível geral do terreno.

Tabela 1 - Parâmetros para cálculo do Fator de Rugosidade $\left(\mathrm{S}_{2}\right)$

Valores de b para Classes $A, B, C$

\begin{tabular}{|l|c|c|c|c|}
\hline Categoria & $\mathrm{zg}(\mathrm{m})$ & $\mathrm{A}$ & $\mathrm{B}$ & $\mathrm{C}$ \\
\hline 1 & 250 & 1,1 & 1,11 & 1,12 \\
\hline 2 & 300 & 1 & 1 & 1 \\
\hline 3 & 350 & 0,94 & 0,94 & 0,93 \\
\hline 4 & 420 & 0,86 & 0,85 & 0,84 \\
\hline 5 & 500 & 0,74 & 0,73 & 0,71 \\
\hline
\end{tabular}

Valores de $p$ para Classes $A, B, C$

\begin{tabular}{|l|c|c|c|c|}
\hline Categoria & $\mathrm{zg}(\mathrm{m})$ & $\mathrm{A}$ & $\mathrm{B}$ & $\mathrm{C}$ \\
\hline 1 & 250 & 0,06 & 0,065 & 0,07 \\
\hline 2 & 300 & 0,085 & 0,09 & 0,1 \\
\hline 3 & 350 & 0,1 & 0,105 & 0,115 \\
\hline 4 & 420 & 0,12 & 0,125 & 0,135 \\
\hline 5 & 500 & 0,15 & 0,16 & 0,175 \\
\hline
\end{tabular}

Valores de Fr para Classes A,B,C

\begin{tabular}{|l|c|c|c|c|}
\hline Categoria & & A & B & C \\
\hline Fr & & 1 & 0,98 & 0,95 \\
\hline
\end{tabular}




\subsubsection{Fator Estatístico $\left(S_{3}\right)$}

O fator S3 é baseado em conceitos estatísticos e considera o grau de segurança requerido e a vida útil da edificação. O grau de segurança necessário é função da finalidade da edificação, que é classificada pela norma em cinco grupos dentro desse critério. No caso de edificações normais destinadas a moradias, hotéis, etc (grupo 2), adota-se o nível de probabilidade de $63 \%$ e uma vida útil de 50 anos. Na falta de uma norma específica sobre segurança nas edificações ou de indicações correspondentes na norma estrutural, os valores mínimos do fator $\mathrm{S}_{3}$ são indicados na Tabela 5.5 da norma. Pode-se obter ainda, $\mathrm{S}_{3}$ para outros níveis de probabilidade e vida útil a partir da Equação 5.4:

$$
S_{3}=0,54\left[-\frac{\ln \left(1-P_{m}\right)}{m}\right]^{-0,157}
$$

sendo $\mathrm{P}_{\mathrm{m}}$ a probabilidade de ocorrência e $\mathrm{m}$ o período de retorno ou tempo de recorrência.

\section{- Grupo 1}

Edificação cuja ruína total ou parcial pode afetar a segurança ou possibilidade de socorro a pessoas após uma tempestade destrutiva (hospitais, quartéis de bombeiros e de forças de segurança, centrais de comunicações, etc).

$$
\mathrm{S}_{3, \min }=1,10
$$

\section{- Grupo 2}

Edificações para hotéis e residências. Edificações para comércio e indústria com alto fator de ocupação.

$$
\mathrm{S}_{3, \min }=1,00
$$


- Grupo 3

Edificações e instalações industriais com baixo fator de ocupação (depósitos, silos, construções rurais, etc).

$\mathrm{S}_{3, \min }=0,95$

- Grupo 4

Vedações (telhas, vidros, painéis de vedação, etc).

$\mathrm{S}_{3, \min }=0,88$

- Grupo 5

Edificações temporárias. Estruturas dos grupos 1 a 3 durante a construção.

$\mathrm{S}_{3, \min }=0,83$

\subsubsection{Coeficientes aerodinâmicos}

A NBR 6123:1988 especifica coeficientes aerodinâmicos dependente da forma geométrica da estrutura, incluindo sua permeabilidade. Estes coeficientes são então multiplicados pela pressão dinâmica do vento para se obter o carregamento de vento na estrutura.

A pressão dinâmica do vento corresponde à velocidade característica $\mathrm{Vk}$ em condições normais de pressão $(1 \mathrm{~atm}=1013,2 \mathrm{mbar}=101320 \mathrm{~Pa})$ e de temperatura $\left(15^{\circ} \mathrm{C}\right)$, e é dada por:

$$
\mathrm{q}=0,613 \mathrm{~V}_{\mathrm{k}}^{2} \quad\left(\mathrm{q}: \mathrm{N} / \mathrm{m} ; \mathrm{V}_{\mathrm{k}}: \mathrm{m} / \mathrm{s}\right) \quad \text { Eq. } 05.5
$$




\subsection{Pesquisa sobre velocidades básicas do Vento}

Motivado pela cada vez mais freqüente ocorrência de catástrofes climáticas, como os ciclones tropicais ocorridos na região Sul do país (vide Figura 6), foi realizada pesquisa em diversos institutos meteorológicos do país sobre medições de rajadas de vento nas condições estabelecidas pela NBR 6123.

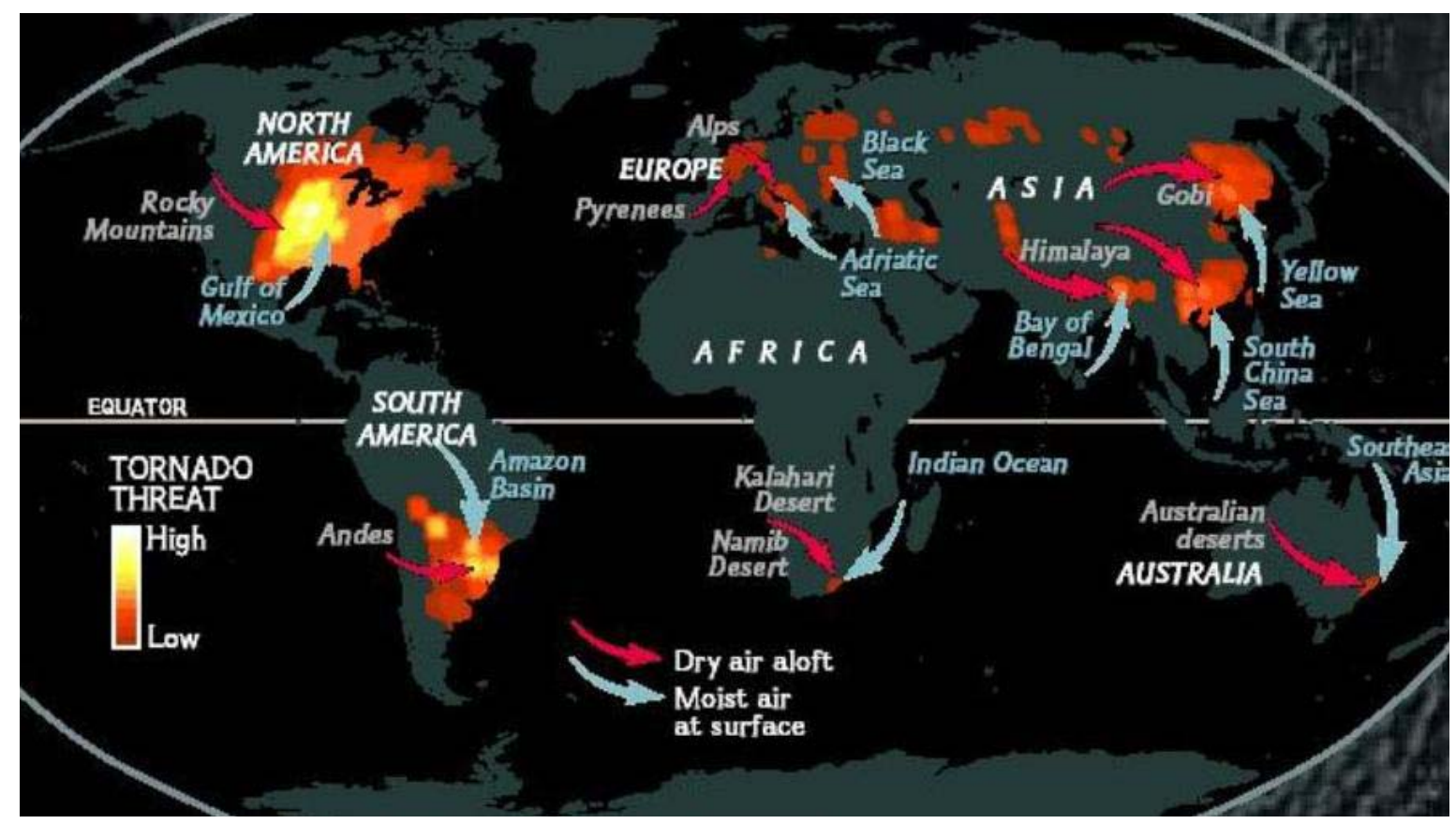

Figura 6 - Mapa mundi de ameaças de tornado

Fonte: http://amora.cap.ufrgs.br/2000/projetos/proj1/torn/Ciclones.htm e http://www.accuweather.com/

Dentre todas as estações meteorológicas pesquisadas, os dados de muitas foram eliminadas por não satisfazerem as condições estabelecidas no capítulo 5.2.1 ou por não terem amostragem suficiente, principalmente nos últimos cinco anos.

Neste sentido, com colaboração do Instituto Nacional de Pesquisas Espaciais (INPE), através do Sistema Nacional de Dados Ambientais (SINDA), foram obtidos com sucesso dados da Estação Meteorológica São Martinho da Serra, localizada no Rio Grande do Sul.

$\mathrm{Na}$ Figura 7 apresentam-se as isopletas fornecidas pela Norma NBR 6123 nas proximidades de São Martinho da Serra, município representado pelo ponto vermelho: 


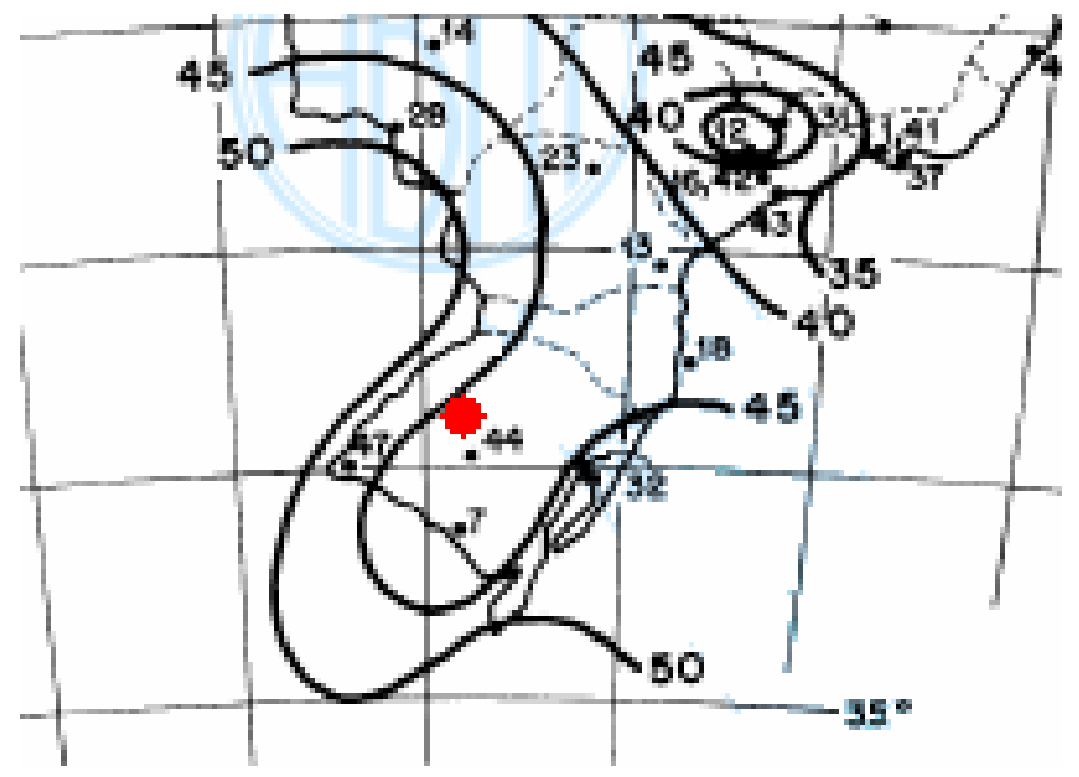

Figura 7 - Isopletas Ampliadas na Região de São Martinho da Serra / RS

Pode-se observar que a velocidades básica prevista em norma para São Martinho da Serra é cerca de $45 \mathrm{~m} / \mathrm{s}$.

Na Tabela 2 resumem-se os valores obtidos na Estação Meteorológica:

\begin{tabular}{|c|c|c|c|c|}
\hline ANO & $\begin{array}{c}\mathrm{N}^{\mathrm{o}} \\
\text { Ocorrências } \\
\mathrm{V}_{0}>45 \mathrm{~m} / \mathrm{s}\end{array}$ & $\begin{array}{c}\mathrm{N}^{\mathrm{o}} \text { Ocorrências } \\
\mathrm{V}_{0}>48 \mathrm{~m} / \mathrm{s}\end{array}$ & $\begin{array}{c}\mathrm{N}^{\circ} \text { Ocorrências } \\
\mathrm{V}_{0} \geq 50 \mathrm{~m} / \mathrm{s}\end{array}$ & $\mathrm{V}_{0}$ máx (m/s) \\
\hline 2010 & 3 & 1 & 1 & 50,2 \\
\hline 2009 & 7 & 5 & 3 & 49,2 \\
\hline 2008 & 12 & 6 & 3 & 51,1 \\
\hline 2007 & 11 & 0 & 0 & 47,7 \\
\hline 2006 & 2 & 0 & 0 & 47,7 \\
\hline
\end{tabular}

Tabela 2 - Ocorrência de Ventos em São Martinho da Serra de 2005 a 2010

Levando em conta as isopletas de norma, em Martinho da Serra uma rajada de vento superior a $45 \mathrm{~m} / \mathrm{s}$ deveria ocorrer em uma oportunidade a cada 50 anos, entretanto registraram-se 38 ocasiões com ventos superiores nos últimos 6 anos. 
Observando-se pontualmente, sem conseguir dados de um conjunto maior de estações, tais dados sugerem que a norma NBR 6123 pode ter seu mapa de isopletas defasado ou ainda que a interpolação entre dados de estações distintas tenha levado a alguma condição desfavorável para esta região.

Continuando a pesquisa, foi realizado um levantamento da quantidade de estações utilizadas para elaborar o mapa de isopletas de alguns códigos nacionais, chegando-se ao seguinte resultado:

\begin{tabular}{|l|c|c|c|}
\hline $\begin{array}{l}\text { UNIDADE } \\
\text { TERRITORIAL }\end{array}$ & EXTENSÃO (km $\left.{ }^{2}\right)$ & $\begin{array}{c}\text { ESTAÇÕES } \\
\text { CONSIDERADAS }\end{array}$ & $\begin{array}{c}\text { DENSIDADE } \\
(\mathbf{k m} \text { / } \mathbf{u n})\end{array}$ \\
\hline África do Sul & 1.221 .037 & 14 & 87.217 \\
\hline Argentina & 2.780 .400 & 29 & 95.876 \\
\hline & & & 173.773 \\
\hline Brasil & 8.514 .876 & 49 & 72.152 \\
\hline Sul & 577.214 & 8 & 84.299 \\
\hline Sudeste & 927.286 & 11 & 268.680 \\
\hline Centro-Oeste & 1.612 .077 & 6 & 351.785 \\
\hline Norte & 3.869 .637 & 11 & 120.090 \\
\hline Nordeste & 1.561 .177 & 13 & \\
\hline
\end{tabular}

Tabela 3 - Estações consideradas no código nacional de ação do vento em estruturas

Observa-se acima que a densidade média de estações no Brasil é muito baixa quando comparada a outros países como África do Sul e Argentina. Entretanto, tal densidade é crítica nas regiões Centro-Oeste, Norte e Nordeste, onde a velocidade básica do vento é menor. A densidade de estações nas Regiões Sul e Sudeste, onde os ventos são mais velozes, é compatível.

Tendo como exemplo o modelo Europeu, onde os códigos foram unificados criando-se um único mapa com isopletas para diversos países, sugere-se uma discussão na próxima atualização da norma levando em conta a contribuição de medições realizadas em países vizinhos para melhorar as isopletas brasileiras, principalmente nas regiões de fronteira.

Outro ponto de discussão interessante envolve ao sistema de medição. No caso supracitado não foi possível determinar a origem das flutuações do vento: térmica ou por agitações mecânicas. 
Levando-se em conta estes resultados, as aplicações com estruturas de membrana serão elaboradas com velocidade básica do vento de $50 \mathrm{~m} / \mathrm{s}$.

\section{FLUIDO-DINÂMICA}

\subsection{Análise computacional}

O problema de escoamento de fluidos é definido pelas leis de conservação da massa, momento e energia. Estas leis são expressas por meio de equações diferenciais que, conforme já mencionado, neste trabalho serão discretizadas com técnica baseada em elementos finitos através do Software Ansys versão 11.

As hipóteses básicas a serem adotadas na modelagem são:

- existe somente uma fase;

- escoamento laminar ou turbulento;

- fluido compressível ou incompressível.

Segundo MANFRIM (2006), até uma velocidade de $300 \mathrm{~km} / \mathrm{h}(83,3 \mathrm{~m} / \mathrm{s})$, o vento pode ser considerado como um fluido incompressível. Considerando que as rajadas máximas observadas no Brasil são consideravelmente menores que este valor, as formulações de fluido-dinâmica serão apresentadas para fluidos incompressíveis.

\subsubsection{Lei da Conservação da Massa}

Num certo intervalo de tempo, a massa de um fluido que entra em volume é igual à massa que sai mais a variação da massa contida no elemento.

Considerando-se um volume finito, no qual escoa um fluido entre as seções S1 e S2 num intervalo de tempo dt, tem-se:

$$
\rho_{1}\left(A_{1} v_{1} d t_{1}\right)=\rho_{2}\left(A_{2} v_{2} d t_{2}\right)
$$


onde:

$\mathrm{A}=$ área de uma superfície plana;

$\mathrm{v}=$ velocidade média do fluido;

$\rho=$ massa específica do fluido.

Quando as partículas de um fluido têm a mesma velocidade num mesmo ponto em uma trajetória independente do tempo, este é dito permanente.

Assim, para um fluido incompressível em regime permanente, simplifica-se a equação acima para:

$$
A_{1} v_{1}=A_{2} v_{2}
$$

\subsubsection{Equação da Continuidade}

Da lei de conservação da massa, facilmente obtém-se a equação da continuidade:

$$
\frac{\partial \rho}{\partial t}+\frac{\partial\left(\rho v_{x}\right)}{\partial x}+\frac{\partial\left(\rho v_{y}\right)}{\partial y}+\frac{\partial\left(\rho v_{z}\right)}{\partial z}=0
$$

onde:

$\mathrm{v}_{\mathrm{x}}, \mathrm{v}_{\mathrm{y}}$ e $\mathrm{v}_{\mathrm{z}}=$ componentes do vetor velocidade nas direções $\mathrm{x}, \mathrm{y}$ e $\mathrm{z}$, respectivamente;

$\rho=$ densidade;

$\mathrm{x}, \mathrm{y}, \mathrm{z}=$ coordenadas Cartesianas globais;

$\mathrm{t}=$ tempo.

A taxa de variação da densidade pode ser substituída pela taxa de variação de pressão usando a seguinte correlação:

$$
\frac{\partial \rho}{\partial t}=\frac{\partial \rho}{\partial P} \frac{\partial P}{\partial t}
$$

onde: $\mathrm{P}=$ pressão.

A solução para a derivada parcial da densidade com relação à pressão é dada pela equação de equilíbrio. 
Se o fluído é compressível, um gás ideal é assumido e a solução é dada por:

$$
\rho=\frac{\rho}{R T} \Rightarrow \frac{\partial \rho}{\partial \rho}=\frac{1}{R T}
$$

onde: $\quad \mathrm{R}=$ constante do gás;

$$
\mathrm{T}=\text { temperatura. }
$$

Se o fluido for incompressível, a solução é dada por:

$$
\frac{d \rho}{d P}=\frac{1}{\beta}
$$

onde: $\quad \beta=$ módulo de "bulk".

O valor padrão de 1015 para $\beta$ implica que para um fluido perfeitamente incompressível, ondas de pressão irão percorrer infinitamente rápidas pelo domínio inteiro do problema. Por exemplo: uma queda no escoamento de massa será observada imediatamente.

\subsubsection{Lei da Conservação dos Momentos}

Em um fluido Newtoniano, a relação entre tensão e taxa de deformação é dada por:

$$
\tau_{i j}=-P \delta_{i j}+\mu\left(\frac{\partial u_{i}}{\partial x_{j}}+\frac{\partial u_{j}}{\partial x_{i}}\right)+\delta_{i j} \lambda \frac{\partial u_{i}}{\partial x_{i}}
$$

onde: $\tau_{\mathrm{ij}}=$ tensor das tensões;

$\mathrm{u}_{\mathrm{i}}=$ velocidades $\left(\mathrm{u}_{1}=\mathrm{v}_{\mathrm{x}}, \mathrm{u}_{2}=\mathrm{v}_{\mathrm{y}}, \mathrm{u}_{3}=\mathrm{v}_{\mathrm{z}}\right)$;

$\mu=$ viscosidade dinâmica;

$\lambda=$ segundo coeficiente da viscosidade. 
O último membro da equação, produto do segundo coeficiente da viscosidade e o divergente da velocidade, é nulo para um fluido de densidade constante e é tão pequeno em fluidos compressíveis que pode ser desprezado.

Esta última equação transforma as equações de momentos nas equações de Navier-Stokes; entretanto, estas ainda serão denominadas equações de momentos em todo este texto.

As equações de momento, sem outras hipóteses que não as propriedades do fluido, são as seguintes:

$$
\begin{aligned}
& \frac{\partial \rho v_{x}}{\partial t}+\frac{\partial\left(\rho v_{x} v_{x}\right)}{\partial x}+\frac{\partial\left(\rho v_{y} v_{x}\right)+\frac{\partial\left(\rho v_{z} v_{x}\right)}{\partial y}=\rho g_{x}-\frac{\partial P}{\partial x}}{\partial z}+R_{x}+\frac{\partial}{\partial x}\left(\mu_{e} \frac{\partial v_{x}}{\partial x}\right)+\frac{\partial}{\partial y}\left(\mu_{e} \frac{\partial v_{x}}{\partial y}\right)+\frac{\partial}{\partial z}\left(\mu_{e} \frac{\partial v_{x}}{\partial z}\right)+\mathrm{T}_{x} \\
& \frac{\partial \rho v_{y}}{\partial t}+\frac{\partial\left(\rho v_{x} v_{y}\right)}{\partial x}+\frac{\partial\left(\rho v_{y} v_{y}\right)}{\partial y}+\frac{\partial\left(\rho v_{z} v_{y}\right)}{\partial z}=\rho g_{y}-\frac{\partial P}{\partial y} \\
& +R_{y}+\frac{\partial}{\partial x}\left(\mu_{e} \frac{\partial v_{y}}{\partial x}\right)+\frac{\partial}{\partial y}\left(\mu_{e} \frac{\partial v_{y}}{\partial y}\right)+\frac{\partial}{\partial z}\left(\mu_{e} \frac{\partial v_{y}}{\partial z}\right)+\mathrm{T}_{y} \\
& \frac{\partial \rho v_{z}}{\partial t}+\frac{\partial\left(\rho v_{x} v_{z}\right)}{\partial x}+\frac{\partial\left(\rho v_{y} v_{z}\right)}{\partial y}+\frac{\partial\left(\rho v_{z} v_{z}\right)}{\partial z}=\rho g_{z}-\frac{\partial P}{\partial z} \\
& +R_{z}+\frac{\partial}{\partial x}\left(\mu_{e} \frac{\partial v_{z}}{\partial x}\right)+\frac{\partial}{\partial y}\left(\mu_{e} \frac{\partial v_{z}}{\partial y}\right)+\frac{\partial}{\partial z}\left(\mu_{e} \frac{\partial v_{z}}{\partial z}\right)+\mathrm{T}_{z}
\end{aligned}
$$

onde: $g_{x}, g_{y}, g_{z}=$ componentes da aceleração devido a gravidade;

$\rho=$ densidade;

$\mu_{\mathrm{e}}=$ viscosidade efetiva;

$\mathrm{R}_{\mathrm{x}}, \mathrm{R}_{\mathrm{y}}, \mathrm{R}_{\mathrm{z}}=$ resistências distribuídas;

$\mathrm{T}_{\mathrm{x}}, \mathrm{T}_{\mathrm{y}}, \mathrm{T}_{\mathrm{z}}=$ termos de perda de viscosidade. 
Para um escoamento laminar, a viscosidade efetiva é simplesmente a viscosidade dinâmica, uma propriedade do fluido. Para um escoamento turbulento esta propriedade será discutida em um capítulo à parte.

Resistências distribuídas podem ser usadas para criar o efeito de algum obstáculo sem modelar sua geometria, como por exemplo um escoamento passando por telas ou poros.

Os termos de perda de viscosidade são eliminados no caso de fluidos incompressíveis. A ordem da derivada é trocada em cada termo, reduzindo o termo para uma derivada da equação da continuidade, que é nula:

$$
\begin{aligned}
& \mathrm{T}_{x}=\frac{\partial}{\partial x}\left(\mu \frac{\partial v_{x}}{\partial x}\right)+\frac{\partial}{\partial y}\left(\mu \frac{\partial v_{y}}{\partial x}\right)+\frac{\partial}{\partial z}\left(\mu \frac{\partial v_{z}}{\partial x}\right) \\
& \mathrm{T}_{y}=\frac{\partial}{\partial x}\left(\mu \frac{\partial v_{x}}{\partial y}\right)+\frac{\partial}{\partial y}\left(\mu \frac{\partial v_{y}}{\partial y}\right)+\frac{\partial}{\partial z}\left(\mu \frac{\partial v_{z}}{\partial y}\right) \\
& \mathrm{T}_{z}=\frac{\partial}{\partial x}\left(\mu \frac{\partial v_{x}}{\partial z}\right)+\frac{\partial}{\partial y}\left(\mu \frac{\partial v_{y}}{\partial z}\right)+\frac{\partial}{\partial z}\left(\mu \frac{\partial v_{z}}{\partial z}\right)
\end{aligned}
$$

A conservação da energia pode ser expressa em termos da temperatura (total) de estagnação, sempre útil em fluidos altamente compressíveis, ou da temperatura estática, apropriada para análises de fluidos incompressíveis em baixa velocidade.

\subsubsection{Equação da Energia Compressivel}

A equação completa da energia é solucionada para fluidos compressíveis através da transferência de calor.

Em termos da temperatura total (ou de estagnação), a equação da energia é:

$$
\begin{aligned}
& \frac{\partial}{\partial t}\left(\rho C_{p} \mathrm{~T}_{0}\right)+\frac{\partial}{\partial x}\left(\rho v_{x} C_{p} \mathrm{~T}_{0}\right)+\frac{\partial}{\partial y}\left(\rho v_{y} C_{p} \mathrm{~T}_{0}\right)+\frac{\partial}{\partial z}\left(\rho v_{z} C_{p} \mathrm{~T}_{0}\right)= \\
& \frac{\partial}{\partial x}\left(\mathrm{~K} \frac{\partial \mathrm{T}_{0}}{\partial x}\right)+\frac{\partial}{\partial y}\left(\mathrm{~K} \frac{\partial \mathrm{T}_{0}}{\partial y}\right)+\frac{\partial}{\partial z}\left(\mathrm{~K} \frac{\partial \mathrm{T}_{0}}{\partial z}\right)+W^{v}+E^{k}+Q_{v}+\Phi+\frac{\partial P}{\partial t}
\end{aligned}
$$

onde: $\mathrm{C}_{\mathrm{p}}=$ calor específico;

$\mathrm{T}_{\mathrm{o}}=$ temperatura total (ou de estagnação); 
$\mathrm{K}=$ condutividade térmica;

$\mathrm{W}^{\mathrm{v}}=$ termo de trabalho viscoso;

$\mathrm{Q}_{\mathrm{v}}=$ fonte de calor volumétrico;

$\Phi=$ termo de dissipação viscosa (geração de calor);

$\mathrm{E}^{\mathrm{k}}=$ energia cinética.

A temperatura estática é calculada através da temperatura total e da energia cinética:

$$
\mathrm{T}=\mathrm{T}_{0}-\frac{v^{2}}{2 C_{p}}
$$

onde: $\quad \mathrm{T}=$ temperatura estática;

$$
\mathrm{v}=\text { norma do vetor velocidade do fluido. }
$$

Nos nós em que não se tem material fluido, as temperaturas estática e total são as mesmas.

O trabalho viscoso é dado por:

$$
W^{v}=u_{j} \mu\left[\frac{\partial}{\partial x_{j}} \frac{\partial u_{j}}{\partial x_{j}}+\frac{\partial}{\partial x_{k}} \frac{\partial u_{k}}{\partial x_{j}}\right]
$$

A energia cinética é dada por:

$$
\mathrm{E}^{k}=-\frac{\partial}{\partial x}\left[\frac{K}{C_{p}} \frac{\partial}{\partial x}\left(\frac{1}{2}\left|v^{2}\right|\right)\right]-\frac{\partial}{\partial y}\left[\frac{K}{C_{p}} \frac{\partial}{\partial y}\left(\frac{1}{2}\left|v^{2}\right|\right)\right]-\frac{\partial}{\partial z}\left[\frac{K}{C_{p}} \frac{\partial}{\partial z}\left(\frac{1}{2}\left|v^{2}\right|\right)\right]
$$

Finalmente, a dissipação viscosa é dada por:

$$
\Phi=\mu\left(\frac{\partial u_{i}}{\partial x_{k}}+\frac{\partial u_{k}}{\partial x_{i}}\right) \frac{\partial u_{i}}{\partial x_{k}}
$$




\subsubsection{Equação da Energia Incompressivel}

A equação completa da energia para fluidos incompressíveis é derivada daquela para fluidos compressíveis, removendo-se o trabalho viscoso $\left(\mathrm{W}^{\mathrm{v}}\right)$, o trabalho da pressão, a dissipação viscosa e a Energia Cinética. Como a Energia Cinética é desprezada, a temperatura estática e a temperatura total são as mesmas. Assim, a equação da energia fica com a forma da equação de transporte térmico para a temperatura estática:

$$
\begin{aligned}
& \frac{\partial}{\partial t}\left(\rho C_{p} \mathrm{~T}\right)+\frac{\partial}{\partial x}\left(\rho v_{x} C_{p} \mathrm{~T}\right)+\frac{\partial}{\partial y}\left(\rho v_{y} C_{p} \mathrm{~T}\right)+\frac{\partial}{\partial z}\left(\rho v_{z} C_{p} \mathrm{~T}\right) \\
& =\frac{\partial}{\partial x}\left(K \frac{\partial T}{\partial x}\right)+\frac{\partial}{\partial y}\left(K \frac{\partial T}{\partial y}\right)+\frac{\partial}{\partial z}\left(K \frac{\partial T}{\partial z}\right)+Q_{v}
\end{aligned}
$$

\subsubsection{Turbulência}

Se os efeitos inerciais são grandes quando comparados aos efeitos da viscosidade, o escoamento se torna turbulento, avaliação esta subjetiva e depende da escolha e sensibilidade do engenheiro.

A turbulência significa que a velocidade instantânea está flutuando em cada ponto no campo de escoamento. Portanto, a velocidade é expressa em termos de um valor médio e uma componente flutuante:

$$
v_{x}=\bar{v}_{x}+v_{x}^{\prime}
$$

onde:

$$
\begin{aligned}
& \bar{v}_{x}=\text { componente absoluta da velocidade na direção } \mathrm{x} \\
& v_{x}^{\prime}=\text { componente flutuante da velocidade na direção } \mathrm{x} .
\end{aligned}
$$

Se esta expressão for usada para obter a velocidade instantânea nas equações de Navier-Stokes, as equações devem ser integradas no tempo. Importante notar que a integração no tempo de uma componente flutuante é nula e da velocidade instantânea é o valor médio absoluto. 
O intervalo de tempo para a integração explícita é escolhido arbitrariamente, de forma que seja pequeno o suficiente para que os deslocamentos transcorridos durante o intervalo não afetem a integração.

$$
\frac{1}{\delta t} \int_{0}^{\delta t} v_{x}^{\prime} d t=0 \quad \therefore \quad \frac{1}{\delta t} \int_{0}^{\delta t} v_{x} d t=\bar{v}_{x}
$$

Após a substituição da equação acima na lei da conservação dos momentos, a interpolação no tempo leva a termos adicionais. Considerando que as velocidades na equação dos momentos são as médias, indicaremo-nas pela barra superior.

$$
\begin{aligned}
& o_{x}^{R}=-\frac{\partial}{\partial x}\left(\overline{\rho v_{x}^{\prime} v_{x}^{\prime}}\right)-\frac{\partial}{\partial y}\left(\overline{\rho v_{x}^{\prime} v_{y}^{\prime}}\right)-\frac{\partial}{\partial z}\left(\overline{\rho v_{x}^{\prime} v_{z}^{\prime}}\right) \\
& o_{y}^{R}=-\frac{\partial}{\partial x}\left(\overline{\rho v_{y}^{\prime} v_{x}^{\prime}}\right)-\frac{\partial}{\partial y}\left(\overline{\rho v_{y}^{\prime} v_{y}^{\prime}}\right)-\frac{\partial}{\partial z}\left(\overline{\rho v_{y}^{\prime} v_{z}^{\prime}}\right) \\
& o_{z}^{R}=-\frac{\partial}{\partial x}\left(\overline{\rho v_{z}^{\prime} v_{x}^{\prime}}\right)-\frac{\partial}{\partial y}\left(\overline{\rho v_{z}^{\prime} v_{y}^{\prime}}\right)-\frac{\partial}{\partial z}\left(\overline{\rho v_{z}^{\prime} v_{z}^{\prime}}\right)
\end{aligned}
$$

onde: $\sigma^{\mathrm{R}}=$ termos de tensões de Reynolds.

Considerando a viscosidade dinâmica para a modelagem da turbulência, os membros são colocados em forma de uma tensão viscosa proporcional a um coeficiente desconhecido, a viscosidade turbulenta:

$$
-\overline{\rho v_{x} v_{y}}=\mu_{t} \frac{\partial v_{x}}{\partial y}
$$

A vantagem deste artifício é a semelhança de representação de $\sigma^{\mathrm{R}}$ em termos com mesma forma que a equação original.

Os dois termos podem ser combinados se uma viscosidade efetiva for definida como a soma da viscosidade laminar e a turbulenta:

$$
\mu_{e}=\mu+\mu_{t}
$$

Assim, a solução do problema turbulento é dada pela solução da turbulência viscosa. 
Observe que nem a tensão de Reynolds nem os termos de fluxo de calor turbulento contém uma densidade flutuante. Isto decorre da aplicação da interpolação de Favre. Basicamente esta técnica pondera cada termo pela densidade média para criar uma variável $\varphi$ que não contem densidade flutuante.

O Ansys fornece oito métodos diferentes de resolução do problema turbulento, a saber:

- Standard k-ع Model (Método k- $\varepsilon$ Padrão);

- Zero Equation Model (Método Raiz da Equação);

- RNG - (Re-normalized Group Model);

- NKE - (New k- $\varepsilon$ Model due to Shih);

- GIR - (Model due to Girimaji);

- SZL - (Shi, Zhu, Lumley Model);

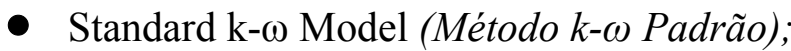

- SST - Shear Stress Transport Model (Método da Translação da Tensão de Cisalhamento).

O método mais simples é o Raiz da Equação em que a viscosidade turbulenta é calculada por:

$$
\mu_{t}=\rho L_{s}^{2} \sqrt{\Phi}
$$

onde: $\mu_{\mathrm{t}}=$ viscosidade turbulenta;

$\Phi=$ dissipação viscosa ;

$\mathrm{Ls}=\mathrm{Lx} \quad$ se $\mathrm{Lx}>0$

= MÍNIMO [ 0,4 Ln; 0,09 Lc ] se Lx $\leq 0 ;$

$\mathrm{Lx}=$ escala do comprimento;

$\mathrm{Ln}=$ menor distância do nó até o muro mais próximo ;

$\mathrm{Lc}=$ comprimento de escala característico (maior valor de Ln encontrado). 
RNG, NKE, GIR e SZL são variações das duas equações do Método k- $\varepsilon$ Padrão. Nestes métodos, a viscosidade turbulenta é calculada em função dos parâmetros turbulentos de energia cinética " $k$ " e a taxa de dissipação e " $\varepsilon$ ". Nos métodos RNG e Padrão, C $\mu$ é constante, enquanto é variável nos outros dois.

$$
\mu_{t}=C_{\mu} \rho \frac{k^{2}}{\varepsilon}
$$

onde: $\mathrm{C} \mu=$ constante de turbulência;

$\mathrm{k}=$ energia cinética turbulenta;

$\varepsilon=$ taxa de dissipação cinética turbulenta.

Finalmente, os dois últimos modelos são Método k- $\omega$ Padrão e SST:

$$
\mu_{t}=\rho \frac{k}{\omega}
$$

Onde $\omega$ é a taxa de dissipação específica, definida por:

$$
\omega=\frac{\varepsilon}{C_{\mu} k}
$$

O método k- $\varepsilon$ e suas extensões implicam na solução de derivadas parciais para energia cinética turbulenta e sua taxa de dissipação, enquanto que o k- $\omega$ e SST implicam na solução de derivadas parciais para energia cinética turbulenta e a taxa de dissipação específica.

\subsection{Ensaios em Túnel de Vento}

Os ensaios em túnel de vento fornecem coeficientes aerodinâmicos estáticos ou dinâmicos (conforme projeto do túnel e do modelo ensaiado), geralmente coeficientes de pressão, em modelos reduzidos da estrutura.

Historicamente, os primeiros ensaios para determinação dos esforços devidos ao vento em edificações datam de 1891, na Austrália, embora os primeiros túneis aerodinâmicos pareçam ter sido construídos na Inglaterra em 1871. Além dos australianos William Charles Kernot e James Mann, também foram pioneiros em ensaios em túneis 
aerodinâmicos o dinamarquês Irminger e o francês Alexandre Gustave Eiffel. Embora tenham o mesmo princípio - ar soprando sobre um objeto - há diferenças entre os túneis.

Os túneis aerodinâmicos são aqueles nos quais o escoamento tem velocidade uniforme e pouca turbulência, normalmente usados para automóveis e aviões. Os túneis de vento, denominação que no Brasil é mais utilizada quando o túnel tem capacidade para simular as principais características dos ventos naturais, tais como variação de velocidade com a altura, escala e intensidade da turbulência, além de outros fenômenos.

Os túneis de vento têm a forma de um corredor e podem ser de circuito aberto ou fechado. No circuito aberto, em uma das extremidades está o gerador de corrente de ar e a outra é o local de saída. Por ter uma configuração mais simples, tem menor custo, mas tem a desvantagem de ser muito influenciado por intempéries e perturbações externas, especialmente rajadas. Já nos túneis de circuito fechado os ventos circulam em corredor com forma de anel.

As principais instalações brasileiras encontram-se no Instituto de Aeronáutica (IAE), no Instituto Tecnológico de Aeronáutica (ITA), no Laboratório Feng e no Instituto de Estudos Avançados (IEAv). Destacam-se ainda o Laboratório de Aerodinâmica das Construções (LAC) da UFRGS, do Instituto de Pesquisas Tecnológicas do Estado de São Paulo (IPT) e a Escola de Engenharia da Universidade de São Paulo (USP), em São Carlos, entre outros.

Os modelos reduzidos são construídos rígidos nos ensaios estáticos e com flexibilidade, amortecimento e distribuição de massa proporcional nos ensaios dinâmicos. Os modelos têm escala compatível com o tamanho do túnel disponível para o ensaio, ao longo do qual são instalados sensores, como tomadas de pressão, além dos pontos críticos apontados pelo projetista. 

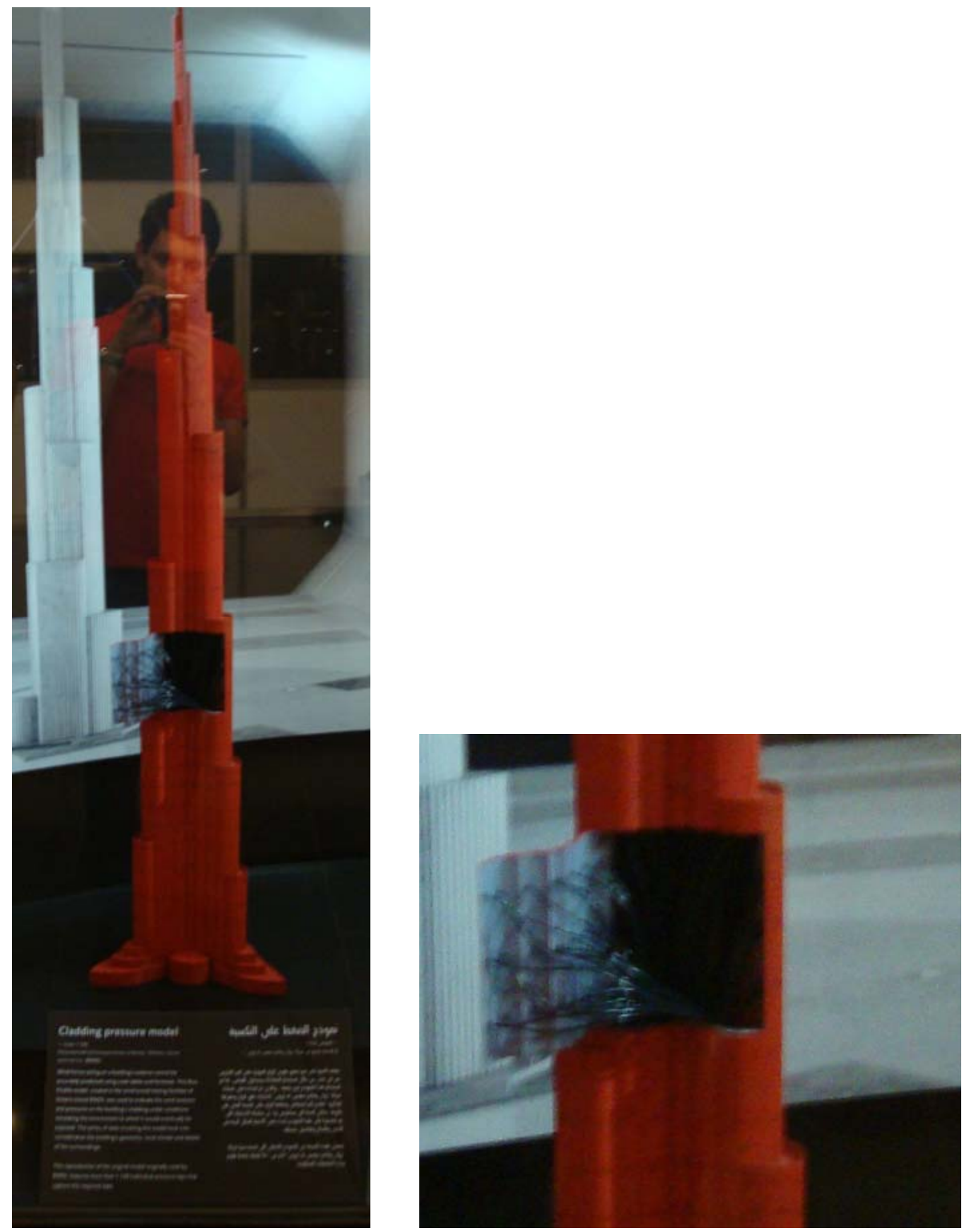

Figura 8 - Um dos 40 modelos para ensaio em túnel de vento do Burj Khalifa

Foto tirada no museu de visitação do edifício Burj Khalifa em 2011, com 828 m de altura

Como pode-se observar na Figura 8, as tomadas de pressão para o edifício Burj Khalifa foram montadas internamente ao modelo. No caso de membranas, pelo fato de geralmente não haver espaço para embutir as tomadas de pressão, as tomadas de pressão podem ficar aparentes, por fora da estrutura. Sugere-se como tema de pesquisas futuras a influência das tomadas de pressão nos resultados.

É comum realizar ensaios em túnel de vento para estruturas de grande porte, que requerem informações acuradas em cargas de vento estáticas e dinâmicas para evitar o uso 
de coeficientes aerodinâmicos exagerados e melhorar a segurança, como pontes de grandes vãos, prédios muito altos, estádios, arenas, etc.

Muitas vezes, os resultados dos ensaios levam aos projetistas a realizarem alterações significativas no projeto, a tal ponto que exigem a execução novos ensaios. Todo este processo tem custo elevado e prazos longos. Um ensaio convencional custa em torno de trinta e oito mil reais no Brasil e o dobro ou mais no exterior. O preço pode variar conforme a quantidade de coeficientes aerodinâmicos solicitados e a complexidade da estrutura.

O elevado custo destes ensaios pode ser facilmente justificado para estruturas de grande porte com a redução nos esforços solicitantes, e por conseqüência, no consumo de material.

Atualmente, com os prazos para projeto e construção muito curtos, têm-se buscado alternativas para a análise dinâmica de estruturas. Com o avanço da tecnologia computacional, a análise dinâmica computacional dos fluidos (CFD) tem grande potencial para disputar o mercado.

Por enquanto, um estudo computacional também consome muito tempo, principalmente com a modelagem, que pode levar mais tempo do que a execução do ensaio em túnel de vento. Com os estudos crescentes na área e a elaboração de softwares mais práticos e ágeis, este cenário tende a mudar no futuro. 


\section{APLICAÇÕES}

Neste capítulo serão apresentadas aplicações da teoria acima explanada, através do uso do software ANSYS versão 11, ambiente de simulação Multiphysics, disponibilizada nos laboratórios da USP.

Inicialmente serão apresentadas aplicações básicas, com benchmarks clássicos da fluido-dinâmica. Posteriormente serão apresentadas duas aplicações com acoplamento parcial à uma estrutura de membrana.

Nestas últimas aplicações serão apresentados: form-finding e ação do vento em situações de rajada de $50 \mathrm{~m} / \mathrm{s}$ através de CFD.

As estruturas de membrana foram modeladas tridimensionalmente através do elemento "Shell 41", o qual permite-se trabalhar exclusivamente à tração ou à tração e compressão. Simplificadamente, os materiais adotados foram isótropos lineares, com relação constitutiva elástica.

Nas aplicações tridimensionais, o fluido (vento) foi modelado com elemento fluido "FLUID142", com propriedades de densidade e viscosidade adotadas igual a 1,23 $\mathrm{kg} / \mathrm{m}^{3}$ e 1,79 x 10-5 $\mathrm{kg} / \mathrm{m}^{3} . \mathrm{s}$, respectivamente. Para as aplicações bidimensionais, o elemento utlizado foi o "FLUID141".

A configuração do modelo levou em conta princípios análogos ao de um túnel de vento, com o domínio determinado em função da estrutura de membrana a ser estudada. A seção transversal do domínio computacional junto à estrutura deve ser grande o suficiente para não influenciar nas condições de escoamento.

Segundo KUPZOK (2009) apud FRANKE (2006), recomenda-se que a altura do domínio seja determinada entre 5 e $6 H_{\text {máx }}$. sendo $\mathrm{H}_{\text {máx }}$ a máxima altura da estrutura. Lateralmente, recomenda-se a distância de $5 H_{m a ́ x}$ a partir dos extremos da estrutura.

Denominando-se a área da seção transversal do domínio por $\mathrm{A}_{\mathrm{QS}}$, segundo KUPZOK (2009), recomenda-se que em CFD a razão dada por $\mathrm{H}_{\text {máx }}{ }^{2} / \mathrm{A}_{\mathrm{QS}}$ tenha valor máximo de 3\%. Em cada aplicação aqui estudada, apresentaremos a verificação da referida recomendação. 
Ainda tratando-se do domínio, recomenda-se que a distância da entrada do escoamento até o primeiro do nó da estrutura seja de $5 H_{\text {máx }}$ e a saída do escoamento de 10 a $15 H_{\text {máx }}$.

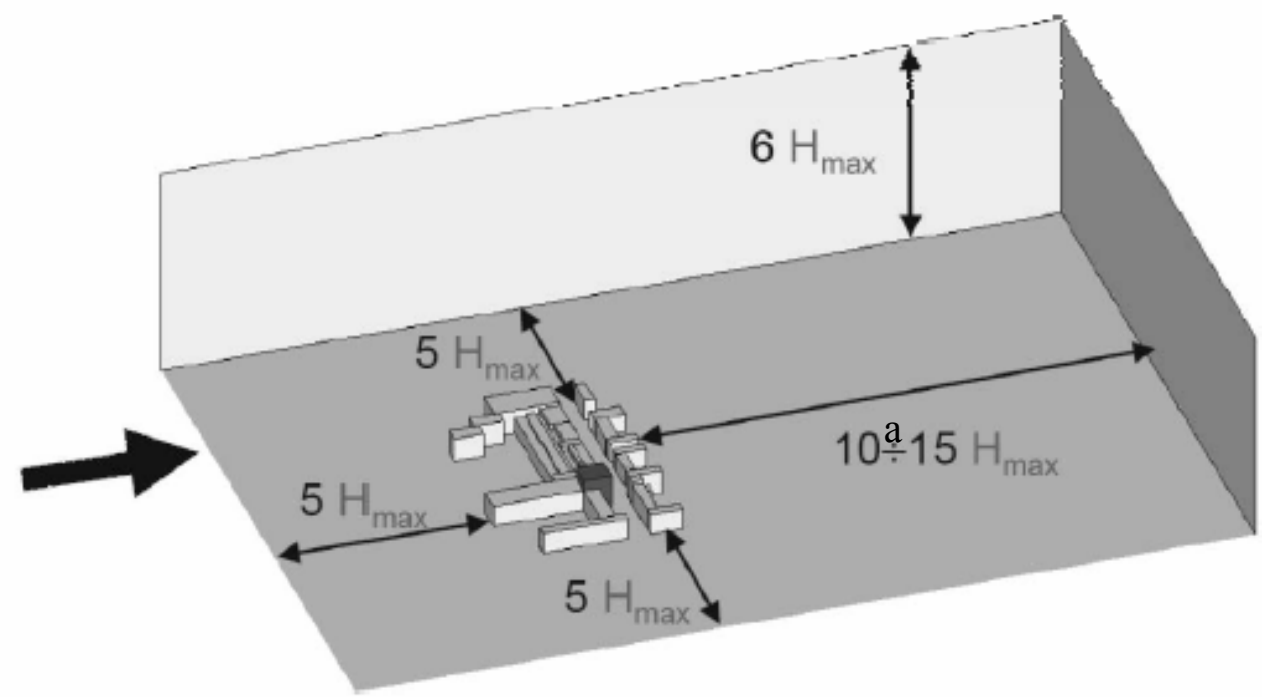

Figura 9 - Domínio recomendado para ação do vento na estrutura

Neste estudo, a rugosidade da estrutura de membrana e do terreno foi desprezada.

\subsection{Fluido sobre Anteparo Circular 2D}

Nesta aplicação foi estudada aplicação de vento com 40 m/s escoando da esquerda para direita, com obstáculo circular:

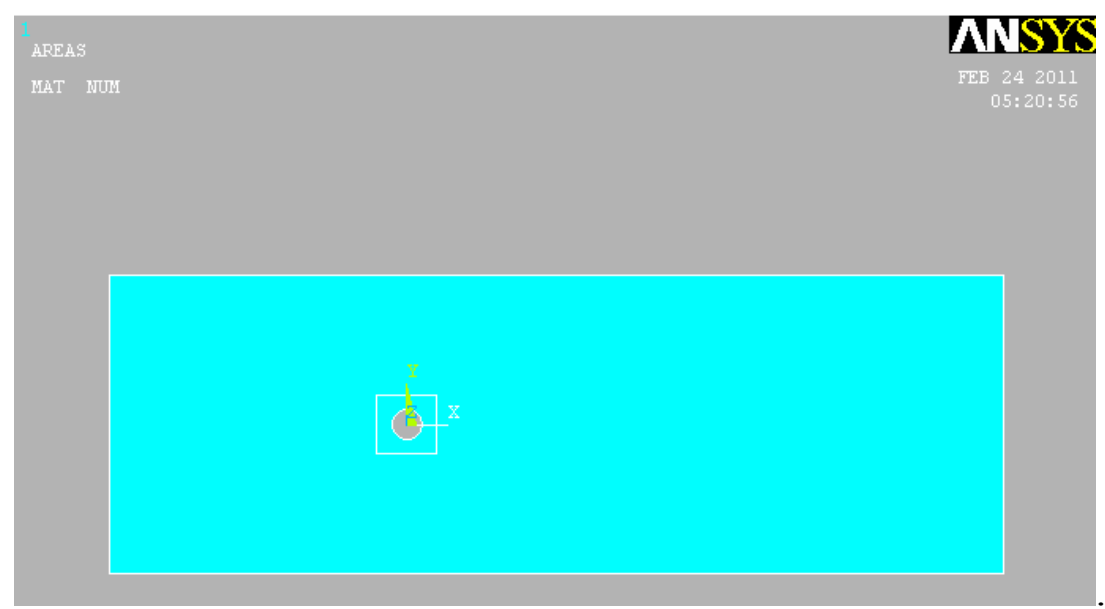

Figura 10 - Domínio adotado na Aplicação 7.1 
As dimensões utilizadas no domínio foram de $30 \mathrm{~m}$ de comprimento, $10 \mathrm{~m}$ de altura e círculo de $1 \mathrm{~m}$ de diâmetro.

$\mathrm{Na}$ seqüência foi lançada uma malha composta por 3592 elementos finitos triangulares e 1876 nós, sendo mais densa na região de interesse próxima ao obstáculo:

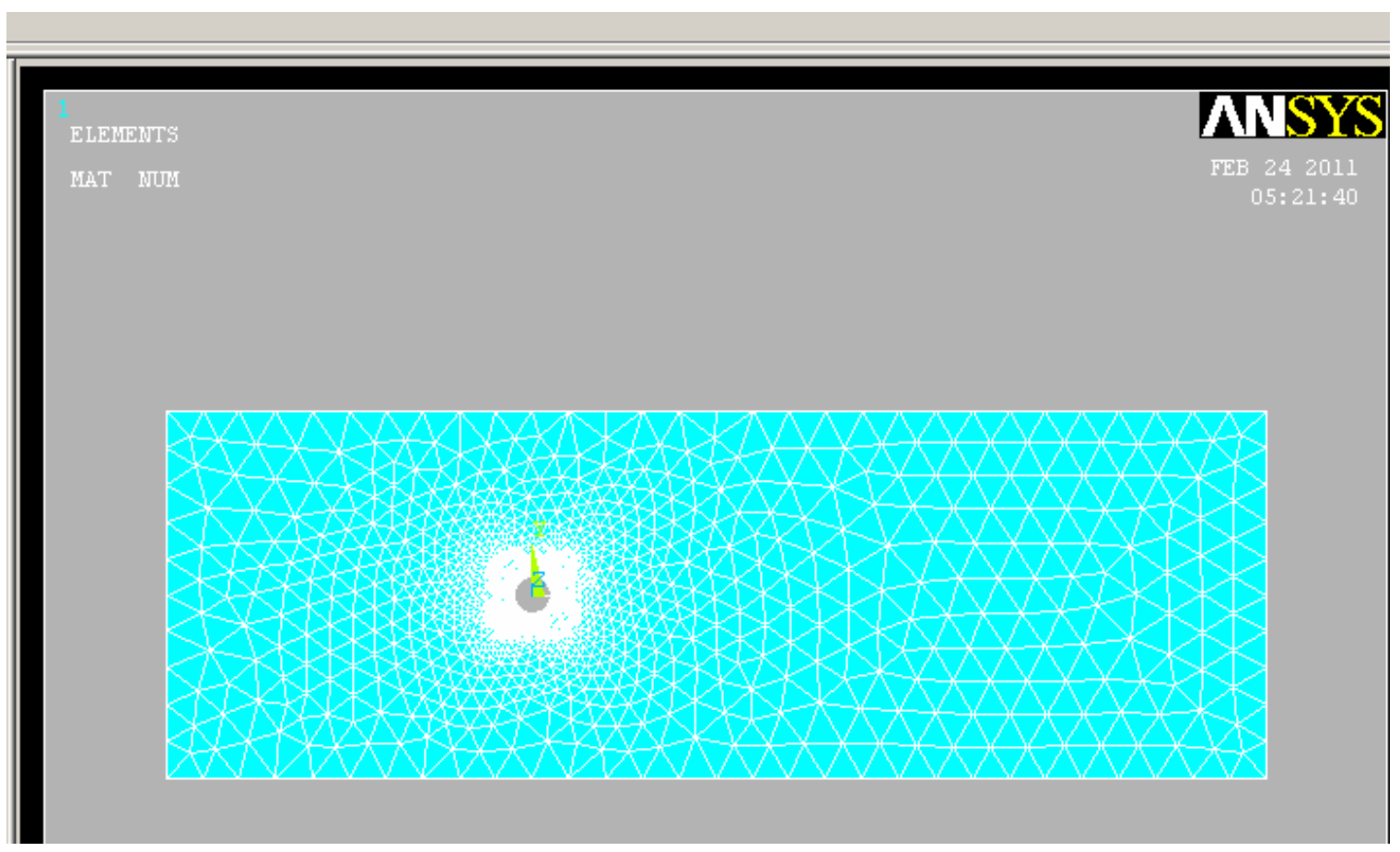

Figura 11 - Malha adotada

Conforme norma NBR 6123, para superfície lisa e altura muito maior que o diâmetro $(\mathrm{h} / \mathrm{d}=10)$ os resultados esperados são fornecidos pela Tabela 4. 


\begin{tabular}{|c|c|c|c|c|}
\hline \multirow{3}{*}{$\beta$} & \multicolumn{4}{|c|}{ Coeficiente de pressão externa $c_{p e}$} \\
\hline & \multicolumn{2}{|c|}{$\begin{array}{l}\text { Superficie rugosa } \\
\text { ou com saliências }\end{array}$} & \multicolumn{2}{|c|}{ Superficie lisa } \\
\hline & $h / d=10$ & $\mathrm{~h} / \mathrm{d} \leq 2,5$ & $\mathrm{~h} / \mathrm{d}=10$ & $\mathrm{~h} / \mathrm{d} \leq 2,5$ \\
\hline $0^{\circ}$ & $+1,0$ & $+1,0$ & $+1,0$ & $+1,0$ \\
\hline $10^{\circ}$ & $+0,9$ & $+0,9$ & $+0,9$ & $+0,9$ \\
\hline $20^{\circ}$ & $+0,7$ & $+0,7$ & $+0,7$ & $+0,7$ \\
\hline $30^{\circ}$ & $+0,4$ & $+0,4$ & $+0,35$ & $+0,35$ \\
\hline $40^{\circ}$ & 0 & 0 & 0 & 0 \\
\hline $50^{\circ}$ & $-0,5$ & $-0,4$ & $-0,7$ & $-0,5$ \\
\hline $60^{\circ}$ & $-0,95$ & $-0,8$ & $-1,2$ & $-1,05$ \\
\hline $70^{\circ}$ & $-1,25$ & $-1,1$ & $-1,4$ & $-1,25$ \\
\hline $80^{\circ}$ & $-1,2$ & $-1,05$ & $-1,45$ & $-1,3$ \\
\hline $90^{\circ}$ & $-1,0$ & $-0,85$ & $-1,4$ & $-1,2$ \\
\hline $100^{\circ}$ & $-0,8$ & $-0,65$ & $-1,1$ & $-0,85$ \\
\hline $120^{\circ}$ & $-0,5$ & $-0,35$ & $-0,6$ & $-0,4$ \\
\hline $140^{\circ}$ & $-0,4$ & $-0,3$ & $-0,35$ & $-0,25$ \\
\hline $160^{\circ}$ & $-0,4$ & $-0,3$ & $-0,35$ & $-0,25$ \\
\hline $180^{\circ}$ & $-0,4$ & $-0,3$ & -0.35 & $-0,25$ \\
\hline
\end{tabular}

Tabela 4 - Distribuição das pressões externas em edificações cilíndricas segundo a NBR 6123

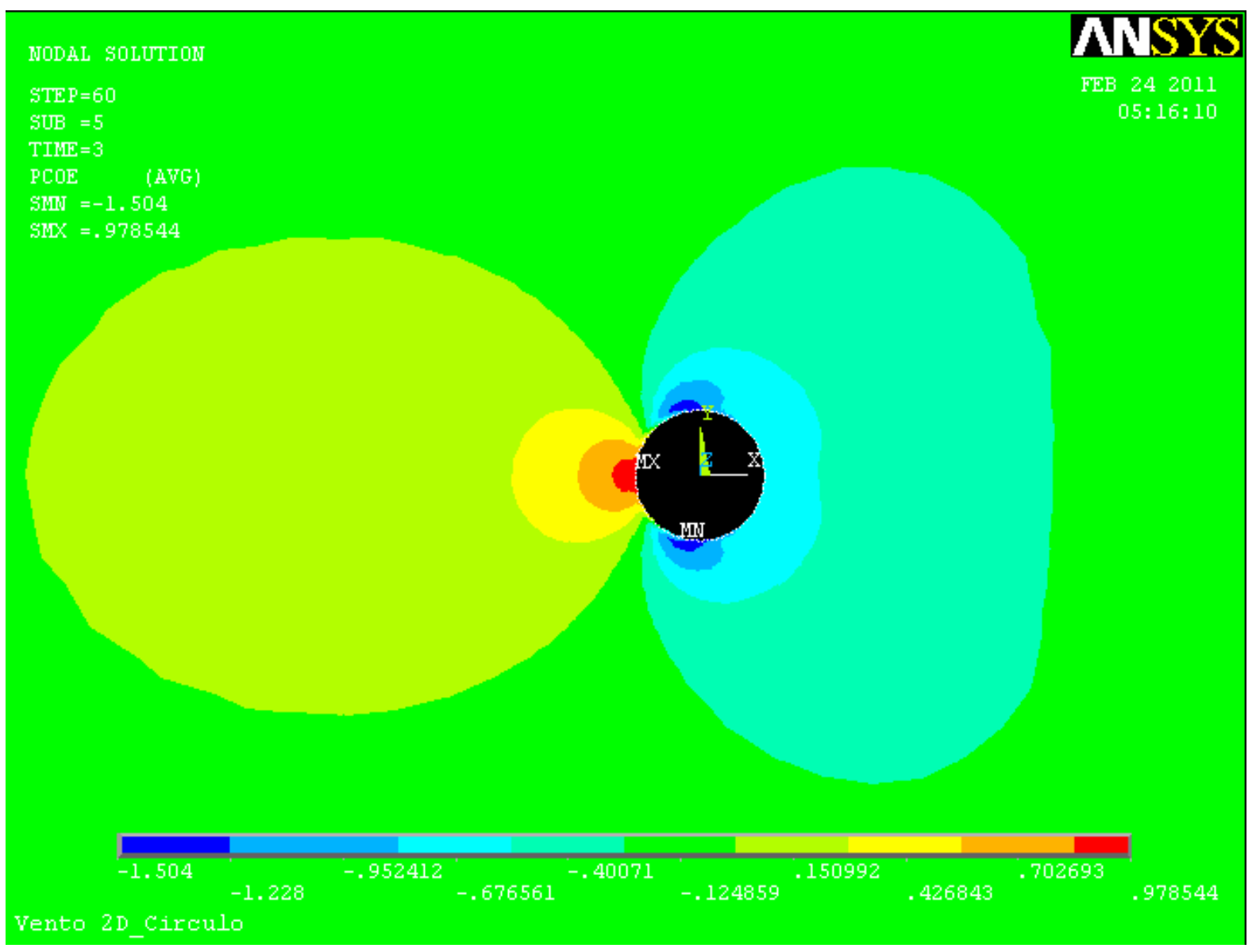

Figura 12 - Coeficientes de pressão obtidos no Ansys 
Como pode observar, os valores encontrados pelo Ansys confirmam os valores esperados por norma.

Na Figura 13 apresentam-se os valores obtidos para velocidade do vento no entorno do círculo:

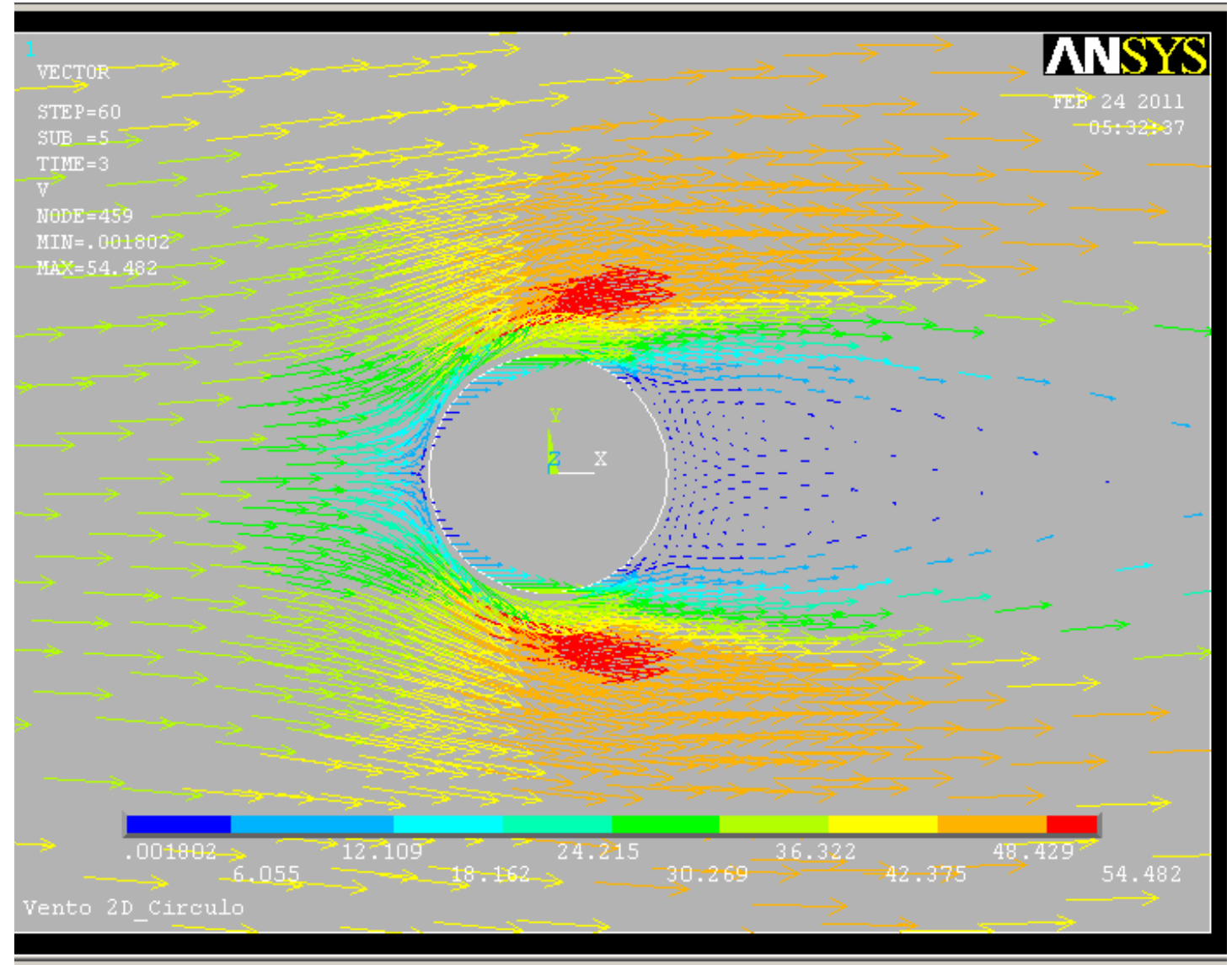

Figura 13 - Velocidade do vento no entorno do anteparo circular (m/s)

Como pode-se observar, a velocidade do vento máxima chega a 54,482 m/s. Também pode-se notar a formação de vórtices logo após a passagem. 


\subsection{Vento sobre Perfil I}

Analogamente à aplicação anterior, esta aplicação solucionará o problema do vento escoando a $25 \mathrm{~m} / \mathrm{s}$ por um perfil I de 1 metro de altura.

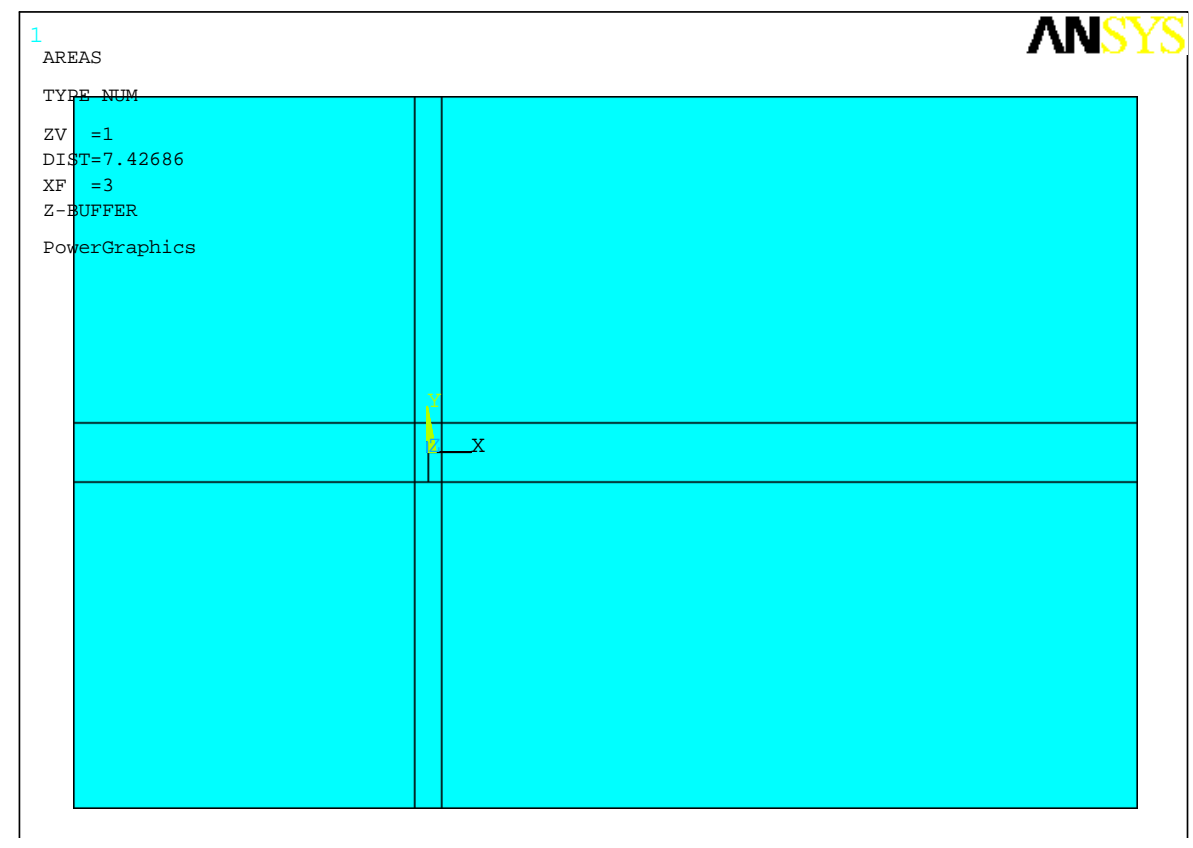

Figura 14 - Geometria do modelo para vento em Perfil I

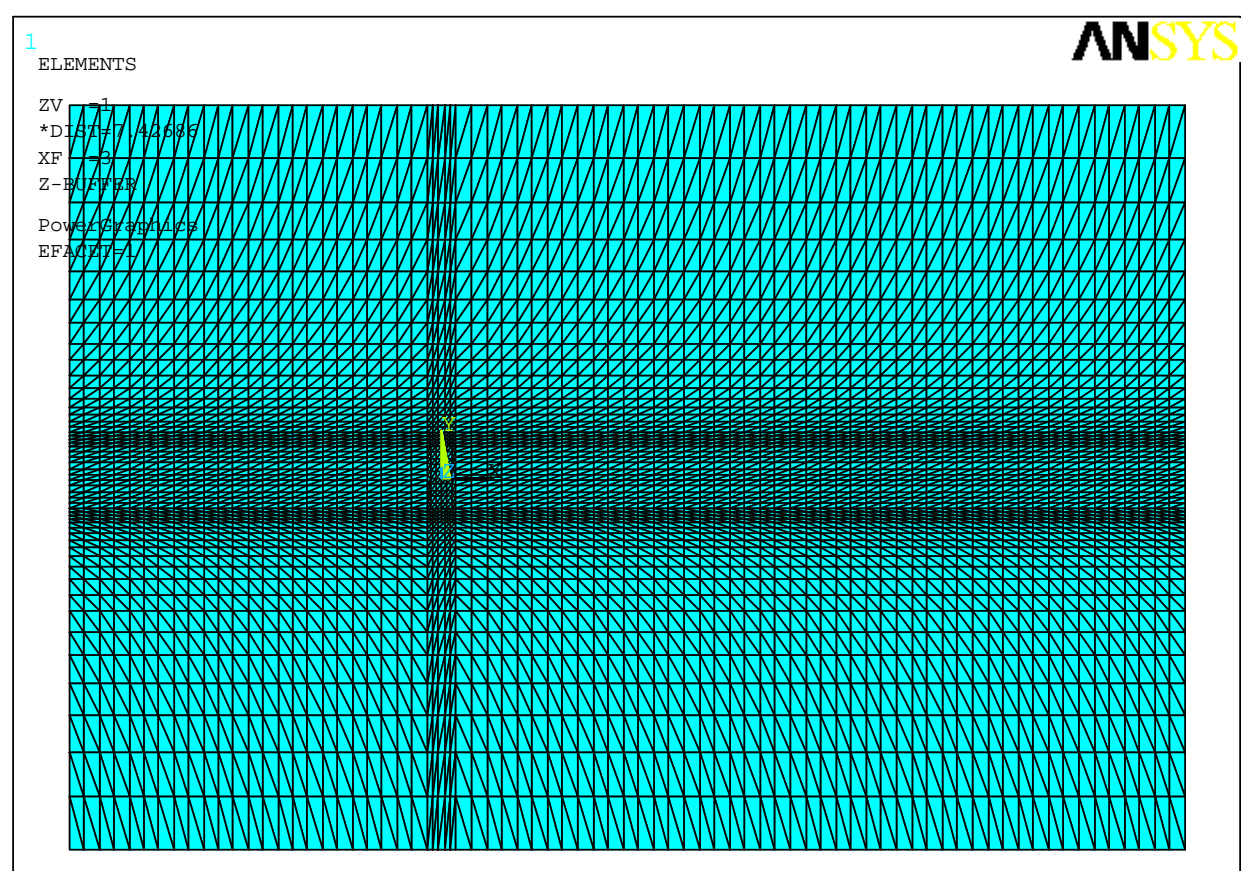

Figura 15 - Malha adotada para vento em Perfil I 
A malha adotada é composta por 8656 elementos triangulares e 4636 nós, sendo mais refinada próximo ao perfil I:

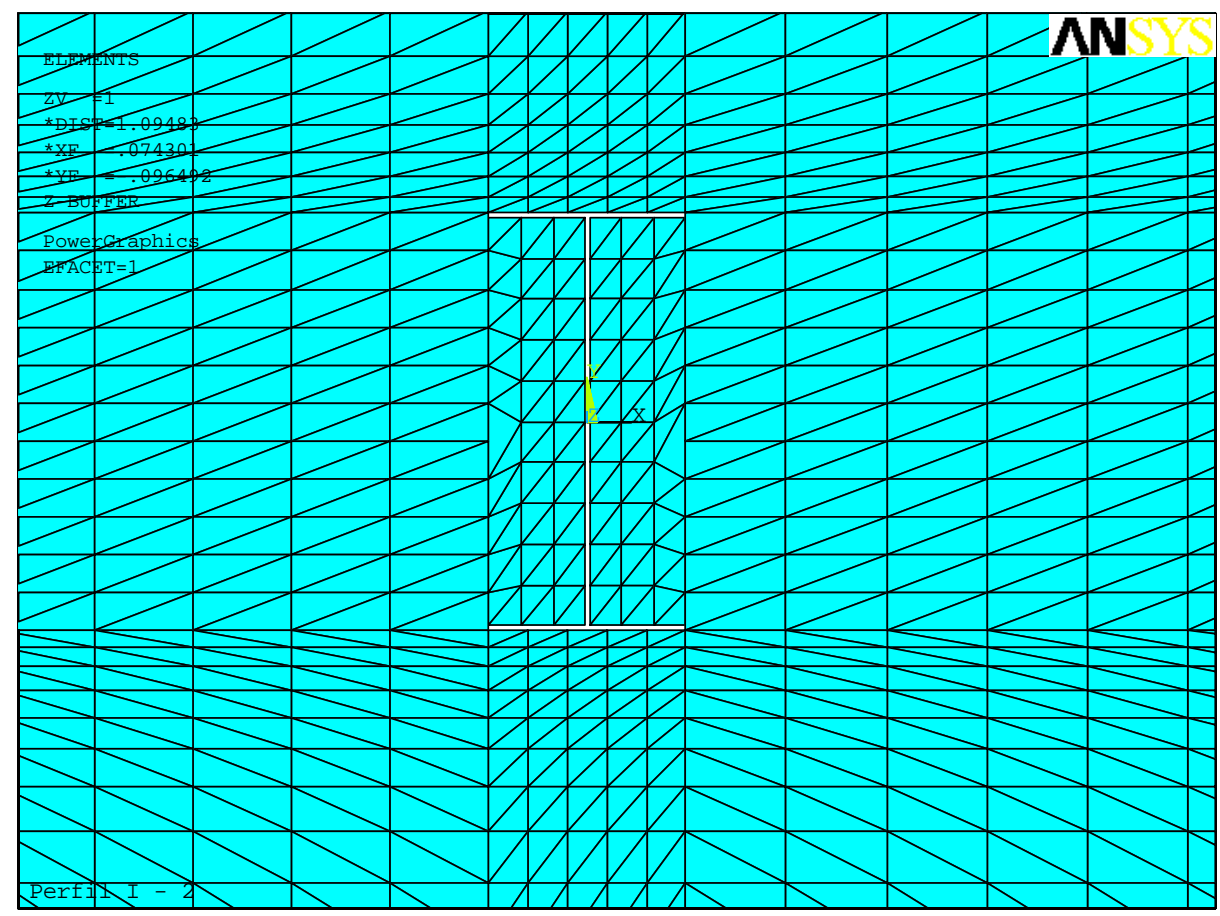

Figura 16 - Malha próxima ao Perfil I

De acordo com a NBR 6123, tabela 12, os coeficientes de pressão esperados são de:

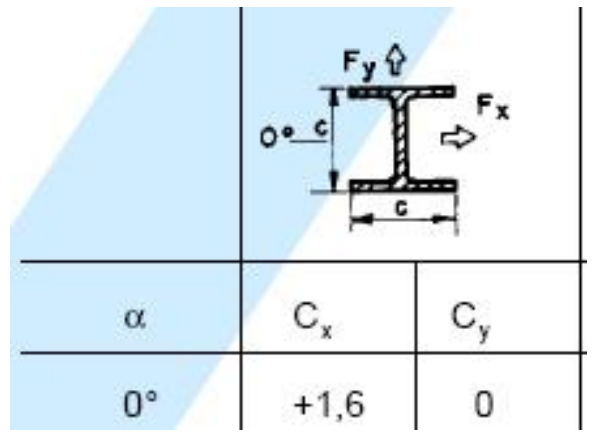

Figura 17 - Coeficientes de pressão esperados

Nas Figuras 18 a 20 são apresentados os valores de coeficiente de pressão, bem como as velocidades máximas encontradas: 


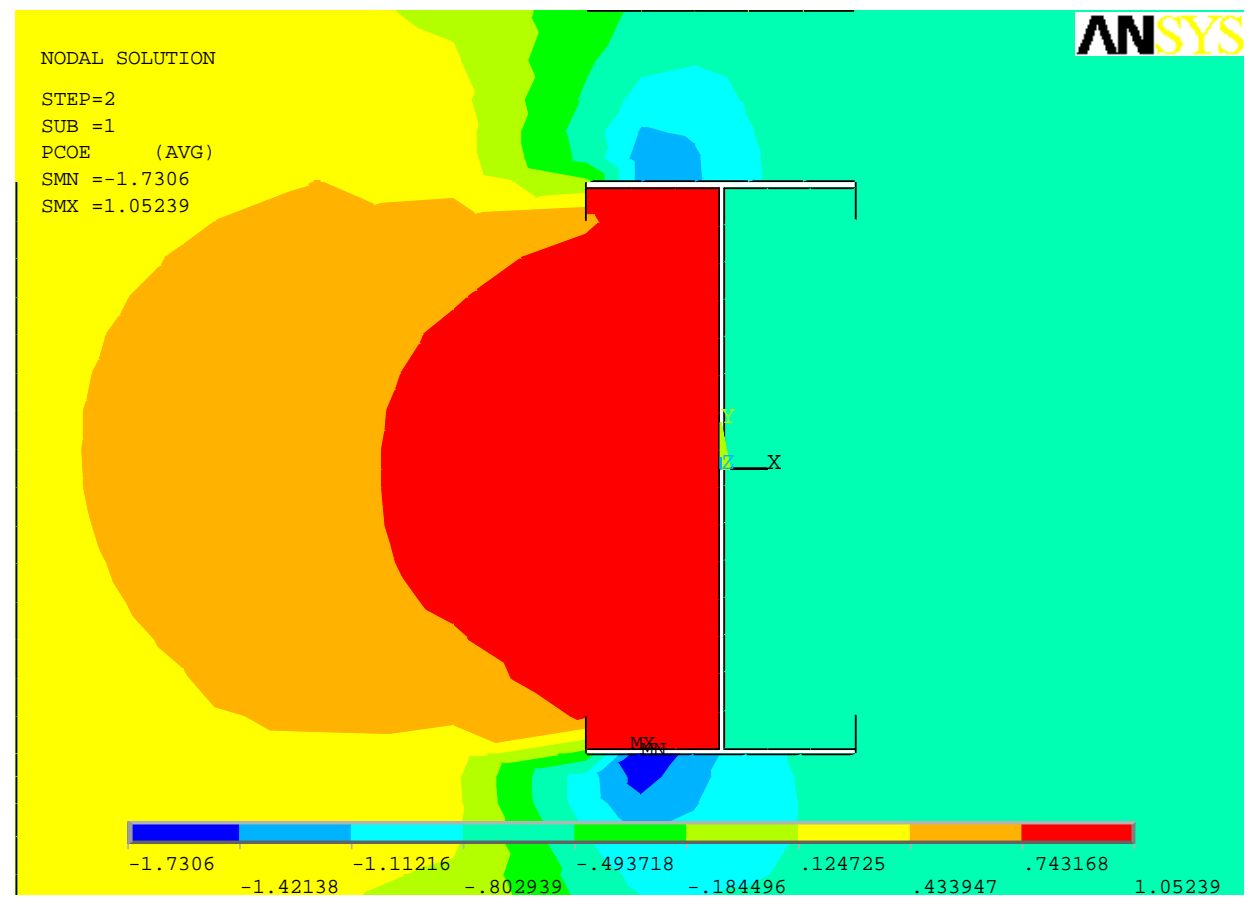

Figura 18 - Resultado dos coeficientes de pressão

Somando-se os valores de pressão e sucção na alma, à barlavento e sotavento respectivamente, chegamos a um coeficiente $\mathrm{Cx}=1,615$, equivalente ao valor proposto pela NBR 6123.

Adicionalmente, somando os valores encontrados para a mesa superior e inferior, temos um coeficiente $\mathrm{Cy} \sim 0$ conforme esperado.

Abaixo seguem resultados da velocidade do vento. Observa-se claramente a formação de vórtices após o perfil, chegando a velocidade absoluta de $31 \mathrm{~m} / \mathrm{s}$ (24\% mais rápido que o escoamento original). 


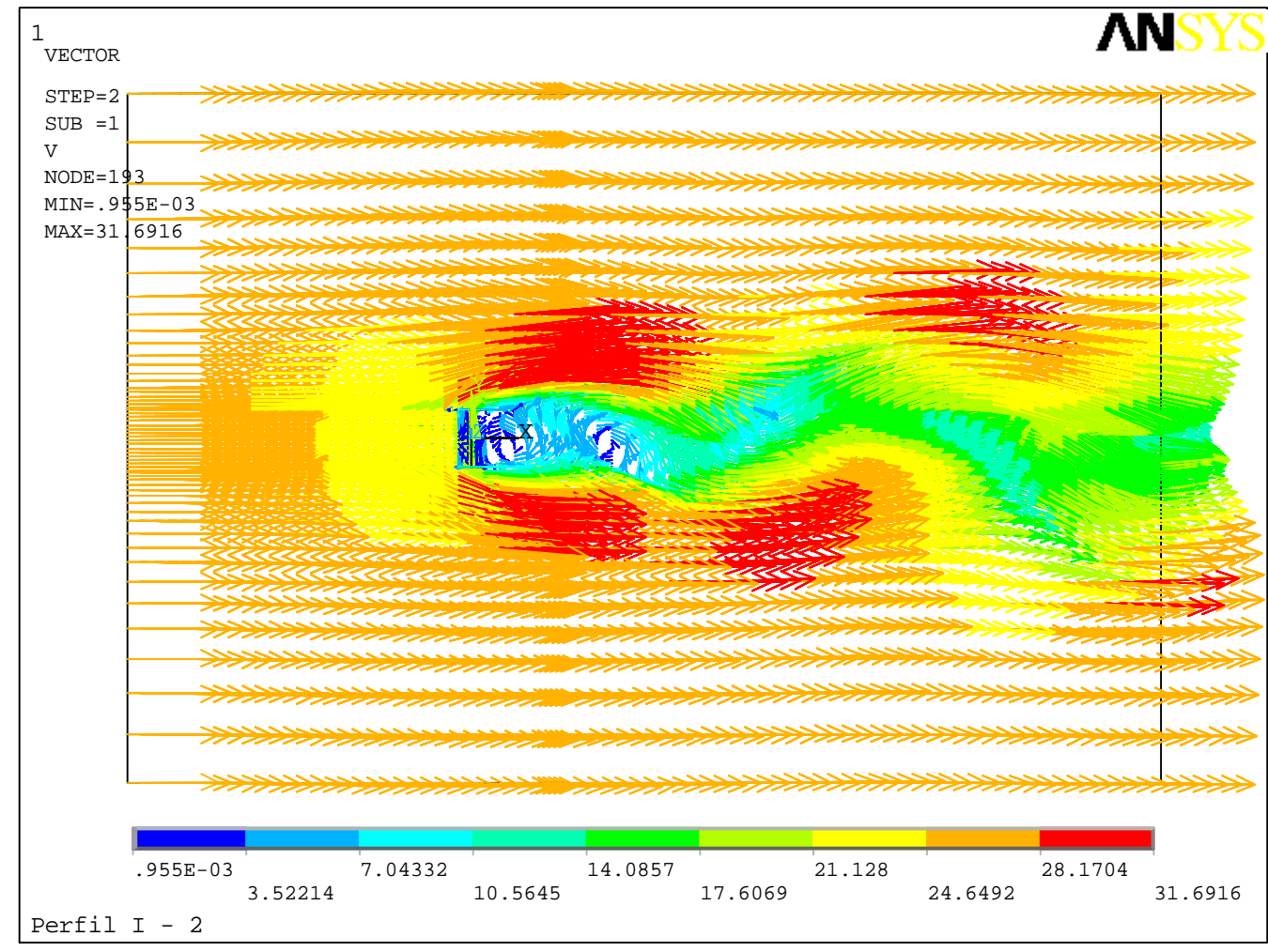

Figura 19 - Resultado de velocidades do vento $(\mathrm{m} / \mathrm{s})$

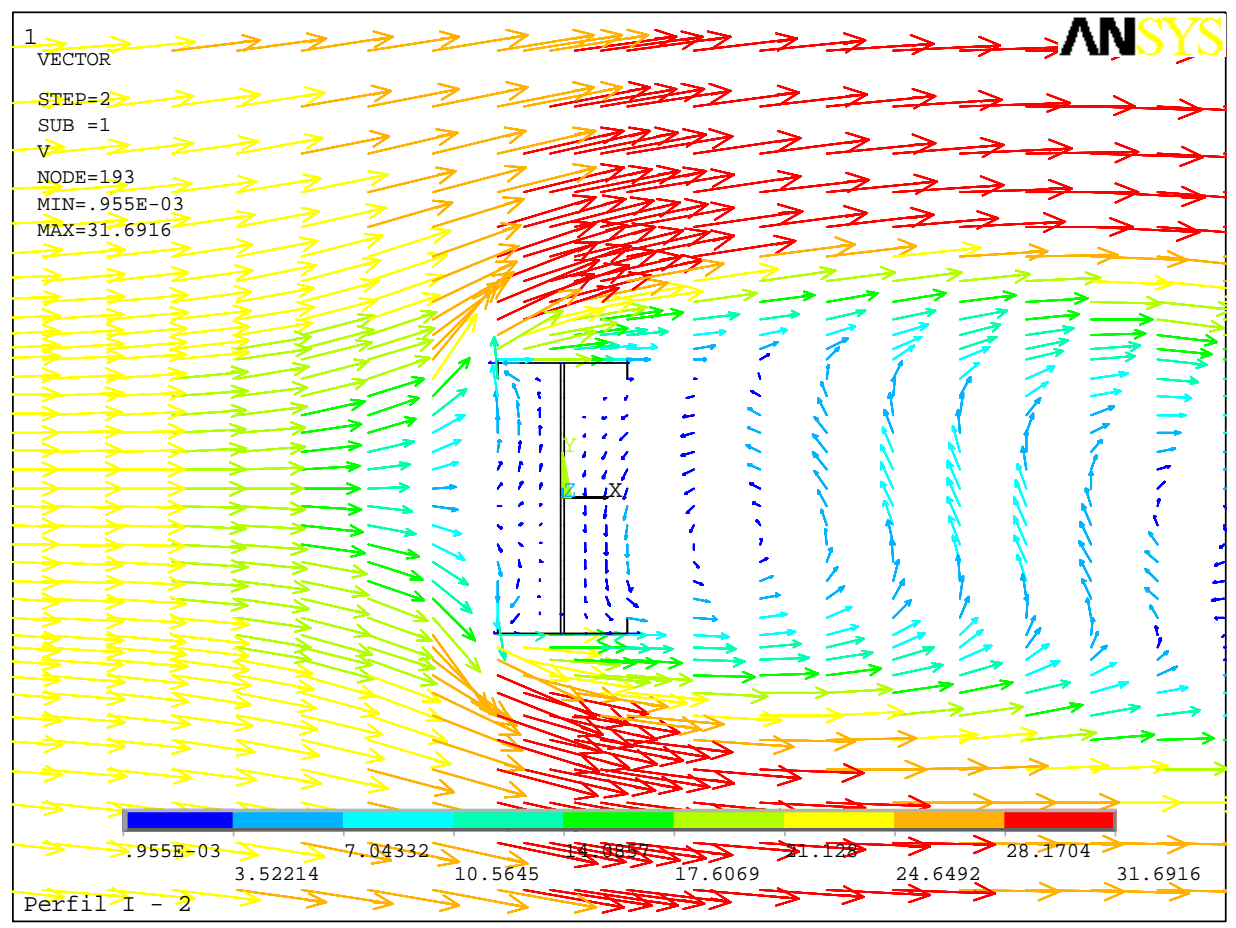

Figura 20 - Velocidades do vento próximas ao Perfil I (m/s) 


\subsection{Vento sobre muro}

Nesta aplicação será apresentada estrutura de vertical com pequena espessura, como um muro, se assemelhando a modelagem bidimensional de uma membrana vertical e plana.

\subsubsection{Dados Iniciais}

Adotou-se para o problema um muro de $4 \mathrm{~m}$ de altura, posicionada no chão e espessura infinitesimal.

O modelo foi concebido por uma área retangular de $60 \times 24 \mathrm{~m} 2$, com o muro posicionado a $20 \mathrm{~m}$ da entrada do escoamento conforme figura abaixo:

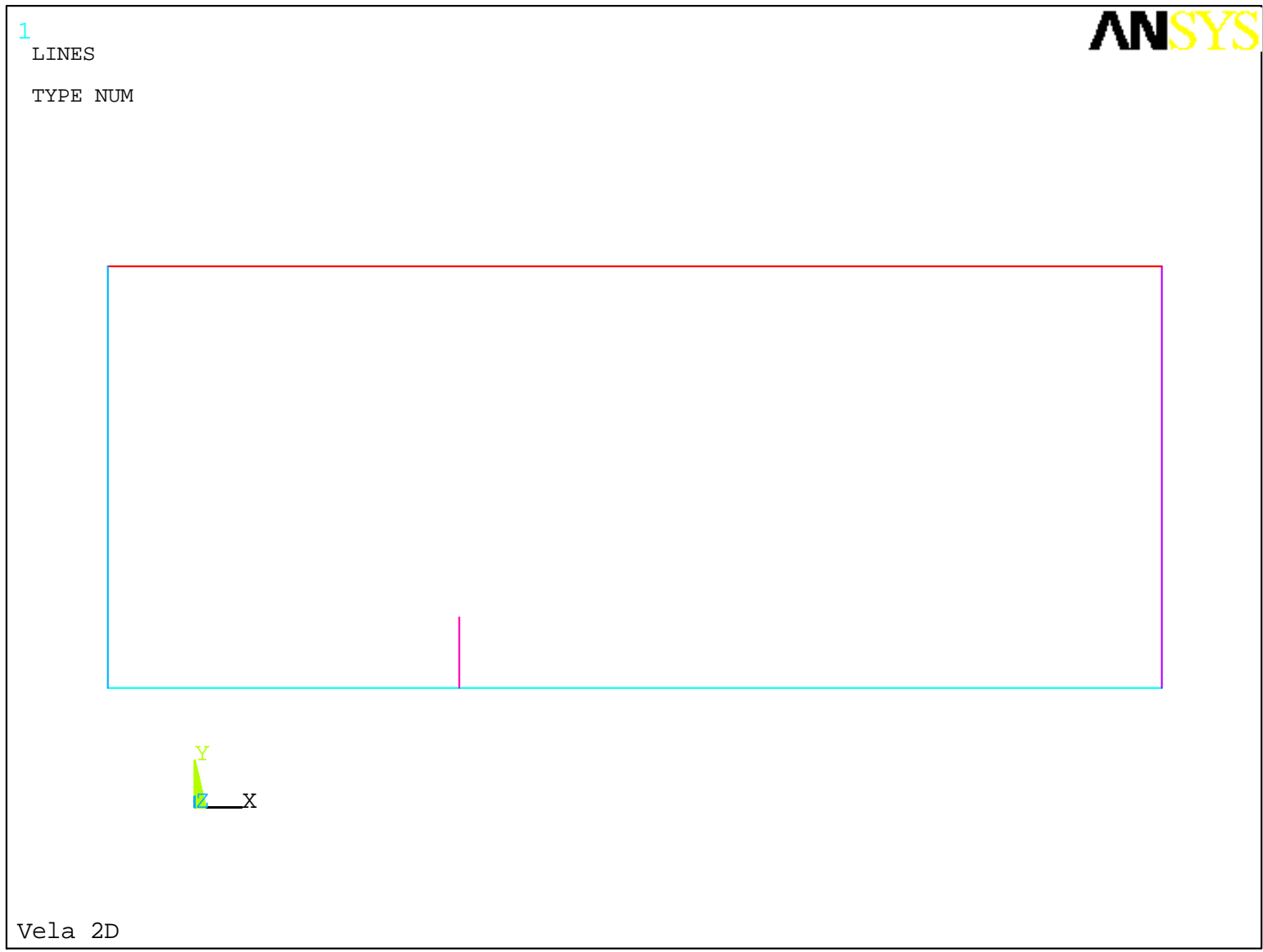

Figura 21 - Geometria do Modelo 
Para o vento foi gerado modelo com 2692 nós e 5086 elementos:

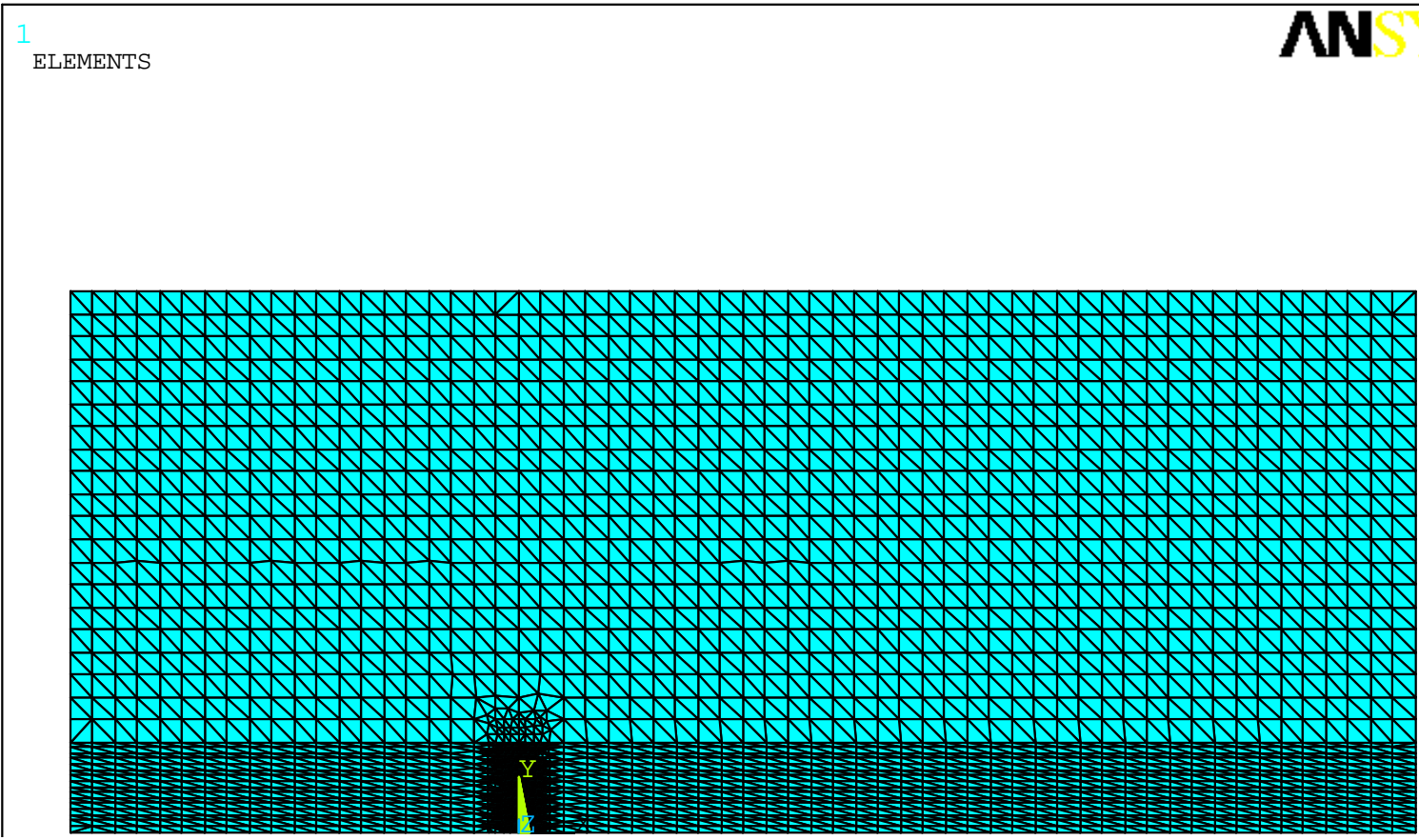

Figura 22 - Malha de Elementos Finitos

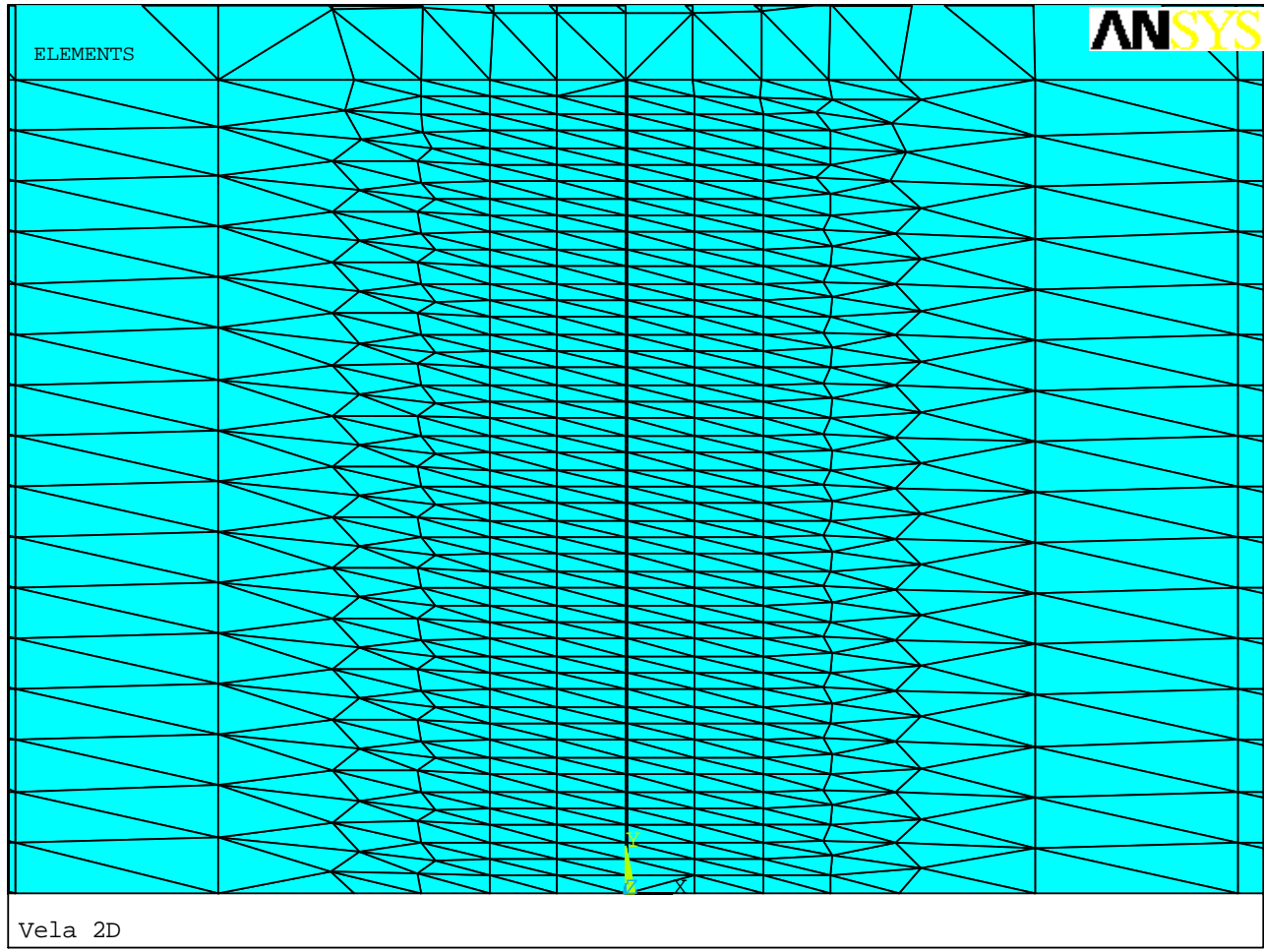

Figura 23 - Refinamento da malha em torno do muro 


\subsubsection{Ação do Vento}

Aplicando-se vento Vx de 50m/s na superfície de barlavento, e considerando-se a malha de elementos da estrutura como indeslocáveis e indeformáveis, obteve-se os seguintes resultados:

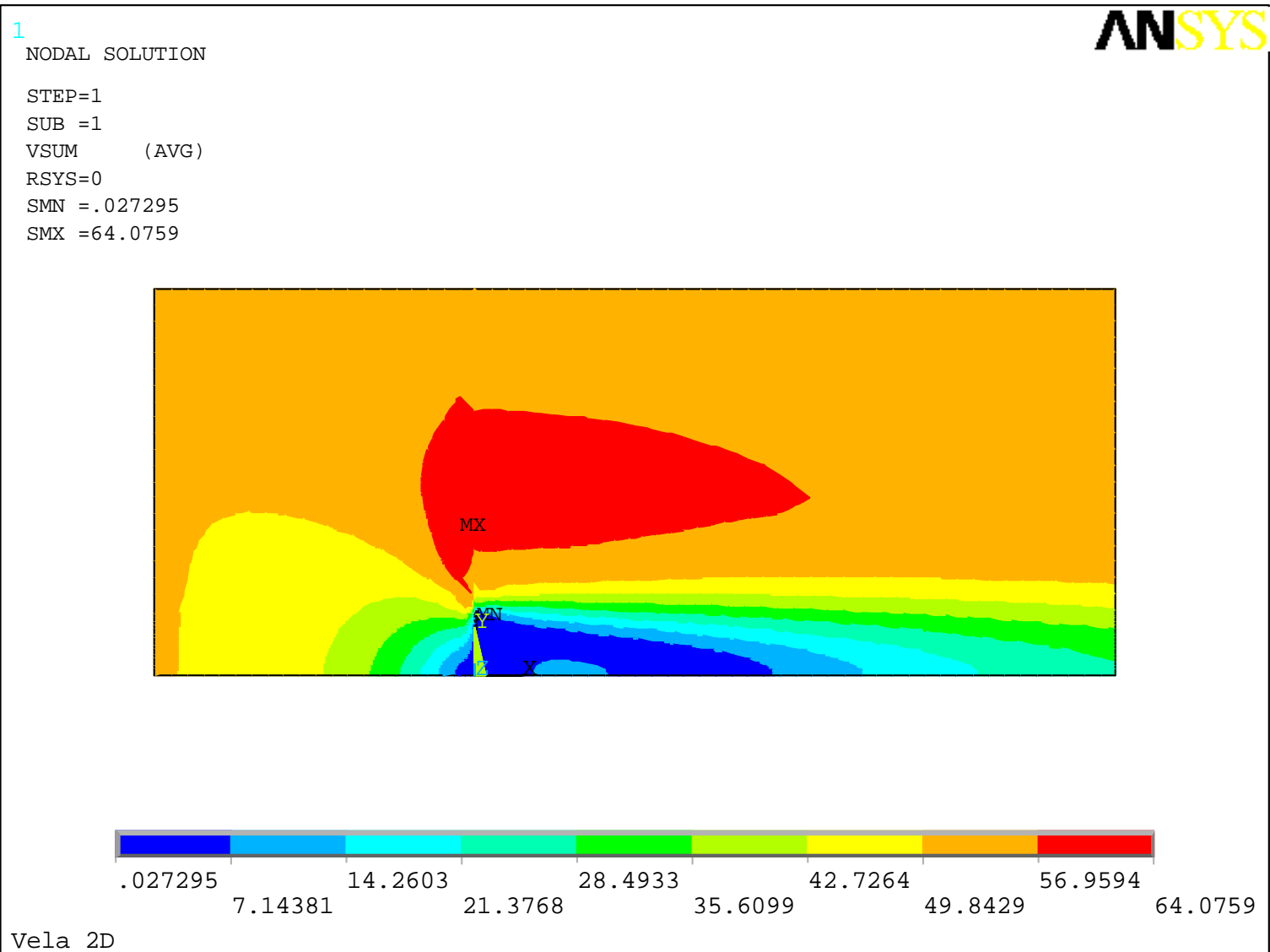

Figura 24 - Valores de velocidade do vento do modelo $(\mathrm{m} / \mathrm{s})$ 


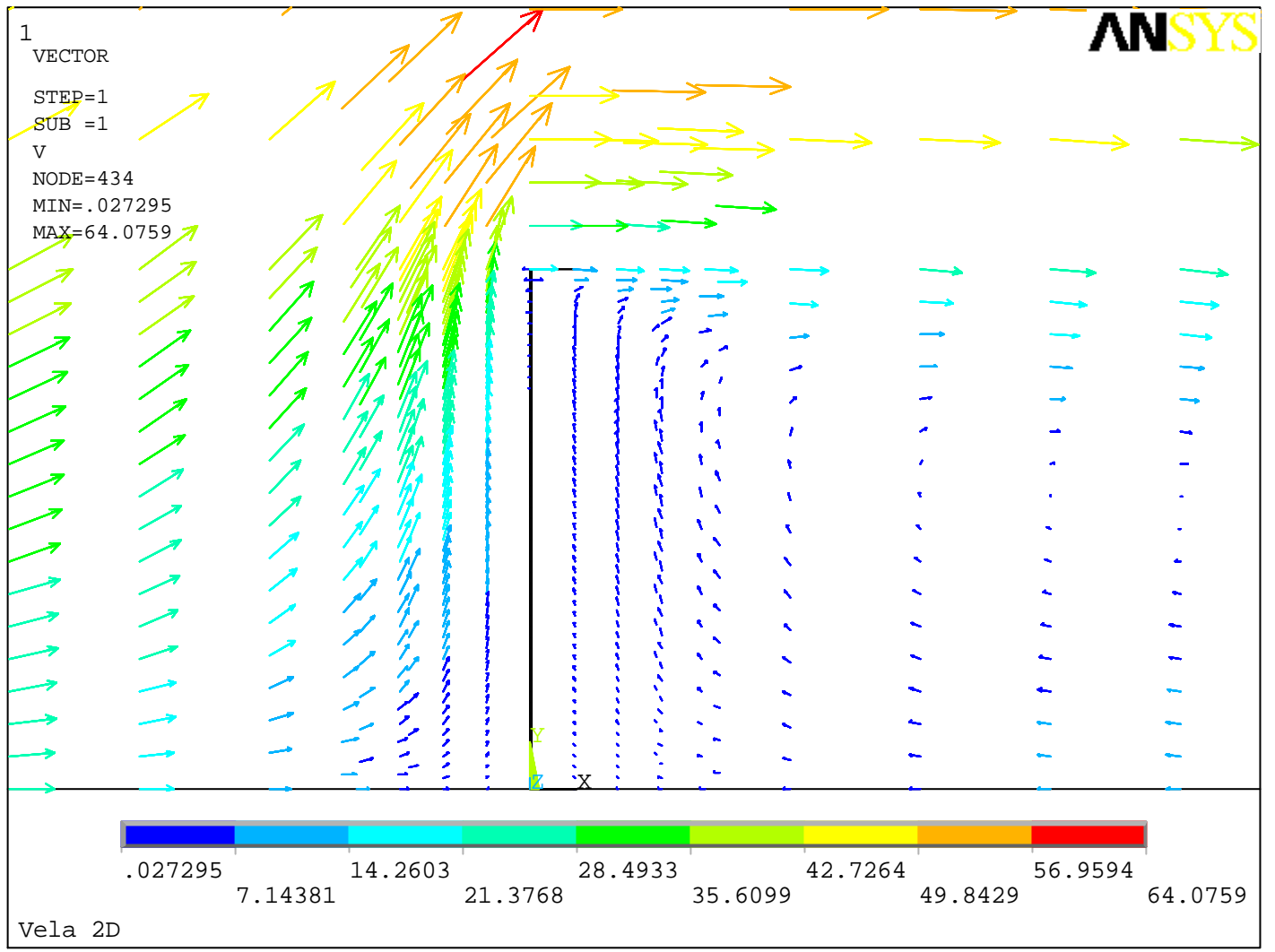

Figura 25 - Vetores velocidade no entorno do muro $(\mathrm{m} / \mathrm{s})$

A velocidade máxima encontrada foi de $64 \mathrm{~m} / \mathrm{s}$, havendo formação de vórtices imediatamente a sotavento da estrutura. Abaixo seguem valores de pressão e coeficientes de pressão: 


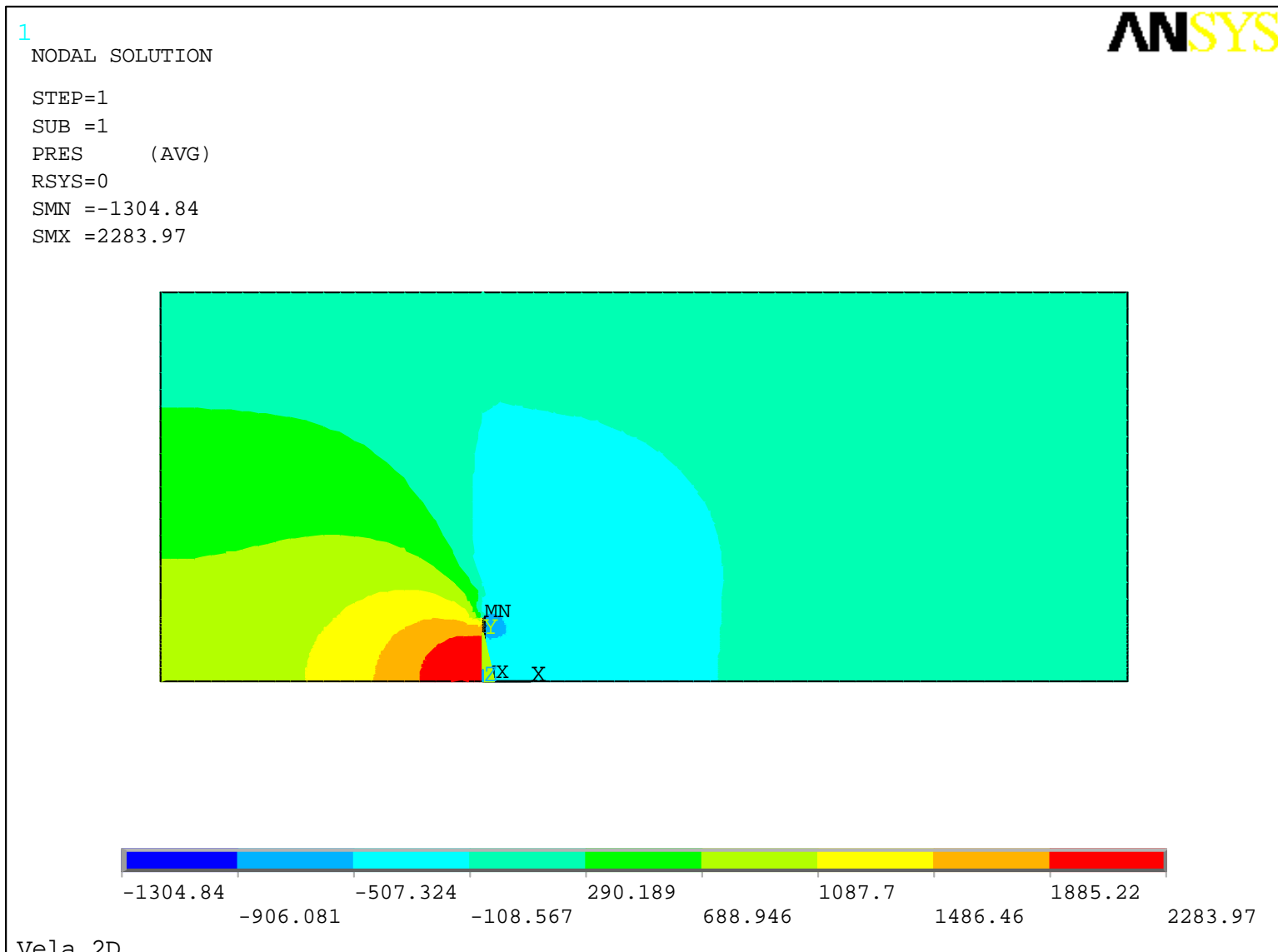

Figura 26 - Valores de pressão do vento (N/m²) 


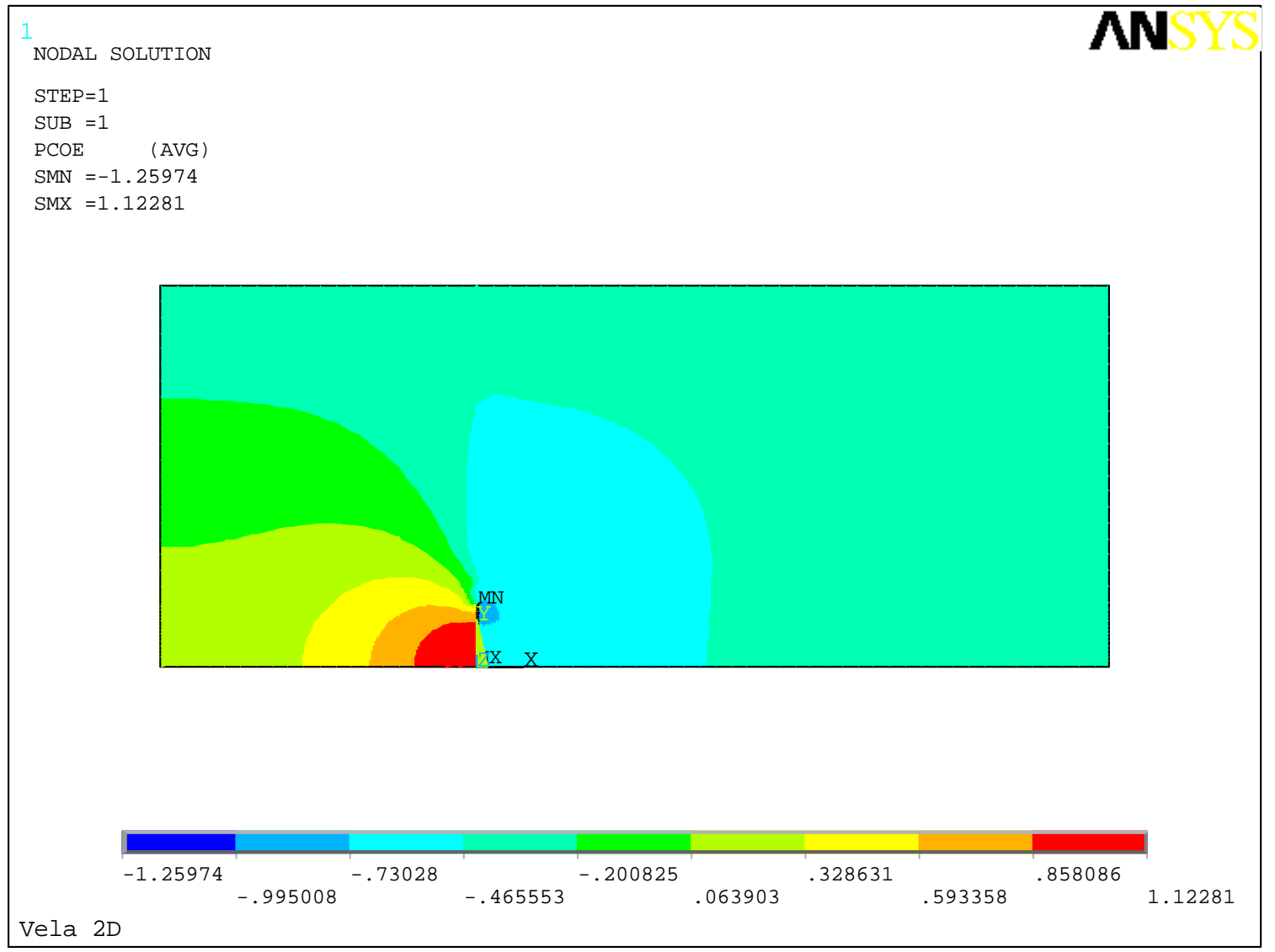

Figura 27 - Coeficientes de pressão do vento

Com exceção do pé do muro, onde os coeficientes de pressão somados ao de sucção se apresentaram maiores $(\mathrm{c} \sim 1,5)$, os valores acima obtidos são compatíveis com os apresentados pela norma NBR 6123 para muros de comprimento infinito $(\mathrm{c} \sim 1,2)$ :

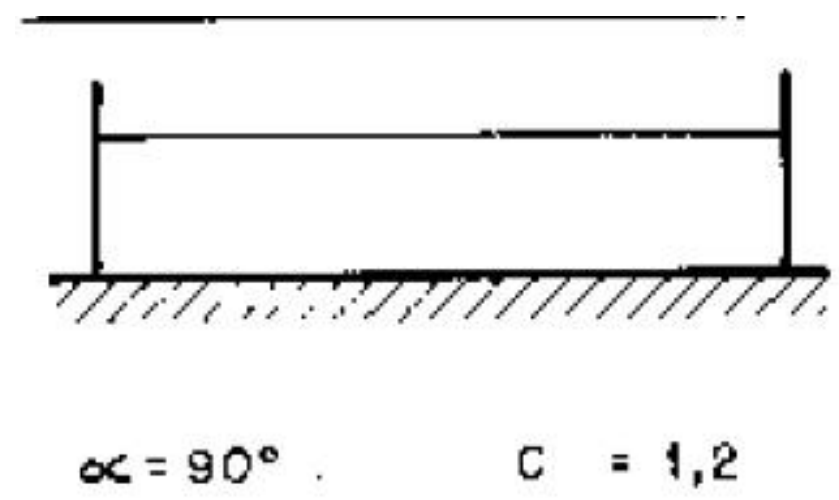




\subsection{Vento sobre Edificação em 3D}

Nesta aplicação foi elaborado um modelo tridimensional, na tentativa de representar uma edificação térrea com cobertura em duas águas, com dimensões típicas de uma residência unifamiliar popular, com ação de vento de $40 \mathrm{~m} / \mathrm{s}$ conforme benchmark disponível em MANFRIM (2006).

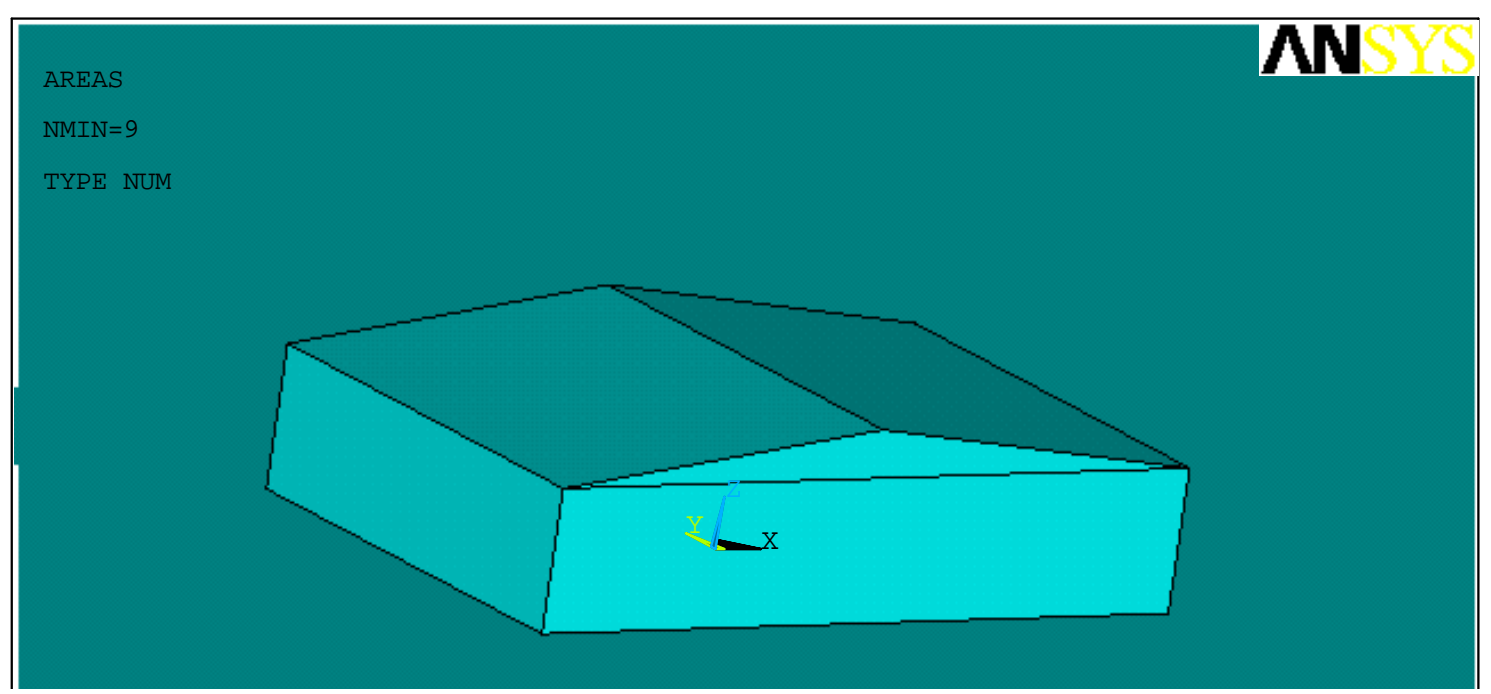

Figura 28 - Geometria da edificação modelada

Nesta aplicação a edificação está envolvida em um volume de controle que é definido de acordo com dimensões da edificação. Segundo MANFRIM (2006), com uma relação entre volume de controle e edificação maior que 3, os coeficientes de pressão não sofreram influência do volume de controle. Assim, o volume de controle foi discretizado fazendo-se uso de elementos triangulares, com densidade maior próximo à edificação.

Esta modelagem contém 19018 nós e 101347 elementos conforme ilustrado a seguir: 


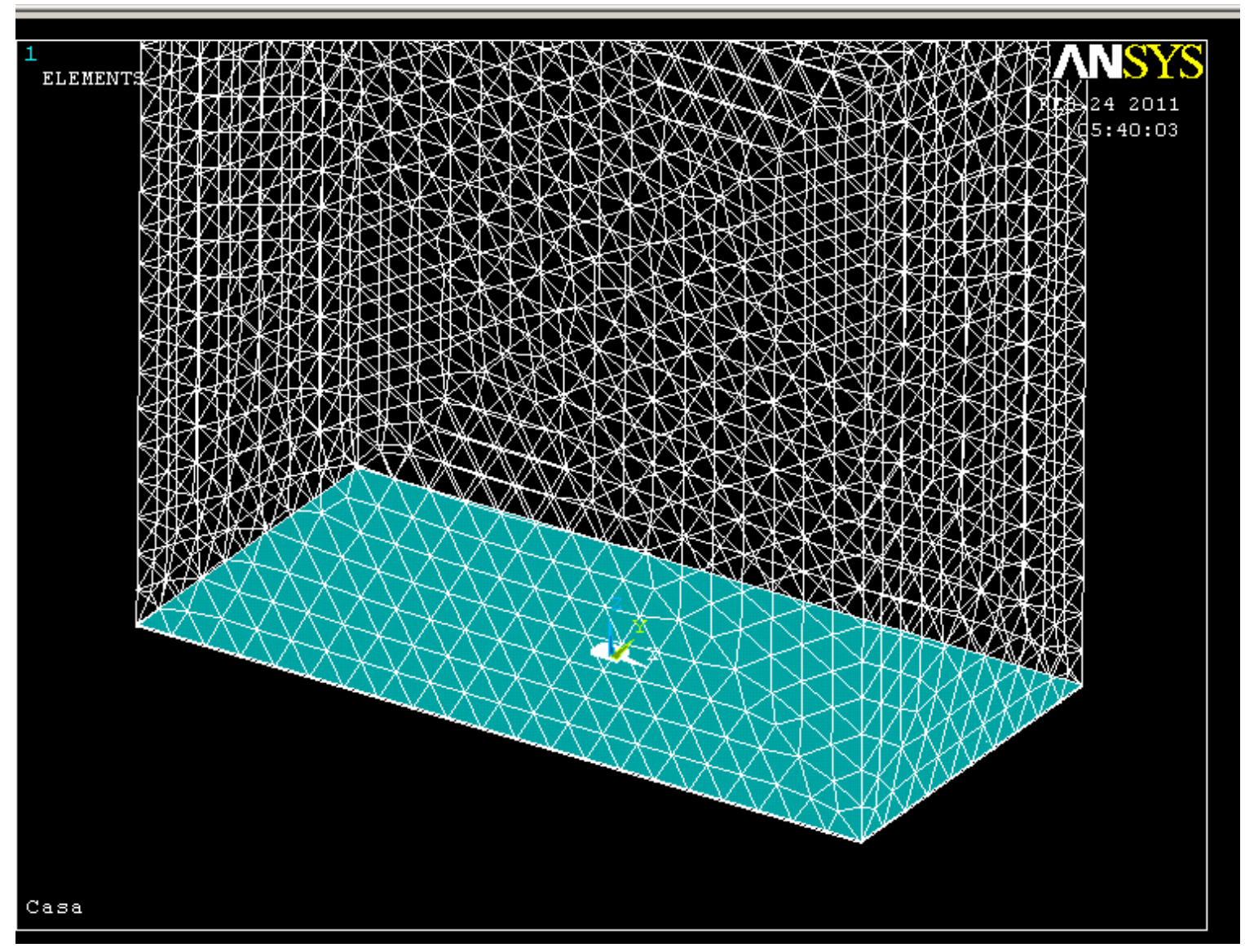

Figura 29 - Malha para Residência em 3D

As condições de contorno neste exemplo são de:

- velocidade de entrada $\mathrm{Vx}=40 \mathrm{~m} / \mathrm{s}$ na superfície à barlavento;

- condição de não deslizamento da superfície inferior, atribuindo-se velocidades $\mathrm{Vy}=\mathrm{Vz}=0$;

- pressão nula nas superfícies superior, laterais e à sotavento. 


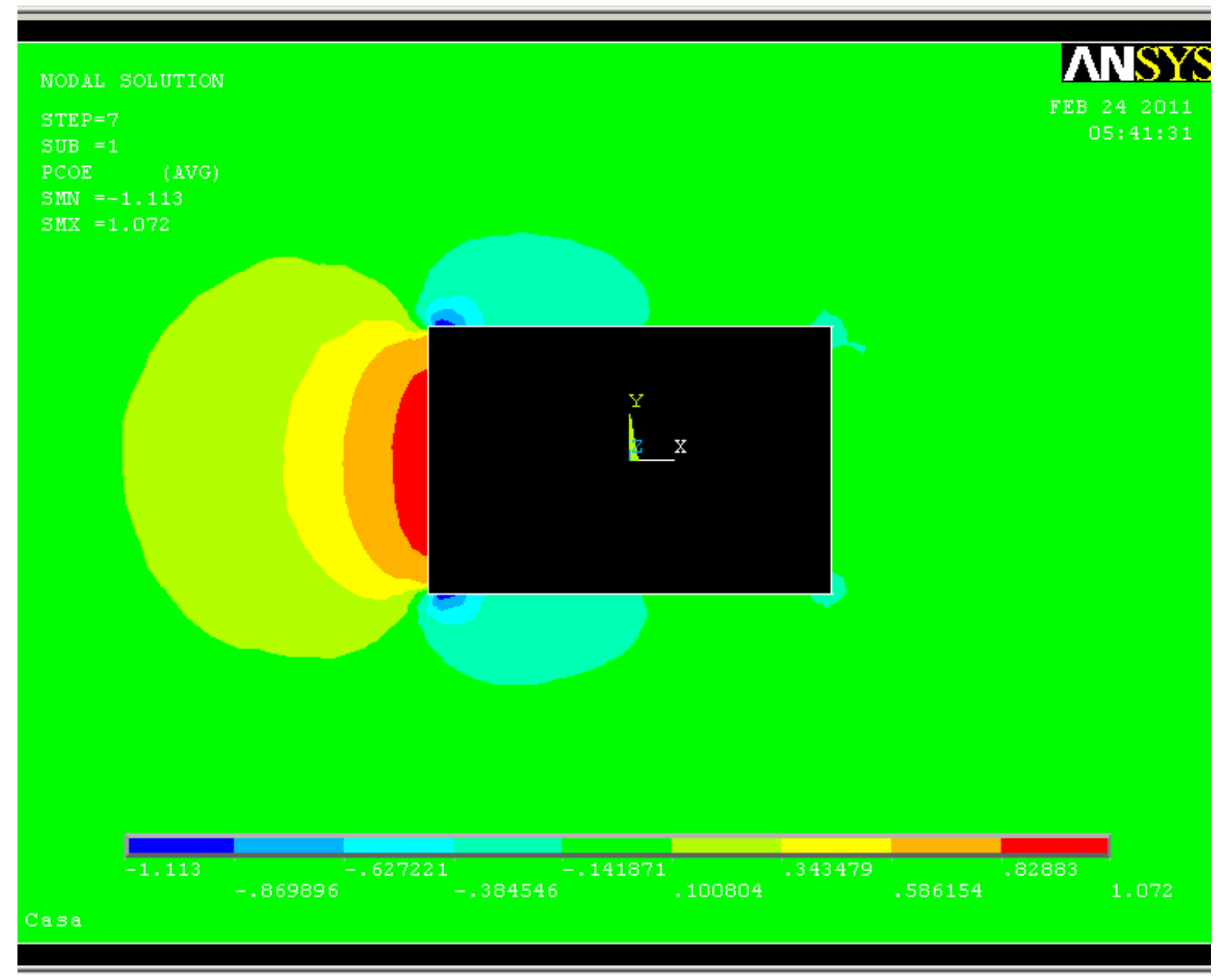

Figura 30 - Coeficientes de Pressão em Plano próximo ao Piso 


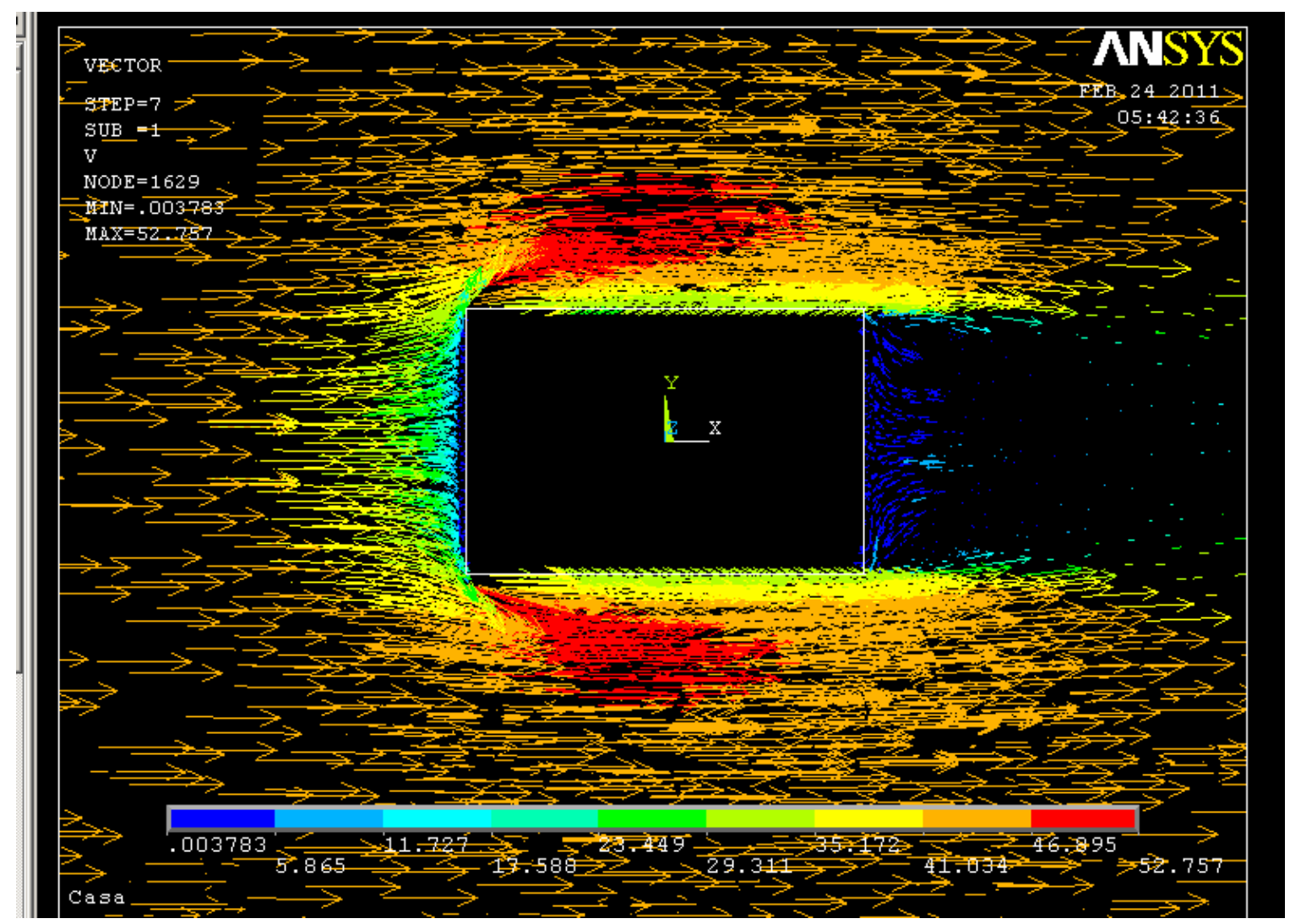

Figura 31 - Valores de Velocidade do Vento próximo ao nível do Piso (m/s)

Os valores obtidos são coerentes com os coeficientes de pressão apresentados em Norma, bem como com os resultados de aplicação apresentada por MANFRIM (2006), exceto por pequenas variações decorridos em pontos de transição entre parede e telhado e parede e piso, ocorridos por razões de simplificações de norma. 


\subsection{Parabolóide Hiperbólico}

Nesta aplicação será apresentada estrutura de membrana com tecido recoberto com PVC, considerado isotrópico. Para a busca da forma foram aplicadas tensões de retesamento iniciais consistentes com a Classe de Resistência e espessura de membrana adotadas.

\subsubsection{Busca da Forma}

A forma da estrutura inicial foi arbitrada com projeção horizontal quadrada, já em configuração de equilíbrio. Através dos quatro pontos representados abaixo, foram determinadas 4 retas que definiram as bordas da membrana. Os pontos 1 e 3 foram elevados a 2,5m de altura e os pontos 2 e 4 foram estabelecidos na cota $0,5 \mathrm{~m}$. O modelo levou em conta o fechamento lateral (vedação) em todo perímetro.

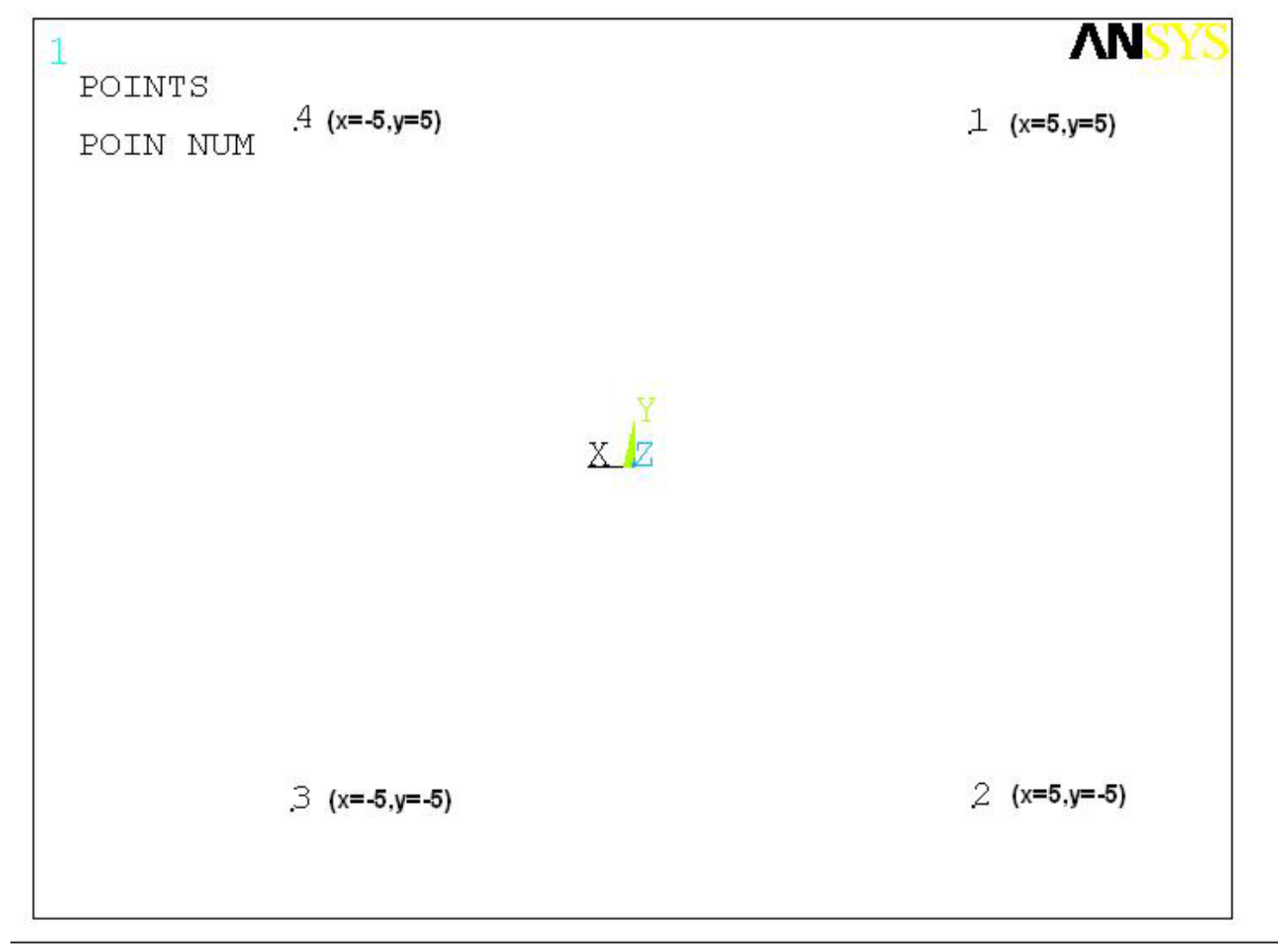

Figura 32 - KeyPoints Básicos para Projeto do Parabolóide 


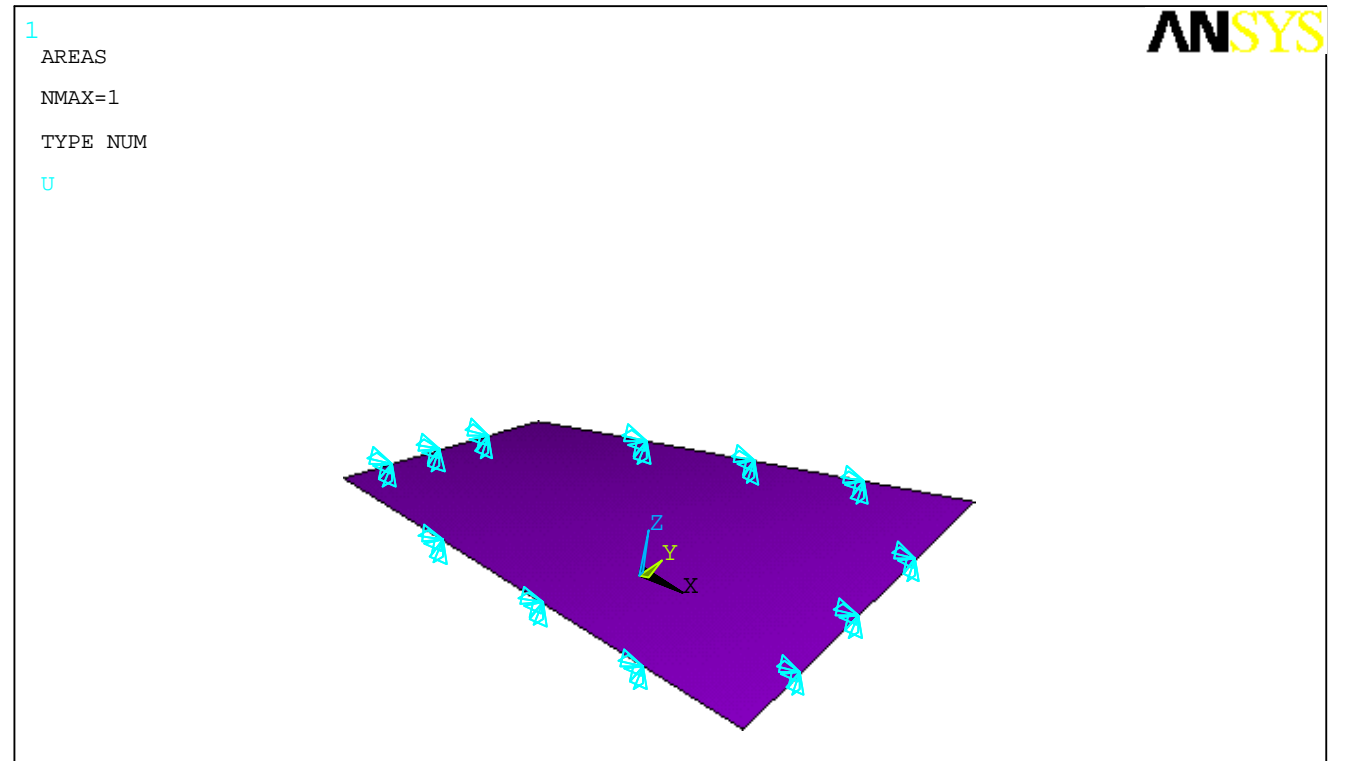

Figura 33 - Superfície Inicial gerada pelo Software

A malha de elementos finitos foi gerada através de uma área mapeada, fazendo-se uso de 800 elementos triangulares e 441 nós.

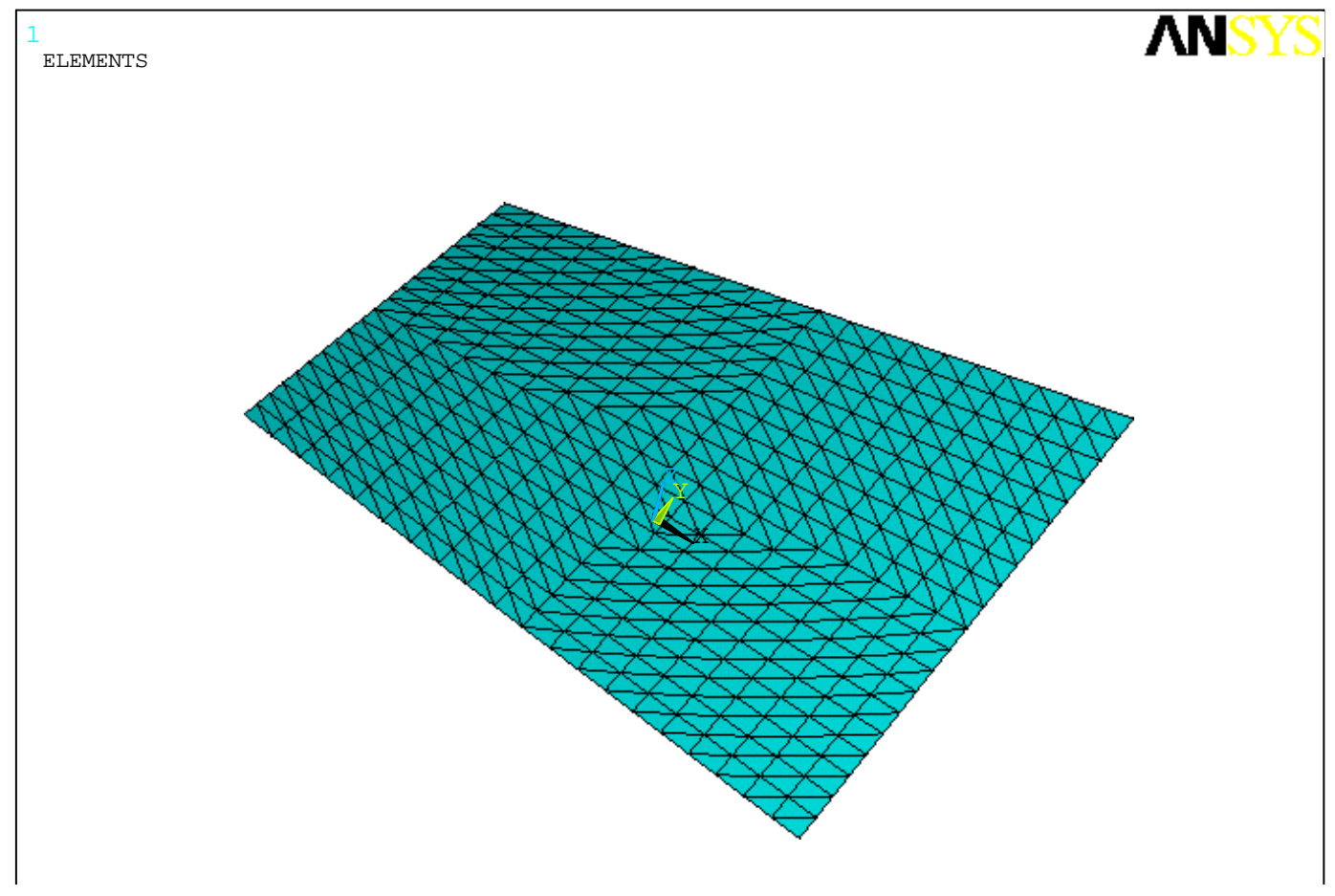

Figura 34 - Malha inicial de elementos finitos 
Conforme apresentado, as curvaturas são idênticas nas direções de cumeeira e talvegue da membrana. Em conseqüência, ao se impor um campo de tensões iniciais uniforme, os deslocamentos apresentaram-se muito pequenos em comparação com a configuração inicial. Observação: no primeiro processo de busca da forma foi utilizado um módulo de elasticidade mais baixo que o usual das membranas de $\mathrm{PVC}$, de forma a concluir a busca da forma mais rapidamente.

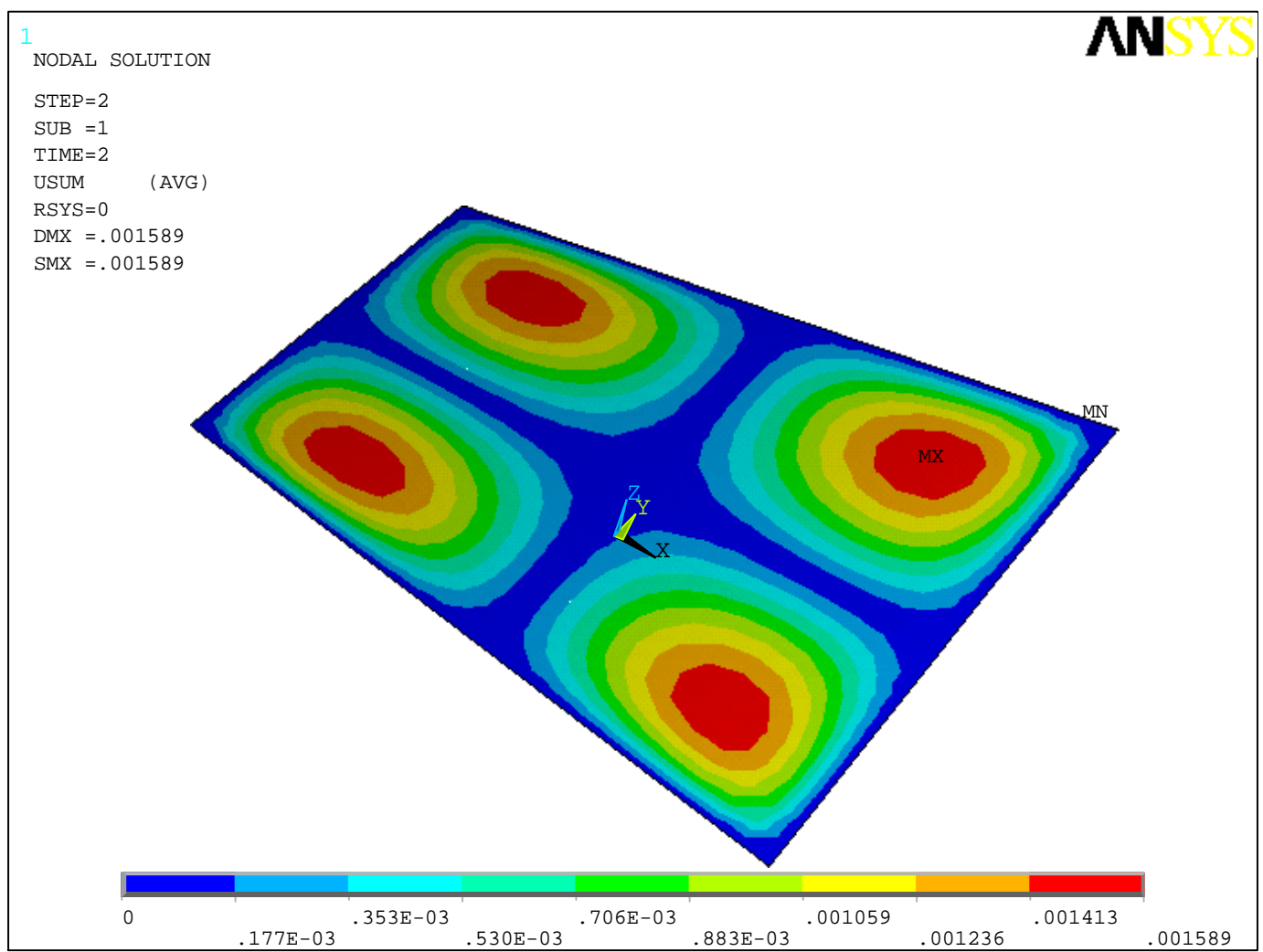

Figura 35 - Deslocamentos provocados pela condição inicial de retesamento (m)

Visto os deslocamentos provocados pelo retesamento inicial serem desprezáveis (menos de $2 \mathrm{~mm}$ ), não foram processadas novas iterações, apenas atualizou-se o módulo de elasticidade.

Com isto, atualizou-se a malha de elementos para a configuração deformada (busca da forma), para então serem aplicados os carregamentos de vento. 


\subsubsection{Ação do Vento}

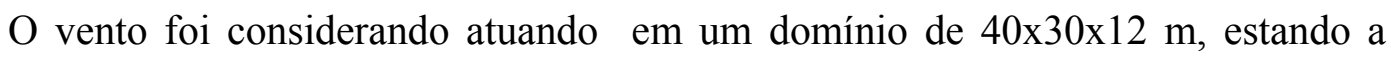
entrada de ar à $20 \mathrm{~m}$ do centróide do parabolóide. Este domínio foi discretizado com elementos tridimensionais, que juntamente com a membrana somou 19239 elementos e 4089 nós.

Aplicando-se vento $\mathrm{Vx}$ de $50 \mathrm{~m} / \mathrm{s}$ na superfície de barlavento, e considerando-se vedação total sob a membrana (fechamento lateral) e malha de elementos da estrutura de membrana como indeslocáveis e indeformáveis, obteve-se os seguintes resultados:

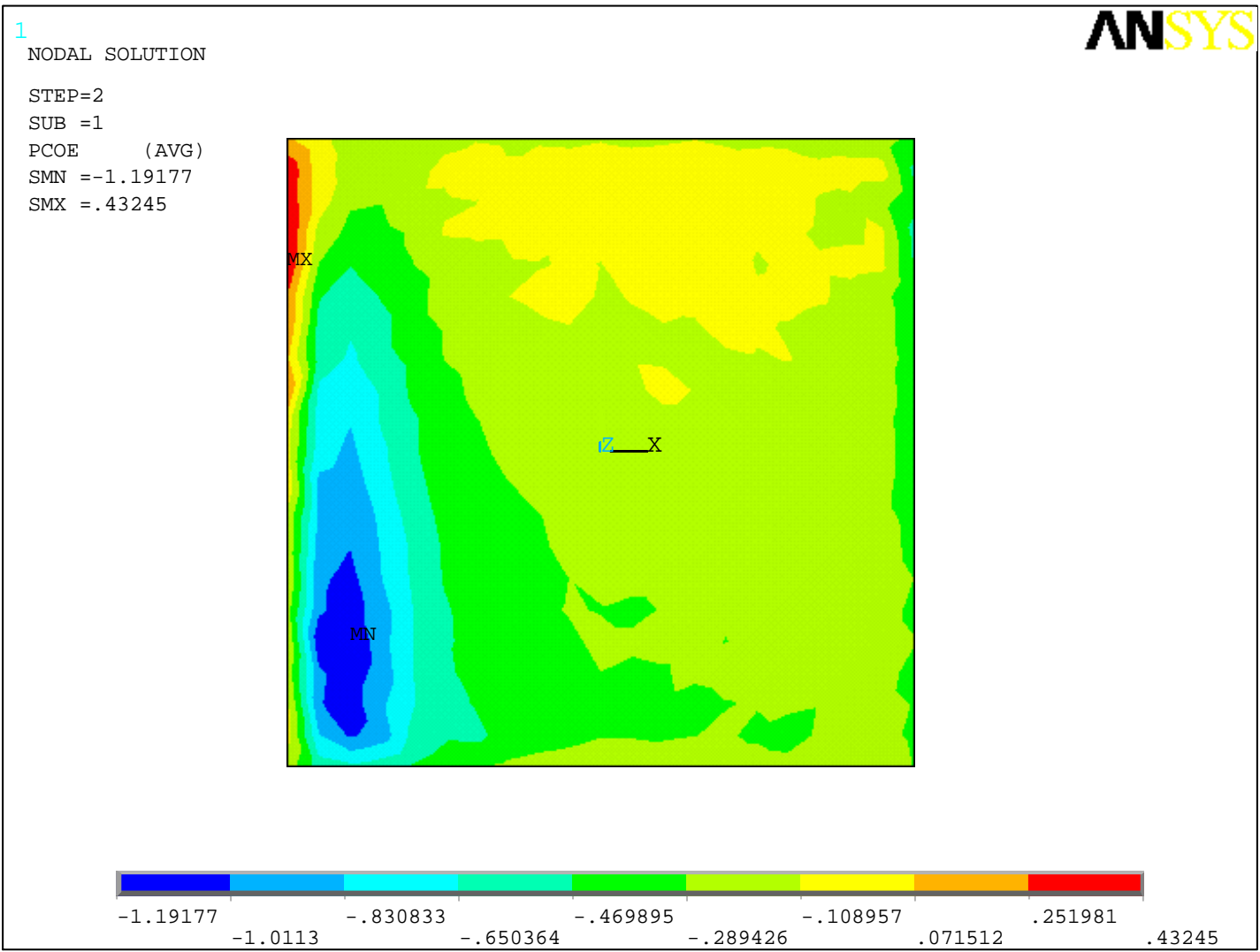

Figura 36 - Coeficientes de pressão sobre a membrana em $1^{\text {a }}$ FSI 


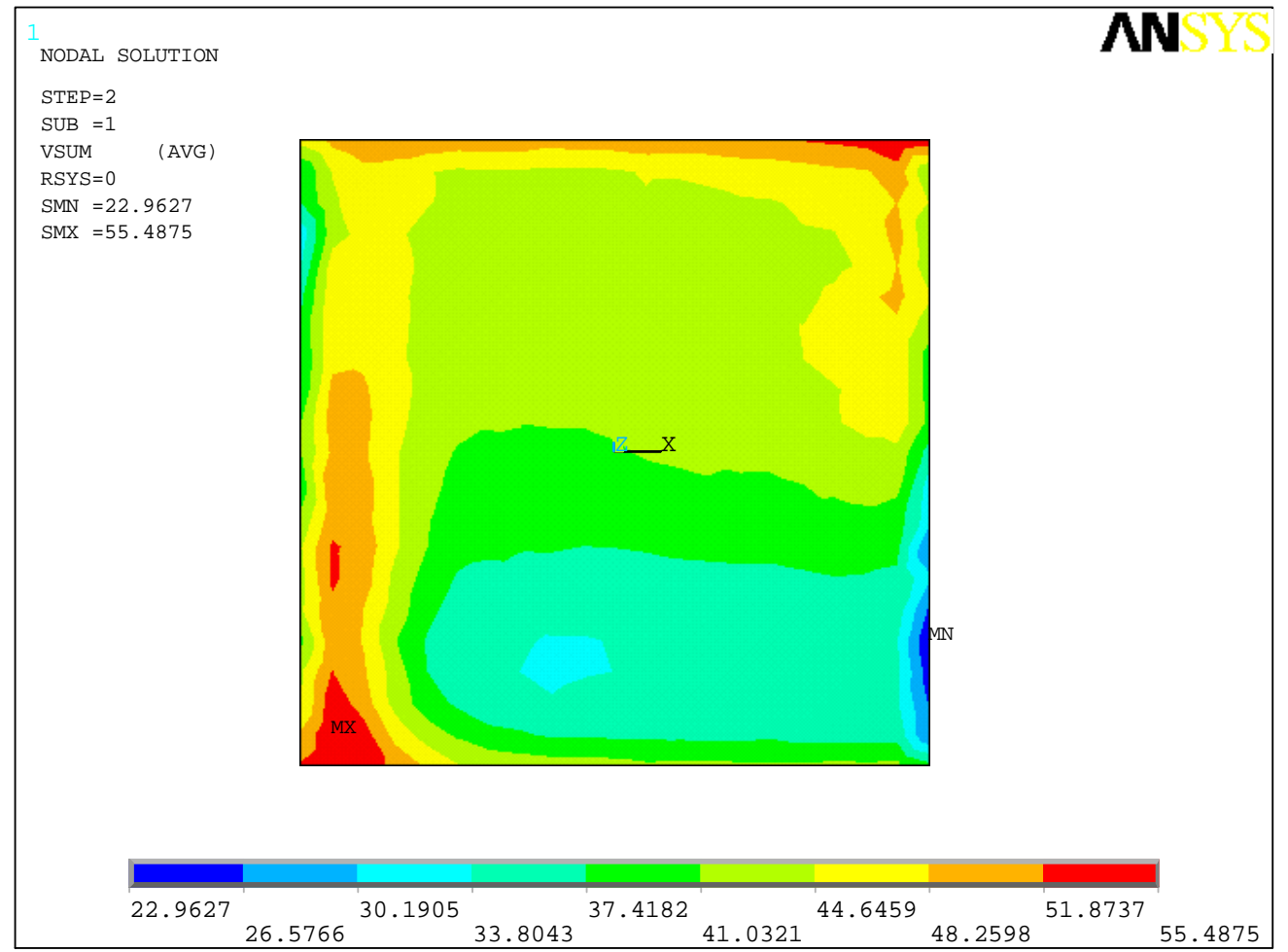

Figura 37 - Velocidades máximas de vento no entorno da membrana (m/s)

Com estes dados, aplicou-se as pressões de vento na membrana, calculando-se os seguintes deslocamentos:

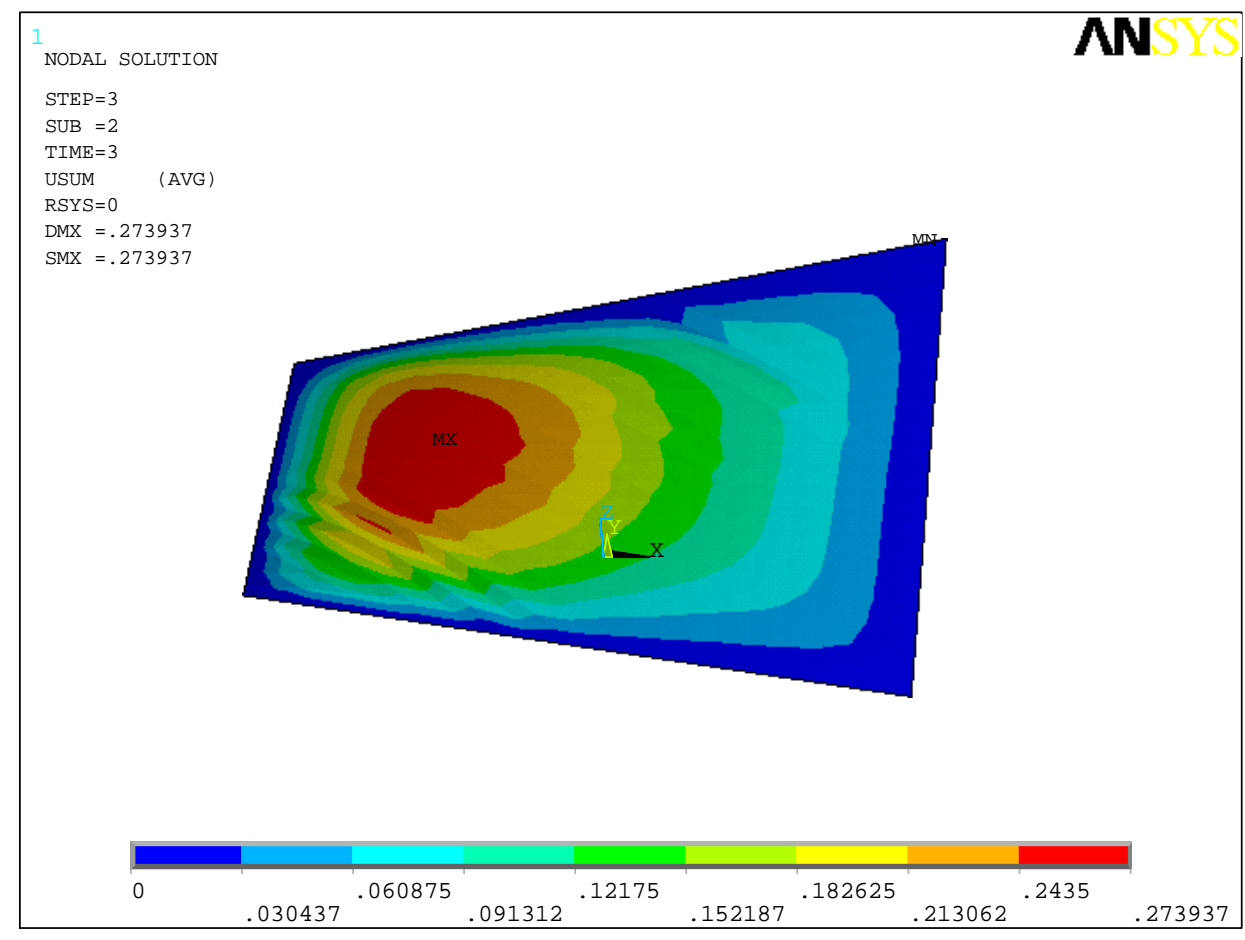

Figura 38 - Norma dos deslocamentos na $1^{\mathrm{a}}$ iteração (m) 


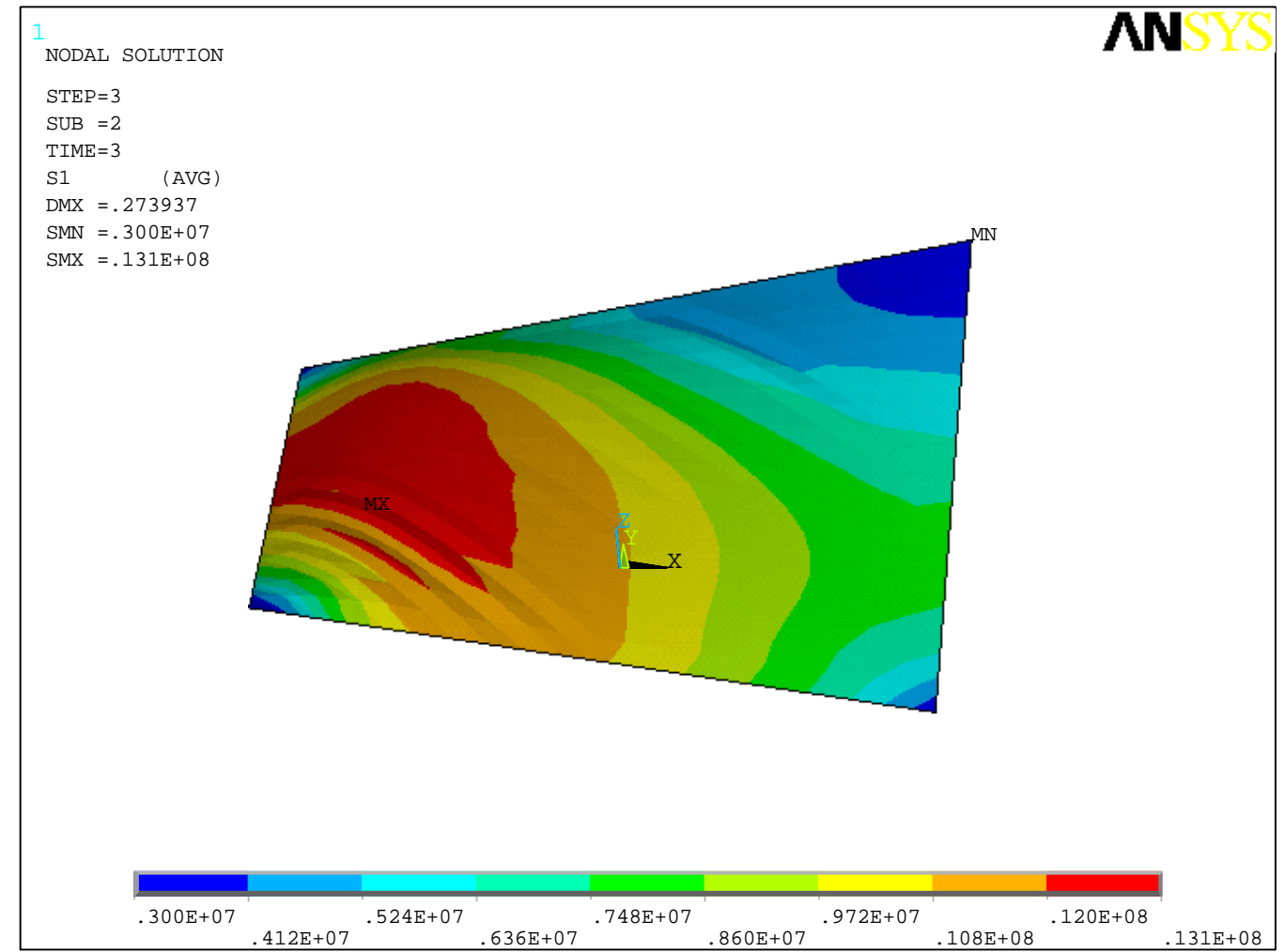

Figura 39 - Tensões principais devido ao vento em $1^{\mathrm{a}}$ iteração $\left(\mathrm{N} / \mathrm{m}^{2}\right)$

Nesta etapa, o engenheiro estrutural poderia avaliar a necessidade de aumento no retesamento da membrana, de forma a evitar instabilidades. Neste trabalho, o retesamento inicial adotado será mantido para efeitos de continuidade com o acoplamento parcial.

Finalmente, os deslocamentos apresentados acima foram atualizados como configuração inicial de um sistema para nova análise de fluido-dinâmica: 


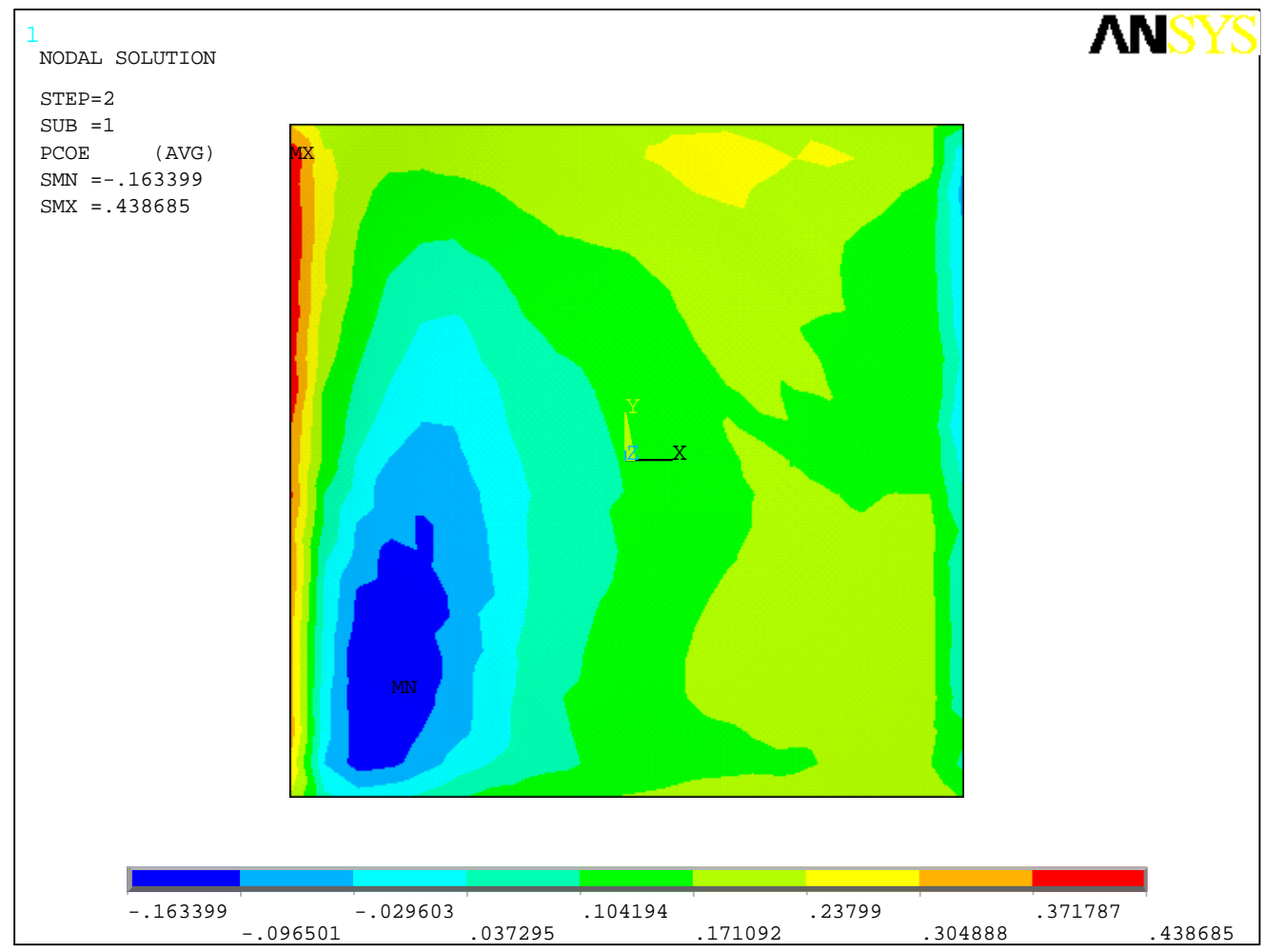

Figura 40 - Coeficientes de pressão sobre a membrana na $2^{\mathrm{a}}$ interação

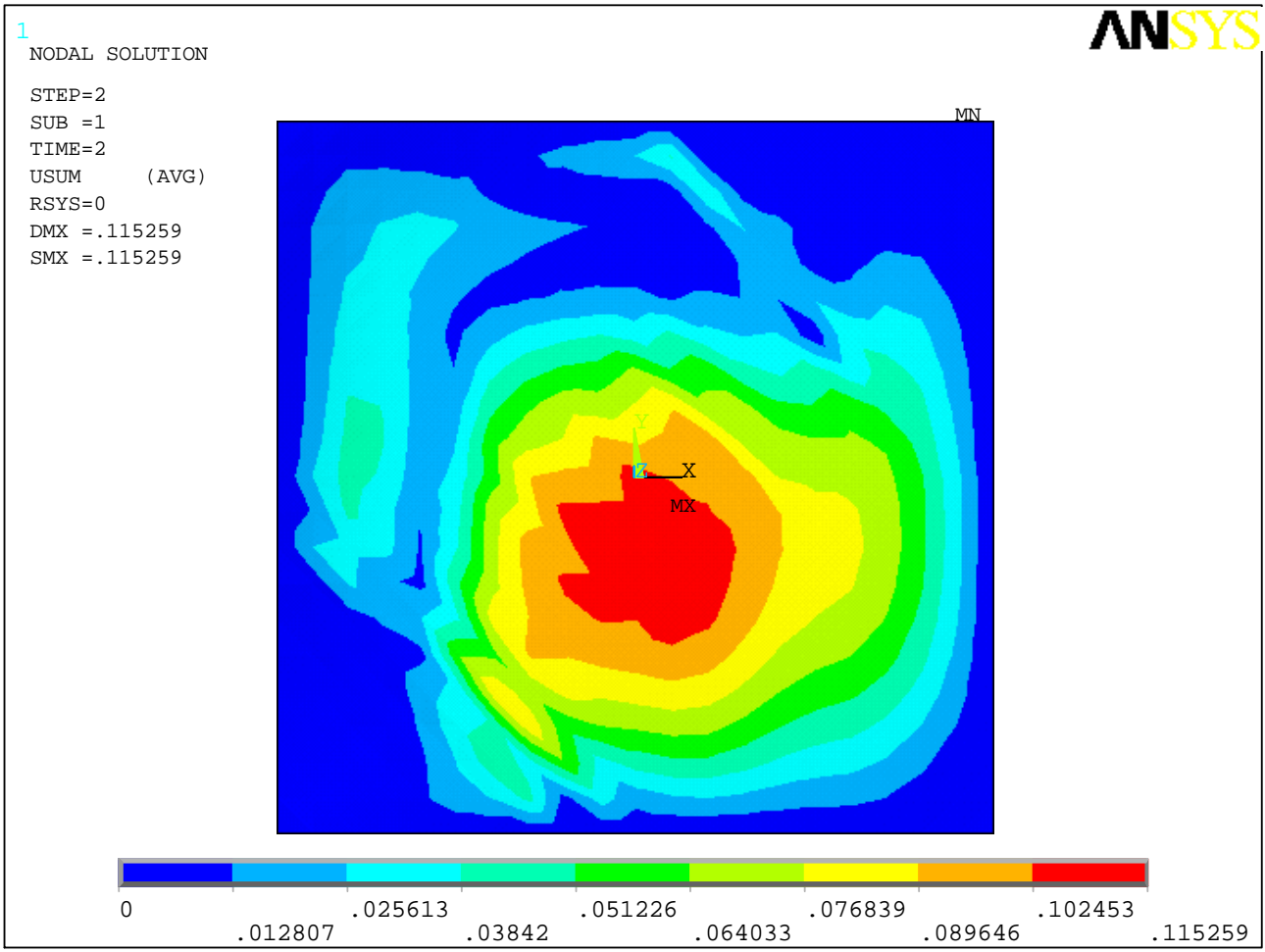

Figura 41 - Norma dos deslocamentos da membrana na $2^{\mathrm{a}}$ interação (m) 


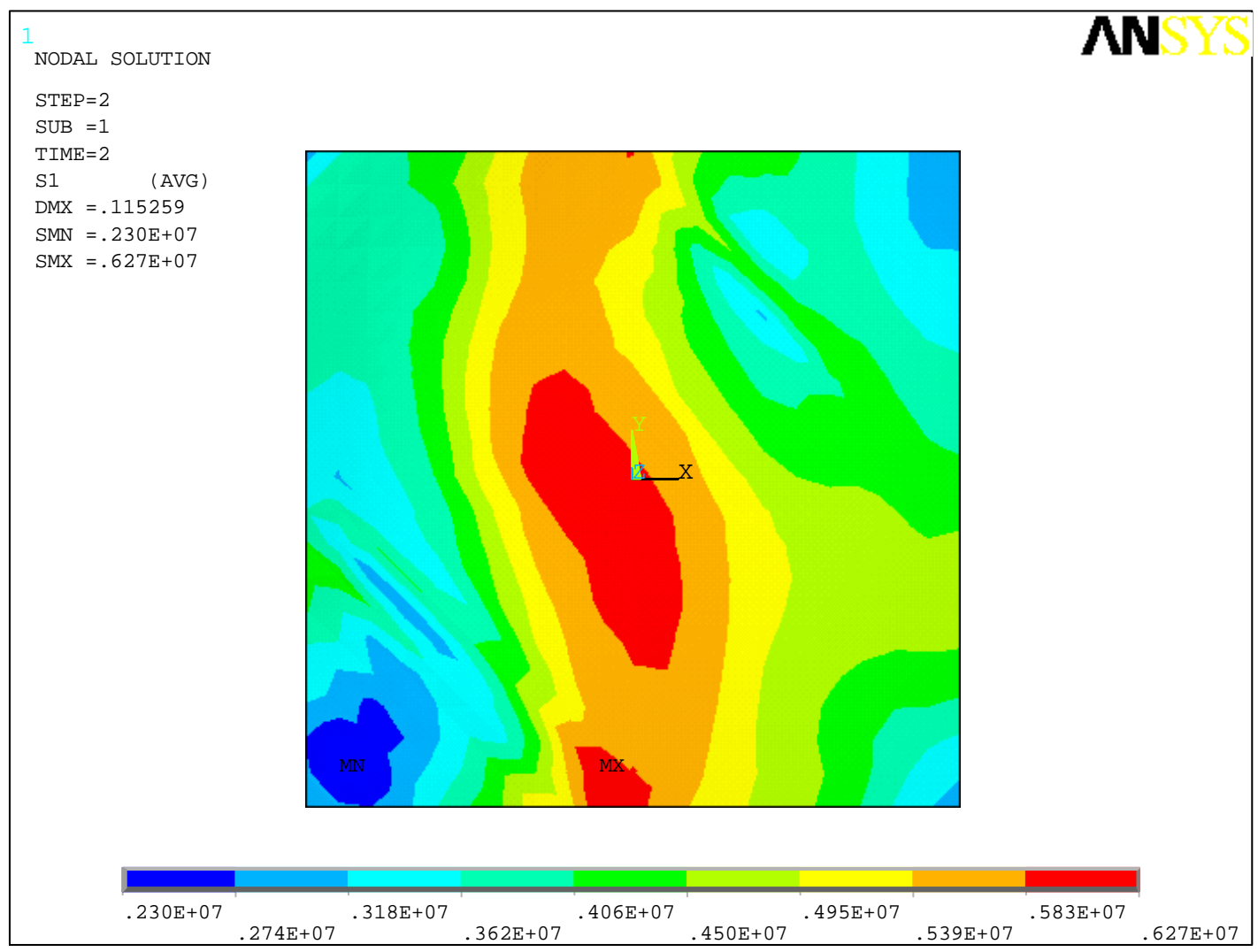

Figura 42 - Tensões principais da membrana na $2^{\mathrm{a}}$ interação (N/m²)

Observando os resultados acima, pode-se perceber que os coeficientes de pressão alteraram significativamente, tendo para sucção máxima $\mathrm{c}=-0,16$ enquanto na primeira interação tínhamos -1,20. Além disto, os deslocamentos máximos foram significativamente menores. Isto ocorreu porque os deslocamentos de sucção na $2^{\mathrm{a}}$ interação foram mais importantes no centro da membrana, enquanto na $1^{\mathrm{a}}$ interação mais próximo a barlavento. Fato decorrente do comportamento estrutural da membrana mencionado em capítulo anterior, havendo acomodação de deformações para uma configuração equilibrada.

Atualizando o modelo com esta configuração deformada, processou-se então a $3^{\text {a }}$ interação. 


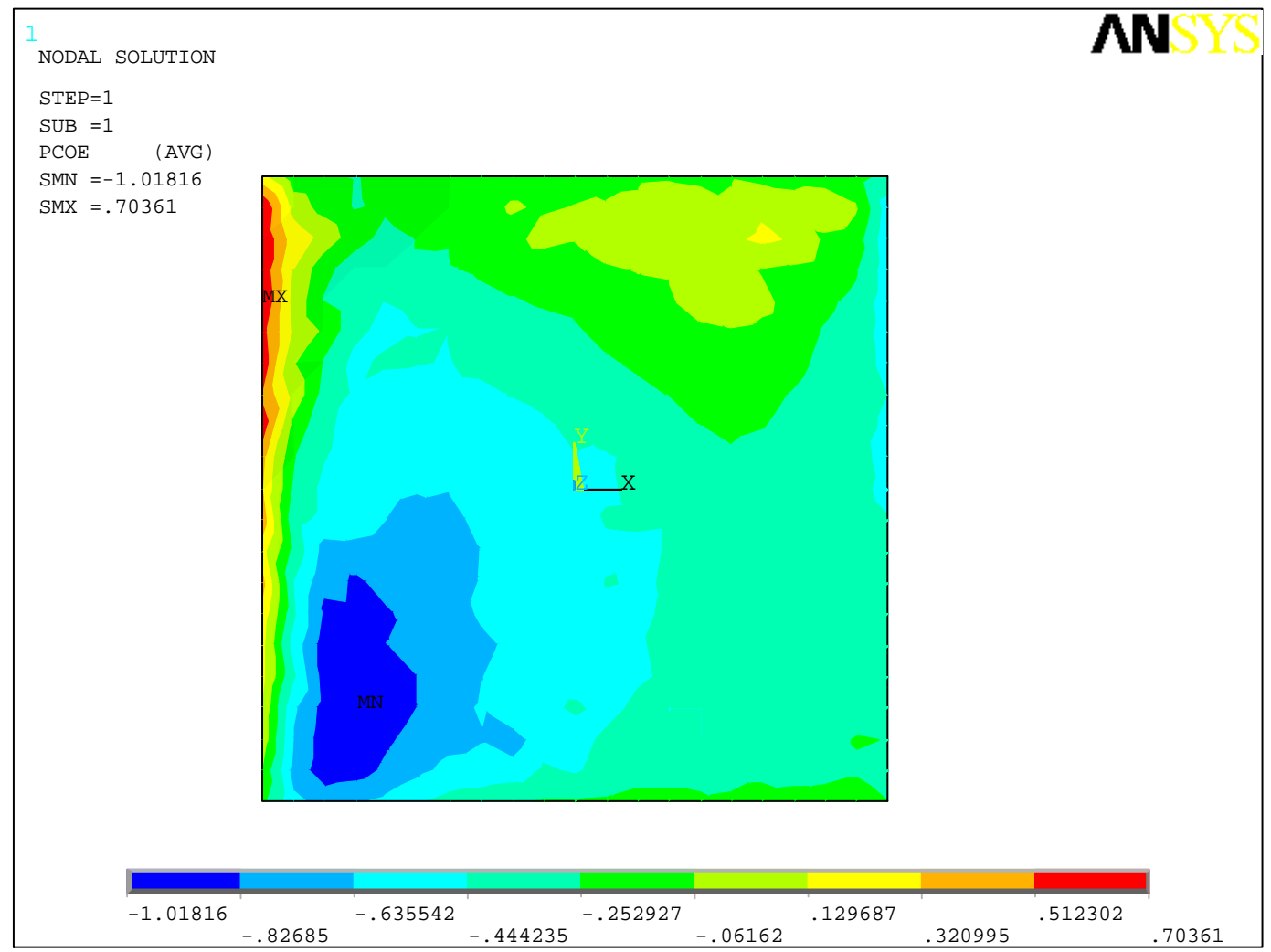

Figura 43 - Coeficientes de pressão sobre a membrana na $3^{\mathrm{a}}$ interação

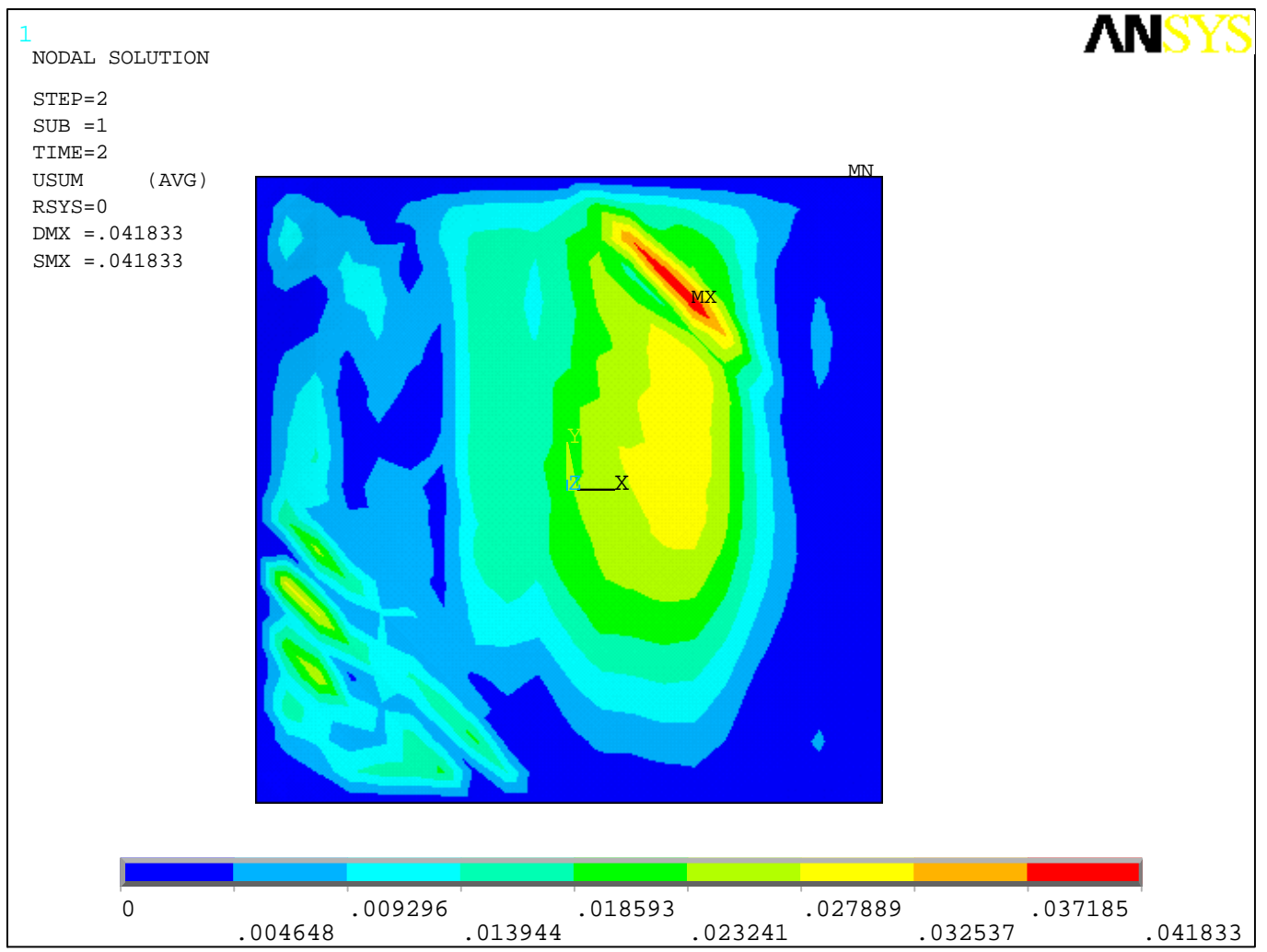

Figura 44 - Norma dos deslocamentos da membrana na $3^{\text {a }}$ interação (m) 


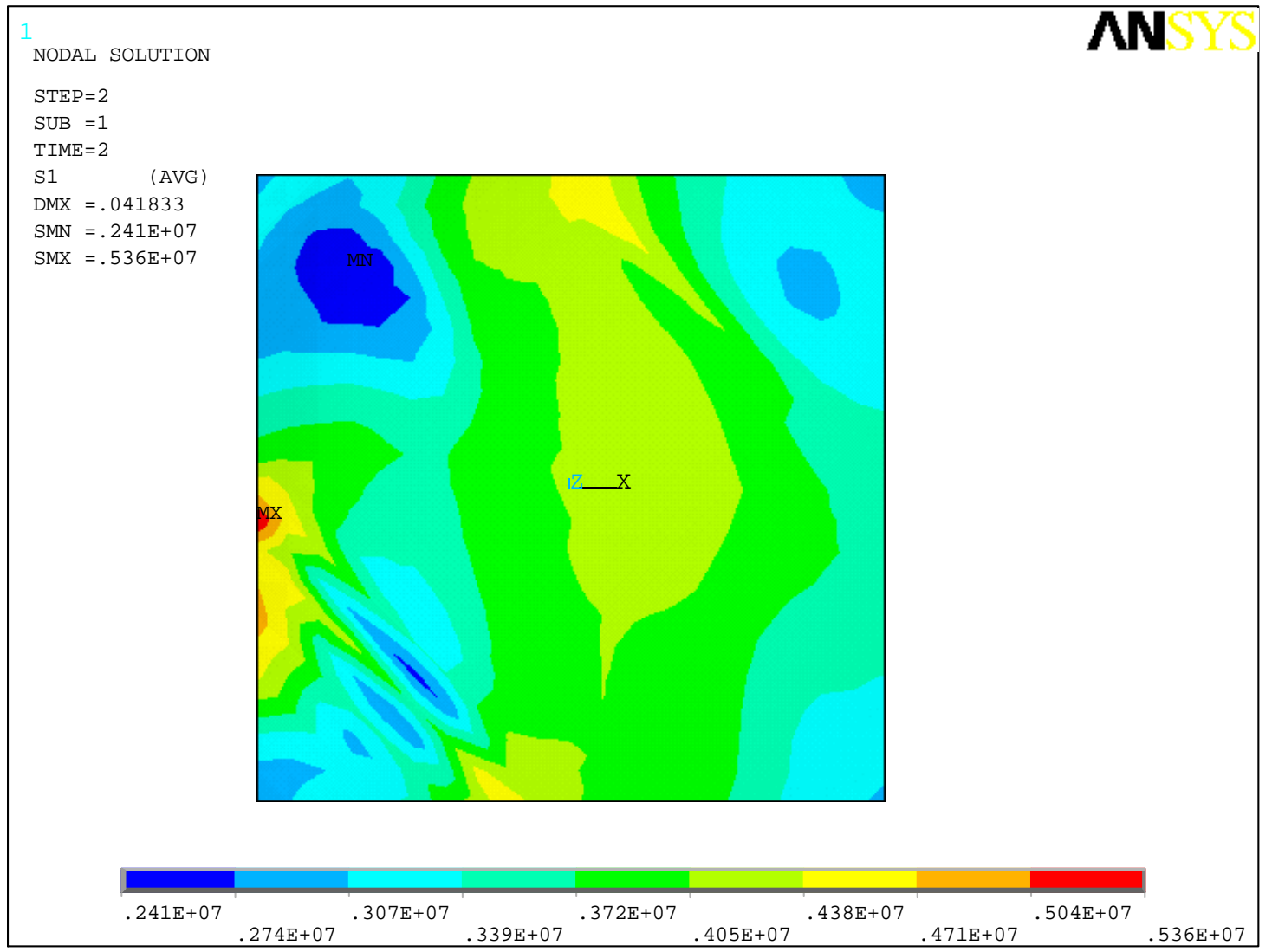

Figura 45 - Tensões principais da membrana na $3^{\mathrm{a}}$ interação $\left(\mathrm{N} / \mathrm{m}^{2}\right)$

Ao longo das três interações, pode-se observar uma variação no sinal dos coeficientes de pressão, principalmente próximo ao vértice inferior esquerdo. Na primeira interação, a forma da membrana decorria em uma região de baixa pressão, havendo sucção e formando-se uma "corcunda". Esta geometria transformou a região para zona de pressão. Finalmente, na última interação, tivemos novamente sucção. Apesar deste efeito, observouse campos de tensões mais homogêneos e tensões menores que nas primeiras interação. Com esta tendência, julgou-se que não seriam necessárias novas interações para definir o dimensionamento da estrutura.

Esta aplicação sugere que, apesar de a mudança de forma da membrana alterar o escoamento e por conseqüência os coeficientes de pressão, a consideração de membranas como estruturas indeformáveis e indeslocáveis para o carregamento de vento é favorável a segurança. 
Caso o engenheiro estrutural decida alterar a espessura ou retesamento inicial da estrutura, de forma a se obter um campo mais uniforme de tensões, pode-se aproveitar os coeficientes de pressão da primeira interação e processar novamente os subseqüentes. 


\subsection{Conóide}

Nesta aplicação será apresentada uma estrutura de membrana conoidal, também de tecido recoberto com PVC e considerado isotrópico.

\subsubsection{Busca da Forma}

A forma da estrutura inicial foi arbitrada como um tronco de cone com diâmetro de base de $10 \mathrm{~m}$ e de topo $2,5 \mathrm{~m}$. A altura adotada foi de $3 \mathrm{~m}$.

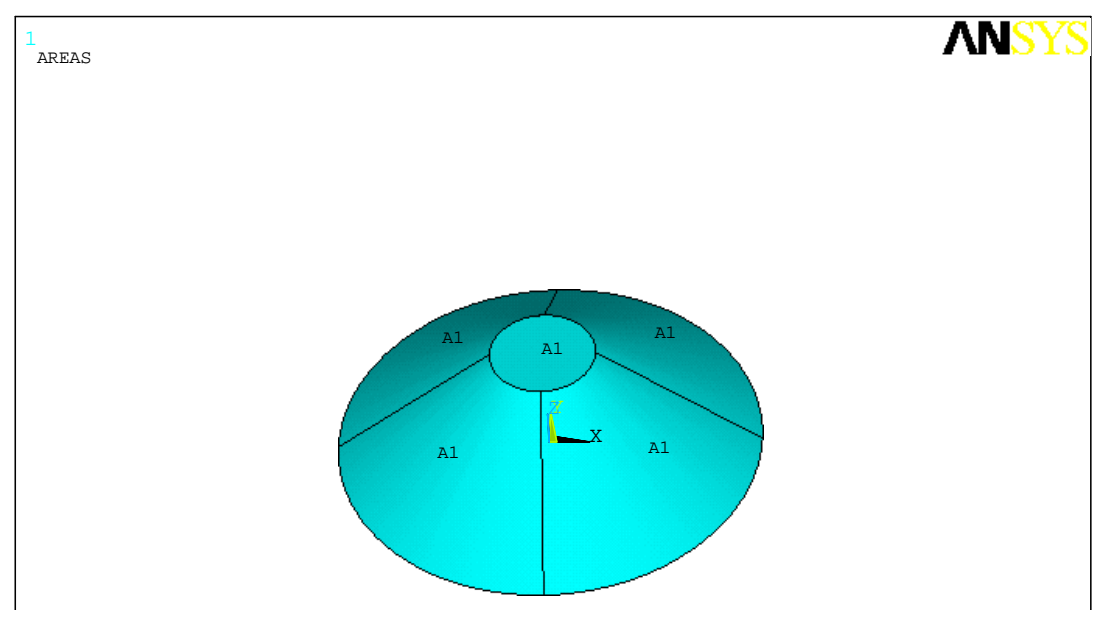

Figura 46 - Geometria inicial adotada para o conóide

Neste tronco de cone, foi lançada uma malha de elementos finitos triangulares na área de contorno, fazendo-se uso de 2400 elementos triangulares e 1260 nós. 


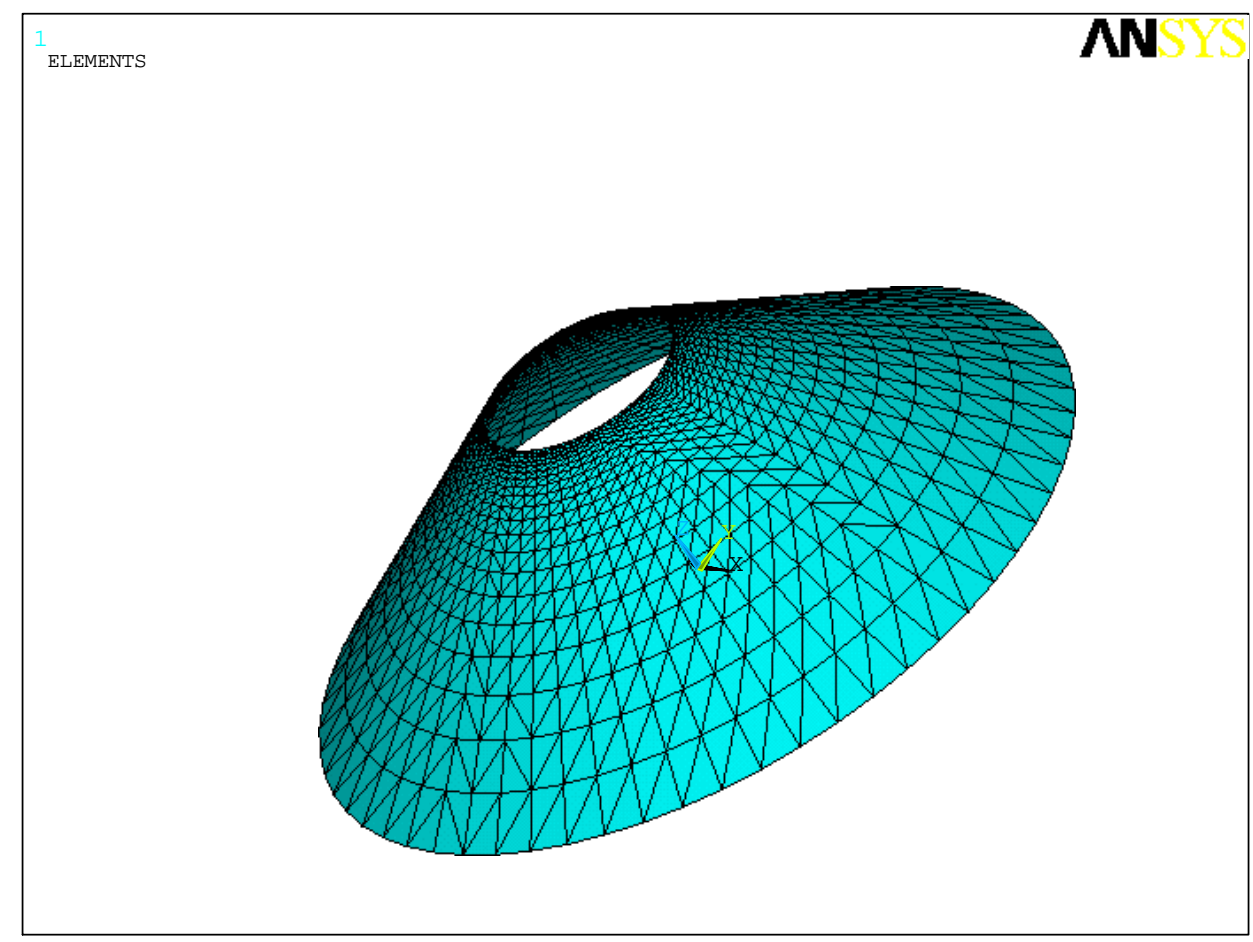

Figura 47 - Malha de elementos finitos do conóide

Abaixo seguem resultados ao se impor um campo de tensões iniciais uniforme utilizado um módulo de elasticidade mais baixo que o usual das membranas de PVC, de forma a concluir a busca da forma mais rapidamente.:

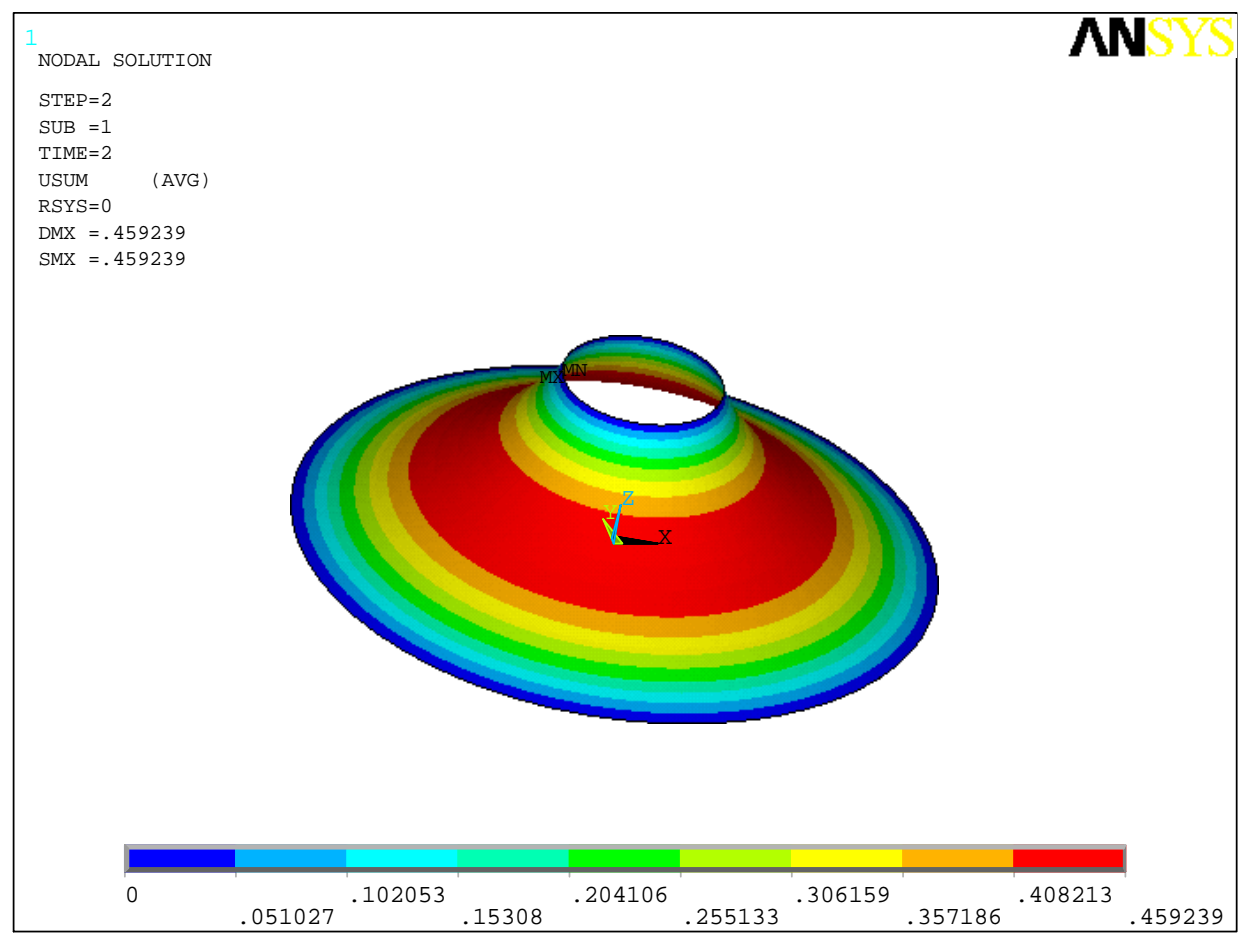

Figura 48 - Norma dos deslocamentos pela condição inicial de retesamento (m) 
Em um segundo processamento para busca da forma, utilizou-se um módulo de elasticidade em concordância com as propriedades de uma membrana de PVC.

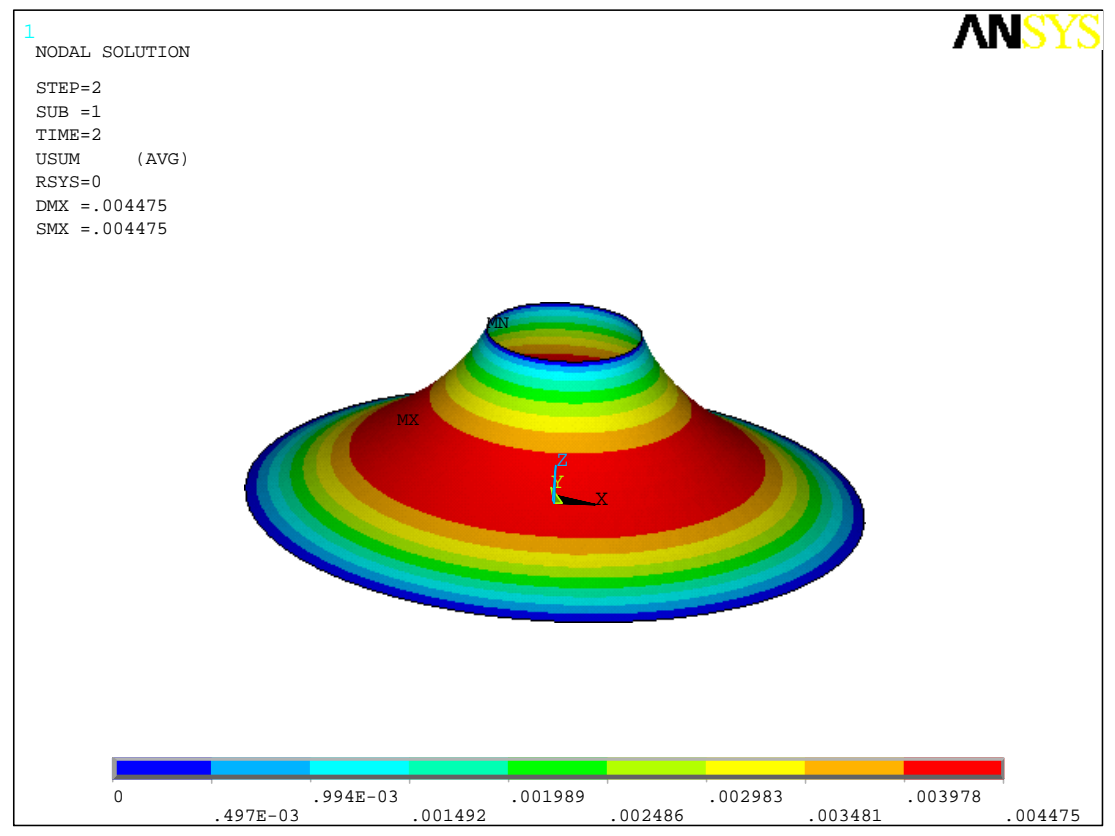

Figura 49 - Norma dos deslocamentos provocados no $2^{\circ}$ processamento da busca da forma (m)

Com deslocamentos inferiores a $1 \mathrm{~mm}$, encerrou-se o processo de busca da forma. Interessante observar que o conóide acima não é uma forma minimal, visto as tensões principais não serem uniformes na superfície da membrana.

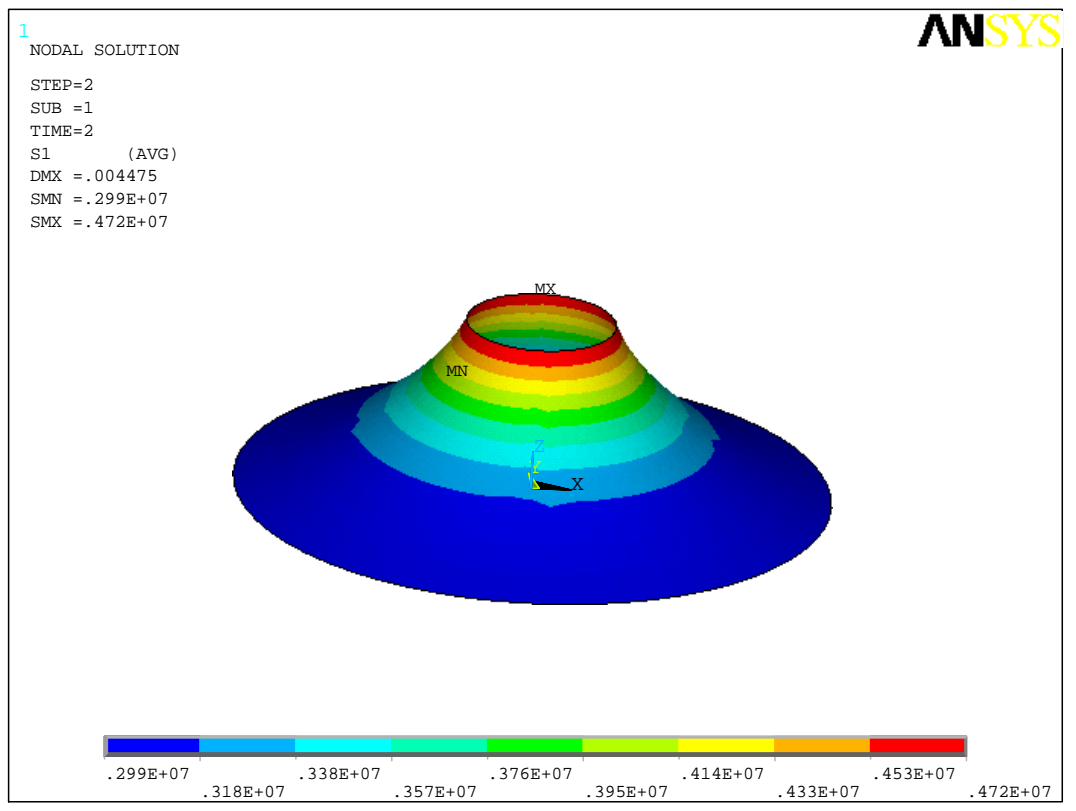

Figura 50 - Tensões principais devido retesamento incial $\left(\mathrm{N} / \mathbf{m}^{2}\right)$

96 


\subsubsection{Ação do Vento}

O domínio de vento adotado foi de um paralelepípedo de 55 × 40 × 15m, estando a entrada de ar à $20 \mathrm{~m}$ do centro do conóide.

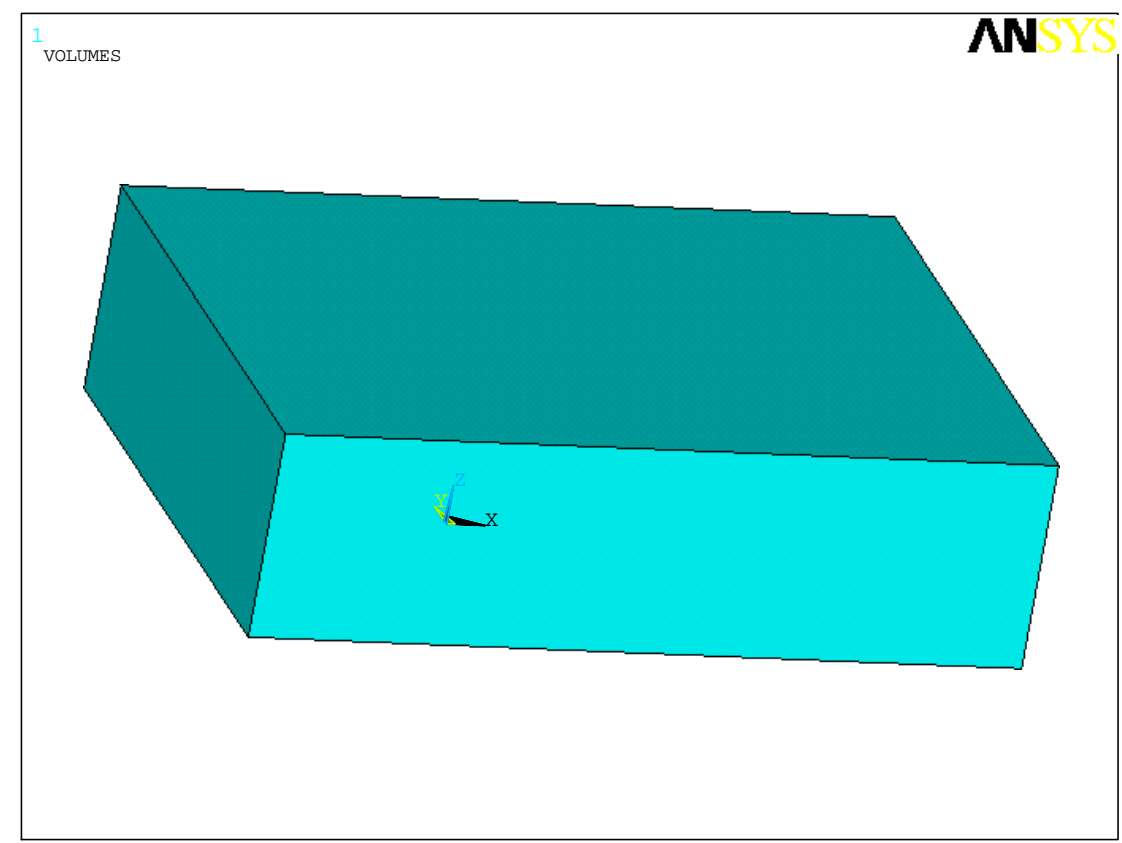

Figura 51 - Domínio de atuação do vento

A modelagem do vento, juntamente com a membrana, totalizou 32413 elementos e 6553 nós.

Aplicando-se vento Vx de $50 \mathrm{~m} / \mathrm{s}$ na superfície de barlavento, e considerando-se vedação total no topo do conóide, obteve-se os seguintes resultados: 


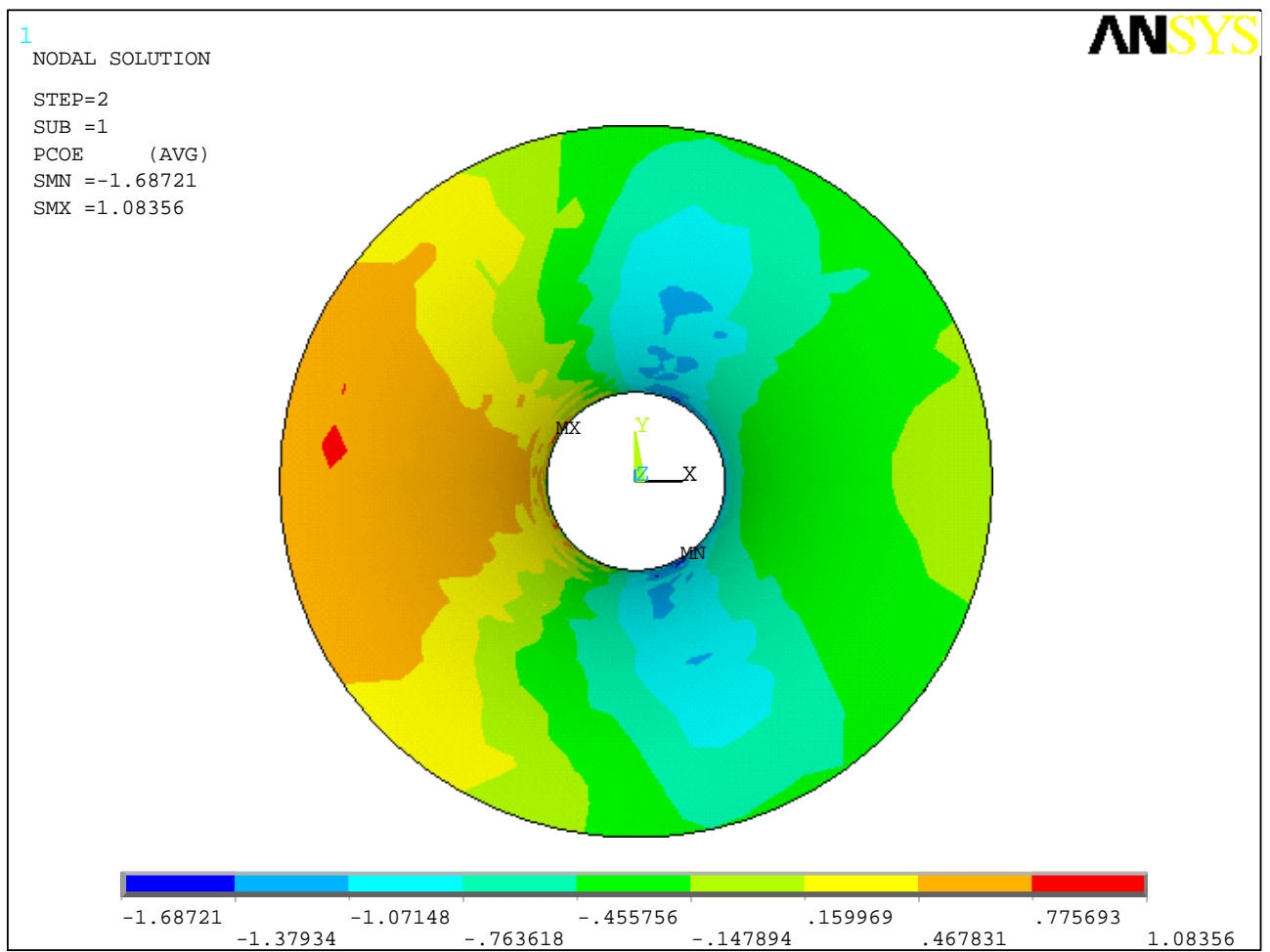

Figura 52 - Coeficientes de pressão sobre a membrana em $1^{\mathrm{a}}$ interação

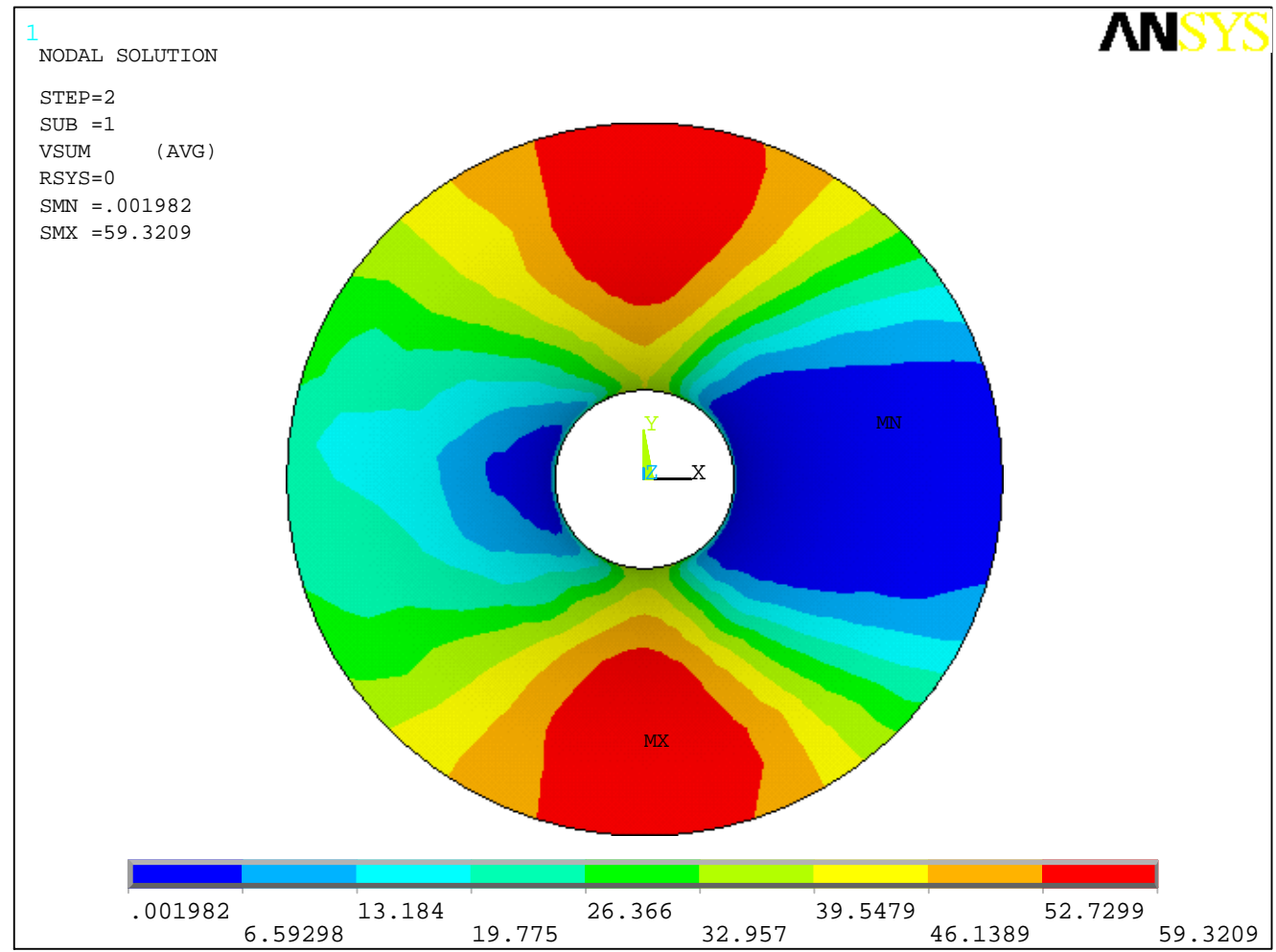

Figura 53 - Velocidades máximas de vento no entorno da membrana (m/s) 


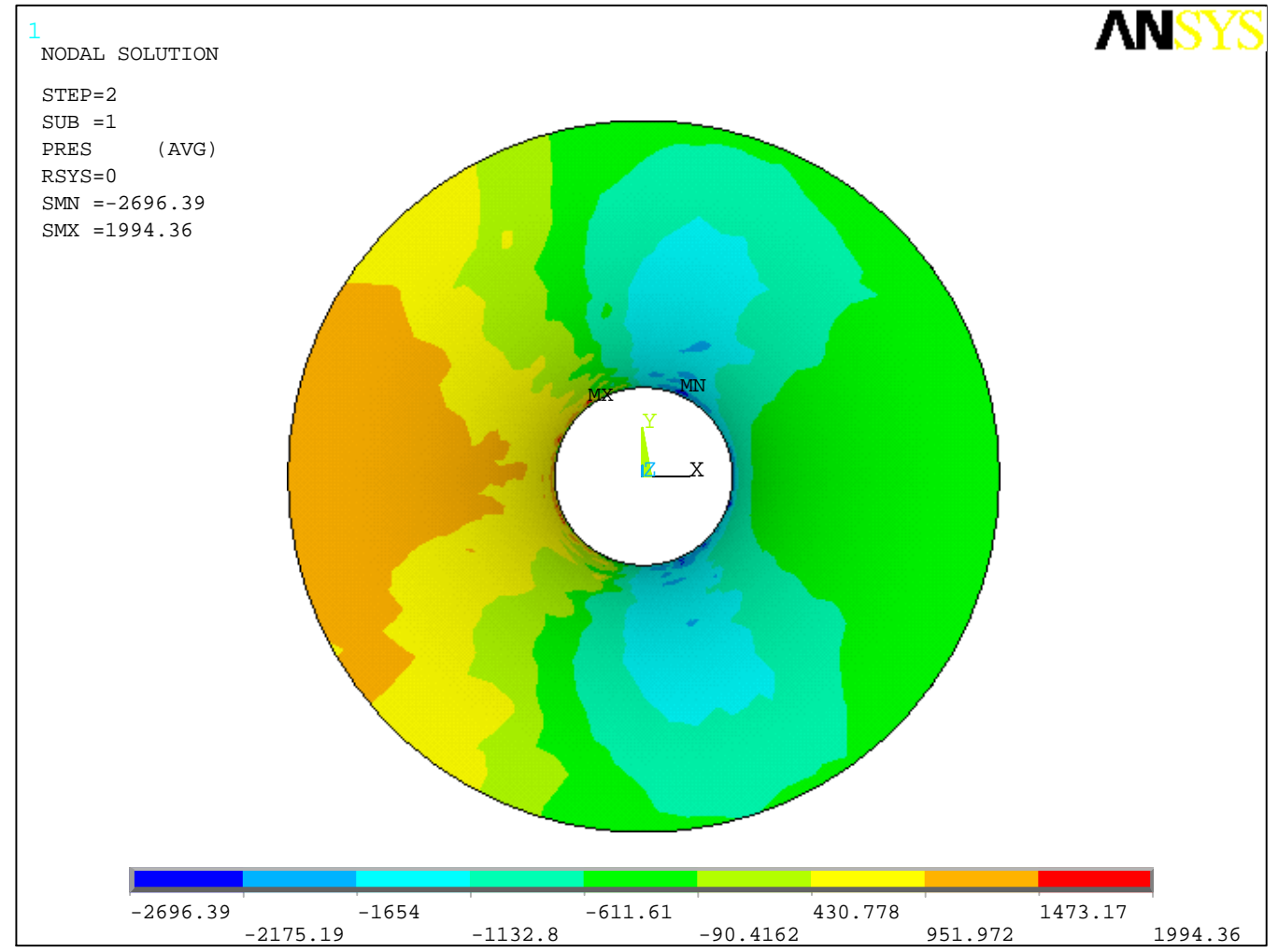

Figura 54 - Pressão do vento na $1^{\mathrm{a}}$ interação $\left(\mathrm{N} / \mathrm{m}^{2}\right)$

Avaliando que as pressões na membrana não se alteraram significativamente no seu estado indeformado.

Com estes dados, aplicou-se as pressões e sucções na membrana, calculando-se os seguintes deslocamentos em z: 


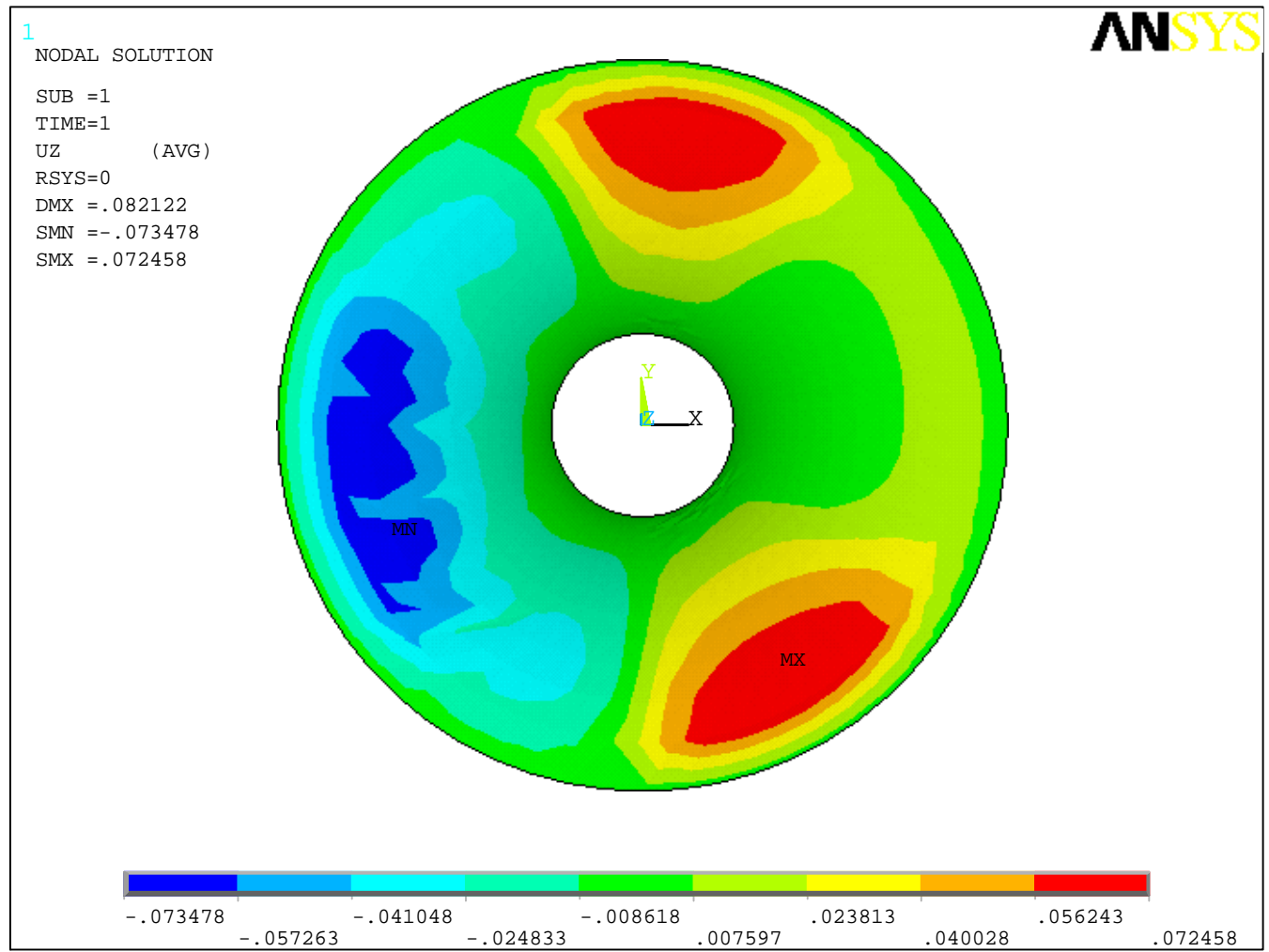

Figura 55 - Deslocamentos em z devido ao vento na $1^{\mathrm{a}}$ interação (m)

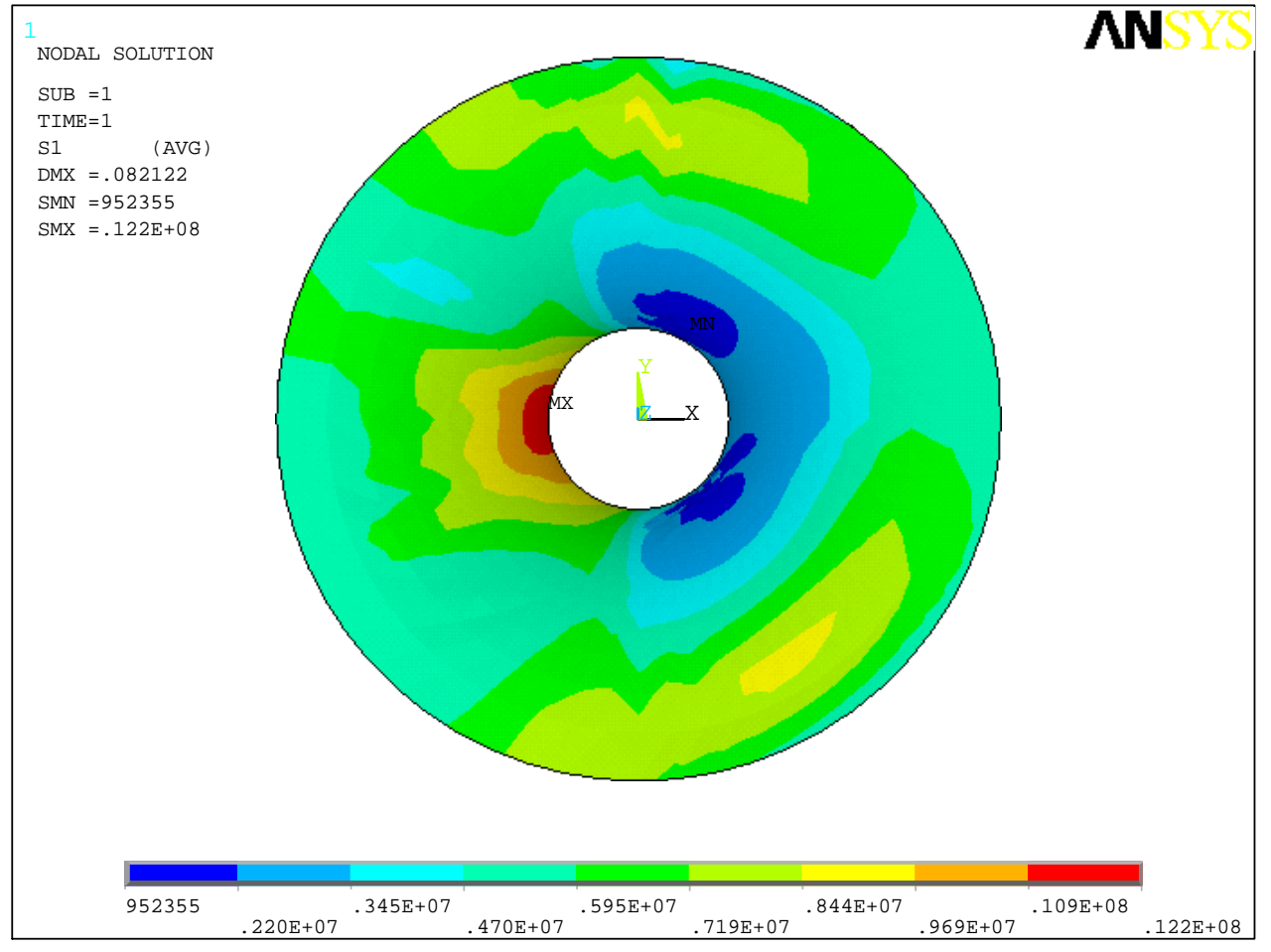

Figura 56 - Tensões principais da membrana $\left(\mathrm{N} / \mathrm{m}^{2}\right)$ 
Fazendo uma nova iteração, temos:

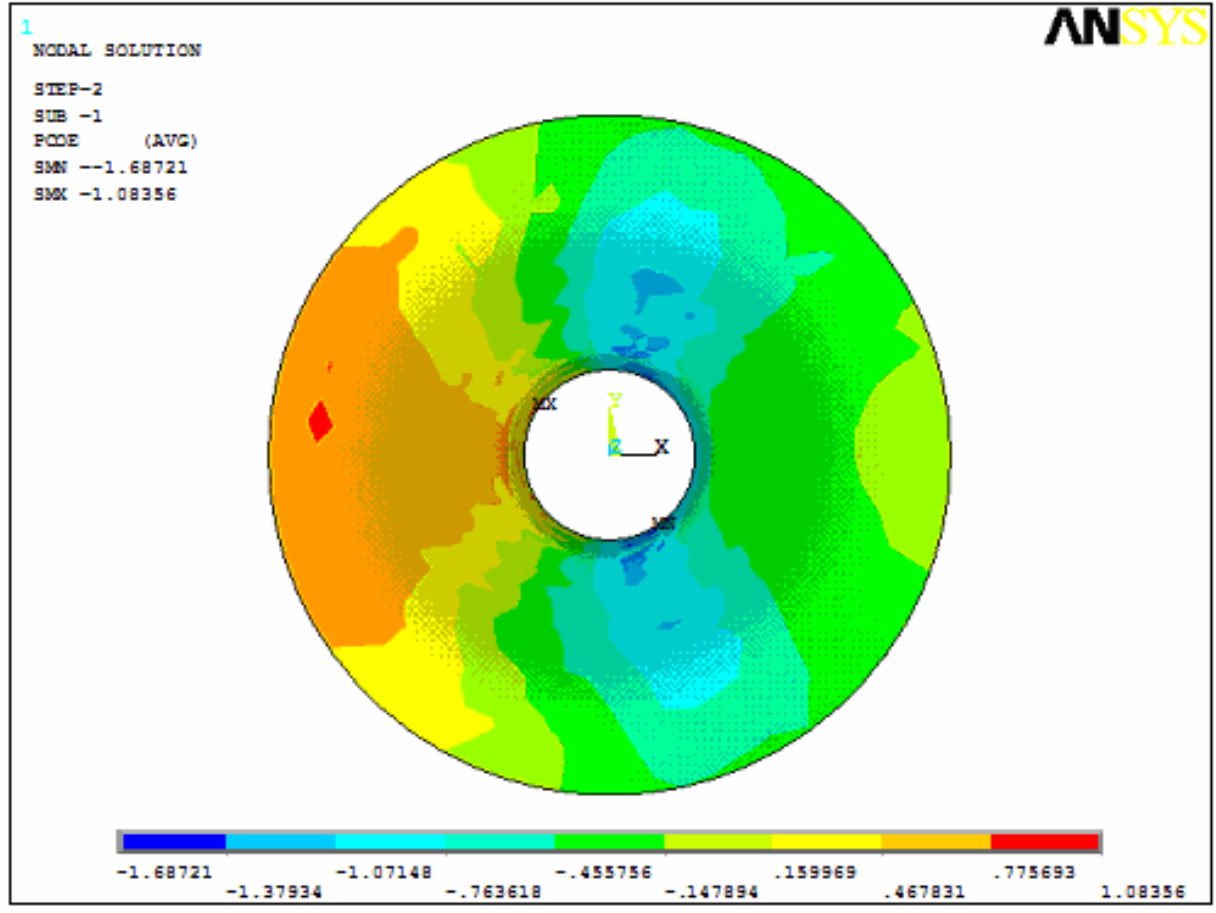

Figura 57 - Coeficientes de pressão na $2^{\mathrm{a}}$ interação

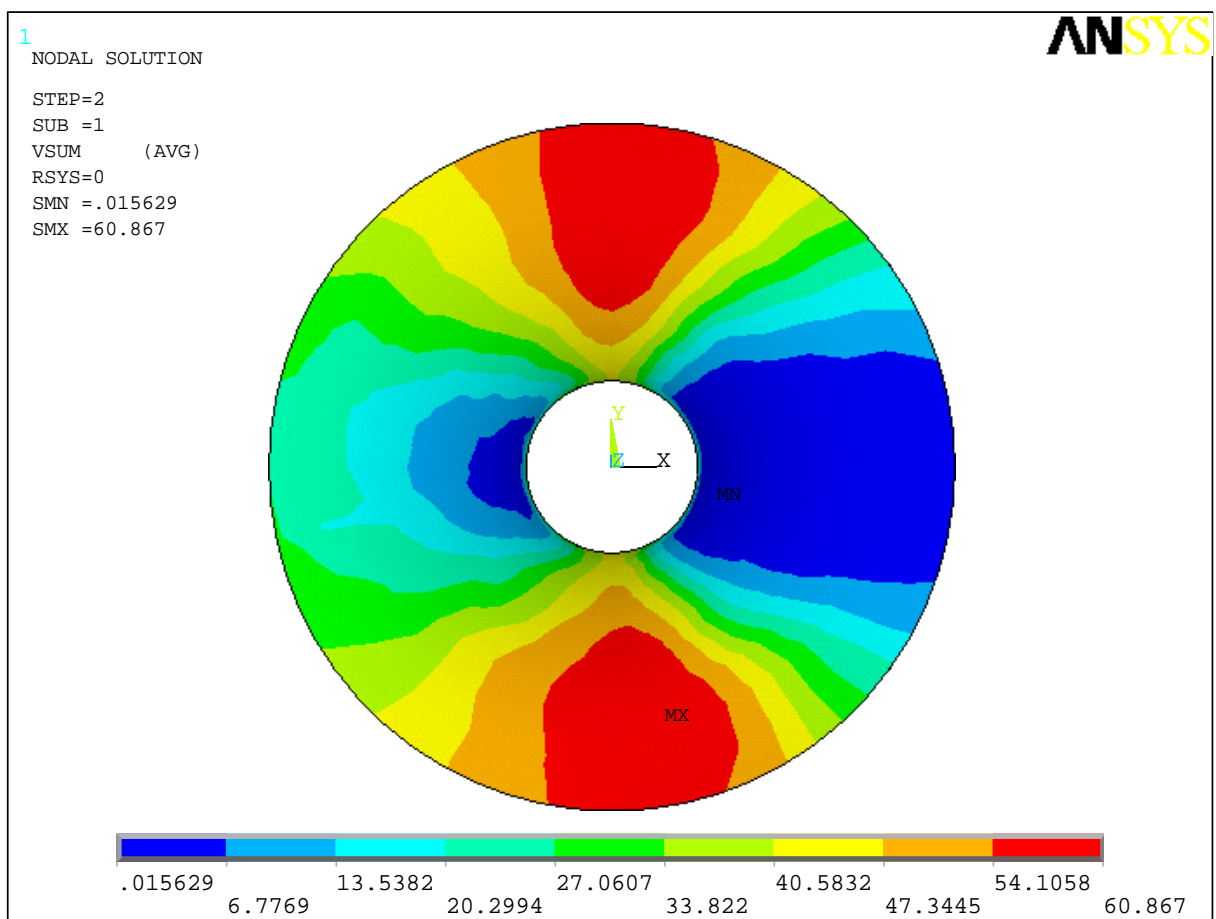

Figura 58 - Velocidades máximas no entorno do conóide na $2^{\mathrm{a}}$ interação $(\mathrm{m} / \mathrm{s})$ 


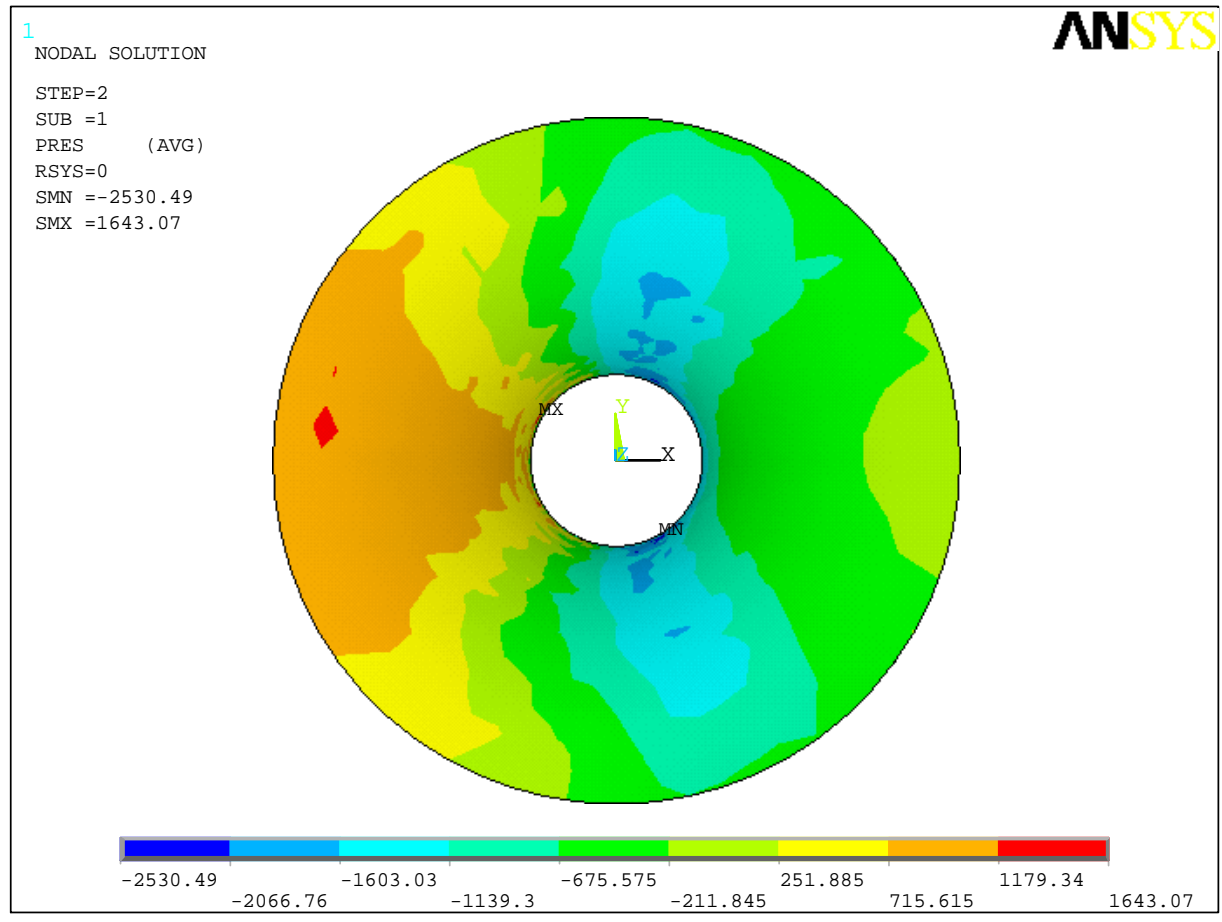

Figura 59 - Pressão do vento na $2^{\mathrm{a}}$ interação $\left(\mathrm{N} / \mathrm{m}^{2}\right)$

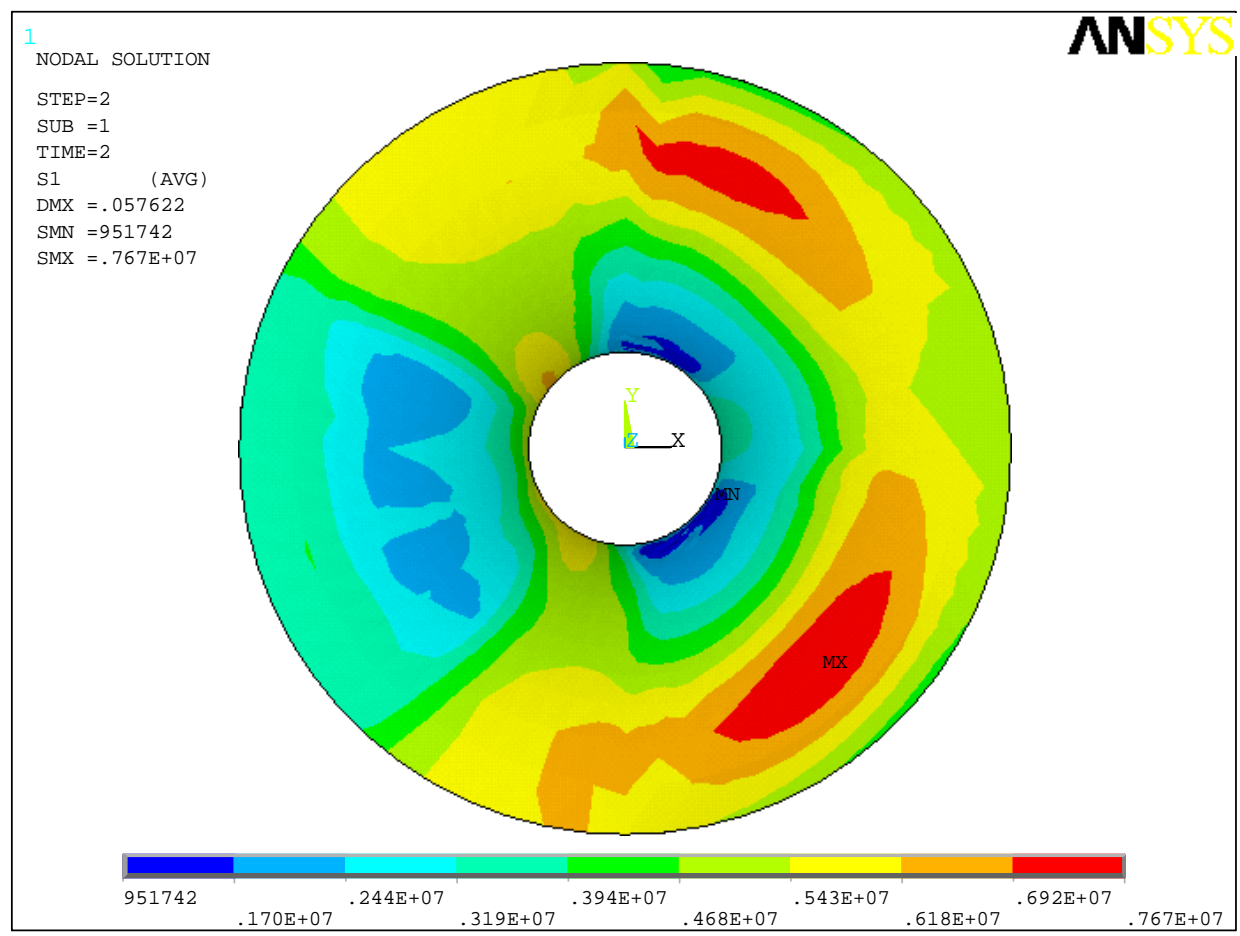

Figura 60 - Tensões principais na $2^{\mathrm{a}}$ iteração $\left(\mathrm{N} / \mathbf{m}^{2}\right)$

Assim como na aplicação anterior, as deformações da membrana devido ao carregamento alteraram o escoamento. Entretanto, as tensões encontradas na estrutura 
tenderam a se uniformizar, sugerindo novamente que a acomodação de forma da membrana é favorável no dimensionamento.

Abaixo seguem resultados para uma terceira interação:

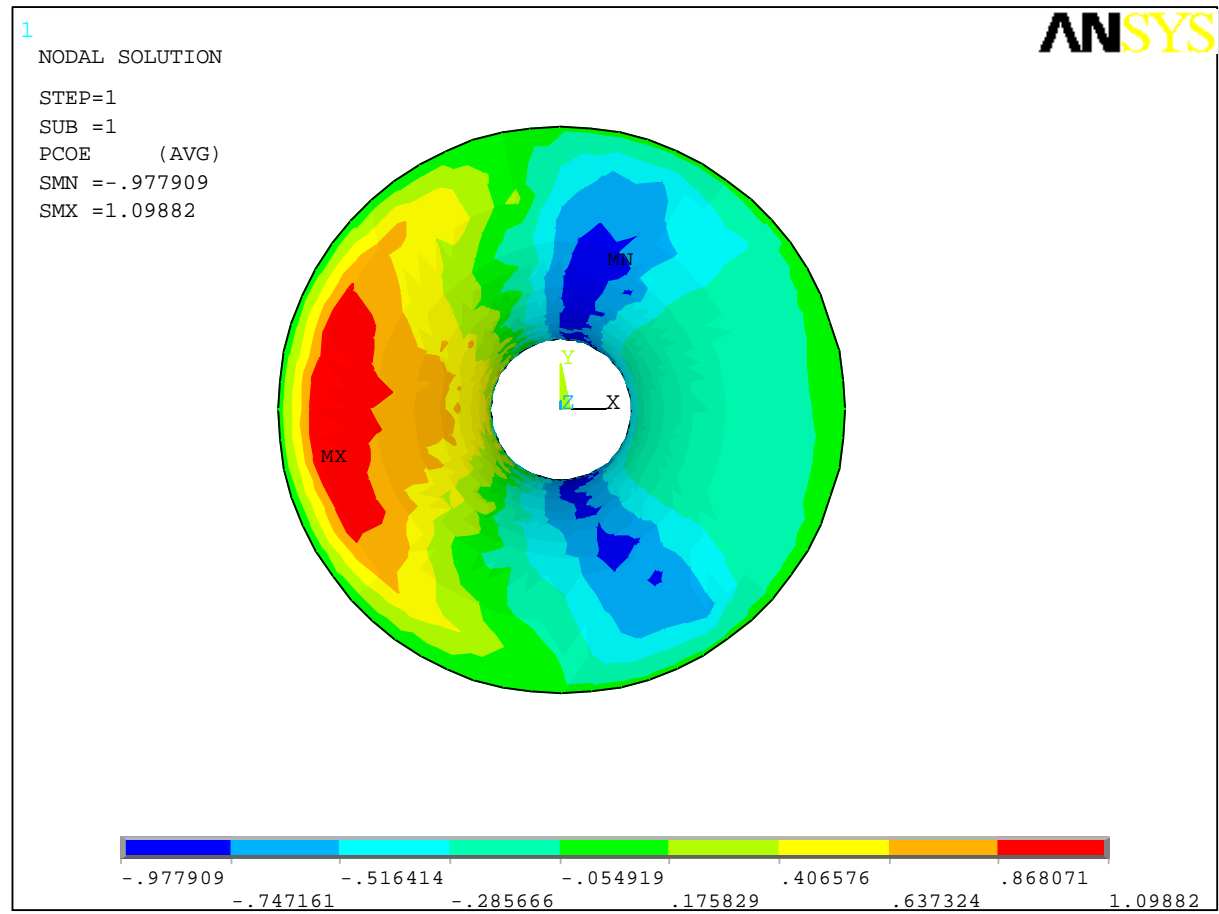

Figura 61 - Coeficientes de pressão na $3^{\mathrm{a}}$ interação

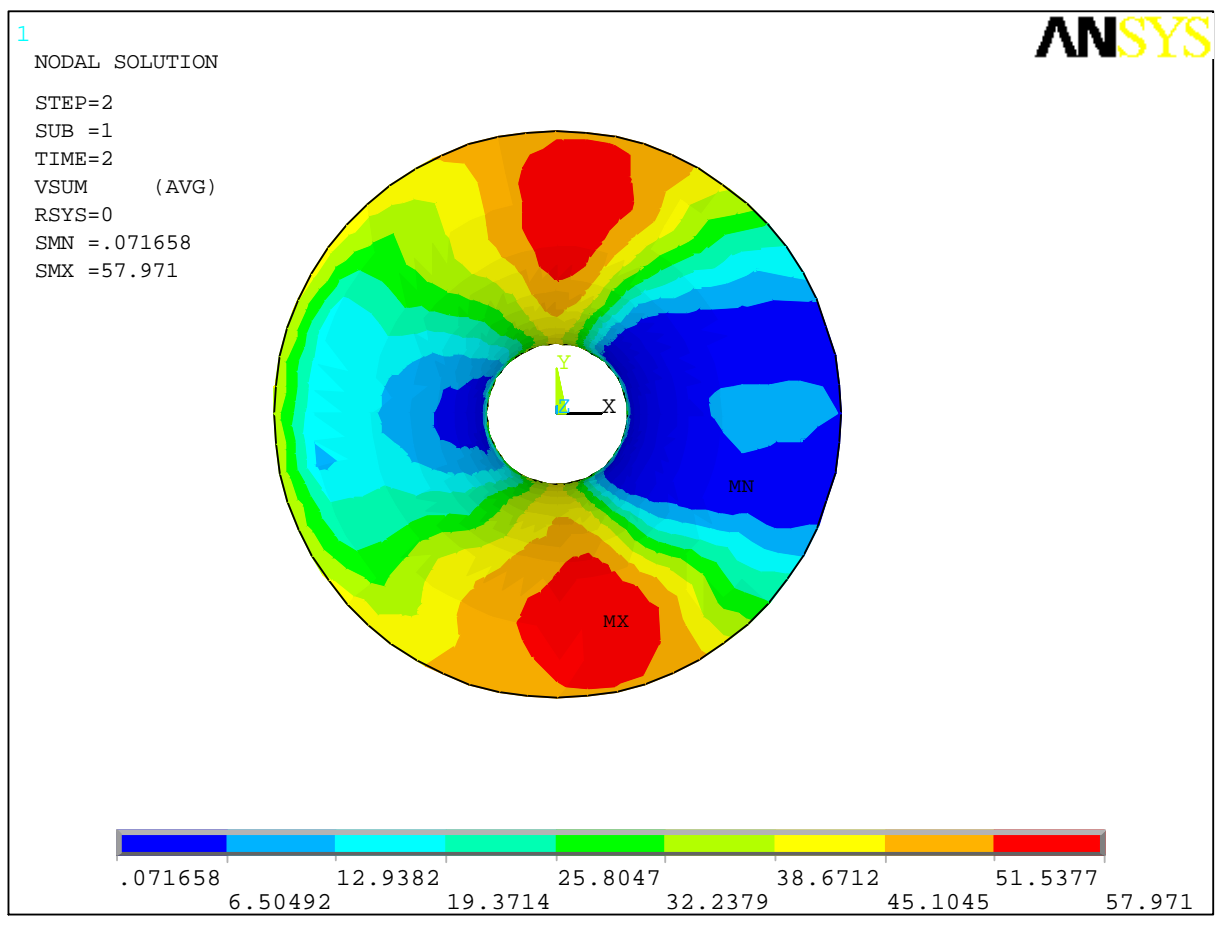

Figura 62 - Velocidades máximas no entorno do conóide na $3^{\mathrm{a}}$ interação $(\mathrm{m} / \mathrm{s})$ 


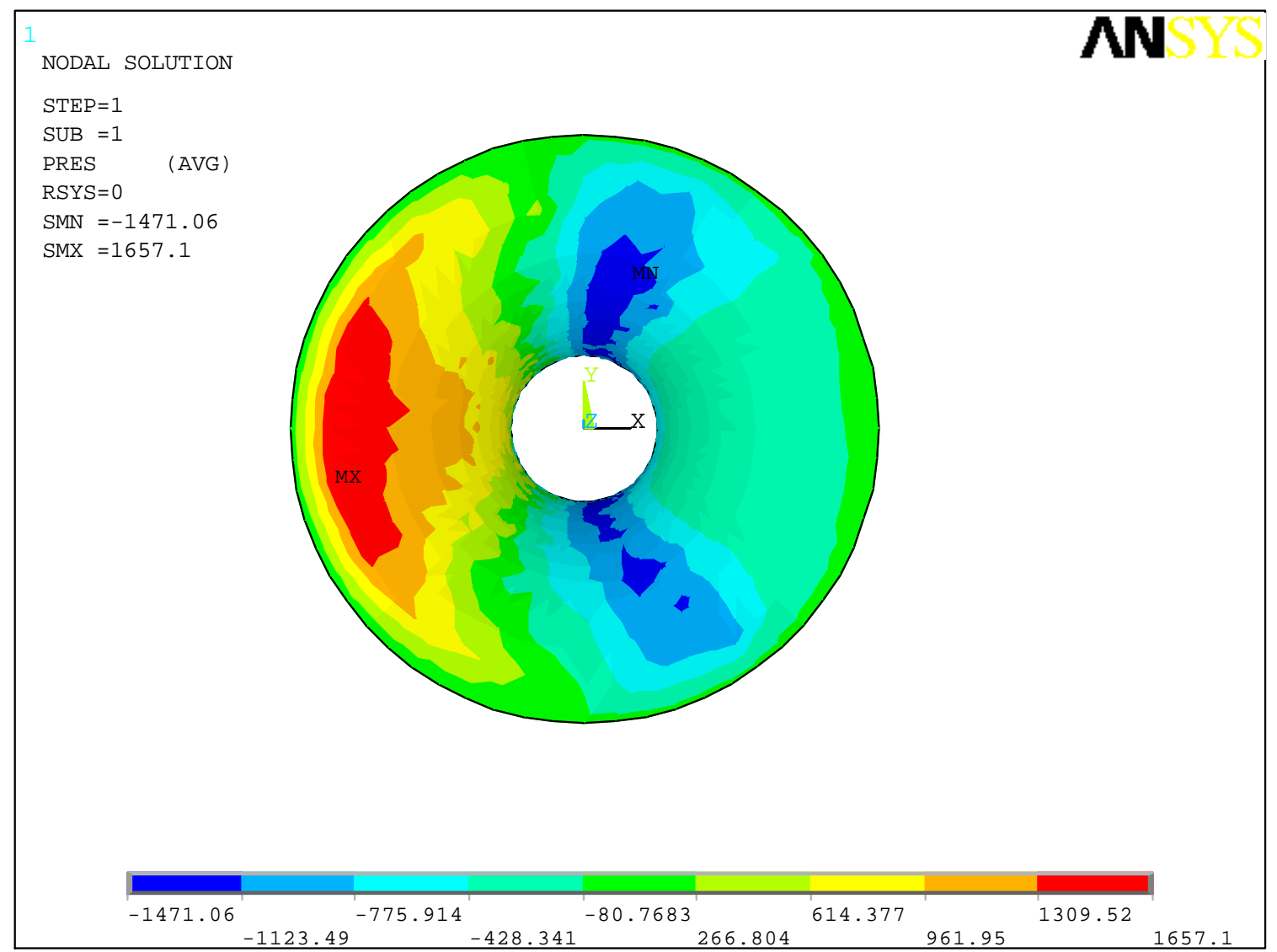

Figura 63 - Pressão do vento na $3^{\mathrm{a}}$ interação $\left(\mathrm{N} / \mathrm{m}^{2}\right)$

Como pode-se observar, da segunda para terceira interação não houveram alterações significativas no escoamento e nas pressões do vento. Também observou-se tensões principais menores que na $1^{\mathrm{a}}$ interação.

Desta forma, havendo condições de se definir o dimensionamento não foram processadas novas interações. 


\section{CONCLUSÕES}

Este trabalho tratou da ação do vento em tensoestruturas, apresentando primeiramente os conceitos e equações básicas sobre tensoestruturas e vento. Foi destinado um capítulo a parte para apresentar as leis físicas e equações gerais para tratativa computacional do problemas de dinâmica dos fluidos. Finalmente, foram apresentadas aplicações, primeiramente básicas e sem acoplamento visando somente assegurar o correto manuseio da ferramenta computacional. Posteriormente, foram apresentadas aplicações com acoplamento parcial a estruturas de membrana.

Dentre os estudos sobre o vento, observou-se importância na checagem das velocidades básicas de vento utilizadas pelo código nacional brasileiro, de forma a garantir a segurança prevista nas edificações. A discussão sobre a cooperação e utilização de registros meteorológicos de países vizinhos é válida para melhoria das isopletas nacionais principalmente nas regiões de fronteira.

Através das aplicações, também reforçou-se a importância do estudo e avanço tecnológico na tratativa da ação do vento em membranas, tanto para segurança como por razões econômicas. Não sendo ainda possível a abordagem no código nacional para ação do vento em tensoestuturas, as aplicações computacionais demonstraram a viabilidade de uma alternativa aos ensaios em túnel de vento.

A modelagem numérica em elementos finitos de estruturas de membrana sob ação de ventos mostrou viável tecnicamente para as condições e simplificações estabelecidas, havendo semelhança entre a modelagem do domínio do fluido com o túnel de vento. A maior desvantagem mostrou-se no demasiado tempo para modelagem, atualizações de malha e ajustes em parâmetros de convergência. Entretanto, com os avanços computacionais, novos métodos e novos softwares, tal prejuízo tende a ser revertido.

Ainda, nas aplicações apresentadas neste trabalho o comportamento estrutural da membrana apresentando grandes deformações para equilibrar-se com o carregamento

solicitante mostrou-se favorável à segurança quando do acoplamento parcial. É oportuno 
aplicar em trabalhos futuros a fluido-dinâmica com acoplamento total e resolver problemas mais complexos da interação fluido-estrutura para confirmar os resultados aqui obtidos.

Finalmente, sugere-se a elaboração de um código nacional para tratamento de estruturas de membrana abordando e prescrevendo condições de projeto, execução, durabilidade, materiais, manutenção e segurança. Visto que a bibliografia disponível e amplamente utilizada é proveniente dos Estados Unidos, Europa e Japão, onde existem grandes diferenças culturais e econômicas. 


\section{BIBLIOGRAFIA}

[1] ABNT. NBR 6123: Forças devidas ao vento em edificações. Rio de Janeiro: Associação Brasileira de Normas Técnicas, 1988.

[2] BARNES, M. R., "Form-Finding and Analysis of Prestressed Nets and Membranes". Computers \& Structures, v.30, n.3, p. 685-695, 1998.

[3] BATHE, K. J., "Finite element procedures”, Prentice Hall, 1996.

[4] BATHE, K. J.; ZHANG, H.; WHANG, M. H.; "Finite element analysis of incompressible and compressible fluid flows with free surfaces and structural interaction", Computers \& Structures, Vol. 56, 1995.

[5] BATHE, K. J.; ZHANG, H.; SHANHONG, J.; "Finite element analysis of fluid fully coupled with structural interactions", Computers \& Structures, Vol. 72, 1999.

[6] BLESSMANN, J. “Aerodinâmica das Construções”. Porto Alegre: Sagra, 1991.

[7] BLESSMANN, J. "Introdução ao estudo das ações dinâmicas do vento". 1 ed. Porto Alegre, 1998.

[8] BLETZINGER, K-U. "Form Finding and Optimization of Membranes and Minimal Surfaces”. Lyngby, Denmark: Technical University of Denmark, 1998.

[9] BLETZINGER, K-U.; WÜCHNER, R.; DAOUD F.; CAMPRUBÍ, “Computational Methods for Form Finding and Optimization of Shells and Membranes." Computer Methods in Applied Mechanics and Engineering, v. 194, n. 30-33, 2005.

[10] KUPZOK, A. M., "Modeling the Interaction of Wind and Membrane Structures by Numerical Simulation”. Dissertação para Universidade de Munique, Alemanha, 2009.

[11] LOREDO-SOUZA A. M., DAVENPORT, A.G., "Wind Tunnel Aeroelastic Studies on the Behavior of Two Parallel Cables." Journal of Wind Engineering and Industrial Aerodynamics, 2002.

[12] MANFRIM, S. T. "Estudo Numérico para a Determinação das Pressões Devidas à Ação do Vento em Edifícios Industriais”, Dissertação (mestrado em Engenharia Civil) - Faculdade de Engenharia de Ilha Solteira, 2006. 
[13] PAULETTI, R. M. O. “An Extension of the Force Density Procedure to Membrane Structures." In: IASS - INTERNATIONAL ASSOCIATION FOR SHELL AND SPATIAL STRUCTURES SYMPOSIUM - New Olympics, New Shell and Spatial Structures, 18. 2006.

[14] PAUlETTI, R. M. O. "História, Análise e Projeto de Estruturas Retesadas", Livre docência - Escola Politécnica, Universidade de São Paulo, 2003.

[15] PAULETTI, R. M. O. “Análise de Estrututas Retesadas.” Notas de Aula da Escola Politécnica, Universidade de São Paulo, 2008.

[16] SOUZA, D. C. B., "Sobre a Busca de Superfícies Minimais e Seu Emprego nas Estruturas de Membrana". Dissertação (mestrado em Engenharia Civil) - Escola Politécnica, Universidade de São Paulo, 2008.

[17] TABARROK, B.; QIN Z., "Dynamic Analysis of Tension Structures.” Computers \& Structures, v.62, n.3, 1997.

[18] TABARROK, B.; QIN Z., "Nonlinear Analysis of Tension Structures.” Computers \& Structures, v.45, n.5-6, 1992.

[19] VÁZQUEZ, J. G. V. "Nonlinear Analysis of Orthotropic Membrane and Shell Structures Including Fluid-Structure Interaction". Universitat Politècnica de Catalunya, 2007.

[20] ZIENKIEWICZ, O.; C.TAYLOR R.L., "The finite element method", vol. 1, Butterworth-Heinemann, 2000.

[21] ZIENKIEWICZ, O.; C.TAYLOR R.L., "The finite element method", vol. 2, Butterworth-Heinemann, 2000.

[22] ZIENKIEWICZ, O.; C.TAYLOR R.L., "The finite element method", vol. 3, Butterworth-Heinemann, 2000. 


\section{ANEXO 01 - RAJADAS DE VENTO REGISTRADAS}

EM SÃO MARTINHO DA SERRA / RS

No disco encontram-se compilados os valores medidos de rajadas de vento em São Martinho da Serra / RS: 


\section{ANEXO 01 - RAJADAS DE VENTO REGISTRADAS EM SÃO MARTINHO DA SERRA / RS}

\begin{tabular}{|c|c|}
\hline DataHora & VelVentoMax \\
\hline 2010-12-31 21:00:00.0 & 23.7 \\
\hline 2010-12-31 18:00:00.0 & 25.2 \\
\hline 2010-12-31 15:00:00.0 & 27.7 \\
\hline 2010-12-31 12:00:00.0 & 29.2 \\
\hline 2010-12-31 09:00:00.0 & 24.6 \\
\hline 2010-12-31 06:00:00.0 & 19.9 \\
\hline 2010-12-31 03:00:00.0 & 17.6 \\
\hline 2010-12-31 00:00:00.0 & \\
\hline 2010-12-30 21:00:00.0 & 29.6 \\
\hline 2010-12-30 18:00:00.0 & 24.3 \\
\hline 2010-12-30 15:00:00.0 & 25.4 \\
\hline 2010-12-30 12:00:00.0 & 29.0 \\
\hline 2010-12-30 09:00:00.0 & 17.7 \\
\hline 2010-12-30 06:00:00.0 & 17.6 \\
\hline 2010-12-30 03:00:00.0 & 19.4 \\
\hline 2010-12-30 00:00:00.0 & 28.4 \\
\hline 2010-12-29 21:00:00.0 & 28.6 \\
\hline 2010-12-29 18:00:00.0 & 24.1 \\
\hline 2010-12-29 15:00:00.0 & 19.7 \\
\hline 2010-12-29 12:00:00.0 & 21.2 \\
\hline 2010-12-29 09:00:00.0 & 18.4 \\
\hline 2010-12-29 06:00:00.0 & 13.8 \\
\hline 2010-12-29 03:00:00.0 & 18.6 \\
\hline 2010-12-29 00:00:00.0 & \\
\hline 2010-12-28 21:00:00.0 & 23.0 \\
\hline 2010-12-28 18:00:00.0 & 18.6 \\
\hline 2010-12-28 15:00:00.0 & \\
\hline 2010-12-28 12:00:00.0 & 19.2 \\
\hline 2010-12-28 09:00:00.0 & 13.3 \\
\hline 2010-12-28 06:00:00.0 & 13.7 \\
\hline 2010-12-28 03:00:00.0 & 15.9 \\
\hline 2010-12-28 00:00:00.0 & 22.2 \\
\hline 2010-12-27 21:00:00.0 & 21.5 \\
\hline 2010-12-27 18:00:00.0 & 20.1 \\
\hline 2010-12-27 15:00:00.0 & 16.9 \\
\hline 2010-12-27 12:00:00.0 & 14.9 \\
\hline 2010-12-27 09:00:00.0 & 12.9 \\
\hline 2010-12-27 06:00:00.0 & 17.7 \\
\hline 2010-12-27 03:00:00.0 & 20.1 \\
\hline 2010-12-27 00:00:00.0 & 27.5 \\
\hline 2010-12-26 21:00:00.0 & 27.1 \\
\hline 2010-12-26 18:00:00.0 & 27.5 \\
\hline 2010-12-26 15:00:00.0 & 27.4 \\
\hline 2010-12-26 12:00:00.0 & 14.5 \\
\hline 2010-12-26 09:00:00.0 & 16.2 \\
\hline 2010-12-26 06:00:00.0 & 16.2 \\
\hline 2010-12-26 03:00:00.0 & 17.6 \\
\hline $2010-12-26$ 00:00:00.0 & 13.4 \\
\hline 2010-12-25 21:00:00.0 & 19.1 \\
\hline 2010-12-25 18:00:00.0 & 16.2 \\
\hline 2010-12-25 15:00:00.0 & 9.0 \\
\hline 2010-12-25 12:00:00.0 & 9.0 \\
\hline 2010-12-25 09:00:00.0 & 13.7 \\
\hline 2010-12-25 06:00:00.0 & 15.4 \\
\hline 2010-12-25 03:00:00.0 & 34.9 \\
\hline 2010-12-25 00:00:00.0 & 25.1 \\
\hline 2010-12-24 21:00:00.0 & 24.6 \\
\hline 2010-12-24 18:00:00.0 & 25.6 \\
\hline 2010-12-24 15:00:00.0 & 21.6 \\
\hline 2010-12-24 12:00:00.0 & 20.0 \\
\hline 2010-12-24 09:00:00.0 & 19.3 \\
\hline 2010-12-24 06:00:00.0 & 17.7 \\
\hline 2010-12-24 03:00:00.0 & 16.2 \\
\hline 2010-12-24 00:00:00.0 & 16.0 \\
\hline 2010-12-23 21:00:00.0 & 20.5 \\
\hline 2010-12-23 18:00:00.0 & 19.3 \\
\hline 2010-12-23 15:00:00.0 & 18.4 \\
\hline 2010-12-23 12:00:00.0 & 19.7 \\
\hline 2010-12-23 09:00:00.0 & 17.6 \\
\hline
\end{tabular}

\begin{tabular}{|c|c|}
\hline DataHora & VelVentoMax \\
\hline 2010-12-23 06:00:00.0 & 15.6 \\
\hline 2010-12-23 03:00:00.0 & 18.8 \\
\hline 2010-12-23 00:00:00.0 & 20.7 \\
\hline 2010-12-22 21:00:00.0 & 18.1 \\
\hline 2010-12-22 18:00:00.0 & 16.0 \\
\hline 2010-12-22 15:00:00.0 & 15.3 \\
\hline 2010-12-22 12:00:00.0 & 16.0 \\
\hline 2010-12-22 09:00:00.0 & 14.0 \\
\hline 2010-12-22 06:00:00.0 & \\
\hline 2010-12-22 03:00:00.0 & \\
\hline 2010-12-22 00:00:00.0 & 19.1 \\
\hline 2010-12-21 21:00:00.0 & 18.3 \\
\hline 2010-12-21 18:00:00.0 & 18.6 \\
\hline 2010-12-21 15:00:00.0 & 13.4 \\
\hline 2010-12-21 12:00:00.0 & 13.5 \\
\hline 2010-12-21 09:00:00.0 & 12.0 \\
\hline 2010-12-21 06:00:00.0 & \\
\hline 2010-12-21 03:00:00.0 & 10.1 \\
\hline 2010-12-21 00:00:00.0 & 12.8 \\
\hline 2010-12-20 21:00:00.0 & 17.6 \\
\hline 2010-12-20 18:00:00.0 & 14.8 \\
\hline 2010-12-20 15:00:00.0 & 14.8 \\
\hline 2010-12-20 12:00:00.0 & 19.4 \\
\hline 2010-12-20 09:00:00.0 & 33.1 \\
\hline 2010-12-20 06:00:00.0 & 20.2 \\
\hline 2010-12-20 03:00:00.0 & 21.4 \\
\hline 2010-12-20 00:00:00.0 & \\
\hline 2010-12-19 21:00:00.0 & 30.7 \\
\hline 2010-12-19 18:00:00.0 & 20.5 \\
\hline 2010-12-19 15:00:00.0 & 20.1 \\
\hline 2010-12-19 12:00:00.0 & 23.5 \\
\hline 2010-12-19 09:00:00.0 & 22.0 \\
\hline 2010-12-19 06:00:00.0 & 22.2 \\
\hline 2010-12-19 03:00:00.0 & 23.7 \\
\hline 2010-12-19 00:00:00.0 & 26.7 \\
\hline 2010-12-18 21:00:00.0 & 15.3 \\
\hline 2010-12-18 18:00:00.0 & 13.6 \\
\hline 2010-12-18 15:00:00.0 & 14.6 \\
\hline 2010-12-18 12:00:00.0 & 10.9 \\
\hline 2010-12-18 09:00:00.0 & 12.8 \\
\hline 2010-12-18 06:00:00.0 & 15.8 \\
\hline 2010-12-18 03:00:00.0 & \\
\hline 2010-12-18 00:00:00.0 & 14.8 \\
\hline 2010-12-17 21:00:00.0 & 18.6 \\
\hline 2010-12-17 18:00:00.0 & 13.9 \\
\hline 2010-12-17 15:00:00.0 & 14.3 \\
\hline 2010-12-17 12:00:00.0 & 43.8 \\
\hline 2010-12-17 09:00:00.0 & 13.2 \\
\hline 2010-12-17 06:00:00.0 & 16.8 \\
\hline 2010-12-17 03:00:00.0 & 21.9 \\
\hline 2010-12-17 00:00:00.0 & 27.8 \\
\hline 2010-12-16 21:00:00.0 & 24.2 \\
\hline 2010-12-16 18:00:00.0 & 22.8 \\
\hline 2010-12-16 15:00:00.0 & 27.6 \\
\hline 2010-12-16 12:00:00.0 & 27.6 \\
\hline 2010-12-16 09:00:00.0 & 25.4 \\
\hline 2010-12-16 06:00:00.0 & \\
\hline 2010-12-16 03:00:00.0 & 18.2 \\
\hline 2010-12-16 00:00:00.0 & 24.4 \\
\hline 2010-12-15 21:00:00.0 & 22.9 \\
\hline 2010-12-15 18:00:00.0 & 20.6 \\
\hline 2010-12-15 15:00:00.0 & 19.0 \\
\hline 2010-12-15 12:00:00.0 & 7.9 \\
\hline 2010-12-15 09:00:00.0 & 14.7 \\
\hline 2010-12-15 06:00:00.0 & 18.2 \\
\hline 2010-12-15 03:00:00.0 & 15.3 \\
\hline 2010-12-15 00:00:00.0 & 15.6 \\
\hline 2010-12-14 21:00:00.0 & 16.9 \\
\hline 2010-12-14 18:00:00.0 & 17.6 \\
\hline
\end{tabular}

\begin{tabular}{|c|c|}
\hline DataHora & VelVentoMax \\
\hline 2010-12-14 15:00:00.0 & 14.3 \\
\hline 2010-12-14 12:00:00.0 & 10.2 \\
\hline 2010-12-14 09:00:00.0 & 11.2 \\
\hline 2010-12-14 06:00:00.0 & 10.4 \\
\hline 2010-12-14 03:00:00.0 & \\
\hline 2010-12-14 00:00:00.0 & \\
\hline 2010-12-13 21:00:00.0 & 27.0 \\
\hline 2010-12-13 18:00:00.0 & 29.6 \\
\hline 2010-12-13 15:00:00.0 & 28.6 \\
\hline 2010-12-13 12:00:00.0 & 22.4 \\
\hline 2010-12-13 09:00:00.0 & 22.4 \\
\hline 2010-12-13 06:00:00.0 & 31.6 \\
\hline 2010-12-13 03:00:00.0 & 31.2 \\
\hline 2010-12-13 00:00:00.0 & \\
\hline 2010-12-12 21:00:00.0 & 33.9 \\
\hline 2010-12-12 18:00:00.0 & 28.1 \\
\hline 2010-12-12 15:00:00.0 & 34.9 \\
\hline 2010-12-12 12:00:00.0 & 19.8 \\
\hline 2010-12-12 09:00:00.0 & 16.3 \\
\hline 2010-12-12 06:00:00.0 & 22.6 \\
\hline 2010-12-12 03:00:00.0 & 26.9 \\
\hline 2010-12-12 00:00:00.0 & \\
\hline 2010-12-11 21:00:00.0 & 24.1 \\
\hline 2010-12-11 18:00:00.0 & 24.7 \\
\hline 2010-12-11 15:00:00.0 & 25.8 \\
\hline 2010-12-11 12:00:00.0 & 18.7 \\
\hline 2010-12-11 09:00:00.0 & 13.7 \\
\hline 2010-12-11 06:00:00.0 & 16.3 \\
\hline 2010-12-11 03:00:00.0 & 24.8 \\
\hline 2010-12-11 00:00:00.0 & \\
\hline 2010-12-10 21:00:00.0 & 23.4 \\
\hline 2010-12-10 18:00:00.0 & 22.7 \\
\hline 2010-12-10 15:00:00.0 & 23.7 \\
\hline 2010-12-10 12:00:00.0 & 26.2 \\
\hline 2010-12-10 09:00:00.0 & 24.9 \\
\hline 2010-12-10 06:00:00.0 & 22.3 \\
\hline 2010-12-10 03:00:00.0 & 19.6 \\
\hline 2010-12-10 00:00:00.0 & \\
\hline 2010-12-09 21:00:00.0 & 27.7 \\
\hline 2010-12-09 18:00:00.0 & 17.7 \\
\hline 2010-12-09 15:00:00.0 & 20.8 \\
\hline 2010-12-09 12:00:00.0 & 23.9 \\
\hline 2010-12-09 09:00:00.0 & \\
\hline 2010-12-09 06:00:00.0 & 14.3 \\
\hline 2010-12-09 03:00:00.0 & \\
\hline 2010-12-09 00:00:00.0 & \\
\hline 2010-12-08 21:00:00.0 & 29.5 \\
\hline 2010-12-08 18:00:00.0 & 34.2 \\
\hline 2010-12-08 15:00:00.0 & 26.6 \\
\hline 2010-12-08 12:00:00.0 & \\
\hline 2010-12-08 09:00:00.0 & 13.6 \\
\hline 2010-12-08 06:00:00.0 & 13.7 \\
\hline 2010-12-08 03:00:00.0 & \\
\hline 2010-12-08 00:00:00.0 & \\
\hline 2010-12-07 21:00:00.0 & 21.4 \\
\hline 2010-12-07 18:00:00.0 & 23.9 \\
\hline 2010-12-07 15:00:00.0 & 20.4 \\
\hline 2010-12-07 12:00:00.0 & \\
\hline 2010-12-07 09:00:00.0 & 19.2 \\
\hline 2010-12-07 06:00:00.0 & 20.5 \\
\hline 2010-12-07 03:00:00.0 & \\
\hline 2010-12-07 00:00:00.0 & 20.1 \\
\hline 2010-12-06 21:00:00.0 & 20.4 \\
\hline 2010-12-06 18:00:00.0 & 21.2 \\
\hline 2010-12-06 15:00:00.0 & 24.9 \\
\hline 2010-12-06 12:00:00.0 & 28.1 \\
\hline 2010-12-06 09:00:00.0 & 15.9 \\
\hline 2010-12-06 06:00:00.0 & 17.5 \\
\hline 2010-12-06 03:00:00.0 & \\
\hline
\end{tabular}




\section{ANEXO 01 - RAJADAS DE VENTO REGISTRADAS EM SÃO MARTINHO DA SERRA / RS}

\begin{tabular}{|c|c|}
\hline \multirow{2}{*}{$\begin{array}{l}\text { DataHora } \\
\text { 2010-12-06 00:00:00.0 }\end{array}$} & VelVentoMax \\
\hline & \\
\hline 2010-12-05 21:00:00.0 & 25.5 \\
\hline 2010-12-05 18:00:00.0 & 28.9 \\
\hline 2010-12-05 15:00:00.0 & \\
\hline 2010-12-05 12:00:00.0 & \\
\hline 2010-12-05 09:00:00.0 & 13.1 \\
\hline 2010-12-05 06:00:00.0 & 8.8 \\
\hline 2010-12-05 03:00:00.0 & \\
\hline 2010-12-05 00:00:00.0 & 9.5 \\
\hline 2010-12-04 21:00:00.0 & 13.5 \\
\hline 2010-12-04 18:00:00.0 & 22.2 \\
\hline 2010-12-04 15:00:00.0 & 27.9 \\
\hline 2010-12-04 12:00:00.0 & \\
\hline 2010-12-04 09:00:00.0 & 23.1 \\
\hline 2010-12-04 06:00:00.0 & 22.5 \\
\hline 2010-12-04 03:00:00.0 & \\
\hline 2010-12-04 00:00:00.0 & \\
\hline 2010-12-03 21:00:00.0 & 24.3 \\
\hline 2010-12-03 18:00:00.0 & 18.3 \\
\hline 2010-12-03 15:00:00.0 & \\
\hline 2010-12-03 12:00:00.0 & \\
\hline 2010-12-03 09:00:00.0 & 16.1 \\
\hline 2010-12-03 06:00:00.0 & 23.7 \\
\hline 2010-12-03 03:00:00.0 & \\
\hline 2010-12-03 00:00:00.0 & 26.7 \\
\hline 2010-12-02 21:00:00.0 & 6.9 \\
\hline 2010-12-02 18:00:00.0 & 17.6 \\
\hline 2010-12-02 15:00:00.0 & 22.6 \\
\hline 2010-12-02 12:00:00.0 & 22.7 \\
\hline 2010-12-02 09:00:00.0 & 21.9 \\
\hline 2010-12-02 06:00:00.0 & 22.3 \\
\hline 2010-12-02 03:00:00.0 & \\
\hline 2010-12-02 00:00:00.0 & 20.3 \\
\hline 2010-12-01 21:00:00.0 & 20.6 \\
\hline 2010-12-01 18:00:00.0 & 22.1 \\
\hline 2010-12-01 15:00:00.0 & 24.1 \\
\hline 2010-12-01 12:00:00.0 & 24.1 \\
\hline 2010-12-01 09:00:00.0 & 17.9 \\
\hline 2010-12-01 06:00:00.0 & \\
\hline 2010-12-01 03:00:00.0 & \\
\hline 2010-12-01 00:00:00.0 & 11.8 \\
\hline 2010-11-30 21:00:00.0 & 17.1 \\
\hline 2010-11-30 18:00:00.0 & 19.0 \\
\hline 2010-11-30 15:00:00.0 & \\
\hline 2010-11-30 12:00:00.0 & 12.0 \\
\hline 2010-11-30 09:00:00.0 & 7.2 \\
\hline 2010-11-30 06:00:00.0 & 10.1 \\
\hline 2010-11-30 03:00:00.0 & \\
\hline 2010-11-30 00:00:00.0 & 12.3 \\
\hline 2010-11-29 21:00:00.0 & 16.5 \\
\hline 2010-11-29 18:00:00.0 & \\
\hline 2010-11-29 15:00:00.0 & \\
\hline 2010-11-29 12:00:00.0 & 15.2 \\
\hline 2010-11-29 09:00:00.0 & 11.4 \\
\hline 2010-11-29 06:00:00.0 & \\
\hline 2010-11-29 03:00:00.0 & 16.5 \\
\hline 2010-11-29 00:00:00.0 & 24.7 \\
\hline 2010-11-28 21:00:00.0 & 28.8 \\
\hline 2010-11-28 18:00:00.0 & \\
\hline 2010-11-28 15:00:00.0 & 25.7 \\
\hline 2010-11-28 12:00:00.0 & 17.5 \\
\hline 2010-11-28 09:00:00.0 & 14.8 \\
\hline 2010-11-28 06:00:00.0 & \\
\hline 2010-11-28 03:00:00.0 & \\
\hline 2010-11-28 00:00:00.0 & 17.0 \\
\hline 2010-11-27 21:00:00.0 & 18.4 \\
\hline 2010-11-27 18:00:00.0 & \\
\hline 2010-11-27 15:00:00.0 & 14.0 \\
\hline 2010-11-27 12:00:00.0 & 16.8 \\
\hline
\end{tabular}

\begin{tabular}{|c|c|}
\hline DataHora & VelVentoMax \\
\hline 2010-11-27 09:00:00.0 & 19.0 \\
\hline 2010-11-27 06:00:00.0 & \\
\hline 2010-11-27 03:00:00.0 & 15.8 \\
\hline 2010-11-27 00:00:00.0 & 16.1 \\
\hline 2010-11-26 21:00:00.0 & 18.7 \\
\hline 2010-11-26 18:00:00.0 & \\
\hline 2010-11-26 15:00:00.0 & 15.8 \\
\hline 2010-11-26 12:00:00.0 & 19.4 \\
\hline 2010-11-26 09:00:00.0 & 19.7 \\
\hline 2010-11-26 06:00:00.0 & \\
\hline 2010-11-26 03:00:00.0 & \\
\hline 2010-11-26 00:00:00.0 & 19.6 \\
\hline 2010-11-25 21:00:00.0 & 22.2 \\
\hline 2010-11-25 18:00:00.0 & \\
\hline 2010-11-25 15:00:00.0 & 18.5 \\
\hline 2010-11-25 12:00:00.0 & 14.0 \\
\hline 2010-11-25 09:00:00.0 & 17.1 \\
\hline 2010-11-25 06:00:00.0 & \\
\hline 2010-11-25 03:00:00.0 & 21.9 \\
\hline 2010-11-25 00:00:00.0 & 19.6 \\
\hline 2010-11-24 21:00:00.0 & 20.8 \\
\hline 2010-11-24 18:00:00.0 & 19.2 \\
\hline 2010-11-24 15:00:00.0 & 23.7 \\
\hline 2010-11-24 12:00:00.0 & 3.0 \\
\hline 2010-11-24 09:00:00.0 & 23.0 \\
\hline 2010-11-24 06:00:00.0 & \\
\hline 2010-11-24 03:00:00.0 & 18.3 \\
\hline 2010-11-24 00:00:00.0 & 29.3 \\
\hline 2010-11-23 21:00:00.0 & \\
\hline 2010-11-23 18:00:00.0 & \\
\hline 2010-11-23 15:00:00.0 & 22.7 \\
\hline 2010-11-23 12:00:00.0 & 20.8 \\
\hline 2010-11-23 09:00:00.0 & \\
\hline 2010-11-23 06:00:00.0 & \\
\hline 2010-11-23 03:00:00.0 & 20.6 \\
\hline 2010-11-23 00:00:00.0 & 20.2 \\
\hline 2010-11-22 21:00:00.0 & 22.9 \\
\hline 2010-11-22 18:00:00.0 & 22.5 \\
\hline 2010-11-22 15:00:00.0 & 17.7 \\
\hline 2010-11-22 12:00:00.0 & 15.7 \\
\hline 2010-11-22 09:00:00.0 & 16.2 \\
\hline 2010-11-22 06:00:00.0 & \\
\hline 2010-11-22 03:00:00.0 & 21.0 \\
\hline 2010-11-22 00:00:00.0 & 23.6 \\
\hline 2010-11-21 21:00:00.0 & 18.9 \\
\hline 2010-11-21 18:00:00.0 & \\
\hline 2010-11-21 15:00:00.0 & 16.9 \\
\hline 2010-11-21 12:00:00.0 & 13.1 \\
\hline 2010-11-21 09:00:00.0 & \\
\hline 2010-11-21 06:00:00.0 & 14.1 \\
\hline 2010-11-21 03:00:00.0 & 15.5 \\
\hline 2010-11-21 00:00:00.0 & 22.7 \\
\hline 2010-11-20 21:00:00.0 & \\
\hline 2010-11-20 18:00:00.0 & 22.2 \\
\hline 2010-11-20 15:00:00.0 & 18.6 \\
\hline 2010-11-20 12:00:00.0 & 22.4 \\
\hline 2010-11-20 09:00:00.0 & \\
\hline 2010-11-20 06:00:00.0 & 18.1 \\
\hline 2010-11-20 03:00:00.0 & 14.5 \\
\hline 2010-11-20 00:00:00.0 & 17.3 \\
\hline 2010-11-19 21:00:00.0 & 17.3 \\
\hline 2010-11-19 18:00:00.0 & 28.3 \\
\hline 2010-11-19 15:00:00.0 & 18.6 \\
\hline 2010-11-19 12:00:00.0 & \\
\hline 2010-11-19 09:00:00.0 & \\
\hline 2010-11-19 06:00:00.0 & \\
\hline 2010-11-19 03:00:00.0 & 10.4 \\
\hline 2010-11-19 00:00:00.0 & 13.9 \\
\hline 2010-11-18 21:00:00.0 & 22.7 \\
\hline
\end{tabular}

\begin{tabular}{|c|c|}
\hline DataHora & VelVentoMax \\
\hline 2010-11-18 18:00:00.0 & 20.1 \\
\hline 2010-11-18 15:00:00.0 & 17.5 \\
\hline 2010-11-18 12:00:00.0 & 10.3 \\
\hline 2010-11-18 09:00:00.0 & 5.4 \\
\hline 2010-11-18 06:00:00.0 & 4.9 \\
\hline 2010-11-18 03:00:00.0 & 8.8 \\
\hline 2010-11-18 00:00:00.0 & 16.0 \\
\hline 2010-11-17 21:00:00.0 & \\
\hline 2010-11-17 18:00:00.0 & 20.6 \\
\hline 2010-11-17 15:00:00.0 & 19.0 \\
\hline 2010-11-17 12:00:00.0 & 12.2 \\
\hline 2010-11-17 09:00:00.0 & 4.9 \\
\hline 2010-11-17 06:00:00.0 & 8.7 \\
\hline 2010-11-17 03:00:00.0 & 10.2 \\
\hline 2010-11-17 00:00:00.0 & 19.1 \\
\hline 2010-11-16 21:00:00.0 & \\
\hline 2010-11-16 18:00:00.0 & 26.0 \\
\hline 2010-11-16 15:00:00.0 & 18.1 \\
\hline 2010-11-16 12:00:00.0 & 14.9 \\
\hline 2010-11-16 09:00:00.0 & 11.9 \\
\hline 2010-11-16 06:00:00.0 & 14.5 \\
\hline 2010-11-16 03:00:00.0 & 16.8 \\
\hline 2010-11-16 00:00:00.0 & 17.3 \\
\hline 2010-11-15 21:00:00.0 & \\
\hline 2010-11-15 18:00:00.0 & 19.2 \\
\hline 2010-11-15 15:00:00.0 & 15.6 \\
\hline 2010-11-15 12:00:00.0 & 19.3 \\
\hline 2010-11-15 09:00:00.0 & 18.0 \\
\hline 2010-11-15 06:00:00.0 & 15.4 \\
\hline 2010-11-15 03:00:00.0 & 20.4 \\
\hline 2010-11-15 00:00:00.0 & 18.2 \\
\hline 2010-11-14 21:00:00.0 & 20.2 \\
\hline 2010-11-14 18:00:00.0 & 26.6 \\
\hline 2010-11-14 15:00:00.0 & 16.2 \\
\hline 2010-11-14 12:00:00.0 & 35.2 \\
\hline 2010-11-14 09:00:00.0 & 18.5 \\
\hline 2010-11-14 06:00:00.0 & 19.4 \\
\hline 2010-11-14 03:00:00.0 & 17.4 \\
\hline 2010-11-14 00:00:00.0 & \\
\hline 2010-11-13 21:00:00.0 & 23.0 \\
\hline 2010-11-13 18:00:00.0 & 24.4 \\
\hline 2010-11-13 15:00:00.0 & 17.3 \\
\hline 2010-11-13 12:00:00.0 & 20.9 \\
\hline 2010-11-13 09:00:00.0 & 14.6 \\
\hline 2010-11-13 06:00:00.0 & 15.6 \\
\hline 2010-11-13 03:00:00.0 & 17.7 \\
\hline 2010-11-13 00:00:00.0 & \\
\hline 2010-11-12 21:00:00.0 & \\
\hline 2010-11-12 18:00:00.0 & 22.7 \\
\hline 2010-11-12 15:00:00.0 & 25.3 \\
\hline 2010-11-12 12:00:00.0 & \\
\hline 2010-11-12 09:00:00.0 & 13.7 \\
\hline 2010-11-12 06:00:00.0 & 16.3 \\
\hline 2010-11-12 03:00:00.0 & 13.8 \\
\hline 2010-11-12 00:00:00.0 & \\
\hline 2010-11-11 21:00:00.0 & 20.5 \\
\hline 2010-11-11 18:00:00.0 & 20.0 \\
\hline 2010-11-11 15:00:00.0 & 13.2 \\
\hline 2010-11-11 12:00:00.0 & 15.1 \\
\hline 2010-11-11 09:00:00.0 & 8.9 \\
\hline 2010-11-11 06:00:00.0 & 9.2 \\
\hline 2010-11-11 03:00:00.0 & 8.5 \\
\hline 2010-11-11 00:00:00.0 & \\
\hline 2010-11-10 21:00:00.0 & 26.4 \\
\hline 2010-11-10 18:00:00.0 & 25.0 \\
\hline 2010-11-10 15:00:00.0 & 25.0 \\
\hline 2010-11-10 12:00:00.0 & \\
\hline 2010-11-10 09:00:00.0 & 10.0 \\
\hline 2010-11-10 06:00:00.0 & 23.3 \\
\hline
\end{tabular}




\section{ANEXO 01 - RAJADAS DE VENTO REGISTRADAS EM SÃO MARTINHO DA SERRA / RS}

\begin{tabular}{|c|c|}
\hline DataHora & VelVentoMax \\
\hline 2010-11-10 03:00:00.0 & 24.4 \\
\hline 2010-11-10 00:00:00.0 & \\
\hline 2010-11-09 21:00:00.0 & 25.8 \\
\hline 2010-11-09 18:00:00.0 & 20.0 \\
\hline 2010-11-09 15:00:00.0 & 44.8 \\
\hline 2010-11-09 12:00:00.0 & 24.9 \\
\hline 2010-11-09 09:00:00.0 & 15.2 \\
\hline 2010-11-09 06:00:00.0 & 11.6 \\
\hline 2010-11-09 03:00:00.0 & 13.9 \\
\hline 2010-11-09 00:00:00.0 & \\
\hline 2010-11-08 21:00:00.0 & 24.2 \\
\hline 2010-11-08 18:00:00.0 & 26.9 \\
\hline 2010-11-08 15:00:00.0 & \\
\hline 2010-11-08 12:00:00.0 & 25.2 \\
\hline 2010-11-08 09:00:00.0 & 18.0 \\
\hline 2010-11-08 06:00:00.0 & 15.2 \\
\hline 2010-11-08 03:00:00.0 & 14.6 \\
\hline 2010-11-08 00:00:00.0 & \\
\hline 2010-11-07 21:00:00.0 & 15.4 \\
\hline 2010-11-07 18:00:00.0 & 24.8 \\
\hline 2010-11-07 15:00:00.0 & 18.8 \\
\hline 2010-11-07 12:00:00.0 & 16.6 \\
\hline 2010-11-07 09:00:00.0 & 13.9 \\
\hline 2010-11-07 06:00:00.0 & 11.7 \\
\hline 2010-11-07 03:00:00.0 & \\
\hline 2010-11-07 00:00:00.0 & \\
\hline 2010-11-06 21:00:00.0 & 22.6 \\
\hline 2010-11-06 18:00:00.0 & 24.5 \\
\hline 2010-11-06 15:00:00.0 & \\
\hline 2010-11-06 12:00:00.0 & 14.4 \\
\hline 2010-11-06 09:00:00.0 & 7.9 \\
\hline 2010-11-06 06:00:00.0 & 7.9 \\
\hline 2010-11-06 03:00:00.0 & \\
\hline 2010-11-06 00:00:00.0 & \\
\hline 2010-11-05 21:00:00.0 & 14.8 \\
\hline 2010-11-05 18:00:00.0 & 9.6 \\
\hline 2010-11-05 15:00:00.0 & 17.2 \\
\hline 2010-11-05 12:00:00.0 & 23.0 \\
\hline 2010-11-05 09:00:00.0 & 26.2 \\
\hline 2010-11-05 06:00:00.0 & 15.5 \\
\hline 2010-11-05 03:00:00.0 & \\
\hline 2010-11-05 00:00:00.0 & \\
\hline 2010-11-04 21:00:00.0 & 17.8 \\
\hline 2010-11-04 18:00:00.0 & 36.5 \\
\hline 2010-11-04 15:00:00.0 & 19.8 \\
\hline 2010-11-04 12:00:00.0 & 4.1 \\
\hline 2010-11-04 09:00:00.0 & 16.8 \\
\hline 2010-11-04 06:00:00.0 & 15.1 \\
\hline 2010-11-04 03:00:00.0 & \\
\hline 2010-11-04 00:00:00.0 & \\
\hline 2010-11-03 21:00:00.0 & 13.0 \\
\hline 2010-11-03 18:00:00.0 & 16.7 \\
\hline 2010-11-03 15:00:00.0 & 20.5 \\
\hline 2010-11-03 12:00:00.0 & 13.7 \\
\hline 2010-11-03 09:00:00.0 & 11.9 \\
\hline 2010-11-03 06:00:00.0 & \\
\hline 2010-11-03 03:00:00.0 & \\
\hline 2010-11-03 00:00:00.0 & 7.3 \\
\hline 2010-11-02 21:00:00.0 & 19.9 \\
\hline 2010-11-02 18:00:00.0 & 20.4 \\
\hline 2010-11-02 15:00:00.0 & 19.5 \\
\hline 2010-11-02 12:00:00.0 & 12.9 \\
\hline 2010-11-02 09:00:00.0 & 8.1 \\
\hline 2010-11-02 06:00:00.0 & 10.0 \\
\hline 2010-11-02 03:00:00.0 & \\
\hline 2010-11-02 00:00:00.0 & 17.3 \\
\hline 2010-11-01 21:00:00.0 & 24.9 \\
\hline 2010-11-01 18:00:00.0 & 25.6 \\
\hline 2010-11-01 15:00:00.0 & 23.4 \\
\hline
\end{tabular}

\begin{tabular}{|c|c|}
\hline DataHora & VelVentoMax \\
\hline 2010-11-01 12:00:00.0 & 20.8 \\
\hline 2010-11-01 09:00:00.0 & 7.5 \\
\hline 2010-11-01 06:00:00.0 & \\
\hline 2010-11-01 03:00:00.0 & \\
\hline 2010-11-01 00:00:00.0 & \\
\hline 2010-10-31 21:00:00.0 & 30.9 \\
\hline 2010-10-31 18:00:00.0 & \\
\hline 2010-10-31 15:00:00.0 & 39.4 \\
\hline 2010-10-31 12:00:00.0 & 18.0 \\
\hline 2010-10-31 09:00:00.0 & 18.0 \\
\hline 2010-10-31 06:00:00.0 & \\
\hline 2010-10-31 03:00:00.0 & \\
\hline 2010-10-31 00:00:00.0 & 13.1 \\
\hline 2010-10-30 21:00:00.0 & 27.1 \\
\hline 2010-10-30 18:00:00.0 & 21.9 \\
\hline 2010-10-30 15:00:00.0 & 24.9 \\
\hline 2010-10-30 12:00:00.0 & 14.9 \\
\hline 2010-10-30 09:00:00.0 & 14.8 \\
\hline 2010-10-30 06:00:00.0 & \\
\hline 2010-10-30 03:00:00.0 & \\
\hline 2010-10-30 00:00:00.0 & 20.7 \\
\hline 2010-10-29 21:00:00.0 & 23.0 \\
\hline 2010-10-29 18:00:00.0 & 26.4 \\
\hline 2010-10-29 15:00:00.0 & 24.1 \\
\hline 2010-10-29 12:00:00.0 & 26.8 \\
\hline 2010-10-29 09:00:00.0 & 23.6 \\
\hline 2010-10-29 06:00:00.0 & 17.3 \\
\hline 2010-10-29 03:00:00.0 & 15.1 \\
\hline 2010-10-29 00:00:00.0 & \\
\hline 2010-10-28 21:00:00.0 & 18.4 \\
\hline 2010-10-28 18:00:00.0 & 18.6 \\
\hline 2010-10-28 15:00:00.0 & 15.6 \\
\hline 2010-10-28 12:00:00.0 & 6.0 \\
\hline 2010-10-28 09:00:00.0 & 14.7 \\
\hline 2010-10-28 06:00:00.0 & 21.4 \\
\hline 2010-10-28 03:00:00.0 & 16.4 \\
\hline 2010-10-28 00:00:00.0 & \\
\hline 2010-10-27 21:00:00.0 & 2.9 \\
\hline 2010-10-27 18:00:00.0 & 18.3 \\
\hline 2010-10-27 15:00:00.0 & 19.6 \\
\hline 2010-10-27 12:00:00.0 & 15.0 \\
\hline 2010-10-27 09:00:00.0 & 10.6 \\
\hline 2010-10-27 06:00:00.0 & 13.6 \\
\hline 2010-10-27 03:00:00.0 & 8.7 \\
\hline 2010-10-27 00:00:00.0 & \\
\hline 2010-10-26 21:00:00.0 & \\
\hline 2010-10-26 18:00:00.0 & 27.6 \\
\hline 2010-10-26 15:00:00.0 & 23.7 \\
\hline 2010-10-26 12:00:00.0 & 14.3 \\
\hline 2010-10-26 09:00:00.0 & 6.4 \\
\hline 2010-10-26 06:00:00.0 & 8.2 \\
\hline 2010-10-26 03:00:00.0 & 11.8 \\
\hline 2010-10-26 00:00:00.0 & 32.8 \\
\hline 2010-10-25 21:00:00.0 & 20.3 \\
\hline 2010-10-25 18:00:00.0 & 20.8 \\
\hline 2010-10-25 15:00:00.0 & 19.0 \\
\hline 2010-10-25 12:00:00.0 & 15.4 \\
\hline 2010-10-25 09:00:00.0 & \\
\hline 2010-10-25 06:00:00.0 & 12.1 \\
\hline 2010-10-25 03:00:00.0 & 9.6 \\
\hline 2010-10-25 00:00:00.0 & \\
\hline 2010-10-24 21:00:00.0 & 15.5 \\
\hline 2010-10-24 18:00:00.0 & 2.7 \\
\hline 2010-10-24 15:00:00.0 & 12.5 \\
\hline 2010-10-24 12:00:00.0 & 14.2 \\
\hline 2010-10-24 09:00:00.0 & \\
\hline 2010-10-24 06:00:00.0 & \\
\hline 2010-10-24 03:00:00.0 & 34.1 \\
\hline 2010-10-24 00:00:00.0 & \\
\hline
\end{tabular}

\begin{tabular}{|c|c|}
\hline DataHora & VelVentoMax \\
\hline 2010-10-23 21:00:00.0 & 16.9 \\
\hline 2010-10-23 18:00:00.0 & 17.9 \\
\hline 2010-10-23 15:00:00.0 & 4.6 \\
\hline 2010-10-23 12:00:00.0 & 3.2 \\
\hline 2010-10-23 09:00:00.0 & 14.5 \\
\hline 2010-10-23 06:00:00.0 & 17.9 \\
\hline 2010-10-23 03:00:00.0 & 7.9 \\
\hline 2010-10-23 00:00:00.0 & \\
\hline 2010-10-22 21:00:00.0 & \\
\hline 2010-10-22 18:00:00.0 & 14.2 \\
\hline 2010-10-22 15:00:00.0 & 17.3 \\
\hline 2010-10-22 12:00:00.0 & 13.7 \\
\hline 2010-10-22 09:00:00.0 & 17.0 \\
\hline 2010-10-22 06:00:00.0 & 15.6 \\
\hline 2010-10-22 03:00:00.0 & 16.5 \\
\hline 2010-10-22 00:00:00.0 & 27.1 \\
\hline 2010-10-21 21:00:00.0 & 12.8 \\
\hline 2010-10-21 18:00:00.0 & 16.2 \\
\hline 2010-10-21 15:00:00.0 & 20.5 \\
\hline 2010-10-21 12:00:00.0 & 20.9 \\
\hline 2010-10-21 09:00:00.0 & 19.9 \\
\hline 2010-10-21 06:00:00.0 & 22.0 \\
\hline 2010-10-21 03:00:00.0 & \\
\hline 2010-10-21 00:00:00.0 & \\
\hline 2010-10-20 21:00:00.0 & 18.7 \\
\hline 2010-10-20 18:00:00.0 & 13.0 \\
\hline 2010-10-20 15:00:00.0 & 18.6 \\
\hline 2010-10-20 12:00:00.0 & 24.9 \\
\hline 2010-10-20 09:00:00.0 & 24.1 \\
\hline 2010-10-20 06:00:00.0 & 15.7 \\
\hline 2010-10-20 03:00:00.0 & 14.4 \\
\hline 2010-10-20 00:00:00.0 & \\
\hline 2010-10-19 21:00:00.0 & 18.0 \\
\hline 2010-10-19 18:00:00.0 & 12.8 \\
\hline 2010-10-19 15:00:00.0 & 19.6 \\
\hline 2010-10-19 12:00:00.0 & 19.3 \\
\hline 2010-10-19 09:00:00.0 & 14.0 \\
\hline 2010-10-19 06:00:00.0 & 9.2 \\
\hline 2010-10-19 03:00:00.0 & 8.5 \\
\hline 2010-10-19 00:00:00.0 & \\
\hline 2010-10-18 21:00:00.0 & 15.7 \\
\hline 2010-10-18 18:00:00.0 & 17.0 \\
\hline 2010-10-18 15:00:00.0 & 17.0 \\
\hline 2010-10-18 12:00:00.0 & 10.8 \\
\hline 2010-10-18 09:00:00.0 & 10.0 \\
\hline 2010-10-18 06:00:00.0 & 7.5 \\
\hline 2010-10-18 03:00:00.0 & 11.3 \\
\hline 2010-10-18 00:00:00.0 & 16.7 \\
\hline 2010-10-17 21:00:00.0 & 17.6 \\
\hline 2010-10-17 18:00:00.0 & 17.6 \\
\hline 2010-10-17 15:00:00.0 & 18.7 \\
\hline 2010-10-17 12:00:00.0 & 15.4 \\
\hline 2010-10-17 09:00:00.0 & 18.2 \\
\hline 2010-10-17 06:00:00.0 & 20.7 \\
\hline 2010-10-17 03:00:00.0 & 15.2 \\
\hline 2010-10-17 00:00:00.0 & \\
\hline 2010-10-16 21:00:00.0 & 20.1 \\
\hline 2010-10-16 18:00:00.0 & 20.8 \\
\hline 2010-10-16 15:00:00.0 & 20.5 \\
\hline 2010-10-16 12:00:00.0 & 14.8 \\
\hline 2010-10-16 09:00:00.0 & 14.9 \\
\hline 2010-10-16 06:00:00.0 & 10.9 \\
\hline 2010-10-16 03:00:00.0 & 6.3 \\
\hline 2010-10-16 00:00:00.0 & \\
\hline 2010-10-15 21:00:00.0 & 12.0 \\
\hline 2010-10-15 18:00:00.0 & 34.2 \\
\hline 2010-10-15 15:00:00.0 & 31.0 \\
\hline 2010-10-15 12:00:00.0 & 12.6 \\
\hline 2010-10-15 09:00:00.0 & 16.9 \\
\hline
\end{tabular}




\section{ANEXO 01 - RAJADAS DE VENTO REGISTRADAS EM SÃO MARTINHO DA SERRA / RS}

\begin{tabular}{|c|c|}
\hline DataHora & VelVentoMax \\
\hline 2010-10-15 06:00:00.0 & 14.6 \\
\hline 2010-10-15 03:00:00.0 & 12.4 \\
\hline 2010-10-15 00:00:00.0 & \\
\hline 2010-10-14 21:00:00.0 & 22.6 \\
\hline 2010-10-14 18:00:00.0 & 31.2 \\
\hline 2010-10-14 15:00:00.0 & 29.6 \\
\hline 2010-10-14 12:00:00.0 & \\
\hline 2010-10-14 09:00:00.0 & 16.8 \\
\hline 2010-10-14 06:00:00.0 & 34.4 \\
\hline 2010-10-14 03:00:00.0 & \\
\hline 2010-10-14 00:00:00.0 & 13.4 \\
\hline 2010-10-13 21:00:00.0 & 17.8 \\
\hline 2010-10-13 18:00:00.0 & 22.3 \\
\hline 2010-10-13 15:00:00.0 & \\
\hline 2010-10-13 12:00:00.0 & 28.8 \\
\hline 2010-10-13 09:00:00.0 & 27.3 \\
\hline 2010-10-13 06:00:00.0 & 24.2 \\
\hline 2010-10-13 03:00:00.0 & \\
\hline 2010-10-13 00:00:00.0 & 14.9 \\
\hline 2010-10-12 21:00:00.0 & 19.2 \\
\hline 2010-10-12 18:00:00.0 & 24.1 \\
\hline 2010-10-12 15:00:00.0 & 25.7 \\
\hline 2010-10-12 12:00:00.0 & 7.3 \\
\hline 2010-10-12 09:00:00.0 & 20.0 \\
\hline 2010-10-12 06:00:00.0 & 17.6 \\
\hline 2010-10-12 03:00:00.0 & 13.3 \\
\hline 2010-10-12 00:00:00.0 & 19.5 \\
\hline 2010-10-11 21:00:00.0 & 22.2 \\
\hline 2010-10-11 18:00:00.0 & 21.4 \\
\hline 2010-10-11 15:00:00.0 & 25.5 \\
\hline 2010-10-11 12:00:00.0 & 25.1 \\
\hline 2010-10-11 09:00:00.0 & 15.1 \\
\hline 2010-10-11 06:00:00.0 & 17.1 \\
\hline 2010-10-11 03:00:00.0 & \\
\hline 2010-10-11 00:00:00.0 & 14.8 \\
\hline 2010-10-10 21:00:00.0 & 26.1 \\
\hline 2010-10-10 18:00:00.0 & 27.2 \\
\hline 2010-10-10 15:00:00.0 & 26.8 \\
\hline 2010-10-10 12:00:00.0 & 25.0 \\
\hline 2010-10-10 09:00:00.0 & 17.5 \\
\hline 2010-10-10 06:00:00.0 & 16.7 \\
\hline 2010-10-10 03:00:00.0 & \\
\hline 2010-10-10 00:00:00.0 & 19.0 \\
\hline 2010-10-09 21:00:00.0 & 22.5 \\
\hline 2010-10-09 18:00:00.0 & 21.6 \\
\hline 2010-10-09 15:00:00.0 & 18.2 \\
\hline 2010-10-09 12:00:00.0 & 3.0 \\
\hline 2010-10-09 09:00:00.0 & 11.5 \\
\hline 2010-10-09 06:00:00.0 & 18.6 \\
\hline 2010-10-09 03:00:00.0 & 16.8 \\
\hline 2010-10-09 00:00:00.0 & 10.9 \\
\hline 2010-10-08 21:00:00.0 & 17.4 \\
\hline 2010-10-08 18:00:00.0 & 18.5 \\
\hline 2010-10-08 15:00:00.0 & 21.7 \\
\hline 2010-10-08 12:00:00.0 & 22.9 \\
\hline 2010-10-08 09:00:00.0 & 26.4 \\
\hline 2010-10-08 06:00:00.0 & 23.7 \\
\hline 2010-10-08 03:00:00.0 & 18.4 \\
\hline 2010-10-08 00:00:00.0 & 23.7 \\
\hline 2010-10-07 21:00:00.0 & 35.0 \\
\hline 2010-10-07 18:00:00.0 & \\
\hline 2010-10-07 15:00:00.0 & \\
\hline 2010-10-07 12:00:00.0 & 28.2 \\
\hline 2010-10-07 09:00:00.0 & 29.8 \\
\hline 2010-10-07 06:00:00.0 & 12.2 \\
\hline 2010-10-07 03:00:00.0 & 23.5 \\
\hline 2010-10-07 00:00:00.0 & 4.3 \\
\hline 2010-10-06 21:00:00.0 & 14.1 \\
\hline 2010-10-06 18:00:00.0 & \\
\hline
\end{tabular}

\begin{tabular}{|c|c|}
\hline DataHora & VelVentoMax \\
\hline 2010-10-06 15:00:00.0 & 18.7 \\
\hline 2010-10-06 12:00:00.0 & 20.7 \\
\hline 2010-10-06 09:00:00.0 & 34.7 \\
\hline 2010-10-06 06:00:00.0 & 14.8 \\
\hline 2010-10-06 03:00:00.0 & 14.6 \\
\hline 2010-10-06 00:00:00.0 & 12.3 \\
\hline 2010-10-05 21:00:00.0 & 14.2 \\
\hline 2010-10-05 18:00:00.0 & 10.6 \\
\hline 2010-10-05 15:00:00.0 & 27.1 \\
\hline 2010-10-05 12:00:00.0 & 6.1 \\
\hline 2010-10-05 09:00:00.0 & 6.6 \\
\hline 2010-10-05 06:00:00.0 & 7.6 \\
\hline 2010-10-05 03:00:00.0 & 6.7 \\
\hline 2010-10-05 00:00:00.0 & 8.1 \\
\hline 2010-10-04 21:00:00.0 & 14.2 \\
\hline 2010-10-04 18:00:00.0 & \\
\hline 2010-10-04 15:00:00.0 & 12.7 \\
\hline 2010-10-04 12:00:00.0 & 41.9 \\
\hline 2010-10-04 09:00:00.0 & 17.0 \\
\hline 2010-10-04 06:00:00.0 & 13.5 \\
\hline 2010-10-04 03:00:00.0 & \\
\hline 2010-10-04 00:00:00.0 & 16.6 \\
\hline 2010-10-03 21:00:00.0 & 17.0 \\
\hline 2010-10-03 18:00:00.0 & 26.7 \\
\hline 2010-10-03 15:00:00.0 & 21.7 \\
\hline 2010-10-03 12:00:00.0 & 17.3 \\
\hline 2010-10-03 09:00:00.0 & 14.3 \\
\hline 2010-10-03 06:00:00.0 & 8.2 \\
\hline 2010-10-03 03:00:00.0 & 7.0 \\
\hline 2010-10-03 00:00:00.0 & 9.7 \\
\hline 2010-10-02 21:00:00.0 & 21.1 \\
\hline 2010-10-02 18:00:00.0 & \\
\hline 2010-10-02 15:00:00.0 & 34.8 \\
\hline 2010-10-02 12:00:00.0 & 18.7 \\
\hline 2010-10-02 09:00:00.0 & 14.0 \\
\hline 2010-10-02 06:00:00.0 & 22.4 \\
\hline 2010-10-02 03:00:00.0 & 18.5 \\
\hline 2010-10-02 00:00:00.0 & 15.6 \\
\hline 2010-10-01 21:00:00.0 & 20.4 \\
\hline 2010-10-01 18:00:00.0 & 20.5 \\
\hline 2010-10-01 15:00:00.0 & 32.3 \\
\hline 2010-10-01 12:00:00.0 & 24.0 \\
\hline 2010-10-01 09:00:00.0 & 15.2 \\
\hline 2010-10-01 06:00:00.0 & 13.1 \\
\hline 2010-10-01 03:00:00.0 & 9.6 \\
\hline 2010-10-01 00:00:00.0 & 9.3 \\
\hline 2010-09-30 21:00:00.0 & 12.5 \\
\hline 2010-09-30 18:00:00.0 & 16.1 \\
\hline 2010-09-30 15:00:00.0 & 16.3 \\
\hline 2010-09-30 12:00:00.0 & 13.9 \\
\hline 2010-09-30 09:00:00.0 & 8.6 \\
\hline 2010-09-30 06:00:00.0 & 15.8 \\
\hline 2010-09-30 03:00:00.0 & 13.4 \\
\hline 2010-09-30 00:00:00.0 & 15.6 \\
\hline 2010-09-29 21:00:00.0 & 23.7 \\
\hline 2010-09-29 18:00:00.0 & 24.5 \\
\hline 2010-09-29 15:00:00.0 & 22.4 \\
\hline 2010-09-29 12:00:00.0 & 8.8 \\
\hline 2010-09-29 09:00:00.0 & 21.7 \\
\hline 2010-09-29 06:00:00.0 & 10.8 \\
\hline 2010-09-29 03:00:00.0 & 12.6 \\
\hline 2010-09-29 00:00:00.0 & 10.5 \\
\hline 2010-09-28 21:00:00.0 & 12.6 \\
\hline 2010-09-28 18:00:00.0 & 21.7 \\
\hline 2010-09-28 15:00:00.0 & 20.9 \\
\hline 2010-09-28 12:00:00.0 & 24.9 \\
\hline 2010-09-28 09:00:00.0 & 22.8 \\
\hline 2010-09-28 06:00:00.0 & 21.8 \\
\hline 2010-09-28 03:00:00.0 & 20.5 \\
\hline
\end{tabular}

\begin{tabular}{|c|c|}
\hline DataHora & VelVentoMax \\
\hline 2010-09-28 00:00:00.0 & 21.8 \\
\hline 2010-09-27 21:00:00.0 & 22.1 \\
\hline 2010-09-27 18:00:00.0 & 18.6 \\
\hline 2010-09-27 15:00:00.0 & \\
\hline 2010-09-27 12:00:00.0 & 22.0 \\
\hline 2010-09-27 09:00:00.0 & 19.8 \\
\hline 2010-09-27 06:00:00.0 & 19.7 \\
\hline 2010-09-27 03:00:00.0 & 21.3 \\
\hline 2010-09-27 00:00:00.0 & \\
\hline 2010-09-26 21:00:00.0 & 21.0 \\
\hline 2010-09-26 18:00:00.0 & 19.6 \\
\hline 2010-09-26 15:00:00.0 & 17.7 \\
\hline 2010-09-26 12:00:00.0 & 20.3 \\
\hline 2010-09-26 09:00:00.0 & 21.8 \\
\hline 2010-09-26 06:00:00.0 & 20.9 \\
\hline 2010-09-26 03:00:00.0 & 18.6 \\
\hline 2010-09-26 00:00:00.0 & 14.7 \\
\hline 2010-09-25 21:00:00.0 & 33.6 \\
\hline 2010-09-25 18:00:00.0 & 13.0 \\
\hline 2010-09-25 15:00:00.0 & 13.2 \\
\hline 2010-09-25 12:00:00.0 & 14.0 \\
\hline 2010-09-25 09:00:00.0 & 11.7 \\
\hline 2010-09-25 06:00:00.0 & 15.1 \\
\hline 2010-09-25 03:00:00.0 & 13.6 \\
\hline 2010-09-25 00:00:00.0 & 11.5 \\
\hline 2010-09-24 21:00:00.0 & 15.1 \\
\hline 2010-09-24 18:00:00.0 & 19.9 \\
\hline 2010-09-24 15:00:00.0 & 19.2 \\
\hline 2010-09-24 12:00:00.0 & 14.3 \\
\hline 2010-09-24 09:00:00.0 & 23.8 \\
\hline 2010-09-24 06:00:00.0 & 23.8 \\
\hline 2010-09-24 03:00:00.0 & 21.0 \\
\hline 2010-09-24 00:00:00.0 & 3.5 \\
\hline 2010-09-23 21:00:00.0 & 13.6 \\
\hline 2010-09-23 18:00:00.0 & 13.8 \\
\hline 2010-09-23 15:00:00.0 & 37.5 \\
\hline 2010-09-23 12:00:00.0 & \\
\hline 2010-09-23 09:00:00.0 & 13.3 \\
\hline 2010-09-23 06:00:00.0 & 16.4 \\
\hline 2010-09-23 03:00:00.0 & \\
\hline 2010-09-23 00:00:00.0 & \\
\hline 2010-09-22 21:00:00.0 & 29.8 \\
\hline 2010-09-22 18:00:00.0 & 28.4 \\
\hline 2010-09-22 15:00:00.0 & 35.6 \\
\hline 2010-09-22 12:00:00.0 & 38.2 \\
\hline 2010-09-22 09:00:00.0 & 28.4 \\
\hline 2010-09-22 06:00:00.0 & 35.0 \\
\hline 2010-09-22 03:00:00.0 & 45.1 \\
\hline 2010-09-22 00:00:00.0 & \\
\hline 2010-09-21 21:00:00.0 & 44.5 \\
\hline 2010-09-21 18:00:00.0 & 32.1 \\
\hline 2010-09-21 15:00:00.0 & 24.0 \\
\hline 2010-09-21 12:00:00.0 & 35.0 \\
\hline 2010-09-21 09:00:00.0 & 25.4 \\
\hline 2010-09-21 06:00:00.0 & 34.1 \\
\hline 2010-09-21 03:00:00.0 & 21.6 \\
\hline 2010-09-21 00:00:00.0 & \\
\hline 2010-09-20 21:00:00.0 & 19.4 \\
\hline 2010-09-20 18:00:00.0 & 20.8 \\
\hline 2010-09-20 15:00:00.0 & 23.5 \\
\hline 2010-09-20 12:00:00.0 & 24.8 \\
\hline 2010-09-20 09:00:00.0 & 21.8 \\
\hline 2010-09-20 06:00:00.0 & 21.0 \\
\hline 2010-09-20 03:00:00.0 & 17.0 \\
\hline 2010-09-20 00:00:00.0 & \\
\hline 2010-09-19 21:00:00.0 & 30.5 \\
\hline 2010-09-19 18:00:00.0 & 17.7 \\
\hline 2010-09-19 15:00:00.0 & 28.2 \\
\hline 2010-09-19 12:00:00.0 & 29.5 \\
\hline
\end{tabular}




\section{ANEXO 01 - RAJADAS DE VENTO REGISTRADAS EM SÃO MARTINHO DA SERRA / RS}

\begin{tabular}{|c|c|}
\hline DataHora & VelVentoMax \\
\hline 2010-09-19 09:00:00.0 & 23.2 \\
\hline 2010-09-19 06:00:00.0 & 21.5 \\
\hline 2010-09-19 03:00:00.0 & 25.0 \\
\hline 2010-09-19 00:00:00.0 & \\
\hline 2010-09-18 21:00:00.0 & 19.5 \\
\hline 2010-09-18 18:00:00.0 & 18.2 \\
\hline 2010-09-18 15:00:00.0 & \\
\hline 2010-09-18 12:00:00.0 & 22.0 \\
\hline 2010-09-18 09:00:00.0 & 20.0 \\
\hline 2010-09-18 06:00:00.0 & 15.4 \\
\hline 2010-09-18 03:00:00.0 & 13.8 \\
\hline 2010-09-18 00:00:00.0 & 13.1 \\
\hline 2010-09-17 21:00:00.0 & 18.8 \\
\hline 2010-09-17 18:00:00.0 & 18.5 \\
\hline 2010-09-17 15:00:00.0 & \\
\hline 2010-09-17 12:00:00.0 & 25.0 \\
\hline 2010-09-17 09:00:00.0 & 23.7 \\
\hline 2010-09-17 06:00:00.0 & 14.9 \\
\hline 2010-09-17 03:00:00.0 & \\
\hline 2010-09-17 00:00:00.0 & \\
\hline 2010-09-16 21:00:00.0 & \\
\hline 2010-09-16 09:00:00.0 & 23.7 \\
\hline 2010-09-16 06:00:00.0 & \\
\hline 2010-09-16 03:00:00.0 & \\
\hline 2010-09-16 00:00:00.0 & \\
\hline 2010-09-15 21:00:00.0 & 27.1 \\
\hline 2010-09-15 18:00:00.0 & 27.1 \\
\hline 2010-09-15 15:00:00.0 & 29.8 \\
\hline 2010-09-15 12:00:00.0 & 10.0 \\
\hline 2010-09-15 09:00:00.0 & 12.2 \\
\hline 2010-09-15 06:00:00.0 & 12.0 \\
\hline 2010-09-15 03:00:00.0 & \\
\hline 2010-09-15 00:00:00.0 & \\
\hline 2010-09-14 21:00:00.0 & 26.3 \\
\hline 2010-09-14 18:00:00.0 & \\
\hline 2010-09-14 15:00:00.0 & \\
\hline 2010-09-14 12:00:00.0 & 37.2 \\
\hline 2010-09-14 09:00:00.0 & 37.1 \\
\hline 2010-09-14 06:00:00.0 & 37.6 \\
\hline 2010-09-14 03:00:00.0 & \\
\hline 2010-09-14 00:00:00.0 & 28.9 \\
\hline 2010-09-13 21:00:00.0 & 15.9 \\
\hline 2010-09-13 18:00:00.0 & 27.4 \\
\hline 2010-09-13 15:00:00.0 & \\
\hline 2010-09-13 12:00:00.0 & 23.1 \\
\hline 2010-09-13 09:00:00.0 & 25.3 \\
\hline 2010-09-13 06:00:00.0 & 34.8 \\
\hline 2010-09-13 03:00:00.0 & \\
\hline 2010-09-13 00:00:00.0 & 20.6 \\
\hline 2010-09-12 21:00:00.0 & 21.1 \\
\hline 2010-09-12 18:00:00.0 & 30.5 \\
\hline 2010-09-12 15:00:00.0 & \\
\hline 2010-09-12 12:00:00.0 & 25.0 \\
\hline 2010-09-12 09:00:00.0 & 25.7 \\
\hline 2010-09-12 06:00:00.0 & \\
\hline 2010-09-12 03:00:00.0 & \\
\hline 2010-09-12 00:00:00.0 & \\
\hline 2010-09-11 21:00:00.0 & 19.2 \\
\hline 2010-09-11 18:00:00.0 & 16.1 \\
\hline 2010-09-11 15:00:00.0 & 13.3 \\
\hline 2010-09-11 12:00:00.0 & 17.1 \\
\hline 2010-09-11 09:00:00.0 & 27.7 \\
\hline 2010-09-11 06:00:00.0 & 27.5 \\
\hline 2010-09-11 03:00:00.0 & \\
\hline 2010-09-11 00:00:00.0 & \\
\hline 2010-09-10 21:00:00.0 & 31.3 \\
\hline 2010-09-10 18:00:00.0 & 23.3 \\
\hline 2010-09-10 15:00:00.0 & 24.0 \\
\hline 2010-09-10 12:00:00.0 & 27.2 \\
\hline
\end{tabular}

\begin{tabular}{|c|c|}
\hline DataHora & VelVentoMax \\
\hline 2010-09-10 09:00:00.0 & 28.8 \\
\hline 2010-09-10 06:00:00.0 & \\
\hline 2010-09-10 03:00:00.0 & \\
\hline 2010-09-10 00:00:00.0 & \\
\hline 2010-09-09 21:00:00.0 & 15.6 \\
\hline 2010-09-09 18:00:00.0 & 21.6 \\
\hline 2010-09-09 15:00:00.0 & 24.3 \\
\hline 2010-09-09 12:00:00.0 & 27.1 \\
\hline 2010-09-09 09:00:00.0 & 19.2 \\
\hline 2010-09-09 06:00:00.0 & 17.4 \\
\hline 2010-09-09 03:00:00.0 & \\
\hline 2010-09-09 00:00:00.0 & 16.0 \\
\hline 2010-09-08 21:00:00.0 & 15.7 \\
\hline 2010-09-08 18:00:00.0 & 18.5 \\
\hline 2010-09-08 15:00:00.0 & 22.0 \\
\hline 2010-09-08 12:00:00.0 & 19.7 \\
\hline 2010-09-08 09:00:00.0 & 19.2 \\
\hline 2010-09-08 06:00:00.0 & \\
\hline 2010-09-08 03:00:00.0 & \\
\hline 2010-09-08 00:00:00.0 & \\
\hline 2010-09-07 21:00:00.0 & 15.7 \\
\hline 2010-09-07 18:00:00.0 & \\
\hline 2010-09-07 15:00:00.0 & 27.1 \\
\hline 2010-09-07 12:00:00.0 & 3.1 \\
\hline 2010-09-07 09:00:00.0 & 12.8 \\
\hline 2010-09-07 06:00:00.0 & 13.7 \\
\hline 2010-09-07 03:00:00.0 & \\
\hline 2010-09-07 00:00:00.0 & \\
\hline 2010-09-06 21:00:00.0 & 15.9 \\
\hline 2010-09-06 18:00:00.0 & 17.8 \\
\hline 2010-09-06 15:00:00.0 & 23.1 \\
\hline 2010-09-06 12:00:00.0 & 22.8 \\
\hline 2010-09-06 09:00:00.0 & 12.3 \\
\hline 2010-09-06 06:00:00.0 & 11.5 \\
\hline 2010-09-06 03:00:00.0 & \\
\hline 2010-09-06 00:00:00.0 & \\
\hline 2010-09-05 21:00:00.0 & 15.5 \\
\hline 2010-09-05 18:00:00.0 & 17.5 \\
\hline 2010-09-05 15:00:00.0 & 17.0 \\
\hline 2010-09-05 12:00:00.0 & 15.6 \\
\hline 2010-09-05 09:00:00.0 & 11.9 \\
\hline 2010-09-05 06:00:00.0 & 11.3 \\
\hline 2010-09-05 03:00:00.0 & \\
\hline 2010-09-05 00:00:00.0 & 29.1 \\
\hline 2010-09-04 21:00:00.0 & 33.6 \\
\hline 2010-09-04 18:00:00.0 & 33.5 \\
\hline 2010-09-04 15:00:00.0 & 26.7 \\
\hline 2010-09-04 12:00:00.0 & 24.7 \\
\hline 2010-09-04 09:00:00.0 & 24.7 \\
\hline 2010-09-04 06:00:00.0 & 23.1 \\
\hline 2010-09-04 03:00:00.0 & \\
\hline 2010-09-04 00:00:00.0 & \\
\hline 2010-09-03 21:00:00.0 & 19.7 \\
\hline 2010-09-03 18:00:00.0 & 23.7 \\
\hline 2010-09-03 15:00:00.0 & 20.0 \\
\hline 2010-09-03 12:00:00.0 & 22.7 \\
\hline 2010-09-03 09:00:00.0 & 23.0 \\
\hline 2010-09-03 06:00:00.0 & \\
\hline 2010-09-03 03:00:00.0 & \\
\hline 2010-09-03 00:00:00.0 & \\
\hline 2010-09-02 21:00:00.0 & 16.5 \\
\hline 2010-09-02 18:00:00.0 & 15.6 \\
\hline 2010-09-02 15:00:00.0 & 11.9 \\
\hline 2010-09-02 12:00:00.0 & 9.7 \\
\hline 2010-09-02 09:00:00.0 & 10.8 \\
\hline 2010-09-02 06:00:00.0 & 16.6 \\
\hline 2010-09-02 03:00:00.0 & \\
\hline 2010-09-02 00:00:00.0 & \\
\hline 2010-09-01 21:00:00.0 & 22.4 \\
\hline
\end{tabular}

\begin{tabular}{|c|c|}
\hline DataHora & VelVentoMax \\
\hline 2010-09-01 18:00:00.0 & 11.1 \\
\hline 2010-09-01 15:00:00.0 & 11.8 \\
\hline 2010-09-01 12:00:00.0 & 24.3 \\
\hline 2010-09-01 09:00:00.0 & 27.4 \\
\hline 2010-09-01 06:00:00.0 & 26.8 \\
\hline 2010-09-01 03:00:00.0 & \\
\hline 2010-09-01 00:00:00.0 & \\
\hline 2010-08-31 21:00:00.0 & 19.8 \\
\hline 2010-08-31 18:00:00.0 & 23.3 \\
\hline 2010-08-31 15:00:00.0 & 27.7 \\
\hline 2010-08-31 12:00:00.0 & 30.6 \\
\hline 2010-08-31 09:00:00.0 & \\
\hline 2010-08-31 06:00:00.0 & 27.3 \\
\hline 2010-08-31 03:00:00.0 & \\
\hline 2010-08-31 00:00:00.0 & \\
\hline 2010-08-30 21:00:00.0 & 20.9 \\
\hline 2010-08-30 18:00:00.0 & 22.8 \\
\hline 2010-08-30 15:00:00.0 & 26.5 \\
\hline 2010-08-30 12:00:00.0 & 31.1 \\
\hline 2010-08-30 09:00:00.0 & 29.4 \\
\hline 2010-08-30 06:00:00.0 & 27.0 \\
\hline 2010-08-30 03:00:00.0 & \\
\hline 2010-08-30 00:00:00.0 & \\
\hline 2010-08-29 21:00:00.0 & 19.8 \\
\hline $2010-08-29$ 18:00:00.0 & 23.6 \\
\hline 2010-08-29 15:00:00.0 & 34.5 \\
\hline 2010-08-29 12:00:00.0 & 26.2 \\
\hline 2010-08-29 09:00:00.0 & 31.3 \\
\hline 2010-08-29 06:00:00.0 & 23.7 \\
\hline 2010-08-29 03:00:00.0 & \\
\hline 2010-08-29 00:00:00.0 & \\
\hline 2010-08-28 21:00:00.0 & 5.0 \\
\hline 2010-08-28 18:00:00.0 & 19.6 \\
\hline 2010-08-28 15:00:00.0 & 2.8 \\
\hline 2010-08-28 12:00:00.0 & 23.4 \\
\hline 2010-08-28 09:00:00.0 & 29.7 \\
\hline 2010-08-28 06:00:00.0 & 29.2 \\
\hline 2010-08-28 03:00:00.0 & \\
\hline 2010-08-28 00:00:00.0 & \\
\hline 2010-08-27 21:00:00.0 & 21.0 \\
\hline 2010-08-27 18:00:00.0 & 23.4 \\
\hline 2010-08-27 15:00:00.0 & \\
\hline 2010-08-27 12:00:00.0 & 17.8 \\
\hline 2010-08-27 09:00:00.0 & \\
\hline 2010-08-27 06:00:00.0 & 23.1 \\
\hline 2010-08-27 03:00:00.0 & \\
\hline 2010-08-27 00:00:00.0 & \\
\hline 2010-08-26 21:00:00.0 & 18.8 \\
\hline 2010-08-26 18:00:00.0 & 14.7 \\
\hline 2010-08-26 15:00:00.0 & 15.2 \\
\hline 2010-08-26 12:00:00.0 & 12.4 \\
\hline 2010-08-26 09:00:00.0 & 8.5 \\
\hline 2010-08-26 06:00:00.0 & 14.7 \\
\hline 2010-08-26 03:00:00.0 & \\
\hline 2010-08-26 00:00:00.0 & 15.7 \\
\hline 2010-08-25 21:00:00.0 & 14.7 \\
\hline 2010-08-25 18:00:00.0 & 20.2 \\
\hline 2010-08-25 15:00:00.0 & \\
\hline 2010-08-25 12:00:00.0 & 23.7 \\
\hline 2010-08-25 09:00:00.0 & 22.2 \\
\hline 2010-08-25 06:00:00.0 & 19.5 \\
\hline 2010-08-25 03:00:00.0 & \\
\hline 2010-08-25 00:00:00.0 & 26.2 \\
\hline 2010-08-24 21:00:00.0 & 18.6 \\
\hline 2010-08-24 18:00:00.0 & 21.2 \\
\hline $2010-08-24$ 15:00:00.0 & 21.6 \\
\hline 2010-08-24 12:00:00.0 & 12.5 \\
\hline 2010-08-24 09:00:00.0 & 23.2 \\
\hline 2010-08-24 06:00:00.0 & 24.7 \\
\hline
\end{tabular}




\section{ANEXO 01 - RAJADAS DE VENTO REGISTRADAS EM SÃO MARTINHO DA SERRA / RS}

\begin{tabular}{|c|c|}
\hline DataHora & VelVentoMax \\
\hline \multicolumn{2}{|l|}{ 2010-08-24 03:00:00.0 } \\
\hline 2010-08-24 00:00:00.0 & 19.4 \\
\hline 2010-08-23 21:00:00.0 & 25.1 \\
\hline 2010-08-23 18:00:00.0 & 30.7 \\
\hline 2010-08-23 15:00:00.0 & 28.0 \\
\hline 2010-08-23 12:00:00.0 & 25.7 \\
\hline 2010-08-23 09:00:00.0 & 22.1 \\
\hline 2010-08-23 06:00:00.0 & 19.6 \\
\hline \multicolumn{2}{|l|}{ 2010-08-23 03:00:00.0 } \\
\hline 2010-08-23 00:00:00.0 & 17.9 \\
\hline 2010-08-22 21:00:00.0 & 23.3 \\
\hline 2010-08-22 18:00:00.0 & 26.3 \\
\hline 2010-08-22 15:00:00.0 & 18.7 \\
\hline 2010-08-22 12:00:00.0 & 19.1 \\
\hline 2010-08-22 09:00:00.0 & 17.6 \\
\hline 2010-08-22 06:00:00.0 & 16.9 \\
\hline \multicolumn{2}{|l|}{ 2010-08-22 03:00:00.0 } \\
\hline 2010-08-22 00:00:00.0 & 15.7 \\
\hline 2010-08-21 21:00:00.0 & 2.5 \\
\hline 2010-08-21 18:00:00.0 & 17.8 \\
\hline \multicolumn{2}{|l|}{ 2010-08-21 15:00:00.0 } \\
\hline 2010-08-21 12:00:00.0 & 18.6 \\
\hline 2010-08-21 09:00:00.0 & 16.8 \\
\hline 2010-08-21 06:00:00.0 & 16.3 \\
\hline \multicolumn{2}{|l|}{ 2010-08-21 03:00:00.0 } \\
\hline 2010-08-21 00:00:00.0 & 11.2 \\
\hline 2010-08-20 21:00:00.0 & 9.0 \\
\hline 2010-08-20 18:00:00.0 & 12.6 \\
\hline \multicolumn{2}{|l|}{ 2010-08-20 15:00:00.0 } \\
\hline 2010-08-20 12:00:00.0 & 27.6 \\
\hline 2010-08-20 09:00:00.0 & 9.4 \\
\hline \multicolumn{2}{|l|}{ 2010-08-20 06:00:00.0 } \\
\hline \multicolumn{2}{|l|}{ 2010-08-20 03:00:00.0 } \\
\hline 2010-08-20 00:00:00.0 & 13.6 \\
\hline 2010-08-19 21:00:00.0 & 16.5 \\
\hline 2010-08-19 18:00:00.0 & 10.5 \\
\hline \multicolumn{2}{|l|}{ 2010-08-19 15:00:00.0 } \\
\hline \multicolumn{2}{|l|}{ 2010-08-19 12:00:00.0 } \\
\hline \multicolumn{2}{|l|}{ 2010-08-19 09:00:00.0 } \\
\hline 2010-08-18 12:00:00.0 & 27.3 \\
\hline 2010-08-18 09:00:00.0 & 22.8 \\
\hline 2010-08-18 06:00:00.0 & 22.2 \\
\hline \multicolumn{2}{|l|}{ 2010-08-18 03:00:00.0 } \\
\hline 2010-08-18 00:00:00.0 & 18.0 \\
\hline 2010-08-17 21:00:00.0 & 16.7 \\
\hline 2010-08-17 18:00:00.0 & 22.8 \\
\hline \multicolumn{2}{|l|}{ 2010-08-17 15:00:00.0 } \\
\hline 2010-08-17 12:00:00.0 & 26.2 \\
\hline 2010-08-17 09:00:00.0 & 25.1 \\
\hline 2010-08-17 06:00:00.0 & \\
\hline 2010-08-17 03:00:00.0 & 19.9 \\
\hline 2010-08-17 00:00:00.0 & 19.6 \\
\hline 2010-08-16 21:00:00.0 & 20.0 \\
\hline 2010-08-16 18:00:00.0 & 30.4 \\
\hline 2010-08-16 15:00:00.0 & 33.0 \\
\hline 2010-08-16 12:00:00.0 & 23.0 \\
\hline 2010-08-16 09:00:00.0 & \\
\hline 2010-08-16 06:00:00.0 & 21.6 \\
\hline 2010-08-16 03:00:00.0 & 19.2 \\
\hline 2010-08-16 00:00:00.0 & 16.2 \\
\hline 2010-08-15 21:00:00.0 & 19.9 \\
\hline 2010-08-15 18:00:00.0 & 21.8 \\
\hline 2010-08-15 15:00:00.0 & 18.9 \\
\hline 2010-08-15 12:00:00.0 & 16.3 \\
\hline 2010-08-15 09:00:00.0 & 15.3 \\
\hline 2010-08-15 06:00:00.0 & \\
\hline 2010-08-15 03:00:00.0 & 7.6 \\
\hline 2010-08-15 00:00:00.0 & 13.8 \\
\hline 2010-08-14 21:00:00.0 & 12.5 \\
\hline
\end{tabular}

\begin{tabular}{|c|c|}
\hline DataHora & VelVentoMax \\
\hline 2010-08-14 18:00:00.0 & 17.6 \\
\hline 2010-08-14 15:00:00.0 & 15.9 \\
\hline 2010-08-14 12:00:00.0 & 16.4 \\
\hline 2010-08-14 09:00:00.0 & \\
\hline 2010-08-14 06:00:00.0 & \\
\hline 2010-08-14 03:00:00.0 & 18.3 \\
\hline 2010-08-14 00:00:00.0 & 18.7 \\
\hline 2010-08-13 21:00:00.0 & 26.2 \\
\hline 2010-08-13 18:00:00.0 & 28.4 \\
\hline 2010-08-13 15:00:00.0 & 26.7 \\
\hline 2010-08-13 12:00:00.0 & 34.1 \\
\hline 2010-08-13 09:00:00.0 & 37.0 \\
\hline 2010-08-13 06:00:00.0 & 31.7 \\
\hline 2010-08-13 03:00:00.0 & 35.1 \\
\hline 2010-08-13 00:00:00.0 & 27.5 \\
\hline 2010-08-12 21:00:00.0 & 33.1 \\
\hline 2010-08-12 18:00:00.0 & 35.3 \\
\hline 2010-08-12 15:00:00.0 & 34.2 \\
\hline 2010-08-12 12:00:00.0 & 31.1 \\
\hline 2010-08-12 09:00:00.0 & 41.5 \\
\hline 2010-08-12 06:00:00.0 & 28.9 \\
\hline 2010-08-12 03:00:00.0 & 27.1 \\
\hline 2010-08-12 00:00:00.0 & 24.8 \\
\hline 2010-08-11 21:00:00.0 & 23.3 \\
\hline 2010-08-11 18:00:00.0 & 23.1 \\
\hline 2010-08-11 15:00:00.0 & 25.9 \\
\hline 2010-08-11 12:00:00.0 & 25.7 \\
\hline 2010-08-11 09:00:00.0 & \\
\hline 2010-08-11 06:00:00.0 & 21.5 \\
\hline 2010-08-11 03:00:00.0 & \\
\hline 2010-08-11 00:00:00.0 & 15.3 \\
\hline 2010-08-10 21:00:00.0 & 22.6 \\
\hline 2010-08-10 18:00:00.0 & 27.5 \\
\hline 2010-08-10 15:00:00.0 & 31.8 \\
\hline 2010-08-10 12:00:00.0 & \\
\hline 2010-08-10 09:00:00.0 & 16.1 \\
\hline 2010-08-10 06:00:00.0 & 15.1 \\
\hline 2010-08-10 03:00:00.0 & 17.8 \\
\hline 2010-08-10 00:00:00.0 & \\
\hline 2010-08-09 21:00:00.0 & 21.8 \\
\hline 2010-08-09 18:00:00.0 & 21.3 \\
\hline 2010-08-09 15:00:00.0 & 21.1 \\
\hline 2010-08-09 12:00:00.0 & 15.5 \\
\hline 2010-08-09 09:00:00.0 & \\
\hline 2010-08-09 06:00:00.0 & 15.8 \\
\hline 2010-08-09 03:00:00.0 & 16.2 \\
\hline 2010-08-09 00:00:00.0 & 18.2 \\
\hline 2010-08-08 21:00:00.0 & 20.2 \\
\hline 2010-08-08 18:00:00.0 & 19.2 \\
\hline 2010-08-08 15:00:00.0 & 10.9 \\
\hline 2010-08-08 12:00:00.0 & 10.9 \\
\hline 2010-08-08 09:00:00.0 & 8.9 \\
\hline 2010-08-08 06:00:00.0 & 9.9 \\
\hline 2010-08-08 03:00:00.0 & 12.3 \\
\hline 2010-08-08 00:00:00.0 & 10.6 \\
\hline 2010-08-07 21:00:00.0 & 10.8 \\
\hline 2010-08-07 18:00:00.0 & 14.7 \\
\hline 2010-08-07 15:00:00.0 & 15.7 \\
\hline 2010-08-07 12:00:00.0 & 17.1 \\
\hline 2010-08-07 09:00:00.0 & 17.6 \\
\hline 2010-08-07 06:00:00.0 & 17.3 \\
\hline 2010-08-07 03:00:00.0 & 14.6 \\
\hline 2010-08-07 00:00:00.0 & 10.8 \\
\hline 2010-08-06 21:00:00.0 & 21.4 \\
\hline 2010-08-06 18:00:00.0 & 21.8 \\
\hline 2010-08-06 15:00:00.0 & 23.1 \\
\hline 2010-08-06 12:00:00.0 & 21.1 \\
\hline 2010-08-06 09:00:00.0 & 20.5 \\
\hline 2010-08-06 06:00:00.0 & 18.9 \\
\hline
\end{tabular}

\begin{tabular}{|c|c|}
\hline DataHora & VelVentoMax \\
\hline 2010-08-06 03:00:00.0 & 21.6 \\
\hline 2010-08-06 00:00:00.0 & 19.6 \\
\hline 2010-08-05 21:00:00.0 & \\
\hline 2010-08-05 18:00:00.0 & 15.0 \\
\hline 2010-08-05 15:00:00.0 & 17.5 \\
\hline 2010-08-05 12:00:00.0 & \\
\hline 2010-08-05 09:00:00.0 & 11.6 \\
\hline 2010-08-05 06:00:00.0 & 8.0 \\
\hline 2010-08-05 03:00:00.0 & 11.6 \\
\hline 2010-08-05 00:00:00.0 & \\
\hline 2010-08-04 21:00:00.0 & 18.9 \\
\hline 2010-08-04 18:00:00.0 & 23.5 \\
\hline 2010-08-04 15:00:00.0 & 24.4 \\
\hline 2010-08-04 12:00:00.0 & 17.9 \\
\hline 2010-08-04 09:00:00.0 & 23.0 \\
\hline 2010-08-04 06:00:00.0 & 17.6 \\
\hline 2010-08-04 03:00:00.0 & 13.7 \\
\hline 2010-08-04 00:00:00.0 & 8.2 \\
\hline 2010-08-03 21:00:00.0 & 20.6 \\
\hline 2010-08-03 18:00:00.0 & 26.9 \\
\hline 2010-08-03 15:00:00.0 & 24.7 \\
\hline 2010-08-03 12:00:00.0 & 16.1 \\
\hline 2010-08-03 09:00:00.0 & 13.8 \\
\hline 2010-08-03 06:00:00.0 & 13.9 \\
\hline 2010-08-03 03:00:00.0 & 14.5 \\
\hline 2010-08-03 00:00:00.0 & \\
\hline 2010-08-02 21:00:00.0 & 20.1 \\
\hline 2010-08-02 18:00:00.0 & 19.7 \\
\hline 2010-08-02 15:00:00.0 & 20.1 \\
\hline 2010-08-02 12:00:00.0 & 18.3 \\
\hline 2010-08-02 09:00:00.0 & 13.5 \\
\hline 2010-08-02 06:00:00.0 & 14.4 \\
\hline 2010-08-02 03:00:00.0 & \\
\hline 2010-08-02 00:00:00.0 & \\
\hline 2010-08-01 21:00:00.0 & 23.0 \\
\hline 2010-08-01 18:00:00.0 & 21.2 \\
\hline 2010-08-01 15:00:00.0 & 16.4 \\
\hline 2010-08-01 12:00:00.0 & 9.3 \\
\hline 2010-08-01 09:00:00.0 & 9.7 \\
\hline 2010-08-01 06:00:00.0 & 12.4 \\
\hline 2010-08-01 03:00:00.0 & 18.9 \\
\hline 2010-08-01 00:00:00.0 & \\
\hline 2010-07-31 21:00:00.0 & 24.3 \\
\hline 2010-07-31 18:00:00.0 & 24.3 \\
\hline 2010-07-31 15:00:00.0 & 14.6 \\
\hline 2010-07-31 12:00:00.0 & 39.8 \\
\hline 2010-07-31 09:00:00.0 & 31.6 \\
\hline 2010-07-31 06:00:00.0 & 35.1 \\
\hline 2010-07-31 03:00:00.0 & 30.2 \\
\hline 2010-07-31 00:00:00.0 & \\
\hline 2010-07-30 21:00:00.0 & 26.8 \\
\hline 2010-07-30 18:00:00.0 & 30.9 \\
\hline 2010-07-30 15:00:00.0 & 23.5 \\
\hline 2010-07-30 12:00:00.0 & 20.2 \\
\hline 2010-07-30 09:00:00.0 & 19.7 \\
\hline 2010-07-30 06:00:00.0 & 18.1 \\
\hline 2010-07-30 03:00:00.0 & \\
\hline 2010-07-30 00:00:00.0 & \\
\hline 2010-07-29 21:00:00.0 & 11.5 \\
\hline 2010-07-29 18:00:00.0 & 13.9 \\
\hline 2010-07-29 15:00:00.0 & 16.4 \\
\hline 2010-07-29 12:00:00.0 & 16.3 \\
\hline 2010-07-29 09:00:00.0 & 8.7 \\
\hline 2010-07-29 06:00:00.0 & 6.1 \\
\hline 2010-07-29 03:00:00.0 & \\
\hline 2010-07-29 00:00:00.0 & \\
\hline 2010-07-28 21:00:00.0 & 20.5 \\
\hline 2010-07-28 18:00:00.0 & 17.8 \\
\hline 2010-07-28 15:00:00.0 & 17.1 \\
\hline
\end{tabular}




\section{ANEXO 01 - RAJADAS DE VENTO REGISTRADAS EM SÃO MARTINHO DA SERRA / RS}

\begin{tabular}{|c|c|}
\hline DataHora & VelVentoMax \\
\hline 2010-07-28 12:00:00.0 & 22.5 \\
\hline 2010-07-28 09:00:00.0 & 24.9 \\
\hline 2010-07-28 06:00:00.0 & 25.9 \\
\hline 2010-07-28 03:00:00.0 & \\
\hline 2010-07-28 00:00:00.0 & \\
\hline 2010-07-27 21:00:00.0 & 21.0 \\
\hline 2010-07-27 18:00:00.0 & 21.0 \\
\hline 2010-07-27 15:00:00.0 & \\
\hline 2010-07-27 12:00:00.0 & 17.8 \\
\hline 2010-07-27 09:00:00.0 & 12.3 \\
\hline 2010-07-27 06:00:00.0 & 11.9 \\
\hline 2010-07-27 03:00:00.0 & \\
\hline 2010-07-27 00:00:00.0 & \\
\hline 2010-07-26 21:00:00.0 & 19.9 \\
\hline 2010-07-26 18:00:00.0 & 20.3 \\
\hline 2010-07-26 15:00:00.0 & 16.1 \\
\hline 2010-07-26 12:00:00.0 & 10.6 \\
\hline 2010-07-26 09:00:00.0 & 8.8 \\
\hline 2010-07-26 06:00:00.0 & 10.4 \\
\hline 2010-07-26 03:00:00.0 & \\
\hline 2010-07-26 00:00:00.0 & \\
\hline 2010-07-25 21:00:00.0 & 23.7 \\
\hline 2010-07-25 18:00:00.0 & 30.6 \\
\hline 2010-07-25 15:00:00.0 & 28.6 \\
\hline 2010-07-25 12:00:00.0 & 23.9 \\
\hline 2010-07-25 09:00:00.0 & 21.0 \\
\hline 2010-07-25 06:00:00.0 & 32.3 \\
\hline 2010-07-25 03:00:00.0 & \\
\hline 2010-07-25 00:00:00.0 & \\
\hline 2010-07-24 21:00:00.0 & 23.6 \\
\hline 2010-07-24 18:00:00.0 & 27.1 \\
\hline 2010-07-24 15:00:00.0 & 26.4 \\
\hline 2010-07-24 12:00:00.0 & 32.3 \\
\hline 2010-07-24 09:00:00.0 & 28.7 \\
\hline 2010-07-24 06:00:00.0 & 24.4 \\
\hline 2010-07-24 03:00:00.0 & \\
\hline 2010-07-24 00:00:00.0 & \\
\hline 2010-07-23 21:00:00.0 & 14.6 \\
\hline 2010-07-23 18:00:00.0 & 17.8 \\
\hline 2010-07-23 15:00:00.0 & 16.5 \\
\hline 2010-07-23 12:00:00.0 & 5.0 \\
\hline 2010-07-23 09:00:00.0 & 10.6 \\
\hline 2010-07-23 06:00:00.0 & 8.3 \\
\hline 2010-07-23 03:00:00.0 & \\
\hline 2010-07-23 00:00:00.0 & \\
\hline 2010-07-22 21:00:00.0 & 17.5 \\
\hline 2010-07-22 18:00:00.0 & 13.9 \\
\hline 2010-07-22 15:00:00.0 & 13.9 \\
\hline 2010-07-22 12:00:00.0 & 11.1 \\
\hline 2010-07-22 09:00:00.0 & 14.8 \\
\hline 2010-07-22 06:00:00.0 & 18.0 \\
\hline 2010-07-22 03:00:00.0 & \\
\hline 2010-07-22 00:00:00.0 & \\
\hline 2010-07-21 21:00:00.0 & 18.7 \\
\hline 2010-07-21 18:00:00.0 & 28.0 \\
\hline 2010-07-21 15:00:00.0 & 27.2 \\
\hline 2010-07-21 12:00:00.0 & 19.3 \\
\hline 2010-07-21 09:00:00.0 & 17.9 \\
\hline 2010-07-21 06:00:00.0 & 18.9 \\
\hline 2010-07-21 03:00:00.0 & \\
\hline 2010-07-21 00:00:00.0 & \\
\hline 2010-07-20 21:00:00.0 & 12.8 \\
\hline 2010-07-20 18:00:00.0 & 12.7 \\
\hline 2010-07-20 15:00:00.0 & 16.6 \\
\hline 2010-07-20 12:00:00.0 & 18.8 \\
\hline 2010-07-20 09:00:00.0 & 13.2 \\
\hline 2010-07-20 06:00:00.0 & 20.1 \\
\hline 2010-07-20 03:00:00.0 & \\
\hline 2010-07-20 00:00:00.0 & \\
\hline
\end{tabular}

\begin{tabular}{|c|c|}
\hline DataHora & VelVentoMax \\
\hline 2010-07-19 21:00:00.0 & 33.9 \\
\hline 2010-07-19 18:00:00.0 & 29.6 \\
\hline 2010-07-19 15:00:00.0 & 28.8 \\
\hline 2010-07-19 12:00:00.0 & 28.7 \\
\hline 2010-07-19 09:00:00.0 & 23.4 \\
\hline 2010-07-19 06:00:00.0 & 13.3 \\
\hline 2010-07-19 03:00:00.0 & \\
\hline 2010-07-19 00:00:00.0 & \\
\hline 2010-07-18 21:00:00.0 & 19.5 \\
\hline 2010-07-18 18:00:00.0 & 24.2 \\
\hline 2010-07-18 15:00:00.0 & 22.0 \\
\hline 2010-07-18 12:00:00.0 & 21.9 \\
\hline 2010-07-18 09:00:00.0 & 23.3 \\
\hline 2010-07-18 06:00:00.0 & 22.1 \\
\hline 2010-07-18 03:00:00.0 & \\
\hline 2010-07-18 00:00:00.0 & \\
\hline 2010-07-17 21:00:00.0 & 28.8 \\
\hline 2010-07-17 18:00:00.0 & 22.0 \\
\hline 2010-07-17 15:00:00.0 & 27.3 \\
\hline 2010-07-17 12:00:00.0 & 30.0 \\
\hline 2010-07-17 09:00:00.0 & 28.4 \\
\hline 2010-07-17 06:00:00.0 & 28.8 \\
\hline 2010-07-17 03:00:00.0 & \\
\hline 2010-07-17 00:00:00.0 & \\
\hline 2010-07-16 21:00:00.0 & \\
\hline 2010-07-16 18:00:00.0 & 26.7 \\
\hline 2010-07-16 15:00:00.0 & 26.2 \\
\hline 2010-07-16 12:00:00.0 & 18.4 \\
\hline 2010-07-16 09:00:00.0 & 11.2 \\
\hline 2010-07-16 06:00:00.0 & 14.5 \\
\hline 2010-07-16 03:00:00.0 & \\
\hline 2010-07-16 00:00:00.0 & \\
\hline 2010-07-15 21:00:00.0 & 16.3 \\
\hline 2010-07-15 18:00:00.0 & 19.2 \\
\hline 2010-07-15 15:00:00.0 & 14.9 \\
\hline 2010-07-15 12:00:00.0 & 8.0 \\
\hline 2010-07-15 09:00:00.0 & \\
\hline 2010-07-15 06:00:00.0 & \\
\hline 2010-07-15 03:00:00.0 & \\
\hline 2010-07-14 21:00:00.0 & 19.8 \\
\hline 2010-07-14 18:00:00.0 & 21.1 \\
\hline 2010-07-14 15:00:00.0 & 22.7 \\
\hline 2010-07-14 12:00:00.0 & 9.9 \\
\hline 2010-07-14 09:00:00.0 & 10.1 \\
\hline 2010-07-14 06:00:00.0 & 8.6 \\
\hline 2010-07-14 03:00:00.0 & \\
\hline 2010-07-14 00:00:00.0 & \\
\hline 2010-07-13 21:00:00.0 & 20.2 \\
\hline 2010-07-13 18:00:00.0 & 24.1 \\
\hline 2010-07-13 15:00:00.0 & 20.5 \\
\hline 2010-07-13 12:00:00.0 & 10.4 \\
\hline 2010-07-13 09:00:00.0 & \\
\hline 2010-07-13 06:00:00.0 & 19.6 \\
\hline 2010-07-13 03:00:00.0 & \\
\hline 2010-07-13 00:00:00.0 & \\
\hline 2010-07-12 21:00:00.0 & 24.9 \\
\hline 2010-07-12 18:00:00.0 & 22.8 \\
\hline 2010-07-12 15:00:00.0 & 23.2 \\
\hline 2010-07-12 12:00:00.0 & 31.4 \\
\hline 2010-07-12 09:00:00.0 & 42.9 \\
\hline 2010-07-12 06:00:00.0 & \\
\hline 2010-07-12 03:00:00.0 & \\
\hline 2010-07-12 00:00:00.0 & \\
\hline 2010-07-11 21:00:00.0 & 26.8 \\
\hline 2010-07-11 18:00:00.0 & 26.1 \\
\hline 2010-07-11 15:00:00.0 & 26.8 \\
\hline 2010-07-11 12:00:00.0 & 21.8 \\
\hline 2010-07-11 09:00:00.0 & \\
\hline 2010-07-11 06:00:00.0 & 21.9 \\
\hline
\end{tabular}

\begin{tabular}{|c|c|}
\hline \multirow{3}{*}{$\begin{array}{l}\text { DataHora } \\
2010-07-11 \text { 03:00:00.0 } \\
2010-07-11 \quad 00 \cdot 00 \cdot 00.0\end{array}$} & VelVentoMax \\
\hline & \\
\hline & \\
\hline 2010-07-10 21:00:00.0 & 12.7 \\
\hline 2010-07-10 18:00:00.0 & 19.6 \\
\hline 2010-07-10 15:00:00.0 & 19.2 \\
\hline 2010-07-10 12:00:00.0 & 19.0 \\
\hline 2010-07-10 09:00:00.0 & \\
\hline 2010-07-10 06:00:00.0 & 15.0 \\
\hline 2010-07-10 03:00:00.0 & \\
\hline 2010-07-10 00:00:00.0 & \\
\hline 2010-07-09 21:00:00.0 & 12.3 \\
\hline 2010-07-09 18:00:00.0 & 21.3 \\
\hline 2010-07-09 15:00:00.0 & 18.7 \\
\hline 2010-07-09 12:00:00.0 & 32.8 \\
\hline 2010-07-09 09:00:00.0 & 31.7 \\
\hline 2010-07-09 06:00:00.0 & 25.8 \\
\hline 2010-07-09 03:00:00.0 & \\
\hline 2010-07-09 00:00:00.0 & \\
\hline 2010-07-08 21:00:00.0 & 16.7 \\
\hline 2010-07-08 18:00:00.0 & 15.9 \\
\hline 2010-07-08 15:00:00.0 & 11.9 \\
\hline 2010-07-08 12:00:00.0 & 13.0 \\
\hline 2010-07-08 09:00:00.0 & 15.8 \\
\hline 2010-07-08 06:00:00.0 & 19.4 \\
\hline 2010-07-08 03:00:00.0 & \\
\hline 2010-07-08 00:00:00.0 & 12.6 \\
\hline 2010-07-07 21:00:00.0 & 41.0 \\
\hline 2010-07-07 18:00:00.0 & 36.1 \\
\hline 2010-07-07 15:00:00.0 & 34.0 \\
\hline 2010-07-07 12:00:00.0 & \\
\hline 2010-07-07 09:00:00.0 & 21.7 \\
\hline 2010-07-07 06:00:00.0 & 16.2 \\
\hline 2010-07-07 03:00:00.0 & \\
\hline 2010-07-07 00:00:00.0 & \\
\hline 2010-07-06 21:00:00.0 & 21.1 \\
\hline 2010-07-06 18:00:00.0 & 26.8 \\
\hline 2010-07-06 15:00:00.0 & \\
\hline 2010-07-06 12:00:00.0 & \\
\hline 2010-07-06 09:00:00.0 & 19.9 \\
\hline 2010-07-06 06:00:00.0 & 20.9 \\
\hline 2010-07-06 03:00:00.0 & \\
\hline 2010-07-06 00:00:00.0 & 16.9 \\
\hline 2010-07-05 21:00:00.0 & 18.1 \\
\hline 2010-07-05 18:00:00.0 & 23.3 \\
\hline 2010-07-05 15:00:00.0 & 26.6 \\
\hline 2010-07-05 12:00:00.0 & 23.6 \\
\hline 2010-07-05 09:00:00.0 & 20.2 \\
\hline 2010-07-05 06:00:00.0 & 19.1 \\
\hline 2010-07-05 03:00:00.0 & \\
\hline 2010-07-05 00:00:00.0 & 16.6 \\
\hline 2010-07-04 21:00:00.0 & 16.1 \\
\hline 2010-07-04 18:00:00.0 & 17.4 \\
\hline 2010-07-04 15:00:00.0 & 19.8 \\
\hline 2010-07-04 12:00:00.0 & 19.7 \\
\hline 2010-07-04 09:00:00.0 & 17.9 \\
\hline 2010-07-04 06:00:00.0 & 17.0 \\
\hline 2010-07-04 03:00:00.0 & \\
\hline 2010-07-04 00:00:00.0 & 14.5 \\
\hline 2010-07-03 21:00:00.0 & 11.2 \\
\hline 2010-07-03 18:00:00.0 & 13.6 \\
\hline 2010-07-03 15:00:00.0 & \\
\hline 2010-07-03 12:00:00.0 & \\
\hline 2010-07-03 09:00:00.0 & 12.1 \\
\hline 2010-07-03 06:00:00.0 & 13.6 \\
\hline 2010-07-03 03:00:00.0 & \\
\hline 2010-07-03 00:00:00.0 & 16.7 \\
\hline 2010-07-02 21:00:00.0 & 31.8 \\
\hline 2010-07-02 18:00:00.0 & 16.7 \\
\hline 2010-07-02 15:00:00.0 & \\
\hline
\end{tabular}




\section{ANEXO 01 - RAJADAS DE VENTO REGISTRADAS EM SÃO MARTINHO DA SERRA / RS}

\begin{tabular}{|c|c|}
\hline DataHora & VelVentoMax \\
\hline 2010-07-02 12:00:00.0 & 17.6 \\
\hline 2010-07-02 09:00:00.0 & 16.5 \\
\hline 2010-07-02 06:00:00.0 & 15.0 \\
\hline 2010-07-02 03:00:00.0 & 14.2 \\
\hline 2010-07-02 00:00:00.0 & 14.6 \\
\hline 2010-07-01 21:00:00.0 & 16.1 \\
\hline 2010-07-01 18:00:00.0 & 18.6 \\
\hline 2010-07-01 15:00:00.0 & \\
\hline 2010-07-01 12:00:00.0 & 17.7 \\
\hline 2010-07-01 09:00:00.0 & 17.1 \\
\hline 2010-07-01 06:00:00.0 & 17.8 \\
\hline 2010-07-01 03:00:00.0 & 16.6 \\
\hline 2010-07-01 00:00:00.0 & 10.5 \\
\hline 2010-06-30 21:00:00.0 & 8.8 \\
\hline 2010-06-30 18:00:00.0 & 12.5 \\
\hline 2010-06-30 15:00:00.0 & 16.6 \\
\hline 2010-06-30 12:00:00.0 & 18.9 \\
\hline 2010-06-30 09:00:00.0 & 16.4 \\
\hline 2010-06-30 06:00:00.0 & \\
\hline 2010-06-30 03:00:00.0 & 7.5 \\
\hline 2010-06-30 00:00:00.0 & 7.5 \\
\hline 2010-06-29 21:00:00.0 & 10.1 \\
\hline 2010-06-29 18:00:00.0 & 9.5 \\
\hline 2010-06-29 15:00:00.0 & \\
\hline 2010-06-29 12:00:00.0 & 9.7 \\
\hline 2010-06-29 09:00:00.0 & 11.2 \\
\hline 2010-06-29 06:00:00.0 & 14.5 \\
\hline 2010-06-29 03:00:00.0 & 13.6 \\
\hline 2010-06-29 00:00:00.0 & 14.1 \\
\hline 2010-06-28 21:00:00.0 & 12.9 \\
\hline 2010-06-28 18:00:00.0 & 12.6 \\
\hline 2010-06-28 15:00:00.0 & \\
\hline 2010-06-28 12:00:00.0 & 15.0 \\
\hline 2010-06-28 09:00:00.0 & 17.8 \\
\hline 2010-06-28 06:00:00.0 & 20.4 \\
\hline 2010-06-28 03:00:00.0 & 20.8 \\
\hline 2010-06-28 00:00:00.0 & 17.8 \\
\hline 2010-06-27 21:00:00.0 & 35.3 \\
\hline 2010-06-27 18:00:00.0 & 26.4 \\
\hline 2010-06-27 15:00:00.0 & 25.5 \\
\hline 2010-06-27 12:00:00.0 & 23.6 \\
\hline 2010-06-27 09:00:00.0 & 22.6 \\
\hline 2010-06-27 06:00:00.0 & 19.6 \\
\hline 2010-06-27 03:00:00.0 & 16.8 \\
\hline 2010-06-27 00:00:00.0 & 18.3 \\
\hline 2010-06-26 21:00:00.0 & 16.3 \\
\hline 2010-06-26 18:00:00.0 & 17.6 \\
\hline 2010-06-26 15:00:00.0 & 20.6 \\
\hline 2010-06-26 12:00:00.0 & 22.5 \\
\hline 2010-06-26 09:00:00.0 & 22.8 \\
\hline 2010-06-26 06:00:00.0 & 24.9 \\
\hline 2010-06-26 03:00:00.0 & 23.8 \\
\hline 2010-06-26 00:00:00.0 & 40.0 \\
\hline 2010-06-25 21:00:00.0 & 33.1 \\
\hline 2010-06-25 18:00:00.0 & 23.6 \\
\hline 2010-06-25 15:00:00.0 & 29.9 \\
\hline 2010-06-25 12:00:00.0 & 27.6 \\
\hline 2010-06-25 09:00:00.0 & 22.5 \\
\hline 2010-06-25 06:00:00.0 & 18.1 \\
\hline 2010-06-25 03:00:00.0 & 12.8 \\
\hline 2010-06-25 00:00:00.0 & 13.3 \\
\hline 2010-06-24 21:00:00.0 & 18.5 \\
\hline 2010-06-24 18:00:00.0 & 27.7 \\
\hline 2010-06-24 15:00:00.0 & 25.5 \\
\hline 2010-06-24 12:00:00.0 & 31.5 \\
\hline 2010-06-24 09:00:00.0 & 25.7 \\
\hline 2010-06-24 06:00:00.0 & 24.4 \\
\hline 2010-06-24 03:00:00.0 & 23.5 \\
\hline 2010-06-24 00:00:00.0 & 26.0 \\
\hline
\end{tabular}

\begin{tabular}{|c|c|}
\hline DataHora & VelVentoMax \\
\hline 2010-06-23 21:00:00.0 & 24.3 \\
\hline 2010-06-23 18:00:00.0 & 25.7 \\
\hline 2010-06-23 15:00:00.0 & 27.4 \\
\hline 2010-06-23 12:00:00.0 & 26.1 \\
\hline 2010-06-23 09:00:00.0 & 24.2 \\
\hline 2010-06-23 06:00:00.0 & 19.2 \\
\hline 2010-06-23 03:00:00.0 & 13.3 \\
\hline 2010-06-23 00:00:00.0 & 10.3 \\
\hline 2010-06-22 21:00:00.0 & 13.6 \\
\hline 2010-06-22 18:00:00.0 & 14.4 \\
\hline 2010-06-22 15:00:00.0 & 16.1 \\
\hline 2010-06-22 12:00:00.0 & 16.5 \\
\hline 2010-06-22 09:00:00.0 & 11.6 \\
\hline 2010-06-22 06:00:00.0 & 12.5 \\
\hline 2010-06-22 03:00:00.0 & \\
\hline 2010-06-22 00:00:00.0 & 9.2 \\
\hline 2010-06-21 21:00:00.0 & 13.3 \\
\hline 2010-06-21 18:00:00.0 & 17.4 \\
\hline 2010-06-21 15:00:00.0 & 13.8 \\
\hline 2010-06-21 12:00:00.0 & 7.0 \\
\hline 2010-06-21 09:00:00.0 & 4.5 \\
\hline 2010-06-21 06:00:00.0 & 4.5 \\
\hline 2010-06-21 03:00:00.0 & 10.2 \\
\hline 2010-06-21 00:00:00.0 & 10.6 \\
\hline 2010-06-20 21:00:00.0 & 11.1 \\
\hline 2010-06-20 18:00:00.0 & 17.4 \\
\hline 2010-06-20 15:00:00.0 & 9.4 \\
\hline 2010-06-20 12:00:00.0 & 31.1 \\
\hline 2010-06-20 09:00:00.0 & 40.9 \\
\hline 2010-06-20 06:00:00.0 & 7.3 \\
\hline 2010-06-20 03:00:00.0 & 17.2 \\
\hline 2010-06-20 00:00:00.0 & 26.2 \\
\hline 2010-06-19 21:00:00.0 & 18.4 \\
\hline 2010-06-19 18:00:00.0 & 14.1 \\
\hline 2010-06-19 15:00:00.0 & 13.1 \\
\hline 2010-06-19 12:00:00.0 & 29.1 \\
\hline 2010-06-19 09:00:00.0 & 1.5 \\
\hline 2010-06-19 06:00:00.0 & 26.5 \\
\hline 2010-06-19 03:00:00.0 & 28.5 \\
\hline 2010-06-19 00:00:00.0 & 23.1 \\
\hline 2010-06-18 21:00:00.0 & 26.2 \\
\hline 2010-06-18 18:00:00.0 & 27.7 \\
\hline 2010-06-18 15:00:00.0 & 19.2 \\
\hline 2010-06-18 12:00:00.0 & 14.4 \\
\hline 2010-06-18 09:00:00.0 & 26.0 \\
\hline 2010-06-18 06:00:00.0 & 23.9 \\
\hline 2010-06-18 03:00:00.0 & 24.1 \\
\hline 2010-06-18 00:00:00.0 & 21.1 \\
\hline 2010-06-17 21:00:00.0 & 17.9 \\
\hline 2010-06-17 18:00:00.0 & 28.9 \\
\hline 2010-06-17 15:00:00.0 & \\
\hline 2010-06-17 12:00:00.0 & \\
\hline 2010-06-17 09:00:00.0 & \\
\hline 2010-06-17 06:00:00.0 & 16.1 \\
\hline 2010-06-17 03:00:00.0 & 27.2 \\
\hline 2010-06-17 00:00:00.0 & 28.9 \\
\hline 2010-06-16 21:00:00.0 & 20.9 \\
\hline 2010-06-16 18:00:00.0 & 25.3 \\
\hline 2010-06-16 15:00:00.0 & 25.5 \\
\hline 2010-06-16 12:00:00.0 & 24.2 \\
\hline 2010-06-16 09:00:00.0 & 19.6 \\
\hline 2010-06-16 06:00:00.0 & 13.9 \\
\hline 2010-06-16 03:00:00.0 & 15.2 \\
\hline 2010-06-16 00:00:00.0 & 12.5 \\
\hline 2010-06-15 21:00:00.0 & 16.9 \\
\hline 2010-06-15 18:00:00.0 & 17.4 \\
\hline 2010-06-15 15:00:00.0 & 13.5 \\
\hline 2010-06-15 12:00:00.0 & 14.5 \\
\hline 2010-06-15 09:00:00.0 & 15.6 \\
\hline
\end{tabular}

\begin{tabular}{|c|c|}
\hline DataHora & VelVentoMax \\
\hline 2010-06-15 06:00:00.0 & 19.8 \\
\hline 2010-06-15 03:00:00.0 & 20.9 \\
\hline 2010-06-15 00:00:00.0 & 3.6 \\
\hline 2010-06-14 21:00:00.0 & 23.2 \\
\hline 2010-06-14 18:00:00.0 & 25.3 \\
\hline 2010-06-14 15:00:00.0 & 27.1 \\
\hline 2010-06-14 12:00:00.0 & 23.8 \\
\hline 2010-06-14 09:00:00.0 & 23.4 \\
\hline 2010-06-14 06:00:00.0 & 22.0 \\
\hline 2010-06-14 03:00:00.0 & 21.8 \\
\hline 2010-06-14 00:00:00.0 & 15.3 \\
\hline 2010-06-13 21:00:00.0 & 20.7 \\
\hline 2010-06-13 18:00:00.0 & 23.0 \\
\hline 2010-06-13 15:00:00.0 & 27.4 \\
\hline 2010-06-13 12:00:00.0 & 25.5 \\
\hline 2010-06-13 09:00:00.0 & 22.7 \\
\hline 2010-06-13 06:00:00.0 & 26.0 \\
\hline 2010-06-13 03:00:00.0 & 27.6 \\
\hline 2010-06-13 00:00:00.0 & \\
\hline 2010-06-12 21:00:00.0 & 22.9 \\
\hline 2010-06-12 18:00:00.0 & 25.4 \\
\hline 2010-06-12 15:00:00.0 & 29.3 \\
\hline 2010-06-12 12:00:00.0 & 26.1 \\
\hline 2010-06-12 09:00:00.0 & 23.8 \\
\hline 2010-06-12 06:00:00.0 & 23.8 \\
\hline 2010-06-12 03:00:00.0 & 22.3 \\
\hline 2010-06-12 00:00:00.0 & 18.6 \\
\hline 2010-06-11 21:00:00.0 & 25.0 \\
\hline 2010-06-11 18:00:00.0 & 29.5 \\
\hline 2010-06-11 15:00:00.0 & 30.8 \\
\hline 2010-06-11 12:00:00.0 & 24.3 \\
\hline 2010-06-11 09:00:00.0 & 24.2 \\
\hline 2010-06-11 06:00:00.0 & 19.8 \\
\hline 2010-06-11 03:00:00.0 & 16.1 \\
\hline 2010-06-11 00:00:00.0 & \\
\hline 2010-06-10 21:00:00.0 & 21.5 \\
\hline 2010-06-10 18:00:00.0 & 21.5 \\
\hline 2010-06-10 15:00:00.0 & 23.8 \\
\hline 2010-06-10 12:00:00.0 & 18.5 \\
\hline 2010-06-10 09:00:00.0 & 17.4 \\
\hline 2010-06-10 06:00:00.0 & 17.1 \\
\hline 2010-06-10 03:00:00.0 & \\
\hline 2010-06-10 00:00:00.0 & \\
\hline 2010-06-09 21:00:00.0 & 19.1 \\
\hline 2010-06-09 18:00:00.0 & 22.6 \\
\hline 2010-06-09 15:00:00.0 & \\
\hline 2010-06-09 12:00:00.0 & 22.9 \\
\hline 2010-06-09 09:00:00.0 & 21.6 \\
\hline 2010-06-09 06:00:00.0 & 14.8 \\
\hline 2010-06-09 03:00:00.0 & \\
\hline 2010-06-09 00:00:00.0 & 19.0 \\
\hline 2010-06-08 21:00:00.0 & 19.6 \\
\hline 2010-06-08 18:00:00.0 & 20.7 \\
\hline 2010-06-08 15:00:00.0 & 23.0 \\
\hline 2010-06-08 12:00:00.0 & 17.9 \\
\hline 2010-06-08 09:00:00.0 & 12.2 \\
\hline 2010-06-08 06:00:00.0 & 12.2 \\
\hline 2010-06-08 03:00:00.0 & \\
\hline 2010-06-08 00:00:00.0 & 11.7 \\
\hline 2010-06-07 21:00:00.0 & 10.0 \\
\hline 2010-06-07 18:00:00.0 & 12.6 \\
\hline 2010-06-07 15:00:00.0 & 9.9 \\
\hline 2010-06-07 12:00:00.0 & 10.5 \\
\hline 2010-06-07 09:00:00.0 & 9.3 \\
\hline 2010-06-07 06:00:00.0 & 8.8 \\
\hline 2010-06-07 03:00:00.0 & \\
\hline 2010-06-07 00:00:00.0 & \\
\hline 2010-06-06 21:00:00.0 & 9.7 \\
\hline 2010-06-06 18:00:00.0 & 31.2 \\
\hline
\end{tabular}




\section{ANEXO 01 - RAJADAS DE VENTO REGISTRADAS EM SÃO MARTINHO DA SERRA / RS}

\begin{tabular}{|c|c|}
\hline \multirow{2}{*}{ DataHora } & VelVentoMax \\
\hline & \\
\hline 2010-06-06 12:00:00.0 & \\
\hline 2010-06-06 09:00:00.0 & 6.7 \\
\hline 2010-06-06 06:00:00.0 & \\
\hline 2010-06-06 03:00:00.0 & \\
\hline 2010-06-06 00:00:00.0 & \\
\hline 2010-06-05 21:00:00.0 & 17.7 \\
\hline 2010-06-05 18:00:00.0 & 20.1 \\
\hline 2010-06-05 15:00:00.0 & 18.2 \\
\hline 2010-06-05 12:00:00.0 & 20.6 \\
\hline 2010-06-05 09:00:00.0 & 18.1 \\
\hline 2010-06-05 06:00:00.0 & 19.5 \\
\hline 2010-06-05 03:00:00.0 & \\
\hline 2010-06-05 00:00:00.0 & \\
\hline 2010-06-04 21:00:00.0 & \\
\hline 2010-06-04 18:00:00.0 & 15.7 \\
\hline 2010-06-04 15:00:00.0 & 7.7 \\
\hline 2010-06-04 12:00:00.0 & 6.8 \\
\hline 2010-06-04 09:00:00.0 & \\
\hline 2010-06-04 06:00:00.0 & \\
\hline 2010-06-04 03:00:00.0 & \\
\hline 2010-06-04 00:00:00.0 & 11.1 \\
\hline 2010-06-03 21:00:00.0 & \\
\hline 2010-06-03 18:00:00.0 & \\
\hline 2010-06-03 15:00:00.0 & \\
\hline 2010-06-03 12:00:00.0 & 39.6 \\
\hline 2010-06-03 09:00:00.0 & \\
\hline 2010-06-03 06:00:00.0 & \\
\hline 2010-06-03 03:00:00.0 & \\
\hline 2010-06-03 00:00:00.0 & 13.1 \\
\hline 2010-06-02 21:00:00.0 & \\
\hline 2010-06-02 18:00:00.0 & \\
\hline 2010-06-02 15:00:00.0 & 22.6 \\
\hline 2010-06-02 12:00:00.0 & 19.5 \\
\hline 2010-06-02 09:00:00.0 & 18.2 \\
\hline 2010-06-02 06:00:00.0 & \\
\hline 2010-06-02 03:00:00.0 & \\
\hline 2010-06-02 00:00:00.0 & \\
\hline 2010-06-01 21:00:00.0 & 17.1 \\
\hline 2010-06-01 18:00:00.0 & 22.0 \\
\hline 2010-06-01 15:00:00.0 & 23.5 \\
\hline 2010-06-01 12:00:00.0 & 9.2 \\
\hline 2010-06-01 09:00:00.0 & 9.2 \\
\hline 2010-06-01 06:00:00.0 & 9.5 \\
\hline 2010-06-01 03:00:00.0 & \\
\hline 2010-06-01 00:00:00.0 & \\
\hline 2010-05-31 21:00:00.0 & 11.5 \\
\hline 2010-05-31 18:00:00.0 & 15.4 \\
\hline 2010-05-31 15:00:00.0 & 18.1 \\
\hline 2010-05-31 12:00:00.0 & 17.8 \\
\hline 2010-05-31 09:00:00.0 & 20.0 \\
\hline 2010-05-31 06:00:00.0 & \\
\hline 2010-05-31 03:00:00.0 & \\
\hline 2010-05-31 00:00:00.0 & 34.0 \\
\hline 2010-05-30 21:00:00.0 & \\
\hline 2010-05-30 18:00:00.0 & \\
\hline 2010-05-30 15:00:00.0 & 28.3 \\
\hline 2010-05-30 12:00:00.0 & 28.0 \\
\hline 2010-05-30 09:00:00.0 & 25.5 \\
\hline 2010-05-30 06:00:00.0 & 26.0 \\
\hline 2010-05-30 03:00:00.0 & \\
\hline 2010-05-30 00:00:00.0 & 12.9 \\
\hline 2010-05-29 21:00:00.0 & 9.0 \\
\hline 2010-05-29 18:00:00.0 & 14.6 \\
\hline 2010-05-29 15:00:00.0 & 16.2 \\
\hline 2010-05-29 12:00:00.0 & 16.8 \\
\hline 2010-05-29 09:00:00.0 & 17.1 \\
\hline 2010-05-29 06:00:00.0 & 18.3 \\
\hline 2010-05-29 03:00:00.0 & \\
\hline
\end{tabular}

\begin{tabular}{|c|c|}
\hline DataHora & VelVentoMax \\
\hline 2010-05-29 00:00:00.0 & 14.1 \\
\hline 2010-05-28 21:00:00.0 & 18.4 \\
\hline 2010-05-28 18:00:00.0 & 19.7 \\
\hline 2010-05-28 15:00:00.0 & 24.1 \\
\hline 2010-05-28 12:00:00.0 & 16.7 \\
\hline 2010-05-28 09:00:00.0 & 21.5 \\
\hline 2010-05-28 06:00:00.0 & \\
\hline 2010-05-28 03:00:00.0 & \\
\hline 2010-05-28 00:00:00.0 & 22.7 \\
\hline 2010-05-27 21:00:00.0 & 24.5 \\
\hline 2010-05-27 18:00:00.0 & 23.5 \\
\hline $2010-05-27$ 15:00:00.0 & 25.3 \\
\hline 2010-05-27 12:00:00.0 & 21.0 \\
\hline 2010-05-27 09:00:00.0 & 20.0 \\
\hline 2010-05-27 06:00:00.0 & 15.5 \\
\hline 2010-05-27 03:00:00.0 & \\
\hline 2010-05-27 00:00:00.0 & \\
\hline 2010-05-26 21:00:00.0 & 12.1 \\
\hline 2010-05-26 18:00:00.0 & 14.0 \\
\hline 2010-05-26 15:00:00.0 & 13.6 \\
\hline 2010-05-26 12:00:00.0 & 14.3 \\
\hline 2010-05-26 09:00:00.0 & 9.5 \\
\hline 2010-05-26 06:00:00.0 & 5.8 \\
\hline 2010-05-26 03:00:00.0 & \\
\hline 2010-05-26 00:00:00.0 & 10.6 \\
\hline 2010-05-25 21:00:00.0 & 15.4 \\
\hline 2010-05-25 18:00:00.0 & \\
\hline 2010-05-25 15:00:00.0 & 14.1 \\
\hline 2010-05-25 12:00:00.0 & 18.7 \\
\hline 2010-05-25 09:00:00.0 & 22.1 \\
\hline 2010-05-25 06:00:00.0 & 15.3 \\
\hline 2010-05-25 03:00:00.0 & 9.0 \\
\hline 2010-05-25 00:00:00.0 & 7.2 \\
\hline 2010-05-24 21:00:00.0 & 15.9 \\
\hline 2010-05-24 18:00:00.0 & 16.5 \\
\hline 2010-05-24 15:00:00.0 & 23.4 \\
\hline 2010-05-24 12:00:00.0 & 21.3 \\
\hline 2010-05-24 09:00:00.0 & 18.5 \\
\hline 2010-05-24 06:00:00.0 & 21.2 \\
\hline 2010-05-24 03:00:00.0 & \\
\hline 2010-05-24 00:00:00.0 & \\
\hline 2010-05-23 21:00:00.0 & 22.3 \\
\hline 2010-05-23 18:00:00.0 & 23.0 \\
\hline 2010-05-23 15:00:00.0 & 24.1 \\
\hline 2010-05-23 12:00:00.0 & 21.2 \\
\hline 2010-05-23 09:00:00.0 & 27.0 \\
\hline 2010-05-23 06:00:00.0 & 17.4 \\
\hline 2010-05-23 03:00:00.0 & \\
\hline 2010-05-23 00:00:00.0 & \\
\hline 2010-05-22 21:00:00.0 & 18.6 \\
\hline 2010-05-22 18:00:00.0 & 21.9 \\
\hline 2010-05-22 15:00:00.0 & 25.1 \\
\hline 2010-05-22 12:00:00.0 & 22.7 \\
\hline 2010-05-22 09:00:00.0 & 17.0 \\
\hline 2010-05-22 06:00:00.0 & 17.9 \\
\hline 2010-05-22 03:00:00.0 & \\
\hline 2010-05-22 00:00:00.0 & 17.9 \\
\hline 2010-05-21 21:00:00.0 & 16.4 \\
\hline 2010-05-21 18:00:00.0 & 18.2 \\
\hline 2010-05-21 15:00:00.0 & 20.9 \\
\hline 2010-05-21 12:00:00.0 & 18.1 \\
\hline 2010-05-21 09:00:00.0 & 6.8 \\
\hline 2010-05-21 06:00:00.0 & 6.2 \\
\hline 2010-05-21 03:00:00.0 & \\
\hline 2010-05-21 00:00:00.0 & 9.1 \\
\hline 2010-05-20 21:00:00.0 & 10.5 \\
\hline 2010-05-20 18:00:00.0 & 9.0 \\
\hline 2010-05-20 15:00:00.0 & 8.3 \\
\hline 2010-05-20 12:00:00.0 & 7.5 \\
\hline
\end{tabular}

\begin{tabular}{|c|c|}
\hline DataHora & VelVentoMax \\
\hline 2010-05-20 09:00:00.0 & 30.1 \\
\hline 2010-05-20 06:00:00.0 & 10.6 \\
\hline 2010-05-20 03:00:00.0 & \\
\hline 2010-05-20 00:00:00.0 & 12.6 \\
\hline 2010-05-19 21:00:00.0 & 12.4 \\
\hline 2010-05-19 18:00:00.0 & 11.3 \\
\hline 2010-05-19 15:00:00.0 & 11.1 \\
\hline 2010-05-19 12:00:00.0 & 14.7 \\
\hline 2010-05-19 09:00:00.0 & 22.0 \\
\hline 2010-05-19 06:00:00.0 & 20.5 \\
\hline 2010-05-19 03:00:00.0 & \\
\hline 2010-05-19 00:00:00.0 & 15.0 \\
\hline 2010-05-18 21:00:00.0 & 12.3 \\
\hline 2010-05-18 18:00:00.0 & 13.5 \\
\hline 2010-05-18 15:00:00.0 & 19.0 \\
\hline 2010-05-18 12:00:00.0 & 19.7 \\
\hline 2010-05-18 09:00:00.0 & 32.8 \\
\hline 2010-05-18 06:00:00.0 & 28.5 \\
\hline 2010-05-18 03:00:00.0 & \\
\hline 2010-05-18 00:00:00.0 & 26.3 \\
\hline 2010-05-17 21:00:00.0 & 24.1 \\
\hline 2010-05-17 18:00:00.0 & 23.0 \\
\hline 2010-05-17 15:00:00.0 & \\
\hline 2010-05-17 12:00:00.0 & \\
\hline 2010-05-17 09:00:00.0 & 23.0 \\
\hline 2010-05-17 06:00:00.0 & \\
\hline 2010-05-17 03:00:00.0 & \\
\hline 2010-05-17 00:00:00.0 & \\
\hline 2010-05-16 21:00:00.0 & 16.7 \\
\hline 2010-05-16 18:00:00.0 & 21.7 \\
\hline 2010-05-16 15:00:00.0 & 19.6 \\
\hline 2010-05-16 12:00:00.0 & 21.1 \\
\hline 2010-05-16 09:00:00.0 & 7.0 \\
\hline 2010-05-16 06:00:00.0 & 8.8 \\
\hline 2010-05-16 03:00:00.0 & \\
\hline 2010-05-16 00:00:00.0 & 5.7 \\
\hline 2010-05-15 21:00:00.0 & 13.4 \\
\hline 2010-05-15 18:00:00.0 & 17.4 \\
\hline 2010-05-15 15:00:00.0 & \\
\hline 2010-05-15 12:00:00.0 & 19.0 \\
\hline 2010-05-15 09:00:00.0 & 18.0 \\
\hline 2010-05-15 06:00:00.0 & 13.7 \\
\hline 2010-05-15 03:00:00.0 & \\
\hline 2010-05-15 00:00:00.0 & 14.1 \\
\hline 2010-05-14 21:00:00.0 & 19.6 \\
\hline 2010-05-14 18:00:00.0 & 23.0 \\
\hline 2010-05-14 15:00:00.0 & \\
\hline 2010-05-14 12:00:00.0 & 44.2 \\
\hline 2010-05-14 09:00:00.0 & 16.4 \\
\hline 2010-05-14 06:00:00.0 & 17.3 \\
\hline 2010-05-14 03:00:00.0 & \\
\hline 2010-05-14 00:00:00.0 & 13.8 \\
\hline 2010-05-13 21:00:00.0 & 13.7 \\
\hline 2010-05-13 18:00:00.0 & 14.9 \\
\hline 2010-05-13 15:00:00.0 & 18.2 \\
\hline 2010-05-13 12:00:00.0 & 18.4 \\
\hline 2010-05-13 09:00:00.0 & 12.0 \\
\hline 2010-05-13 06:00:00.0 & 11.7 \\
\hline 2010-05-13 03:00:00.0 & 13.5 \\
\hline 2010-05-13 00:00:00.0 & 10.8 \\
\hline 2010-05-12 21:00:00.0 & 24.5 \\
\hline 2010-05-12 18:00:00.0 & 29.8 \\
\hline 2010-05-12 15:00:00.0 & 26.2 \\
\hline 2010-05-12 12:00:00.0 & 17.1 \\
\hline 2010-05-12 09:00:00.0 & 16.3 \\
\hline 2010-05-12 06:00:00.0 & \\
\hline 2010-05-12 03:00:00.0 & \\
\hline 2010-05-12 00:00:00.0 & 15.5 \\
\hline 2010-05-11 21:00:00.0 & 21.8 \\
\hline
\end{tabular}




\section{ANEXO 01 - RAJADAS DE VENTO REGISTRADAS EM SÃO MARTINHO DA SERRA / RS}

\begin{tabular}{|c|c|}
\hline DataHora & VelVentoMax \\
\hline 2010-05-11 18:00:00.0 & 27.4 \\
\hline 2010-05-11 15:00:00.0 & \\
\hline 2010-05-11 12:00:00.0 & 20.3 \\
\hline 2010-05-11 09:00:00.0 & 20.5 \\
\hline 2010-05-11 06:00:00.0 & 18.1 \\
\hline 2010-05-11 03:00:00.0 & 21.7 \\
\hline 2010-05-11 00:00:00.0 & 21.5 \\
\hline 2010-05-10 21:00:00.0 & 19.3 \\
\hline 2010-05-10 18:00:00.0 & 17.2 \\
\hline 2010-05-10 15:00:00.0 & \\
\hline 2010-05-10 12:00:00.0 & 17.0 \\
\hline 2010-05-10 09:00:00.0 & 15.1 \\
\hline 2010-05-10 06:00:00.0 & 10.9 \\
\hline 2010-05-10 03:00:00.0 & \\
\hline 2010-05-10 00:00:00.0 & 9.8 \\
\hline 2010-05-09 21:00:00.0 & 17.4 \\
\hline 2010-05-09 18:00:00.0 & 23.4 \\
\hline 2010-05-09 15:00:00.0 & \\
\hline 2010-05-09 12:00:00.0 & 20.4 \\
\hline 2010-05-09 09:00:00.0 & 20.1 \\
\hline 2010-05-09 06:00:00.0 & 18.6 \\
\hline 2010-05-09 03:00:00.0 & 14.7 \\
\hline 2010-05-09 00:00:00.0 & 8.7 \\
\hline 2010-05-08 21:00:00.0 & 22.1 \\
\hline 2010-05-08 18:00:00.0 & 23.0 \\
\hline 2010-05-08 15:00:00.0 & \\
\hline 2010-05-08 12:00:00.0 & 17.0 \\
\hline 2010-05-08 09:00:00.0 & 18.8 \\
\hline 2010-05-08 06:00:00.0 & 20.2 \\
\hline 2010-05-08 03:00:00.0 & 20.0 \\
\hline 2010-05-08 00:00:00.0 & 14.2 \\
\hline 2010-05-07 21:00:00.0 & 19.8 \\
\hline 2010-05-07 18:00:00.0 & 23.4 \\
\hline 2010-05-07 15:00:00.0 & \\
\hline 2010-05-07 12:00:00.0 & 22.0 \\
\hline 2010-05-07 09:00:00.0 & 15.8 \\
\hline 2010-05-07 06:00:00.0 & \\
\hline 2010-05-07 03:00:00.0 & 10.6 \\
\hline 2010-05-07 00:00:00.0 & 8.5 \\
\hline 2010-05-06 21:00:00.0 & 16.2 \\
\hline 2010-05-06 18:00:00.0 & 18.1 \\
\hline 2010-05-06 15:00:00.0 & 18.5 \\
\hline 2010-05-06 12:00:00.0 & 15.8 \\
\hline 2010-05-06 09:00:00.0 & 15.4 \\
\hline 2010-05-06 06:00:00.0 & 13.5 \\
\hline 2010-05-06 03:00:00.0 & 12.5 \\
\hline 2010-05-06 00:00:00.0 & 11.5 \\
\hline 2010-05-05 21:00:00.0 & 32.2 \\
\hline 2010-05-05 18:00:00.0 & 11.5 \\
\hline 2010-05-05 15:00:00.0 & 43.7 \\
\hline 2010-05-05 12:00:00.0 & 6.7 \\
\hline 2010-05-05 09:00:00.0 & 5.7 \\
\hline 2010-05-05 06:00:00.0 & 5.8 \\
\hline 2010-05-05 03:00:00.0 & 6.5 \\
\hline 2010-05-05 00:00:00.0 & 8.5 \\
\hline 2010-05-04 21:00:00.0 & 17.9 \\
\hline 2010-05-04 18:00:00.0 & \\
\hline 2010-05-04 15:00:00.0 & 26.1 \\
\hline 2010-05-04 12:00:00.0 & 25.6 \\
\hline 2010-05-04 09:00:00.0 & \\
\hline 2010-05-04 06:00:00.0 & 27.2 \\
\hline 2010-05-04 03:00:00.0 & 19.1 \\
\hline 2010-05-04 00:00:00.0 & 19.8 \\
\hline 2010-05-03 21:00:00.0 & \\
\hline 2010-05-03 18:00:00.0 & 23.8 \\
\hline 2010-05-03 15:00:00.0 & 19.9 \\
\hline 2010-05-03 12:00:00.0 & 18.2 \\
\hline 2010-05-03 09:00:00.0 & 15.5 \\
\hline 2010-05-03 06:00:00.0 & 16.6 \\
\hline
\end{tabular}

\begin{tabular}{|c|c|}
\hline DataHora & VelVentoMax \\
\hline 2010-05-03 03:00:00.0 & 15.6 \\
\hline 2010-05-03 00:00:00.0 & \\
\hline 2010-05-02 21:00:00.0 & 29.9 \\
\hline 2010-05-02 18:00:00.0 & 16.9 \\
\hline 2010-05-02 15:00:00.0 & 17.8 \\
\hline 2010-05-02 12:00:00.0 & 15.0 \\
\hline 2010-05-02 09:00:00.0 & 13.6 \\
\hline 2010-05-02 06:00:00.0 & 12.1 \\
\hline 2010-05-02 03:00:00.0 & 11.6 \\
\hline 2010-05-02 00:00:00.0 & 13.2 \\
\hline 2010-05-01 21:00:00.0 & 10.0 \\
\hline 2010-05-01 18:00:00.0 & 12.3 \\
\hline 2010-05-01 15:00:00.0 & 12.0 \\
\hline 2010-05-01 12:00:00.0 & 8.2 \\
\hline 2010-05-01 09:00:00.0 & 7.7 \\
\hline 2010-05-01 06:00:00.0 & 6.2 \\
\hline 2010-05-01 03:00:00.0 & 8.5 \\
\hline 2010-05-01 00:00:00.0 & 7.6 \\
\hline 2010-04-30 21:00:00.0 & 12.0 \\
\hline 2010-04-30 18:00:00.0 & 10.4 \\
\hline 2010-04-30 15:00:00.0 & 10.2 \\
\hline 2010-04-30 12:00:00.0 & 13.8 \\
\hline 2010-04-30 09:00:00.0 & 14.9 \\
\hline 2010-04-30 06:00:00.0 & 15.3 \\
\hline 2010-04-30 03:00:00.0 & 12.4 \\
\hline 2010-04-30 00:00:00.0 & 10.9 \\
\hline 2010-04-29 21:00:00.0 & 15.1 \\
\hline 2010-04-29 18:00:00.0 & 17.2 \\
\hline 2010-04-29 15:00:00.0 & 13.5 \\
\hline 2010-04-29 12:00:00.0 & 11.5 \\
\hline 2010-04-29 09:00:00.0 & 11.2 \\
\hline 2010-04-29 06:00:00.0 & 11.0 \\
\hline 2010-04-29 03:00:00.0 & 10.7 \\
\hline 2010-04-29 00:00:00.0 & 11.1 \\
\hline 2010-04-28 21:00:00.0 & \\
\hline 2010-04-28 18:00:00.0 & 15.8 \\
\hline 2010-04-28 15:00:00.0 & 10.6 \\
\hline 2010-04-28 12:00:00.0 & 11.4 \\
\hline 2010-04-28 09:00:00.0 & 10.7 \\
\hline 2010-04-28 06:00:00.0 & 10.9 \\
\hline 2010-04-28 03:00:00.0 & 8.9 \\
\hline 2010-04-28 00:00:00.0 & \\
\hline 2010-04-27 21:00:00.0 & 11.0 \\
\hline 2010-04-27 18:00:00.0 & 12.9 \\
\hline 2010-04-27 15:00:00.0 & 11.1 \\
\hline 2010-04-27 12:00:00.0 & 10.6 \\
\hline 2010-04-27 09:00:00.0 & 9.6 \\
\hline 2010-04-27 06:00:00.0 & 10.6 \\
\hline 2010-04-27 03:00:00.0 & 17.6 \\
\hline 2010-04-27 00:00:00.0 & 17.6 \\
\hline 2010-04-26 21:00:00.0 & 20.9 \\
\hline 2010-04-26 18:00:00.0 & 20.6 \\
\hline 2010-04-26 15:00:00.0 & 20.5 \\
\hline 2010-04-26 12:00:00.0 & 16.8 \\
\hline 2010-04-26 09:00:00.0 & 16.7 \\
\hline 2010-04-26 06:00:00.0 & 9.1 \\
\hline 2010-04-26 03:00:00.0 & 16.6 \\
\hline 2010-04-26 00:00:00.0 & 20.9 \\
\hline 2010-04-25 21:00:00.0 & 17.2 \\
\hline 2010-04-25 18:00:00.0 & 25.0 \\
\hline 2010-04-25 15:00:00.0 & 13.0 \\
\hline 2010-04-25 12:00:00.0 & 12.7 \\
\hline 2010-04-25 09:00:00.0 & 15.7 \\
\hline 2010-04-25 06:00:00.0 & 17.5 \\
\hline 2010-04-25 03:00:00.0 & 21.2 \\
\hline 2010-04-25 00:00:00.0 & \\
\hline 2010-04-24 21:00:00.0 & 21.3 \\
\hline 2010-04-24 18:00:00.0 & 22.0 \\
\hline 2010-04-24 15:00:00.0 & 28.3 \\
\hline
\end{tabular}

\begin{tabular}{|c|c|}
\hline DataHora & VelVentoMax \\
\hline 2010-04-24 12:00:00.0 & 26.5 \\
\hline 2010-04-24 09:00:00.0 & 19.7 \\
\hline 2010-04-24 06:00:00.0 & 17.3 \\
\hline 2010-04-24 03:00:00.0 & 18.0 \\
\hline 2010-04-24 00:00:00.0 & \\
\hline 2010-04-23 21:00:00.0 & 23.4 \\
\hline 2010-04-23 18:00:00.0 & 26.5 \\
\hline 2010-04-23 15:00:00.0 & 30.6 \\
\hline 2010-04-23 12:00:00.0 & 26.4 \\
\hline 2010-04-23 09:00:00.0 & 28.5 \\
\hline 2010-04-23 06:00:00.0 & 27.1 \\
\hline 2010-04-23 03:00:00.0 & 26.2 \\
\hline 2010-04-23 00:00:00.0 & \\
\hline 2010-04-22 21:00:00.0 & 23.9 \\
\hline 2010-04-22 18:00:00.0 & 30.4 \\
\hline 2010-04-22 15:00:00.0 & 22.4 \\
\hline 2010-04-22 12:00:00.0 & 27.1 \\
\hline 2010-04-22 09:00:00.0 & 17.2 \\
\hline 2010-04-22 06:00:00.0 & 8.5 \\
\hline 2010-04-22 03:00:00.0 & 8.5 \\
\hline 2010-04-22 00:00:00.0 & 8.9 \\
\hline 2010-04-21 21:00:00.0 & 16.8 \\
\hline 2010-04-21 18:00:00.0 & 20.4 \\
\hline 2010-04-21 15:00:00.0 & 29.2 \\
\hline 2010-04-21 12:00:00.0 & 20.7 \\
\hline 2010-04-21 09:00:00.0 & 16.4 \\
\hline 2010-04-21 06:00:00.0 & 18.6 \\
\hline 2010-04-21 03:00:00.0 & 30.3 \\
\hline 2010-04-21 00:00:00.0 & 27.9 \\
\hline 2010-04-20 21:00:00.0 & 27.2 \\
\hline 2010-04-20 18:00:00.0 & 31.0 \\
\hline 2010-04-20 15:00:00.0 & 19.8 \\
\hline 2010-04-20 12:00:00.0 & 28.3 \\
\hline 2010-04-20 09:00:00.0 & 39.2 \\
\hline 2010-04-20 06:00:00.0 & 26.5 \\
\hline 2010-04-20 03:00:00.0 & 5.6 \\
\hline 2010-04-20 00:00:00.0 & 13.7 \\
\hline 2010-04-19 21:00:00.0 & 20.4 \\
\hline 2010-04-19 18:00:00.0 & 20.7 \\
\hline 2010-04-19 15:00:00.0 & \\
\hline 2010-04-19 12:00:00.0 & 16.7 \\
\hline 2010-04-19 09:00:00.0 & 15.4 \\
\hline 2010-04-19 06:00:00.0 & 17.0 \\
\hline 2010-04-19 03:00:00.0 & \\
\hline 2010-04-19 00:00:00.0 & 14.6 \\
\hline 2010-04-18 21:00:00.0 & 21.2 \\
\hline 2010-04-18 18:00:00.0 & 29.1 \\
\hline 2010-04-18 15:00:00.0 & \\
\hline 2010-04-18 12:00:00.0 & 16.6 \\
\hline 2010-04-18 09:00:00.0 & 16.5 \\
\hline 2010-04-18 06:00:00.0 & 16.8 \\
\hline 2010-04-18 03:00:00.0 & 16.1 \\
\hline 2010-04-18 00:00:00.0 & 14.3 \\
\hline 2010-04-17 21:00:00.0 & 15.6 \\
\hline 2010-04-17 18:00:00.0 & 19.2 \\
\hline 2010-04-17 15:00:00.0 & 14.0 \\
\hline 2010-04-17 12:00:00.0 & 14.8 \\
\hline 2010-04-17 09:00:00.0 & 16.0 \\
\hline 2010-04-17 06:00:00.0 & 14.0 \\
\hline 2010-04-17 03:00:00.0 & \\
\hline 2010-04-17 00:00:00.0 & 8.3 \\
\hline 2010-04-16 21:00:00.0 & 19.7 \\
\hline 2010-04-16 18:00:00.0 & 20.9 \\
\hline 2010-04-16 15:00:00.0 & \\
\hline 2010-04-16 12:00:00.0 & 7.0 \\
\hline 2010-04-16 09:00:00.0 & 7.7 \\
\hline 2010-04-16 06:00:00.0 & 10.0 \\
\hline 2010-04-16 03:00:00.0 & 8.8 \\
\hline 2010-04-16 00:00:00.0 & 13.6 \\
\hline
\end{tabular}




\section{ANEXO 01 - RAJADAS DE VENTO REGISTRADAS EM SÃO MARTINHO DA SERRA / RS}

\begin{tabular}{|c|c|}
\hline DataHora & VelVentoMax \\
\hline 2010-04-15 21:00:00.0 & 17.3 \\
\hline 2010-04-15 18:00:00.0 & 34.1 \\
\hline 2010-04-15 15:00:00.0 & \\
\hline 2010-04-15 12:00:00.0 & 17.6 \\
\hline 2010-04-15 09:00:00.0 & 18.9 \\
\hline 2010-04-15 06:00:00.0 & 7.8 \\
\hline 2010-04-15 03:00:00.0 & \\
\hline 2010-04-15 00:00:00.0 & 12.0 \\
\hline 2010-04-14 21:00:00.0 & 27.8 \\
\hline 2010-04-14 18:00:00.0 & 27.3 \\
\hline 2010-04-14 15:00:00.0 & 25.6 \\
\hline 2010-04-14 12:00:00.0 & 23.8 \\
\hline 2010-04-14 09:00:00.0 & 17.0 \\
\hline 2010-04-14 06:00:00.0 & 14.5 \\
\hline 2010-04-14 03:00:00.0 & 12.2 \\
\hline 2010-04-14 00:00:00.0 & \\
\hline 2010-04-13 21:00:00.0 & 17.8 \\
\hline 2010-04-13 18:00:00.0 & 25.6 \\
\hline 2010-04-13 15:00:00.0 & 21.4 \\
\hline 2010-04-13 12:00:00.0 & 20.2 \\
\hline 2010-04-13 09:00:00.0 & 17.0 \\
\hline 2010-04-13 06:00:00.0 & \\
\hline 2010-04-13 03:00:00.0 & 12.4 \\
\hline 2010-04-13 00:00:00.0 & 11.3 \\
\hline 2010-04-12 21:00:00.0 & 15.3 \\
\hline 2010-04-12 18:00:00.0 & 20.8 \\
\hline 2010-04-12 15:00:00.0 & 18.2 \\
\hline 2010-04-12 12:00:00.0 & 18.2 \\
\hline 2010-04-12 09:00:00.0 & 15.0 \\
\hline 2010-04-12 06:00:00.0 & 24.6 \\
\hline 2010-04-12 03:00:00.0 & 14.4 \\
\hline 2010-04-12 00:00:00.0 & 12.2 \\
\hline 2010-04-11 21:00:00.0 & 24.1 \\
\hline 2010-04-11 18:00:00.0 & 20.0 \\
\hline 2010-04-11 15:00:00.0 & 42.8 \\
\hline 2010-04-11 12:00:00.0 & 25.0 \\
\hline 2010-04-11 09:00:00.0 & 17.7 \\
\hline 2010-04-11 06:00:00.0 & 15.4 \\
\hline 2010-04-11 03:00:00.0 & 16.3 \\
\hline 2010-04-11 00:00:00.0 & 14.9 \\
\hline 2010-04-10 21:00:00.0 & 16.0 \\
\hline 2010-04-10 18:00:00.0 & 15.8 \\
\hline 2010-04-10 15:00:00.0 & 17.3 \\
\hline 2010-04-10 12:00:00.0 & 18.4 \\
\hline 2010-04-10 09:00:00.0 & 14.4 \\
\hline 2010-04-10 06:00:00.0 & 17.1 \\
\hline 2010-04-10 03:00:00.0 & 16.5 \\
\hline 2010-04-10 00:00:00.0 & 15.1 \\
\hline 2010-04-09 21:00:00.0 & 3.3 \\
\hline 2010-04-09 18:00:00.0 & 24.1 \\
\hline 2010-04-09 15:00:00.0 & 24.1 \\
\hline 2010-04-09 12:00:00.0 & 22.8 \\
\hline 2010-04-09 09:00:00.0 & 12.4 \\
\hline 2010-04-09 06:00:00.0 & 11.9 \\
\hline 2010-04-09 03:00:00.0 & 12.8 \\
\hline 2010-04-09 00:00:00.0 & 11.6 \\
\hline 2010-04-08 21:00:00.0 & 18.8 \\
\hline 2010-04-08 18:00:00.0 & 26.3 \\
\hline 2010-04-08 15:00:00.0 & 24.9 \\
\hline 2010-04-08 12:00:00.0 & 17.9 \\
\hline 2010-04-08 09:00:00.0 & 8.4 \\
\hline 2010-04-08 06:00:00.0 & 10.9 \\
\hline 2010-04-08 03:00:00.0 & 10.8 \\
\hline 2010-04-08 00:00:00.0 & 13.3 \\
\hline 2010-04-07 21:00:00.0 & 23.2 \\
\hline 2010-04-07 18:00:00.0 & 24.1 \\
\hline 2010-04-07 15:00:00.0 & 28.1 \\
\hline 2010-04-07 12:00:00.0 & 13.0 \\
\hline 2010-04-07 09:00:00.0 & 11.5 \\
\hline
\end{tabular}

\begin{tabular}{|c|c|}
\hline DataHora & VelVentoMax \\
\hline 2010-04-07 06:00:00.0 & 13.6 \\
\hline 2010-04-07 03:00:00.0 & 19.1 \\
\hline 2010-04-07 00:00:00.0 & 19.0 \\
\hline 2010-04-06 21:00:00.0 & 22.8 \\
\hline 2010-04-06 18:00:00.0 & 25.1 \\
\hline 2010-04-06 15:00:00.0 & 22.5 \\
\hline 2010-04-06 12:00:00.0 & 17.4 \\
\hline 2010-04-06 09:00:00.0 & 8.0 \\
\hline 2010-04-06 06:00:00.0 & 9.1 \\
\hline 2010-04-06 03:00:00.0 & 11.3 \\
\hline 2010-04-06 00:00:00.0 & 13.6 \\
\hline 2010-04-05 21:00:00.0 & 26.7 \\
\hline 2010-04-05 18:00:00.0 & 26.9 \\
\hline 2010-04-05 15:00:00.0 & 26.5 \\
\hline 2010-04-05 12:00:00.0 & 17.2 \\
\hline 2010-04-05 09:00:00.0 & 21.5 \\
\hline 2010-04-05 06:00:00.0 & 24.4 \\
\hline 2010-04-05 03:00:00.0 & 22.9 \\
\hline 2010-04-05 00:00:00.0 & 23.5 \\
\hline 2010-04-04 21:00:00.0 & 25.8 \\
\hline 2010-04-04 18:00:00.0 & 23.9 \\
\hline 2010-04-04 15:00:00.0 & 27.1 \\
\hline 2010-04-04 12:00:00.0 & 25.1 \\
\hline 2010-04-04 09:00:00.0 & 23.6 \\
\hline 2010-04-04 06:00:00.0 & 19.4 \\
\hline 2010-04-04 03:00:00.0 & 28.8 \\
\hline 2010-04-04 00:00:00.0 & \\
\hline 2010-04-03 21:00:00.0 & 28.8 \\
\hline 2010-04-03 18:00:00.0 & 21.8 \\
\hline 2010-04-03 15:00:00.0 & 14.4 \\
\hline 2010-04-03 12:00:00.0 & 14.2 \\
\hline 2010-04-03 09:00:00.0 & 17.0 \\
\hline 2010-04-03 06:00:00.0 & 12.8 \\
\hline 2010-04-03 03:00:00.0 & 10.8 \\
\hline 2010-04-03 00:00:00.0 & \\
\hline 2010-04-02 21:00:00.0 & 44.7 \\
\hline 2010-04-02 18:00:00.0 & 19.4 \\
\hline 2010-04-02 15:00:00.0 & 16.3 \\
\hline 2010-04-02 12:00:00.0 & 15.0 \\
\hline 2010-04-02 09:00:00.0 & \\
\hline 2010-04-02 06:00:00.0 & 12.8 \\
\hline 2010-04-02 03:00:00.0 & 13.8 \\
\hline 2010-04-02 00:00:00.0 & 12.2 \\
\hline 2010-04-01 21:00:00.0 & 14.1 \\
\hline 2010-04-01 18:00:00.0 & 19.6 \\
\hline 2010-04-01 15:00:00.0 & 4.8 \\
\hline 2010-04-01 12:00:00.0 & 15.5 \\
\hline 2010-04-01 09:00:00.0 & 17.3 \\
\hline 2010-04-01 06:00:00.0 & 19.6 \\
\hline 2010-04-01 03:00:00.0 & 18.1 \\
\hline 2010-04-01 00:00:00.0 & 14.2 \\
\hline 2010-03-31 21:00:00.0 & 3.3 \\
\hline 2010-03-31 18:00:00.0 & 15.7 \\
\hline 2010-03-31 15:00:00.0 & 16.8 \\
\hline 2010-03-31 12:00:00.0 & 17.1 \\
\hline 2010-03-31 09:00:00.0 & 14.2 \\
\hline 2010-03-31 06:00:00.0 & 12.0 \\
\hline 2010-03-31 03:00:00.0 & 12.0 \\
\hline 2010-03-31 00:00:00.0 & 13.3 \\
\hline 2010-03-30 21:00:00.0 & 15.7 \\
\hline 2010-03-30 18:00:00.0 & 17.2 \\
\hline 2010-03-30 15:00:00.0 & 13.2 \\
\hline 2010-03-30 12:00:00.0 & 11.1 \\
\hline 2010-03-30 09:00:00.0 & 11.4 \\
\hline 2010-03-30 06:00:00.0 & 12.7 \\
\hline 2010-03-30 03:00:00.0 & \\
\hline 2010-03-30 00:00:00.0 & 15.5 \\
\hline 2010-03-29 21:00:00.0 & 20.5 \\
\hline 2010-03-29 18:00:00.0 & 14.3 \\
\hline
\end{tabular}

\begin{tabular}{|c|c|}
\hline DataHora & VelVentoMax \\
\hline 2010-03-29 15:00:00.0 & 16.2 \\
\hline 2010-03-29 12:00:00.0 & 19.2 \\
\hline 2010-03-29 09:00:00.0 & 19.5 \\
\hline 2010-03-29 06:00:00.0 & 20.6 \\
\hline 2010-03-29 03:00:00.0 & 18.8 \\
\hline 2010-03-29 00:00:00.0 & \\
\hline 2010-03-28 21:00:00.0 & 25.0 \\
\hline 2010-03-28 18:00:00.0 & 21.5 \\
\hline 2010-03-28 15:00:00.0 & 22.0 \\
\hline 2010-03-28 12:00:00.0 & 21.7 \\
\hline 2010-03-28 09:00:00.0 & 14.6 \\
\hline 2010-03-28 06:00:00.0 & 17.7 \\
\hline 2010-03-28 03:00:00.0 & 23.0 \\
\hline 2010-03-28 00:00:00.0 & 25.1 \\
\hline 2010-03-27 21:00:00.0 & 24.8 \\
\hline 2010-03-27 18:00:00.0 & 23.1 \\
\hline 2010-03-27 15:00:00.0 & 24.4 \\
\hline 2010-03-27 12:00:00.0 & 24.3 \\
\hline 2010-03-27 09:00:00.0 & 24.9 \\
\hline 2010-03-27 06:00:00.0 & 18.7 \\
\hline 2010-03-27 03:00:00.0 & \\
\hline 2010-03-27 00:00:00.0 & 19.9 \\
\hline 2010-03-26 21:00:00.0 & 23.6 \\
\hline 2010-03-26 18:00:00.0 & 24.6 \\
\hline 2010-03-26 15:00:00.0 & \\
\hline 2010-03-26 12:00:00.0 & 22.2 \\
\hline 2010-03-26 09:00:00.0 & 22.3 \\
\hline 2010-03-26 06:00:00.0 & 20.4 \\
\hline 2010-03-26 03:00:00.0 & 22.4 \\
\hline 2010-03-26 00:00:00.0 & 29.7 \\
\hline 2010-03-25 21:00:00.0 & 30.6 \\
\hline 2010-03-25 18:00:00.0 & 29.9 \\
\hline 2010-03-25 15:00:00.0 & 33.6 \\
\hline 2010-03-25 12:00:00.0 & 28.8 \\
\hline 2010-03-25 09:00:00.0 & 24.0 \\
\hline 2010-03-25 06:00:00.0 & 27.3 \\
\hline 2010-03-25 03:00:00.0 & 26.2 \\
\hline 2010-03-25 00:00:00.0 & 22.4 \\
\hline 2010-03-24 21:00:00.0 & 27.2 \\
\hline 2010-03-24 18:00:00.0 & 25.9 \\
\hline 2010-03-24 15:00:00.0 & 25.5 \\
\hline 2010-03-24 12:00:00.0 & 22.4 \\
\hline 2010-03-24 09:00:00.0 & 15.0 \\
\hline 2010-03-24 06:00:00.0 & 15.3 \\
\hline 2010-03-24 03:00:00.0 & \\
\hline 2010-03-24 00:00:00.0 & 18.1 \\
\hline 2010-03-23 21:00:00.0 & 15.8 \\
\hline 2010-03-23 18:00:00.0 & 24.8 \\
\hline 2010-03-23 15:00:00.0 & \\
\hline 2010-03-23 12:00:00.0 & 15.7 \\
\hline 2010-03-23 09:00:00.0 & 17.5 \\
\hline 2010-03-23 06:00:00.0 & 12.6 \\
\hline 2010-03-23 03:00:00.0 & 9.0 \\
\hline 2010-03-23 00:00:00.0 & 8.5 \\
\hline 2010-03-22 21:00:00.0 & 18.7 \\
\hline 2010-03-22 18:00:00.0 & 18.3 \\
\hline 2010-03-22 15:00:00.0 & \\
\hline 2010-03-22 12:00:00.0 & 18.7 \\
\hline 2010-03-22 09:00:00.0 & 31.7 \\
\hline 2010-03-22 06:00:00.0 & 30.2 \\
\hline 2010-03-22 03:00:00.0 & \\
\hline 2010-03-22 00:00:00.0 & 19.3 \\
\hline 2010-03-21 21:00:00.0 & 19.3 \\
\hline 2010-03-21 18:00:00.0 & 19.4 \\
\hline 2010-03-21 15:00:00.0 & 17.2 \\
\hline 2010-03-21 12:00:00.0 & 18.0 \\
\hline 2010-03-21 09:00:00.0 & 13.2 \\
\hline 2010-03-21 06:00:00.0 & 15.5 \\
\hline 2010-03-21 03:00:00.0 & 15.1 \\
\hline
\end{tabular}




\section{ANEXO 01 - RAJADAS DE VENTO REGISTRADAS EM SÃO MARTINHO DA SERRA / RS}

\begin{tabular}{|c|c|}
\hline DataHora & VelVentoMax \\
\hline 2010-03-21 00:00:00.0 & 11.8 \\
\hline 2010-03-20 21:00:00.0 & 17.5 \\
\hline 2010-03-20 18:00:00.0 & 17.7 \\
\hline 2010-03-20 15:00:00.0 & \\
\hline 2010-03-20 12:00:00.0 & 7.3 \\
\hline 2010-03-20 09:00:00.0 & 12.1 \\
\hline 2010-03-20 06:00:00.0 & 10.4 \\
\hline 2010-03-20 03:00:00.0 & 12.1 \\
\hline 2010-03-20 00:00:00.0 & 9.5 \\
\hline 2010-03-19 21:00:00.0 & 20.9 \\
\hline 2010-03-19 18:00:00.0 & 21.9 \\
\hline 2010-03-19 15:00:00.0 & 16.6 \\
\hline 2010-03-19 12:00:00.0 & 17.5 \\
\hline 2010-03-19 09:00:00.0 & 13.5 \\
\hline 2010-03-19 06:00:00.0 & 15.8 \\
\hline 2010-03-19 03:00:00.0 & 14.4 \\
\hline 2010-03-19 00:00:00.0 & 9.8 \\
\hline 2010-03-18 21:00:00.0 & 14.4 \\
\hline 2010-03-18 18:00:00.0 & 17.4 \\
\hline 2010-03-18 15:00:00.0 & 17.8 \\
\hline 2010-03-18 12:00:00.0 & 23.0 \\
\hline 2010-03-18 09:00:00.0 & 21.3 \\
\hline 2010-03-18 06:00:00.0 & 15.0 \\
\hline 2010-03-18 03:00:00.0 & 18.7 \\
\hline 2010-03-18 00:00:00.0 & 12.7 \\
\hline 2010-03-17 21:00:00.0 & 17.8 \\
\hline 2010-03-17 18:00:00.0 & 19.6 \\
\hline 2010-03-17 15:00:00.0 & 23.6 \\
\hline 2010-03-17 12:00:00.0 & 25.2 \\
\hline 2010-03-17 09:00:00.0 & 19.6 \\
\hline 2010-03-17 06:00:00.0 & 19.6 \\
\hline 2010-03-17 03:00:00.0 & 16.8 \\
\hline 2010-03-17 00:00:00.0 & 12.8 \\
\hline 2010-03-16 21:00:00.0 & 18.0 \\
\hline 2010-03-16 18:00:00.0 & 27.1 \\
\hline 2010-03-16 15:00:00.0 & 22.0 \\
\hline 2010-03-16 12:00:00.0 & 11.4 \\
\hline 2010-03-16 09:00:00.0 & 10.9 \\
\hline 2010-03-16 06:00:00.0 & 11.5 \\
\hline 2010-03-16 03:00:00.0 & 12.4 \\
\hline 2010-03-16 00:00:00.0 & 7.7 \\
\hline 2010-03-15 21:00:00.0 & 13.6 \\
\hline 2010-03-15 18:00:00.0 & 18.1 \\
\hline 2010-03-15 15:00:00.0 & 14.5 \\
\hline 2010-03-15 12:00:00.0 & 17.6 \\
\hline 2010-03-15 09:00:00.0 & 15.2 \\
\hline 2010-03-15 06:00:00.0 & 14.4 \\
\hline 2010-03-15 03:00:00.0 & 12.4 \\
\hline 2010-03-15 00:00:00.0 & 12.9 \\
\hline 2010-03-14 21:00:00.0 & 15.9 \\
\hline 2010-03-14 18:00:00.0 & \\
\hline 2010-03-14 15:00:00.0 & 17.1 \\
\hline 2010-03-14 12:00:00.0 & 16.9 \\
\hline 2010-03-14 09:00:00.0 & 18.5 \\
\hline 2010-03-14 06:00:00.0 & 16.0 \\
\hline 2010-03-14 03:00:00.0 & 11.0 \\
\hline 2010-03-14 00:00:00.0 & 12.1 \\
\hline 2010-03-13 21:00:00.0 & 20.0 \\
\hline 2010-03-13 18:00:00.0 & 23.1 \\
\hline 2010-03-13 15:00:00.0 & 13.1 \\
\hline 2010-03-13 12:00:00.0 & 16.9 \\
\hline 2010-03-13 09:00:00.0 & \\
\hline 2010-03-13 06:00:00.0 & 9.4 \\
\hline 2010-03-13 03:00:00.0 & 9.8 \\
\hline 2010-03-13 00:00:00.0 & 9.8 \\
\hline 2010-03-12 21:00:00.0 & 15.6 \\
\hline 2010-03-12 18:00:00.0 & 17.7 \\
\hline 2010-03-12 15:00:00.0 & 12.8 \\
\hline 2010-03-12 12:00:00.0 & 8.3 \\
\hline
\end{tabular}

\begin{tabular}{|c|c|}
\hline DataHora & VelVentoMax \\
\hline 2010-03-12 09:00:00.0 & 7.3 \\
\hline 2010-03-12 06:00:00.0 & 7.4 \\
\hline 2010-03-12 03:00:00.0 & 9.4 \\
\hline 2010-03-12 00:00:00.0 & 14.1 \\
\hline 2010-03-11 21:00:00.0 & 20.7 \\
\hline 2010-03-11 18:00:00.0 & 22.9 \\
\hline 2010-03-11 15:00:00.0 & 19.6 \\
\hline 2010-03-11 12:00:00.0 & 8.4 \\
\hline 2010-03-11 09:00:00.0 & 7.4 \\
\hline 2010-03-11 06:00:00.0 & 8.2 \\
\hline 2010-03-11 03:00:00.0 & 8.2 \\
\hline 2010-03-11 00:00:00.0 & 10.0 \\
\hline 2010-03-10 21:00:00.0 & 14.1 \\
\hline 2010-03-10 18:00:00.0 & 13.6 \\
\hline 2010-03-10 15:00:00.0 & 11.6 \\
\hline 2010-03-10 12:00:00.0 & 8.8 \\
\hline 2010-03-10 09:00:00.0 & 12.4 \\
\hline 2010-03-10 06:00:00.0 & 10.3 \\
\hline 2010-03-10 03:00:00.0 & 9.5 \\
\hline 2010-03-10 00:00:00.0 & 9.3 \\
\hline 2010-03-09 21:00:00.0 & 18.1 \\
\hline 2010-03-09 18:00:00.0 & 18.8 \\
\hline 2010-03-09 15:00:00.0 & 21.3 \\
\hline 2010-03-09 12:00:00.0 & 18.7 \\
\hline 2010-03-09 09:00:00.0 & 14.7 \\
\hline 2010-03-09 06:00:00.0 & 11.7 \\
\hline 2010-03-09 03:00:00.0 & 12.2 \\
\hline 2010-03-09 00:00:00.0 & 10.8 \\
\hline 2010-03-08 21:00:00.0 & 23.1 \\
\hline 2010-03-08 18:00:00.0 & 16.5 \\
\hline 2010-03-08 15:00:00.0 & 17.3 \\
\hline 2010-03-08 12:00:00.0 & 16.7 \\
\hline 2010-03-08 09:00:00.0 & 12.6 \\
\hline 2010-03-08 06:00:00.0 & 12.6 \\
\hline 2010-03-08 03:00:00.0 & 12.9 \\
\hline 2010-03-08 00:00:00.0 & 10.4 \\
\hline 2010-03-07 21:00:00.0 & 17.4 \\
\hline 2010-03-07 18:00:00.0 & 16.8 \\
\hline 2010-03-07 15:00:00.0 & 18.5 \\
\hline 2010-03-07 12:00:00.0 & 12.4 \\
\hline 2010-03-07 09:00:00.0 & 9.6 \\
\hline 2010-03-07 06:00:00.0 & 8.9 \\
\hline 2010-03-07 03:00:00.0 & 10.2 \\
\hline 2010-03-07 00:00:00.0 & 15.2 \\
\hline 2010-03-06 21:00:00.0 & 19.3 \\
\hline 2010-03-06 18:00:00.0 & 20.5 \\
\hline 2010-03-06 15:00:00.0 & 16.6 \\
\hline 2010-03-06 12:00:00.0 & 6.4 \\
\hline 2010-03-06 09:00:00.0 & 11.0 \\
\hline 2010-03-06 06:00:00.0 & 14.8 \\
\hline 2010-03-06 03:00:00.0 & 8.7 \\
\hline 2010-03-06 00:00:00.0 & 13.9 \\
\hline 2010-03-05 21:00:00.0 & 20.2 \\
\hline 2010-03-05 18:00:00.0 & 23.3 \\
\hline 2010-03-05 15:00:00.0 & 18.1 \\
\hline 2010-03-05 12:00:00.0 & 12.6 \\
\hline 2010-03-05 09:00:00.0 & 12.8 \\
\hline 2010-03-05 06:00:00.0 & 14.3 \\
\hline 2010-03-05 03:00:00.0 & 12.6 \\
\hline 2010-03-05 00:00:00.0 & 8.7 \\
\hline 2010-03-04 21:00:00.0 & 12.2 \\
\hline 2010-03-04 18:00:00.0 & 16.7 \\
\hline 2010-03-04 15:00:00.0 & 19.5 \\
\hline 2010-03-04 12:00:00.0 & 21.3 \\
\hline 2010-03-04 09:00:00.0 & 16.8 \\
\hline 2010-03-04 06:00:00.0 & 14.9 \\
\hline 2010-03-04 03:00:00.0 & 13.6 \\
\hline 2010-03-04 00:00:00.0 & 12.8 \\
\hline 2010-03-03 21:00:00.0 & 12.6 \\
\hline
\end{tabular}

\begin{tabular}{|c|c|}
\hline DataHora & VelVentoMax \\
\hline 2010-03-03 18:00:00.0 & 19.1 \\
\hline 2010-03-03 15:00:00.0 & 24.3 \\
\hline 2010-03-03 12:00:00.0 & 23.8 \\
\hline 2010-03-03 09:00:00.0 & 22.1 \\
\hline 2010-03-03 06:00:00.0 & 15.0 \\
\hline 2010-03-03 03:00:00.0 & 13.8 \\
\hline 2010-03-03 00:00:00.0 & 14.1 \\
\hline 2010-03-02 21:00:00.0 & 12.1 \\
\hline 2010-03-02 18:00:00.0 & 12.9 \\
\hline 2010-03-02 15:00:00.0 & 19.4 \\
\hline 2010-03-02 12:00:00.0 & 21.2 \\
\hline 2010-03-02 09:00:00.0 & 19.8 \\
\hline 2010-03-02 06:00:00.0 & 11.2 \\
\hline 2010-03-02 03:00:00.0 & 11.9 \\
\hline 2010-03-02 00:00:00.0 & 13.3 \\
\hline 2010-03-01 21:00:00.0 & 14.4 \\
\hline 2010-03-01 18:00:00.0 & 14.4 \\
\hline 2010-03-01 15:00:00.0 & 23.5 \\
\hline 2010-03-01 12:00:00.0 & 23.5 \\
\hline 2010-03-01 09:00:00.0 & 19.0 \\
\hline 2010-03-01 06:00:00.0 & 15.4 \\
\hline 2010-03-01 03:00:00.0 & 14.1 \\
\hline 2010-03-01 00:00:00.0 & 3.8 \\
\hline 2010-02-28 21:00:00.0 & 20.5 \\
\hline 2010-02-28 18:00:00.0 & 18.3 \\
\hline 2010-02-28 15:00:00.0 & 16.7 \\
\hline 2010-02-28 12:00:00.0 & 21.6 \\
\hline 2010-02-28 09:00:00.0 & \\
\hline 2010-02-28 06:00:00.0 & 12.4 \\
\hline 2010-02-28 03:00:00.0 & \\
\hline 2010-02-28 00:00:00.0 & 39.1 \\
\hline 2010-02-27 21:00:00.0 & \\
\hline 2010-02-27 18:00:00.0 & 13.0 \\
\hline 2010-02-27 15:00:00.0 & \\
\hline 2010-02-27 12:00:00.0 & 21.0 \\
\hline 2010-02-27 09:00:00.0 & 17.6 \\
\hline 2010-02-27 06:00:00.0 & 19.4 \\
\hline 2010-02-27 03:00:00.0 & 14.6 \\
\hline 2010-02-27 00:00:00.0 & 14.3 \\
\hline 2010-02-26 21:00:00.0 & 16.0 \\
\hline 2010-02-26 18:00:00.0 & 17.1 \\
\hline 2010-02-26 15:00:00.0 & 20.5 \\
\hline 2010-02-26 12:00:00.0 & 22.7 \\
\hline 2010-02-26 09:00:00.0 & 16.2 \\
\hline 2010-02-26 06:00:00.0 & 13.2 \\
\hline 2010-02-26 03:00:00.0 & \\
\hline 2010-02-26 00:00:00.0 & 14.5 \\
\hline 2010-02-25 21:00:00.0 & 18.0 \\
\hline 2010-02-25 18:00:00.0 & 21.2 \\
\hline 2010-02-25 15:00:00.0 & \\
\hline $2010-02-25$ 12:00:00.0 & 21.5 \\
\hline 2010-02-25 09:00:00.0 & 20.8 \\
\hline 2010-02-25 06:00:00.0 & 20.8 \\
\hline 2010-02-25 03:00:00.0 & 17.3 \\
\hline 2010-02-25 00:00:00.0 & 15.9 \\
\hline 2010-02-24 21:00:00.0 & 21.2 \\
\hline 2010-02-24 18:00:00.0 & 22.0 \\
\hline 2010-02-24 15:00:00.0 & 22.9 \\
\hline 2010-02-24 12:00:00.0 & 21.6 \\
\hline 2010-02-24 09:00:00.0 & 12.7 \\
\hline 2010-02-24 06:00:00.0 & \\
\hline 2010-02-24 03:00:00.0 & 13.9 \\
\hline 2010-02-24 00:00:00.0 & 9.2 \\
\hline 2010-02-23 21:00:00.0 & 22.6 \\
\hline 2010-02-23 18:00:00.0 & 22.3 \\
\hline $2010-02-23$ 15:00:00.0 & 25.8 \\
\hline 2010-02-23 12:00:00.0 & 19.1 \\
\hline 2010-02-23 09:00:00.0 & 21.3 \\
\hline 2010-02-23 06:00:00.0 & 20.2 \\
\hline
\end{tabular}




\section{ANEXO 01 - RAJADAS DE VENTO REGISTRADAS EM SÃO MARTINHO DA SERRA / RS}

\begin{tabular}{|c|c|}
\hline \multirow{2}{*}{$\begin{array}{l}\text { DataHora } \\
\text { 2010-02-23 03:00:00.0 }\end{array}$} & VelVentoMax \\
\hline & \\
\hline 2010-02-23 00:00:00.0 & 25.6 \\
\hline 2010-02-22 21:00:00.0 & 50.2 \\
\hline 2010-02-22 18:00:00.0 & 21.5 \\
\hline 2010-02-22 15:00:00.0 & 17.7 \\
\hline 2010-02-22 12:00:00.0 & 16.1 \\
\hline 2010-02-22 09:00:00.0 & 17.7 \\
\hline 2010-02-22 06:00:00.0 & 19.5 \\
\hline 2010-02-22 03:00:00.0 & 18.5 \\
\hline 2010-02-22 00:00:00.0 & 29.7 \\
\hline 2010-02-21 21:00:00.0 & 32.6 \\
\hline 2010-02-21 18:00:00.0 & 25.3 \\
\hline 2010-02-21 15:00:00.0 & 24.6 \\
\hline 2010-02-21 12:00:00.0 & 17.1 \\
\hline 2010-02-21 09:00:00.0 & 13.2 \\
\hline 2010-02-21 06:00:00.0 & \\
\hline 2010-02-21 03:00:00.0 & 14.6 \\
\hline 2010-02-21 00:00:00.0 & 9.0 \\
\hline 2010-02-20 21:00:00.0 & 15.5 \\
\hline 2010-02-20 18:00:00.0 & 21.2 \\
\hline 2010-02-20 15:00:00.0 & 26.1 \\
\hline 2010-02-20 12:00:00.0 & 25.3 \\
\hline 2010-02-20 09:00:00.0 & 17.2 \\
\hline 2010-02-20 06:00:00.0 & 17.5 \\
\hline 2010-02-20 03:00:00.0 & 17.1 \\
\hline 2010-02-20 00:00:00.0 & 14.6 \\
\hline 2010-02-19 21:00:00.0 & 17.5 \\
\hline 2010-02-19 18:00:00.0 & 21.1 \\
\hline 2010-02-19 15:00:00.0 & 19.9 \\
\hline 2010-02-19 12:00:00.0 & 26.1 \\
\hline 2010-02-19 09:00:00.0 & 23.8 \\
\hline 2010-02-19 06:00:00.0 & 22.3 \\
\hline 2010-02-19 03:00:00.0 & \\
\hline 2010-02-19 00:00:00.0 & \\
\hline 2010-02-18 21:00:00.0 & 20.4 \\
\hline 2010-02-18 18:00:00.0 & 18.5 \\
\hline 2010-02-18 15:00:00.0 & 23.4 \\
\hline 2010-02-18 12:00:00.0 & 16.7 \\
\hline 2010-02-18 09:00:00.0 & 24.5 \\
\hline 2010-02-18 06:00:00.0 & 47.4 \\
\hline 2010-02-18 03:00:00.0 & 24.1 \\
\hline 2010-02-18 00:00:00.0 & 18.9 \\
\hline 2010-02-17 21:00:00.0 & 20.0 \\
\hline 2010-02-17 18:00:00.0 & 22.5 \\
\hline 2010-02-17 15:00:00.0 & 23.2 \\
\hline 2010-02-17 12:00:00.0 & 31.8 \\
\hline 2010-02-17 09:00:00.0 & 23.6 \\
\hline 2010-02-17 06:00:00.0 & 20.9 \\
\hline 2010-02-17 03:00:00.0 & 23.0 \\
\hline 2010-02-17 00:00:00.0 & 25.3 \\
\hline 2010-02-16 21:00:00.0 & 23.7 \\
\hline 2010-02-16 18:00:00.0 & 29.9 \\
\hline 2010-02-16 15:00:00.0 & 19.8 \\
\hline 2010-02-16 12:00:00.0 & 22.3 \\
\hline 2010-02-16 09:00:00.0 & 19.7 \\
\hline 2010-02-16 06:00:00.0 & 19.6 \\
\hline 2010-02-16 03:00:00.0 & 13.0 \\
\hline 2010-02-16 00:00:00.0 & 16.8 \\
\hline 2010-02-15 21:00:00.0 & 14.9 \\
\hline 2010-02-15 18:00:00.0 & 10.5 \\
\hline 2010-02-15 15:00:00.0 & 15.4 \\
\hline 2010-02-15 12:00:00.0 & 14.0 \\
\hline 2010-02-15 09:00:00.0 & 7.3 \\
\hline 2010-02-15 06:00:00.0 & 10.6 \\
\hline 2010-02-15 03:00:00.0 & 13.0 \\
\hline 2010-02-15 00:00:00.0 & 11.1 \\
\hline 2010-02-14 21:00:00.0 & 31.4 \\
\hline 2010-02-14 18:00:00.0 & 13.1 \\
\hline 2010-02-14 15:00:00.0 & 20.6 \\
\hline
\end{tabular}

\begin{tabular}{|c|c|}
\hline DataHora & VelVentoMax \\
\hline 2010-02-14 12:00:00.0 & 20.6 \\
\hline 2010-02-14 09:00:00.0 & 17.1 \\
\hline 2010-02-14 06:00:00.0 & 14.8 \\
\hline 2010-02-14 03:00:00.0 & 16.4 \\
\hline 2010-02-14 00:00:00.0 & 30.7 \\
\hline 2010-02-13 21:00:00.0 & 15.8 \\
\hline 2010-02-13 18:00:00.0 & 19.0 \\
\hline 2010-02-13 15:00:00.0 & 20.3 \\
\hline 2010-02-13 12:00:00.0 & 0.6 \\
\hline 2010-02-13 09:00:00.0 & 13.2 \\
\hline 2010-02-13 06:00:00.0 & 15.3 \\
\hline 2010-02-13 03:00:00.0 & 14.4 \\
\hline 2010-02-13 00:00:00.0 & 10.8 \\
\hline 2010-02-12 21:00:00.0 & 26.6 \\
\hline 2010-02-12 18:00:00.0 & 37.0 \\
\hline 2010-02-12 15:00:00.0 & 12.2 \\
\hline 2010-02-12 12:00:00.0 & 15.3 \\
\hline 2010-02-12 09:00:00.0 & 12.6 \\
\hline 2010-02-12 06:00:00.0 & 13.2 \\
\hline 2010-02-12 03:00:00.0 & 13.7 \\
\hline 2010-02-12 00:00:00.0 & 11.5 \\
\hline 2010-02-11 21:00:00.0 & 15.9 \\
\hline 2010-02-11 18:00:00.0 & 10.9 \\
\hline 2010-02-11 15:00:00.0 & 18.5 \\
\hline 2010-02-11 12:00:00.0 & 19.7 \\
\hline 2010-02-11 09:00:00.0 & 17.0 \\
\hline 2010-02-11 06:00:00.0 & 15.2 \\
\hline 2010-02-11 03:00:00.0 & 17.9 \\
\hline 2010-02-11 00:00:00.0 & 14.5 \\
\hline 2010-02-10 21:00:00.0 & 19.9 \\
\hline 2010-02-10 18:00:00.0 & 18.6 \\
\hline 2010-02-10 15:00:00.0 & 13.9 \\
\hline 2010-02-10 12:00:00.0 & 19.4 \\
\hline 2010-02-10 09:00:00.0 & 17.9 \\
\hline 2010-02-10 06:00:00.0 & 19.8 \\
\hline 2010-02-10 03:00:00.0 & 14.3 \\
\hline 2010-02-10 00:00:00.0 & 11.4 \\
\hline 2010-02-09 21:00:00.0 & 14.4 \\
\hline 2010-02-09 18:00:00.0 & 13.5 \\
\hline 2010-02-09 15:00:00.0 & \\
\hline 2010-02-09 12:00:00.0 & 18.1 \\
\hline 2010-02-09 09:00:00.0 & 10.0 \\
\hline 2010-02-09 06:00:00.0 & 12.6 \\
\hline 2010-02-09 03:00:00.0 & 11.7 \\
\hline 2010-02-09 00:00:00.0 & 12.1 \\
\hline 2010-02-08 21:00:00.0 & 23.5 \\
\hline 2010-02-08 18:00:00.0 & 23.4 \\
\hline 2010-02-08 15:00:00.0 & 29.7 \\
\hline 2010-02-08 12:00:00.0 & 1.4 \\
\hline 2010-02-08 09:00:00.0 & \\
\hline 2010-02-08 06:00:00.0 & 17.0 \\
\hline 2010-02-08 03:00:00.0 & 16.3 \\
\hline 2010-02-08 00:00:00.0 & 17.6 \\
\hline 2010-02-07 21:00:00.0 & 22.3 \\
\hline 2010-02-07 18:00:00.0 & 22.5 \\
\hline 2010-02-07 15:00:00.0 & 19.5 \\
\hline 2010-02-07 12:00:00.0 & 15.2 \\
\hline 2010-02-07 09:00:00.0 & 18.7 \\
\hline 2010-02-07 06:00:00.0 & 17.5 \\
\hline 2010-02-07 03:00:00.0 & 18.7 \\
\hline 2010-02-07 00:00:00.0 & 13.4 \\
\hline 2010-02-06 21:00:00.0 & 24.7 \\
\hline 2010-02-06 18:00:00.0 & 22.4 \\
\hline 2010-02-06 15:00:00.0 & 24.1 \\
\hline 2010-02-06 12:00:00.0 & 23.4 \\
\hline 2010-02-06 09:00:00.0 & 21.1 \\
\hline 2010-02-06 06:00:00.0 & 19.4 \\
\hline 2010-02-06 03:00:00.0 & 22.7 \\
\hline 2010-02-06 00:00:00.0 & 12.9 \\
\hline
\end{tabular}

\begin{tabular}{|c|c|}
\hline DataHora & VelVentoMax \\
\hline 2010-02-05 21:00:00.0 & 18.5 \\
\hline 2010-02-05 18:00:00.0 & 21.7 \\
\hline 2010-02-05 15:00:00.0 & 21.6 \\
\hline 2010-02-05 12:00:00.0 & 18.9 \\
\hline 2010-02-05 09:00:00.0 & 13.0 \\
\hline 2010-02-05 06:00:00.0 & 20.4 \\
\hline 2010-02-05 03:00:00.0 & 21.2 \\
\hline 2010-02-05 00:00:00.0 & 16.6 \\
\hline 2010-02-04 21:00:00.0 & 4.4 \\
\hline 2010-02-04 18:00:00.0 & 22.1 \\
\hline 2010-02-04 15:00:00.0 & 20.6 \\
\hline 2010-02-04 12:00:00.0 & 31.5 \\
\hline 2010-02-04 09:00:00.0 & 24.4 \\
\hline 2010-02-04 06:00:00.0 & 23.0 \\
\hline 2010-02-04 03:00:00.0 & 16.3 \\
\hline 2010-02-04 00:00:00.0 & 18.6 \\
\hline 2010-02-03 21:00:00.0 & 30.1 \\
\hline 2010-02-03 18:00:00.0 & 39.9 \\
\hline 2010-02-03 15:00:00.0 & 36.3 \\
\hline 2010-02-03 12:00:00.0 & 29.2 \\
\hline 2010-02-03 09:00:00.0 & 22.9 \\
\hline 2010-02-03 06:00:00.0 & 21.1 \\
\hline 2010-02-03 03:00:00.0 & 16.3 \\
\hline 2010-02-03 00:00:00.0 & 14.0 \\
\hline 2010-02-02 21:00:00.0 & 20.5 \\
\hline 2010-02-02 18:00:00.0 & 26.1 \\
\hline 2010-02-02 15:00:00.0 & 26.1 \\
\hline 2010-02-02 12:00:00.0 & 22.0 \\
\hline 2010-02-02 09:00:00.0 & 15.1 \\
\hline 2010-02-02 06:00:00.0 & 18.6 \\
\hline 2010-02-02 03:00:00.0 & \\
\hline 2010-02-02 00:00:00.0 & 7.5 \\
\hline 2010-02-01 21:00:00.0 & 17.4 \\
\hline 2010-02-01 18:00:00.0 & 23.2 \\
\hline 2010-02-01 15:00:00.0 & 23.3 \\
\hline 2010-02-01 12:00:00.0 & 16.6 \\
\hline 2010-02-01 09:00:00.0 & 15.5 \\
\hline 2010-02-01 06:00:00.0 & 15.4 \\
\hline 2010-02-01 03:00:00.0 & 15.2 \\
\hline 2010-02-01 00:00:00.0 & 13.0 \\
\hline 2010-01-31 21:00:00.0 & 9.7 \\
\hline 2010-01-31 18:00:00.0 & 15.2 \\
\hline 2010-01-31 15:00:00.0 & \\
\hline 2010-01-31 12:00:00.0 & 15.4 \\
\hline 2010-01-31 09:00:00.0 & 14.1 \\
\hline 2010-01-31 06:00:00.0 & 13.1 \\
\hline 2010-01-31 03:00:00.0 & 12.6 \\
\hline 2010-01-31 00:00:00.0 & 12.5 \\
\hline 2010-01-30 21:00:00.0 & \\
\hline 2010-01-30 18:00:00.0 & \\
\hline 2010-01-30 15:00:00.0 & \\
\hline 2010-01-30 12:00:00.0 & 13.6 \\
\hline 2010-01-30 09:00:00.0 & 12.0 \\
\hline 2010-01-30 06:00:00.0 & 14.0 \\
\hline 2010-01-30 03:00:00.0 & 15.2 \\
\hline 2010-01-30 00:00:00.0 & 8.1 \\
\hline 2010-01-29 21:00:00.0 & 11.4 \\
\hline 2010-01-29 18:00:00.0 & 30.9 \\
\hline 2010-01-29 15:00:00.0 & 15.4 \\
\hline 2010-01-29 12:00:00.0 & 32.0 \\
\hline 2010-01-29 09:00:00.0 & 9.9 \\
\hline 2010-01-29 06:00:00.0 & 15.2 \\
\hline 2010-01-29 03:00:00.0 & 14.0 \\
\hline 2010-01-29 00:00:00.0 & 9.8 \\
\hline 2010-01-28 21:00:00.0 & 27.9 \\
\hline 2010-01-28 18:00:00.0 & 27.5 \\
\hline 2010-01-28 15:00:00.0 & 13.2 \\
\hline 2010-01-28 12:00:00.0 & 14.5 \\
\hline 2010-01-28 09:00:00.0 & 15.0 \\
\hline
\end{tabular}




\section{ANEXO 01 - RAJADAS DE VENTO REGISTRADAS EM SÃO MARTINHO DA SERRA / RS}

\begin{tabular}{|c|c|}
\hline DataHora & VelVentoMax \\
\hline 2010-01-28 06:00:00.0 & 15.6 \\
\hline 2010-01-28 03:00:00.0 & 17.1 \\
\hline 2010-01-28 00:00:00.0 & 23.9 \\
\hline 2010-01-27 21:00:00.0 & 24.2 \\
\hline 2010-01-27 18:00:00.0 & 6.0 \\
\hline 2010-01-27 15:00:00.0 & 14.0 \\
\hline 2010-01-27 12:00:00.0 & 27.1 \\
\hline 2010-01-27 09:00:00.0 & 11.3 \\
\hline 2010-01-27 06:00:00.0 & 12.5 \\
\hline 2010-01-27 03:00:00.0 & 14.4 \\
\hline 2010-01-27 00:00:00.0 & 17.3 \\
\hline 2010-01-26 21:00:00.0 & 19.7 \\
\hline 2010-01-26 18:00:00.0 & 14.3 \\
\hline 2010-01-26 15:00:00.0 & 21.2 \\
\hline 2010-01-26 12:00:00.0 & 20.6 \\
\hline 2010-01-26 09:00:00.0 & 15.7 \\
\hline 2010-01-26 06:00:00.0 & 12.8 \\
\hline 2010-01-26 03:00:00.0 & 12.9 \\
\hline 2010-01-26 00:00:00.0 & 17.4 \\
\hline 2010-01-25 21:00:00.0 & 16.4 \\
\hline 2010-01-25 18:00:00.0 & 3.4 \\
\hline 2010-01-25 15:00:00.0 & 13.7 \\
\hline 2010-01-25 12:00:00.0 & 13.7 \\
\hline 2010-01-25 09:00:00.0 & 9.4 \\
\hline 2010-01-25 06:00:00.0 & 18.2 \\
\hline 2010-01-25 03:00:00.0 & 17.1 \\
\hline 2010-01-25 00:00:00.0 & 19.0 \\
\hline 2010-01-24 21:00:00.0 & 21.8 \\
\hline 2010-01-24 18:00:00.0 & 13.9 \\
\hline 2010-01-24 15:00:00.0 & 21.6 \\
\hline 2010-01-24 12:00:00.0 & 21.7 \\
\hline 2010-01-24 09:00:00.0 & 11.7 \\
\hline 2010-01-24 06:00:00.0 & 14.0 \\
\hline 2010-01-24 03:00:00.0 & 18.3 \\
\hline 2010-01-24 00:00:00.0 & 22.7 \\
\hline 2010-01-23 21:00:00.0 & 23.9 \\
\hline 2010-01-23 18:00:00.0 & 18.7 \\
\hline 2010-01-23 15:00:00.0 & 17.8 \\
\hline 2010-01-23 12:00:00.0 & 18.1 \\
\hline 2010-01-23 09:00:00.0 & 18.4 \\
\hline 2010-01-23 06:00:00.0 & 14.3 \\
\hline 2010-01-23 03:00:00.0 & 14.5 \\
\hline 2010-01-23 00:00:00.0 & 20.3 \\
\hline 2010-01-22 21:00:00.0 & 21.3 \\
\hline 2010-01-22 18:00:00.0 & 18.6 \\
\hline 2010-01-22 15:00:00.0 & 16.0 \\
\hline 2010-01-22 12:00:00.0 & 15.6 \\
\hline 2010-01-22 09:00:00.0 & 39.7 \\
\hline 2010-01-22 06:00:00.0 & 8.7 \\
\hline 2010-01-22 03:00:00.0 & 8.8 \\
\hline 2010-01-22 00:00:00.0 & 19.1 \\
\hline 2010-01-21 21:00:00.0 & \\
\hline 2010-01-21 18:00:00.0 & 23.0 \\
\hline 2010-01-21 15:00:00.0 & 23.0 \\
\hline 2010-01-21 12:00:00.0 & 13.9 \\
\hline 2010-01-21 09:00:00.0 & 11.7 \\
\hline 2010-01-21 06:00:00.0 & 13.0 \\
\hline 2010-01-21 03:00:00.0 & 10.8 \\
\hline 2010-01-21 00:00:00.0 & 17.8 \\
\hline 2010-01-20 21:00:00.0 & 23.7 \\
\hline 2010-01-20 18:00:00.0 & 21.6 \\
\hline 2010-01-20 15:00:00.0 & 17.1 \\
\hline 2010-01-20 12:00:00.0 & 17.1 \\
\hline 2010-01-20 09:00:00.0 & 18.2 \\
\hline 2010-01-20 06:00:00.0 & 12.0 \\
\hline 2010-01-20 03:00:00.0 & 13.2 \\
\hline 2010-01-20 00:00:00.0 & 20.4 \\
\hline 2010-01-19 21:00:00.0 & 23.0 \\
\hline 2010-01-19 18:00:00.0 & 26.7 \\
\hline
\end{tabular}

\begin{tabular}{|c|c|}
\hline DataHora & VelVentoMax \\
\hline 2010-01-19 15:00:00.0 & 19.0 \\
\hline 2010-01-19 12:00:00.0 & 19.2 \\
\hline 2010-01-19 09:00:00.0 & 30.8 \\
\hline 2010-01-19 06:00:00.0 & 35.2 \\
\hline 2010-01-19 03:00:00.0 & 18.0 \\
\hline 2010-01-19 00:00:00.0 & 19.2 \\
\hline 2010-01-18 21:00:00.0 & \\
\hline 2010-01-18 18:00:00.0 & \\
\hline 2010-01-18 15:00:00.0 & \\
\hline 2010-01-18 12:00:00.0 & 35.3 \\
\hline 2010-01-18 09:00:00.0 & 15.2 \\
\hline 2010-01-18 06:00:00.0 & 18.0 \\
\hline 2010-01-18 03:00:00.0 & 14.3 \\
\hline 2010-01-18 00:00:00.0 & 15.5 \\
\hline 2010-01-17 21:00:00.0 & 16.5 \\
\hline 2010-01-17 18:00:00.0 & 17.6 \\
\hline 2010-01-17 15:00:00.0 & 16.0 \\
\hline 2010-01-17 12:00:00.0 & 10.4 \\
\hline 2010-01-17 09:00:00.0 & 14.3 \\
\hline 2010-01-17 06:00:00.0 & 19.7 \\
\hline 2010-01-17 03:00:00.0 & 21.2 \\
\hline 2010-01-17 00:00:00.0 & 21.3 \\
\hline 2010-01-16 21:00:00.0 & 27.7 \\
\hline 2010-01-16 18:00:00.0 & 34.1 \\
\hline 2010-01-16 15:00:00.0 & 28.4 \\
\hline 2010-01-16 12:00:00.0 & 23.6 \\
\hline 2010-01-16 09:00:00.0 & 22.3 \\
\hline 2010-01-16 06:00:00.0 & 20.2 \\
\hline 2010-01-16 03:00:00.0 & 14.9 \\
\hline 2010-01-16 00:00:00.0 & 20.6 \\
\hline 2010-01-15 21:00:00.0 & 21.3 \\
\hline 2010-01-15 18:00:00.0 & 27.1 \\
\hline 2010-01-15 15:00:00.0 & 21.3 \\
\hline 2010-01-15 12:00:00.0 & 21.4 \\
\hline 2010-01-15 09:00:00.0 & 16.7 \\
\hline 2010-01-15 06:00:00.0 & 13.5 \\
\hline 2010-01-15 03:00:00.0 & 14.9 \\
\hline 2010-01-15 00:00:00.0 & 18.7 \\
\hline 2010-01-14 21:00:00.0 & 19.6 \\
\hline 2010-01-14 18:00:00.0 & 21.6 \\
\hline 2010-01-14 15:00:00.0 & 21.3 \\
\hline 2010-01-14 12:00:00.0 & 27.1 \\
\hline 2010-01-14 09:00:00.0 & 4.0 \\
\hline 2010-01-14 06:00:00.0 & 6.1 \\
\hline 2010-01-14 03:00:00.0 & 8.7 \\
\hline 2010-01-14 00:00:00.0 & 15.1 \\
\hline 2010-01-13 21:00:00.0 & 18.5 \\
\hline 2010-01-13 18:00:00.0 & 16.0 \\
\hline 2010-01-13 15:00:00.0 & 25.8 \\
\hline 2010-01-13 12:00:00.0 & 25.6 \\
\hline 2010-01-13 09:00:00.0 & 19.8 \\
\hline 2010-01-13 06:00:00.0 & 18.1 \\
\hline 2010-01-13 03:00:00.0 & 22.1 \\
\hline 2010-01-13 00:00:00.0 & 28.5 \\
\hline 2010-01-12 21:00:00.0 & 25.5 \\
\hline 2010-01-12 18:00:00.0 & 34.5 \\
\hline 2010-01-12 15:00:00.0 & 29.7 \\
\hline 2010-01-12 12:00:00.0 & 27.8 \\
\hline 2010-01-12 09:00:00.0 & 18.1 \\
\hline 2010-01-12 06:00:00.0 & 20.2 \\
\hline 2010-01-12 03:00:00.0 & 18.5 \\
\hline 2010-01-12 00:00:00.0 & 16.0 \\
\hline 2010-01-11 21:00:00.0 & 17.2 \\
\hline 2010-01-11 18:00:00.0 & 13.4 \\
\hline 2010-01-11 15:00:00.0 & 14.1 \\
\hline 2010-01-11 12:00:00.0 & 10.1 \\
\hline 2010-01-11 09:00:00.0 & 7.7 \\
\hline 2010-01-11 06:00:00.0 & 8.9 \\
\hline 2010-01-11 03:00:00.0 & \\
\hline
\end{tabular}

\begin{tabular}{|c|c|}
\hline DataHora & VelVentoMax \\
\hline 2010-01-11 00:00:00.0 & 7.1 \\
\hline 2010-01-10 21:00:00.0 & 9.7 \\
\hline 2010-01-10 18:00:00.0 & 28.8 \\
\hline 2010-01-10 15:00:00.0 & 21.6 \\
\hline 2010-01-10 12:00:00.0 & 14.4 \\
\hline 2010-01-10 09:00:00.0 & 12.4 \\
\hline 2010-01-10 06:00:00.0 & 15.4 \\
\hline 2010-01-10 03:00:00.0 & 12.8 \\
\hline 2010-01-10 00:00:00.0 & 13.0 \\
\hline 2010-01-09 21:00:00.0 & 11.3 \\
\hline 2010-01-09 18:00:00.0 & 14.6 \\
\hline 2010-01-09 15:00:00.0 & 20.0 \\
\hline 2010-01-09 12:00:00.0 & 23.8 \\
\hline 2010-01-09 09:00:00.0 & 23.0 \\
\hline 2010-01-09 06:00:00.0 & 15.4 \\
\hline 2010-01-09 03:00:00.0 & \\
\hline 2010-01-09 00:00:00.0 & 17.7 \\
\hline 2010-01-08 21:00:00.0 & 20.8 \\
\hline 2010-01-08 18:00:00.0 & 19.4 \\
\hline 2010-01-08 15:00:00.0 & 28.7 \\
\hline 2010-01-08 12:00:00.0 & 29.4 \\
\hline 2010-01-08 09:00:00.0 & 24.8 \\
\hline 2010-01-08 06:00:00.0 & 27.0 \\
\hline 2010-01-08 03:00:00.0 & 25.0 \\
\hline 2010-01-08 00:00:00.0 & 25.8 \\
\hline 2010-01-07 21:00:00.0 & 21.6 \\
\hline 2010-01-07 18:00:00.0 & 21.6 \\
\hline 2010-01-07 15:00:00.0 & 22.4 \\
\hline 2010-01-07 12:00:00.0 & 20.2 \\
\hline 2010-01-07 09:00:00.0 & 24.0 \\
\hline 2010-01-07 06:00:00.0 & 27.1 \\
\hline 2010-01-07 03:00:00.0 & 24.7 \\
\hline 2010-01-07 00:00:00.0 & 29.3 \\
\hline 2010-01-06 21:00:00.0 & 21.6 \\
\hline 2010-01-06 18:00:00.0 & 27.4 \\
\hline 2010-01-06 15:00:00.0 & 24.4 \\
\hline 2010-01-06 12:00:00.0 & 39.1 \\
\hline 2010-01-06 09:00:00.0 & 22.4 \\
\hline 2010-01-06 06:00:00.0 & 13.3 \\
\hline 2010-01-06 03:00:00.0 & 15.0 \\
\hline 2010-01-06 00:00:00.0 & 10.2 \\
\hline 2010-01-05 21:00:00.0 & 20.7 \\
\hline 2010-01-05 18:00:00.0 & 30.8 \\
\hline 2010-01-05 15:00:00.0 & 20.5 \\
\hline 2010-01-05 12:00:00.0 & 19.7 \\
\hline 2010-01-05 09:00:00.0 & 16.5 \\
\hline 2010-01-05 06:00:00.0 & 18.4 \\
\hline 2010-01-05 03:00:00.0 & 27.5 \\
\hline 2010-01-05 00:00:00.0 & 26.6 \\
\hline 2010-01-04 21:00:00.0 & 21.3 \\
\hline 2010-01-04 18:00:00.0 & 24.8 \\
\hline 2010-01-04 15:00:00.0 & 22.0 \\
\hline 2010-01-04 12:00:00.0 & 23.4 \\
\hline 2010-01-04 09:00:00.0 & 27.6 \\
\hline 2010-01-04 06:00:00.0 & 29.8 \\
\hline 2010-01-04 03:00:00.0 & \\
\hline 2010-01-04 00:00:00.0 & 32.2 \\
\hline 2010-01-03 21:00:00.0 & 28.8 \\
\hline 2010-01-03 18:00:00.0 & 16.0 \\
\hline 2010-01-03 15:00:00.0 & 19.0 \\
\hline 2010-01-03 12:00:00.0 & 21.0 \\
\hline 2010-01-03 09:00:00.0 & 32.8 \\
\hline 2010-01-03 06:00:00.0 & 19.7 \\
\hline 2010-01-03 03:00:00.0 & \\
\hline 2010-01-03 00:00:00.0 & 18.9 \\
\hline 2010-01-02 21:00:00.0 & 21.8 \\
\hline 2010-01-02 18:00:00.0 & 21.5 \\
\hline 2010-01-02 15:00:00.0 & 16.0 \\
\hline 2010-01-02 12:00:00.0 & 11.8 \\
\hline
\end{tabular}




\section{ANEXO 01 - RAJADAS DE VENTO REGISTRADAS EM SÃO MARTINHO DA SERRA / RS}

\begin{tabular}{|c|c|}
\hline DataHora & VelVentoMax \\
\hline 2010-01-02 09:00:00.0 & 14.5 \\
\hline 2010-01-02 06:00:00.0 & 15.3 \\
\hline 2010-01-02 03:00:00.0 & 14.9 \\
\hline 2010-01-02 00:00:00.0 & 17.4 \\
\hline 2010-01-01 21:00:00.0 & 21.2 \\
\hline 2010-01-01 18:00:00.0 & 19.1 \\
\hline 2010-01-01 15:00:00.0 & 22.4 \\
\hline 2010-01-01 12:00:00.0 & 34.7 \\
\hline 2010-01-01 09:00:00.0 & 24.1 \\
\hline 2010-01-01 06:00:00.0 & \\
\hline 2010-01-01 03:00:00.0 & \\
\hline 2010-01-01 00:00:00.0 & 32.1 \\
\hline 2009-12-31 21:00:00.0 & 31.3 \\
\hline 2009-12-31 18:00:00.0 & \\
\hline 2009-12-31 15:00:00.0 & 29.4 \\
\hline 2009-12-31 12:00:00.0 & 24.6 \\
\hline 2009-12-31 09:00:00.0 & 18.3 \\
\hline 2009-12-31 06:00:00.0 & \\
\hline 2009-12-31 03:00:00.0 & 26.7 \\
\hline 2009-12-31 00:00:00.0 & 23.7 \\
\hline 2009-12-30 21:00:00.0 & 25.3 \\
\hline 2009-12-30 18:00:00.0 & 21.7 \\
\hline 2009-12-30 15:00:00.0 & 18.4 \\
\hline 2009-12-30 12:00:00.0 & 16.0 \\
\hline 2009-12-30 09:00:00.0 & 6.2 \\
\hline 2009-12-30 06:00:00.0 & 13.4 \\
\hline 2009-12-30 03:00:00.0 & \\
\hline 2009-12-30 00:00:00.0 & 9.7 \\
\hline 2009-12-29 21:00:00.0 & 27.1 \\
\hline 2009-12-29 18:00:00.0 & \\
\hline 2009-12-29 15:00:00.0 & 12.4 \\
\hline 2009-12-29 12:00:00.0 & \\
\hline 2009-12-29 09:00:00.0 & 15.9 \\
\hline 2009-12-29 06:00:00.0 & 15.6 \\
\hline 2009-12-29 03:00:00.0 & \\
\hline 2009-12-29 00:00:00.0 & 18.8 \\
\hline 2009-12-28 21:00:00.0 & 17.3 \\
\hline 2009-12-28 18:00:00.0 & 15.9 \\
\hline 2009-12-28 15:00:00.0 & 14.2 \\
\hline 2009-12-28 12:00:00.0 & 13.8 \\
\hline 2009-12-28 09:00:00.0 & 16.3 \\
\hline 2009-12-28 06:00:00.0 & 15.2 \\
\hline 2009-12-28 03:00:00.0 & 14.2 \\
\hline 2009-12-28 00:00:00.0 & 14.2 \\
\hline 2009-12-27 21:00:00.0 & 21.3 \\
\hline 2009-12-27 18:00:00.0 & 18.8 \\
\hline 2009-12-27 15:00:00.0 & 18.1 \\
\hline 2009-12-27 12:00:00.0 & 8.8 \\
\hline 2009-12-27 09:00:00.0 & 8.0 \\
\hline 2009-12-27 06:00:00.0 & 19.6 \\
\hline 2009-12-27 03:00:00.0 & 22.8 \\
\hline 2009-12-27 00:00:00.0 & 9.7 \\
\hline 2009-12-26 21:00:00.0 & 42.3 \\
\hline 2009-12-26 18:00:00.0 & \\
\hline 2009-12-26 15:00:00.0 & 16.7 \\
\hline 2009-12-26 12:00:00.0 & 43.5 \\
\hline 2009-12-26 09:00:00.0 & 13.3 \\
\hline 2009-12-26 06:00:00.0 & \\
\hline 2009-12-26 03:00:00.0 & 8.0 \\
\hline 2009-12-26 00:00:00.0 & 15.1 \\
\hline 2009-12-25 21:00:00.0 & 14.7 \\
\hline 2009-12-25 18:00:00.0 & 15.0 \\
\hline 2009-12-25 15:00:00.0 & 13.4 \\
\hline 2009-12-25 12:00:00.0 & 9.8 \\
\hline 2009-12-25 09:00:00.0 & \\
\hline 2009-12-25 06:00:00.0 & 17.7 \\
\hline 2009-12-25 03:00:00.0 & 24.4 \\
\hline 2009-12-25 00:00:00.0 & 13.8 \\
\hline 2009-12-24 21:00:00.0 & 21.2 \\
\hline
\end{tabular}

\begin{tabular}{|c|c|}
\hline DataHora & VelVentoMax \\
\hline 2009-12-24 18:00:00.0 & 18.1 \\
\hline 2009-12-24 15:00:00.0 & 16.5 \\
\hline 2009-12-24 12:00:00.0 & 14.7 \\
\hline 2009-12-24 09:00:00.0 & 16.6 \\
\hline 2009-12-24 06:00:00.0 & 19.4 \\
\hline 2009-12-24 03:00:00.0 & \\
\hline 2009-12-24 00:00:00.0 & 17.5 \\
\hline 2009-12-23 21:00:00.0 & 24.3 \\
\hline 2009-12-23 18:00:00.0 & 27.8 \\
\hline 2009-12-23 15:00:00.0 & 16.1 \\
\hline 2009-12-23 12:00:00.0 & 19.7 \\
\hline 2009-12-23 09:00:00.0 & 17.2 \\
\hline 2009-12-23 06:00:00.0 & 17.4 \\
\hline 2009-12-23 03:00:00.0 & 18.4 \\
\hline 2009-12-23 00:00:00.0 & 19.9 \\
\hline 2009-12-22 21:00:00.0 & 20.3 \\
\hline 2009-12-22 18:00:00.0 & 17.1 \\
\hline 2009-12-22 15:00:00.0 & 18.4 \\
\hline 2009-12-22 12:00:00.0 & 16.2 \\
\hline 2009-12-22 09:00:00.0 & 14.9 \\
\hline 2009-12-22 06:00:00.0 & 12.2 \\
\hline 2009-12-22 03:00:00.0 & 15.3 \\
\hline 2009-12-22 00:00:00.0 & 17.4 \\
\hline 2009-12-21 21:00:00.0 & 18.8 \\
\hline 2009-12-21 18:00:00.0 & 22.9 \\
\hline 2009-12-21 15:00:00.0 & 17.6 \\
\hline 2009-12-21 12:00:00.0 & 35.4 \\
\hline 2009-12-21 09:00:00.0 & 18.9 \\
\hline 2009-12-21 06:00:00.0 & 18.2 \\
\hline 2009-12-21 03:00:00.0 & 16.6 \\
\hline 2009-12-21 00:00:00.0 & 15.0 \\
\hline 2009-12-20 21:00:00.0 & 29.5 \\
\hline 2009-12-20 18:00:00.0 & 24.3 \\
\hline 2009-12-20 15:00:00.0 & 25.9 \\
\hline 2009-12-20 12:00:00.0 & 17.7 \\
\hline 2009-12-20 09:00:00.0 & \\
\hline 2009-12-20 06:00:00.0 & 11.5 \\
\hline 2009-12-20 03:00:00.0 & 12.6 \\
\hline 2009-12-20 00:00:00.0 & 14.9 \\
\hline 2009-12-19 21:00:00.0 & 17.0 \\
\hline 2009-12-19 18:00:00.0 & 19.4 \\
\hline 2009-12-19 15:00:00.0 & 26.5 \\
\hline 2009-12-19 12:00:00.0 & 28.3 \\
\hline 2009-12-19 09:00:00.0 & 20.0 \\
\hline 2009-12-19 06:00:00.0 & 20.8 \\
\hline 2009-12-19 03:00:00.0 & 16.3 \\
\hline 2009-12-19 00:00:00.0 & 21.4 \\
\hline 2009-12-18 21:00:00.0 & 21.2 \\
\hline 2009-12-18 18:00:00.0 & 15.5 \\
\hline 2009-12-18 15:00:00.0 & 23.3 \\
\hline 2009-12-18 12:00:00.0 & 25.7 \\
\hline 2009-12-18 09:00:00.0 & 26.5 \\
\hline 2009-12-18 06:00:00.0 & 21.4 \\
\hline 2009-12-18 03:00:00.0 & \\
\hline 2009-12-18 00:00:00.0 & 22.9 \\
\hline 2009-12-17 21:00:00.0 & 24.8 \\
\hline 2009-12-17 18:00:00.0 & 26.0 \\
\hline 2009-12-17 15:00:00.0 & 26.5 \\
\hline 2009-12-17 12:00:00.0 & 24.0 \\
\hline 2009-12-17 09:00:00.0 & 6.5 \\
\hline 2009-12-17 06:00:00.0 & 18.5 \\
\hline 2009-12-17 03:00:00.0 & 28.0 \\
\hline 2009-12-17 00:00:00.0 & 27.7 \\
\hline 2009-12-16 21:00:00.0 & 5.7 \\
\hline 2009-12-16 18:00:00.0 & 29.1 \\
\hline 2009-12-16 15:00:00.0 & 5.9 \\
\hline 2009-12-16 12:00:00.0 & 20.3 \\
\hline 2009-12-16 09:00:00.0 & 18.2 \\
\hline 2009-12-16 06:00:00.0 & 15.2 \\
\hline
\end{tabular}

\begin{tabular}{|c|c|}
\hline DataHora & VelVentoMax \\
\hline 2009-12-16 03:00:00.0 & 14.4 \\
\hline 2009-12-16 00:00:00.0 & 18.8 \\
\hline 2009-12-15 21:00:00.0 & 17.8 \\
\hline 2009-12-15 18:00:00.0 & 14.7 \\
\hline 2009-12-15 15:00:00.0 & 12.8 \\
\hline 2009-12-15 12:00:00.0 & 2.9 \\
\hline 2009-12-15 09:00:00.0 & 9.5 \\
\hline 2009-12-15 06:00:00.0 & 10.3 \\
\hline 2009-12-15 03:00:00.0 & 11.6 \\
\hline 2009-12-15 00:00:00.0 & 13.9 \\
\hline 2009-12-14 21:00:00.0 & 21.5 \\
\hline 2009-12-14 18:00:00.0 & 17.8 \\
\hline 2009-12-14 15:00:00.0 & \\
\hline 2009-12-14 12:00:00.0 & 17.5 \\
\hline 2009-12-14 09:00:00.0 & 16.1 \\
\hline 2009-12-14 06:00:00.0 & 36.3 \\
\hline 2009-12-14 03:00:00.0 & 13.5 \\
\hline 2009-12-14 00:00:00.0 & 14.7 \\
\hline 2009-12-13 21:00:00.0 & 14.8 \\
\hline 2009-12-13 18:00:00.0 & 14.4 \\
\hline 2009-12-13 15:00:00.0 & 13.1 \\
\hline 2009-12-13 12:00:00.0 & 12.7 \\
\hline 2009-12-13 09:00:00.0 & 13.1 \\
\hline 2009-12-13 06:00:00.0 & 8.8 \\
\hline 2009-12-13 03:00:00.0 & \\
\hline 2009-12-13 00:00:00.0 & 22.1 \\
\hline 2009-12-12 21:00:00.0 & 32.3 \\
\hline 2009-12-12 18:00:00.0 & 27.5 \\
\hline 2009-12-12 15:00:00.0 & \\
\hline 2009-12-12 12:00:00.0 & 29.1 \\
\hline 2009-12-12 09:00:00.0 & 24.7 \\
\hline 2009-12-12 06:00:00.0 & 16.7 \\
\hline 2009-12-12 03:00:00.0 & 23.8 \\
\hline 2009-12-12 00:00:00.0 & 21.6 \\
\hline 2009-12-11 21:00:00.0 & 24.2 \\
\hline 2009-12-11 18:00:00.0 & 20.7 \\
\hline 2009-12-11 15:00:00.0 & 27.1 \\
\hline 2009-12-11 12:00:00.0 & 19.0 \\
\hline 2009-12-11 09:00:00.0 & 11.6 \\
\hline 2009-12-11 06:00:00.0 & 13.9 \\
\hline 2009-12-11 03:00:00.0 & 11.1 \\
\hline 2009-12-11 00:00:00.0 & 16.3 \\
\hline 2009-12-10 21:00:00.0 & 17.5 \\
\hline 2009-12-10 18:00:00.0 & \\
\hline 2009-12-10 15:00:00.0 & 13.3 \\
\hline 2009-12-10 12:00:00.0 & 16.7 \\
\hline 2009-12-10 09:00:00.0 & 16.7 \\
\hline 2009-12-10 06:00:00.0 & 14.3 \\
\hline 2009-12-10 03:00:00.0 & 14.9 \\
\hline 2009-12-10 00:00:00.0 & 22.0 \\
\hline 2009-12-09 21:00:00.0 & 25.9 \\
\hline 2009-12-09 18:00:00.0 & 20.4 \\
\hline 2009-12-09 15:00:00.0 & 14.4 \\
\hline 2009-12-09 12:00:00.0 & 16.2 \\
\hline 2009-12-09 09:00:00.0 & 18.5 \\
\hline 2009-12-09 06:00:00.0 & 21.1 \\
\hline 2009-12-09 03:00:00.0 & 26.9 \\
\hline 2009-12-09 00:00:00.0 & 28.1 \\
\hline 2009-12-08 21:00:00.0 & 21.8 \\
\hline 2009-12-08 18:00:00.0 & 19.6 \\
\hline 2009-12-08 15:00:00.0 & 21.3 \\
\hline 2009-12-08 12:00:00.0 & 22.6 \\
\hline 2009-12-08 09:00:00.0 & 20.7 \\
\hline 2009-12-08 06:00:00.0 & 21.7 \\
\hline 2009-12-08 03:00:00.0 & 23.6 \\
\hline 2009-12-08 00:00:00.0 & 26.6 \\
\hline 2009-12-07 21:00:00.0 & 31.4 \\
\hline 2009-12-07 18:00:00.0 & \\
\hline 2009-12-07 15:00:00.0 & 25.7 \\
\hline
\end{tabular}




\section{ANEXO 01 - RAJADAS DE VENTO REGISTRADAS EM SÃO MARTINHO DA SERRA / RS}

\begin{tabular}{|c|c|}
\hline DataHora & VelVentoMax \\
\hline 2009-12-07 12:00:00.0 & 23.5 \\
\hline 2009-12-07 09:00:00.0 & 17.6 \\
\hline 2009-12-07 06:00:00.0 & 21.9 \\
\hline 2009-12-07 03:00:00.0 & 23.1 \\
\hline 2009-12-07 00:00:00.0 & 23.3 \\
\hline 2009-12-06 21:00:00.0 & 9.5 \\
\hline 2009-12-06 18:00:00.0 & 14.8 \\
\hline 2009-12-06 15:00:00.0 & 36.6 \\
\hline 2009-12-06 12:00:00.0 & 18.1 \\
\hline 2009-12-06 09:00:00.0 & 19.1 \\
\hline 2009-12-06 06:00:00.0 & 26.9 \\
\hline 2009-12-06 03:00:00.0 & 13.9 \\
\hline 2009-12-06 00:00:00.0 & 11.0 \\
\hline 2009-12-05 21:00:00.0 & 18.9 \\
\hline 2009-12-05 18:00:00.0 & 25.5 \\
\hline 2009-12-05 15:00:00.0 & 24.7 \\
\hline 2009-12-05 12:00:00.0 & 26.5 \\
\hline 2009-12-05 09:00:00.0 & 22.5 \\
\hline 2009-12-05 06:00:00.0 & 22.1 \\
\hline 2009-12-05 03:00:00.0 & 23.7 \\
\hline 2009-12-05 00:00:00.0 & 22.4 \\
\hline 2009-12-04 21:00:00.0 & 22.8 \\
\hline 2009-12-04 18:00:00.0 & 20.9 \\
\hline 2009-12-04 15:00:00.0 & 22.6 \\
\hline 2009-12-04 12:00:00.0 & 26.1 \\
\hline 2009-12-04 09:00:00.0 & 35.0 \\
\hline 2009-12-04 06:00:00.0 & 24.9 \\
\hline 2009-12-04 03:00:00.0 & 21.3 \\
\hline 2009-12-04 00:00:00.0 & 20.8 \\
\hline 2009-12-03 21:00:00.0 & 31.7 \\
\hline 2009-12-03 18:00:00.0 & 27.3 \\
\hline 2009-12-03 15:00:00.0 & 24.0 \\
\hline 2009-12-03 12:00:00.0 & 18.1 \\
\hline 2009-12-03 09:00:00.0 & 33.2 \\
\hline 2009-12-03 06:00:00.0 & 15.9 \\
\hline 2009-12-03 03:00:00.0 & 9.3 \\
\hline 2009-12-03 00:00:00.0 & 9.3 \\
\hline 2009-12-02 21:00:00.0 & 15.3 \\
\hline 2009-12-02 18:00:00.0 & 30.6 \\
\hline 2009-12-02 15:00:00.0 & 32.6 \\
\hline 2009-12-02 12:00:00.0 & 22.4 \\
\hline 2009-12-02 09:00:00.0 & 21.8 \\
\hline 2009-12-02 06:00:00.0 & 19.9 \\
\hline 2009-12-02 03:00:00.0 & 18.0 \\
\hline 2009-12-02 00:00:00.0 & 18.0 \\
\hline 2009-12-01 21:00:00.0 & 16.0 \\
\hline 2009-12-01 18:00:00.0 & 3.6 \\
\hline 2009-12-01 15:00:00.0 & 14.7 \\
\hline 2009-12-01 12:00:00.0 & 19.6 \\
\hline 2009-12-01 09:00:00.0 & 3.7 \\
\hline 2009-12-01 06:00:00.0 & 26.8 \\
\hline 2009-12-01 03:00:00.0 & 28.1 \\
\hline 2009-12-01 00:00:00.0 & 18.7 \\
\hline 2010-11-30 21:00:00.0 & 17.1 \\
\hline 2010-11-30 18:00:00.0 & 19.0 \\
\hline 2010-11-30 15:00:00.0 & \\
\hline 2010-11-30 12:00:00.0 & 12.0 \\
\hline 2010-11-30 09:00:00.0 & 7.2 \\
\hline 2010-11-30 06:00:00.0 & 10.1 \\
\hline 2010-11-30 03:00:00.0 & \\
\hline 2010-11-30 00:00:00.0 & 12.3 \\
\hline 2010-11-29 21:00:00.0 & 16.5 \\
\hline 2010-11-29 18:00:00.0 & \\
\hline 2010-11-29 15:00:00.0 & \\
\hline 2010-11-29 12:00:00.0 & 15.2 \\
\hline 2010-11-29 09:00:00.0 & 11.4 \\
\hline 2010-11-29 06:00:00.0 & \\
\hline 2010-11-29 03:00:00.0 & 16.5 \\
\hline 2010-11-29 00:00:00.0 & 24.7 \\
\hline
\end{tabular}

\begin{tabular}{|c|c|}
\hline DataHora & VelVentoMax \\
\hline 2010-11-28 21:00:00.0 & 28.8 \\
\hline \multicolumn{2}{|l|}{ 2010-11-28 18:00:00.0 } \\
\hline 2010-11-28 15:00:00.0 & 25.7 \\
\hline 2010-11-28 12:00:00.0 & 17.5 \\
\hline 2010-11-28 09:00:00.0 & 14.8 \\
\hline \multicolumn{2}{|l|}{ 2010-11-28 06:00:00.0 } \\
\hline \multicolumn{2}{|l|}{ 2010-11-28 03:00:00.0 } \\
\hline 2010-11-28 00:00:00.0 & 17.0 \\
\hline 2010-11-27 21:00:00.0 & 18.4 \\
\hline \multicolumn{2}{|l|}{ 2010-11-27 18:00:00.0 } \\
\hline 2010-11-27 15:00:00.0 & 14.0 \\
\hline 2010-11-27 12:00:00.0 & 16.8 \\
\hline 2010-11-27 09:00:00.0 & 19.0 \\
\hline \multicolumn{2}{|l|}{ 2010-11-27 06:00:00.0 } \\
\hline 2010-11-27 03:00:00.0 & 15.8 \\
\hline 2010-11-27 00:00:00.0 & 16.1 \\
\hline 2010-11-26 21:00:00.0 & 18.7 \\
\hline \multicolumn{2}{|l|}{ 2010-11-26 18:00:00.0 } \\
\hline 2010-11-26 15:00:00.0 & 15.8 \\
\hline 2010-11-26 12:00:00.0 & 19.4 \\
\hline 2010-11-26 09:00:00.0 & 19.7 \\
\hline \multicolumn{2}{|l|}{ 2010-11-26 06:00:00.0 } \\
\hline \multicolumn{2}{|l|}{ 2010-11-26 03:00:00.0 } \\
\hline 2010-11-26 00:00:00.0 & 19.6 \\
\hline 2010-11-25 21:00:00.0 & 22.2 \\
\hline \multicolumn{2}{|l|}{ 2010-11-25 18:00:00.0 } \\
\hline 2010-11-25 15:00:00.0 & 18.5 \\
\hline 2010-11-25 12:00:00.0 & 14.0 \\
\hline 2010-11-25 09:00:00.0 & 17.1 \\
\hline \multicolumn{2}{|l|}{ 2010-11-25 06:00:00.0 } \\
\hline 2010-11-25 03:00:00.0 & 21.9 \\
\hline 2010-11-25 00:00:00.0 & 19.6 \\
\hline 2010-11-24 21:00:00.0 & 20.8 \\
\hline 2010-11-24 18:00:00.0 & 19.2 \\
\hline 2010-11-24 15:00:00.0 & 23.7 \\
\hline 2010-11-24 12:00:00.0 & 3.0 \\
\hline 2010-11-24 09:00:00.0 & 23.0 \\
\hline \multicolumn{2}{|l|}{ 2010-11-24 06:00:00.0 } \\
\hline 2010-11-24 03:00:00.0 & 18.3 \\
\hline 2010-11-24 00:00:00.0 & 29.3 \\
\hline \multicolumn{2}{|l|}{ 2010-11-23 21:00:00.0 } \\
\hline \multicolumn{2}{|l|}{ 2010-11-23 18:00:00.0 } \\
\hline 2010-11-23 15:00:00.0 & 22.7 \\
\hline 2010-11-23 12:00:00.0 & 20.8 \\
\hline 2010-11-23 09:00:00.0 & \\
\hline 2010-11-23 06:00:00.0 & \\
\hline 2010-11-23 03:00:00.0 & 20.6 \\
\hline 2010-11-23 00:00:00.0 & 20.2 \\
\hline 2010-11-22 21:00:00.0 & 22.9 \\
\hline 2010-11-22 18:00:00.0 & 22.5 \\
\hline 2010-11-22 15:00:00.0 & 17.7 \\
\hline 2010-11-22 12:00:00.0 & 15.7 \\
\hline 2010-11-22 09:00:00.0 & 16.2 \\
\hline 2010-11-22 06:00:00.0 & \\
\hline 2010-11-22 03:00:00.0 & 21.0 \\
\hline 2010-11-22 00:00:00.0 & 23.6 \\
\hline 2010-11-21 21:00:00.0 & 18.9 \\
\hline 2010-11-21 18:00:00.0 & \\
\hline 2010-11-21 15:00:00.0 & 16.9 \\
\hline 2010-11-21 12:00:00.0 & 13.1 \\
\hline 2010-11-21 09:00:00.0 & \\
\hline 2010-11-21 06:00:00.0 & 14.1 \\
\hline 2010-11-21 03:00:00.0 & 15.5 \\
\hline 2010-11-21 00:00:00.0 & 22.7 \\
\hline 2010-11-20 21:00:00.0 & \\
\hline 2010-11-20 18:00:00.0 & 22.2 \\
\hline 2010-11-20 15:00:00.0 & 18.6 \\
\hline 2010-11-20 12:00:00.0 & 22.4 \\
\hline 2010-11-20 09:00:00.0 & \\
\hline
\end{tabular}

\begin{tabular}{|c|c|}
\hline DataHora & VelVentoMax \\
\hline 2010-11-20 06:00:00.0 & 18.1 \\
\hline 2010-11-20 03:00:00.0 & 14.5 \\
\hline 2010-11-20 00:00:00.0 & 17.3 \\
\hline 2010-11-19 21:00:00.0 & 17.3 \\
\hline 2010-11-19 18:00:00.0 & 28.3 \\
\hline 2010-11-19 15:00:00.0 & 18.6 \\
\hline 2010-11-19 12:00:00.0 & \\
\hline 2010-11-19 09:00:00.0 & \\
\hline 2010-11-19 06:00:00.0 & \\
\hline 2010-11-19 03:00:00.0 & 10.4 \\
\hline 2010-11-19 00:00:00.0 & 13.9 \\
\hline 2010-11-18 21:00:00.0 & 22.7 \\
\hline 2010-11-18 18:00:00.0 & 20.1 \\
\hline 2010-11-18 15:00:00.0 & 17.5 \\
\hline 2010-11-18 12:00:00.0 & 10.3 \\
\hline 2010-11-18 09:00:00.0 & 5.4 \\
\hline 2010-11-18 06:00:00.0 & 4.9 \\
\hline 2010-11-18 03:00:00.0 & 8.8 \\
\hline 2010-11-18 00:00:00.0 & 16.0 \\
\hline 2010-11-17 21:00:00.0 & \\
\hline 2010-11-17 18:00:00.0 & 20.6 \\
\hline 2010-11-17 15:00:00.0 & 19.0 \\
\hline 2010-11-17 12:00:00.0 & 12.2 \\
\hline 2010-11-17 09:00:00.0 & 4.9 \\
\hline 2010-11-17 06:00:00.0 & 8.7 \\
\hline 2010-11-17 03:00:00.0 & 10.2 \\
\hline 2010-11-17 00:00:00.0 & 19.1 \\
\hline 2010-11-16 21:00:00.0 & \\
\hline 2010-11-16 18:00:00.0 & 26.0 \\
\hline 2010-11-16 15:00:00.0 & 18.1 \\
\hline 2010-11-16 12:00:00.0 & 14.9 \\
\hline 2010-11-16 09:00:00.0 & 11.9 \\
\hline 2010-11-16 06:00:00.0 & 14.5 \\
\hline 2010-11-16 03:00:00.0 & 16.8 \\
\hline 2010-11-16 00:00:00.0 & 17.3 \\
\hline 2010-11-15 21:00:00.0 & \\
\hline 2010-11-15 18:00:00.0 & 19.2 \\
\hline 2010-11-15 15:00:00.0 & 15.6 \\
\hline 2010-11-15 12:00:00.0 & 19.3 \\
\hline 2010-11-15 09:00:00.0 & 18.0 \\
\hline 2010-11-15 06:00:00.0 & 15.4 \\
\hline 2010-11-15 03:00:00.0 & 20.4 \\
\hline 2010-11-15 00:00:00.0 & 18.2 \\
\hline 2010-11-14 21:00:00.0 & 20.2 \\
\hline 2010-11-14 18:00:00.0 & 26.6 \\
\hline 2010-11-14 15:00:00.0 & 16.2 \\
\hline 2010-11-14 12:00:00.0 & 35.2 \\
\hline 2010-11-14 09:00:00.0 & 18.5 \\
\hline 2010-11-14 06:00:00.0 & 19.4 \\
\hline 2010-11-14 03:00:00.0 & 17.4 \\
\hline 2010-11-14 00:00:00.0 & \\
\hline 2010-11-13 21:00:00.0 & 23.0 \\
\hline 2010-11-13 18:00:00.0 & 24.4 \\
\hline 2010-11-13 15:00:00.0 & 17.3 \\
\hline 2010-11-13 12:00:00.0 & 20.9 \\
\hline 2010-11-13 09:00:00.0 & 14.6 \\
\hline 2010-11-13 06:00:00.0 & 15.6 \\
\hline 2010-11-13 03:00:00.0 & 17.7 \\
\hline 2010-11-13 00:00:00.0 & \\
\hline 2010-11-12 21:00:00.0 & \\
\hline 2010-11-12 18:00:00.0 & 22.7 \\
\hline 2010-11-12 15:00:00.0 & 25.3 \\
\hline 2010-11-12 12:00:00.0 & \\
\hline 2010-11-12 09:00:00.0 & 13.7 \\
\hline 2010-11-12 06:00:00.0 & 16.3 \\
\hline 2010-11-12 03:00:00.0 & 13.8 \\
\hline 2010-11-12 00:00:00.0 & \\
\hline 2010-11-11 21:00:00.0 & 20.5 \\
\hline 2010-11-11 18:00:00.0 & 20.0 \\
\hline
\end{tabular}




\section{ANEXO 01 - RAJADAS DE VENTO REGISTRADAS EM SÃO MARTINHO DA SERRA / RS}

\begin{tabular}{|c|c|}
\hline DataHora & VelVentoMax \\
\hline 2010-11-11 15:00:00.0 & 13.2 \\
\hline 2010-11-11 12:00:00.0 & 15.1 \\
\hline 2010-11-11 09:00:00.0 & 8.9 \\
\hline 2010-11-11 06:00:00.0 & 9.2 \\
\hline 2010-11-11 03:00:00.0 & 8.5 \\
\hline \multicolumn{2}{|l|}{ 2010-11-11 00:00:00.0 } \\
\hline 2010-11-10 21:00:00.0 & 26.4 \\
\hline 2010-11-10 18:00:00.0 & 25.0 \\
\hline 2010-11-10 15:00:00.0 & 25.0 \\
\hline \multicolumn{2}{|l|}{ 2010-11-10 12:00:00.0 } \\
\hline 2010-11-10 09:00:00.0 & 10.0 \\
\hline 2010-11-10 06:00:00.0 & 23.3 \\
\hline 2010-11-10 03:00:00.0 & 24.4 \\
\hline \multicolumn{2}{|l|}{ 2010-11-10 00:00:00.0 } \\
\hline 2010-11-09 21:00:00.0 & 25.8 \\
\hline 2010-11-09 18:00:00.0 & 20.0 \\
\hline 2010-11-09 15:00:00.0 & 44.8 \\
\hline 2010-11-09 12:00:00.0 & 24.9 \\
\hline 2010-11-09 09:00:00.0 & 15.2 \\
\hline 2010-11-09 06:00:00.0 & 11.6 \\
\hline 2010-11-09 03:00:00.0 & 13.9 \\
\hline \multicolumn{2}{|l|}{ 2010-11-09 00:00:00.0 } \\
\hline 2010-11-08 21:00:00.0 & 24.2 \\
\hline 2010-11-08 18:00:00.0 & 26.9 \\
\hline \multicolumn{2}{|l|}{ 2010-11-08 15:00:00.0 } \\
\hline 2010-11-08 12:00:00.0 & 25.2 \\
\hline 2010-11-08 09:00:00.0 & 18.0 \\
\hline 2010-11-08 06:00:00.0 & 15.2 \\
\hline 2010-11-08 03:00:00.0 & 14.6 \\
\hline \multicolumn{2}{|l|}{ 2010-11-08 00:00:00.0 } \\
\hline 2010-11-07 21:00:00.0 & 15.4 \\
\hline 2010-11-07 18:00:00.0 & 24.8 \\
\hline 2010-11-07 15:00:00.0 & 18.8 \\
\hline 2010-11-07 12:00:00.0 & 16.6 \\
\hline 2010-11-07 09:00:00.0 & 13.9 \\
\hline 2010-11-07 06:00:00.0 & 11.7 \\
\hline \multicolumn{2}{|l|}{ 2010-11-07 03:00:00.0 } \\
\hline \multicolumn{2}{|l|}{ 2010-11-07 00:00:00.0 } \\
\hline 2010-11-06 21:00:00.0 & 22.6 \\
\hline 2010-11-06 18:00:00.0 & 24.5 \\
\hline \multicolumn{2}{|l|}{ 2010-11-06 15:00:00.0 } \\
\hline 2010-11-06 12:00:00.0 & 14.4 \\
\hline 2010-11-06 09:00:00.0 & 7.9 \\
\hline 2010-11-06 06:00:00.0 & 7.9 \\
\hline \multicolumn{2}{|l|}{ 2010-11-06 03:00:00.0 } \\
\hline \multicolumn{2}{|l|}{ 2010-11-06 00:00:00.0 } \\
\hline 2010-11-05 21:00:00.0 & 14.8 \\
\hline 2010-11-05 18:00:00.0 & 9.6 \\
\hline 2010-11-05 15:00:00.0 & 17.2 \\
\hline 2010-11-05 12:00:00.0 & 23.0 \\
\hline 2010-11-05 09:00:00.0 & 26.2 \\
\hline 2010-11-05 06:00:00.0 & 15.5 \\
\hline \multicolumn{2}{|l|}{ 2010-11-05 03:00:00.0 } \\
\hline \multicolumn{2}{|l|}{ 2010-11-05 00:00:00.0 } \\
\hline 2010-11-04 21:00:00.0 & 17.8 \\
\hline 2010-11-04 18:00:00.0 & 36.5 \\
\hline 2010-11-04 15:00:00.0 & 19.8 \\
\hline 2010-11-04 12:00:00.0 & 4.1 \\
\hline 2010-11-04 09:00:00.0 & 16.8 \\
\hline 2010-11-04 06:00:00.0 & 15.1 \\
\hline 2010-11-04 03:00:00.0 & \\
\hline 2010-11-04 00:00:00.0 & \\
\hline 2010-11-03 21:00:00.0 & 13.0 \\
\hline 2010-11-03 18:00:00.0 & 16.7 \\
\hline 2010-11-03 15:00:00.0 & 20.5 \\
\hline 2010-11-03 12:00:00.0 & 13.7 \\
\hline 2010-11-03 09:00:00.0 & 11.9 \\
\hline 2010-11-03 06:00:00.0 & \\
\hline 2010-11-03 03:00:00.0 & \\
\hline
\end{tabular}

\begin{tabular}{|c|c|}
\hline DataHora & VelVentoMax \\
\hline 2010-11-03 00:00:00.0 & 7.3 \\
\hline 2010-11-02 21:00:00.0 & 19.9 \\
\hline 2010-11-02 18:00:00.0 & 20.4 \\
\hline 2010-11-02 15:00:00.0 & 19.5 \\
\hline 2010-11-02 12:00:00.0 & 12.9 \\
\hline 2010-11-02 09:00:00.0 & 8.1 \\
\hline 2010-11-02 06:00:00.0 & 10.0 \\
\hline 2010-11-02 03:00:00.0 & \\
\hline 2010-11-02 00:00:00.0 & 17.3 \\
\hline 2010-11-01 21:00:00.0 & 24.9 \\
\hline 2010-11-01 18:00:00.0 & 25.6 \\
\hline 2010-11-01 15:00:00.0 & 23.4 \\
\hline 2010-11-01 12:00:00.0 & 20.8 \\
\hline 2010-11-01 09:00:00.0 & 7.5 \\
\hline 2010-11-01 06:00:00.0 & \\
\hline 2010-11-01 03:00:00.0 & \\
\hline 2010-11-01 00:00:00.0 & \\
\hline 2009-10-31 21:00:00.0 & \\
\hline 2009-10-31 18:00:00.0 & 19.2 \\
\hline 2009-10-31 15:00:00.0 & 16.2 \\
\hline 2009-10-31 12:00:00.0 & 19.4 \\
\hline 2009-10-31 09:00:00.0 & 16.8 \\
\hline 2009-10-31 06:00:00.0 & 16.9 \\
\hline 2009-10-31 03:00:00.0 & 17.0 \\
\hline 2009-10-31 00:00:00.0 & 10.5 \\
\hline 2009-10-30 21:00:00.0 & 14.7 \\
\hline 2009-10-30 18:00:00.0 & 21.3 \\
\hline 2009-10-30 15:00:00.0 & 22.8 \\
\hline 2009-10-30 12:00:00.0 & 19.1 \\
\hline 2009-10-30 09:00:00.0 & 16.1 \\
\hline 2009-10-30 06:00:00.0 & 17.0 \\
\hline 2009-10-30 03:00:00.0 & 16.0 \\
\hline 2009-10-30 00:00:00.0 & 11.9 \\
\hline 2009-10-29 21:00:00.0 & 14.3 \\
\hline 2009-10-29 18:00:00.0 & 11.4 \\
\hline 2009-10-29 15:00:00.0 & 10.2 \\
\hline 2009-10-29 12:00:00.0 & 15.9 \\
\hline 2009-10-29 09:00:00.0 & 15.2 \\
\hline 2009-10-29 06:00:00.0 & 16.3 \\
\hline 2009-10-29 03:00:00.0 & \\
\hline 2009-10-29 00:00:00.0 & \\
\hline 2009-10-28 21:00:00.0 & \\
\hline 2009-10-28 18:00:00.0 & 14.7 \\
\hline 2009-10-28 15:00:00.0 & 43.8 \\
\hline 2009-10-28 12:00:00.0 & 24.3 \\
\hline 2009-10-28 09:00:00.0 & 25.4 \\
\hline 2009-10-28 06:00:00.0 & 23.6 \\
\hline 2009-10-28 03:00:00.0 & 18.5 \\
\hline 2009-10-28 00:00:00.0 & 20.8 \\
\hline 2009-10-27 21:00:00.0 & 21.1 \\
\hline 2009-10-27 18:00:00.0 & 19.5 \\
\hline 2009-10-27 15:00:00.0 & 20.9 \\
\hline 2009-10-27 12:00:00.0 & 24.1 \\
\hline 2009-10-27 09:00:00.0 & 14.7 \\
\hline 2009-10-27 06:00:00.0 & 16.7 \\
\hline 2009-10-27 03:00:00.0 & 26.1 \\
\hline 2009-10-27 00:00:00.0 & \\
\hline 2009-10-26 21:00:00.0 & 26.3 \\
\hline 2009-10-26 18:00:00.0 & 42.7 \\
\hline 2009-10-26 15:00:00.0 & 27.8 \\
\hline 2009-10-26 12:00:00.0 & 31.2 \\
\hline 2009-10-26 09:00:00.0 & 21.9 \\
\hline 2009-10-26 06:00:00.0 & 7.0 \\
\hline 2009-10-26 03:00:00.0 & \\
\hline 2009-10-26 00:00:00.0 & 10.4 \\
\hline 2009-10-25 21:00:00.0 & 18.1 \\
\hline 2009-10-25 18:00:00.0 & 23.5 \\
\hline 2009-10-25 15:00:00.0 & 29.5 \\
\hline 2009-10-25 12:00:00.0 & 28.6 \\
\hline
\end{tabular}

\begin{tabular}{|c|c|}
\hline DataHora & VelVentoMax \\
\hline 2009-10-25 09:00:00.0 & 26.7 \\
\hline 2009-10-25 06:00:00.0 & 23.0 \\
\hline 2009-10-25 03:00:00.0 & 19.9 \\
\hline 2009-10-25 00:00:00.0 & 23.6 \\
\hline 2009-10-24 21:00:00.0 & 20.8 \\
\hline 2009-10-24 18:00:00.0 & 35.1 \\
\hline 2009-10-24 15:00:00.0 & 37.8 \\
\hline 2009-10-24 12:00:00.0 & 35.4 \\
\hline 2009-10-24 09:00:00.0 & 21.2 \\
\hline 2009-10-24 06:00:00.0 & 17.2 \\
\hline 2009-10-24 03:00:00.0 & 17.1 \\
\hline 2009-10-24 00:00:00.0 & 12.7 \\
\hline 2009-10-23 21:00:00.0 & 18.5 \\
\hline 2009-10-23 18:00:00.0 & 17.7 \\
\hline 2009-10-23 15:00:00.0 & \\
\hline 2009-10-23 12:00:00.0 & 17.3 \\
\hline 2009-10-23 09:00:00.0 & 18.0 \\
\hline 2009-10-23 06:00:00.0 & 19.8 \\
\hline 2009-10-23 03:00:00.0 & 14.1 \\
\hline 2009-10-23 00:00:00.0 & 10.9 \\
\hline 2009-10-22 21:00:00.0 & 13.1 \\
\hline 2009-10-22 18:00:00.0 & 15.1 \\
\hline 2009-10-22 15:00:00.0 & 15.9 \\
\hline 2009-10-22 12:00:00.0 & 7.7 \\
\hline 2009-10-22 09:00:00.0 & 11.8 \\
\hline 2009-10-22 06:00:00.0 & 14.7 \\
\hline 2009-10-22 03:00:00.0 & 12.8 \\
\hline 2009-10-22 00:00:00.0 & 23.0 \\
\hline 2009-10-21 21:00:00.0 & 16.3 \\
\hline 2009-10-21 18:00:00.0 & 15.5 \\
\hline 2009-10-21 15:00:00.0 & 21.5 \\
\hline 2009-10-21 12:00:00.0 & 25.1 \\
\hline 2009-10-21 09:00:00.0 & 20.5 \\
\hline 2009-10-21 06:00:00.0 & 14.9 \\
\hline 2009-10-21 03:00:00.0 & 12.8 \\
\hline 2009-10-21 00:00:00.0 & 11.4 \\
\hline 2009-10-20 21:00:00.0 & 14.6 \\
\hline 2009-10-20 18:00:00.0 & 17.1 \\
\hline 2009-10-20 15:00:00.0 & \\
\hline 2009-10-20 12:00:00.0 & 2.7 \\
\hline 2009-10-20 09:00:00.0 & 8.1 \\
\hline 2009-10-20 06:00:00.0 & \\
\hline 2009-10-20 03:00:00.0 & \\
\hline 2009-10-20 00:00:00.0 & 14.1 \\
\hline 2009-10-19 21:00:00.0 & 16.8 \\
\hline 2009-10-19 18:00:00.0 & 14.8 \\
\hline 2009-10-19 15:00:00.0 & 9.2 \\
\hline 2009-10-19 12:00:00.0 & 14.9 \\
\hline 2009-10-19 09:00:00.0 & 16.5 \\
\hline 2009-10-19 06:00:00.0 & 17.7 \\
\hline 2009-10-19 03:00:00.0 & 20.4 \\
\hline 2009-10-19 00:00:00.0 & 17.7 \\
\hline 2009-10-18 21:00:00.0 & 21.9 \\
\hline 2009-10-18 18:00:00.0 & 21.8 \\
\hline 2009-10-18 15:00:00.0 & 21.3 \\
\hline 2009-10-18 12:00:00.0 & 20.2 \\
\hline 2009-10-18 09:00:00.0 & 21.1 \\
\hline 2009-10-18 06:00:00.0 & 18.8 \\
\hline 2009-10-18 03:00:00.0 & 13.4 \\
\hline 2009-10-18 00:00:00.0 & 12.6 \\
\hline 2009-10-17 21:00:00.0 & 17.4 \\
\hline 2009-10-17 18:00:00.0 & 16.7 \\
\hline 2009-10-17 15:00:00.0 & 15.0 \\
\hline 2009-10-17 12:00:00.0 & 12.9 \\
\hline 2009-10-17 09:00:00.0 & 14.8 \\
\hline 2009-10-17 06:00:00.0 & 17.4 \\
\hline 2009-10-17 03:00:00.0 & 15.4 \\
\hline 2009-10-17 00:00:00.0 & 16.2 \\
\hline 2009-10-16 21:00:00.0 & 21.0 \\
\hline
\end{tabular}




\section{ANEXO 01 - RAJADAS DE VENTO REGISTRADAS EM SÃO MARTINHO DA SERRA / RS}

\begin{tabular}{|c|c|}
\hline DataHora & VelVentoMax \\
\hline 2009-10-16 18:00:00.0 & 22.8 \\
\hline 2009-10-16 15:00:00.0 & 27.1 \\
\hline 2009-10-16 12:00:00.0 & 24.6 \\
\hline 2009-10-16 09:00:00.0 & 24.3 \\
\hline 2009-10-16 06:00:00.0 & 22.8 \\
\hline 2009-10-16 03:00:00.0 & 27.2 \\
\hline 2009-10-16 00:00:00.0 & 15.0 \\
\hline 2009-10-15 21:00:00.0 & 21.2 \\
\hline 2009-10-15 18:00:00.0 & 20.5 \\
\hline 2009-10-15 15:00:00.0 & 19.0 \\
\hline 2009-10-15 12:00:00.0 & 22.1 \\
\hline 2009-10-15 09:00:00.0 & 24.9 \\
\hline 2009-10-15 06:00:00.0 & 22.0 \\
\hline 2009-10-15 03:00:00.0 & 23.4 \\
\hline 2009-10-15 00:00:00.0 & 23.7 \\
\hline 2009-10-14 21:00:00.0 & 15.2 \\
\hline 2009-10-14 18:00:00.0 & 12.2 \\
\hline 2009-10-14 15:00:00.0 & 14.8 \\
\hline 2009-10-14 12:00:00.0 & 16.0 \\
\hline 2009-10-14 09:00:00.0 & 16.6 \\
\hline 2009-10-14 06:00:00.0 & 15.3 \\
\hline 2009-10-14 03:00:00.0 & 12.4 \\
\hline 2009-10-14 00:00:00.0 & 27.1 \\
\hline 2009-10-13 21:00:00.0 & 2.0 \\
\hline 2009-10-13 18:00:00.0 & 14.0 \\
\hline 2009-10-13 15:00:00.0 & 13.1 \\
\hline 2009-10-13 12:00:00.0 & 9.3 \\
\hline 2009-10-13 09:00:00.0 & 9.2 \\
\hline 2009-10-13 06:00:00.0 & 9.4 \\
\hline 2009-10-13 03:00:00.0 & 7.8 \\
\hline 2009-10-13 00:00:00.0 & 15.8 \\
\hline 2009-10-12 21:00:00.0 & 23.3 \\
\hline 2009-10-12 18:00:00.0 & 22.6 \\
\hline 2009-10-12 15:00:00.0 & 16.2 \\
\hline 2009-10-12 12:00:00.0 & 14.5 \\
\hline 2009-10-12 09:00:00.0 & 23.4 \\
\hline 2009-10-12 06:00:00.0 & 19.7 \\
\hline 2009-10-12 03:00:00.0 & 12.5 \\
\hline 2009-10-12 00:00:00.0 & 17.0 \\
\hline 2009-10-11 21:00:00.0 & 32.4 \\
\hline 2009-10-11 18:00:00.0 & 28.8 \\
\hline 2009-10-11 15:00:00.0 & 34.7 \\
\hline 2009-10-11 12:00:00.0 & 40.3 \\
\hline 2009-10-11 09:00:00.0 & 29.9 \\
\hline 2009-10-11 06:00:00.0 & 28.1 \\
\hline 2009-10-11 03:00:00.0 & 22.2 \\
\hline 2009-10-11 00:00:00.0 & 18.8 \\
\hline 2009-10-10 21:00:00.0 & 26.0 \\
\hline 2009-10-10 18:00:00.0 & 21.0 \\
\hline 2009-10-10 15:00:00.0 & 23.7 \\
\hline 2009-10-10 12:00:00.0 & 25.4 \\
\hline 2009-10-10 09:00:00.0 & 23.7 \\
\hline 2009-10-10 06:00:00.0 & 24.3 \\
\hline 2009-10-10 03:00:00.0 & 22.8 \\
\hline 2009-10-10 00:00:00.0 & 22.1 \\
\hline 2009-10-09 21:00:00.0 & 19.6 \\
\hline 2009-10-09 18:00:00.0 & 16.3 \\
\hline 2009-10-09 15:00:00.0 & 15.8 \\
\hline 2009-10-09 12:00:00.0 & 24.6 \\
\hline 2009-10-09 09:00:00.0 & 18.3 \\
\hline 2009-10-09 06:00:00.0 & 17.3 \\
\hline 2009-10-09 03:00:00.0 & 15.0 \\
\hline 2009-10-09 00:00:00.0 & 19.8 \\
\hline 2009-10-08 21:00:00.0 & 20.2 \\
\hline 2009-10-08 18:00:00.0 & 19.2 \\
\hline 2009-10-08 15:00:00.0 & 22.0 \\
\hline 2009-10-08 12:00:00.0 & 48.4 \\
\hline 2009-10-08 09:00:00.0 & 22.3 \\
\hline 2009-10-08 06:00:00.0 & 17.6 \\
\hline
\end{tabular}

\begin{tabular}{|c|c|}
\hline DataHora & VelVentoMax \\
\hline 2009-10-08 03:00:00.0 & \\
\hline 2009-10-08 00:00:00.0 & 23.4 \\
\hline 2009-10-07 21:00:00.0 & \\
\hline 2009-10-07 18:00:00.0 & 19.9 \\
\hline 2009-10-07 15:00:00.0 & 25.0 \\
\hline 2009-10-07 12:00:00.0 & 24.0 \\
\hline 2009-10-07 09:00:00.0 & 24.5 \\
\hline 2009-10-07 06:00:00.0 & 25.9 \\
\hline 2009-10-07 03:00:00.0 & 24.9 \\
\hline 2009-10-07 00:00:00.0 & 13.6 \\
\hline 2009-10-06 21:00:00.0 & 29.3 \\
\hline 2009-10-06 18:00:00.0 & 27.0 \\
\hline 2009-10-06 15:00:00.0 & 42.6 \\
\hline 2009-10-06 12:00:00.0 & 13.9 \\
\hline 2009-10-06 09:00:00.0 & 15.4 \\
\hline 2009-10-06 06:00:00.0 & 47.2 \\
\hline 2009-10-06 03:00:00.0 & 39.9 \\
\hline 2009-10-06 00:00:00.0 & 33.4 \\
\hline 2009-10-05 21:00:00.0 & 39.8 \\
\hline 2009-10-05 18:00:00.0 & 43.4 \\
\hline 2009-10-05 15:00:00.0 & 39.3 \\
\hline 2009-10-05 12:00:00.0 & 38.4 \\
\hline 2009-10-05 09:00:00.0 & 24.9 \\
\hline 2009-10-05 06:00:00.0 & 21.1 \\
\hline 2009-10-05 03:00:00.0 & 17.8 \\
\hline 2009-10-05 00:00:00.0 & 16.8 \\
\hline 2009-10-04 21:00:00.0 & 18.4 \\
\hline 2009-10-04 18:00:00.0 & 15.2 \\
\hline 2009-10-04 15:00:00.0 & 35.0 \\
\hline 2009-10-04 12:00:00.0 & 22.8 \\
\hline 2009-10-04 09:00:00.0 & 23.3 \\
\hline 2009-10-04 06:00:00.0 & 19.8 \\
\hline 2009-10-04 03:00:00.0 & 18.1 \\
\hline 2009-10-04 00:00:00.0 & 14.4 \\
\hline 2009-10-03 21:00:00.0 & 14.9 \\
\hline 2009-10-03 18:00:00.0 & 16.3 \\
\hline 2009-10-03 15:00:00.0 & 17.3 \\
\hline 2009-10-03 12:00:00.0 & 19.0 \\
\hline 2009-10-03 09:00:00.0 & 13.4 \\
\hline 2009-10-03 06:00:00.0 & 12.8 \\
\hline 2009-10-03 03:00:00.0 & 11.1 \\
\hline 2009-10-03 00:00:00.0 & 10.7 \\
\hline 2009-10-02 21:00:00.0 & 14.4 \\
\hline 2009-10-02 18:00:00.0 & 27.1 \\
\hline 2009-10-02 15:00:00.0 & 11.6 \\
\hline 2009-10-02 12:00:00.0 & 15.9 \\
\hline 2009-10-02 09:00:00.0 & 11.7 \\
\hline 2009-10-02 06:00:00.0 & 15.0 \\
\hline 2009-10-02 03:00:00.0 & 18.1 \\
\hline 2009-10-02 00:00:00.0 & 24.8 \\
\hline 2009-10-01 21:00:00.0 & 28.0 \\
\hline 2009-10-01 18:00:00.0 & 27.4 \\
\hline 2009-10-01 15:00:00.0 & 23.0 \\
\hline 2009-10-01 12:00:00.0 & 21.1 \\
\hline 2009-10-01 09:00:00.0 & 25.5 \\
\hline 2009-10-01 06:00:00.0 & 22.8 \\
\hline 2009-10-01 03:00:00.0 & 24.4 \\
\hline 2009-10-01 00:00:00.0 & 20.9 \\
\hline 2009-09-30 21:00:00.0 & 22.4 \\
\hline 2009-09-30 18:00:00.0 & 28.1 \\
\hline 2009-09-30 15:00:00.0 & 33.5 \\
\hline 2009-09-30 12:00:00.0 & 28.9 \\
\hline 2009-09-30 09:00:00.0 & 11.7 \\
\hline 2009-09-30 06:00:00.0 & 9.9 \\
\hline 2009-09-30 03:00:00.0 & \\
\hline 2009-09-30 00:00:00.0 & 13.6 \\
\hline 2009-09-29 21:00:00.0 & 23.3 \\
\hline 2009-09-29 18:00:00.0 & 26.0 \\
\hline 2009-09-29 15:00:00.0 & \\
\hline
\end{tabular}

\begin{tabular}{|c|c|}
\hline DataHora & VelVentoMax \\
\hline 2009-09-29 12:00:00.0 & 20.3 \\
\hline 2009-09-29 09:00:00.0 & 23.9 \\
\hline 2009-09-29 06:00:00.0 & 17.2 \\
\hline 2009-09-29 03:00:00.0 & 13.6 \\
\hline 2009-09-29 00:00:00.0 & 22.3 \\
\hline 2009-09-28 21:00:00.0 & 21.7 \\
\hline 2009-09-28 18:00:00.0 & 22.7 \\
\hline $2009-09-28$ 15:00:00.0 & 24.0 \\
\hline 2009-09-28 12:00:00.0 & 31.4 \\
\hline 2009-09-28 09:00:00.0 & 26.3 \\
\hline $2009-09-28$ 06:00:00.0 & 28.6 \\
\hline 2009-09-28 03:00:00.0 & 24.0 \\
\hline 2009-09-28 00:00:00.0 & 11.3 \\
\hline 2009-09-27 21:00:00.0 & 22.5 \\
\hline 2009-09-27 18:00:00.0 & 28.8 \\
\hline $2009-09-27$ 15:00:00.0 & 29.9 \\
\hline 2009-09-27 12:00:00.0 & 19.7 \\
\hline 2009-09-27 09:00:00.0 & 21.0 \\
\hline 2009-09-27 06:00:00.0 & 18.6 \\
\hline 2009-09-27 03:00:00.0 & \\
\hline 2009-09-27 00:00:00.0 & 17.4 \\
\hline 2009-09-26 21:00:00.0 & 19.9 \\
\hline $2009-09-26$ 18:00:00.0 & 20.9 \\
\hline 2009-09-26 15:00:00.0 & 19.9 \\
\hline 2009-09-26 12:00:00.0 & 6.0 \\
\hline 2009-09-26 09:00:00.0 & 20.7 \\
\hline 2009-09-26 06:00:00.0 & 22.7 \\
\hline 2009-09-26 03:00:00.0 & 17.5 \\
\hline 2009-09-26 00:00:00.0 & 31.1 \\
\hline 2009-09-25 21:00:00.0 & 18.9 \\
\hline 2009-09-25 18:00:00.0 & 18.6 \\
\hline $2009-09-25$ 15:00:00.0 & 20.7 \\
\hline 2009-09-25 12:00:00.0 & 36.1 \\
\hline 2009-09-25 09:00:00.0 & 19.2 \\
\hline $2009-09-25$ 06:00:00.0 & 15.8 \\
\hline 2009-09-25 03:00:00.0 & 10.5 \\
\hline 2009-09-25 00:00:00.0 & 10.5 \\
\hline 2009-09-24 21:00:00.0 & 16.8 \\
\hline 2009-09-24 18:00:00.0 & 33.4 \\
\hline $2009-09-24$ 15:00:00.0 & 22.5 \\
\hline 2009-09-24 12:00:00.0 & 33.7 \\
\hline 2009-09-24 09:00:00.0 & 12.1 \\
\hline 2009-09-24 06:00:00.0 & 45.1 \\
\hline 2009-09-24 03:00:00.0 & 17.9 \\
\hline 2009-09-24 00:00:00.0 & 23.7 \\
\hline 2009-09-23 21:00:00.0 & 21.6 \\
\hline 2009-09-23 18:00:00.0 & 28.9 \\
\hline 2009-09-23 15:00:00.0 & 28.9 \\
\hline $2009-09-23$ 12:00:00.0 & 33.4 \\
\hline 2009-09-23 09:00:00.0 & 19.7 \\
\hline 2009-09-23 06:00:00.0 & \\
\hline 2009-09-23 03:00:00.0 & 18.5 \\
\hline 2009-09-23 00:00:00.0 & 17.0 \\
\hline $2009-09-22$ 21:00:00.0 & 48.9 \\
\hline 2009-09-22 18:00:00.0 & 16.2 \\
\hline 2009-09-22 15:00:00.0 & 21.4 \\
\hline 2009-09-22 12:00:00.0 & 19.2 \\
\hline 2009-09-22 09:00:00.0 & 18.8 \\
\hline 2009-09-22 06:00:00.0 & 21.3 \\
\hline 2009-09-22 03:00:00.0 & 18.4 \\
\hline 2009-09-22 00:00:00.0 & 11.9 \\
\hline 2009-09-21 21:00:00.0 & 31.8 \\
\hline 2009-09-21 18:00:00.0 & 17.7 \\
\hline $2009-09-21$ 15:00:00.0 & 21.3 \\
\hline 2009-09-21 12:00:00.0 & 3.6 \\
\hline 2009-09-21 09:00:00.0 & 9.2 \\
\hline 2009-09-21 06:00:00.0 & 6.5 \\
\hline 2009-09-21 03:00:00.0 & 6.5 \\
\hline 2009-09-21 00:00:00.0 & 5.2 \\
\hline
\end{tabular}




\section{ANEXO 01 - RAJADAS DE VENTO REGISTRADAS EM SÃO MARTINHO DA SERRA / RS}

\begin{tabular}{|c|c|}
\hline DataHora & VelVentoMax \\
\hline 2009-09-20 21:00:00.0 & 14.3 \\
\hline 2009-09-20 18:00:00.0 & 17.7 \\
\hline 2009-09-20 15:00:00.0 & 18.1 \\
\hline 2009-09-20 12:00:00.0 & 4.0 \\
\hline 2009-09-20 09:00:00.0 & 7.8 \\
\hline 2009-09-20 06:00:00.0 & 9.3 \\
\hline 2009-09-20 03:00:00.0 & 9.7 \\
\hline 2009-09-20 00:00:00.0 & 13.8 \\
\hline 2009-09-19 21:00:00.0 & 24.1 \\
\hline 2009-09-19 18:00:00.0 & 27.5 \\
\hline 2009-09-19 15:00:00.0 & 27.4 \\
\hline 2009-09-19 12:00:00.0 & 25.9 \\
\hline 2009-09-19 09:00:00.0 & 19.2 \\
\hline 2009-09-19 06:00:00.0 & \\
\hline 2009-09-19 03:00:00.0 & 24.3 \\
\hline 2009-09-19 00:00:00.0 & 24.3 \\
\hline 2009-09-18 21:00:00.0 & 22.4 \\
\hline 2009-09-18 18:00:00.0 & 21.4 \\
\hline 2009-09-18 15:00:00.0 & 23.5 \\
\hline 2009-09-18 12:00:00.0 & 27.1 \\
\hline 2009-09-18 09:00:00.0 & 29.7 \\
\hline 2009-09-18 06:00:00.0 & 25.6 \\
\hline 2009-09-18 03:00:00.0 & 24.8 \\
\hline 2009-09-18 00:00:00.0 & 32.4 \\
\hline 2009-09-17 21:00:00.0 & 26.5 \\
\hline 2009-09-17 18:00:00.0 & 22.1 \\
\hline 2009-09-17 15:00:00.0 & 19.8 \\
\hline 2009-09-17 12:00:00.0 & 22.9 \\
\hline 2009-09-17 09:00:00.0 & 18.0 \\
\hline 2009-09-17 06:00:00.0 & 22.3 \\
\hline 2009-09-17 03:00:00.0 & 15.2 \\
\hline 2009-09-17 00:00:00.0 & 23.4 \\
\hline 2009-09-16 21:00:00.0 & 27.1 \\
\hline 2009-09-16 18:00:00.0 & 25.9 \\
\hline 2009-09-16 15:00:00.0 & 25.6 \\
\hline 2009-09-16 12:00:00.0 & 11.0 \\
\hline 2009-09-16 09:00:00.0 & 17.8 \\
\hline 2009-09-16 06:00:00.0 & 16.7 \\
\hline 2009-09-16 03:00:00.0 & 18.0 \\
\hline 2009-09-16 00:00:00.0 & 14.1 \\
\hline 2009-09-15 21:00:00.0 & 11.0 \\
\hline 2009-09-15 18:00:00.0 & 15.3 \\
\hline 2009-09-15 15:00:00.0 & \\
\hline 2009-09-14 06:00:00.0 & 20.3 \\
\hline 2009-09-14 03:00:00.0 & 19.7 \\
\hline 2009-09-14 00:00:00.0 & 16.8 \\
\hline 2009-09-13 21:00:00.0 & 18.4 \\
\hline 2009-09-13 18:00:00.0 & 18.3 \\
\hline 2009-09-13 15:00:00.0 & 6.1 \\
\hline 2009-09-13 12:00:00.0 & 16.5 \\
\hline 2009-09-13 09:00:00.0 & 11.2 \\
\hline 2009-09-13 06:00:00.0 & 14.4 \\
\hline 2009-09-13 03:00:00.0 & 19.1 \\
\hline 2009-09-13 00:00:00.0 & 15.7 \\
\hline 2009-09-12 21:00:00.0 & 16.4 \\
\hline 2009-09-12 18:00:00.0 & 33.9 \\
\hline 2009-09-12 15:00:00.0 & 31.1 \\
\hline 2009-09-12 12:00:00.0 & 30.5 \\
\hline 2009-09-12 09:00:00.0 & 23.5 \\
\hline 2009-09-12 06:00:00.0 & 17.6 \\
\hline 2009-09-12 03:00:00.0 & 22.0 \\
\hline 2009-09-12 00:00:00.0 & 22.3 \\
\hline 2009-09-11 21:00:00.0 & 24.5 \\
\hline 2009-09-11 18:00:00.0 & 28.2 \\
\hline 2009-09-11 15:00:00.0 & 28.9 \\
\hline 2009-09-11 12:00:00.0 & 30.2 \\
\hline 2009-09-11 09:00:00.0 & 29.9 \\
\hline 2009-09-11 06:00:00.0 & 34.7 \\
\hline 2009-09-11 03:00:00.0 & 34.0 \\
\hline
\end{tabular}

\begin{tabular}{|c|c|}
\hline DataHora & VelVentoMax \\
\hline 2009-09-11 00:00:00.0 & 21.6 \\
\hline 2009-09-10 21:00:00.0 & 25.7 \\
\hline 2009-09-10 18:00:00.0 & 24.5 \\
\hline 2009-09-10 15:00:00.0 & 29.5 \\
\hline 2009-09-10 12:00:00.0 & 23.2 \\
\hline 2009-09-10 09:00:00.0 & 19.0 \\
\hline 2009-09-10 06:00:00.0 & 20.9 \\
\hline 2009-09-10 03:00:00.0 & 13.1 \\
\hline 2009-09-10 00:00:00.0 & 8.5 \\
\hline 2009-09-09 21:00:00.0 & 17.3 \\
\hline 2009-09-09 18:00:00.0 & 19.1 \\
\hline 2009-09-09 15:00:00.0 & 22.5 \\
\hline 2009-09-09 12:00:00.0 & 20.6 \\
\hline 2009-09-09 09:00:00.0 & 37.6 \\
\hline 2009-09-09 06:00:00.0 & 16.7 \\
\hline 2009-09-09 03:00:00.0 & 15.3 \\
\hline 2009-09-09 00:00:00.0 & 18.3 \\
\hline 2009-09-08 21:00:00.0 & \\
\hline 2009-09-08 18:00:00.0 & \\
\hline 2009-09-08 15:00:00.0 & \\
\hline 2009-09-08 12:00:00.0 & 23.1 \\
\hline 2009-09-08 09:00:00.0 & 41.8 \\
\hline 2009-09-08 06:00:00.0 & 29.5 \\
\hline 2009-09-08 03:00:00.0 & 18.6 \\
\hline 2009-09-08 00:00:00.0 & 26.5 \\
\hline 2009-09-07 21:00:00.0 & 7.3 \\
\hline 2009-09-07 18:00:00.0 & 28.7 \\
\hline 2009-09-07 15:00:00.0 & 31.1 \\
\hline 2009-09-07 12:00:00.0 & 26.3 \\
\hline 2009-09-07 09:00:00.0 & 20.6 \\
\hline 2009-09-07 06:00:00.0 & 17.9 \\
\hline 2009-09-07 03:00:00.0 & 17.7 \\
\hline 2009-09-07 00:00:00.0 & 12.8 \\
\hline 2009-09-06 21:00:00.0 & 15.4 \\
\hline 2009-09-06 18:00:00.0 & 15.8 \\
\hline 2009-09-06 15:00:00.0 & 18.6 \\
\hline 2009-09-06 12:00:00.0 & 18.0 \\
\hline 2009-09-06 09:00:00.0 & 14.9 \\
\hline 2009-09-06 06:00:00.0 & 18.6 \\
\hline 2009-09-06 03:00:00.0 & \\
\hline 2009-09-06 00:00:00.0 & 11.4 \\
\hline 2009-09-05 21:00:00.0 & 8.3 \\
\hline 2009-09-05 18:00:00.0 & 9.3 \\
\hline 2009-09-05 15:00:00.0 & 12.0 \\
\hline 2009-09-05 12:00:00.0 & 10.8 \\
\hline 2009-09-05 09:00:00.0 & 9.3 \\
\hline 2009-09-05 06:00:00.0 & 7.6 \\
\hline 2009-09-05 03:00:00.0 & 7.1 \\
\hline 2009-09-05 00:00:00.0 & 18.2 \\
\hline 2009-09-04 21:00:00.0 & 18.2 \\
\hline 2009-09-04 18:00:00.0 & 22.5 \\
\hline 2009-09-04 15:00:00.0 & \\
\hline 2009-09-04 12:00:00.0 & 16.1 \\
\hline 2009-09-04 09:00:00.0 & 13.2 \\
\hline 2009-09-04 06:00:00.0 & 15.5 \\
\hline 2009-09-04 03:00:00.0 & 13.1 \\
\hline 2009-09-04 00:00:00.0 & 27.0 \\
\hline 2009-09-03 21:00:00.0 & 34.9 \\
\hline 2009-09-03 18:00:00.0 & 30.2 \\
\hline 2009-09-03 15:00:00.0 & \\
\hline 2009-09-03 12:00:00.0 & 19.0 \\
\hline 2009-09-03 09:00:00.0 & 21.2 \\
\hline 2009-09-03 06:00:00.0 & 30.5 \\
\hline 2009-09-03 03:00:00.0 & \\
\hline 2009-09-03 00:00:00.0 & 24.1 \\
\hline 2009-09-02 21:00:00.0 & 25.9 \\
\hline 2009-09-02 18:00:00.0 & 28.2 \\
\hline 2009-09-02 15:00:00.0 & 23.5 \\
\hline 2009-09-02 12:00:00.0 & 36.5 \\
\hline
\end{tabular}

\begin{tabular}{|c|c|}
\hline DataHora & VelVentoMax \\
\hline 2009-09-02 09:00:00.0 & 31.5 \\
\hline 2009-09-02 06:00:00.0 & 32.4 \\
\hline 2009-09-02 03:00:00.0 & 32.7 \\
\hline 2009-09-02 00:00:00.0 & 41.0 \\
\hline 2009-09-01 21:00:00.0 & 27.3 \\
\hline 2009-09-01 18:00:00.0 & 29.8 \\
\hline 2009-09-01 15:00:00.0 & 25.5 \\
\hline 2009-09-01 12:00:00.0 & 28.9 \\
\hline 2009-09-01 09:00:00.0 & 27.8 \\
\hline 2009-09-01 06:00:00.0 & 16.2 \\
\hline 2009-09-01 03:00:00.0 & 15.6 \\
\hline 2009-09-01 00:00:00.0 & 11.8 \\
\hline 2009-08-31 21:00:00.0 & 34.1 \\
\hline 2009-08-31 18:00:00.0 & 26.5 \\
\hline 2009-08-31 15:00:00.0 & \\
\hline 2009-08-31 12:00:00.0 & 19.3 \\
\hline 2009-08-31 09:00:00.0 & 16.8 \\
\hline 2009-08-31 06:00:00.0 & 16.6 \\
\hline 2009-08-31 03:00:00.0 & 19.3 \\
\hline 2009-08-31 00:00:00.0 & 15.1 \\
\hline 2009-08-30 21:00:00.0 & 18.9 \\
\hline 2009-08-30 18:00:00.0 & 25.7 \\
\hline 2009-08-30 15:00:00.0 & 25.0 \\
\hline 2009-08-30 12:00:00.0 & 21.8 \\
\hline 2009-08-30 09:00:00.0 & 20.3 \\
\hline 2009-08-30 06:00:00.0 & 22.0 \\
\hline 2009-08-30 03:00:00.0 & 17.1 \\
\hline 2009-08-30 00:00:00.0 & 16.0 \\
\hline 2009-08-29 21:00:00.0 & 23.3 \\
\hline 2009-08-29 18:00:00.0 & 30.8 \\
\hline 2009-08-29 15:00:00.0 & 31.9 \\
\hline 2009-08-29 12:00:00.0 & 20.6 \\
\hline 2009-08-29 09:00:00.0 & 21.1 \\
\hline 2009-08-29 06:00:00.0 & 18.7 \\
\hline 2009-08-29 03:00:00.0 & \\
\hline 2009-08-29 00:00:00.0 & 15.0 \\
\hline 2009-08-28 21:00:00.0 & 15.5 \\
\hline $2009-08-28$ 18:00:00.0 & 19.3 \\
\hline 2009-08-28 15:00:00.0 & 16.5 \\
\hline 2009-08-28 12:00:00.0 & 15.8 \\
\hline 2009-08-28 09:00:00.0 & 13.6 \\
\hline 2009-08-28 06:00:00.0 & 8.9 \\
\hline 2009-08-28 03:00:00.0 & 6.7 \\
\hline 2009-08-28 00:00:00.0 & 7.7 \\
\hline 2009-08-27 21:00:00.0 & 14.2 \\
\hline 2009-08-27 18:00:00.0 & 16.8 \\
\hline 2009-08-27 15:00:00.0 & 13.6 \\
\hline 2009-08-27 12:00:00.0 & 13.6 \\
\hline 2009-08-27 09:00:00.0 & 14.1 \\
\hline 2009-08-27 06:00:00.0 & 13.7 \\
\hline 2009-08-27 03:00:00.0 & 12.6 \\
\hline 2009-08-27 00:00:00.0 & 10.5 \\
\hline 2009-08-26 21:00:00.0 & 11.0 \\
\hline 2009-08-26 18:00:00.0 & 17.3 \\
\hline 2009-08-26 15:00:00.0 & 20.4 \\
\hline 2009-08-26 12:00:00.0 & 19.3 \\
\hline 2009-08-26 09:00:00.0 & 19.2 \\
\hline 2009-08-26 06:00:00.0 & 19.2 \\
\hline 2009-08-26 03:00:00.0 & 13.7 \\
\hline 2009-08-26 00:00:00.0 & 10.1 \\
\hline 2009-08-25 21:00:00.0 & 15.6 \\
\hline 2009-08-25 18:00:00.0 & 14.5 \\
\hline 2009-08-25 15:00:00.0 & 21.7 \\
\hline 2009-08-25 12:00:00.0 & 20.8 \\
\hline 2009-08-25 09:00:00.0 & 16.6 \\
\hline 2009-08-25 06:00:00.0 & 16.7 \\
\hline 2009-08-25 03:00:00.0 & 16.0 \\
\hline 2009-08-25 00:00:00.0 & 15.2 \\
\hline 2009-08-24 21:00:00.0 & 19.0 \\
\hline
\end{tabular}




\section{ANEXO 01 - RAJADAS DE VENTO REGISTRADAS EM SÃO MARTINHO DA SERRA / RS}

\begin{tabular}{|c|c|}
\hline DataHora & VelVentoMax \\
\hline 2009-08-24 18:00:00.0 & 19.4 \\
\hline 2009-08-24 15:00:00.0 & 19.1 \\
\hline 2009-08-24 12:00:00.0 & 11.9 \\
\hline 2009-08-24 09:00:00.0 & 13.2 \\
\hline 2009-08-24 06:00:00.0 & 11.9 \\
\hline 2009-08-24 03:00:00.0 & 12.9 \\
\hline 2009-08-24 00:00:00.0 & 9.3 \\
\hline 2009-08-23 21:00:00.0 & 27.1 \\
\hline 2009-08-23 18:00:00.0 & 14.4 \\
\hline 2009-08-23 15:00:00.0 & 22.1 \\
\hline 2009-08-23 12:00:00.0 & 22.0 \\
\hline 2009-08-23 09:00:00.0 & 22.5 \\
\hline 2009-08-23 06:00:00.0 & 23.4 \\
\hline 2009-08-23 03:00:00.0 & 15.9 \\
\hline 2009-08-23 00:00:00.0 & 11.1 \\
\hline 2009-08-22 21:00:00.0 & 17.2 \\
\hline 2009-08-22 18:00:00.0 & 20.1 \\
\hline 2009-08-22 15:00:00.0 & 24.2 \\
\hline 2009-08-22 12:00:00.0 & 22.5 \\
\hline 2009-08-22 09:00:00.0 & 20.0 \\
\hline 2009-08-22 06:00:00.0 & 14.8 \\
\hline 2009-08-22 03:00:00.0 & 13.8 \\
\hline 2009-08-22 00:00:00.0 & 12.7 \\
\hline 2009-08-21 21:00:00.0 & 13.3 \\
\hline 2009-08-21 18:00:00.0 & \\
\hline 2009-08-21 15:00:00.0 & 20.8 \\
\hline 2009-08-21 12:00:00.0 & 18.9 \\
\hline 2009-08-21 09:00:00.0 & 18.3 \\
\hline 2009-08-21 06:00:00.0 & 10.0 \\
\hline 2009-08-21 03:00:00.0 & 19.0 \\
\hline 2009-08-21 00:00:00.0 & 22.7 \\
\hline 2009-08-20 21:00:00.0 & 22.9 \\
\hline 2009-08-20 18:00:00.0 & 23.5 \\
\hline 2009-08-20 15:00:00.0 & 21.2 \\
\hline 2009-08-20 12:00:00.0 & 19.4 \\
\hline 2009-08-20 09:00:00.0 & 20.6 \\
\hline 2009-08-20 06:00:00.0 & 13.2 \\
\hline 2009-08-20 03:00:00.0 & 12.6 \\
\hline 2009-08-20 00:00:00.0 & 11.4 \\
\hline 2009-08-19 21:00:00.0 & 31.5 \\
\hline 2009-08-19 18:00:00.0 & 20.9 \\
\hline 2009-08-19 15:00:00.0 & 23.7 \\
\hline 2009-08-19 12:00:00.0 & 19.8 \\
\hline 2009-08-19 09:00:00.0 & 14.8 \\
\hline 2009-08-19 06:00:00.0 & 38.1 \\
\hline 2009-08-19 03:00:00.0 & 44.7 \\
\hline 2009-08-19 00:00:00.0 & 32.2 \\
\hline 2009-08-18 21:00:00.0 & 25.4 \\
\hline 2009-08-18 18:00:00.0 & 26.8 \\
\hline 2009-08-18 15:00:00.0 & 29.6 \\
\hline 2009-08-18 12:00:00.0 & 28.9 \\
\hline 2009-08-18 09:00:00.0 & \\
\hline 2009-08-18 06:00:00.0 & 27.9 \\
\hline 2009-08-18 03:00:00.0 & 24.4 \\
\hline 2009-08-18 00:00:00.0 & 22.9 \\
\hline 2009-08-17 21:00:00.0 & 27.7 \\
\hline 2009-08-17 18:00:00.0 & 20.5 \\
\hline 2009-08-17 15:00:00.0 & 23.0 \\
\hline 2009-08-17 12:00:00.0 & 19.4 \\
\hline 2009-08-17 09:00:00.0 & 30.6 \\
\hline 2009-08-17 06:00:00.0 & 18.1 \\
\hline 2009-08-17 03:00:00.0 & 20.2 \\
\hline 2009-08-17 00:00:00.0 & 24.8 \\
\hline 2009-08-16 21:00:00.0 & 32.6 \\
\hline 2009-08-16 18:00:00.0 & 37.7 \\
\hline 2009-08-16 15:00:00.0 & 35.4 \\
\hline 2009-08-16 12:00:00.0 & 29.7 \\
\hline 2009-08-16 09:00:00.0 & 28.4 \\
\hline 2009-08-16 06:00:00.0 & 25.7 \\
\hline
\end{tabular}

\begin{tabular}{|c|c|}
\hline DataHora & VelVentoMax \\
\hline 2009-08-16 03:00:00.0 & 18.9 \\
\hline 2009-08-16 00:00:00.0 & 20.4 \\
\hline 2009-08-15 21:00:00.0 & 27.7 \\
\hline 2009-08-15 18:00:00.0 & 30.8 \\
\hline 2009-08-15 15:00:00.0 & 31.6 \\
\hline 2009-08-15 12:00:00.0 & 23.7 \\
\hline 2009-08-15 09:00:00.0 & 22.9 \\
\hline 2009-08-15 06:00:00.0 & 19.2 \\
\hline 2009-08-15 03:00:00.0 & 20.1 \\
\hline 2009-08-15 00:00:00.0 & 16.6 \\
\hline 2009-08-14 21:00:00.0 & 19.7 \\
\hline 2009-08-14 18:00:00.0 & 18.0 \\
\hline 2009-08-14 15:00:00.0 & 21.2 \\
\hline 2009-08-14 12:00:00.0 & 18.1 \\
\hline 2009-08-14 09:00:00.0 & 17.6 \\
\hline 2009-08-14 06:00:00.0 & 14.9 \\
\hline 2009-08-14 03:00:00.0 & 11.0 \\
\hline 2009-08-14 00:00:00.0 & 5.9 \\
\hline 2009-08-13 21:00:00.0 & 17.4 \\
\hline 2009-08-13 18:00:00.0 & 19.1 \\
\hline 2009-08-13 15:00:00.0 & 26.8 \\
\hline 2009-08-13 12:00:00.0 & 23.0 \\
\hline 2009-08-13 09:00:00.0 & 25.1 \\
\hline 2009-08-13 06:00:00.0 & 21.7 \\
\hline 2009-08-13 03:00:00.0 & 23.2 \\
\hline 2009-08-13 00:00:00.0 & 18.3 \\
\hline 2009-08-12 21:00:00.0 & 14.6 \\
\hline 2009-08-12 18:00:00.0 & 17.0 \\
\hline 2009-08-12 15:00:00.0 & 18.8 \\
\hline 2009-08-12 12:00:00.0 & 19.7 \\
\hline 2009-08-12 09:00:00.0 & 17.0 \\
\hline 2009-08-12 06:00:00.0 & 17.4 \\
\hline 2009-08-12 03:00:00.0 & 16.6 \\
\hline 2009-08-12 00:00:00.0 & 14.1 \\
\hline 2009-08-11 21:00:00.0 & 12.2 \\
\hline 2009-08-11 18:00:00.0 & 15.0 \\
\hline 2009-08-11 15:00:00.0 & \\
\hline 2009-08-11 12:00:00.0 & 12.3 \\
\hline 2009-08-11 06:00:00.0 & 7.1 \\
\hline 2009-08-11 03:00:00.0 & 6.6 \\
\hline 2009-08-11 00:00:00.0 & 8.2 \\
\hline 2009-08-10 12:00:00.0 & 20.2 \\
\hline 2009-08-10 09:00:00.0 & 30.6 \\
\hline 2009-08-10 06:00:00.0 & 44.5 \\
\hline 2009-08-10 03:00:00.0 & \\
\hline 2009-08-10 00:00:00.0 & 27.6 \\
\hline 2009-08-09 21:00:00.0 & 31.8 \\
\hline 2009-08-09 18:00:00.0 & 40.9 \\
\hline 2009-08-09 15:00:00.0 & 31.4 \\
\hline 2009-08-09 12:00:00.0 & 27.6 \\
\hline 2009-08-09 09:00:00.0 & 27.3 \\
\hline 2009-08-09 06:00:00.0 & 26.3 \\
\hline 2009-08-09 03:00:00.0 & 26.0 \\
\hline 2009-08-09 00:00:00.0 & 35.7 \\
\hline 2009-08-08 21:00:00.0 & 36.0 \\
\hline 2009-08-08 18:00:00.0 & 20.4 \\
\hline 2009-08-08 15:00:00.0 & 14.8 \\
\hline 2009-08-08 12:00:00.0 & 16.4 \\
\hline 2009-08-08 06:00:00.0 & 14.7 \\
\hline 2009-08-08 03:00:00.0 & 15.3 \\
\hline 2009-08-08 00:00:00.0 & 14.5 \\
\hline 2009-08-07 21:00:00.0 & 19.3 \\
\hline 2009-08-07 18:00:00.0 & 21.2 \\
\hline 2009-08-07 15:00:00.0 & 27.8 \\
\hline 2009-08-07 12:00:00.0 & 24.2 \\
\hline 2009-08-07 09:00:00.0 & 21.5 \\
\hline 2009-08-07 06:00:00.0 & 14.4 \\
\hline 2009-08-07 03:00:00.0 & 10.2 \\
\hline 2009-08-07 00:00:00.0 & 10.2 \\
\hline
\end{tabular}

\begin{tabular}{|c|c|}
\hline DataHora & VelVentoMax \\
\hline 2009-08-06 21:00:00.0 & 16.3 \\
\hline 2009-08-06 18:00:00.0 & 19.8 \\
\hline 2009-08-06 15:00:00.0 & 24.9 \\
\hline 2009-08-06 12:00:00.0 & 21.6 \\
\hline 2009-08-06 09:00:00.0 & 25.2 \\
\hline 2009-08-06 06:00:00.0 & 23.3 \\
\hline 2009-08-06 03:00:00.0 & 16.7 \\
\hline 2009-08-06 00:00:00.0 & 15.8 \\
\hline 2009-08-05 21:00:00.0 & 30.0 \\
\hline $2009-08-05$ 18:00:00.0 & \\
\hline $2009-08-05$ 15:00:00.0 & 25.9 \\
\hline 2009-08-05 12:00:00.0 & 22.2 \\
\hline 2009-08-05 09:00:00.0 & 20.4 \\
\hline 2009-08-05 06:00:00.0 & 20.4 \\
\hline 2009-08-05 03:00:00.0 & \\
\hline 2009-08-05 00:00:00.0 & 20.2 \\
\hline 2009-08-04 21:00:00.0 & 17.9 \\
\hline 2009-08-04 18:00:00.0 & 18.5 \\
\hline 2009-08-04 15:00:00.0 & 24.0 \\
\hline 2009-08-04 06:00:00.0 & 23.2 \\
\hline 2009-08-04 03:00:00.0 & 15.3 \\
\hline 2009-08-04 00:00:00.0 & 18.4 \\
\hline 2009-08-03 21:00:00.0 & 11.7 \\
\hline 2009-08-03 18:00:00.0 & 11.2 \\
\hline $2009-08-03$ 15:00:00.0 & 13.8 \\
\hline 2009-08-03 12:00:00.0 & 11.7 \\
\hline 2009-08-03 09:00:00.0 & 17.1 \\
\hline 2009-08-03 06:00:00.0 & 21.9 \\
\hline 2009-08-03 03:00:00.0 & 23.4 \\
\hline 2009-08-03 00:00:00.0 & 26.4 \\
\hline 2009-08-02 21:00:00.0 & 30.3 \\
\hline 2009-08-02 18:00:00.0 & 29.8 \\
\hline 2009-08-02 12:00:00.0 & 11.4 \\
\hline 2009-08-02 09:00:00.0 & 16.4 \\
\hline 2009-08-02 06:00:00.0 & 19.0 \\
\hline 2009-08-02 03:00:00.0 & 18.8 \\
\hline 2009-08-02 00:00:00.0 & 18.1 \\
\hline 2009-08-01 21:00:00.0 & 21.0 \\
\hline 2009-08-01 18:00:00.0 & 15.1 \\
\hline 2009-08-01 15:00:00.0 & 16.8 \\
\hline 2009-08-01 09:00:00.0 & 18.0 \\
\hline 2009-08-01 03:00:00.0 & 18.6 \\
\hline 2009-07-31 21:00:00.0 & 23.3 \\
\hline 2009-07-31 18:00:00.0 & \\
\hline 2009-07-31 15:00:00.0 & 25.1 \\
\hline 2009-07-31 12:00:00.0 & 21.6 \\
\hline 2009-07-31 09:00:00.0 & 18.7 \\
\hline 2009-07-31 06:00:00.0 & \\
\hline 2009-07-31 03:00:00.0 & 15.4 \\
\hline 2009-07-31 00:00:00.0 & 15.8 \\
\hline 2009-07-30 21:00:00.0 & 21.3 \\
\hline 2009-07-30 18:00:00.0 & 18.5 \\
\hline 2009-07-30 15:00:00.0 & 18.3 \\
\hline $2009-07-3012: 00: 00.0$ & 11.9 \\
\hline 2009-07-30 09:00:00.0 & \\
\hline 2009-07-30 06:00:00.0 & 9.1 \\
\hline 2009-07-30 03:00:00.0 & 8.0 \\
\hline 2009-07-30 00:00:00.0 & 7.7 \\
\hline 2009-07-29 21:00:00.0 & 16.1 \\
\hline 2009-07-29 18:00:00.0 & 17.6 \\
\hline 2009-07-29 15:00:00.0 & 27.1 \\
\hline $2009-07-2912: 00: 00.0$ & 4.6 \\
\hline 2009-07-29 09:00:00.0 & 10.4 \\
\hline 2009-07-29 06:00:00.0 & 9.2 \\
\hline 2009-07-29 03:00:00.0 & 11.8 \\
\hline 2009-07-29 00:00:00.0 & 14.8 \\
\hline 2009-07-28 21:00:00.0 & 20.5 \\
\hline $2009-07-28$ 18:00:00.0 & 15.9 \\
\hline 2009-07-28 15:00:00.0 & 19.3 \\
\hline
\end{tabular}




\section{ANEXO 01 - RAJADAS DE VENTO REGISTRADAS EM SÃO MARTINHO DA SERRA / RS}

\begin{tabular}{|c|c|}
\hline DataHora & VelVentoMax \\
\hline 2009-07-28 12:00:00.0 & 15.6 \\
\hline 2009-07-28 09:00:00.0 & \\
\hline 2009-07-28 06:00:00.0 & 16.9 \\
\hline 2009-07-28 03:00:00.0 & 11.1 \\
\hline 2009-07-28 00:00:00.0 & 10.5 \\
\hline 2009-07-27 21:00:00.0 & 12.9 \\
\hline 2009-07-27 18:00:00.0 & 13.1 \\
\hline 2009-07-27 15:00:00.0 & 13.2 \\
\hline 2009-07-27 12:00:00.0 & 8.8 \\
\hline 2009-07-27 09:00:00.0 & \\
\hline 2009-07-27 06:00:00.0 & 6.5 \\
\hline 2009-07-27 03:00:00.0 & 9.6 \\
\hline 2009-07-27 00:00:00.0 & 6.7 \\
\hline 2009-07-26 21:00:00.0 & 13.3 \\
\hline 2009-07-26 18:00:00.0 & 16.9 \\
\hline 2009-07-26 15:00:00.0 & 27.5 \\
\hline 2009-07-26 12:00:00.0 & 3.8 \\
\hline 2009-07-26 09:00:00.0 & 5.5 \\
\hline 2009-07-26 06:00:00.0 & 8.4 \\
\hline 2009-07-26 03:00:00.0 & 9.6 \\
\hline 2009-07-26 00:00:00.0 & 8.0 \\
\hline 2009-07-25 21:00:00.0 & 12.2 \\
\hline 2009-07-25 18:00:00.0 & 12.5 \\
\hline 2009-07-25 15:00:00.0 & 9.1 \\
\hline 2009-07-25 12:00:00.0 & 7.1 \\
\hline 2009-07-25 09:00:00.0 & 5.2 \\
\hline 2009-07-25 06:00:00.0 & 5.8 \\
\hline 2009-07-25 03:00:00.0 & 5.9 \\
\hline 2009-07-25 00:00:00.0 & 7.8 \\
\hline 2009-07-24 21:00:00.0 & 15.6 \\
\hline 2009-07-24 18:00:00.0 & 20.2 \\
\hline 2009-07-24 15:00:00.0 & 21.1 \\
\hline 2009-07-24 12:00:00.0 & 10.4 \\
\hline 2009-07-24 09:00:00.0 & \\
\hline 2009-07-24 06:00:00.0 & 11.1 \\
\hline 2009-07-24 03:00:00.0 & 12.1 \\
\hline 2009-07-24 00:00:00.0 & 13.4 \\
\hline 2009-07-23 21:00:00.0 & 21.3 \\
\hline 2009-07-23 18:00:00.0 & 21.3 \\
\hline 2009-07-23 15:00:00.0 & 18.2 \\
\hline 2009-07-23 12:00:00.0 & 48.4 \\
\hline 2009-07-23 09:00:00.0 & 21.2 \\
\hline 2009-07-23 06:00:00.0 & 16.2 \\
\hline 2009-07-23 03:00:00.0 & 15.1 \\
\hline 2009-07-22 21:00:00.0 & 20.8 \\
\hline 2009-07-22 18:00:00.0 & 21.8 \\
\hline 2009-07-22 15:00:00.0 & 20.3 \\
\hline 2009-07-22 12:00:00.0 & 18.9 \\
\hline 2009-07-22 09:00:00.0 & 23.8 \\
\hline 2009-07-22 06:00:00.0 & 26.1 \\
\hline 2009-07-22 03:00:00.0 & 41.1 \\
\hline 2009-07-22 00:00:00.0 & 41.5 \\
\hline 2009-07-21 21:00:00.0 & 40.0 \\
\hline 2009-07-21 18:00:00.0 & 34.9 \\
\hline 2009-07-21 15:00:00.0 & 34.9 \\
\hline 2009-07-21 12:00:00.0 & 27.8 \\
\hline 2009-07-21 09:00:00.0 & 28.0 \\
\hline 2009-07-21 06:00:00.0 & 23.5 \\
\hline 2009-07-21 03:00:00.0 & 23.4 \\
\hline 2009-07-21 00:00:00.0 & 20.3 \\
\hline 2009-07-20 21:00:00.0 & 16.2 \\
\hline 2009-07-20 18:00:00.0 & 20.8 \\
\hline 2009-07-20 15:00:00.0 & 28.2 \\
\hline 2009-07-20 12:00:00.0 & 20.1 \\
\hline 2009-07-20 09:00:00.0 & 22.8 \\
\hline 2009-07-20 06:00:00.0 & 19.0 \\
\hline 2009-07-20 00:00:00.0 & 17.5 \\
\hline 2009-07-19 21:00:00.0 & 21.0 \\
\hline 2009-07-19 18:00:00.0 & 24.1 \\
\hline
\end{tabular}

\begin{tabular}{|c|c|}
\hline DataHora & VelVentoMax \\
\hline 2009-07-19 15:00:00.0 & 28.2 \\
\hline 2009-07-19 12:00:00.0 & 16.3 \\
\hline 2009-07-19 09:00:00.0 & 15.2 \\
\hline 2009-07-19 06:00:00.0 & 10.0 \\
\hline 2009-07-19 03:00:00.0 & \\
\hline 2009-07-19 00:00:00.0 & \\
\hline 2009-07-18 21:00:00.0 & 12.0 \\
\hline 2009-07-18 18:00:00.0 & 9.8 \\
\hline 2009-07-18 15:00:00.0 & 9.0 \\
\hline 2009-07-18 12:00:00.0 & 10.7 \\
\hline 2009-07-18 09:00:00.0 & 14.5 \\
\hline 2009-07-18 06:00:00.0 & 9.0 \\
\hline 2009-07-18 03:00:00.0 & 15.1 \\
\hline 2009-07-18 00:00:00.0 & 15.2 \\
\hline 2009-07-17 21:00:00.0 & 12.7 \\
\hline 2009-07-17 18:00:00.0 & 27.1 \\
\hline 2009-07-17 15:00:00.0 & 14.1 \\
\hline 2009-07-17 12:00:00.0 & 10.2 \\
\hline 2009-07-17 09:00:00.0 & 7.2 \\
\hline 2009-07-17 06:00:00.0 & 7.7 \\
\hline 2009-07-17 03:00:00.0 & 9.0 \\
\hline 2009-07-16 21:00:00.0 & 9.3 \\
\hline 2009-07-16 18:00:00.0 & 30.2 \\
\hline 2009-07-16 15:00:00.0 & 28.0 \\
\hline 2009-07-16 12:00:00.0 & 7.4 \\
\hline 2009-07-16 09:00:00.0 & 10.5 \\
\hline 2009-07-16 06:00:00.0 & 13.1 \\
\hline 2009-07-16 03:00:00.0 & 11.1 \\
\hline 2009-07-15 21:00:00.0 & 8.3 \\
\hline 2009-07-15 18:00:00.0 & 19.0 \\
\hline 2009-07-15 15:00:00.0 & \\
\hline 2009-07-15 12:00:00.0 & \\
\hline 2009-07-15 09:00:00.0 & 17.7 \\
\hline 2009-07-15 06:00:00.0 & 18.5 \\
\hline 2009-07-15 00:00:00.0 & 11.9 \\
\hline 2009-07-14 21:00:00.0 & 13.7 \\
\hline 2009-07-14 18:00:00.0 & 18.3 \\
\hline 2009-07-14 15:00:00.0 & \\
\hline 2009-07-14 12:00:00.0 & \\
\hline 2009-07-14 09:00:00.0 & 10.2 \\
\hline 2009-07-14 06:00:00.0 & 10.9 \\
\hline 2009-07-14 03:00:00.0 & 13.3 \\
\hline 2009-07-14 00:00:00.0 & 12.1 \\
\hline 2009-07-13 21:00:00.0 & 17.7 \\
\hline 2009-07-13 18:00:00.0 & 24.8 \\
\hline 2009-07-13 15:00:00.0 & \\
\hline 2009-07-13 12:00:00.0 & 16.4 \\
\hline 2009-07-13 09:00:00.0 & 17.5 \\
\hline 2009-07-13 06:00:00.0 & \\
\hline 2009-07-13 03:00:00.0 & \\
\hline 2009-07-13 00:00:00.0 & 12.8 \\
\hline 2009-07-12 21:00:00.0 & 15.8 \\
\hline 2009-07-12 18:00:00.0 & 16.0 \\
\hline 2009-07-12 15:00:00.0 & \\
\hline 2009-07-12 12:00:00.0 & 14.7 \\
\hline 2009-07-12 09:00:00.0 & 8.2 \\
\hline 2009-07-12 06:00:00.0 & 4.3 \\
\hline 2009-07-12 03:00:00.0 & 10.5 \\
\hline 2009-07-12 00:00:00.0 & \\
\hline 2009-07-11 21:00:00.0 & 23.5 \\
\hline 2009-07-11 18:00:00.0 & 5.6 \\
\hline 2009-07-11 15:00:00.0 & 20.7 \\
\hline 2009-07-11 12:00:00.0 & 21.5 \\
\hline 2009-07-11 09:00:00.0 & 17.4 \\
\hline 2009-07-11 06:00:00.0 & 17.4 \\
\hline 2009-07-11 03:00:00.0 & 14.9 \\
\hline 2009-07-11 00:00:00.0 & 15.3 \\
\hline 2009-07-10 21:00:00.0 & 15.2 \\
\hline 2009-07-10 18:00:00.0 & 3.1 \\
\hline
\end{tabular}

\begin{tabular}{|c|c|}
\hline \multirow{2}{*}{$\begin{array}{l}\text { DataHora } \\
2009-07-10 \text { 15:00:00.0 }\end{array}$} & VelVentoMax \\
\hline & \\
\hline 2009-07-10 12:00:00.0 & 12.5 \\
\hline 2009-07-10 09:00:00.0 & 11.1 \\
\hline 2009-07-10 06:00:00.0 & 10.2 \\
\hline 2009-07-10 03:00:00.0 & 11.0 \\
\hline 2009-07-10 00:00:00.0 & 12.2 \\
\hline 2009-07-09 21:00:00.0 & 14.6 \\
\hline 2009-07-09 18:00:00.0 & 21.5 \\
\hline 2009-07-09 15:00:00.0 & \\
\hline 2009-07-09 12:00:00.0 & 19.9 \\
\hline 2009-07-09 09:00:00.0 & 20.5 \\
\hline 2009-07-09 06:00:00.0 & 6.9 \\
\hline 2009-07-09 03:00:00.0 & 18.6 \\
\hline 2009-07-09 00:00:00.0 & \\
\hline 2009-07-08 21:00:00.0 & 18.5 \\
\hline 2009-07-08 18:00:00.0 & 14.4 \\
\hline 2009-07-08 15:00:00.0 & 12.0 \\
\hline 2009-07-08 12:00:00.0 & 48.8 \\
\hline 2009-07-08 09:00:00.0 & 32.3 \\
\hline 2009-07-08 06:00:00.0 & 15.3 \\
\hline 2009-07-08 03:00:00.0 & 14.2 \\
\hline 2009-07-08 00:00:00.0 & 15.7 \\
\hline 2009-07-07 21:00:00.0 & 16.8 \\
\hline 2009-07-07 18:00:00.0 & 17.8 \\
\hline 2009-07-07 15:00:00.0 & 18.9 \\
\hline 2009-07-07 12:00:00.0 & 18.7 \\
\hline 2009-07-07 09:00:00.0 & 23.1 \\
\hline 2009-07-07 06:00:00.0 & 17.4 \\
\hline 2009-07-07 03:00:00.0 & 34.2 \\
\hline 2009-07-07 00:00:00.0 & 23.8 \\
\hline 2009-07-06 21:00:00.0 & 19.5 \\
\hline 2009-07-06 18:00:00.0 & 20.1 \\
\hline 2009-07-06 15:00:00.0 & 19.1 \\
\hline 2009-07-06 12:00:00.0 & 20.6 \\
\hline 2009-07-06 09:00:00.0 & 20.5 \\
\hline 2009-07-06 06:00:00.0 & 19.0 \\
\hline 2009-07-06 03:00:00.0 & 20.0 \\
\hline 2009-07-06 00:00:00.0 & 17.9 \\
\hline 2009-07-05 21:00:00.0 & 18.1 \\
\hline 2009-07-05 18:00:00.0 & 19.3 \\
\hline 2009-07-05 15:00:00.0 & 21.6 \\
\hline 2009-07-05 12:00:00.0 & 22.2 \\
\hline 2009-07-05 09:00:00.0 & 23.7 \\
\hline 2009-07-05 06:00:00.0 & 25.4 \\
\hline 2009-07-05 03:00:00.0 & 24.6 \\
\hline 2009-07-05 00:00:00.0 & 21.1 \\
\hline 2009-07-04 21:00:00.0 & 20.2 \\
\hline 2009-07-04 18:00:00.0 & 23.3 \\
\hline 2009-07-04 15:00:00.0 & 21.1 \\
\hline 2009-07-04 12:00:00.0 & 19.2 \\
\hline 2009-07-04 09:00:00.0 & 19.0 \\
\hline 2009-07-04 06:00:00.0 & 17.8 \\
\hline 2009-07-04 03:00:00.0 & 12.5 \\
\hline 2009-07-04 00:00:00.0 & 8.9 \\
\hline 2009-07-03 21:00:00.0 & 29.8 \\
\hline 2009-07-03 18:00:00.0 & 11.8 \\
\hline 2009-07-03 15:00:00.0 & 7.3 \\
\hline 2009-07-03 12:00:00.0 & 30.3 \\
\hline 2009-07-03 09:00:00.0 & 6.4 \\
\hline 2009-07-03 06:00:00.0 & 3.7 \\
\hline 2009-07-03 03:00:00.0 & 3.3 \\
\hline 2009-07-03 00:00:00.0 & 5.7 \\
\hline 2009-07-02 21:00:00.0 & \\
\hline 2009-07-02 18:00:00.0 & 19.9 \\
\hline 2009-07-02 15:00:00.0 & 18.4 \\
\hline 2009-07-02 12:00:00.0 & 3.6 \\
\hline 2009-07-02 09:00:00.0 & \\
\hline 2009-07-02 06:00:00.0 & 6.8 \\
\hline 2009-07-02 03:00:00.0 & 5.6 \\
\hline
\end{tabular}




\section{ANEXO 01 - RAJADAS DE VENTO REGISTRADAS EM SÃO MARTINHO DA SERRA / RS}

\begin{tabular}{|c|c|}
\hline DataHora & VelVentoMax \\
\hline 2009-07-02 00:00:00.0 & 8.6 \\
\hline 2009-07-01 21:00:00.0 & 10.6 \\
\hline 2009-07-01 18:00:00.0 & 29.9 \\
\hline 2009-07-01 15:00:00.0 & 28.1 \\
\hline 2009-07-01 12:00:00.0 & 8.0 \\
\hline 2009-07-01 09:00:00.0 & 8.6 \\
\hline 2009-07-01 06:00:00.0 & 7.9 \\
\hline 2009-07-01 03:00:00.0 & 8.9 \\
\hline 2009-07-01 00:00:00.0 & 12.0 \\
\hline 2009-06-30 21:00:00.0 & 25.5 \\
\hline 2009-06-30 18:00:00.0 & 27.4 \\
\hline 2009-06-30 12:00:00.0 & 18.9 \\
\hline 2009-06-30 09:00:00.0 & 20.8 \\
\hline 2009-06-30 06:00:00.0 & 16.0 \\
\hline 2009-06-30 03:00:00.0 & 27.7 \\
\hline 2009-06-30 00:00:00.0 & 28.7 \\
\hline 2009-06-29 21:00:00.0 & 3.8 \\
\hline 2009-06-29 18:00:00.0 & 49.2 \\
\hline 2009-06-29 15:00:00.0 & 44.2 \\
\hline 2009-06-29 12:00:00.0 & 33.9 \\
\hline 2009-06-29 09:00:00.0 & 27.1 \\
\hline 2009-06-29 06:00:00.0 & 26.4 \\
\hline 2009-06-29 03:00:00.0 & 23.4 \\
\hline 2009-06-28 21:00:00.0 & 20.7 \\
\hline 2009-06-28 18:00:00.0 & 23.4 \\
\hline 2009-06-28 12:00:00.0 & 22.3 \\
\hline 2009-06-28 09:00:00.0 & 24.1 \\
\hline 2009-06-28 06:00:00.0 & 23.7 \\
\hline 2009-06-28 03:00:00.0 & 21.5 \\
\hline \multicolumn{2}{|l|}{ 2009-06-28 00:00:00.0 } \\
\hline 2009-06-27 21:00:00.0 & 15.0 \\
\hline 2009-06-27 18:00:00.0 & 15.7 \\
\hline 2009-06-27 15:00:00.0 & 21.5 \\
\hline 2009-06-27 12:00:00.0 & 20.8 \\
\hline 2009-06-27 09:00:00.0 & 17.0 \\
\hline 2009-06-27 03:00:00.0 & 18.7 \\
\hline 2009-06-27 00:00:00.0 & 16.3 \\
\hline 2009-06-26 21:00:00.0 & 16.4 \\
\hline 2009-06-26 15:00:00.0 & 21.7 \\
\hline 2009-06-26 12:00:00.0 & 16.8 \\
\hline 2009-06-26 09:00:00.0 & 15.3 \\
\hline 2009-06-26 06:00:00.0 & 12.7 \\
\hline 2009-06-26 03:00:00.0 & 11.4 \\
\hline \multicolumn{2}{|l|}{ 2009-06-26 00:00:00.0 } \\
\hline 2009-06-25 21:00:00.0 & 15.8 \\
\hline 2009-06-25 12:00:00.0 & 13.0 \\
\hline 2009-06-25 09:00:00.0 & 9.6 \\
\hline 2009-06-25 06:00:00.0 & 11.1 \\
\hline 2009-06-25 03:00:00.0 & 11.2 \\
\hline 2009-06-25 00:00:00.0 & 11.5 \\
\hline \multicolumn{2}{|l|}{ 2009-06-24 21:00:00.0 } \\
\hline 2009-06-24 18:00:00.0 & 18.8 \\
\hline 2009-06-24 15:00:00.0 & 18.7 \\
\hline 2009-06-24 12:00:00.0 & 15.3 \\
\hline 2009-06-24 09:00:00.0 & 14.3 \\
\hline 2009-06-24 06:00:00.0 & 19.3 \\
\hline 2009-06-24 03:00:00.0 & 21.0 \\
\hline \multicolumn{2}{|l|}{ 2009-06-24 00:00:00.0 } \\
\hline 2009-06-23 21:00:00.0 & 28.0 \\
\hline 2009-06-23 18:00:00.0 & 17.9 \\
\hline 2009-06-23 15:00:00.0 & 14.3 \\
\hline 2009-06-23 12:00:00.0 & 19.6 \\
\hline 2009-06-23 09:00:00.0 & 19.2 \\
\hline 2009-06-23 06:00:00.0 & 20.0 \\
\hline 2009-06-23 03:00:00.0 & 16.4 \\
\hline 2009-06-23 00:00:00.0 & 17.1 \\
\hline 2009-06-22 18:00:00.0 & 33.8 \\
\hline 2009-06-22 15:00:00.0 & \\
\hline 2009-06-22 12:00:00.0 & 16.9 \\
\hline
\end{tabular}

\begin{tabular}{|c|c|}
\hline DataHora & VelVentoMax \\
\hline 2009-06-22 09:00:00.0 & 10.8 \\
\hline 2009-06-22 06:00:00.0 & 7.5 \\
\hline 2009-06-21 21:00:00.0 & 13.6 \\
\hline 2009-06-21 18:00:00.0 & 21.7 \\
\hline 2009-06-21 15:00:00.0 & 25.4 \\
\hline 2009-06-21 12:00:00.0 & 21.9 \\
\hline 2009-06-21 09:00:00.0 & 18.6 \\
\hline 2009-06-21 06:00:00.0 & 17.2 \\
\hline 2009-06-21 03:00:00.0 & 20.6 \\
\hline 2009-06-21 00:00:00.0 & 19.2 \\
\hline 2009-06-20 18:00:00.0 & 20.8 \\
\hline 2009-06-20 15:00:00.0 & 23.1 \\
\hline 2009-06-20 12:00:00.0 & 20.0 \\
\hline 2009-06-20 09:00:00.0 & 18.2 \\
\hline 2009-06-20 06:00:00.0 & 19.9 \\
\hline 2009-06-20 03:00:00.0 & 18.3 \\
\hline 2009-06-20 00:00:00.0 & 21.5 \\
\hline 2009-06-19 21:00:00.0 & 21.2 \\
\hline 2009-06-19 18:00:00.0 & 23.9 \\
\hline 2009-06-19 15:00:00.0 & 24.5 \\
\hline 2009-06-19 12:00:00.0 & 38.5 \\
\hline 2009-06-19 06:00:00.0 & 15.9 \\
\hline 2009-06-19 03:00:00.0 & 16.9 \\
\hline 2009-06-19 00:00:00.0 & 12.2 \\
\hline 2009-06-18 21:00:00.0 & 17.5 \\
\hline 2009-06-18 12:00:00.0 & 22.6 \\
\hline 2009-06-18 09:00:00.0 & 17.0 \\
\hline 2009-06-18 06:00:00.0 & 14.2 \\
\hline 2009-06-18 03:00:00.0 & \\
\hline 2009-06-18 00:00:00.0 & 11.5 \\
\hline 2009-06-17 21:00:00.0 & \\
\hline 2009-06-17 18:00:00.0 & \\
\hline 2009-06-17 15:00:00.0 & \\
\hline 2009-06-17 12:00:00.0 & 7.9 \\
\hline 2009-06-17 09:00:00.0 & 4.2 \\
\hline 2009-06-17 06:00:00.0 & 7.7 \\
\hline 2009-06-17 03:00:00.0 & 10.2 \\
\hline 2009-06-17 00:00:00.0 & 15.2 \\
\hline 2009-06-16 18:00:00.0 & 19.4 \\
\hline 2009-06-16 15:00:00.0 & \\
\hline 2009-06-16 09:00:00.0 & 8.8 \\
\hline 2009-06-16 06:00:00.0 & 10.8 \\
\hline 2009-06-16 03:00:00.0 & 9.9 \\
\hline 2009-06-16 00:00:00.0 & 9.9 \\
\hline 2009-06-15 21:00:00.0 & 10.3 \\
\hline 2009-06-15 15:00:00.0 & 20.6 \\
\hline 2009-06-15 12:00:00.0 & 19.3 \\
\hline 2009-06-15 09:00:00.0 & 20.0 \\
\hline 2009-06-15 06:00:00.0 & \\
\hline 2009-06-15 03:00:00.0 & 19.8 \\
\hline 2009-06-14 21:00:00.0 & 17.2 \\
\hline 2009-06-14 18:00:00.0 & 22.2 \\
\hline 2009-06-14 15:00:00.0 & 23.2 \\
\hline 2009-06-14 12:00:00.0 & 21.3 \\
\hline 2009-06-14 09:00:00.0 & 23.0 \\
\hline 2009-06-14 06:00:00.0 & 22.3 \\
\hline 2009-06-14 03:00:00.0 & 16.8 \\
\hline 2009-06-14 00:00:00.0 & 14.2 \\
\hline 2009-06-13 21:00:00.0 & 14.3 \\
\hline 2009-06-13 18:00:00.0 & 22.8 \\
\hline 2009-06-13 15:00:00.0 & 23.6 \\
\hline 2009-06-13 12:00:00.0 & 18.0 \\
\hline 2009-06-13 09:00:00.0 & 14.0 \\
\hline 2009-06-13 06:00:00.0 & 11.8 \\
\hline 2009-06-13 03:00:00.0 & \\
\hline 2009-06-13 00:00:00.0 & 13.2 \\
\hline 2009-06-12 21:00:00.0 & 20.7 \\
\hline 2009-06-12 18:00:00.0 & 19.5 \\
\hline 2009-06-12 15:00:00.0 & 14.6 \\
\hline
\end{tabular}

\begin{tabular}{|c|c|}
\hline DataHora & VelVentoMax \\
\hline 2009-06-12 12:00:00.0 & 10.5 \\
\hline 2009-06-12 09:00:00.0 & 9.2 \\
\hline 2009-06-12 06:00:00.0 & 10.1 \\
\hline 2009-06-12 03:00:00.0 & 9.8 \\
\hline 2009-06-12 00:00:00.0 & 3.0 \\
\hline 2009-06-11 21:00:00.0 & 12.7 \\
\hline 2009-06-11 18:00:00.0 & 18.0 \\
\hline 2009-06-11 15:00:00.0 & 29.1 \\
\hline 2009-06-11 12:00:00.0 & 10.6 \\
\hline 2009-06-11 09:00:00.0 & 12.9 \\
\hline 2009-06-11 06:00:00.0 & 5.5 \\
\hline 2009-06-11 03:00:00.0 & 9.4 \\
\hline 2009-06-11 00:00:00.0 & 8.7 \\
\hline 2009-06-10 21:00:00.0 & 10.2 \\
\hline 2009-06-10 18:00:00.0 & 11.7 \\
\hline 2009-06-10 15:00:00.0 & 11.8 \\
\hline 2009-06-10 12:00:00.0 & 8.2 \\
\hline 2009-06-10 09:00:00.0 & 9.8 \\
\hline 2009-06-10 06:00:00.0 & 8.9 \\
\hline 2009-06-10 03:00:00.0 & 6.0 \\
\hline 2009-06-10 00:00:00.0 & 7.5 \\
\hline 2009-06-09 21:00:00.0 & 9.7 \\
\hline 2009-06-09 18:00:00.0 & 10.2 \\
\hline 2009-06-09 15:00:00.0 & 10.0 \\
\hline 2009-06-09 12:00:00.0 & 21.5 \\
\hline 2009-06-09 09:00:00.0 & 7.6 \\
\hline 2009-06-09 06:00:00.0 & 7.6 \\
\hline 2009-06-09 03:00:00.0 & 17.6 \\
\hline 2009-06-09 00:00:00.0 & 18.7 \\
\hline 2009-06-08 21:00:00.0 & 11.4 \\
\hline 2009-06-08 18:00:00.0 & 12.4 \\
\hline 2009-06-08 15:00:00.0 & 21.2 \\
\hline 2009-06-08 12:00:00.0 & 21.0 \\
\hline 2009-06-08 09:00:00.0 & 20.9 \\
\hline 2009-06-08 06:00:00.0 & 20.9 \\
\hline 2009-06-08 03:00:00.0 & 20.0 \\
\hline 2009-06-08 00:00:00.0 & 16.6 \\
\hline 2009-06-07 21:00:00.0 & 14.7 \\
\hline 2009-06-07 18:00:00.0 & 14.8 \\
\hline 2009-06-07 15:00:00.0 & 24.0 \\
\hline 2009-06-07 12:00:00.0 & 20.5 \\
\hline 2009-06-07 09:00:00.0 & 15.0 \\
\hline 2009-06-07 06:00:00.0 & 10.8 \\
\hline 2009-06-07 03:00:00.0 & 11.8 \\
\hline 2009-06-07 00:00:00.0 & 7.0 \\
\hline 2009-06-06 21:00:00.0 & 13.6 \\
\hline 2009-06-06 18:00:00.0 & 15.7 \\
\hline 2009-06-06 15:00:00.0 & 15.3 \\
\hline 2009-06-06 12:00:00.0 & 8.4 \\
\hline 2009-06-06 09:00:00.0 & 10.3 \\
\hline 2009-06-06 06:00:00.0 & 7.4 \\
\hline 2009-06-06 03:00:00.0 & 13.9 \\
\hline 2009-06-06 00:00:00.0 & 13.7 \\
\hline 2009-06-05 21:00:00.0 & 23.4 \\
\hline 2009-06-05 18:00:00.0 & 26.2 \\
\hline 2009-06-05 15:00:00.0 & 21.6 \\
\hline 2009-06-05 12:00:00.0 & 9.6 \\
\hline 2009-06-05 09:00:00.0 & \\
\hline 2009-06-05 06:00:00.0 & 10.9 \\
\hline 2009-06-05 03:00:00.0 & 10.1 \\
\hline 2009-06-05 00:00:00.0 & 9.9 \\
\hline 2009-06-04 21:00:00.0 & 19.8 \\
\hline 2009-06-04 18:00:00.0 & 27.4 \\
\hline 2009-06-04 15:00:00.0 & 24.2 \\
\hline 2009-06-04 12:00:00.0 & 16.5 \\
\hline 2009-06-04 09:00:00.0 & 17.0 \\
\hline 2009-06-04 06:00:00.0 & 15.8 \\
\hline 2009-06-04 03:00:00.0 & 12.0 \\
\hline 2009-06-04 00:00:00.0 & 10.1 \\
\hline
\end{tabular}




\section{ANEXO 01 - RAJADAS DE VENTO REGISTRADAS EM SÃO MARTINHO DA SERRA / RS}

\begin{tabular}{|c|c|}
\hline DataHora & VelVentoMax \\
\hline 2009-06-03 21:00:00.0 & 14.8 \\
\hline 2009-06-03 18:00:00.0 & 16.1 \\
\hline 2009-06-03 15:00:00.0 & 16.2 \\
\hline 2009-06-03 12:00:00.0 & 7.9 \\
\hline 2009-06-03 09:00:00.0 & 4.8 \\
\hline 2009-06-03 06:00:00.0 & 5.7 \\
\hline 2009-06-03 03:00:00.0 & 6.2 \\
\hline 2009-06-03 00:00:00.0 & 10.0 \\
\hline 2009-06-02 21:00:00.0 & 24.2 \\
\hline 2009-06-02 18:00:00.0 & 22.9 \\
\hline 2009-06-02 15:00:00.0 & 25.1 \\
\hline 2009-06-02 12:00:00.0 & 16.0 \\
\hline 2009-06-02 09:00:00.0 & 41.9 \\
\hline 2009-06-02 06:00:00.0 & 9.1 \\
\hline 2009-06-02 03:00:00.0 & 11.2 \\
\hline 2009-06-02 00:00:00.0 & 16.6 \\
\hline 2009-06-01 21:00:00.0 & 21.2 \\
\hline 2009-06-01 18:00:00.0 & 23.5 \\
\hline 2009-06-01 15:00:00.0 & 19.2 \\
\hline 2009-06-01 12:00:00.0 & 21.6 \\
\hline 2009-06-01 09:00:00.0 & 18.3 \\
\hline 2009-06-01 06:00:00.0 & 17.6 \\
\hline 2009-06-01 03:00:00.0 & 19.8 \\
\hline 2009-06-01 00:00:00.0 & \\
\hline 2009-05-31 21:00:00.0 & 23.9 \\
\hline 2009-05-31 18:00:00.0 & 27.0 \\
\hline 2009-05-31 15:00:00.0 & 26.0 \\
\hline 2009-05-31 12:00:00.0 & 28.8 \\
\hline 2009-05-31 09:00:00.0 & 25.1 \\
\hline 2009-05-31 06:00:00.0 & 32.0 \\
\hline 2009-05-31 03:00:00.0 & 28.1 \\
\hline 2009-05-31 00:00:00.0 & 30.5 \\
\hline 2009-05-30 21:00:00.0 & 25.0 \\
\hline 2009-05-30 18:00:00.0 & 23.8 \\
\hline 2009-05-30 15:00:00.0 & 37.2 \\
\hline 2009-05-30 12:00:00.0 & 32.1 \\
\hline 2009-05-30 09:00:00.0 & 30.1 \\
\hline 2009-05-30 06:00:00.0 & 20.3 \\
\hline 2009-05-30 03:00:00.0 & 21.4 \\
\hline 2009-05-30 00:00:00.0 & 24.3 \\
\hline 2009-05-29 21:00:00.0 & 22.8 \\
\hline 2009-05-29 18:00:00.0 & 26.0 \\
\hline 2009-05-29 15:00:00.0 & 25.1 \\
\hline 2009-05-29 12:00:00.0 & \\
\hline 2009-05-29 09:00:00.0 & 15.5 \\
\hline 2009-05-29 06:00:00.0 & 17.1 \\
\hline 2009-05-29 03:00:00.0 & 18.9 \\
\hline 2009-05-29 00:00:00.0 & 13.8 \\
\hline 2009-05-28 21:00:00.0 & 16.9 \\
\hline 2009-05-28 18:00:00.0 & 19.9 \\
\hline 2009-05-28 15:00:00.0 & 19.6 \\
\hline 2009-05-28 12:00:00.0 & 21.9 \\
\hline 2009-05-28 09:00:00.0 & \\
\hline 2009-05-28 06:00:00.0 & 17.3 \\
\hline 2009-05-28 03:00:00.0 & 17.1 \\
\hline 2009-05-28 00:00:00.0 & 11.1 \\
\hline 2009-05-27 21:00:00.0 & 20.1 \\
\hline 2009-05-27 18:00:00.0 & 18.0 \\
\hline 2009-05-27 15:00:00.0 & 21.2 \\
\hline 2009-05-27 12:00:00.0 & 18.2 \\
\hline 2009-05-27 09:00:00.0 & 19.5 \\
\hline 2009-05-27 06:00:00.0 & 19.7 \\
\hline 2009-05-27 03:00:00.0 & \\
\hline 2009-05-27 00:00:00.0 & \\
\hline 2009-05-26 21:00:00.0 & 21.3 \\
\hline 2009-05-26 18:00:00.0 & 15.0 \\
\hline 2009-05-26 15:00:00.0 & 14.5 \\
\hline 2009-05-26 12:00:00.0 & 12.5 \\
\hline 2009-05-26 09:00:00.0 & 6.8 \\
\hline
\end{tabular}

\begin{tabular}{|c|c|}
\hline DataHora & VelVentoMax \\
\hline 2009-05-26 06:00:00.0 & 7.2 \\
\hline 2009-05-26 03:00:00.0 & 5.0 \\
\hline 2009-05-26 00:00:00.0 & 8.0 \\
\hline 2009-05-25 21:00:00.0 & 15.7 \\
\hline 2009-05-25 18:00:00.0 & 16.0 \\
\hline 2009-05-25 15:00:00.0 & 16.8 \\
\hline 2009-05-25 12:00:00.0 & 17.5 \\
\hline 2009-05-25 09:00:00.0 & 19.2 \\
\hline 2009-05-25 06:00:00.0 & 21.8 \\
\hline 2009-05-25 03:00:00.0 & 19.5 \\
\hline 2009-05-25 00:00:00.0 & 14.4 \\
\hline 2009-05-24 21:00:00.0 & 15.5 \\
\hline 2009-05-24 18:00:00.0 & 16.8 \\
\hline 2009-05-24 15:00:00.0 & 17.3 \\
\hline 2009-05-24 12:00:00.0 & 16.9 \\
\hline 2009-05-24 09:00:00.0 & 15.1 \\
\hline 2009-05-24 06:00:00.0 & 14.8 \\
\hline 2009-05-24 03:00:00.0 & 13.4 \\
\hline 2009-05-24 00:00:00.0 & 11.4 \\
\hline 2009-05-23 21:00:00.0 & 10.8 \\
\hline 2009-05-23 18:00:00.0 & 22.3 \\
\hline 2009-05-23 15:00:00.0 & 19.0 \\
\hline 2009-05-23 12:00:00.0 & 17.1 \\
\hline 2009-05-23 09:00:00.0 & 18.0 \\
\hline 2009-05-23 06:00:00.0 & 14.7 \\
\hline 2009-05-23 03:00:00.0 & 14.5 \\
\hline 2009-05-23 00:00:00.0 & 11.2 \\
\hline 2009-05-22 21:00:00.0 & 12.8 \\
\hline 2009-05-22 18:00:00.0 & 16.6 \\
\hline 2009-05-22 15:00:00.0 & 14.5 \\
\hline 2009-05-22 12:00:00.0 & 18.2 \\
\hline 2009-05-22 09:00:00.0 & 15.2 \\
\hline 2009-05-22 06:00:00.0 & 16.1 \\
\hline 2009-05-22 03:00:00.0 & 15.6 \\
\hline 2009-05-22 00:00:00.0 & 12.6 \\
\hline 2009-05-21 21:00:00.0 & 13.7 \\
\hline 2009-05-21 18:00:00.0 & 20.3 \\
\hline 2009-05-21 15:00:00.0 & 19.8 \\
\hline 2009-05-21 12:00:00.0 & 19.6 \\
\hline 2009-05-21 09:00:00.0 & 17.3 \\
\hline 2009-05-21 06:00:00.0 & \\
\hline 2009-05-21 03:00:00.0 & 15.1 \\
\hline 2009-05-21 00:00:00.0 & 14.2 \\
\hline 2009-05-20 21:00:00.0 & 12.7 \\
\hline 2009-05-20 18:00:00.0 & 12.7 \\
\hline 2009-05-20 15:00:00.0 & \\
\hline 2009-05-20 12:00:00.0 & 19.5 \\
\hline 2009-05-20 09:00:00.0 & 20.0 \\
\hline 2009-05-20 06:00:00.0 & \\
\hline 2009-05-20 03:00:00.0 & 20.5 \\
\hline 2009-05-20 00:00:00.0 & 15.4 \\
\hline 2009-05-19 21:00:00.0 & 13.7 \\
\hline 2009-05-19 18:00:00.0 & 18.4 \\
\hline 2009-05-19 15:00:00.0 & 17.6 \\
\hline 2009-05-19 12:00:00.0 & 15.8 \\
\hline 2009-05-19 09:00:00.0 & 17.7 \\
\hline 2009-05-19 06:00:00.0 & \\
\hline 2009-05-19 03:00:00.0 & 13.0 \\
\hline 2009-05-19 00:00:00.0 & 17.8 \\
\hline 2009-05-18 21:00:00.0 & 10.1 \\
\hline 2009-05-18 18:00:00.0 & \\
\hline 2009-05-18 15:00:00.0 & 16.2 \\
\hline 2009-05-18 12:00:00.0 & 15.3 \\
\hline 2009-05-18 09:00:00.0 & 15.5 \\
\hline 2009-05-18 06:00:00.0 & 16.4 \\
\hline 2009-05-18 03:00:00.0 & 15.0 \\
\hline 2009-05-18 00:00:00.0 & 15.9 \\
\hline 2009-05-17 21:00:00.0 & 13.0 \\
\hline 2009-05-17 18:00:00.0 & \\
\hline
\end{tabular}

\begin{tabular}{|c|c|}
\hline DataHora & VelVentoMax \\
\hline 2009-05-17 15:00:00.0 & 20.4 \\
\hline 2009-05-17 12:00:00.0 & 19.7 \\
\hline 2009-05-17 09:00:00.0 & 19.7 \\
\hline 2009-05-17 06:00:00.0 & 19.4 \\
\hline 2009-05-17 03:00:00.0 & 19.1 \\
\hline 2009-05-17 00:00:00.0 & 16.8 \\
\hline 2009-05-16 21:00:00.0 & 11.5 \\
\hline 2009-05-16 18:00:00.0 & 10.4 \\
\hline 2009-05-16 15:00:00.0 & 32.2 \\
\hline $2009-05-16$ 12:00:00.0 & 9.4 \\
\hline 2009-05-16 09:00:00.0 & \\
\hline 2009-05-16 06:00:00.0 & 6.0 \\
\hline 2009-05-16 03:00:00.0 & 4.4 \\
\hline 2009-05-16 00:00:00.0 & 7.0 \\
\hline 2009-05-15 21:00:00.0 & 17.1 \\
\hline 2009-05-15 18:00:00.0 & 19.7 \\
\hline 2009-05-15 15:00:00.0 & 18.9 \\
\hline 2009-05-15 12:00:00.0 & 19.0 \\
\hline 2009-05-15 09:00:00.0 & \\
\hline 2009-05-15 06:00:00.0 & 24.0 \\
\hline 2009-05-15 03:00:00.0 & 28.6 \\
\hline 2009-05-15 00:00:00.0 & 17.0 \\
\hline 2009-05-14 21:00:00.0 & 26.9 \\
\hline 2009-05-14 18:00:00.0 & \\
\hline 2009-05-14 15:00:00.0 & 27.5 \\
\hline 2009-05-14 12:00:00.0 & 25.0 \\
\hline 2009-05-14 09:00:00.0 & \\
\hline 2009-05-14 06:00:00.0 & \\
\hline 2009-05-14 03:00:00.0 & 17.1 \\
\hline 2009-05-14 00:00:00.0 & 11.6 \\
\hline 2009-05-13 21:00:00.0 & 29.9 \\
\hline 2009-05-13 18:00:00.0 & 29.1 \\
\hline 2009-05-13 15:00:00.0 & 25.4 \\
\hline 2009-05-13 12:00:00.0 & \\
\hline 2009-05-13 09:00:00.0 & \\
\hline 2009-05-13 06:00:00.0 & 21.5 \\
\hline 2009-05-13 03:00:00.0 & 19.6 \\
\hline $2009-05-13$ 00:00:00.0 & 19.4 \\
\hline 2009-05-12 21:00:00.0 & 24.4 \\
\hline 2009-05-12 18:00:00.0 & 20.1 \\
\hline 2009-05-12 15:00:00.0 & 32.7 \\
\hline 2009-05-12 12:00:00.0 & 13.8 \\
\hline 2009-05-12 09:00:00.0 & 14.1 \\
\hline 2009-05-12 06:00:00.0 & 14.9 \\
\hline 2009-05-12 03:00:00.0 & 13.4 \\
\hline 2009-05-12 00:00:00.0 & 11.4 \\
\hline 2009-05-11 21:00:00.0 & 20.7 \\
\hline 2009-05-11 18:00:00.0 & 36.7 \\
\hline 2009-05-11 15:00:00.0 & 17.1 \\
\hline 2009-05-11 12:00:00.0 & 14.7 \\
\hline 2009-05-11 09:00:00.0 & 14.7 \\
\hline 2009-05-11 06:00:00.0 & 16.4 \\
\hline 2009-05-11 03:00:00.0 & 15.6 \\
\hline 2009-05-11 00:00:00.0 & \\
\hline 2009-05-10 21:00:00.0 & 16.3 \\
\hline 2009-05-10 18:00:00.0 & 15.4 \\
\hline 2009-05-10 15:00:00.0 & 16.1 \\
\hline 2009-05-10 12:00:00.0 & 15.0 \\
\hline 2009-05-10 09:00:00.0 & 16.6 \\
\hline 2009-05-10 06:00:00.0 & 19.4 \\
\hline 2009-05-10 03:00:00.0 & 16.3 \\
\hline 2009-05-10 00:00:00.0 & 13.9 \\
\hline 2009-05-09 21:00:00.0 & 15.7 \\
\hline 2009-05-09 18:00:00.0 & 15.9 \\
\hline 2009-05-09 15:00:00.0 & 11.4 \\
\hline 2009-05-09 12:00:00.0 & 12.5 \\
\hline 2009-05-09 09:00:00.0 & 14.8 \\
\hline 2009-05-09 06:00:00.0 & 8.3 \\
\hline 2009-05-09 03:00:00.0 & 20.4 \\
\hline
\end{tabular}




\section{ANEXO 01 - RAJADAS DE VENTO REGISTRADAS EM SÃO MARTINHO DA SERRA / RS}

\begin{tabular}{|c|c|}
\hline DataHora & VelVentoMax \\
\hline 2009-05-09 00:00:00.0 & 17.7 \\
\hline 2009-05-08 21:00:00.0 & 11.8 \\
\hline 2009-05-08 18:00:00.0 & 13.1 \\
\hline 2009-05-08 15:00:00.0 & 15.1 \\
\hline 2009-05-08 12:00:00.0 & 10.9 \\
\hline 2009-05-08 09:00:00.0 & 10.1 \\
\hline 2009-05-08 06:00:00.0 & 14.4 \\
\hline 2009-05-08 03:00:00.0 & 15.1 \\
\hline 2009-05-08 00:00:00.0 & 10.3 \\
\hline 2009-05-07 21:00:00.0 & 10.7 \\
\hline 2009-05-07 18:00:00.0 & 12.1 \\
\hline 2009-05-07 15:00:00.0 & 15.1 \\
\hline 2009-05-07 12:00:00.0 & 17.1 \\
\hline 2009-05-07 09:00:00.0 & 12.6 \\
\hline 2009-05-07 06:00:00.0 & 9.3 \\
\hline 2009-05-07 03:00:00.0 & 9.6 \\
\hline 2009-05-07 00:00:00.0 & 5.9 \\
\hline 2009-05-06 21:00:00.0 & 4.1 \\
\hline 2009-05-06 18:00:00.0 & 20.1 \\
\hline 2009-05-06 15:00:00.0 & 18.4 \\
\hline 2009-05-06 12:00:00.0 & 6.4 \\
\hline 2009-05-06 09:00:00.0 & 12.2 \\
\hline 2009-05-06 06:00:00.0 & 7.7 \\
\hline 2009-05-06 03:00:00.0 & 23.9 \\
\hline 2009-05-06 00:00:00.0 & 28.1 \\
\hline 2009-05-05 21:00:00.0 & 25.4 \\
\hline 2009-05-05 18:00:00.0 & 24.2 \\
\hline 2009-05-05 15:00:00.0 & 21.9 \\
\hline 2009-05-05 12:00:00.0 & 11.1 \\
\hline 2009-05-05 09:00:00.0 & 12.7 \\
\hline 2009-05-05 06:00:00.0 & 14.3 \\
\hline 2009-05-05 03:00:00.0 & 13.1 \\
\hline 2009-05-05 00:00:00.0 & 10.8 \\
\hline 2009-05-04 21:00:00.0 & 13.7 \\
\hline 2009-05-04 18:00:00.0 & 14.7 \\
\hline 2009-05-04 15:00:00.0 & 22.2 \\
\hline 2009-05-04 12:00:00.0 & 19.9 \\
\hline 2009-05-04 09:00:00.0 & \\
\hline 2009-05-04 06:00:00.0 & 15.9 \\
\hline 2009-05-04 03:00:00.0 & 17.5 \\
\hline 2009-05-04 00:00:00.0 & \\
\hline 2009-05-03 21:00:00.0 & 7.1 \\
\hline 2009-05-03 18:00:00.0 & 27.1 \\
\hline 2009-05-03 15:00:00.0 & 18.1 \\
\hline 2009-05-03 12:00:00.0 & 19.7 \\
\hline 2009-05-03 09:00:00.0 & 15.6 \\
\hline 2009-05-03 06:00:00.0 & 12.9 \\
\hline 2009-05-03 03:00:00.0 & 11.3 \\
\hline 2009-05-03 00:00:00.0 & 9.8 \\
\hline 2009-05-02 21:00:00.0 & 13.2 \\
\hline 2009-05-02 18:00:00.0 & 29.2 \\
\hline 2009-05-02 15:00:00.0 & 21.3 \\
\hline 2009-05-02 12:00:00.0 & 21.1 \\
\hline 2009-05-02 09:00:00.0 & \\
\hline 2009-05-02 06:00:00.0 & 18.0 \\
\hline 2009-05-02 03:00:00.0 & 18.4 \\
\hline 2009-05-02 00:00:00.0 & 16.6 \\
\hline 2009-05-01 21:00:00.0 & 36.5 \\
\hline 2009-05-01 18:00:00.0 & 13.8 \\
\hline 2009-05-01 15:00:00.0 & 33.2 \\
\hline 2009-05-01 12:00:00.0 & 20.2 \\
\hline 2009-05-01 09:00:00.0 & 14.4 \\
\hline 2009-05-01 06:00:00.0 & 15.9 \\
\hline 2009-05-01 03:00:00.0 & 9.6 \\
\hline 2009-05-01 00:00:00.0 & 9.6 \\
\hline 2009-04-30 21:00:00.0 & 32.3 \\
\hline 2009-04-30 18:00:00.0 & 22.2 \\
\hline 2009-04-30 15:00:00.0 & 26.5 \\
\hline 2009-04-30 12:00:00.0 & 22.2 \\
\hline
\end{tabular}

\begin{tabular}{|c|c|}
\hline DataHora & VelVentoMax \\
\hline 2009-04-30 09:00:00.0 & 16.2 \\
\hline 2009-04-30 06:00:00.0 & 8.9 \\
\hline 2009-04-30 03:00:00.0 & 12.8 \\
\hline 2009-04-30 00:00:00.0 & 11.8 \\
\hline 2009-04-29 21:00:00.0 & 18.6 \\
\hline 2009-04-29 18:00:00.0 & 18.2 \\
\hline 2009-04-29 15:00:00.0 & 14.1 \\
\hline 2009-04-28 12:00:00.0 & 13.6 \\
\hline 2009-04-28 09:00:00.0 & 13.8 \\
\hline 2009-04-28 06:00:00.0 & 10.4 \\
\hline 2009-04-28 03:00:00.0 & 10.6 \\
\hline 2009-04-28 00:00:00.0 & 11.4 \\
\hline 2009-04-27 21:00:00.0 & 18.3 \\
\hline 2009-04-27 18:00:00.0 & 17.4 \\
\hline 2009-04-27 15:00:00.0 & 30.0 \\
\hline 2009-04-27 12:00:00.0 & 13.8 \\
\hline 2009-04-27 09:00:00.0 & 12.0 \\
\hline 2009-04-27 06:00:00.0 & 13.4 \\
\hline 2009-04-27 03:00:00.0 & 15.0 \\
\hline 2009-04-27 00:00:00.0 & 8.8 \\
\hline 2009-04-26 21:00:00.0 & 16.6 \\
\hline 2009-04-26 18:00:00.0 & 23.9 \\
\hline 2009-04-26 15:00:00.0 & 4.1 \\
\hline 2009-04-26 12:00:00.0 & 28.1 \\
\hline 2009-04-26 09:00:00.0 & 7.1 \\
\hline 2009-04-26 06:00:00.0 & \\
\hline 2009-04-26 03:00:00.0 & 9.8 \\
\hline 2009-04-26 00:00:00.0 & 10.5 \\
\hline 2009-04-25 21:00:00.0 & 16.6 \\
\hline 2009-04-25 18:00:00.0 & 15.6 \\
\hline 2009-04-25 15:00:00.0 & 34.0 \\
\hline 2009-04-25 12:00:00.0 & 16.9 \\
\hline 2009-04-25 09:00:00.0 & 16.4 \\
\hline 2009-04-25 06:00:00.0 & 13.3 \\
\hline 2009-04-25 03:00:00.0 & 13.5 \\
\hline 2009-04-25 00:00:00.0 & 11.9 \\
\hline 2009-04-24 21:00:00.0 & 17.9 \\
\hline 2009-04-24 18:00:00.0 & 19.0 \\
\hline 2009-04-24 15:00:00.0 & 18.2 \\
\hline 2009-04-24 12:00:00.0 & 15.0 \\
\hline 2009-04-24 09:00:00.0 & 6.6 \\
\hline 2009-04-24 06:00:00.0 & 10.0 \\
\hline 2009-04-24 03:00:00.0 & 7.1 \\
\hline 2009-04-24 00:00:00.0 & 8.9 \\
\hline 2009-04-23 21:00:00.0 & 14.6 \\
\hline 2009-04-23 18:00:00.0 & 27.1 \\
\hline 2009-04-23 15:00:00.0 & 11.5 \\
\hline 2009-04-23 12:00:00.0 & 11.1 \\
\hline 2009-04-23 09:00:00.0 & 9.5 \\
\hline 2009-04-23 06:00:00.0 & 7.7 \\
\hline 2009-04-23 03:00:00.0 & 13.3 \\
\hline 2009-04-23 00:00:00.0 & 10.9 \\
\hline 2009-04-22 21:00:00.0 & 40.6 \\
\hline 2009-04-22 18:00:00.0 & 9.5 \\
\hline 2009-04-22 15:00:00.0 & 14.7 \\
\hline 2009-04-22 12:00:00.0 & 12.6 \\
\hline 2009-04-22 09:00:00.0 & 16.4 \\
\hline 2009-04-22 06:00:00.0 & 14.6 \\
\hline 2009-04-22 03:00:00.0 & 15.3 \\
\hline 2009-04-22 00:00:00.0 & 13.3 \\
\hline 2009-04-21 21:00:00.0 & 5.8 \\
\hline 2009-04-21 18:00:00.0 & 15.2 \\
\hline 2009-04-21 15:00:00.0 & 19.7 \\
\hline 2009-04-21 12:00:00.0 & 16.7 \\
\hline 2009-04-21 09:00:00.0 & 18.3 \\
\hline 2009-04-21 06:00:00.0 & 19.7 \\
\hline 2009-04-21 03:00:00.0 & 18.2 \\
\hline 2009-04-21 00:00:00.0 & 15.4 \\
\hline 2009-04-20 21:00:00.0 & 19.9 \\
\hline
\end{tabular}

\begin{tabular}{|c|c|}
\hline DataHora & VelVentoMax \\
\hline 2009-04-20 18:00:00.0 & 17.8 \\
\hline 2009-04-20 15:00:00.0 & 22.8 \\
\hline 2009-04-20 12:00:00.0 & 21.1 \\
\hline 2009-04-20 09:00:00.0 & 13.3 \\
\hline 2009-04-20 06:00:00.0 & 11.3 \\
\hline 2009-04-20 03:00:00.0 & 11.9 \\
\hline 2009-04-20 00:00:00.0 & 16.0 \\
\hline 2009-04-19 21:00:00.0 & 15.8 \\
\hline 2009-04-19 18:00:00.0 & 19.9 \\
\hline 2009-04-19 15:00:00.0 & 21.1 \\
\hline 2009-04-19 12:00:00.0 & 27.1 \\
\hline 2009-04-19 09:00:00.0 & 8.2 \\
\hline 2009-04-19 06:00:00.0 & 9.7 \\
\hline 2009-04-19 03:00:00.0 & 8.7 \\
\hline 2009-04-19 00:00:00.0 & 8.1 \\
\hline 2009-04-18 21:00:00.0 & 22.6 \\
\hline 2009-04-18 18:00:00.0 & 25.7 \\
\hline 2009-04-18 15:00:00.0 & 24.3 \\
\hline 2009-04-18 12:00:00.0 & 11.7 \\
\hline 2009-04-18 09:00:00.0 & 8.8 \\
\hline 2009-04-18 06:00:00.0 & 12.9 \\
\hline 2009-04-18 03:00:00.0 & 11.8 \\
\hline 2009-04-18 00:00:00.0 & 11.0 \\
\hline 2009-04-17 21:00:00.0 & \\
\hline 2009-04-17 18:00:00.0 & 15.3 \\
\hline 2009-04-17 15:00:00.0 & 14.4 \\
\hline 2009-04-17 12:00:00.0 & 17.6 \\
\hline 2009-04-17 09:00:00.0 & 16.5 \\
\hline 2009-04-17 06:00:00.0 & 17.9 \\
\hline 2009-04-17 03:00:00.0 & 17.5 \\
\hline 2009-04-17 00:00:00.0 & 12.9 \\
\hline 2009-04-16 21:00:00.0 & 16.8 \\
\hline 2009-04-16 18:00:00.0 & 13.1 \\
\hline 2009-04-16 15:00:00.0 & 20.8 \\
\hline 2009-04-16 12:00:00.0 & 21.9 \\
\hline 2009-04-16 09:00:00.0 & 19.9 \\
\hline 2009-04-16 06:00:00.0 & 15.8 \\
\hline 2009-04-16 03:00:00.0 & 14.8 \\
\hline 2009-04-16 00:00:00.0 & 13.5 \\
\hline 2009-04-15 21:00:00.0 & \\
\hline 2009-04-15 18:00:00.0 & 20.5 \\
\hline 2009-04-15 15:00:00.0 & 41.6 \\
\hline 2009-04-15 12:00:00.0 & 18.6 \\
\hline 2009-04-15 09:00:00.0 & 15.9 \\
\hline 2009-04-15 06:00:00.0 & 21.0 \\
\hline 2009-04-15 03:00:00.0 & 23.2 \\
\hline 2009-04-15 00:00:00.0 & 21.4 \\
\hline 2009-04-14 21:00:00.0 & 20.3 \\
\hline 2009-04-14 18:00:00.0 & 20.4 \\
\hline 2009-04-14 15:00:00.0 & 16.4 \\
\hline 2009-04-14 12:00:00.0 & 8.9 \\
\hline 2009-04-14 09:00:00.0 & \\
\hline 2009-04-14 06:00:00.0 & 10.6 \\
\hline 2009-04-14 03:00:00.0 & 11.7 \\
\hline 2009-04-14 00:00:00.0 & 8.7 \\
\hline 2009-04-13 21:00:00.0 & 16.3 \\
\hline 2009-04-13 18:00:00.0 & 18.7 \\
\hline 2009-04-13 15:00:00.0 & 13.5 \\
\hline 2009-04-13 12:00:00.0 & 16.1 \\
\hline 2009-04-13 09:00:00.0 & \\
\hline 2009-04-13 06:00:00.0 & 20.1 \\
\hline 2009-04-13 03:00:00.0 & 19.7 \\
\hline 2009-04-13 00:00:00.0 & 14.8 \\
\hline 2009-04-12 21:00:00.0 & 18.6 \\
\hline 2009-04-12 18:00:00.0 & 2.8 \\
\hline 2009-04-12 15:00:00.0 & 23.2 \\
\hline 2009-04-12 12:00:00.0 & 22.2 \\
\hline 2009-04-12 09:00:00.0 & \\
\hline 2009-04-12 06:00:00.0 & 13.5 \\
\hline
\end{tabular}




\section{ANEXO 01 - RAJADAS DE VENTO REGISTRADAS EM SÃO MARTINHO DA SERRA / RS}

\begin{tabular}{|c|c|}
\hline DataHora & VelVentoMax \\
\hline 2009-04-12 03:00:00.0 & 14.1 \\
\hline 2009-04-12 00:00:00.0 & 12.7 \\
\hline 2009-04-11 21:00:00.0 & 17.0 \\
\hline 2009-04-11 18:00:00.0 & 6.9 \\
\hline 2009-04-11 15:00:00.0 & 6.9 \\
\hline 2009-04-11 12:00:00.0 & 10.6 \\
\hline 2009-04-11 09:00:00.0 & 10.4 \\
\hline 2009-04-11 06:00:00.0 & 6.1 \\
\hline 2009-04-11 03:00:00.0 & 7.1 \\
\hline 2009-04-11 00:00:00.0 & 6.6 \\
\hline 2009-04-10 21:00:00.0 & 18.3 \\
\hline 2009-04-10 18:00:00.0 & 21.4 \\
\hline 2009-04-10 15:00:00.0 & 16.9 \\
\hline 2009-04-10 12:00:00.0 & 13.4 \\
\hline 2009-04-10 09:00:00.0 & 9.5 \\
\hline 2009-04-10 06:00:00.0 & 10.6 \\
\hline 2009-04-10 03:00:00.0 & 15.7 \\
\hline 2009-04-10 00:00:00.0 & 10.4 \\
\hline 2009-04-09 21:00:00.0 & 22.7 \\
\hline 2009-04-09 18:00:00.0 & 22.9 \\
\hline 2009-04-09 15:00:00.0 & 18.8 \\
\hline 2009-04-09 12:00:00.0 & 13.2 \\
\hline 2009-04-09 09:00:00.0 & 16.5 \\
\hline 2009-04-09 06:00:00.0 & 13.0 \\
\hline 2009-04-09 03:00:00.0 & 24.4 \\
\hline 2009-04-09 00:00:00.0 & \\
\hline 2009-04-08 21:00:00.0 & 23.7 \\
\hline 2009-04-08 18:00:00.0 & 24.4 \\
\hline 2009-04-08 15:00:00.0 & 20.5 \\
\hline 2009-04-08 12:00:00.0 & 11.5 \\
\hline 2009-04-08 09:00:00.0 & \\
\hline 2009-04-08 06:00:00.0 & 8.1 \\
\hline 2009-04-08 03:00:00.0 & 8.0 \\
\hline 2009-04-08 00:00:00.0 & 9.5 \\
\hline 2009-04-07 21:00:00.0 & 16.7 \\
\hline 2009-04-07 18:00:00.0 & 22.9 \\
\hline 2009-04-07 15:00:00.0 & 18.0 \\
\hline 2009-04-07 12:00:00.0 & 16.9 \\
\hline 2009-04-07 09:00:00.0 & 12.6 \\
\hline 2009-04-07 06:00:00.0 & 0.5 \\
\hline 2009-04-07 03:00:00.0 & 10.7 \\
\hline 2009-04-07 00:00:00.0 & 12.2 \\
\hline 2009-04-06 21:00:00.0 & 16.7 \\
\hline 2009-04-06 18:00:00.0 & 16.9 \\
\hline 2009-04-06 15:00:00.0 & 13.1 \\
\hline 2009-04-06 12:00:00.0 & 16.1 \\
\hline 2009-04-06 09:00:00.0 & 19.0 \\
\hline 2009-04-06 06:00:00.0 & 21.3 \\
\hline 2009-04-06 03:00:00.0 & 19.8 \\
\hline 2009-04-06 00:00:00.0 & 18.7 \\
\hline 2009-04-05 21:00:00.0 & 16.1 \\
\hline 2009-04-05 18:00:00.0 & 15.7 \\
\hline 2009-04-05 15:00:00.0 & 33.2 \\
\hline 2009-04-05 12:00:00.0 & 5.6 \\
\hline 2009-04-05 09:00:00.0 & 19.2 \\
\hline 2009-04-05 06:00:00.0 & 22.6 \\
\hline 2009-04-05 03:00:00.0 & 21.6 \\
\hline 2009-04-05 00:00:00.0 & 16.2 \\
\hline 2009-04-04 21:00:00.0 & 29.2 \\
\hline 2009-04-04 18:00:00.0 & 31.9 \\
\hline 2009-04-04 15:00:00.0 & 29.8 \\
\hline 2009-04-04 12:00:00.0 & 24.5 \\
\hline 2009-04-04 09:00:00.0 & 22.5 \\
\hline 2009-04-04 06:00:00.0 & 21.5 \\
\hline 2009-04-04 03:00:00.0 & \\
\hline 2009-04-04 00:00:00.0 & 36.6 \\
\hline 2009-04-03 21:00:00.0 & 28.2 \\
\hline 2009-04-03 18:00:00.0 & 16.7 \\
\hline 2009-04-03 15:00:00.0 & 17.2 \\
\hline
\end{tabular}

\begin{tabular}{|c|c|}
\hline DataHora & VelVentoMax \\
\hline 2009-04-03 12:00:00.0 & 21.6 \\
\hline 2009-04-03 09:00:00.0 & 19.2 \\
\hline 2009-04-03 06:00:00.0 & 19.2 \\
\hline 2009-04-03 03:00:00.0 & 17.8 \\
\hline 2009-04-03 00:00:00.0 & 14.6 \\
\hline 2009-04-02 21:00:00.0 & 16.0 \\
\hline 2009-04-02 18:00:00.0 & 14.6 \\
\hline 2009-04-02 15:00:00.0 & 21.9 \\
\hline 2009-04-02 12:00:00.0 & 22.8 \\
\hline 2009-04-02 09:00:00.0 & 19.5 \\
\hline 2009-04-02 06:00:00.0 & 18.1 \\
\hline 2009-04-02 03:00:00.0 & 15.5 \\
\hline 2009-04-02 00:00:00.0 & 17.6 \\
\hline 2009-04-01 21:00:00.0 & 22.2 \\
\hline 2009-04-01 18:00:00.0 & 23.6 \\
\hline 2009-04-01 15:00:00.0 & 23.1 \\
\hline 2009-04-01 12:00:00.0 & 20.5 \\
\hline 2009-04-01 09:00:00.0 & 18.1 \\
\hline 2009-04-01 06:00:00.0 & 17.1 \\
\hline 2009-04-01 03:00:00.0 & 22.0 \\
\hline 2009-04-01 00:00:00.0 & 26.6 \\
\hline 2009-03-31 21:00:00.0 & 21.5 \\
\hline 2009-03-31 18:00:00.0 & 21.6 \\
\hline 2009-03-31 15:00:00.0 & 11.3 \\
\hline 2009-03-31 12:00:00.0 & 9.9 \\
\hline 2009-03-31 09:00:00.0 & 10.2 \\
\hline 2009-03-31 06:00:00.0 & 9.1 \\
\hline 2009-03-31 03:00:00.0 & 9.3 \\
\hline 2009-03-31 00:00:00.0 & 9.6 \\
\hline 2009-03-30 21:00:00.0 & 9.9 \\
\hline 2009-03-30 18:00:00.0 & \\
\hline 2009-03-30 15:00:00.0 & 15.1 \\
\hline 2009-03-30 12:00:00.0 & 19.4 \\
\hline 2009-03-30 09:00:00.0 & 15.1 \\
\hline 2009-03-30 06:00:00.0 & 14.5 \\
\hline 2009-03-30 03:00:00.0 & 11.6 \\
\hline 2009-03-30 00:00:00.0 & 9.2 \\
\hline 2009-03-29 21:00:00.0 & 12.0 \\
\hline 2009-03-29 18:00:00.0 & \\
\hline 2009-03-29 15:00:00.0 & 14.3 \\
\hline 2009-03-29 12:00:00.0 & 12.9 \\
\hline 2009-03-29 09:00:00.0 & 11.8 \\
\hline 2009-03-29 06:00:00.0 & 12.8 \\
\hline 2009-03-29 03:00:00.0 & 8.0 \\
\hline 2009-03-29 00:00:00.0 & 7.2 \\
\hline 2009-03-28 21:00:00.0 & 15.7 \\
\hline 2009-03-28 18:00:00.0 & 18.1 \\
\hline 2009-03-28 15:00:00.0 & 13.8 \\
\hline 2009-03-28 12:00:00.0 & 9.2 \\
\hline 2009-03-28 09:00:00.0 & 9.1 \\
\hline 2009-03-28 06:00:00.0 & 13.1 \\
\hline 2009-03-28 03:00:00.0 & 14.9 \\
\hline 2009-03-28 00:00:00.0 & 14.7 \\
\hline 2009-03-27 21:00:00.0 & 16.5 \\
\hline 2009-03-27 18:00:00.0 & 18.8 \\
\hline 2009-03-27 15:00:00.0 & 12.9 \\
\hline 2009-03-27 12:00:00.0 & 8.4 \\
\hline 2009-03-27 09:00:00.0 & 9.7 \\
\hline 2009-03-27 06:00:00.0 & 12.9 \\
\hline 2009-03-27 03:00:00.0 & 18.7 \\
\hline 2009-03-27 00:00:00.0 & 20.7 \\
\hline 2009-03-26 21:00:00.0 & 43.2 \\
\hline 2009-03-26 18:00:00.0 & 42.2 \\
\hline 2009-03-26 15:00:00.0 & 21.7 \\
\hline 2009-03-26 12:00:00.0 & 22.0 \\
\hline 2009-03-26 09:00:00.0 & 20.6 \\
\hline 2009-03-26 06:00:00.0 & 19.5 \\
\hline 2009-03-26 03:00:00.0 & 15.7 \\
\hline 2009-03-26 00:00:00.0 & 15.3 \\
\hline
\end{tabular}

\begin{tabular}{|c|c|}
\hline DataHora & VelVentoMax \\
\hline 2009-03-25 21:00:00.0 & 18.7 \\
\hline 2009-03-25 18:00:00.0 & 16.7 \\
\hline 2009-03-25 15:00:00.0 & 22.8 \\
\hline 2009-03-25 12:00:00.0 & 22.1 \\
\hline 2009-03-25 09:00:00.0 & \\
\hline 2009-03-25 06:00:00.0 & 13.2 \\
\hline 2009-03-25 03:00:00.0 & 19.5 \\
\hline 2009-03-25 00:00:00.0 & 22.2 \\
\hline 2009-03-24 21:00:00.0 & 25.2 \\
\hline 2009-03-24 18:00:00.0 & 25.9 \\
\hline 2009-03-24 15:00:00.0 & 23.8 \\
\hline 2009-03-24 12:00:00.0 & 20.0 \\
\hline 2009-03-24 09:00:00.0 & 16.0 \\
\hline 2009-03-24 06:00:00.0 & 13.7 \\
\hline 2009-03-24 03:00:00.0 & 15.6 \\
\hline 2009-03-24 00:00:00.0 & 15.8 \\
\hline 2009-03-23 21:00:00.0 & 21.3 \\
\hline 2009-03-23 18:00:00.0 & 18.9 \\
\hline 2009-03-23 15:00:00.0 & 11.1 \\
\hline 2009-03-23 12:00:00.0 & 14.2 \\
\hline 2009-03-23 09:00:00.0 & \\
\hline 2009-03-23 06:00:00.0 & 7.9 \\
\hline 2009-03-23 03:00:00.0 & 9.8 \\
\hline 2009-03-23 00:00:00.0 & 9.2 \\
\hline 2009-03-22 21:00:00.0 & 17.1 \\
\hline 2009-03-22 18:00:00.0 & 16.8 \\
\hline 2009-03-22 15:00:00.0 & 12.7 \\
\hline 2009-03-22 12:00:00.0 & 13.3 \\
\hline 2009-03-22 09:00:00.0 & \\
\hline 2009-03-22 06:00:00.0 & 9.5 \\
\hline 2009-03-22 03:00:00.0 & 9.9 \\
\hline 2009-03-22 00:00:00.0 & 11.0 \\
\hline 2009-03-21 21:00:00.0 & 15.3 \\
\hline 2009-03-21 18:00:00.0 & 16.1 \\
\hline 2009-03-21 15:00:00.0 & 12.6 \\
\hline 2009-03-21 12:00:00.0 & 11.1 \\
\hline 2009-03-21 09:00:00.0 & 5.2 \\
\hline 2009-03-21 06:00:00.0 & 12.2 \\
\hline 2009-03-21 03:00:00.0 & 12.0 \\
\hline 2009-03-21 00:00:00.0 & 9.5 \\
\hline 2009-03-20 21:00:00.0 & 13.8 \\
\hline 2009-03-20 18:00:00.0 & 17.6 \\
\hline 2009-03-20 15:00:00.0 & 22.2 \\
\hline 2009-03-20 12:00:00.0 & 18.6 \\
\hline 2009-03-20 09:00:00.0 & 19.6 \\
\hline 2009-03-20 06:00:00.0 & 15.4 \\
\hline 2009-03-20 03:00:00.0 & 15.3 \\
\hline 2009-03-20 00:00:00.0 & 15.0 \\
\hline 2009-03-19 21:00:00.0 & 15.0 \\
\hline 2009-03-19 18:00:00.0 & 14.5 \\
\hline 2009-03-19 15:00:00.0 & 23.5 \\
\hline 2009-03-19 12:00:00.0 & 21.0 \\
\hline 2009-03-19 09:00:00.0 & 20.3 \\
\hline 2009-03-19 06:00:00.0 & 19.2 \\
\hline 2009-03-19 03:00:00.0 & 15.1 \\
\hline 2009-03-19 00:00:00.0 & 15.9 \\
\hline 2009-03-18 21:00:00.0 & 20.3 \\
\hline 2009-03-18 18:00:00.0 & 23.2 \\
\hline 2009-03-18 15:00:00.0 & 20.0 \\
\hline 2009-03-18 12:00:00.0 & 22.2 \\
\hline 2009-03-18 09:00:00.0 & 11.3 \\
\hline 2009-03-18 06:00:00.0 & 14.8 \\
\hline 2009-03-18 03:00:00.0 & 11.1 \\
\hline 2009-03-18 00:00:00.0 & 12.3 \\
\hline 2009-03-17 21:00:00.0 & 17.6 \\
\hline 2009-03-17 18:00:00.0 & 19.6 \\
\hline 2009-03-17 15:00:00.0 & 14.4 \\
\hline 2009-03-17 12:00:00.0 & 24.5 \\
\hline 2009-03-17 09:00:00.0 & 42.3 \\
\hline
\end{tabular}




\section{ANEXO 01 - RAJADAS DE VENTO REGISTRADAS EM SÃO MARTINHO DA SERRA / RS}

\begin{tabular}{|c|c|}
\hline DataHora & VelVentoMax \\
\hline 2009-03-17 06:00:00.0 & 19.8 \\
\hline 2009-03-17 03:00:00.0 & 19.1 \\
\hline 2009-03-17 00:00:00.0 & 11.2 \\
\hline 2009-03-16 21:00:00.0 & 10.9 \\
\hline 2009-03-16 18:00:00.0 & 12.2 \\
\hline 2009-03-16 15:00:00.0 & 17.6 \\
\hline 2009-03-16 12:00:00.0 & 14.8 \\
\hline 2009-03-16 09:00:00.0 & 11.7 \\
\hline 2009-03-16 06:00:00.0 & 9.5 \\
\hline 2009-03-16 03:00:00.0 & 10.4 \\
\hline 2009-03-16 00:00:00.0 & 17.2 \\
\hline 2009-03-15 21:00:00.0 & 24.1 \\
\hline 2009-03-15 18:00:00.0 & 20.4 \\
\hline 2009-03-15 15:00:00.0 & 36.5 \\
\hline 2009-03-15 12:00:00.0 & 31.8 \\
\hline 2009-03-15 09:00:00.0 & 10.6 \\
\hline 2009-03-15 06:00:00.0 & 9.6 \\
\hline 2009-03-15 03:00:00.0 & 9.4 \\
\hline 2009-03-15 00:00:00.0 & 6.4 \\
\hline 2009-03-14 21:00:00.0 & 17.7 \\
\hline 2009-03-14 18:00:00.0 & 15.5 \\
\hline 2009-03-14 15:00:00.0 & 5.3 \\
\hline 2009-03-14 12:00:00.0 & 7.4 \\
\hline 2009-03-14 09:00:00.0 & 5.2 \\
\hline 2009-03-14 06:00:00.0 & 5.8 \\
\hline 2009-03-14 03:00:00.0 & 5.3 \\
\hline 2009-03-14 00:00:00.0 & 14.3 \\
\hline 2009-03-13 21:00:00.0 & 22.6 \\
\hline 2009-03-13 18:00:00.0 & 20.9 \\
\hline 2009-03-13 15:00:00.0 & 18.7 \\
\hline 2009-03-13 12:00:00.0 & 30.3 \\
\hline 2009-03-13 09:00:00.0 & 6.9 \\
\hline 2009-03-13 06:00:00.0 & 4.5 \\
\hline 2009-03-13 03:00:00.0 & 5.0 \\
\hline 2009-03-13 00:00:00.0 & 10.6 \\
\hline 2009-03-12 21:00:00.0 & 30.9 \\
\hline 2009-03-12 18:00:00.0 & 17.2 \\
\hline 2009-03-12 15:00:00.0 & 14.6 \\
\hline 2009-03-12 12:00:00.0 & 11.1 \\
\hline 2009-03-12 09:00:00.0 & 12.3 \\
\hline 2009-03-12 06:00:00.0 & 12.3 \\
\hline 2009-03-12 03:00:00.0 & 15.1 \\
\hline 2009-03-12 00:00:00.0 & 14.4 \\
\hline 2009-03-11 21:00:00.0 & 12.7 \\
\hline 2009-03-11 18:00:00.0 & 17.6 \\
\hline 2009-03-11 15:00:00.0 & 17.0 \\
\hline 2009-03-11 12:00:00.0 & 18.3 \\
\hline 2009-03-11 09:00:00.0 & \\
\hline 2009-03-11 06:00:00.0 & 19.7 \\
\hline 2009-03-11 03:00:00.0 & 20.9 \\
\hline 2009-03-11 00:00:00.0 & 21.4 \\
\hline 2009-03-10 21:00:00.0 & 22.4 \\
\hline 2009-03-10 18:00:00.0 & 24.5 \\
\hline 2009-03-10 15:00:00.0 & 29.1 \\
\hline 2009-03-10 12:00:00.0 & 25.4 \\
\hline 2009-03-10 09:00:00.0 & 17.8 \\
\hline 2009-03-10 06:00:00.0 & 19.5 \\
\hline 2009-03-10 03:00:00.0 & 24.4 \\
\hline 2009-03-10 00:00:00.0 & 25.1 \\
\hline 2009-03-09 21:00:00.0 & 22.9 \\
\hline 2009-03-09 18:00:00.0 & 20.7 \\
\hline 2009-03-09 15:00:00.0 & 15.9 \\
\hline 2009-03-09 12:00:00.0 & 16.9 \\
\hline 2009-03-09 09:00:00.0 & 20.3 \\
\hline 2009-03-09 06:00:00.0 & 12.6 \\
\hline 2009-03-09 03:00:00.0 & 13.4 \\
\hline 2009-03-09 00:00:00.0 & 18.6 \\
\hline 2009-03-08 21:00:00.0 & \\
\hline 2009-03-08 18:00:00.0 & 19.5 \\
\hline
\end{tabular}

\begin{tabular}{|c|c|}
\hline DataHora & VelVentoMax \\
\hline 2009-03-08 15:00:00.0 & 18.2 \\
\hline 2009-03-08 12:00:00.0 & 13.1 \\
\hline 2009-03-08 09:00:00.0 & 9.0 \\
\hline 2009-03-08 06:00:00.0 & 12.6 \\
\hline 2009-03-08 03:00:00.0 & 14.3 \\
\hline 2009-03-08 00:00:00.0 & 16.9 \\
\hline 2009-03-07 21:00:00.0 & 18.5 \\
\hline 2009-03-07 18:00:00.0 & 30.9 \\
\hline 2009-03-07 15:00:00.0 & 14.2 \\
\hline 2009-03-07 12:00:00.0 & 16.9 \\
\hline 2009-03-07 09:00:00.0 & 44.1 \\
\hline 2009-03-07 06:00:00.0 & 17.2 \\
\hline 2009-03-07 03:00:00.0 & 11.6 \\
\hline 2009-03-07 00:00:00.0 & 13.6 \\
\hline 2009-03-06 21:00:00.0 & 17.0 \\
\hline 2009-03-06 18:00:00.0 & 14.2 \\
\hline 2009-03-06 15:00:00.0 & 17.9 \\
\hline 2009-03-06 12:00:00.0 & 17.4 \\
\hline 2009-03-06 09:00:00.0 & 11.5 \\
\hline 2009-03-06 06:00:00.0 & 13.8 \\
\hline 2009-03-06 03:00:00.0 & 37.4 \\
\hline 2009-03-06 00:00:00.0 & 15.3 \\
\hline 2009-03-05 21:00:00.0 & 22.5 \\
\hline 2009-03-05 18:00:00.0 & \\
\hline 2009-03-05 15:00:00.0 & 23.7 \\
\hline 2009-03-05 12:00:00.0 & 20.8 \\
\hline 2009-03-05 09:00:00.0 & 22.2 \\
\hline 2009-03-05 06:00:00.0 & 19.5 \\
\hline 2009-03-05 03:00:00.0 & 44.9 \\
\hline 2009-03-05 00:00:00.0 & 38.8 \\
\hline 2009-03-04 21:00:00.0 & 26.9 \\
\hline 2009-03-04 18:00:00.0 & 28.8 \\
\hline 2009-03-04 15:00:00.0 & 21.7 \\
\hline 2009-03-04 12:00:00.0 & 19.3 \\
\hline 2009-03-04 09:00:00.0 & 20.5 \\
\hline 2009-03-04 06:00:00.0 & 20.9 \\
\hline 2009-03-04 03:00:00.0 & 16.8 \\
\hline 2009-03-04 00:00:00.0 & 12.0 \\
\hline 2009-03-03 21:00:00.0 & 13.7 \\
\hline 2009-03-03 18:00:00.0 & 16.2 \\
\hline 2009-03-03 15:00:00.0 & 19.1 \\
\hline 2009-03-03 12:00:00.0 & 15.7 \\
\hline 2009-03-03 09:00:00.0 & 13.0 \\
\hline 2009-03-03 06:00:00.0 & 14.2 \\
\hline 2009-03-03 03:00:00.0 & 10.9 \\
\hline 2009-03-03 00:00:00.0 & 7.0 \\
\hline 2009-03-02 21:00:00.0 & 31.2 \\
\hline 2009-03-02 18:00:00.0 & 19.6 \\
\hline 2009-03-02 15:00:00.0 & 17.9 \\
\hline 2009-03-02 12:00:00.0 & 25.4 \\
\hline 2009-03-02 09:00:00.0 & 19.1 \\
\hline 2009-03-02 06:00:00.0 & \\
\hline 2009-03-02 03:00:00.0 & 17.3 \\
\hline 2009-03-02 00:00:00.0 & 21.7 \\
\hline 2009-03-01 21:00:00.0 & 18.2 \\
\hline 2009-03-01 18:00:00.0 & 22.8 \\
\hline 2009-03-01 15:00:00.0 & 21.2 \\
\hline 2009-03-01 12:00:00.0 & 12.9 \\
\hline 2009-03-01 09:00:00.0 & 14.7 \\
\hline 2009-03-01 06:00:00.0 & 17.5 \\
\hline 2009-03-01 03:00:00.0 & 16.9 \\
\hline 2009-03-01 00:00:00.0 & 14.0 \\
\hline 2009-02-28 21:00:00.0 & 13.5 \\
\hline 2009-02-28 18:00:00.0 & 14.2 \\
\hline 2009-02-28 15:00:00.0 & 22.2 \\
\hline 2009-02-28 12:00:00.0 & 25.1 \\
\hline 2009-02-28 09:00:00.0 & 18.5 \\
\hline 2009-02-28 06:00:00.0 & 14.0 \\
\hline 2009-02-28 03:00:00.0 & 14.2 \\
\hline
\end{tabular}

\begin{tabular}{|c|c|}
\hline DataHora & VelVentoMax \\
\hline 2009-02-28 00:00:00.0 & 20.2 \\
\hline 2009-02-27 21:00:00.0 & \\
\hline 2009-02-27 18:00:00.0 & 22.8 \\
\hline 2009-02-27 15:00:00.0 & 20.0 \\
\hline 2009-02-27 12:00:00.0 & 14.4 \\
\hline 2009-02-27 09:00:00.0 & 6.0 \\
\hline 2009-02-27 06:00:00.0 & 11.8 \\
\hline 2009-02-27 03:00:00.0 & 15.0 \\
\hline 2009-02-27 00:00:00.0 & 19.7 \\
\hline 2009-02-26 21:00:00.0 & \\
\hline 2009-02-26 18:00:00.0 & 17.7 \\
\hline 2009-02-26 15:00:00.0 & 27.1 \\
\hline 2009-02-26 12:00:00.0 & 36.9 \\
\hline 2009-02-26 09:00:00.0 & 14.2 \\
\hline 2009-02-26 06:00:00.0 & 10.6 \\
\hline 2009-02-26 03:00:00.0 & 14.0 \\
\hline 2009-02-26 00:00:00.0 & 20.2 \\
\hline 2009-02-25 21:00:00.0 & 19.2 \\
\hline 2009-02-25 18:00:00.0 & 22.1 \\
\hline 2009-02-25 15:00:00.0 & 25.5 \\
\hline 2009-02-25 12:00:00.0 & 20.8 \\
\hline 2009-02-25 09:00:00.0 & \\
\hline 2009-02-25 06:00:00.0 & 11.9 \\
\hline 2009-02-25 03:00:00.0 & 19.0 \\
\hline 2009-02-25 00:00:00.0 & 22.4 \\
\hline 2009-02-24 21:00:00.0 & 24.9 \\
\hline 2009-02-24 18:00:00.0 & 25.4 \\
\hline 2009-02-24 15:00:00.0 & 23.2 \\
\hline 2009-02-24 12:00:00.0 & 21.8 \\
\hline 2009-02-24 09:00:00.0 & 17.3 \\
\hline 2009-02-24 06:00:00.0 & 16.0 \\
\hline 2009-02-24 03:00:00.0 & 9.8 \\
\hline 2009-02-24 00:00:00.0 & 16.1 \\
\hline 2009-02-23 21:00:00.0 & 14.3 \\
\hline 2009-02-23 18:00:00.0 & 15.5 \\
\hline 2009-02-23 15:00:00.0 & 19.9 \\
\hline 2009-02-23 12:00:00.0 & 11.4 \\
\hline 2009-02-23 09:00:00.0 & \\
\hline 2009-02-23 06:00:00.0 & 16.3 \\
\hline 2009-02-23 03:00:00.0 & 38.7 \\
\hline 2009-02-23 00:00:00.0 & 15.5 \\
\hline 2009-02-22 21:00:00.0 & 19.8 \\
\hline 2009-02-22 18:00:00.0 & 20.8 \\
\hline 2009-02-22 15:00:00.0 & 19.2 \\
\hline 2009-02-22 12:00:00.0 & 15.9 \\
\hline 2009-02-22 09:00:00.0 & 12.2 \\
\hline 2009-02-22 06:00:00.0 & 13.3 \\
\hline 2009-02-22 03:00:00.0 & 12.0 \\
\hline 2009-02-22 00:00:00.0 & 11.2 \\
\hline 2009-02-21 21:00:00.0 & 10.5 \\
\hline 2009-02-21 18:00:00.0 & 30.8 \\
\hline 2009-02-21 15:00:00.0 & 1.2 \\
\hline 2009-02-21 12:00:00.0 & 29.6 \\
\hline 2009-02-21 09:00:00.0 & 17.8 \\
\hline 2009-02-21 06:00:00.0 & 18.3 \\
\hline 2009-02-21 03:00:00.0 & 23.6 \\
\hline 2009-02-21 00:00:00.0 & 17.0 \\
\hline 2009-02-20 21:00:00.0 & 4.1 \\
\hline 2009-02-20 18:00:00.0 & 20.6 \\
\hline 2009-02-20 15:00:00.0 & 21.3 \\
\hline 2009-02-20 12:00:00.0 & 19.6 \\
\hline 2009-02-20 09:00:00.0 & \\
\hline 2009-02-20 06:00:00.0 & 27.9 \\
\hline 2009-02-20 03:00:00.0 & 19.0 \\
\hline 2009-02-20 00:00:00.0 & 20.5 \\
\hline 2009-02-19 21:00:00.0 & 28.6 \\
\hline 2009-02-19 18:00:00.0 & 16.3 \\
\hline 2009-02-19 15:00:00.0 & 20.8 \\
\hline 2009-02-19 12:00:00.0 & 29.3 \\
\hline
\end{tabular}




\section{ANEXO 01 - RAJADAS DE VENTO REGISTRADAS EM SÃO MARTINHO DA SERRA / RS}

\begin{tabular}{|c|c|}
\hline DataHora & VelVentoMax \\
\hline 2009-02-19 09:00:00.0 & 19.3 \\
\hline 2009-02-19 06:00:00.0 & 20.3 \\
\hline 2009-02-19 03:00:00.0 & 12.8 \\
\hline 2009-02-19 00:00:00.0 & 19.6 \\
\hline 2009-02-18 21:00:00.0 & 19.2 \\
\hline 2009-02-18 18:00:00.0 & 17.7 \\
\hline 2009-02-18 15:00:00.0 & 17.9 \\
\hline 2009-02-18 12:00:00.0 & 11.9 \\
\hline 2009-02-18 09:00:00.0 & 14.2 \\
\hline 2009-02-18 06:00:00.0 & 13.7 \\
\hline 2009-02-18 03:00:00.0 & 8.0 \\
\hline 2009-02-18 00:00:00.0 & 8.4 \\
\hline 2009-02-17 21:00:00.0 & 5.6 \\
\hline 2009-02-17 18:00:00.0 & 16.5 \\
\hline 2009-02-17 15:00:00.0 & 13.7 \\
\hline 2009-02-17 12:00:00.0 & 11.2 \\
\hline 2009-02-17 09:00:00.0 & 12.4 \\
\hline 2009-02-17 06:00:00.0 & 7.7 \\
\hline 2009-02-17 03:00:00.0 & 7.4 \\
\hline 2009-02-17 00:00:00.0 & 7.0 \\
\hline 2009-02-16 21:00:00.0 & 20.7 \\
\hline 2009-02-16 18:00:00.0 & 18.2 \\
\hline 2009-02-16 15:00:00.0 & 24.4 \\
\hline 2009-02-16 12:00:00.0 & 15.3 \\
\hline 2009-02-16 09:00:00.0 & 19.0 \\
\hline 2009-02-16 06:00:00.0 & 18.5 \\
\hline 2009-02-16 03:00:00.0 & 15.3 \\
\hline 2009-02-16 00:00:00.0 & 18.6 \\
\hline 2009-02-15 21:00:00.0 & 17.3 \\
\hline 2009-02-15 18:00:00.0 & 12.3 \\
\hline 2009-02-15 15:00:00.0 & 19.6 \\
\hline 2009-02-15 12:00:00.0 & 21.3 \\
\hline 2009-02-15 09:00:00.0 & 35.1 \\
\hline 2009-02-15 06:00:00.0 & 17.3 \\
\hline 2009-02-15 03:00:00.0 & 14.6 \\
\hline 2009-02-15 00:00:00.0 & 17.9 \\
\hline 2009-02-14 21:00:00.0 & 18.6 \\
\hline 2009-02-14 18:00:00.0 & \\
\hline 2009-02-14 15:00:00.0 & 14.5 \\
\hline 2009-02-14 12:00:00.0 & 32.8 \\
\hline 2009-02-14 09:00:00.0 & 14.1 \\
\hline 2009-02-14 06:00:00.0 & 15.3 \\
\hline 2009-02-14 03:00:00.0 & \\
\hline 2009-02-14 00:00:00.0 & 15.4 \\
\hline 2009-02-13 21:00:00.0 & 6.1 \\
\hline 2009-02-13 18:00:00.0 & 12.0 \\
\hline 2009-02-13 15:00:00.0 & 18.7 \\
\hline 2009-02-13 12:00:00.0 & 20.9 \\
\hline 2009-02-13 09:00:00.0 & 15.6 \\
\hline 2009-02-13 06:00:00.0 & 16.6 \\
\hline 2009-02-13 03:00:00.0 & 14.5 \\
\hline 2009-02-13 00:00:00.0 & 16.1 \\
\hline 2009-02-12 21:00:00.0 & 20.4 \\
\hline 2009-02-12 18:00:00.0 & 21.0 \\
\hline 2009-02-12 15:00:00.0 & 26.0 \\
\hline 2009-02-12 12:00:00.0 & 21.2 \\
\hline 2009-02-12 09:00:00.0 & 16.5 \\
\hline 2009-02-12 06:00:00.0 & 23.7 \\
\hline 2009-02-12 03:00:00.0 & 21.0 \\
\hline 2009-02-12 00:00:00.0 & 17.8 \\
\hline 2009-02-11 21:00:00.0 & 26.0 \\
\hline 2009-02-11 18:00:00.0 & 25.3 \\
\hline 2009-02-11 15:00:00.0 & 29.4 \\
\hline 2009-02-11 12:00:00.0 & 22.0 \\
\hline 2009-02-11 09:00:00.0 & 35.1 \\
\hline 2009-02-11 06:00:00.0 & 24.0 \\
\hline 2009-02-11 03:00:00.0 & 20.4 \\
\hline 2009-02-11 00:00:00.0 & 26.7 \\
\hline 2009-02-10 21:00:00.0 & 17.3 \\
\hline
\end{tabular}

\begin{tabular}{|c|c|}
\hline DataHora & VelVentoMax \\
\hline 2009-02-10 18:00:00.0 & 20.2 \\
\hline 2009-02-10 15:00:00.0 & 18.5 \\
\hline 2009-02-10 12:00:00.0 & 20.6 \\
\hline 2009-02-10 09:00:00.0 & 16.7 \\
\hline 2009-02-10 06:00:00.0 & 17.3 \\
\hline 2009-02-10 03:00:00.0 & 15.5 \\
\hline 2009-02-10 00:00:00.0 & 15.5 \\
\hline 2009-02-09 21:00:00.0 & 15.5 \\
\hline 2009-02-09 18:00:00.0 & 18.8 \\
\hline 2009-02-09 15:00:00.0 & 15.4 \\
\hline 2009-02-09 12:00:00.0 & 13.5 \\
\hline 2009-02-09 09:00:00.0 & 17.0 \\
\hline 2009-02-09 06:00:00.0 & 20.1 \\
\hline 2009-02-09 03:00:00.0 & 17.9 \\
\hline 2009-02-09 00:00:00.0 & 13.4 \\
\hline 2009-02-08 21:00:00.0 & 15.2 \\
\hline 2009-02-08 18:00:00.0 & 14.5 \\
\hline 2009-02-08 15:00:00.0 & 29.0 \\
\hline 2009-02-08 12:00:00.0 & 14.8 \\
\hline 2009-02-08 09:00:00.0 & 14.7 \\
\hline 2009-02-08 06:00:00.0 & 16.2 \\
\hline 2009-02-08 03:00:00.0 & 12.0 \\
\hline 2009-02-08 00:00:00.0 & 9.6 \\
\hline 2009-02-07 21:00:00.0 & 16.9 \\
\hline 2009-02-07 18:00:00.0 & \\
\hline 2009-02-07 15:00:00.0 & 9.1 \\
\hline 2009-02-07 12:00:00.0 & 8.9 \\
\hline 2009-02-07 09:00:00.0 & 7.9 \\
\hline 2009-02-07 06:00:00.0 & 15.6 \\
\hline 2009-02-07 03:00:00.0 & 8.4 \\
\hline 2009-02-07 00:00:00.0 & 14.6 \\
\hline 2009-02-06 21:00:00.0 & 19.2 \\
\hline 2009-02-06 18:00:00.0 & 18.1 \\
\hline 2009-02-06 15:00:00.0 & 19.6 \\
\hline 2009-02-06 12:00:00.0 & 15.7 \\
\hline 2009-02-06 09:00:00.0 & \\
\hline 2009-02-06 06:00:00.0 & 22.1 \\
\hline 2009-02-06 03:00:00.0 & 15.7 \\
\hline 2009-02-06 00:00:00.0 & 15.9 \\
\hline 2009-02-05 21:00:00.0 & 16.9 \\
\hline 2009-02-05 18:00:00.0 & \\
\hline 2009-02-05 15:00:00.0 & 19.6 \\
\hline 2009-02-05 12:00:00.0 & 21.5 \\
\hline 2009-02-05 09:00:00.0 & 15.8 \\
\hline 2009-02-05 06:00:00.0 & 16.0 \\
\hline 2009-02-05 03:00:00.0 & 25.0 \\
\hline 2009-02-05 00:00:00.0 & 19.6 \\
\hline 2009-02-04 21:00:00.0 & 19.4 \\
\hline 2009-02-04 18:00:00.0 & \\
\hline 2009-02-04 15:00:00.0 & \\
\hline 2009-02-04 12:00:00.0 & 9.6 \\
\hline 2009-02-04 09:00:00.0 & 5.6 \\
\hline 2009-02-04 06:00:00.0 & 6.9 \\
\hline 2009-02-04 03:00:00.0 & 13.8 \\
\hline 2009-02-04 00:00:00.0 & 16.9 \\
\hline 2009-02-03 21:00:00.0 & \\
\hline 2009-02-03 18:00:00.0 & 25.2 \\
\hline 2009-02-03 15:00:00.0 & 28.4 \\
\hline 2009-02-03 12:00:00.0 & 24.9 \\
\hline 2009-02-03 09:00:00.0 & \\
\hline 2009-02-03 06:00:00.0 & \\
\hline 2009-02-03 03:00:00.0 & 26.9 \\
\hline 2009-02-03 00:00:00.0 & 41.7 \\
\hline 2009-02-02 21:00:00.0 & \\
\hline 2009-02-02 18:00:00.0 & \\
\hline 2009-02-02 15:00:00.0 & 22.9 \\
\hline 2009-02-02 12:00:00.0 & 22.3 \\
\hline 2009-02-02 09:00:00.0 & 20.9 \\
\hline 2009-02-02 06:00:00.0 & 12.7 \\
\hline
\end{tabular}

\begin{tabular}{|c|c|}
\hline DataHora & VelVentoMax \\
\hline 2009-02-02 03:00:00.0 & 16.3 \\
\hline 2009-02-02 00:00:00.0 & 17.2 \\
\hline 2009-02-01 21:00:00.0 & 20.2 \\
\hline 2009-02-01 18:00:00.0 & 20.5 \\
\hline 2009-02-01 15:00:00.0 & 20.2 \\
\hline 2009-02-01 12:00:00.0 & 9.4 \\
\hline 2009-02-01 09:00:00.0 & \\
\hline 2009-02-01 06:00:00.0 & 7.8 \\
\hline 2009-02-01 03:00:00.0 & 7.6 \\
\hline 2009-02-01 00:00:00.0 & 16.6 \\
\hline 2009-01-31 21:00:00.0 & \\
\hline 2009-01-31 18:00:00.0 & 30.9 \\
\hline 2009-01-31 15:00:00.0 & 30.4 \\
\hline 2009-01-31 12:00:00.0 & 25.4 \\
\hline 2009-01-31 09:00:00.0 & \\
\hline 2009-01-31 06:00:00.0 & 14.5 \\
\hline 2009-01-31 03:00:00.0 & 20.3 \\
\hline 2009-01-31 00:00:00.0 & 24.5 \\
\hline 2009-01-30 21:00:00.0 & 32.0 \\
\hline 2009-01-30 18:00:00.0 & 33.0 \\
\hline 2009-01-30 15:00:00.0 & 32.6 \\
\hline 2009-01-30 12:00:00.0 & 27.2 \\
\hline 2009-01-30 09:00:00.0 & 22.9 \\
\hline 2009-01-30 06:00:00.0 & 18.4 \\
\hline 2009-01-30 03:00:00.0 & 13.1 \\
\hline 2009-01-30 00:00:00.0 & 12.7 \\
\hline 2009-01-29 21:00:00.0 & 27.9 \\
\hline 2009-01-29 18:00:00.0 & 22.6 \\
\hline 2009-01-29 15:00:00.0 & 22.0 \\
\hline 2009-01-29 12:00:00.0 & 15.2 \\
\hline 2009-01-29 09:00:00.0 & 11.0 \\
\hline 2009-01-29 06:00:00.0 & 13.2 \\
\hline 2009-01-29 03:00:00.0 & 15.8 \\
\hline 2009-01-29 00:00:00.0 & 9.2 \\
\hline 2009-01-28 21:00:00.0 & 15.1 \\
\hline 2009-01-28 18:00:00.0 & 29.7 \\
\hline 2009-01-28 15:00:00.0 & 26.1 \\
\hline 2009-01-28 12:00:00.0 & 20.2 \\
\hline 2009-01-28 09:00:00.0 & 19.0 \\
\hline 2009-01-28 06:00:00.0 & 17.3 \\
\hline 2009-01-28 03:00:00.0 & 19.4 \\
\hline 2009-01-28 00:00:00.0 & 23.1 \\
\hline 2009-01-27 21:00:00.0 & \\
\hline 2009-01-27 18:00:00.0 & 16.3 \\
\hline 2009-01-27 15:00:00.0 & 14.0 \\
\hline 2009-01-27 12:00:00.0 & 15.8 \\
\hline 2009-01-27 09:00:00.0 & 12.3 \\
\hline 2009-01-27 06:00:00.0 & 15.0 \\
\hline 2009-01-27 03:00:00.0 & 15.9 \\
\hline 2009-01-27 00:00:00.0 & 13.4 \\
\hline 2009-01-26 21:00:00.0 & 22.2 \\
\hline 2009-01-26 18:00:00.0 & 25.2 \\
\hline 2009-01-26 15:00:00.0 & 15.7 \\
\hline 2009-01-26 12:00:00.0 & 16.5 \\
\hline 2009-01-26 09:00:00.0 & 27.1 \\
\hline 2009-01-26 06:00:00.0 & 8.3 \\
\hline 2009-01-26 03:00:00.0 & 10.9 \\
\hline 2009-01-26 00:00:00.0 & 10.7 \\
\hline 2009-01-25 21:00:00.0 & 12.9 \\
\hline 2009-01-25 18:00:00.0 & 14.8 \\
\hline 2009-01-25 15:00:00.0 & 21.5 \\
\hline 2009-01-25 12:00:00.0 & 24.4 \\
\hline 2009-01-25 09:00:00.0 & 19.9 \\
\hline 2009-01-25 06:00:00.0 & 20.2 \\
\hline 2009-01-25 03:00:00.0 & 19.0 \\
\hline 2009-01-25 00:00:00.0 & 16.9 \\
\hline 2009-01-24 21:00:00.0 & 17.3 \\
\hline 2009-01-24 18:00:00.0 & 16.9 \\
\hline 2009-01-24 15:00:00.0 & 24.2 \\
\hline
\end{tabular}




\section{ANEXO 01 - RAJADAS DE VENTO REGISTRADAS EM SÃO MARTINHO DA SERRA / RS}

\begin{tabular}{|c|c|}
\hline DataHora & VelVentoMax \\
\hline 2009-01-24 12:00:00.0 & 25.2 \\
\hline 2009-01-24 09:00:00.0 & 24.1 \\
\hline 2009-01-24 06:00:00.0 & 21.6 \\
\hline 2009-01-24 03:00:00.0 & 12.6 \\
\hline 2009-01-24 00:00:00.0 & 17.8 \\
\hline 2009-01-23 21:00:00.0 & 23.1 \\
\hline 2009-01-23 18:00:00.0 & 23.6 \\
\hline 2009-01-23 15:00:00.0 & 25.0 \\
\hline 2009-01-23 12:00:00.0 & 22.8 \\
\hline 2009-01-23 09:00:00.0 & 21.2 \\
\hline 2009-01-23 06:00:00.0 & 17.4 \\
\hline 2009-01-23 03:00:00.0 & 17.8 \\
\hline 2009-01-23 00:00:00.0 & 19.7 \\
\hline 2009-01-22 21:00:00.0 & 21.6 \\
\hline 2009-01-22 18:00:00.0 & 19.2 \\
\hline 2009-01-22 15:00:00.0 & 24.0 \\
\hline 2009-01-22 12:00:00.0 & 25.4 \\
\hline 2009-01-22 09:00:00.0 & 19.0 \\
\hline 2009-01-22 06:00:00.0 & 16.6 \\
\hline 2009-01-22 03:00:00.0 & 16.8 \\
\hline 2009-01-22 00:00:00.0 & 20.7 \\
\hline 2009-01-21 21:00:00.0 & 21.4 \\
\hline 2009-01-21 18:00:00.0 & \\
\hline 2009-01-21 15:00:00.0 & 20.5 \\
\hline 2009-01-21 12:00:00.0 & 19.7 \\
\hline 2009-01-21 09:00:00.0 & 15.2 \\
\hline 2009-01-21 06:00:00.0 & 16.0 \\
\hline 2009-01-21 03:00:00.0 & 15.8 \\
\hline 2009-01-21 00:00:00.0 & 18.8 \\
\hline 2009-01-20 21:00:00.0 & 22.6 \\
\hline 2009-01-20 18:00:00.0 & 20.9 \\
\hline 2009-01-20 15:00:00.0 & \\
\hline 2009-01-20 12:00:00.0 & 13.5 \\
\hline 2009-01-20 09:00:00.0 & 12.5 \\
\hline 2009-01-20 06:00:00.0 & 14.8 \\
\hline 2009-01-20 03:00:00.0 & 11.5 \\
\hline 2009-01-20 00:00:00.0 & 29.9 \\
\hline 2009-01-19 21:00:00.0 & 28.0 \\
\hline 2009-01-19 18:00:00.0 & 32.2 \\
\hline 2009-01-19 15:00:00.0 & \\
\hline 2009-01-19 12:00:00.0 & \\
\hline 2009-01-19 09:00:00.0 & 17.7 \\
\hline 2009-01-19 06:00:00.0 & 17.8 \\
\hline 2009-01-19 03:00:00.0 & 22.3 \\
\hline 2009-01-19 00:00:00.0 & 16.4 \\
\hline 2009-01-18 21:00:00.0 & 23.8 \\
\hline 2009-01-18 18:00:00.0 & 32.2 \\
\hline 2009-01-18 15:00:00.0 & 22.2 \\
\hline 2009-01-18 12:00:00.0 & 15.6 \\
\hline 2009-01-18 09:00:00.0 & 11.7 \\
\hline 2009-01-18 06:00:00.0 & 13.7 \\
\hline 2009-01-18 03:00:00.0 & 16.7 \\
\hline 2009-01-18 00:00:00.0 & 10.9 \\
\hline 2009-01-17 21:00:00.0 & 22.3 \\
\hline 2009-01-17 18:00:00.0 & \\
\hline 2009-01-17 15:00:00.0 & 15.8 \\
\hline 2009-01-17 12:00:00.0 & 12.0 \\
\hline 2009-01-17 09:00:00.0 & 11.0 \\
\hline 2009-01-17 06:00:00.0 & \\
\hline 2009-01-17 03:00:00.0 & 16.9 \\
\hline 2009-01-17 00:00:00.0 & 19.3 \\
\hline 2009-01-16 21:00:00.0 & 19.5 \\
\hline 2009-01-16 18:00:00.0 & 15.5 \\
\hline 2009-01-16 15:00:00.0 & 11.7 \\
\hline 2009-01-16 12:00:00.0 & 16.3 \\
\hline 2009-01-16 09:00:00.0 & 13.1 \\
\hline 2009-01-16 06:00:00.0 & 12.5 \\
\hline 2009-01-16 03:00:00.0 & 10.4 \\
\hline 2009-01-16 00:00:00.0 & 13.1 \\
\hline
\end{tabular}

\begin{tabular}{|c|c|}
\hline DataHora & VelVentoMax \\
\hline 2009-01-15 21:00:00.0 & 17.2 \\
\hline 2009-01-15 18:00:00.0 & 15.3 \\
\hline 2009-01-15 15:00:00.0 & 10.7 \\
\hline 2009-01-15 12:00:00.0 & 11.5 \\
\hline 2009-01-15 09:00:00.0 & 8.5 \\
\hline 2009-01-15 06:00:00.0 & 10.1 \\
\hline 2009-01-15 03:00:00.0 & 15.0 \\
\hline 2009-01-15 00:00:00.0 & 16.5 \\
\hline 2009-01-14 21:00:00.0 & 20.1 \\
\hline 2009-01-14 18:00:00.0 & 21.0 \\
\hline 2009-01-14 15:00:00.0 & 19.4 \\
\hline 2009-01-14 12:00:00.0 & 15.2 \\
\hline 2009-01-14 09:00:00.0 & 14.4 \\
\hline 2009-01-14 06:00:00.0 & 11.9 \\
\hline 2009-01-14 03:00:00.0 & 16.7 \\
\hline 2009-01-14 00:00:00.0 & 27.0 \\
\hline 2009-01-13 21:00:00.0 & 42.1 \\
\hline 2009-01-13 18:00:00.0 & 27.0 \\
\hline 2009-01-13 15:00:00.0 & 36.8 \\
\hline 2009-01-13 12:00:00.0 & 21.0 \\
\hline 2009-01-13 09:00:00.0 & 27.2 \\
\hline 2009-01-13 06:00:00.0 & 37.4 \\
\hline 2009-01-13 03:00:00.0 & 13.2 \\
\hline 2009-01-13 00:00:00.0 & 14.8 \\
\hline 2009-01-12 21:00:00.0 & 16.8 \\
\hline 2009-01-12 18:00:00.0 & 12.1 \\
\hline 2009-01-12 15:00:00.0 & 10.4 \\
\hline 2009-01-12 12:00:00.0 & 7.7 \\
\hline 2009-01-12 09:00:00.0 & 7.8 \\
\hline 2009-01-12 06:00:00.0 & 12.1 \\
\hline 2009-01-12 03:00:00.0 & \\
\hline 2009-01-12 00:00:00.0 & \\
\hline 2009-01-11 21:00:00.0 & \\
\hline 2009-01-11 18:00:00.0 & 20.0 \\
\hline 2009-01-11 15:00:00.0 & 18.4 \\
\hline 2009-01-11 12:00:00.0 & 18.7 \\
\hline 2009-01-11 09:00:00.0 & 18.7 \\
\hline 2009-01-11 06:00:00.0 & 21.0 \\
\hline 2009-01-11 03:00:00.0 & 20.5 \\
\hline 2009-01-11 00:00:00.0 & 35.0 \\
\hline 2009-01-10 21:00:00.0 & 25.4 \\
\hline 2009-01-10 18:00:00.0 & 21.6 \\
\hline 2009-01-10 15:00:00.0 & 34.2 \\
\hline 2009-01-10 12:00:00.0 & 17.9 \\
\hline 2009-01-10 09:00:00.0 & 14.2 \\
\hline 2009-01-10 06:00:00.0 & \\
\hline 2009-01-10 03:00:00.0 & 17.2 \\
\hline 2009-01-10 00:00:00.0 & 22.8 \\
\hline 2009-01-09 21:00:00.0 & 25.2 \\
\hline 2009-01-09 18:00:00.0 & 21.1 \\
\hline 2009-01-09 15:00:00.0 & 19.8 \\
\hline 2009-01-09 12:00:00.0 & 16.0 \\
\hline 2009-01-09 09:00:00.0 & 22.9 \\
\hline 2009-01-09 06:00:00.0 & \\
\hline 2009-01-09 03:00:00.0 & \\
\hline 2009-01-09 00:00:00.0 & \\
\hline 2009-01-08 21:00:00.0 & 35.4 \\
\hline 2009-01-08 18:00:00.0 & 17.4 \\
\hline 2009-01-08 15:00:00.0 & 13.6 \\
\hline 2009-01-08 12:00:00.0 & 17.0 \\
\hline 2009-01-08 09:00:00.0 & 19.3 \\
\hline 2009-01-08 06:00:00.0 & 20.4 \\
\hline 2009-01-08 03:00:00.0 & 17.0 \\
\hline 2009-01-08 00:00:00.0 & 10.0 \\
\hline 2009-01-07 21:00:00.0 & 16.5 \\
\hline 2009-01-07 18:00:00.0 & 24.9 \\
\hline 2009-01-07 15:00:00.0 & 19.7 \\
\hline 2009-01-07 12:00:00.0 & 13.6 \\
\hline 2009-01-07 09:00:00.0 & 16.4 \\
\hline
\end{tabular}

\begin{tabular}{|c|c|}
\hline DataHora & VelVentoMax \\
\hline 2009-01-07 06:00:00.0 & 14.0 \\
\hline 2009-01-07 03:00:00.0 & 13.0 \\
\hline 2009-01-07 00:00:00.0 & 10.4 \\
\hline 2009-01-06 21:00:00.0 & 14.8 \\
\hline 2009-01-06 18:00:00.0 & 17.1 \\
\hline 2009-01-06 15:00:00.0 & 16.3 \\
\hline 2009-01-06 12:00:00.0 & 18.4 \\
\hline 2009-01-06 09:00:00.0 & \\
\hline 2009-01-06 06:00:00.0 & 15.7 \\
\hline 2009-01-06 03:00:00.0 & 16.2 \\
\hline 2009-01-06 00:00:00.0 & 18.0 \\
\hline 2009-01-05 21:00:00.0 & 20.4 \\
\hline 2009-01-05 18:00:00.0 & 19.2 \\
\hline 2009-01-05 15:00:00.0 & 20.9 \\
\hline 2009-01-05 12:00:00.0 & 22.1 \\
\hline 2009-01-05 09:00:00.0 & \\
\hline 2009-01-05 06:00:00.0 & 14.0 \\
\hline 2009-01-05 03:00:00.0 & 13.2 \\
\hline 2009-01-05 00:00:00.0 & 19.0 \\
\hline 2009-01-04 21:00:00.0 & 23.1 \\
\hline 2009-01-04 18:00:00.0 & 22.5 \\
\hline 2009-01-04 15:00:00.0 & 24.1 \\
\hline 2009-01-04 12:00:00.0 & 22.0 \\
\hline 2009-01-04 09:00:00.0 & 11.0 \\
\hline 2009-01-04 06:00:00.0 & 9.4 \\
\hline 2009-01-04 03:00:00.0 & 20.4 \\
\hline 2009-01-04 00:00:00.0 & 25.6 \\
\hline 2009-01-03 21:00:00.0 & 30.1 \\
\hline 2009-01-03 18:00:00.0 & 34.8 \\
\hline 2009-01-03 15:00:00.0 & 39.8 \\
\hline 2009-01-03 12:00:00.0 & 29.1 \\
\hline 2009-01-03 09:00:00.0 & \\
\hline 2009-01-03 06:00:00.0 & 30.1 \\
\hline 2009-01-03 03:00:00.0 & 28.3 \\
\hline 2009-01-03 00:00:00.0 & 28.3 \\
\hline 2009-01-02 21:00:00.0 & 34.2 \\
\hline 2009-01-02 18:00:00.0 & 31.1 \\
\hline 2009-01-02 15:00:00.0 & 27.7 \\
\hline 2009-01-02 12:00:00.0 & 29.4 \\
\hline 2009-01-02 09:00:00.0 & \\
\hline 2009-01-02 06:00:00.0 & 19.8 \\
\hline 2009-01-02 03:00:00.0 & 27.8 \\
\hline 2009-01-02 00:00:00.0 & 32.2 \\
\hline 2009-01-01 21:00:00.0 & 30.4 \\
\hline 2009-01-01 18:00:00.0 & 28.2 \\
\hline 2009-01-01 15:00:00.0 & 30.9 \\
\hline 2009-01-01 12:00:00.0 & 22.3 \\
\hline 2009-01-01 09:00:00.0 & \\
\hline 2009-01-01 06:00:00.0 & 15.7 \\
\hline 2009-01-01 03:00:00.0 & 25.5 \\
\hline 2009-01-01 00:00:00.0 & 20.1 \\
\hline 2008-12-31 21:00:00.0 & 21.7 \\
\hline 2008-12-31 18:00:00.0 & 18.8 \\
\hline 2008-12-31 15:00:00.0 & 15.9 \\
\hline 2008-12-31 12:00:00.0 & 11.1 \\
\hline 2008-12-31 09:00:00.0 & 12.0 \\
\hline 2008-12-31 06:00:00.0 & 16.5 \\
\hline 2008-12-31 03:00:00.0 & 16.2 \\
\hline 2008-12-31 00:00:00.0 & 19.6 \\
\hline 2008-12-30 21:00:00.0 & 33.5 \\
\hline 2008-12-30 18:00:00.0 & 18.1 \\
\hline $2008-12-30$ 15:00:00.0 & 17.2 \\
\hline 2008-12-30 12:00:00.0 & 19.1 \\
\hline 2008-12-30 09:00:00.0 & 12.9 \\
\hline 2008-12-30 06:00:00.0 & 28.2 \\
\hline 2008-12-30 03:00:00.0 & 32.7 \\
\hline 2008-12-30 00:00:00.0 & 25.7 \\
\hline $2008-12-29$ 21:00:00.0 & 26.1 \\
\hline 2008-12-29 18:00:00.0 & 30.1 \\
\hline
\end{tabular}




\section{ANEXO 01 - RAJADAS DE VENTO REGISTRADAS EM SÃO MARTINHO DA SERRA / RS}

\begin{tabular}{|c|c|}
\hline DataHora & VelVentoMax \\
\hline 2008-12-29 15:00:00.0 & 47.8 \\
\hline 2008-12-29 12:00:00.0 & 16.6 \\
\hline 2008-12-29 09:00:00.0 & 11.5 \\
\hline 2008-12-29 06:00:00.0 & 10.3 \\
\hline 2008-12-29 03:00:00.0 & 18.9 \\
\hline 2008-12-29 00:00:00.0 & 13.3 \\
\hline 2008-12-28 21:00:00.0 & 13.7 \\
\hline 2008-12-28 18:00:00.0 & 42.0 \\
\hline 2008-12-28 15:00:00.0 & 21.8 \\
\hline 2008-12-28 12:00:00.0 & 18.2 \\
\hline 2008-12-28 09:00:00.0 & 20.1 \\
\hline 2008-12-28 06:00:00.0 & 40.1 \\
\hline 2008-12-28 03:00:00.0 & 16.6 \\
\hline 2008-12-28 00:00:00.0 & 18.0 \\
\hline 2008-12-27 21:00:00.0 & 21.4 \\
\hline 2008-12-27 18:00:00.0 & 19.1 \\
\hline 2008-12-27 15:00:00.0 & 22.6 \\
\hline 2008-12-27 12:00:00.0 & 25.6 \\
\hline 2008-12-27 09:00:00.0 & 21.6 \\
\hline 2008-12-27 06:00:00.0 & 16.5 \\
\hline 2008-12-27 03:00:00.0 & 18.8 \\
\hline 2008-12-27 00:00:00.0 & 19.8 \\
\hline 2008-12-26 21:00:00.0 & 21.5 \\
\hline 2008-12-26 18:00:00.0 & 22.9 \\
\hline 2008-12-26 15:00:00.0 & 21.9 \\
\hline 2008-12-26 12:00:00.0 & 21.5 \\
\hline 2008-12-26 09:00:00.0 & 11.5 \\
\hline 2008-12-26 06:00:00.0 & 16.2 \\
\hline 2008-12-26 03:00:00.0 & 18.4 \\
\hline 2008-12-26 00:00:00.0 & 14.4 \\
\hline 2008-12-25 21:00:00.0 & 18.2 \\
\hline 2008-12-25 18:00:00.0 & 21.6 \\
\hline 2008-12-25 15:00:00.0 & \\
\hline 2008-12-25 12:00:00.0 & 14.5 \\
\hline 2008-12-25 09:00:00.0 & 15.8 \\
\hline 2008-12-25 06:00:00.0 & 10.1 \\
\hline 2008-12-25 03:00:00.0 & 11.7 \\
\hline 2008-12-25 00:00:00.0 & 10.6 \\
\hline 2008-12-24 21:00:00.0 & 16.4 \\
\hline 2008-12-24 18:00:00.0 & 12.8 \\
\hline 2008-12-24 15:00:00.0 & 10.1 \\
\hline 2008-12-24 12:00:00.0 & 23.5 \\
\hline 2008-12-24 09:00:00.0 & 11.5 \\
\hline 2008-12-24 06:00:00.0 & 11.7 \\
\hline 2008-12-24 03:00:00.0 & 6.5 \\
\hline 2008-12-24 00:00:00.0 & 15.3 \\
\hline 2008-12-23 21:00:00.0 & 19.8 \\
\hline 2008-12-23 18:00:00.0 & 22.8 \\
\hline 2008-12-23 15:00:00.0 & 17.9 \\
\hline 2008-12-23 12:00:00.0 & 19.6 \\
\hline 2008-12-23 09:00:00.0 & 18.5 \\
\hline 2008-12-23 06:00:00.0 & 17.1 \\
\hline 2008-12-23 03:00:00.0 & \\
\hline 2008-12-23 00:00:00.0 & \\
\hline 2008-12-22 21:00:00.0 & \\
\hline 2008-12-22 18:00:00.0 & 23.4 \\
\hline 2008-12-22 15:00:00.0 & \\
\hline 2008-12-22 12:00:00.0 & 21.4 \\
\hline 2008-12-22 09:00:00.0 & 16.4 \\
\hline 2008-12-22 06:00:00.0 & 15.3 \\
\hline 2008-12-22 03:00:00.0 & 13.3 \\
\hline 2008-12-22 00:00:00.0 & 17.5 \\
\hline 2008-12-21 21:00:00.0 & 21.0 \\
\hline 2008-12-21 18:00:00.0 & 15.8 \\
\hline 2008-12-21 15:00:00.0 & 18.2 \\
\hline 2008-12-21 12:00:00.0 & 20.7 \\
\hline 2008-12-21 09:00:00.0 & 19.0 \\
\hline 2008-12-21 06:00:00.0 & \\
\hline 2008-12-21 03:00:00.0 & 16.1 \\
\hline
\end{tabular}

\begin{tabular}{|c|c|}
\hline DataHora & VelVentoMax \\
\hline 2008-12-21 00:00:00.0 & 16.5 \\
\hline 2008-12-20 21:00:00.0 & 21.8 \\
\hline $2008-12-20$ 18:00:00.0 & 19.7 \\
\hline 2008-12-20 15:00:00.0 & 16.2 \\
\hline 2008-12-20 12:00:00.0 & 19.5 \\
\hline 2008-12-20 09:00:00.0 & 17.9 \\
\hline 2008-12-20 06:00:00.0 & 18.7 \\
\hline 2008-12-20 03:00:00.0 & 15.6 \\
\hline 2008-12-20 00:00:00.0 & 18.3 \\
\hline 2008-12-19 21:00:00.0 & 19.3 \\
\hline 2008-12-19 18:00:00.0 & 18.6 \\
\hline 2008-12-19 15:00:00.0 & 16.6 \\
\hline 2008-12-19 12:00:00.0 & 21.7 \\
\hline 2008-12-19 09:00:00.0 & 20.1 \\
\hline 2008-12-19 06:00:00.0 & 23.2 \\
\hline 2008-12-19 03:00:00.0 & 18.3 \\
\hline 2008-12-19 00:00:00.0 & 23.5 \\
\hline 2008-12-18 21:00:00.0 & 24.4 \\
\hline 2008-12-18 18:00:00.0 & 20.8 \\
\hline 2008-12-18 15:00:00.0 & 15.2 \\
\hline 2008-12-18 12:00:00.0 & 3.5 \\
\hline 2008-12-18 09:00:00.0 & 13.9 \\
\hline 2008-12-18 06:00:00.0 & 18.4 \\
\hline 2008-12-18 03:00:00.0 & 14.8 \\
\hline 2008-12-18 00:00:00.0 & 21.2 \\
\hline 2008-12-17 21:00:00.0 & 21.0 \\
\hline 2008-12-17 18:00:00.0 & 20.1 \\
\hline 2008-12-17 15:00:00.0 & 19.5 \\
\hline 2008-12-17 12:00:00.0 & 20.6 \\
\hline 2008-12-17 09:00:00.0 & 16.8 \\
\hline 2008-12-17 06:00:00.0 & 16.1 \\
\hline 2008-12-17 03:00:00.0 & 14.7 \\
\hline 2008-12-17 00:00:00.0 & 27.2 \\
\hline 2008-12-16 21:00:00.0 & 24.7 \\
\hline 2008-12-16 18:00:00.0 & 22.4 \\
\hline 2008-12-16 15:00:00.0 & 20.4 \\
\hline 2008-12-16 12:00:00.0 & 21.7 \\
\hline 2008-12-16 09:00:00.0 & 16.9 \\
\hline 2008-12-16 06:00:00.0 & 14.3 \\
\hline 2008-12-16 03:00:00.0 & 18.4 \\
\hline 2008-12-16 00:00:00.0 & 26.7 \\
\hline 2008-12-15 21:00:00.0 & 21.5 \\
\hline 2008-12-15 18:00:00.0 & 19.4 \\
\hline 2008-12-15 15:00:00.0 & 11.4 \\
\hline 2008-12-15 12:00:00.0 & 11.4 \\
\hline 2008-12-15 09:00:00.0 & 13.0 \\
\hline 2008-12-15 06:00:00.0 & 7.6 \\
\hline 2008-12-15 03:00:00.0 & 8.7 \\
\hline 2008-12-15 00:00:00.0 & 14.0 \\
\hline 2008-12-14 21:00:00.0 & 13.3 \\
\hline 2008-12-14 18:00:00.0 & 21.0 \\
\hline 2008-12-14 15:00:00.0 & 16.6 \\
\hline 2008-12-14 12:00:00.0 & 18.1 \\
\hline 2008-12-14 09:00:00.0 & 17.0 \\
\hline 2008-12-14 06:00:00.0 & 15.4 \\
\hline 2008-12-14 03:00:00.0 & 14.8 \\
\hline 2008-12-14 00:00:00.0 & 24.1 \\
\hline 2008-12-13 21:00:00.0 & 25.5 \\
\hline 2008-12-13 18:00:00.0 & 19.2 \\
\hline 2008-12-13 15:00:00.0 & 19.4 \\
\hline 2008-12-13 12:00:00.0 & 27.0 \\
\hline 2008-12-13 09:00:00.0 & 24.1 \\
\hline 2008-12-13 06:00:00.0 & 21.6 \\
\hline 2008-12-13 03:00:00.0 & 19.1 \\
\hline 2008-12-13 00:00:00.0 & 24.9 \\
\hline 2008-12-12 21:00:00.0 & 24.0 \\
\hline 2008-12-12 18:00:00.0 & 29.7 \\
\hline 2008-12-12 15:00:00.0 & 28.3 \\
\hline 2008-12-12 12:00:00.0 & 28.9 \\
\hline
\end{tabular}

\begin{tabular}{|c|c|}
\hline DataHora & VelVentoMax \\
\hline 2008-12-12 09:00:00.0 & \\
\hline 2008-12-12 06:00:00.0 & 20.4 \\
\hline 2008-12-12 03:00:00.0 & 30.6 \\
\hline 2008-12-12 00:00:00.0 & \\
\hline 2008-12-11 21:00:00.0 & \\
\hline 2008-12-11 18:00:00.0 & 25.0 \\
\hline 2008-12-11 15:00:00.0 & 27.9 \\
\hline 2008-12-11 12:00:00.0 & 32.0 \\
\hline 2008-12-11 09:00:00.0 & 18.6 \\
\hline 2008-12-11 06:00:00.0 & 16.9 \\
\hline 2008-12-11 03:00:00.0 & 22.1 \\
\hline 2008-12-11 00:00:00.0 & 25.4 \\
\hline 2008-12-10 21:00:00.0 & \\
\hline 2008-12-10 18:00:00.0 & 17.7 \\
\hline 2008-12-10 15:00:00.0 & 14.3 \\
\hline 2008-12-10 12:00:00.0 & 10.7 \\
\hline 2008-12-10 09:00:00.0 & 7.2 \\
\hline 2008-12-10 06:00:00.0 & 14.0 \\
\hline 2008-12-10 03:00:00.0 & 18.7 \\
\hline 2008-12-10 00:00:00.0 & 24.2 \\
\hline 2008-12-09 21:00:00.0 & 49.7 \\
\hline 2008-12-09 18:00:00.0 & 0.9 \\
\hline 2008-12-09 15:00:00.0 & 16.9 \\
\hline 2008-12-09 12:00:00.0 & 20.3 \\
\hline 2008-12-09 09:00:00.0 & 16.3 \\
\hline 2008-12-09 06:00:00.0 & 10.2 \\
\hline 2008-12-09 03:00:00.0 & 9.8 \\
\hline 2008-12-09 00:00:00.0 & 12.9 \\
\hline 2008-12-08 21:00:00.0 & 18.6 \\
\hline 2008-12-08 18:00:00.0 & 35.2 \\
\hline 2008-12-08 15:00:00.0 & 20.9 \\
\hline 2008-12-08 12:00:00.0 & 16.3 \\
\hline 2008-12-08 09:00:00.0 & 11.5 \\
\hline 2008-12-08 06:00:00.0 & 12.8 \\
\hline 2008-12-08 03:00:00.0 & 11.4 \\
\hline 2008-12-08 00:00:00.0 & 19.5 \\
\hline 2008-12-07 21:00:00.0 & 20.3 \\
\hline 2008-12-07 18:00:00.0 & 25.0 \\
\hline 2008-12-07 15:00:00.0 & 20.8 \\
\hline 2008-12-07 12:00:00.0 & 21.7 \\
\hline 2008-12-07 09:00:00.0 & 18.1 \\
\hline 2008-12-07 06:00:00.0 & 16.4 \\
\hline 2008-12-07 03:00:00.0 & 13.2 \\
\hline 2008-12-07 00:00:00.0 & 16.8 \\
\hline 2008-12-06 21:00:00.0 & 18.5 \\
\hline 2008-12-06 18:00:00.0 & 15.9 \\
\hline 2008-12-06 15:00:00.0 & 19.5 \\
\hline 2008-12-06 12:00:00.0 & 20.0 \\
\hline 2008-12-06 09:00:00.0 & 14.9 \\
\hline 2008-12-06 06:00:00.0 & 13.3 \\
\hline 2008-12-06 03:00:00.0 & 18.4 \\
\hline 2008-12-06 00:00:00.0 & 18.4 \\
\hline 2008-12-05 21:00:00.0 & 6.8 \\
\hline 2008-12-05 18:00:00.0 & 18.1 \\
\hline 2008-12-05 15:00:00.0 & 20.6 \\
\hline 2008-12-05 12:00:00.0 & 20.8 \\
\hline 2008-12-05 09:00:00.0 & 15.3 \\
\hline 2008-12-05 06:00:00.0 & 14.3 \\
\hline 2008-12-05 03:00:00.0 & 10.3 \\
\hline 2008-12-05 00:00:00.0 & 15.8 \\
\hline 2008-12-04 21:00:00.0 & 19.1 \\
\hline 2008-12-04 18:00:00.0 & 21.9 \\
\hline 2008-12-04 15:00:00.0 & 15.5 \\
\hline 2008-12-04 12:00:00.0 & 14.6 \\
\hline 2008-12-04 09:00:00.0 & 9.7 \\
\hline 2008-12-04 06:00:00.0 & 10.7 \\
\hline 2008-12-04 03:00:00.0 & 6.1 \\
\hline 2008-12-04 00:00:00.0 & 15.7 \\
\hline 2008-12-03 21:00:00.0 & 20.6 \\
\hline
\end{tabular}




\section{ANEXO 01 - RAJADAS DE VENTO REGISTRADAS EM SÃO MARTINHO DA SERRA / RS}

\begin{tabular}{|c|c|}
\hline DataHora & VelVentoMax \\
\hline 2008-12-03 18:00:00.0 & 18.9 \\
\hline 2008-12-03 15:00:00.0 & 18.3 \\
\hline 2008-12-03 12:00:00.0 & 17.3 \\
\hline 2008-12-03 09:00:00.0 & 22.4 \\
\hline 2008-12-03 06:00:00.0 & 22.1 \\
\hline 2008-12-03 03:00:00.0 & 26.3 \\
\hline 2008-12-03 00:00:00.0 & 32.4 \\
\hline 2008-12-02 21:00:00.0 & 24.7 \\
\hline 2008-12-02 18:00:00.0 & 4.5 \\
\hline 2008-12-02 15:00:00.0 & 18.7 \\
\hline 2008-12-02 12:00:00.0 & 12.4 \\
\hline 2008-12-02 09:00:00.0 & 8.1 \\
\hline 2008-12-02 06:00:00.0 & 6.6 \\
\hline 2008-12-02 03:00:00.0 & 7.2 \\
\hline 2008-12-02 00:00:00.0 & 18.4 \\
\hline 2008-12-01 21:00:00.0 & 20.3 \\
\hline 2008-12-01 18:00:00.0 & 22.5 \\
\hline 2008-12-01 15:00:00.0 & 27.2 \\
\hline 2008-12-01 12:00:00.0 & 22.8 \\
\hline 2008-12-01 09:00:00.0 & 19.7 \\
\hline 2008-12-01 06:00:00.0 & 8.7 \\
\hline 2008-12-01 03:00:00.0 & 18.6 \\
\hline 2008-12-01 00:00:00.0 & 12.0 \\
\hline 2008-11-30 21:00:00.0 & 20.9 \\
\hline 2008-11-30 18:00:00.0 & 36.4 \\
\hline 2008-11-30 15:00:00.0 & \\
\hline 2008-11-30 12:00:00.0 & 25.3 \\
\hline 2008-11-30 09:00:00.0 & 18.2 \\
\hline 2008-11-30 06:00:00.0 & 12.9 \\
\hline 2008-11-30 03:00:00.0 & 12.0 \\
\hline 2008-11-30 00:00:00.0 & 15.5 \\
\hline 2008-11-29 21:00:00.0 & 19.1 \\
\hline 2008-11-29 18:00:00.0 & 29.5 \\
\hline 2008-11-29 15:00:00.0 & 21.6 \\
\hline 2008-11-29 12:00:00.0 & 26.5 \\
\hline 2008-11-29 09:00:00.0 & 24.3 \\
\hline 2008-11-29 06:00:00.0 & 23.0 \\
\hline 2008-11-29 03:00:00.0 & 15.7 \\
\hline 2008-11-29 00:00:00.0 & 18.7 \\
\hline 2008-11-28 21:00:00.0 & 21.5 \\
\hline 2008-11-28 18:00:00.0 & 19.9 \\
\hline 2008-11-28 15:00:00.0 & 22.9 \\
\hline 2008-11-28 12:00:00.0 & 25.6 \\
\hline 2008-11-28 09:00:00.0 & 23.4 \\
\hline 2008-11-28 06:00:00.0 & 23.3 \\
\hline 2008-11-28 03:00:00.0 & 17.2 \\
\hline 2008-11-28 00:00:00.0 & 16.2 \\
\hline 2008-11-27 21:00:00.0 & 19.5 \\
\hline 2008-11-27 18:00:00.0 & 15.3 \\
\hline 2008-11-27 15:00:00.0 & \\
\hline 2008-11-27 12:00:00.0 & 29.6 \\
\hline 2008-11-27 09:00:00.0 & 23.6 \\
\hline 2008-11-27 06:00:00.0 & 18.5 \\
\hline 2008-11-27 03:00:00.0 & 20.5 \\
\hline 2008-11-27 00:00:00.0 & 16.3 \\
\hline 2008-11-26 21:00:00.0 & \\
\hline 2008-11-26 18:00:00.0 & \\
\hline 2008-11-26 15:00:00.0 & \\
\hline 2008-11-26 06:00:00.0 & 20.3 \\
\hline 2008-11-26 03:00:00.0 & 16.9 \\
\hline 2008-11-26 00:00:00.0 & 15.7 \\
\hline 2008-11-25 21:00:00.0 & 16.4 \\
\hline 2008-11-25 18:00:00.0 & 13.7 \\
\hline 2008-11-25 15:00:00.0 & 21.8 \\
\hline 2008-11-25 12:00:00.0 & 26.2 \\
\hline 2008-11-25 09:00:00.0 & 25.2 \\
\hline 2008-11-25 06:00:00.0 & 25.5 \\
\hline 2008-11-25 03:00:00.0 & 23.4 \\
\hline 2008-11-25 00:00:00.0 & 20.2 \\
\hline
\end{tabular}

\begin{tabular}{|c|c|}
\hline DataHora & VelVentoMax \\
\hline 2008-11-24 21:00:00.0 & 19.9 \\
\hline 2008-11-24 18:00:00.0 & 18.8 \\
\hline 2008-11-24 15:00:00.0 & 20.6 \\
\hline 2008-11-24 12:00:00.0 & 27.3 \\
\hline 2008-11-24 09:00:00.0 & 24.2 \\
\hline 2008-11-24 06:00:00.0 & 21.8 \\
\hline 2008-11-24 03:00:00.0 & 20.6 \\
\hline 2008-11-24 00:00:00.0 & 24.1 \\
\hline 2008-11-23 21:00:00.0 & 21.8 \\
\hline 2008-11-23 18:00:00.0 & 17.1 \\
\hline 2008-11-23 15:00:00.0 & 25.8 \\
\hline 2008-11-23 12:00:00.0 & 28.6 \\
\hline 2008-11-23 09:00:00.0 & 22.7 \\
\hline 2008-11-23 06:00:00.0 & 24.0 \\
\hline 2008-11-23 03:00:00.0 & 24.8 \\
\hline 2008-11-23 00:00:00.0 & 25.2 \\
\hline 2008-11-22 21:00:00.0 & 25.9 \\
\hline 2008-11-22 18:00:00.0 & 22.1 \\
\hline 2008-11-22 15:00:00.0 & 22.7 \\
\hline 2008-11-22 12:00:00.0 & \\
\hline 2008-11-22 09:00:00.0 & 22.7 \\
\hline 2008-11-22 06:00:00.0 & 30.7 \\
\hline 2008-11-22 03:00:00.0 & 22.4 \\
\hline 2008-11-22 00:00:00.0 & 23.8 \\
\hline 2008-11-21 21:00:00.0 & 24.1 \\
\hline 2008-11-21 18:00:00.0 & 27.5 \\
\hline 2008-11-21 15:00:00.0 & 32.4 \\
\hline 2008-11-21 12:00:00.0 & 31.3 \\
\hline 2008-11-21 09:00:00.0 & 20.8 \\
\hline 2008-11-21 06:00:00.0 & 20.6 \\
\hline 2008-11-21 03:00:00.0 & 22.3 \\
\hline 2008-11-21 00:00:00.0 & 30.8 \\
\hline 2008-11-20 21:00:00.0 & 28.5 \\
\hline 2008-11-20 18:00:00.0 & 25.7 \\
\hline 2008-11-20 15:00:00.0 & 28.1 \\
\hline 2008-11-20 12:00:00.0 & 26.8 \\
\hline 2008-11-20 09:00:00.0 & 17.6 \\
\hline 2008-11-20 06:00:00.0 & 15.4 \\
\hline 2008-11-20 03:00:00.0 & 20.1 \\
\hline 2008-11-20 00:00:00.0 & 30.3 \\
\hline 2008-11-19 21:00:00.0 & 29.2 \\
\hline 2008-11-19 18:00:00.0 & 29.1 \\
\hline 2008-11-19 15:00:00.0 & 29.0 \\
\hline 2008-11-19 12:00:00.0 & 28.6 \\
\hline 2008-11-19 09:00:00.0 & 12.6 \\
\hline 2008-11-19 06:00:00.0 & 18.2 \\
\hline 2008-11-19 03:00:00.0 & 26.4 \\
\hline 2008-11-19 00:00:00.0 & 26.3 \\
\hline 2008-11-18 21:00:00.0 & 41.5 \\
\hline 2008-11-18 18:00:00.0 & 28.5 \\
\hline 2008-11-18 15:00:00.0 & 32.7 \\
\hline 2008-11-18 12:00:00.0 & 28.7 \\
\hline 2008-11-18 09:00:00.0 & 12.8 \\
\hline 2008-11-18 06:00:00.0 & 4.2 \\
\hline 2008-11-18 03:00:00.0 & 14.7 \\
\hline 2008-11-18 00:00:00.0 & 17.5 \\
\hline 2008-11-17 21:00:00.0 & 22.5 \\
\hline 2008-11-17 18:00:00.0 & 21.2 \\
\hline 2008-11-17 15:00:00.0 & 21.2 \\
\hline 2008-11-17 12:00:00.0 & 24.5 \\
\hline 2008-11-17 09:00:00.0 & 16.6 \\
\hline 2008-11-17 06:00:00.0 & 16.1 \\
\hline 2008-11-17 03:00:00.0 & 11.8 \\
\hline 2008-11-17 00:00:00.0 & 17.6 \\
\hline 2008-11-16 21:00:00.0 & 24.7 \\
\hline 2008-11-16 18:00:00.0 & 21.3 \\
\hline 2008-11-16 15:00:00.0 & 21.6 \\
\hline 2008-11-16 12:00:00.0 & 22.0 \\
\hline 2008-11-16 09:00:00.0 & 28.3 \\
\hline
\end{tabular}

\begin{tabular}{|c|c|}
\hline DataHora & VelVentoMax \\
\hline 2008-11-16 06:00:00.0 & 29.9 \\
\hline 2008-11-16 03:00:00.0 & 35.2 \\
\hline 2008-11-16 00:00:00.0 & 32.2 \\
\hline 2008-11-15 21:00:00.0 & 21.6 \\
\hline 2008-11-15 18:00:00.0 & 21.2 \\
\hline 2008-11-15 15:00:00.0 & 16.4 \\
\hline 2008-11-15 12:00:00.0 & 30.1 \\
\hline 2008-11-15 09:00:00.0 & \\
\hline 2008-11-15 06:00:00.0 & 11.9 \\
\hline 2008-11-15 03:00:00.0 & 9.6 \\
\hline 2008-11-15 00:00:00.0 & 13.0 \\
\hline 2008-11-14 21:00:00.0 & 14.5 \\
\hline 2008-11-14 18:00:00.0 & 17.5 \\
\hline 2008-11-14 15:00:00.0 & 12.7 \\
\hline 2008-11-14 12:00:00.0 & 12.9 \\
\hline 2008-11-14 09:00:00.0 & \\
\hline 2008-11-14 06:00:00.0 & 10.8 \\
\hline 2008-11-14 03:00:00.0 & 21.2 \\
\hline 2008-11-14 00:00:00.0 & 20.8 \\
\hline 2008-11-13 21:00:00.0 & 24.7 \\
\hline 2008-11-13 18:00:00.0 & 25.3 \\
\hline 2008-11-13 15:00:00.0 & 23.9 \\
\hline 2008-11-13 12:00:00.0 & 24.5 \\
\hline 2008-11-13 09:00:00.0 & \\
\hline 2008-11-13 06:00:00.0 & 15.1 \\
\hline 2008-11-13 03:00:00.0 & 19.2 \\
\hline 2008-11-13 00:00:00.0 & 21.7 \\
\hline 2008-11-12 21:00:00.0 & 26.2 \\
\hline 2008-11-12 18:00:00.0 & 25.6 \\
\hline 2008-11-12 15:00:00.0 & 27.1 \\
\hline 2008-11-12 12:00:00.0 & 21.6 \\
\hline 2008-11-12 09:00:00.0 & 16.3 \\
\hline 2008-11-12 06:00:00.0 & 17.3 \\
\hline 2008-11-12 03:00:00.0 & 17.9 \\
\hline 2008-11-12 00:00:00.0 & 14.2 \\
\hline 2008-11-11 21:00:00.0 & 26.6 \\
\hline 2008-11-11 18:00:00.0 & 27.1 \\
\hline 2008-11-11 15:00:00.0 & 24.3 \\
\hline 2008-11-11 12:00:00.0 & 27.8 \\
\hline 2008-11-11 09:00:00.0 & 23.6 \\
\hline 2008-11-11 06:00:00.0 & 20.9 \\
\hline 2008-11-11 03:00:00.0 & 19.6 \\
\hline 2008-11-11 00:00:00.0 & 24.9 \\
\hline 2008-11-10 21:00:00.0 & 29.6 \\
\hline 2008-11-10 18:00:00.0 & 24.3 \\
\hline 2008-11-10 15:00:00.0 & 22.9 \\
\hline 2008-11-10 12:00:00.0 & 22.5 \\
\hline 2008-11-10 09:00:00.0 & 19.3 \\
\hline 2008-11-10 06:00:00.0 & 20.7 \\
\hline 2008-11-10 03:00:00.0 & 20.1 \\
\hline 2008-11-10 00:00:00.0 & 23.3 \\
\hline 2008-11-09 21:00:00.0 & 25.0 \\
\hline 2008-11-09 18:00:00.0 & 23.0 \\
\hline 2008-11-09 15:00:00.0 & 20.5 \\
\hline 2008-11-09 12:00:00.0 & 16.9 \\
\hline 2008-11-09 09:00:00.0 & 12.1 \\
\hline 2008-11-09 06:00:00.0 & 14.5 \\
\hline 2008-11-09 03:00:00.0 & 12.8 \\
\hline 2008-11-09 00:00:00.0 & 16.7 \\
\hline 2008-11-08 21:00:00.0 & 19.3 \\
\hline 2008-11-08 18:00:00.0 & 15.5 \\
\hline 2008-11-08 15:00:00.0 & 21.5 \\
\hline 2008-11-08 12:00:00.0 & 24.5 \\
\hline 2008-11-08 09:00:00.0 & 19.2 \\
\hline 2008-11-08 06:00:00.0 & 19.3 \\
\hline 2008-11-08 03:00:00.0 & 17.5 \\
\hline 2008-11-08 00:00:00.0 & 18.4 \\
\hline 2008-11-07 21:00:00.0 & 19.7 \\
\hline 2008-11-07 18:00:00.0 & 22.3 \\
\hline
\end{tabular}




\section{ANEXO 01 - RAJADAS DE VENTO REGISTRADAS EM SÃO MARTINHO DA SERRA / RS}

\begin{tabular}{|c|c|}
\hline DataHora & VelVentoMax \\
\hline 2008-11-07 15:00:00.0 & 19.2 \\
\hline 2008-11-07 12:00:00.0 & 16.1 \\
\hline 2008-11-07 09:00:00.0 & 10.7 \\
\hline 2008-11-07 06:00:00.0 & 14.3 \\
\hline 2008-11-07 03:00:00.0 & 13.9 \\
\hline 2008-11-07 00:00:00.0 & 15.3 \\
\hline 2008-11-06 21:00:00.0 & 20.2 \\
\hline 2008-11-06 18:00:00.0 & 20.3 \\
\hline 2008-11-06 15:00:00.0 & 29.8 \\
\hline 2008-11-06 12:00:00.0 & 14.8 \\
\hline 2008-11-06 09:00:00.0 & 11.8 \\
\hline 2008-11-06 06:00:00.0 & 29.1 \\
\hline 2008-11-06 03:00:00.0 & 21.9 \\
\hline 2008-11-06 00:00:00.0 & 41.4 \\
\hline 2008-11-05 21:00:00.0 & 19.3 \\
\hline 2008-11-05 18:00:00.0 & 16.3 \\
\hline 2008-11-05 15:00:00.0 & 28.8 \\
\hline 2008-11-05 12:00:00.0 & 19.1 \\
\hline 2008-11-05 09:00:00.0 & 19.3 \\
\hline 2008-11-05 06:00:00.0 & 16.6 \\
\hline 2008-11-05 03:00:00.0 & 11.3 \\
\hline 2008-11-05 00:00:00.0 & 11.4 \\
\hline 2008-11-04 21:00:00.0 & 31.3 \\
\hline 2008-11-04 18:00:00.0 & 15.6 \\
\hline 2008-11-04 15:00:00.0 & 13.4 \\
\hline 2008-11-04 12:00:00.0 & 9.1 \\
\hline 2008-11-04 09:00:00.0 & 16.6 \\
\hline 2008-11-04 06:00:00.0 & 13.7 \\
\hline 2008-11-04 03:00:00.0 & 12.2 \\
\hline 2008-11-04 00:00:00.0 & 31.1 \\
\hline 2008-11-03 21:00:00.0 & 15.9 \\
\hline 2008-11-03 18:00:00.0 & 18.2 \\
\hline 2008-11-03 15:00:00.0 & 4.0 \\
\hline 2008-11-03 12:00:00.0 & 14.5 \\
\hline 2008-11-03 09:00:00.0 & 9.5 \\
\hline 2008-11-03 06:00:00.0 & 10.1 \\
\hline 2008-11-03 03:00:00.0 & 10.5 \\
\hline 2008-11-03 00:00:00.0 & 14.1 \\
\hline 2008-11-02 21:00:00.0 & 19.1 \\
\hline 2008-11-02 18:00:00.0 & 17.8 \\
\hline 2008-11-02 15:00:00.0 & 15.0 \\
\hline 2008-11-02 12:00:00.0 & 14.6 \\
\hline 2008-11-02 09:00:00.0 & 12.3 \\
\hline 2008-11-02 06:00:00.0 & 23.9 \\
\hline 2008-11-02 03:00:00.0 & 19.0 \\
\hline 2008-11-02 00:00:00.0 & 17.7 \\
\hline 2008-11-01 21:00:00.0 & 23.3 \\
\hline 2008-11-01 18:00:00.0 & 19.9 \\
\hline 2008-11-01 15:00:00.0 & 19.3 \\
\hline 2008-11-01 12:00:00.0 & 24.4 \\
\hline 2008-11-01 09:00:00.0 & 20.0 \\
\hline 2008-11-01 06:00:00.0 & 17.7 \\
\hline 2008-11-01 03:00:00.0 & 15.7 \\
\hline 2008-11-01 00:00:00.0 & 17.5 \\
\hline 2008-10-31 21:00:00.0 & 17.0 \\
\hline 2008-10-31 18:00:00.0 & 13.3 \\
\hline 2008-10-31 15:00:00.0 & 18.6 \\
\hline 2008-10-31 12:00:00.0 & 18.0 \\
\hline 2008-10-31 09:00:00.0 & 14.4 \\
\hline 2008-10-31 06:00:00.0 & 12.0 \\
\hline 2008-10-31 03:00:00.0 & 9.5 \\
\hline 2008-10-31 00:00:00.0 & 13.3 \\
\hline 2008-10-30 21:00:00.0 & 18.5 \\
\hline 2008-10-30 18:00:00.0 & 19.0 \\
\hline 2008-10-30 15:00:00.0 & 18.6 \\
\hline 2008-10-30 12:00:00.0 & 18.8 \\
\hline 2008-10-30 09:00:00.0 & 20.6 \\
\hline 2008-10-30 06:00:00.0 & 10.4 \\
\hline 2008-10-30 03:00:00.0 & 9.2 \\
\hline
\end{tabular}

\begin{tabular}{|c|c|}
\hline DataHora & VelVentoMax \\
\hline 2008-10-30 00:00:00.0 & 19.6 \\
\hline 2008-10-29 21:00:00.0 & 19.1 \\
\hline 2008-10-29 18:00:00.0 & 4.7 \\
\hline 2008-10-29 15:00:00.0 & 16.6 \\
\hline 2008-10-29 12:00:00.0 & 19.9 \\
\hline 2008-10-29 09:00:00.0 & 29.5 \\
\hline 2008-10-29 06:00:00.0 & \\
\hline 2008-10-29 03:00:00.0 & 34.8 \\
\hline 2008-10-29 00:00:00.0 & 19.9 \\
\hline 2008-10-28 21:00:00.0 & 20.1 \\
\hline 2008-10-28 18:00:00.0 & 19.9 \\
\hline 2008-10-28 15:00:00.0 & 25.3 \\
\hline 2008-10-28 12:00:00.0 & 25.3 \\
\hline 2008-10-28 09:00:00.0 & 21.9 \\
\hline 2008-10-28 06:00:00.0 & 22.0 \\
\hline 2008-10-28 03:00:00.0 & 22.9 \\
\hline 2008-10-28 00:00:00.0 & \\
\hline 2008-10-27 21:00:00.0 & 20.9 \\
\hline 2008-10-27 18:00:00.0 & 20.1 \\
\hline 2008-10-27 15:00:00.0 & 22.1 \\
\hline 2008-10-27 12:00:00.0 & 19.3 \\
\hline 2008-10-27 09:00:00.0 & 13.6 \\
\hline 2008-10-27 06:00:00.0 & 16.4 \\
\hline 2008-10-27 03:00:00.0 & 20.2 \\
\hline 2008-10-27 00:00:00.0 & 18.9 \\
\hline 2008-10-26 21:00:00.0 & 24.7 \\
\hline 2008-10-26 18:00:00.0 & 28.3 \\
\hline 2008-10-26 15:00:00.0 & 23.1 \\
\hline 2008-10-26 12:00:00.0 & 26.2 \\
\hline 2008-10-26 09:00:00.0 & 20.5 \\
\hline 2008-10-26 06:00:00.0 & 38.0 \\
\hline 2008-10-26 03:00:00.0 & 30.8 \\
\hline 2008-10-26 00:00:00.0 & 25.6 \\
\hline 2008-10-25 21:00:00.0 & 21.4 \\
\hline 2008-10-25 18:00:00.0 & 25.1 \\
\hline 2008-10-25 15:00:00.0 & 25.4 \\
\hline 2008-10-25 12:00:00.0 & 20.2 \\
\hline 2008-10-25 09:00:00.0 & 20.2 \\
\hline 2008-10-25 06:00:00.0 & 19.9 \\
\hline 2008-10-25 03:00:00.0 & 17.2 \\
\hline 2008-10-25 00:00:00.0 & 18.6 \\
\hline 2008-10-24 21:00:00.0 & 13.3 \\
\hline 2008-10-24 18:00:00.0 & 13.4 \\
\hline 2008-10-24 15:00:00.0 & 15.3 \\
\hline 2008-10-24 12:00:00.0 & 15.5 \\
\hline 2008-10-24 09:00:00.0 & 15.7 \\
\hline 2008-10-24 06:00:00.0 & 18.0 \\
\hline 2008-10-24 03:00:00.0 & 13.8 \\
\hline 2008-10-24 00:00:00.0 & 15.1 \\
\hline 2008-10-23 21:00:00.0 & 32.7 \\
\hline 2008-10-23 18:00:00.0 & 17.5 \\
\hline 2008-10-23 15:00:00.0 & 22.7 \\
\hline 2008-10-23 12:00:00.0 & 23.7 \\
\hline 2008-10-23 09:00:00.0 & 18.0 \\
\hline 2008-10-23 06:00:00.0 & 25.3 \\
\hline 2008-10-23 03:00:00.0 & 18.6 \\
\hline 2008-10-23 00:00:00.0 & 14.7 \\
\hline 2008-10-22 21:00:00.0 & 30.6 \\
\hline 2008-10-22 18:00:00.0 & 26.9 \\
\hline 2008-10-22 15:00:00.0 & 15.5 \\
\hline 2008-10-22 12:00:00.0 & 18.7 \\
\hline 2008-10-22 09:00:00.0 & \\
\hline 2008-10-22 06:00:00.0 & 32.3 \\
\hline 2008-10-22 03:00:00.0 & 14.4 \\
\hline 2008-10-22 00:00:00.0 & 16.3 \\
\hline 2008-10-21 21:00:00.0 & 27.1 \\
\hline 2008-10-21 18:00:00.0 & 27.8 \\
\hline 2008-10-21 15:00:00.0 & 28.0 \\
\hline 2008-10-21 12:00:00.0 & 24.0 \\
\hline
\end{tabular}

\begin{tabular}{|c|c|}
\hline DataHora & VelVentoMax \\
\hline 2008-10-21 09:00:00.0 & 24.6 \\
\hline 2008-10-21 06:00:00.0 & 21.6 \\
\hline $2008-10-21$ 03:00:00.0 & 17.3 \\
\hline 2008-10-21 00:00:00.0 & 13.3 \\
\hline 2008-10-20 21:00:00.0 & \\
\hline 2008-10-20 18:00:00.0 & 18.2 \\
\hline $2008-10-20$ 15:00:00.0 & 25.5 \\
\hline 2008-10-20 12:00:00.0 & 5.8 \\
\hline 2008-10-20 09:00:00.0 & 22.9 \\
\hline 2008-10-20 06:00:00.0 & 19.5 \\
\hline 2008-10-20 03:00:00.0 & 19.9 \\
\hline 2008-10-20 00:00:00.0 & 17.5 \\
\hline 2008-10-19 21:00:00.0 & \\
\hline 2008-10-19 18:00:00.0 & 13.9 \\
\hline 2008-10-19 15:00:00.0 & 21.5 \\
\hline 2008-10-19 12:00:00.0 & 23.9 \\
\hline 2008-10-19 09:00:00.0 & 17.8 \\
\hline 2008-10-19 06:00:00.0 & 20.7 \\
\hline 2008-10-19 03:00:00.0 & 22.6 \\
\hline 2008-10-19 00:00:00.0 & 18.5 \\
\hline 2008-10-18 21:00:00.0 & 22.2 \\
\hline 2008-10-18 18:00:00.0 & 24.1 \\
\hline 2008-10-18 15:00:00.0 & 24.2 \\
\hline 2008-10-18 12:00:00.0 & 24.9 \\
\hline 2008-10-18 09:00:00.0 & 21.1 \\
\hline 2008-10-18 06:00:00.0 & 18.3 \\
\hline 2008-10-18 03:00:00.0 & 20.5 \\
\hline 2008-10-18 00:00:00.0 & 21.0 \\
\hline 2008-10-17 21:00:00.0 & 23.1 \\
\hline 2008-10-17 18:00:00.0 & 24.3 \\
\hline 2008-10-17 15:00:00.0 & 25.7 \\
\hline 2008-10-17 12:00:00.0 & 24.5 \\
\hline 2008-10-17 09:00:00.0 & 24.2 \\
\hline 2008-10-17 06:00:00.0 & 24.6 \\
\hline 2008-10-17 03:00:00.0 & 26.7 \\
\hline 2008-10-17 00:00:00.0 & 25.8 \\
\hline 2008-10-16 21:00:00.0 & 26.2 \\
\hline 2008-10-16 18:00:00.0 & 25.1 \\
\hline 2008-10-16 15:00:00.0 & 30.2 \\
\hline 2008-10-16 12:00:00.0 & 30.9 \\
\hline 2008-10-16 09:00:00.0 & 19.7 \\
\hline 2008-10-16 06:00:00.0 & 18.9 \\
\hline 2008-10-16 03:00:00.0 & 23.3 \\
\hline 2008-10-16 00:00:00.0 & 21.4 \\
\hline 2008-10-15 21:00:00.0 & 10.2 \\
\hline 2008-10-15 18:00:00.0 & 23.6 \\
\hline 2008-10-15 15:00:00.0 & 34.2 \\
\hline 2008-10-15 12:00:00.0 & 32.9 \\
\hline 2008-10-15 09:00:00.0 & 17.3 \\
\hline 2008-10-15 06:00:00.0 & 10.5 \\
\hline 2008-10-15 03:00:00.0 & 15.4 \\
\hline 2008-10-15 00:00:00.0 & 18.4 \\
\hline 2008-10-14 21:00:00.0 & 29.6 \\
\hline 2008-10-14 18:00:00.0 & 21.2 \\
\hline 2008-10-14 15:00:00.0 & 21.3 \\
\hline 2008-10-14 12:00:00.0 & 24.6 \\
\hline 2008-10-14 09:00:00.0 & 29.2 \\
\hline 2008-10-14 06:00:00.0 & 21.1 \\
\hline 2008-10-14 03:00:00.0 & 25.2 \\
\hline 2008-10-14 00:00:00.0 & 25.4 \\
\hline 2008-10-13 21:00:00.0 & 17.4 \\
\hline 2008-10-13 18:00:00.0 & 37.6 \\
\hline 2008-10-13 15:00:00.0 & 40.6 \\
\hline 2008-10-13 12:00:00.0 & 33.6 \\
\hline 2008-10-13 09:00:00.0 & 21.3 \\
\hline 2008-10-13 06:00:00.0 & 23.2 \\
\hline 2008-10-13 03:00:00.0 & 21.8 \\
\hline 2008-10-13 00:00:00.0 & 17.9 \\
\hline 2008-10-12 21:00:00.0 & 15.7 \\
\hline
\end{tabular}




\section{ANEXO 01 - RAJADAS DE VENTO REGISTRADAS EM SÃO MARTINHO DA SERRA / RS}

\begin{tabular}{|c|c|}
\hline DataHora & VelVentoMax \\
\hline 2008-10-12 18:00:00.0 & 20.8 \\
\hline 2008-10-12 15:00:00.0 & 39.1 \\
\hline 2008-10-12 12:00:00.0 & 17.9 \\
\hline 2008-10-12 09:00:00.0 & 17.6 \\
\hline 2008-10-12 06:00:00.0 & 18.6 \\
\hline 2008-10-12 03:00:00.0 & 20.2 \\
\hline 2008-10-12 00:00:00.0 & 20.9 \\
\hline 2008-10-11 21:00:00.0 & 23.5 \\
\hline 2008-10-11 18:00:00.0 & 23.6 \\
\hline 2008-10-11 15:00:00.0 & 26.4 \\
\hline 2008-10-11 12:00:00.0 & 23.7 \\
\hline 2008-10-11 09:00:00.0 & 30.7 \\
\hline 2008-10-11 06:00:00.0 & 21.8 \\
\hline 2008-10-11 03:00:00.0 & 18.6 \\
\hline 2008-10-11 00:00:00.0 & 12.4 \\
\hline 2008-10-10 21:00:00.0 & 19.8 \\
\hline 2008-10-10 18:00:00.0 & 16.8 \\
\hline 2008-10-10 15:00:00.0 & 23.4 \\
\hline 2008-10-10 12:00:00.0 & 27.8 \\
\hline 2008-10-10 09:00:00.0 & 24.4 \\
\hline 2008-10-10 06:00:00.0 & 22.2 \\
\hline 2008-10-10 03:00:00.0 & 17.9 \\
\hline 2008-10-10 00:00:00.0 & 32.0 \\
\hline 2008-10-09 21:00:00.0 & 19.2 \\
\hline 2008-10-09 18:00:00.0 & 26.1 \\
\hline 2008-10-09 15:00:00.0 & \\
\hline 2008-10-09 12:00:00.0 & 31.7 \\
\hline 2008-10-09 09:00:00.0 & 22.9 \\
\hline 2008-10-09 06:00:00.0 & 22.6 \\
\hline 2008-10-09 03:00:00.0 & 18.8 \\
\hline 2008-10-09 00:00:00.0 & 19.2 \\
\hline 2008-10-08 21:00:00.0 & 26.1 \\
\hline 2008-10-08 18:00:00.0 & 23.2 \\
\hline 2008-10-08 15:00:00.0 & 22.5 \\
\hline 2008-10-08 12:00:00.0 & 25.3 \\
\hline 2008-10-08 09:00:00.0 & 17.6 \\
\hline 2008-10-08 06:00:00.0 & 25.9 \\
\hline 2008-10-08 03:00:00.0 & 28.8 \\
\hline 2008-10-08 00:00:00.0 & 19.2 \\
\hline 2008-10-07 21:00:00.0 & 21.8 \\
\hline 2008-10-07 18:00:00.0 & 21.8 \\
\hline 2008-10-07 15:00:00.0 & 21.1 \\
\hline 2008-10-07 12:00:00.0 & 17.1 \\
\hline 2008-10-07 09:00:00.0 & 6.6 \\
\hline 2008-10-07 06:00:00.0 & 12.6 \\
\hline 2008-10-07 03:00:00.0 & 24.5 \\
\hline 2008-10-07 00:00:00.0 & 2.8 \\
\hline 2008-10-06 21:00:00.0 & 9.9 \\
\hline 2008-10-06 18:00:00.0 & 10.3 \\
\hline 2008-10-06 15:00:00.0 & 14.1 \\
\hline 2008-10-06 12:00:00.0 & 8.1 \\
\hline 2008-10-06 09:00:00.0 & 7.1 \\
\hline 2008-10-06 06:00:00.0 & 6.3 \\
\hline 2008-10-06 03:00:00.0 & 10.8 \\
\hline 2008-10-06 00:00:00.0 & 7.5 \\
\hline 2008-10-05 21:00:00.0 & 12.9 \\
\hline 2008-10-05 18:00:00.0 & 15.6 \\
\hline 2008-10-05 15:00:00.0 & 16.2 \\
\hline 2008-10-05 12:00:00.0 & 15.7 \\
\hline 2008-10-05 09:00:00.0 & 18.5 \\
\hline 2008-10-05 06:00:00.0 & \\
\hline 2008-10-05 03:00:00.0 & 21.7 \\
\hline 2008-10-05 00:00:00.0 & 20.4 \\
\hline 2008-10-04 21:00:00.0 & 21.7 \\
\hline 2008-10-04 18:00:00.0 & 20.2 \\
\hline 2008-10-04 15:00:00.0 & 17.7 \\
\hline 2008-10-04 12:00:00.0 & 22.2 \\
\hline 2008-10-04 09:00:00.0 & 20.8 \\
\hline 2008-10-04 06:00:00.0 & 18.5 \\
\hline
\end{tabular}

\begin{tabular}{|c|c|}
\hline DataHora & VelVentoMax \\
\hline 2008-10-04 03:00:00.0 & \\
\hline 2008-10-04 00:00:00.0 & 13.7 \\
\hline 2008-10-03 21:00:00.0 & 16.4 \\
\hline 2008-10-03 18:00:00.0 & 16.6 \\
\hline 2008-10-03 15:00:00.0 & 20.1 \\
\hline 2008-10-03 12:00:00.0 & 15.2 \\
\hline 2008-10-03 09:00:00.0 & 9.9 \\
\hline 2008-10-03 06:00:00.0 & 9.6 \\
\hline 2008-10-03 03:00:00.0 & 13.3 \\
\hline 2008-10-03 00:00:00.0 & 18.1 \\
\hline 2008-10-02 21:00:00.0 & 27.0 \\
\hline 2008-10-02 18:00:00.0 & 27.8 \\
\hline 2008-10-02 15:00:00.0 & 26.2 \\
\hline 2008-10-02 12:00:00.0 & 21.4 \\
\hline 2008-10-02 09:00:00.0 & 15.1 \\
\hline 2008-10-02 06:00:00.0 & 8.9 \\
\hline 2008-10-02 03:00:00.0 & 13.4 \\
\hline 2008-10-02 00:00:00.0 & 10.5 \\
\hline 2008-10-01 21:00:00.0 & 16.9 \\
\hline 2008-10-01 18:00:00.0 & 13.1 \\
\hline 2008-10-01 15:00:00.0 & 16.0 \\
\hline 2008-10-01 12:00:00.0 & 17.2 \\
\hline 2008-10-01 09:00:00.0 & 18.8 \\
\hline 2008-10-01 06:00:00.0 & 13.5 \\
\hline 2008-10-01 03:00:00.0 & 10.6 \\
\hline 2008-10-01 00:00:00.0 & 6.7 \\
\hline 2008-09-30 21:00:00.0 & 10.0 \\
\hline 2008-09-30 18:00:00.0 & 11.7 \\
\hline 2008-09-30 15:00:00.0 & 29.8 \\
\hline 2008-09-30 12:00:00.0 & 29.5 \\
\hline 2008-09-30 09:00:00.0 & 11.1 \\
\hline 2008-09-30 06:00:00.0 & 14.2 \\
\hline 2008-09-30 03:00:00.0 & 24.7 \\
\hline 2008-09-30 00:00:00.0 & 22.0 \\
\hline 2008-09-29 21:00:00.0 & 47.3 \\
\hline 2008-09-29 18:00:00.0 & 16.6 \\
\hline 2008-09-29 15:00:00.0 & 24.2 \\
\hline 2008-09-29 12:00:00.0 & 25.5 \\
\hline 2008-09-29 09:00:00.0 & 26.5 \\
\hline 2008-09-29 06:00:00.0 & 23.0 \\
\hline 2008-09-29 03:00:00.0 & 17.4 \\
\hline 2008-09-29 00:00:00.0 & 13.4 \\
\hline 2008-09-28 21:00:00.0 & 20.0 \\
\hline 2008-09-28 18:00:00.0 & 25.4 \\
\hline 2008-09-28 15:00:00.0 & 28.9 \\
\hline 2008-09-28 12:00:00.0 & 27.1 \\
\hline 2008-09-28 09:00:00.0 & \\
\hline 2008-09-28 06:00:00.0 & 22.7 \\
\hline 2008-09-28 03:00:00.0 & 19.9 \\
\hline 2008-09-28 00:00:00.0 & 16.9 \\
\hline 2008-09-27 21:00:00.0 & 20.4 \\
\hline 2008-09-27 18:00:00.0 & 22.4 \\
\hline 2008-09-27 15:00:00.0 & 22.2 \\
\hline 2008-09-27 12:00:00.0 & 21.1 \\
\hline 2008-09-27 09:00:00.0 & 16.5 \\
\hline 2008-09-27 06:00:00.0 & 13.9 \\
\hline 2008-09-27 03:00:00.0 & 10.8 \\
\hline 2008-09-27 00:00:00.0 & 13.9 \\
\hline 2008-09-26 21:00:00.0 & 17.0 \\
\hline 2008-09-26 18:00:00.0 & 17.4 \\
\hline 2008-09-26 15:00:00.0 & 33.8 \\
\hline 2008-09-26 12:00:00.0 & 36.7 \\
\hline 2008-09-26 09:00:00.0 & 19.9 \\
\hline 2008-09-26 06:00:00.0 & 18.3 \\
\hline 2008-09-26 03:00:00.0 & 14.4 \\
\hline 2008-09-26 00:00:00.0 & 12.6 \\
\hline 2008-09-25 21:00:00.0 & 15.1 \\
\hline 2008-09-25 18:00:00.0 & 16.4 \\
\hline 2008-09-25 15:00:00.0 & 21.1 \\
\hline
\end{tabular}

\begin{tabular}{|c|c|}
\hline DataHora & VelVentoMax \\
\hline 2008-09-25 12:00:00.0 & 23.9 \\
\hline 2008-09-25 09:00:00.0 & 20.9 \\
\hline 2008-09-25 06:00:00.0 & 21.0 \\
\hline 2008-09-25 03:00:00.0 & 15.2 \\
\hline 2008-09-25 00:00:00.0 & 16.1 \\
\hline 2008-09-24 21:00:00.0 & 35.3 \\
\hline $2008-09-24$ 18:00:00.0 & 22.1 \\
\hline 2008-09-24 15:00:00.0 & 24.9 \\
\hline 2008-09-24 12:00:00.0 & 24.8 \\
\hline 2008-09-24 09:00:00.0 & 23.2 \\
\hline 2008-09-24 06:00:00.0 & 19.1 \\
\hline 2008-09-24 03:00:00.0 & 18.9 \\
\hline 2008-09-24 00:00:00.0 & 16.0 \\
\hline 2008-09-23 21:00:00.0 & 22.4 \\
\hline 2008-09-23 18:00:00.0 & 20.0 \\
\hline 2008-09-23 15:00:00.0 & 20.4 \\
\hline 2008-09-23 12:00:00.0 & 20.8 \\
\hline 2008-09-23 09:00:00.0 & \\
\hline 2008-09-23 06:00:00.0 & 22.4 \\
\hline 2008-09-23 03:00:00.0 & 20.0 \\
\hline 2008-09-23 00:00:00.0 & 16.3 \\
\hline 2008-09-22 21:00:00.0 & 17.7 \\
\hline $2008-09-22$ 18:00:00.0 & 19.4 \\
\hline 2008-09-22 15:00:00.0 & 16.4 \\
\hline 2008-09-22 12:00:00.0 & 17.8 \\
\hline 2008-09-22 09:00:00.0 & 13.5 \\
\hline 2008-09-22 06:00:00.0 & 12.7 \\
\hline 2008-09-22 03:00:00.0 & 13.5 \\
\hline 2008-09-22 00:00:00.0 & 23.3 \\
\hline 2008-09-21 21:00:00.0 & 25.1 \\
\hline 2008-09-21 18:00:00.0 & 29.4 \\
\hline 2008-09-21 15:00:00.0 & 27.6 \\
\hline 2008-09-21 12:00:00.0 & 22.1 \\
\hline 2008-09-21 09:00:00.0 & 27.5 \\
\hline 2008-09-21 06:00:00.0 & 17.0 \\
\hline 2008-09-21 03:00:00.0 & 10.9 \\
\hline 2008-09-21 00:00:00.0 & 9.9 \\
\hline 2008-09-20 21:00:00.0 & 9.7 \\
\hline 2008-09-20 18:00:00.0 & 12.2 \\
\hline 2008-09-20 15:00:00.0 & 11.6 \\
\hline 2008-09-20 12:00:00.0 & 12.2 \\
\hline 2008-09-20 09:00:00.0 & 15.5 \\
\hline 2008-09-20 06:00:00.0 & 9.7 \\
\hline 2008-09-20 03:00:00.0 & 13.1 \\
\hline 2008-09-20 00:00:00.0 & 13.0 \\
\hline 2008-09-19 21:00:00.0 & 20.6 \\
\hline 2008-09-19 18:00:00.0 & 25.9 \\
\hline 2008-09-19 15:00:00.0 & 28.4 \\
\hline 2008-09-19 12:00:00.0 & 27.0 \\
\hline 2008-09-19 09:00:00.0 & 22.6 \\
\hline 2008-09-19 06:00:00.0 & 18.9 \\
\hline 2008-09-19 03:00:00.0 & 15.4 \\
\hline 2008-09-19 00:00:00.0 & \\
\hline 2008-09-18 21:00:00.0 & 21.0 \\
\hline 2008-09-18 18:00:00.0 & 19.8 \\
\hline 2008-09-18 15:00:00.0 & 23.2 \\
\hline 2008-09-18 12:00:00.0 & 24.0 \\
\hline 2008-09-18 09:00:00.0 & 19.3 \\
\hline 2008-09-18 06:00:00.0 & 21.0 \\
\hline 2008-09-18 03:00:00.0 & 24.0 \\
\hline 2008-09-18 00:00:00.0 & 20.4 \\
\hline 2008-09-17 21:00:00.0 & 20.5 \\
\hline 2008-09-17 18:00:00.0 & 16.6 \\
\hline 2008-09-17 15:00:00.0 & 21.3 \\
\hline 2008-09-17 12:00:00.0 & 23.4 \\
\hline 2008-09-17 09:00:00.0 & 40.6 \\
\hline 2008-09-17 06:00:00.0 & 18.1 \\
\hline 2008-09-17 03:00:00.0 & 17.8 \\
\hline 2008-09-17 00:00:00.0 & 19.0 \\
\hline
\end{tabular}




\section{ANEXO 01 - RAJADAS DE VENTO REGISTRADAS EM SÃO MARTINHO DA SERRA / RS}

\begin{tabular}{|c|c|}
\hline DataHora & VelVentoMax \\
\hline 2008-09-16 21:00:00.0 & 24.4 \\
\hline 2008-09-16 18:00:00.0 & 20.5 \\
\hline 2008-09-16 15:00:00.0 & 19.8 \\
\hline 2008-09-16 12:00:00.0 & 19.9 \\
\hline 2008-09-16 09:00:00.0 & 11.2 \\
\hline 2008-09-16 06:00:00.0 & 11.5 \\
\hline 2008-09-16 03:00:00.0 & 13.3 \\
\hline 2008-09-16 00:00:00.0 & 13.4 \\
\hline 2008-09-15 21:00:00.0 & 18.2 \\
\hline 2008-09-15 18:00:00.0 & 14.0 \\
\hline 2008-09-15 15:00:00.0 & 16.7 \\
\hline 2008-09-15 12:00:00.0 & 3.5 \\
\hline 2008-09-15 09:00:00.0 & 7.7 \\
\hline 2008-09-15 06:00:00.0 & 10.3 \\
\hline 2008-09-15 03:00:00.0 & 10.7 \\
\hline 2008-09-15 00:00:00.0 & 7.9 \\
\hline 2008-09-14 21:00:00.0 & 17.1 \\
\hline 2008-09-14 18:00:00.0 & 36.5 \\
\hline 2008-09-14 15:00:00.0 & 13.7 \\
\hline 2008-09-14 12:00:00.0 & 7.2 \\
\hline 2008-09-14 09:00:00.0 & 6.2 \\
\hline 2008-09-14 06:00:00.0 & 6.0 \\
\hline 2008-09-14 03:00:00.0 & 18.1 \\
\hline 2008-09-14 00:00:00.0 & 9.2 \\
\hline 2008-09-13 21:00:00.0 & 16.9 \\
\hline 2008-09-13 18:00:00.0 & 19.7 \\
\hline 2008-09-13 15:00:00.0 & 22.5 \\
\hline 2008-09-13 12:00:00.0 & 17.3 \\
\hline 2008-09-13 09:00:00.0 & 14.9 \\
\hline 2008-09-13 06:00:00.0 & 9.9 \\
\hline 2008-09-13 03:00:00.0 & 18.3 \\
\hline 2008-09-13 00:00:00.0 & 22.5 \\
\hline 2008-09-12 21:00:00.0 & 21.9 \\
\hline 2008-09-12 18:00:00.0 & 18.9 \\
\hline 2008-09-12 15:00:00.0 & 34.8 \\
\hline 2008-09-12 12:00:00.0 & 20.0 \\
\hline 2008-09-12 09:00:00.0 & 20.7 \\
\hline 2008-09-12 06:00:00.0 & 15.3 \\
\hline 2008-09-12 03:00:00.0 & 18.1 \\
\hline 2008-09-12 00:00:00.0 & 30.1 \\
\hline 2008-09-11 21:00:00.0 & 19.1 \\
\hline 2008-09-11 18:00:00.0 & 20.2 \\
\hline 2008-09-11 15:00:00.0 & 24.2 \\
\hline 2008-09-11 12:00:00.0 & 37.5 \\
\hline 2008-09-11 09:00:00.0 & 27.7 \\
\hline 2008-09-11 06:00:00.0 & 21.4 \\
\hline 2008-09-11 03:00:00.0 & 23.5 \\
\hline 2008-09-11 00:00:00.0 & 20.4 \\
\hline 2008-09-10 21:00:00.0 & 19.6 \\
\hline 2008-09-10 18:00:00.0 & 29.2 \\
\hline 2008-09-10 15:00:00.0 & 16.9 \\
\hline 2008-09-10 12:00:00.0 & 16.9 \\
\hline 2008-09-10 09:00:00.0 & 16.9 \\
\hline 2008-09-10 06:00:00.0 & 15.9 \\
\hline 2008-09-10 03:00:00.0 & 16.8 \\
\hline 2008-09-10 00:00:00.0 & 27.1 \\
\hline 2008-09-09 21:00:00.0 & 14.6 \\
\hline 2008-09-09 18:00:00.0 & 14.1 \\
\hline 2008-09-09 15:00:00.0 & 22.3 \\
\hline 2008-09-09 12:00:00.0 & 23.0 \\
\hline 2008-09-09 09:00:00.0 & 34.0 \\
\hline 2008-09-09 06:00:00.0 & 18.7 \\
\hline 2008-09-09 03:00:00.0 & 20.6 \\
\hline 2008-09-09 00:00:00.0 & 15.2 \\
\hline 2008-09-08 21:00:00.0 & 15.8 \\
\hline 2008-09-08 18:00:00.0 & 20.5 \\
\hline 2008-09-08 15:00:00.0 & 21.3 \\
\hline 2008-09-08 12:00:00.0 & 11.7 \\
\hline 2008-09-08 09:00:00.0 & 9.4 \\
\hline
\end{tabular}

\begin{tabular}{|c|c|}
\hline DataHora & VelVentoMax \\
\hline 2008-09-08 06:00:00.0 & 10.3 \\
\hline 2008-09-08 03:00:00.0 & 7.0 \\
\hline 2008-09-08 00:00:00.0 & 8.7 \\
\hline 2008-09-07 21:00:00.0 & 18.9 \\
\hline 2008-09-07 18:00:00.0 & 21.2 \\
\hline 2008-09-07 15:00:00.0 & 22.6 \\
\hline 2008-09-07 12:00:00.0 & 22.8 \\
\hline 2008-09-07 09:00:00.0 & 24.8 \\
\hline 2008-09-07 06:00:00.0 & 22.8 \\
\hline 2008-09-07 03:00:00.0 & 22.3 \\
\hline 2008-09-07 00:00:00.0 & 22.3 \\
\hline 2008-09-06 21:00:00.0 & 25.8 \\
\hline 2008-09-06 18:00:00.0 & 28.2 \\
\hline 2008-09-06 15:00:00.0 & 19.0 \\
\hline 2008-09-06 12:00:00.0 & 14.6 \\
\hline 2008-09-06 09:00:00.0 & 17.6 \\
\hline 2008-09-06 06:00:00.0 & 17.5 \\
\hline 2008-09-06 03:00:00.0 & 22.9 \\
\hline 2008-09-06 00:00:00.0 & 23.5 \\
\hline 2008-09-05 21:00:00.0 & 32.0 \\
\hline 2008-09-05 18:00:00.0 & 32.2 \\
\hline 2008-09-05 15:00:00.0 & 32.8 \\
\hline 2008-09-05 12:00:00.0 & 33.3 \\
\hline 2008-09-05 09:00:00.0 & 32.6 \\
\hline 2008-09-05 06:00:00.0 & 33.5 \\
\hline 2008-09-05 03:00:00.0 & 30.1 \\
\hline 2008-09-05 00:00:00.0 & 23.3 \\
\hline 2008-09-04 21:00:00.0 & 26.6 \\
\hline 2008-09-04 18:00:00.0 & 29.3 \\
\hline 2008-09-04 15:00:00.0 & 30.9 \\
\hline 2008-09-04 12:00:00.0 & 30.2 \\
\hline 2008-09-04 09:00:00.0 & 27.0 \\
\hline 2008-09-04 06:00:00.0 & 31.7 \\
\hline 2008-09-04 03:00:00.0 & 31.8 \\
\hline 2008-09-04 00:00:00.0 & 27.7 \\
\hline 2008-09-03 21:00:00.0 & \\
\hline 2008-09-03 18:00:00.0 & 33.1 \\
\hline 2008-09-03 15:00:00.0 & 33.8 \\
\hline 2008-09-03 12:00:00.0 & 29.3 \\
\hline 2008-09-03 09:00:00.0 & 28.4 \\
\hline 2008-09-03 06:00:00.0 & 28.3 \\
\hline 2008-09-03 03:00:00.0 & 24.7 \\
\hline 2008-09-03 00:00:00.0 & 19.5 \\
\hline 2008-09-02 21:00:00.0 & 21.8 \\
\hline 2008-09-02 18:00:00.0 & 26.6 \\
\hline 2008-09-02 15:00:00.0 & 26.5 \\
\hline 2008-09-02 12:00:00.0 & 25.5 \\
\hline 2008-09-02 09:00:00.0 & \\
\hline 2008-09-02 06:00:00.0 & 19.4 \\
\hline 2008-09-02 03:00:00.0 & 18.3 \\
\hline 2008-09-02 00:00:00.0 & 16.4 \\
\hline 2008-09-01 21:00:00.0 & 16.0 \\
\hline 2008-09-01 18:00:00.0 & 18.5 \\
\hline 2008-09-01 15:00:00.0 & 26.2 \\
\hline 2008-09-01 12:00:00.0 & 23.0 \\
\hline 2008-09-01 09:00:00.0 & 22.0 \\
\hline 2008-09-01 06:00:00.0 & 18.4 \\
\hline 2008-09-01 03:00:00.0 & 18.2 \\
\hline 2008-09-01 00:00:00.0 & 16.2 \\
\hline 2008-08-31 21:00:00.0 & 15.7 \\
\hline 2008-08-31 18:00:00.0 & 17.3 \\
\hline 2008-08-31 15:00:00.0 & 22.1 \\
\hline 2008-08-31 12:00:00.0 & 21.3 \\
\hline 2008-08-31 09:00:00.0 & 21.2 \\
\hline 2008-08-31 06:00:00.0 & 17.3 \\
\hline 2008-08-31 03:00:00.0 & 14.4 \\
\hline 2008-08-31 00:00:00.0 & 11.0 \\
\hline 2008-08-30 21:00:00.0 & \\
\hline 2008-08-30 18:00:00.0 & 15.7 \\
\hline
\end{tabular}

\begin{tabular}{|c|c|}
\hline DataHora & VelVentoMax \\
\hline 2008-08-30 15:00:00.0 & 17.5 \\
\hline 2008-08-30 12:00:00.0 & 12.0 \\
\hline 2008-08-30 09:00:00.0 & 11.9 \\
\hline 2008-08-30 06:00:00.0 & 7.2 \\
\hline 2008-08-30 03:00:00.0 & 6.4 \\
\hline 2008-08-30 00:00:00.0 & 3.2 \\
\hline 2008-08-29 21:00:00.0 & 18.9 \\
\hline $2008-08-29$ 18:00:00.0 & 24.3 \\
\hline $2008-08-29$ 15:00:00.0 & 19.9 \\
\hline $2008-08-29$ 12:00:00.0 & 14.1 \\
\hline $2008-08-29$ 09:00:00.0 & \\
\hline $2008-08-2906: 00: 00.0$ & 25.0 \\
\hline 2008-08-29 03:00:00.0 & 31.8 \\
\hline 2008-08-29 00:00:00.0 & 36.7 \\
\hline 2008-08-28 21:00:00.0 & 19.2 \\
\hline $2008-08-28$ 18:00:00.0 & 19.8 \\
\hline 2008-08-28 15:00:00.0 & 45.3 \\
\hline 2008-08-28 12:00:00.0 & 28.2 \\
\hline 2008-08-28 09:00:00.0 & 26.1 \\
\hline 2008-08-28 06:00:00.0 & 24.3 \\
\hline 2008-08-28 03:00:00.0 & 18.6 \\
\hline $2008-08-28$ 00:00:00.0 & 18.6 \\
\hline $2008-08-27$ 21:00:00.0 & 24.0 \\
\hline 2008-08-27 18:00:00.0 & 24.5 \\
\hline $2008-08-27$ 15:00:00.0 & 20.3 \\
\hline 2008-08-27 12:00:00.0 & 21.6 \\
\hline 2008-08-27 09:00:00.0 & 21.8 \\
\hline 2008-08-27 06:00:00.0 & 23.5 \\
\hline 2008-08-27 03:00:00.0 & 23.9 \\
\hline 2008-08-27 00:00:00.0 & 14.9 \\
\hline 2008-08-26 21:00:00.0 & 33.5 \\
\hline 2008-08-26 18:00:00.0 & 19.0 \\
\hline 2008-08-26 15:00:00.0 & 18.6 \\
\hline $2008-08-26$ 12:00:00.0 & 22.7 \\
\hline $2008-08-26$ 09:00:00.0 & 21.9 \\
\hline 2008-08-26 06:00:00.0 & 20.8 \\
\hline 2008-08-26 03:00:00.0 & 16.5 \\
\hline $2008-08-2600: 00: 00.0$ & 13.0 \\
\hline 2008-08-25 21:00:00.0 & 11.0 \\
\hline $2008-08-25$ 18:00:00.0 & 14.5 \\
\hline $2008-08-25$ 15:00:00.0 & 13.5 \\
\hline $2008-08-25$ 12:00:00.0 & 9.1 \\
\hline 2008-08-25 09:00:00.0 & \\
\hline 2008-08-25 06:00:00.0 & 9.3 \\
\hline 2008-08-25 03:00:00.0 & 10.4 \\
\hline 2008-08-25 00:00:00.0 & 9.4 \\
\hline 2008-08-24 21:00:00.0 & 32.6 \\
\hline 2008-08-24 18:00:00.0 & 23.0 \\
\hline $2008-08-24$ 15:00:00.0 & 24.5 \\
\hline 2008-08-24 12:00:00.0 & 23.6 \\
\hline $2008-08-24$ 09:00:00.0 & 23.5 \\
\hline 2008-08-24 06:00:00.0 & 26.1 \\
\hline 2008-08-24 03:00:00.0 & 27.3 \\
\hline $2008-08-24$ 00:00:00.0 & 24.9 \\
\hline 2008-08-23 21:00:00.0 & 23.7 \\
\hline $2008-08-23$ 18:00:00.0 & 24.5 \\
\hline $2008-08-23$ 15:00:00.0 & 29.8 \\
\hline 2008-08-23 12:00:00.0 & 24.4 \\
\hline 2008-08-23 09:00:00.0 & 19.6 \\
\hline 2008-08-23 06:00:00.0 & 15.8 \\
\hline 2008-08-23 03:00:00.0 & 13.1 \\
\hline $2008-08-23$ 00:00:00.0 & 11.0 \\
\hline 2008-08-22 21:00:00.0 & 23.5 \\
\hline $2008-08-22$ 18:00:00.0 & 23.0 \\
\hline 2008-08-22 15:00:00.0 & 23.6 \\
\hline $2008-08-22$ 12:00:00.0 & 17.6 \\
\hline 2008-08-22 09:00:00.0 & 19.4 \\
\hline 2008-08-22 06:00:00.0 & 15.7 \\
\hline 2008-08-22 03:00:00.0 & 15.4 \\
\hline
\end{tabular}




\section{ANEXO 01 - RAJADAS DE VENTO REGISTRADAS EM SÃO MARTINHO DA SERRA / RS}

\begin{tabular}{|c|c|}
\hline DataHora & VelVentoMax \\
\hline 2008-08-22 00:00:00.0 & 15.9 \\
\hline 2008-08-21 21:00:00.0 & 17.8 \\
\hline 2008-08-21 18:00:00.0 & 15.5 \\
\hline 2008-08-21 15:00:00.0 & 15.8 \\
\hline 2008-08-21 12:00:00.0 & 16.7 \\
\hline 2008-08-21 09:00:00.0 & 20.1 \\
\hline 2008-08-21 06:00:00.0 & 19.2 \\
\hline 2008-08-21 03:00:00.0 & 14.8 \\
\hline 2008-08-21 00:00:00.0 & 13.9 \\
\hline 2008-08-20 21:00:00.0 & 13.0 \\
\hline 2008-08-20 18:00:00.0 & 16.2 \\
\hline 2008-08-20 15:00:00.0 & 29.6 \\
\hline 2008-08-20 12:00:00.0 & 18.3 \\
\hline 2008-08-20 09:00:00.0 & 17.9 \\
\hline 2008-08-20 06:00:00.0 & 14.9 \\
\hline 2008-08-20 03:00:00.0 & 17.4 \\
\hline 2008-08-20 00:00:00.0 & 15.2 \\
\hline 2008-08-19 21:00:00.0 & 13.7 \\
\hline 2008-08-19 18:00:00.0 & 22.1 \\
\hline 2008-08-19 15:00:00.0 & 23.8 \\
\hline 2008-08-19 12:00:00.0 & 21.3 \\
\hline 2008-08-19 09:00:00.0 & 23.1 \\
\hline 2008-08-19 06:00:00.0 & 24.8 \\
\hline 2008-08-19 03:00:00.0 & 23.8 \\
\hline 2008-08-19 00:00:00.0 & 18.6 \\
\hline 2008-08-18 21:00:00.0 & 20.6 \\
\hline 2008-08-18 18:00:00.0 & 24.5 \\
\hline 2008-08-18 15:00:00.0 & \\
\hline 2008-08-18 12:00:00.0 & 27.4 \\
\hline 2008-08-18 09:00:00.0 & 32.3 \\
\hline 2008-08-18 06:00:00.0 & 30.4 \\
\hline 2008-08-18 03:00:00.0 & 28.7 \\
\hline 2008-08-18 00:00:00.0 & 26.7 \\
\hline 2008-08-17 21:00:00.0 & 24.9 \\
\hline 2008-08-17 18:00:00.0 & 17.3 \\
\hline 2008-08-17 15:00:00.0 & 22.4 \\
\hline 2008-08-17 12:00:00.0 & 14.2 \\
\hline 2008-08-17 09:00:00.0 & 29.4 \\
\hline 2008-08-17 06:00:00.0 & 32.1 \\
\hline 2008-08-17 03:00:00.0 & 30.1 \\
\hline 2008-08-17 00:00:00.0 & 22.2 \\
\hline 2008-08-16 21:00:00.0 & 24.9 \\
\hline 2008-08-16 18:00:00.0 & 31.2 \\
\hline 2008-08-16 15:00:00.0 & 26.1 \\
\hline 2008-08-16 12:00:00.0 & 22.7 \\
\hline 2008-08-16 09:00:00.0 & 21.0 \\
\hline 2008-08-16 06:00:00.0 & 19.1 \\
\hline 2008-08-16 03:00:00.0 & 15.9 \\
\hline 2008-08-16 00:00:00.0 & 14.4 \\
\hline 2008-08-15 21:00:00.0 & 15.3 \\
\hline 2008-08-15 18:00:00.0 & 18.4 \\
\hline 2008-08-15 15:00:00.0 & 17.2 \\
\hline 2008-08-15 12:00:00.0 & 16.4 \\
\hline 2008-08-15 09:00:00.0 & 16.3 \\
\hline 2008-08-15 06:00:00.0 & 16.5 \\
\hline 2008-08-15 03:00:00.0 & 14.6 \\
\hline 2008-08-15 00:00:00.0 & 16.2 \\
\hline 2008-08-14 21:00:00.0 & 14.7 \\
\hline 2008-08-14 18:00:00.0 & 14.5 \\
\hline 2008-08-14 15:00:00.0 & 17.5 \\
\hline 2008-08-14 12:00:00.0 & 17.3 \\
\hline 2008-08-14 09:00:00.0 & 21.7 \\
\hline 2008-08-14 06:00:00.0 & 19.1 \\
\hline 2008-08-14 03:00:00.0 & 20.3 \\
\hline 2008-08-14 00:00:00.0 & 17.9 \\
\hline 2008-08-13 21:00:00.0 & 15.5 \\
\hline 2008-08-13 18:00:00.0 & 18.7 \\
\hline 2008-08-13 15:00:00.0 & 20.7 \\
\hline 2008-08-13 12:00:00.0 & 21.7 \\
\hline
\end{tabular}

\begin{tabular}{|c|c|}
\hline DataHora & VelVentoMax \\
\hline 2008-08-13 09:00:00.0 & 15.2 \\
\hline 2008-08-13 06:00:00.0 & 14.9 \\
\hline 2008-08-13 03:00:00.0 & 10.6 \\
\hline 2008-08-13 00:00:00.0 & 14.5 \\
\hline 2008-08-12 21:00:00.0 & 21.3 \\
\hline 2008-08-12 18:00:00.0 & 20.7 \\
\hline 2008-08-12 15:00:00.0 & 34.8 \\
\hline 2008-08-12 12:00:00.0 & 41.0 \\
\hline 2008-08-12 09:00:00.0 & 49.4 \\
\hline 2008-08-12 06:00:00.0 & 24.1 \\
\hline 2008-08-12 03:00:00.0 & 27.4 \\
\hline 2008-08-12 00:00:00.0 & 22.4 \\
\hline 2008-08-11 21:00:00.0 & 19.1 \\
\hline 2008-08-11 18:00:00.0 & 27.3 \\
\hline 2008-08-11 15:00:00.0 & 25.2 \\
\hline 2008-08-11 12:00:00.0 & 24.6 \\
\hline 2008-08-11 09:00:00.0 & 23.2 \\
\hline 2008-08-11 06:00:00.0 & 22.3 \\
\hline 2008-08-11 03:00:00.0 & 21.3 \\
\hline 2008-08-11 00:00:00.0 & 18.3 \\
\hline 2008-08-10 21:00:00.0 & 19.0 \\
\hline 2008-08-10 18:00:00.0 & 19.3 \\
\hline 2008-08-10 15:00:00.0 & \\
\hline 2008-08-10 12:00:00.0 & \\
\hline 2008-08-10 09:00:00.0 & \\
\hline 2008-08-10 06:00:00.0 & 19.4 \\
\hline 2008-08-10 03:00:00.0 & 17.4 \\
\hline 2008-08-10 00:00:00.0 & 14.4 \\
\hline 2008-08-09 21:00:00.0 & 16.7 \\
\hline 2008-08-09 18:00:00.0 & 17.0 \\
\hline 2008-08-09 15:00:00.0 & 17.9 \\
\hline 2008-08-09 12:00:00.0 & 16.2 \\
\hline 2008-08-09 09:00:00.0 & 15.5 \\
\hline 2008-08-09 06:00:00.0 & 16.2 \\
\hline 2008-08-09 03:00:00.0 & 14.3 \\
\hline 2008-08-09 00:00:00.0 & 12.6 \\
\hline 2008-08-08 21:00:00.0 & 20.7 \\
\hline 2008-08-08 18:00:00.0 & 20.9 \\
\hline 2008-08-08 15:00:00.0 & 21.1 \\
\hline 2008-08-08 12:00:00.0 & 18.1 \\
\hline 2008-08-08 09:00:00.0 & \\
\hline 2008-08-08 06:00:00.0 & 19.6 \\
\hline 2008-08-08 03:00:00.0 & 19.5 \\
\hline 2008-08-08 00:00:00.0 & 10.9 \\
\hline 2008-08-07 21:00:00.0 & 16.9 \\
\hline 2008-08-07 18:00:00.0 & 15.5 \\
\hline 2008-08-07 15:00:00.0 & 9.9 \\
\hline 2008-08-07 12:00:00.0 & 7.9 \\
\hline 2008-08-07 09:00:00.0 & 5.9 \\
\hline 2008-08-07 06:00:00.0 & 14.4 \\
\hline 2008-08-07 03:00:00.0 & 38.5 \\
\hline 2008-08-07 00:00:00.0 & 8.9 \\
\hline 2008-08-06 21:00:00.0 & 11.8 \\
\hline 2008-08-06 18:00:00.0 & 13.6 \\
\hline 2008-08-06 15:00:00.0 & 20.0 \\
\hline 2008-08-06 12:00:00.0 & 13.3 \\
\hline 2008-08-06 09:00:00.0 & 21.9 \\
\hline 2008-08-06 06:00:00.0 & 22.8 \\
\hline 2008-08-06 03:00:00.0 & 13.1 \\
\hline 2008-08-06 00:00:00.0 & 9.8 \\
\hline 2008-08-05 21:00:00.0 & 15.0 \\
\hline 2008-08-05 18:00:00.0 & 19.4 \\
\hline 2008-08-05 15:00:00.0 & 24.8 \\
\hline 2008-08-05 12:00:00.0 & 7.0 \\
\hline 2008-08-05 09:00:00.0 & 8.3 \\
\hline 2008-08-05 06:00:00.0 & 13.6 \\
\hline 2008-08-05 03:00:00.0 & 14.4 \\
\hline 2008-08-05 00:00:00.0 & 16.3 \\
\hline 2008-08-04 21:00:00.0 & 22.4 \\
\hline
\end{tabular}

\begin{tabular}{|c|c|}
\hline DataHora & VelVentoMax \\
\hline 2008-08-04 18:00:00.0 & 21.8 \\
\hline 2008-08-04 15:00:00.0 & 19.1 \\
\hline 2008-08-04 12:00:00.0 & 16.9 \\
\hline 2008-08-04 09:00:00.0 & \\
\hline 2008-08-04 06:00:00.0 & 14.7 \\
\hline 2008-08-04 03:00:00.0 & 8.4 \\
\hline 2008-08-04 00:00:00.0 & 6.0 \\
\hline 2008-08-03 21:00:00.0 & 16.6 \\
\hline 2008-08-03 18:00:00.0 & 19.6 \\
\hline 2008-08-03 15:00:00.0 & 20.1 \\
\hline 2008-08-03 12:00:00.0 & 18.7 \\
\hline 2008-08-03 09:00:00.0 & \\
\hline 2008-08-03 06:00:00.0 & 20.2 \\
\hline 2008-08-03 03:00:00.0 & 6.7 \\
\hline 2008-08-03 00:00:00.0 & 9.1 \\
\hline 2008-08-02 21:00:00.0 & 18.1 \\
\hline 2008-08-02 18:00:00.0 & 22.2 \\
\hline 2008-08-02 15:00:00.0 & 21.4 \\
\hline 2008-08-02 12:00:00.0 & 17.2 \\
\hline 2008-08-02 09:00:00.0 & 11.0 \\
\hline 2008-08-02 06:00:00.0 & 21.8 \\
\hline 2008-08-02 03:00:00.0 & 17.4 \\
\hline 2008-08-02 00:00:00.0 & 35.4 \\
\hline 2008-08-01 21:00:00.0 & 43.4 \\
\hline 2008-08-01 18:00:00.0 & 16.8 \\
\hline 2008-08-01 15:00:00.0 & 18.7 \\
\hline 2008-08-01 12:00:00.0 & 11.6 \\
\hline 2008-08-01 09:00:00.0 & 12.1 \\
\hline 2008-08-01 06:00:00.0 & 10.2 \\
\hline 2008-08-01 03:00:00.0 & 9.7 \\
\hline 2008-08-01 00:00:00.0 & 10.9 \\
\hline 2008-07-31 21:00:00.0 & 29.8 \\
\hline 2008-07-31 18:00:00.0 & 12.6 \\
\hline 2008-07-31 15:00:00.0 & 13.5 \\
\hline 2008-07-31 12:00:00.0 & 11.3 \\
\hline 2008-07-31 09:00:00.0 & 7.2 \\
\hline 2008-07-31 06:00:00.0 & 7.7 \\
\hline 2008-07-31 03:00:00.0 & 4.6 \\
\hline 2008-07-31 00:00:00.0 & 6.9 \\
\hline 2008-07-30 21:00:00.0 & 14.2 \\
\hline 2008-07-30 18:00:00.0 & 14.0 \\
\hline 2008-07-30 15:00:00.0 & 16.3 \\
\hline 2008-07-30 12:00:00.0 & 45.8 \\
\hline 2008-07-30 09:00:00.0 & 8.7 \\
\hline 2008-07-30 06:00:00.0 & 18.8 \\
\hline 2008-07-30 03:00:00.0 & 35.3 \\
\hline 2008-07-30 00:00:00.0 & 23.5 \\
\hline 2008-07-29 21:00:00.0 & 30.7 \\
\hline 2008-07-29 18:00:00.0 & 23.8 \\
\hline $2008-07-29$ 15:00:00.0 & 26.6 \\
\hline 2008-07-29 12:00:00.0 & 25.0 \\
\hline 2008-07-29 09:00:00.0 & 23.7 \\
\hline 2008-07-29 06:00:00.0 & 26.5 \\
\hline 2008-07-29 03:00:00.0 & 18.6 \\
\hline 2008-07-29 00:00:00.0 & 22.9 \\
\hline 2008-07-28 21:00:00.0 & 22.5 \\
\hline $2008-07-28$ 18:00:00.0 & 18.8 \\
\hline 2008-07-28 15:00:00.0 & 19.0 \\
\hline 2008-07-28 12:00:00.0 & 16.4 \\
\hline 2008-07-28 09:00:00.0 & 10.1 \\
\hline 2008-07-28 06:00:00.0 & 15.1 \\
\hline 2008-07-28 03:00:00.0 & 14.6 \\
\hline 2008-07-28 00:00:00.0 & 9.9 \\
\hline 2008-07-27 21:00:00.0 & 9.7 \\
\hline $2008-07-27$ 18:00:00.0 & 15.4 \\
\hline $2008-07-27$ 15:00:00.0 & 18.4 \\
\hline 2008-07-27 12:00:00.0 & 21.1 \\
\hline 2008-07-27 09:00:00.0 & 21.0 \\
\hline 2008-07-27 06:00:00.0 & 20.6 \\
\hline
\end{tabular}




\section{ANEXO 01 - RAJADAS DE VENTO REGISTRADAS EM SÃO MARTINHO DA SERRA / RS}

\begin{tabular}{|c|c|}
\hline DataHora & VelVentoMax \\
\hline 2008-07-27 03:00:00.0 & 20.3 \\
\hline 2008-07-27 00:00:00.0 & 17.1 \\
\hline 2008-07-26 21:00:00.0 & 11.2 \\
\hline 2008-07-26 18:00:00.0 & 15.9 \\
\hline 2008-07-26 15:00:00.0 & 19.1 \\
\hline 2008-07-26 12:00:00.0 & 20.0 \\
\hline 2008-07-26 09:00:00.0 & 19.8 \\
\hline 2008-07-26 06:00:00.0 & 17.6 \\
\hline 2008-07-26 03:00:00.0 & 14.0 \\
\hline 2008-07-26 00:00:00.0 & 11.5 \\
\hline 2008-07-25 21:00:00.0 & 11.2 \\
\hline 2008-07-25 18:00:00.0 & 12.0 \\
\hline 2008-07-25 15:00:00.0 & 15.3 \\
\hline 2008-07-25 12:00:00.0 & 9.9 \\
\hline 2008-07-25 09:00:00.0 & 8.7 \\
\hline 2008-07-25 06:00:00.0 & 13.4 \\
\hline 2008-07-25 03:00:00.0 & 12.8 \\
\hline 2008-07-25 00:00:00.0 & 15.9 \\
\hline 2008-07-24 21:00:00.0 & 22.2 \\
\hline 2008-07-24 18:00:00.0 & 25.5 \\
\hline 2008-07-24 15:00:00.0 & 19.8 \\
\hline 2008-07-24 12:00:00.0 & 19.2 \\
\hline 2008-07-24 09:00:00.0 & 34.1 \\
\hline 2008-07-24 06:00:00.0 & 13.1 \\
\hline 2008-07-24 03:00:00.0 & 14.4 \\
\hline 2008-07-24 00:00:00.0 & 18.4 \\
\hline 2008-07-23 21:00:00.0 & 16.6 \\
\hline 2008-07-23 18:00:00.0 & 15.1 \\
\hline 2008-07-23 15:00:00.0 & 13.4 \\
\hline 2008-07-23 12:00:00.0 & 8.5 \\
\hline 2008-07-23 09:00:00.0 & 8.8 \\
\hline 2008-07-23 06:00:00.0 & 10.6 \\
\hline 2008-07-23 03:00:00.0 & 14.5 \\
\hline 2008-07-23 00:00:00.0 & 19.2 \\
\hline 2008-07-22 21:00:00.0 & 23.2 \\
\hline 2008-07-22 18:00:00.0 & 24.8 \\
\hline 2008-07-22 15:00:00.0 & 24.8 \\
\hline 2008-07-22 12:00:00.0 & 30.9 \\
\hline 2008-07-22 09:00:00.0 & 29.5 \\
\hline 2008-07-22 06:00:00.0 & 31.9 \\
\hline 2008-07-22 03:00:00.0 & 3.3 \\
\hline 2008-07-22 00:00:00.0 & 25.0 \\
\hline 2008-07-21 21:00:00.0 & 19.4 \\
\hline 2008-07-21 18:00:00.0 & 19.1 \\
\hline 2008-07-21 15:00:00.0 & 5.9 \\
\hline 2008-07-21 12:00:00.0 & 21.3 \\
\hline 2008-07-21 09:00:00.0 & 22.3 \\
\hline 2008-07-21 06:00:00.0 & 21.3 \\
\hline 2008-07-21 03:00:00.0 & 21.1 \\
\hline 2008-07-21 00:00:00.0 & 18.5 \\
\hline 2008-07-20 21:00:00.0 & 18.8 \\
\hline 2008-07-20 18:00:00.0 & 20.6 \\
\hline 2008-07-20 15:00:00.0 & 26.5 \\
\hline 2008-07-20 12:00:00.0 & 23.4 \\
\hline 2008-07-20 09:00:00.0 & 21.4 \\
\hline 2008-07-20 06:00:00.0 & 21.9 \\
\hline 2008-07-20 03:00:00.0 & 20.1 \\
\hline 2008-07-20 00:00:00.0 & 19.2 \\
\hline 2008-07-19 21:00:00.0 & 20.7 \\
\hline 2008-07-19 18:00:00.0 & 17.5 \\
\hline 2008-07-19 15:00:00.0 & 18.1 \\
\hline 2008-07-19 12:00:00.0 & 13.3 \\
\hline 2008-07-19 09:00:00.0 & 16.6 \\
\hline 2008-07-19 06:00:00.0 & 11.9 \\
\hline 2008-07-19 03:00:00.0 & 13.8 \\
\hline 2008-07-19 00:00:00.0 & 11.4 \\
\hline 2008-07-18 21:00:00.0 & 18.4 \\
\hline 2008-07-18 18:00:00.0 & 18.1 \\
\hline 2008-07-18 15:00:00.0 & 18.3 \\
\hline
\end{tabular}

\begin{tabular}{|c|c|}
\hline DataHora & VelVentoMax \\
\hline 2008-07-18 12:00:00.0 & 21.4 \\
\hline 2008-07-18 09:00:00.0 & 20.2 \\
\hline 2008-07-18 06:00:00.0 & 20.1 \\
\hline 2008-07-18 03:00:00.0 & 18.2 \\
\hline 2008-07-18 00:00:00.0 & 18.4 \\
\hline 2008-07-17 21:00:00.0 & 29.8 \\
\hline 2008-07-17 18:00:00.0 & 37.2 \\
\hline 2008-07-17 15:00:00.0 & 35.1 \\
\hline 2008-07-17 12:00:00.0 & 21.4 \\
\hline 2008-07-17 09:00:00.0 & 26.5 \\
\hline 2008-07-17 06:00:00.0 & 32.5 \\
\hline 2008-07-17 03:00:00.0 & 24.8 \\
\hline 2008-07-17 00:00:00.0 & 16.0 \\
\hline 2008-07-16 21:00:00.0 & 25.0 \\
\hline 2008-07-16 18:00:00.0 & 30.0 \\
\hline 2008-07-16 15:00:00.0 & 24.9 \\
\hline 2008-07-16 12:00:00.0 & 20.1 \\
\hline 2008-07-16 09:00:00.0 & 18.8 \\
\hline 2008-07-16 06:00:00.0 & 18.4 \\
\hline 2008-07-16 03:00:00.0 & 16.7 \\
\hline 2008-07-16 00:00:00.0 & 13.2 \\
\hline 2008-07-15 21:00:00.0 & 14.6 \\
\hline 2008-07-15 18:00:00.0 & 18.5 \\
\hline 2008-07-15 15:00:00.0 & 17.3 \\
\hline 2008-07-15 12:00:00.0 & 15.2 \\
\hline 2008-07-15 09:00:00.0 & 15.1 \\
\hline 2008-07-15 06:00:00.0 & 13.3 \\
\hline 2008-07-15 03:00:00.0 & 13.4 \\
\hline 2008-07-15 00:00:00.0 & 12.2 \\
\hline 2008-07-14 21:00:00.0 & 12.3 \\
\hline 2008-07-14 18:00:00.0 & 14.4 \\
\hline 2008-07-14 15:00:00.0 & 14.6 \\
\hline 2008-07-14 12:00:00.0 & 13.7 \\
\hline 2008-07-14 09:00:00.0 & 12.4 \\
\hline 2008-07-14 06:00:00.0 & 14.3 \\
\hline 2008-07-14 03:00:00.0 & 15.7 \\
\hline 2008-07-14 00:00:00.0 & 12.2 \\
\hline 2008-07-13 21:00:00.0 & 17.5 \\
\hline 2008-07-13 18:00:00.0 & 22.4 \\
\hline 2008-07-13 15:00:00.0 & 22.5 \\
\hline 2008-07-13 12:00:00.0 & 24.6 \\
\hline 2008-07-13 09:00:00.0 & 23.2 \\
\hline 2008-07-13 06:00:00.0 & 24.6 \\
\hline 2008-07-13 03:00:00.0 & 21.4 \\
\hline 2008-07-13 00:00:00.0 & 18.9 \\
\hline 2008-07-12 21:00:00.0 & 21.7 \\
\hline 2008-07-12 18:00:00.0 & 23.1 \\
\hline 2008-07-12 15:00:00.0 & 23.7 \\
\hline 2008-07-12 12:00:00.0 & 19.5 \\
\hline 2008-07-12 09:00:00.0 & 20.1 \\
\hline 2008-07-12 06:00:00.0 & 19.0 \\
\hline 2008-07-12 03:00:00.0 & 16.2 \\
\hline 2008-07-12 00:00:00.0 & 17.4 \\
\hline 2008-07-11 21:00:00.0 & 17.5 \\
\hline 2008-07-11 18:00:00.0 & 19.5 \\
\hline 2008-07-11 15:00:00.0 & 21.1 \\
\hline 2008-07-11 12:00:00.0 & 19.5 \\
\hline 2008-07-11 09:00:00.0 & 19.6 \\
\hline 2008-07-11 06:00:00.0 & 3.1 \\
\hline 2008-07-11 03:00:00.0 & 15.2 \\
\hline 2008-07-11 00:00:00.0 & 13.5 \\
\hline 2008-07-10 21:00:00.0 & 10.4 \\
\hline 2008-07-10 18:00:00.0 & 8.5 \\
\hline 2008-07-10 15:00:00.0 & 13.5 \\
\hline 2008-07-10 12:00:00.0 & 17.7 \\
\hline 2008-07-10 09:00:00.0 & \\
\hline 2008-07-10 06:00:00.0 & 19.0 \\
\hline 2008-07-10 03:00:00.0 & 16.2 \\
\hline 2008-07-10 00:00:00.0 & 13.7 \\
\hline
\end{tabular}

\begin{tabular}{|c|c|}
\hline DataHora & VelVentoMax \\
\hline 2008-07-09 21:00:00.0 & 12.4 \\
\hline 2008-07-09 18:00:00.0 & 17.5 \\
\hline 2008-07-09 15:00:00.0 & 17.7 \\
\hline 2008-07-09 12:00:00.0 & 16.0 \\
\hline 2008-07-09 09:00:00.0 & 15.1 \\
\hline 2008-07-09 06:00:00.0 & 13.9 \\
\hline 2008-07-09 03:00:00.0 & 12.3 \\
\hline 2008-07-09 00:00:00.0 & 10.4 \\
\hline 2008-07-08 21:00:00.0 & 11.4 \\
\hline 2008-07-08 18:00:00.0 & 10.6 \\
\hline 2008-07-08 15:00:00.0 & 13.1 \\
\hline 2008-07-08 12:00:00.0 & 8.5 \\
\hline 2008-07-08 09:00:00.0 & 19.0 \\
\hline 2008-07-08 06:00:00.0 & 24.6 \\
\hline 2008-07-08 03:00:00.0 & 21.1 \\
\hline 2008-07-08 00:00:00.0 & 13.1 \\
\hline 2008-07-07 21:00:00.0 & 12.6 \\
\hline 2008-07-07 18:00:00.0 & 19.7 \\
\hline 2008-07-07 15:00:00.0 & 25.3 \\
\hline 2008-07-07 12:00:00.0 & 20.3 \\
\hline 2008-07-07 09:00:00.0 & 22.0 \\
\hline 2008-07-07 06:00:00.0 & 18.8 \\
\hline 2008-07-07 03:00:00.0 & 18.7 \\
\hline 2008-07-07 00:00:00.0 & 17.6 \\
\hline 2008-07-06 21:00:00.0 & 16.3 \\
\hline 2008-07-06 18:00:00.0 & 19.4 \\
\hline 2008-07-06 15:00:00.0 & 21.5 \\
\hline 2008-07-06 12:00:00.0 & 19.6 \\
\hline 2008-07-06 09:00:00.0 & 18.1 \\
\hline 2008-07-06 06:00:00.0 & 16.2 \\
\hline 2008-07-06 03:00:00.0 & 17.8 \\
\hline 2008-07-06 00:00:00.0 & 16.7 \\
\hline 2008-07-05 21:00:00.0 & 18.7 \\
\hline 2008-07-05 18:00:00.0 & 20.8 \\
\hline 2008-07-05 15:00:00.0 & 20.6 \\
\hline 2008-07-05 12:00:00.0 & 19.2 \\
\hline 2008-07-05 09:00:00.0 & 17.7 \\
\hline 2008-07-05 06:00:00.0 & 17.9 \\
\hline 2008-07-05 03:00:00.0 & 19.9 \\
\hline 2008-07-05 00:00:00.0 & 15.6 \\
\hline 2008-07-04 21:00:00.0 & 12.8 \\
\hline 2008-07-04 18:00:00.0 & 15.5 \\
\hline 2008-07-04 15:00:00.0 & 19.9 \\
\hline 2008-07-04 12:00:00.0 & 19.0 \\
\hline 2008-07-04 09:00:00.0 & 18.2 \\
\hline 2008-07-04 06:00:00.0 & 15.1 \\
\hline 2008-07-04 03:00:00.0 & 12.6 \\
\hline 2008-07-04 00:00:00.0 & 15.7 \\
\hline 2008-07-03 21:00:00.0 & 17.8 \\
\hline 2008-07-03 18:00:00.0 & 17.6 \\
\hline 2008-07-03 15:00:00.0 & 19.9 \\
\hline 2008-07-03 12:00:00.0 & 32.2 \\
\hline 2008-07-03 09:00:00.0 & 10.7 \\
\hline 2008-07-03 06:00:00.0 & 8.8 \\
\hline 2008-07-03 03:00:00.0 & 11.2 \\
\hline 2008-07-03 00:00:00.0 & 18.6 \\
\hline 2008-07-02 21:00:00.0 & 21.6 \\
\hline 2008-07-02 18:00:00.0 & 21.3 \\
\hline 2008-07-02 15:00:00.0 & 17.0 \\
\hline 2008-07-02 12:00:00.0 & 14.5 \\
\hline 2008-07-02 09:00:00.0 & 18.0 \\
\hline 2008-07-02 06:00:00.0 & 15.6 \\
\hline 2008-07-02 03:00:00.0 & 13.7 \\
\hline 2008-07-02 00:00:00.0 & 12.8 \\
\hline 2008-07-01 21:00:00.0 & 11.5 \\
\hline 2008-07-01 18:00:00.0 & 10.1 \\
\hline 2008-07-01 15:00:00.0 & 10.5 \\
\hline 2008-07-01 12:00:00.0 & 10.2 \\
\hline 2008-07-01 09:00:00.0 & 8.8 \\
\hline
\end{tabular}




\section{ANEXO 01 - RAJADAS DE VENTO REGISTRADAS EM SÃO MARTINHO DA SERRA / RS}

\begin{tabular}{|c|c|}
\hline DataHora & VelVentoMax \\
\hline 2008-07-01 06:00:00.0 & 14.0 \\
\hline 2008-07-01 03:00:00.0 & 10.2 \\
\hline 2008-07-01 00:00:00.0 & 14.1 \\
\hline 2008-06-30 21:00:00.0 & 10.2 \\
\hline 2008-06-30 18:00:00.0 & 27.1 \\
\hline 2008-06-30 15:00:00.0 & 13.7 \\
\hline 2008-06-30 12:00:00.0 & 44.8 \\
\hline 2008-06-30 09:00:00.0 & 12.9 \\
\hline 2008-06-30 06:00:00.0 & 12.7 \\
\hline 2008-06-30 03:00:00.0 & 12.0 \\
\hline 2008-06-30 00:00:00.0 & 15.4 \\
\hline 2008-06-29 21:00:00.0 & 11.8 \\
\hline 2008-06-29 18:00:00.0 & 11.8 \\
\hline 2008-06-29 15:00:00.0 & 9.7 \\
\hline 2008-06-29 12:00:00.0 & 8.5 \\
\hline 2008-06-29 09:00:00.0 & 8.8 \\
\hline 2008-06-29 06:00:00.0 & 8.8 \\
\hline 2008-06-29 03:00:00.0 & 5.2 \\
\hline 2008-06-29 00:00:00.0 & 5.7 \\
\hline 2008-06-28 21:00:00.0 & 9.6 \\
\hline 2008-06-28 18:00:00.0 & 12.1 \\
\hline 2008-06-28 15:00:00.0 & 14.2 \\
\hline 2008-06-28 12:00:00.0 & 13.2 \\
\hline 2008-06-28 09:00:00.0 & 14.1 \\
\hline 2008-06-28 06:00:00.0 & 14.9 \\
\hline 2008-06-28 03:00:00.0 & 14.4 \\
\hline 2008-06-28 00:00:00.0 & 9.2 \\
\hline 2008-06-27 21:00:00.0 & 12.8 \\
\hline 2008-06-27 18:00:00.0 & 9.4 \\
\hline 2008-06-27 15:00:00.0 & 12.2 \\
\hline 2008-06-27 12:00:00.0 & 11.1 \\
\hline 2008-06-27 09:00:00.0 & 29.8 \\
\hline 2008-06-27 06:00:00.0 & 15.8 \\
\hline 2008-06-27 03:00:00.0 & 14.3 \\
\hline 2008-06-27 00:00:00.0 & 18.3 \\
\hline 2008-06-26 21:00:00.0 & 19.1 \\
\hline 2008-06-26 18:00:00.0 & 22.6 \\
\hline 2008-06-26 15:00:00.0 & 24.8 \\
\hline 2008-06-26 12:00:00.0 & 25.0 \\
\hline 2008-06-26 09:00:00.0 & 25.2 \\
\hline 2008-06-26 06:00:00.0 & 20.4 \\
\hline 2008-06-26 03:00:00.0 & 20.1 \\
\hline 2008-06-26 00:00:00.0 & 14.3 \\
\hline 2008-06-25 21:00:00.0 & 15.6 \\
\hline 2008-06-25 18:00:00.0 & 21.5 \\
\hline 2008-06-25 15:00:00.0 & 26.6 \\
\hline 2008-06-25 12:00:00.0 & 19.6 \\
\hline 2008-06-25 09:00:00.0 & 16.9 \\
\hline 2008-06-25 06:00:00.0 & 20.9 \\
\hline 2008-06-25 03:00:00.0 & 15.0 \\
\hline 2008-06-25 00:00:00.0 & 15.4 \\
\hline 2008-06-24 21:00:00.0 & 18.3 \\
\hline 2008-06-24 18:00:00.0 & 20.1 \\
\hline 2008-06-24 15:00:00.0 & 23.1 \\
\hline 2008-06-24 12:00:00.0 & 18.7 \\
\hline 2008-06-24 09:00:00.0 & 19.1 \\
\hline 2008-06-24 06:00:00.0 & 18.5 \\
\hline 2008-06-24 03:00:00.0 & 18.4 \\
\hline 2008-06-24 00:00:00.0 & 24.7 \\
\hline 2008-06-23 21:00:00.0 & 26.8 \\
\hline 2008-06-23 18:00:00.0 & 19.7 \\
\hline 2008-06-23 15:00:00.0 & 19.4 \\
\hline 2008-06-23 12:00:00.0 & 18.6 \\
\hline 2008-06-23 09:00:00.0 & 11.2 \\
\hline 2008-06-23 06:00:00.0 & 11.2 \\
\hline 2008-06-23 03:00:00.0 & 11.7 \\
\hline 2008-06-23 00:00:00.0 & 12.2 \\
\hline 2008-06-22 21:00:00.0 & 11.7 \\
\hline 2008-06-22 18:00:00.0 & 15.4 \\
\hline
\end{tabular}

\begin{tabular}{|c|c|}
\hline DataHora & VelVentoMax \\
\hline 2008-06-22 15:00:00.0 & 27.1 \\
\hline 2008-06-22 12:00:00.0 & 11.0 \\
\hline 2008-06-22 09:00:00.0 & 15.2 \\
\hline 2008-06-22 06:00:00.0 & 14.5 \\
\hline 2008-06-22 03:00:00.0 & 12.7 \\
\hline 2008-06-22 00:00:00.0 & 13.0 \\
\hline 2008-06-21 21:00:00.0 & 14.6 \\
\hline 2008-06-21 18:00:00.0 & 20.1 \\
\hline 2008-06-21 15:00:00.0 & 17.1 \\
\hline 2008-06-21 12:00:00.0 & 18.1 \\
\hline 2008-06-21 09:00:00.0 & 23.2 \\
\hline 2008-06-21 06:00:00.0 & 24.3 \\
\hline 2008-06-21 03:00:00.0 & 27.5 \\
\hline 2008-06-21 00:00:00.0 & 28.6 \\
\hline 2008-06-20 21:00:00.0 & 26.7 \\
\hline 2008-06-20 18:00:00.0 & 24.6 \\
\hline 2008-06-20 15:00:00.0 & 27.6 \\
\hline 2008-06-20 12:00:00.0 & 20.7 \\
\hline 2008-06-20 09:00:00.0 & 21.4 \\
\hline 2008-06-20 06:00:00.0 & 21.2 \\
\hline 2008-06-20 03:00:00.0 & 24.3 \\
\hline 2008-06-20 00:00:00.0 & 22.1 \\
\hline 2008-06-19 21:00:00.0 & 32.9 \\
\hline 2008-06-19 18:00:00.0 & 32.0 \\
\hline 2008-06-19 15:00:00.0 & 23.7 \\
\hline 2008-06-19 12:00:00.0 & 24.8 \\
\hline 2008-06-19 09:00:00.0 & 22.7 \\
\hline 2008-06-19 06:00:00.0 & 20.8 \\
\hline 2008-06-19 03:00:00.0 & 23.2 \\
\hline 2008-06-19 00:00:00.0 & 15.9 \\
\hline 2008-06-18 21:00:00.0 & 13.8 \\
\hline 2008-06-18 18:00:00.0 & 12.4 \\
\hline 2008-06-18 15:00:00.0 & 8.1 \\
\hline 2008-06-18 12:00:00.0 & 9.7 \\
\hline 2008-06-18 09:00:00.0 & 12.3 \\
\hline 2008-06-18 06:00:00.0 & 12.0 \\
\hline 2008-06-18 03:00:00.0 & 10.5 \\
\hline 2008-06-18 00:00:00.0 & 9.7 \\
\hline 2008-06-17 21:00:00.0 & 9.8 \\
\hline 2008-06-17 18:00:00.0 & 11.5 \\
\hline 2008-06-17 15:00:00.0 & 12.3 \\
\hline 2008-06-17 12:00:00.0 & 13.5 \\
\hline 2008-06-17 09:00:00.0 & 8.6 \\
\hline 2008-06-17 06:00:00.0 & 9.0 \\
\hline 2008-06-17 03:00:00.0 & 8.2 \\
\hline 2008-06-17 00:00:00.0 & 7.8 \\
\hline 2008-06-16 21:00:00.0 & 14.5 \\
\hline 2008-06-16 18:00:00.0 & 16.8 \\
\hline 2008-06-16 15:00:00.0 & 9.8 \\
\hline 2008-06-16 12:00:00.0 & 8.1 \\
\hline 2008-06-16 09:00:00.0 & 7.8 \\
\hline 2008-06-16 06:00:00.0 & 14.0 \\
\hline 2008-06-16 03:00:00.0 & 18.2 \\
\hline 2008-06-16 00:00:00.0 & 14.2 \\
\hline 2008-06-15 21:00:00.0 & 32.3 \\
\hline 2008-06-15 18:00:00.0 & 32.7 \\
\hline 2008-06-15 15:00:00.0 & 30.7 \\
\hline 2008-06-15 12:00:00.0 & 17.8 \\
\hline 2008-06-15 09:00:00.0 & 35.1 \\
\hline 2008-06-15 06:00:00.0 & 14.9 \\
\hline 2008-06-15 03:00:00.0 & 14.4 \\
\hline 2008-06-15 00:00:00.0 & 16.8 \\
\hline 2008-06-14 21:00:00.0 & 27.1 \\
\hline 2008-06-14 18:00:00.0 & 28.2 \\
\hline 2008-06-14 15:00:00.0 & 24.9 \\
\hline 2008-06-14 12:00:00.0 & 16.2 \\
\hline 2008-06-14 09:00:00.0 & 11.3 \\
\hline 2008-06-14 06:00:00.0 & 23.0 \\
\hline 2008-06-14 03:00:00.0 & 16.6 \\
\hline
\end{tabular}

\begin{tabular}{|c|c|}
\hline DataHora & VelVentoMax \\
\hline 2008-06-14 00:00:00.0 & 13.0 \\
\hline 2008-06-13 21:00:00.0 & 9.1 \\
\hline 2008-06-13 18:00:00.0 & 10.5 \\
\hline 2008-06-13 15:00:00.0 & 10.6 \\
\hline 2008-06-13 12:00:00.0 & 7.9 \\
\hline 2008-06-13 09:00:00.0 & 6.7 \\
\hline 2008-06-13 06:00:00.0 & 8.0 \\
\hline 2008-06-13 03:00:00.0 & 9.0 \\
\hline 2008-06-13 00:00:00.0 & 8.2 \\
\hline 2008-06-12 21:00:00.0 & 10.7 \\
\hline 2008-06-12 18:00:00.0 & 13.1 \\
\hline 2008-06-12 15:00:00.0 & 10.7 \\
\hline 2008-06-12 12:00:00.0 & 5.8 \\
\hline 2008-06-12 09:00:00.0 & 6.2 \\
\hline 2008-06-12 06:00:00.0 & 7.0 \\
\hline 2008-06-12 03:00:00.0 & 8.4 \\
\hline 2008-06-12 00:00:00.0 & \\
\hline 2008-06-11 21:00:00.0 & 12.6 \\
\hline 2008-06-11 18:00:00.0 & 9.4 \\
\hline 2008-06-11 15:00:00.0 & 9.2 \\
\hline 2008-06-11 12:00:00.0 & 5.7 \\
\hline 2008-06-11 09:00:00.0 & 6.8 \\
\hline 2008-06-11 06:00:00.0 & 6.5 \\
\hline 2008-06-11 03:00:00.0 & 8.2 \\
\hline 2008-06-11 00:00:00.0 & 5.4 \\
\hline 2008-06-10 21:00:00.0 & 18.5 \\
\hline 2008-06-10 18:00:00.0 & 20.0 \\
\hline 2008-06-10 15:00:00.0 & 20.2 \\
\hline 2008-06-10 12:00:00.0 & 16.5 \\
\hline 2008-06-10 09:00:00.0 & 25.7 \\
\hline 2008-06-10 06:00:00.0 & 32.8 \\
\hline 2008-06-10 03:00:00.0 & 35.0 \\
\hline 2008-06-10 00:00:00.0 & 42.4 \\
\hline 2008-06-09 21:00:00.0 & 44.1 \\
\hline 2008-06-09 18:00:00.0 & 24.1 \\
\hline 2008-06-09 15:00:00.0 & 21.7 \\
\hline 2008-06-09 12:00:00.0 & 13.1 \\
\hline 2008-06-09 09:00:00.0 & 19.9 \\
\hline 2008-06-09 06:00:00.0 & 16.1 \\
\hline 2008-06-09 03:00:00.0 & 16.7 \\
\hline 2008-06-09 00:00:00.0 & 15.0 \\
\hline 2008-06-08 21:00:00.0 & 13.9 \\
\hline 2008-06-08 18:00:00.0 & 29.6 \\
\hline 2008-06-08 15:00:00.0 & 14.2 \\
\hline 2008-06-08 12:00:00.0 & 16.1 \\
\hline 2008-06-08 09:00:00.0 & 12.7 \\
\hline 2008-06-08 06:00:00.0 & 16.7 \\
\hline 2008-06-08 03:00:00.0 & 14.4 \\
\hline 2008-06-08 00:00:00.0 & 14.9 \\
\hline 2008-06-07 21:00:00.0 & 12.7 \\
\hline 2008-06-07 18:00:00.0 & 18.1 \\
\hline 2008-06-07 15:00:00.0 & 21.1 \\
\hline 2008-06-07 12:00:00.0 & 20.9 \\
\hline 2008-06-07 09:00:00.0 & 14.9 \\
\hline 2008-06-07 06:00:00.0 & 11.6 \\
\hline 2008-06-07 03:00:00.0 & 9.8 \\
\hline 2008-06-07 00:00:00.0 & 9.1 \\
\hline 2008-06-06 21:00:00.0 & 11.7 \\
\hline 2008-06-06 18:00:00.0 & 20.0 \\
\hline 2008-06-06 15:00:00.0 & 18.9 \\
\hline 2008-06-06 12:00:00.0 & 22.2 \\
\hline 2008-06-06 09:00:00.0 & 22.3 \\
\hline 2008-06-06 06:00:00.0 & 13.0 \\
\hline 2008-06-06 03:00:00.0 & 10.6 \\
\hline 2008-06-06 00:00:00.0 & 9.3 \\
\hline 2008-06-05 21:00:00.0 & 13.8 \\
\hline 2008-06-05 18:00:00.0 & 15.3 \\
\hline 2008-06-05 15:00:00.0 & 16.9 \\
\hline 2008-06-05 12:00:00.0 & 11.8 \\
\hline
\end{tabular}




\section{ANEXO 01 - RAJADAS DE VENTO REGISTRADAS EM SÃO MARTINHO DA SERRA / RS}

\begin{tabular}{|c|c|}
\hline DataHora & VelVentoMax \\
\hline 2008-06-05 09:00:00.0 & 18.6 \\
\hline 2008-06-05 06:00:00.0 & 17.8 \\
\hline 2008-06-05 03:00:00.0 & 16.4 \\
\hline 2008-06-05 00:00:00.0 & 16.4 \\
\hline 2008-06-04 21:00:00.0 & 14.8 \\
\hline 2008-06-04 18:00:00.0 & 18.4 \\
\hline 2008-06-04 15:00:00.0 & 19.0 \\
\hline 2008-06-04 12:00:00.0 & 13.5 \\
\hline 2008-06-04 09:00:00.0 & 16.0 \\
\hline 2008-06-04 06:00:00.0 & 15.7 \\
\hline 2008-06-04 03:00:00.0 & 11.5 \\
\hline 2008-06-04 00:00:00.0 & 13.5 \\
\hline 2008-06-03 21:00:00.0 & 20.2 \\
\hline 2008-06-03 18:00:00.0 & 24.2 \\
\hline 2008-06-03 15:00:00.0 & 28.0 \\
\hline 2008-06-03 12:00:00.0 & 21.5 \\
\hline 2008-06-03 09:00:00.0 & 26.1 \\
\hline 2008-06-03 06:00:00.0 & 17.2 \\
\hline 2008-06-03 03:00:00.0 & 19.9 \\
\hline 2008-06-03 00:00:00.0 & 19.4 \\
\hline 2008-06-02 21:00:00.0 & 16.8 \\
\hline 2008-06-02 18:00:00.0 & 20.1 \\
\hline 2008-06-02 15:00:00.0 & 13.7 \\
\hline 2008-06-02 12:00:00.0 & 17.3 \\
\hline 2008-06-02 09:00:00.0 & 16.1 \\
\hline 2008-06-02 06:00:00.0 & 15.6 \\
\hline 2008-06-02 03:00:00.0 & 15.1 \\
\hline 2008-06-02 00:00:00.0 & 14.1 \\
\hline 2008-06-01 21:00:00.0 & 14.0 \\
\hline 2008-06-01 18:00:00.0 & 11.8 \\
\hline 2008-06-01 15:00:00.0 & 18.0 \\
\hline 2008-06-01 12:00:00.0 & 21.0 \\
\hline 2008-06-01 09:00:00.0 & 21.1 \\
\hline 2008-06-01 06:00:00.0 & 15.6 \\
\hline 2008-06-01 03:00:00.0 & 15.8 \\
\hline 2008-06-01 00:00:00.0 & 19.2 \\
\hline 2008-05-31 21:00:00.0 & \\
\hline 2008-05-31 18:00:00.0 & 16.6 \\
\hline 2008-05-31 15:00:00.0 & 17.3 \\
\hline 2008-05-31 12:00:00.0 & 12.5 \\
\hline 2008-05-31 09:00:00.0 & 9.4 \\
\hline 2008-05-31 06:00:00.0 & 4.5 \\
\hline 2008-05-31 03:00:00.0 & 11.3 \\
\hline 2008-05-31 00:00:00.0 & 8.7 \\
\hline 2008-05-30 21:00:00.0 & 14.5 \\
\hline 2008-05-30 18:00:00.0 & 13.9 \\
\hline 2008-05-30 15:00:00.0 & 16.6 \\
\hline 2008-05-30 12:00:00.0 & 6.9 \\
\hline 2008-05-30 09:00:00.0 & 10.8 \\
\hline 2008-05-30 06:00:00.0 & 13.7 \\
\hline 2008-05-30 03:00:00.0 & 20.7 \\
\hline 2008-05-30 00:00:00.0 & 18.6 \\
\hline 2008-05-29 21:00:00.0 & 22.0 \\
\hline 2008-05-29 18:00:00.0 & 20.1 \\
\hline 2008-05-29 15:00:00.0 & 20.7 \\
\hline 2008-05-29 12:00:00.0 & 23.3 \\
\hline 2008-05-29 09:00:00.0 & 27.5 \\
\hline 2008-05-29 06:00:00.0 & 27.8 \\
\hline 2008-05-29 03:00:00.0 & 27.9 \\
\hline 2008-05-29 00:00:00.0 & 17.1 \\
\hline 2008-05-28 21:00:00.0 & 19.1 \\
\hline 2008-05-28 18:00:00.0 & 17.9 \\
\hline 2008-05-28 15:00:00.0 & 17.6 \\
\hline 2008-05-28 12:00:00.0 & 27.3 \\
\hline 2008-05-28 09:00:00.0 & 38.7 \\
\hline 2008-05-28 06:00:00.0 & 26.6 \\
\hline 2008-05-28 03:00:00.0 & 19.2 \\
\hline 2008-05-28 00:00:00.0 & 13.0 \\
\hline 2008-05-27 21:00:00.0 & 29.1 \\
\hline
\end{tabular}

\begin{tabular}{|c|c|}
\hline DataHora & VelVentoMax \\
\hline 2008-05-27 18:00:00.0 & 50.5 \\
\hline 2008-05-27 15:00:00.0 & 19.3 \\
\hline 2008-05-27 12:00:00.0 & 20.1 \\
\hline 2008-05-27 09:00:00.0 & 17.3 \\
\hline 2008-05-27 06:00:00.0 & 18.8 \\
\hline 2008-05-27 03:00:00.0 & 19.2 \\
\hline 2008-05-27 00:00:00.0 & 12.9 \\
\hline 2008-05-26 21:00:00.0 & 12.6 \\
\hline 2008-05-26 18:00:00.0 & 13.7 \\
\hline 2008-05-26 15:00:00.0 & 15.5 \\
\hline 2008-05-26 12:00:00.0 & 13.0 \\
\hline 2008-05-26 09:00:00.0 & 13.1 \\
\hline 2008-05-26 06:00:00.0 & 13.5 \\
\hline 2008-05-26 03:00:00.0 & 10.2 \\
\hline 2008-05-26 00:00:00.0 & 10.1 \\
\hline 2008-05-25 21:00:00.0 & 11.7 \\
\hline 2008-05-25 18:00:00.0 & \\
\hline 2008-05-25 15:00:00.0 & 14.1 \\
\hline 2008-05-25 12:00:00.0 & 7.3 \\
\hline 2008-05-25 09:00:00.0 & \\
\hline 2008-05-25 06:00:00.0 & 19.5 \\
\hline 2008-05-25 03:00:00.0 & 21.1 \\
\hline 2008-05-25 00:00:00.0 & 21.4 \\
\hline 2008-05-24 21:00:00.0 & 23.9 \\
\hline 2008-05-24 18:00:00.0 & 22.3 \\
\hline 2008-05-24 15:00:00.0 & 21.3 \\
\hline 2008-05-24 12:00:00.0 & 16.9 \\
\hline 2008-05-24 09:00:00.0 & \\
\hline 2008-05-24 06:00:00.0 & 23.3 \\
\hline 2008-05-24 03:00:00.0 & 23.5 \\
\hline 2008-05-24 00:00:00.0 & 13.3 \\
\hline 2008-05-23 21:00:00.0 & 28.8 \\
\hline 2008-05-23 18:00:00.0 & 27.3 \\
\hline 2008-05-23 15:00:00.0 & 21.6 \\
\hline 2008-05-23 12:00:00.0 & 14.3 \\
\hline 2008-05-23 09:00:00.0 & 12.0 \\
\hline 2008-05-23 06:00:00.0 & 11.9 \\
\hline 2008-05-23 03:00:00.0 & 9.5 \\
\hline 2008-05-23 00:00:00.0 & 8.2 \\
\hline 2008-05-22 21:00:00.0 & 20.6 \\
\hline 2008-05-22 18:00:00.0 & 23.5 \\
\hline 2008-05-22 15:00:00.0 & 23.4 \\
\hline 2008-05-22 12:00:00.0 & 20.0 \\
\hline 2008-05-22 09:00:00.0 & 16.1 \\
\hline 2008-05-22 06:00:00.0 & 15.7 \\
\hline 2008-05-22 03:00:00.0 & 14.7 \\
\hline 2008-05-22 00:00:00.0 & 15.4 \\
\hline 2008-05-21 21:00:00.0 & 19.6 \\
\hline 2008-05-21 18:00:00.0 & 29.1 \\
\hline 2008-05-21 15:00:00.0 & 27.9 \\
\hline 2008-05-21 12:00:00.0 & 45.7 \\
\hline 2008-05-21 09:00:00.0 & \\
\hline 2008-05-21 06:00:00.0 & 41.3 \\
\hline 2008-05-21 03:00:00.0 & 16.3 \\
\hline 2008-05-21 00:00:00.0 & 14.7 \\
\hline 2008-05-20 21:00:00.0 & 23.7 \\
\hline 2008-05-20 18:00:00.0 & 28.3 \\
\hline 2008-05-20 15:00:00.0 & 27.6 \\
\hline 2008-05-20 12:00:00.0 & 23.4 \\
\hline 2008-05-20 09:00:00.0 & 17.5 \\
\hline 2008-05-20 06:00:00.0 & 20.5 \\
\hline 2008-05-20 03:00:00.0 & 14.1 \\
\hline 2008-05-20 00:00:00.0 & 11.6 \\
\hline 2008-05-19 21:00:00.0 & 27.0 \\
\hline 2008-05-19 18:00:00.0 & 31.0 \\
\hline 2008-05-19 15:00:00.0 & 26.2 \\
\hline 2008-05-19 12:00:00.0 & 18.3 \\
\hline 2008-05-19 09:00:00.0 & 17.3 \\
\hline 2008-05-19 06:00:00.0 & 16.2 \\
\hline
\end{tabular}

\begin{tabular}{|c|c|}
\hline DataHora & VelVentoMax \\
\hline 2008-05-19 03:00:00.0 & 15.0 \\
\hline 2008-05-19 00:00:00.0 & 11.2 \\
\hline 2008-05-18 21:00:00.0 & 32.5 \\
\hline 2008-05-18 18:00:00.0 & 19.3 \\
\hline 2008-05-18 15:00:00.0 & 18.2 \\
\hline 2008-05-18 12:00:00.0 & 17.6 \\
\hline 2008-05-18 09:00:00.0 & 17.7 \\
\hline 2008-05-18 06:00:00.0 & 15.8 \\
\hline 2008-05-18 03:00:00.0 & 16.2 \\
\hline 2008-05-18 00:00:00.0 & 13.7 \\
\hline 2008-05-17 21:00:00.0 & 15.7 \\
\hline 2008-05-17 18:00:00.0 & 22.1 \\
\hline 2008-05-17 15:00:00.0 & 20.9 \\
\hline 2008-05-17 12:00:00.0 & 20.0 \\
\hline 2008-05-17 09:00:00.0 & 20.8 \\
\hline 2008-05-17 06:00:00.0 & 21.2 \\
\hline 2008-05-17 03:00:00.0 & 18.4 \\
\hline 2008-05-17 00:00:00.0 & 12.2 \\
\hline 2008-05-16 21:00:00.0 & 17.0 \\
\hline 2008-05-16 18:00:00.0 & 15.9 \\
\hline 2008-05-16 15:00:00.0 & 34.4 \\
\hline 2008-05-16 12:00:00.0 & 5.2 \\
\hline 2008-05-16 09:00:00.0 & 4.4 \\
\hline 2008-05-16 06:00:00.0 & 4.3 \\
\hline 2008-05-16 03:00:00.0 & 6.2 \\
\hline 2008-05-16 00:00:00.0 & 7.4 \\
\hline 2008-05-15 21:00:00.0 & 12.8 \\
\hline 2008-05-15 18:00:00.0 & 18.1 \\
\hline 2008-05-15 15:00:00.0 & 18.9 \\
\hline 2008-05-15 12:00:00.0 & 11.3 \\
\hline 2008-05-15 09:00:00.0 & 7.2 \\
\hline 2008-05-15 06:00:00.0 & 4.8 \\
\hline 2008-05-15 03:00:00.0 & 5.3 \\
\hline 2008-05-15 00:00:00.0 & 7.9 \\
\hline 2008-05-14 21:00:00.0 & 16.2 \\
\hline 2008-05-14 18:00:00.0 & 20.7 \\
\hline 2008-05-14 15:00:00.0 & 18.9 \\
\hline 2008-05-14 12:00:00.0 & 51.1 \\
\hline 2008-05-14 09:00:00.0 & 8.6 \\
\hline 2008-05-14 06:00:00.0 & 5.4 \\
\hline 2008-05-14 03:00:00.0 & 6.0 \\
\hline 2008-05-14 00:00:00.0 & 7.9 \\
\hline 2008-05-13 21:00:00.0 & 16.0 \\
\hline 2008-05-13 18:00:00.0 & 27.1 \\
\hline 2008-05-13 15:00:00.0 & 13.7 \\
\hline 2008-05-13 12:00:00.0 & 14.9 \\
\hline 2008-05-13 09:00:00.0 & 16.7 \\
\hline 2008-05-13 06:00:00.0 & 16.6 \\
\hline 2008-05-13 03:00:00.0 & 13.7 \\
\hline 2008-05-13 00:00:00.0 & 10.8 \\
\hline 2008-05-12 21:00:00.0 & 20.4 \\
\hline 2008-05-12 18:00:00.0 & 2.7 \\
\hline 2008-05-12 15:00:00.0 & 17.4 \\
\hline 2008-05-12 12:00:00.0 & 16.7 \\
\hline 2008-05-12 09:00:00.0 & 12.9 \\
\hline 2008-05-12 06:00:00.0 & 16.5 \\
\hline 2008-05-12 03:00:00.0 & 15.3 \\
\hline 2008-05-12 00:00:00.0 & 14.7 \\
\hline 2008-05-11 21:00:00.0 & 19.5 \\
\hline 2008-05-11 18:00:00.0 & 27.1 \\
\hline 2008-05-11 15:00:00.0 & 18.9 \\
\hline 2008-05-11 12:00:00.0 & 17.6 \\
\hline 2008-05-11 09:00:00.0 & 17.6 \\
\hline 2008-05-11 06:00:00.0 & 17.2 \\
\hline 2008-05-11 03:00:00.0 & 15.1 \\
\hline 2008-05-11 00:00:00.0 & 14.5 \\
\hline 2008-05-10 21:00:00.0 & 20.0 \\
\hline 2008-05-10 18:00:00.0 & 18.9 \\
\hline 2008-05-10 15:00:00.0 & 18.4 \\
\hline
\end{tabular}




\section{ANEXO 01 - RAJADAS DE VENTO REGISTRADAS EM SÃO MARTINHO DA SERRA / RS}

\begin{tabular}{|c|c|}
\hline DataHora & VelVentoMax \\
\hline 2008-05-10 12:00:00.0 & 18.1 \\
\hline 2008-05-10 09:00:00.0 & 13.5 \\
\hline 2008-05-10 06:00:00.0 & 13.8 \\
\hline 2008-05-10 03:00:00.0 & \\
\hline 2008-05-10 00:00:00.0 & 11.7 \\
\hline 2008-05-09 21:00:00.0 & 15.4 \\
\hline 2008-05-09 18:00:00.0 & 18.7 \\
\hline 2008-05-09 15:00:00.0 & 20.0 \\
\hline 2008-05-09 12:00:00.0 & 12.8 \\
\hline 2008-05-09 09:00:00.0 & 12.5 \\
\hline 2008-05-09 06:00:00.0 & 16.5 \\
\hline 2008-05-09 03:00:00.0 & 13.1 \\
\hline 2008-05-09 00:00:00.0 & 15.3 \\
\hline 2008-05-08 21:00:00.0 & 16.5 \\
\hline 2008-05-08 18:00:00.0 & 16.0 \\
\hline 2008-05-08 15:00:00.0 & 11.2 \\
\hline 2008-05-08 12:00:00.0 & 7.5 \\
\hline 2008-05-08 09:00:00.0 & 7.8 \\
\hline 2008-05-08 06:00:00.0 & 7.5 \\
\hline 2008-05-08 03:00:00.0 & 8.9 \\
\hline 2008-05-08 00:00:00.0 & 9.6 \\
\hline 2008-05-07 21:00:00.0 & 11.1 \\
\hline 2008-05-07 18:00:00.0 & 12.6 \\
\hline 2008-05-07 15:00:00.0 & 12.8 \\
\hline 2008-05-07 12:00:00.0 & 11.1 \\
\hline 2008-05-07 09:00:00.0 & 12.3 \\
\hline 2008-05-07 06:00:00.0 & \\
\hline 2008-05-07 03:00:00.0 & 9.7 \\
\hline 2008-05-07 00:00:00.0 & 1.7 \\
\hline 2008-05-06 21:00:00.0 & 9.1 \\
\hline 2008-05-06 18:00:00.0 & 14.8 \\
\hline 2008-05-06 15:00:00.0 & 15.3 \\
\hline 2008-05-06 12:00:00.0 & 3.5 \\
\hline 2008-05-06 09:00:00.0 & 4.5 \\
\hline 2008-05-06 06:00:00.0 & 6.0 \\
\hline 2008-05-06 03:00:00.0 & 6.4 \\
\hline 2008-05-06 00:00:00.0 & 7.6 \\
\hline 2008-05-05 21:00:00.0 & 19.7 \\
\hline 2008-05-05 18:00:00.0 & 22.2 \\
\hline 2008-05-05 15:00:00.0 & 17.2 \\
\hline 2008-05-05 12:00:00.0 & 11.1 \\
\hline 2008-05-05 09:00:00.0 & 6.6 \\
\hline 2008-05-05 06:00:00.0 & 5.4 \\
\hline 2008-05-05 03:00:00.0 & 7.6 \\
\hline 2008-05-05 00:00:00.0 & 7.7 \\
\hline 2008-05-04 21:00:00.0 & 14.8 \\
\hline 2008-05-04 18:00:00.0 & 19.5 \\
\hline 2008-05-04 15:00:00.0 & 21.5 \\
\hline 2008-05-04 12:00:00.0 & 17.4 \\
\hline 2008-05-04 09:00:00.0 & \\
\hline 2008-05-04 06:00:00.0 & 24.5 \\
\hline 2008-05-04 03:00:00.0 & 22.3 \\
\hline 2008-05-04 00:00:00.0 & 21.7 \\
\hline 2008-05-03 21:00:00.0 & 39.3 \\
\hline 2008-05-03 18:00:00.0 & 34.2 \\
\hline 2008-05-03 15:00:00.0 & 34.9 \\
\hline 2008-05-03 12:00:00.0 & 31.6 \\
\hline 2008-05-03 09:00:00.0 & 34.0 \\
\hline 2008-05-03 06:00:00.0 & 31.6 \\
\hline 2008-05-03 03:00:00.0 & 28.0 \\
\hline 2008-05-03 00:00:00.0 & 29.3 \\
\hline 2008-05-02 21:00:00.0 & 27.7 \\
\hline 2008-05-02 18:00:00.0 & 25.2 \\
\hline 2008-05-02 15:00:00.0 & 25.6 \\
\hline 2008-05-02 12:00:00.0 & 21.1 \\
\hline 2008-05-02 09:00:00.0 & 16.8 \\
\hline 2008-05-02 06:00:00.0 & 16.6 \\
\hline 2008-05-02 03:00:00.0 & 12.6 \\
\hline 2008-05-02 00:00:00.0 & 15.6 \\
\hline
\end{tabular}

\begin{tabular}{|c|c|}
\hline DataHora & VelVentoMax \\
\hline 2008-05-01 21:00:00.0 & \\
\hline 2008-05-01 18:00:00.0 & 15.9 \\
\hline 2008-05-01 15:00:00.0 & 24.6 \\
\hline 2008-05-01 12:00:00.0 & 28.0 \\
\hline 2008-05-01 09:00:00.0 & 19.4 \\
\hline 2008-05-01 06:00:00.0 & 13.0 \\
\hline 2008-05-01 03:00:00.0 & 13.8 \\
\hline 2008-05-01 00:00:00.0 & 11.3 \\
\hline 2008-04-30 21:00:00.0 & 18.3 \\
\hline 2008-04-30 18:00:00.0 & 21.5 \\
\hline 2008-04-30 15:00:00.0 & 21.5 \\
\hline 2008-04-30 12:00:00.0 & 17.2 \\
\hline 2008-04-30 09:00:00.0 & 17.4 \\
\hline 2008-04-30 06:00:00.0 & 13.5 \\
\hline 2008-04-30 03:00:00.0 & 13.0 \\
\hline 2008-04-30 00:00:00.0 & 19.7 \\
\hline 2008-04-29 21:00:00.0 & 29.4 \\
\hline 2008-04-29 18:00:00.0 & 30.6 \\
\hline 2008-04-29 15:00:00.0 & 30.4 \\
\hline 2008-04-29 12:00:00.0 & 25.8 \\
\hline 2008-04-29 09:00:00.0 & 28.2 \\
\hline 2008-04-29 06:00:00.0 & 12.7 \\
\hline 2008-04-29 03:00:00.0 & 19.0 \\
\hline 2008-04-29 00:00:00.0 & 19.5 \\
\hline 2008-04-28 21:00:00.0 & 20.9 \\
\hline 2008-04-28 18:00:00.0 & 19.6 \\
\hline 2008-04-28 15:00:00.0 & 30.9 \\
\hline 2008-04-28 12:00:00.0 & 21.6 \\
\hline 2008-04-28 09:00:00.0 & 20.3 \\
\hline 2008-04-28 06:00:00.0 & 22.7 \\
\hline 2008-04-28 03:00:00.0 & 25.4 \\
\hline 2008-04-28 00:00:00.0 & 15.0 \\
\hline 2008-04-27 21:00:00.0 & \\
\hline 2008-04-27 18:00:00.0 & 14.0 \\
\hline 2008-04-27 15:00:00.0 & 16.8 \\
\hline 2008-04-27 12:00:00.0 & 16.8 \\
\hline 2008-04-27 09:00:00.0 & 10.1 \\
\hline 2008-04-27 06:00:00.0 & 5.2 \\
\hline 2008-04-27 03:00:00.0 & 7.3 \\
\hline 2008-04-27 00:00:00.0 & 21.2 \\
\hline 2008-04-26 21:00:00.0 & 31.5 \\
\hline 2008-04-26 18:00:00.0 & 22.1 \\
\hline 2008-04-26 15:00:00.0 & 21.0 \\
\hline 2008-04-26 12:00:00.0 & 31.7 \\
\hline 2008-04-26 09:00:00.0 & \\
\hline 2008-04-26 06:00:00.0 & 10.1 \\
\hline 2008-04-26 03:00:00.0 & 9.7 \\
\hline 2008-04-26 00:00:00.0 & 12.9 \\
\hline 2008-04-25 21:00:00.0 & 22.4 \\
\hline 2008-04-25 18:00:00.0 & 22.5 \\
\hline 2008-04-25 15:00:00.0 & 19.9 \\
\hline 2008-04-25 12:00:00.0 & 11.0 \\
\hline 2008-04-25 09:00:00.0 & \\
\hline 2008-04-25 06:00:00.0 & 7.6 \\
\hline 2008-04-25 03:00:00.0 & 7.7 \\
\hline 2008-04-25 00:00:00.0 & 8.2 \\
\hline 2008-04-24 21:00:00.0 & 15.3 \\
\hline 2008-04-24 18:00:00.0 & 15.4 \\
\hline 2008-04-24 15:00:00.0 & 30.1 \\
\hline 2008-04-24 12:00:00.0 & 8.2 \\
\hline 2008-04-24 09:00:00.0 & 30.7 \\
\hline 2008-04-24 06:00:00.0 & 5.4 \\
\hline 2008-04-24 03:00:00.0 & 4.4 \\
\hline 2008-04-24 00:00:00.0 & 5.1 \\
\hline 2008-04-23 21:00:00.0 & 13.0 \\
\hline 2008-04-23 18:00:00.0 & 14.8 \\
\hline 2008-04-23 15:00:00.0 & 13.4 \\
\hline 2008-04-23 12:00:00.0 & 4.2 \\
\hline 2008-04-23 09:00:00.0 & 2.7 \\
\hline
\end{tabular}

\begin{tabular}{|c|c|}
\hline DataHora & VelVentoMax \\
\hline 2008-04-23 06:00:00.0 & 12.3 \\
\hline 2008-04-23 03:00:00.0 & 9.5 \\
\hline $2008-04-23$ 00:00:00.0 & 10.4 \\
\hline 2008-04-22 21:00:00.0 & 14.8 \\
\hline $2008-04-22$ 18:00:00.0 & 17.6 \\
\hline 2008-04-22 15:00:00.0 & 13.9 \\
\hline 2008-04-22 12:00:00.0 & 27.1 \\
\hline 2008-04-22 09:00:00.0 & 11.6 \\
\hline 2008-04-22 06:00:00.0 & 13.5 \\
\hline 2008-04-22 03:00:00.0 & 16.2 \\
\hline $2008-04-22$ 00:00:00.0 & 19.7 \\
\hline $2008-04-21$ 21:00:00.0 & 20.6 \\
\hline 2008-04-21 18:00:00.0 & 22.1 \\
\hline 2008-04-21 15:00:00.0 & 25.1 \\
\hline 2008-04-21 12:00:00.0 & 21.6 \\
\hline 2008-04-21 09:00:00.0 & 15.9 \\
\hline 2008-04-21 06:00:00.0 & 14.6 \\
\hline 2008-04-21 03:00:00.0 & 16.2 \\
\hline 2008-04-21 00:00:00.0 & 20.0 \\
\hline 2008-04-20 21:00:00.0 & 19.5 \\
\hline 2008-04-20 18:00:00.0 & 19.2 \\
\hline $2008-04-2015: 00: 00.0$ & 15.5 \\
\hline $2008-04-2012: 00: 00.0$ & 6.6 \\
\hline 2008-04-20 09:00:00.0 & 12.6 \\
\hline 2008-04-20 06:00:00.0 & 13.0 \\
\hline 2008-04-20 03:00:00.0 & 11.2 \\
\hline 2008-04-20 00:00:00.0 & 11.6 \\
\hline 2008-04-19 21:00:00.0 & 9.6 \\
\hline 2008-04-19 18:00:00.0 & 13.2 \\
\hline 2008-04-19 15:00:00.0 & 19.7 \\
\hline 2008-04-19 12:00:00.0 & 18.7 \\
\hline 2008-04-19 09:00:00.0 & 16.5 \\
\hline 2008-04-19 06:00:00.0 & 15.0 \\
\hline 2008-04-19 03:00:00.0 & 14.8 \\
\hline 2008-04-19 00:00:00.0 & 11.7 \\
\hline 2008-04-18 21:00:00.0 & 15.3 \\
\hline 2008-04-18 18:00:00.0 & 15.0 \\
\hline 2008-04-18 15:00:00.0 & 15.4 \\
\hline 2008-04-18 12:00:00.0 & 14.4 \\
\hline 2008-04-18 09:00:00.0 & 14.7 \\
\hline 2008-04-18 06:00:00.0 & 11.0 \\
\hline 2008-04-18 03:00:00.0 & 9.8 \\
\hline 2008-04-18 00:00:00.0 & 7.7 \\
\hline 2008-04-17 21:00:00.0 & 16.7 \\
\hline 2008-04-17 18:00:00.0 & 15.4 \\
\hline 2008-04-17 15:00:00.0 & 38.5 \\
\hline 2008-04-17 12:00:00.0 & 6.7 \\
\hline 2008-04-17 09:00:00.0 & \\
\hline 2008-04-17 06:00:00.0 & 12.0 \\
\hline 2008-04-17 03:00:00.0 & 11.2 \\
\hline 2008-04-17 00:00:00.0 & 10.6 \\
\hline 2008-04-16 21:00:00.0 & 11.2 \\
\hline 2008-04-16 18:00:00.0 & 14.5 \\
\hline $2008-04-16$ 15:00:00.0 & 18.1 \\
\hline 2008-04-16 12:00:00.0 & 19.1 \\
\hline 2008-04-16 09:00:00.0 & 19.4 \\
\hline 2008-04-16 06:00:00.0 & 17.4 \\
\hline 2008-04-16 03:00:00.0 & 13.7 \\
\hline 2008-04-16 00:00:00.0 & 13.1 \\
\hline 2008-04-15 21:00:00.0 & 16.9 \\
\hline 2008-04-15 18:00:00.0 & 17.0 \\
\hline 2008-04-15 15:00:00.0 & 29.6 \\
\hline 2008-04-15 12:00:00.0 & 7.4 \\
\hline 2008-04-15 09:00:00.0 & 5.7 \\
\hline 2008-04-15 06:00:00.0 & 6.7 \\
\hline 2008-04-15 03:00:00.0 & 6.5 \\
\hline 2008-04-15 00:00:00.0 & 8.4 \\
\hline 2008-04-14 21:00:00.0 & 20.1 \\
\hline 2008-04-14 18:00:00.0 & 21.9 \\
\hline
\end{tabular}




\section{ANEXO 01 - RAJADAS DE VENTO REGISTRADAS EM SÃO MARTINHO DA SERRA / RS}

\begin{tabular}{|c|c|}
\hline DataHora & VelVentoMax \\
\hline 2008-04-14 15:00:00.0 & 22.5 \\
\hline 2008-04-14 12:00:00.0 & 32.6 \\
\hline 2008-04-14 09:00:00.0 & 19.3 \\
\hline 2008-04-14 06:00:00.0 & 18.7 \\
\hline 2008-04-14 03:00:00.0 & 26.2 \\
\hline 2008-04-14 00:00:00.0 & 27.4 \\
\hline 2008-04-13 21:00:00.0 & 24.2 \\
\hline 2008-04-13 18:00:00.0 & 24.5 \\
\hline 2008-04-13 15:00:00.0 & 20.9 \\
\hline 2008-04-13 12:00:00.0 & 18.4 \\
\hline 2008-04-13 09:00:00.0 & 14.5 \\
\hline 2008-04-13 06:00:00.0 & 11.4 \\
\hline 2008-04-13 03:00:00.0 & 16.0 \\
\hline 2008-04-13 00:00:00.0 & 14.1 \\
\hline 2008-04-12 21:00:00.0 & 9.7 \\
\hline 2008-04-12 18:00:00.0 & 9.5 \\
\hline 2008-04-12 15:00:00.0 & 10.3 \\
\hline 2008-04-12 12:00:00.0 & 9.8 \\
\hline 2008-04-12 09:00:00.0 & 17.3 \\
\hline 2008-04-12 06:00:00.0 & 44.4 \\
\hline 2008-04-12 03:00:00.0 & 19.1 \\
\hline 2008-04-12 00:00:00.0 & 30.0 \\
\hline 2008-04-11 21:00:00.0 & 15.1 \\
\hline 2008-04-11 18:00:00.0 & 18.4 \\
\hline 2008-04-11 15:00:00.0 & 27.1 \\
\hline 2008-04-11 12:00:00.0 & 9.7 \\
\hline 2008-04-11 09:00:00.0 & 9.5 \\
\hline 2008-04-11 06:00:00.0 & 9.7 \\
\hline 2008-04-11 03:00:00.0 & 5.6 \\
\hline 2008-04-11 00:00:00.0 & 6.8 \\
\hline 2008-04-10 21:00:00.0 & 13.7 \\
\hline 2008-04-10 18:00:00.0 & 22.9 \\
\hline 2008-04-10 15:00:00.0 & 21.9 \\
\hline 2008-04-10 12:00:00.0 & 11.6 \\
\hline 2008-04-10 09:00:00.0 & 16.8 \\
\hline 2008-04-10 06:00:00.0 & 20.4 \\
\hline 2008-04-10 03:00:00.0 & 28.2 \\
\hline 2008-04-10 00:00:00.0 & 10.9 \\
\hline 2008-04-09 21:00:00.0 & 10.8 \\
\hline 2008-04-09 18:00:00.0 & 16.2 \\
\hline 2008-04-09 15:00:00.0 & 18.0 \\
\hline 2008-04-09 12:00:00.0 & 17.7 \\
\hline 2008-04-09 09:00:00.0 & 18.1 \\
\hline 2008-04-09 06:00:00.0 & 17.4 \\
\hline 2008-04-09 03:00:00.0 & 11.4 \\
\hline 2008-04-09 00:00:00.0 & 8.9 \\
\hline 2008-04-08 21:00:00.0 & 14.8 \\
\hline 2008-04-08 18:00:00.0 & 20.7 \\
\hline 2008-04-08 15:00:00.0 & 15.4 \\
\hline 2008-04-08 12:00:00.0 & 14.4 \\
\hline 2008-04-08 09:00:00.0 & 18.0 \\
\hline 2008-04-08 06:00:00.0 & 16.5 \\
\hline 2008-04-08 03:00:00.0 & 12.7 \\
\hline 2008-04-08 00:00:00.0 & 11.1 \\
\hline 2008-04-07 21:00:00.0 & 16.0 \\
\hline 2008-04-07 18:00:00.0 & 16.7 \\
\hline 2008-04-07 15:00:00.0 & 11.5 \\
\hline 2008-04-07 12:00:00.0 & 13.7 \\
\hline 2008-04-07 09:00:00.0 & 14.6 \\
\hline 2008-04-07 06:00:00.0 & 12.3 \\
\hline 2008-04-07 03:00:00.0 & 11.8 \\
\hline 2008-04-07 00:00:00.0 & 8.2 \\
\hline 2008-04-06 21:00:00.0 & 12.8 \\
\hline 2008-04-06 18:00:00.0 & 15.1 \\
\hline 2008-04-06 15:00:00.0 & 16.3 \\
\hline 2008-04-06 12:00:00.0 & 15.2 \\
\hline 2008-04-06 09:00:00.0 & 16.7 \\
\hline 2008-04-06 06:00:00.0 & 26.9 \\
\hline 2008-04-06 03:00:00.0 & 8.2 \\
\hline
\end{tabular}

\begin{tabular}{|c|c|}
\hline DataHora & VelVentoMax \\
\hline 2008-04-06 00:00:00.0 & 7.5 \\
\hline 2008-04-05 21:00:00.0 & 11.0 \\
\hline 2008-04-05 18:00:00.0 & 14.8 \\
\hline 2008-04-05 15:00:00.0 & 18.2 \\
\hline 2008-04-05 12:00:00.0 & 15.0 \\
\hline 2008-04-05 09:00:00.0 & 6.2 \\
\hline 2008-04-05 06:00:00.0 & 5.0 \\
\hline 2008-04-05 03:00:00.0 & 6.7 \\
\hline 2008-04-05 00:00:00.0 & 7.4 \\
\hline 2008-04-04 21:00:00.0 & 13.6 \\
\hline 2008-04-04 18:00:00.0 & 14.7 \\
\hline 2008-04-04 15:00:00.0 & 4.2 \\
\hline 2008-04-04 12:00:00.0 & 7.5 \\
\hline 2008-04-04 09:00:00.0 & 7.8 \\
\hline 2008-04-04 06:00:00.0 & 8.8 \\
\hline 2008-04-04 03:00:00.0 & 9.3 \\
\hline 2008-04-04 00:00:00.0 & 6.7 \\
\hline 2008-04-03 21:00:00.0 & 16.3 \\
\hline 2008-04-03 18:00:00.0 & 15.5 \\
\hline 2008-04-03 15:00:00.0 & 13.7 \\
\hline 2008-04-03 12:00:00.0 & 12.5 \\
\hline 2008-04-03 09:00:00.0 & 11.5 \\
\hline 2008-04-03 06:00:00.0 & 10.4 \\
\hline 2008-04-03 03:00:00.0 & 20.8 \\
\hline 2008-04-03 00:00:00.0 & 15.3 \\
\hline 2008-04-02 21:00:00.0 & \\
\hline 2008-04-02 18:00:00.0 & 22.0 \\
\hline 2008-04-02 15:00:00.0 & 16.4 \\
\hline 2008-04-02 12:00:00.0 & 6.9 \\
\hline 2008-04-02 09:00:00.0 & 5.8 \\
\hline 2008-04-02 06:00:00.0 & 7.7 \\
\hline 2008-04-02 03:00:00.0 & 5.6 \\
\hline 2008-04-02 00:00:00.0 & 7.4 \\
\hline 2008-04-01 21:00:00.0 & 12.2 \\
\hline 2008-04-01 18:00:00.0 & 27.0 \\
\hline 2008-04-01 15:00:00.0 & 18.4 \\
\hline 2008-04-01 12:00:00.0 & 13.6 \\
\hline 2008-04-01 09:00:00.0 & 13.1 \\
\hline 2008-04-01 06:00:00.0 & 15.5 \\
\hline 2008-04-01 03:00:00.0 & 15.5 \\
\hline 2008-04-01 00:00:00.0 & 20.0 \\
\hline 2008-03-31 21:00:00.0 & 15.0 \\
\hline 2008-03-31 18:00:00.0 & 23.9 \\
\hline 2008-03-31 15:00:00.0 & 27.9 \\
\hline 2008-03-31 12:00:00.0 & 24.4 \\
\hline 2008-03-31 09:00:00.0 & 23.7 \\
\hline 2008-03-31 06:00:00.0 & 19.0 \\
\hline 2008-03-31 03:00:00.0 & 18.6 \\
\hline 2008-03-31 00:00:00.0 & 18.3 \\
\hline 2008-03-30 21:00:00.0 & 18.9 \\
\hline 2008-03-30 18:00:00.0 & 21.2 \\
\hline 2008-03-30 15:00:00.0 & 37.3 \\
\hline 2008-03-30 12:00:00.0 & 29.0 \\
\hline 2008-03-30 09:00:00.0 & 22.2 \\
\hline 2008-03-30 06:00:00.0 & \\
\hline 2008-03-30 03:00:00.0 & 17.1 \\
\hline 2008-03-30 00:00:00.0 & 18.2 \\
\hline 2008-03-29 21:00:00.0 & 9.4 \\
\hline 2008-03-29 18:00:00.0 & 22.9 \\
\hline 2008-03-29 15:00:00.0 & 25.7 \\
\hline 2008-03-29 12:00:00.0 & 31.2 \\
\hline 2008-03-29 09:00:00.0 & 13.5 \\
\hline 2008-03-29 06:00:00.0 & 34.8 \\
\hline 2008-03-29 03:00:00.0 & 19.3 \\
\hline 2008-03-29 00:00:00.0 & 20.2 \\
\hline 2008-03-28 21:00:00.0 & 21.6 \\
\hline 2008-03-28 18:00:00.0 & 20.3 \\
\hline 2008-03-28 15:00:00.0 & 23.2 \\
\hline 2008-03-28 12:00:00.0 & 19.6 \\
\hline
\end{tabular}

\begin{tabular}{|c|c|}
\hline DataHora & VelVentoMax \\
\hline 2008-03-28 09:00:00.0 & 17.8 \\
\hline 2008-03-28 06:00:00.0 & 15.7 \\
\hline 2008-03-28 03:00:00.0 & 18.6 \\
\hline 2008-03-28 00:00:00.0 & 18.8 \\
\hline 2008-03-27 21:00:00.0 & 17.2 \\
\hline 2008-03-27 18:00:00.0 & 43.8 \\
\hline 2008-03-27 15:00:00.0 & 14.9 \\
\hline 2008-03-27 12:00:00.0 & 15.0 \\
\hline 2008-03-27 09:00:00.0 & 14.9 \\
\hline 2008-03-27 06:00:00.0 & 10.4 \\
\hline 2008-03-27 03:00:00.0 & 15.7 \\
\hline 2008-03-27 00:00:00.0 & 6.0 \\
\hline 2008-03-26 21:00:00.0 & 16.7 \\
\hline 2008-03-26 18:00:00.0 & 21.8 \\
\hline 2008-03-26 15:00:00.0 & 21.4 \\
\hline 2008-03-26 12:00:00.0 & 18.7 \\
\hline 2008-03-26 09:00:00.0 & 18.9 \\
\hline 2008-03-26 06:00:00.0 & 19.2 \\
\hline 2008-03-26 03:00:00.0 & 15.2 \\
\hline 2008-03-26 00:00:00.0 & 12.2 \\
\hline 2008-03-25 21:00:00.0 & 14.0 \\
\hline 2008-03-25 18:00:00.0 & 16.1 \\
\hline 2008-03-25 15:00:00.0 & 22.3 \\
\hline 2008-03-25 12:00:00.0 & 43.1 \\
\hline 2008-03-25 09:00:00.0 & 15.2 \\
\hline 2008-03-25 06:00:00.0 & 18.0 \\
\hline 2008-03-25 03:00:00.0 & 12.8 \\
\hline 2008-03-25 00:00:00.0 & 16.0 \\
\hline 2008-03-24 21:00:00.0 & 18.0 \\
\hline 2008-03-24 18:00:00.0 & 20.2 \\
\hline 2008-03-24 15:00:00.0 & 17.1 \\
\hline 2008-03-24 12:00:00.0 & 13.4 \\
\hline 2008-03-24 09:00:00.0 & 12.5 \\
\hline 2008-03-24 06:00:00.0 & 11.3 \\
\hline 2008-03-24 03:00:00.0 & 16.4 \\
\hline 2008-03-24 00:00:00.0 & 17.7 \\
\hline 2008-03-23 21:00:00.0 & 18.2 \\
\hline 2008-03-23 18:00:00.0 & 18.4 \\
\hline 2008-03-23 15:00:00.0 & 21.8 \\
\hline 2008-03-23 12:00:00.0 & 21.1 \\
\hline 2008-03-23 09:00:00.0 & 15.2 \\
\hline 2008-03-23 06:00:00.0 & 18.7 \\
\hline 2008-03-23 03:00:00.0 & 23.2 \\
\hline 2008-03-23 00:00:00.0 & 16.5 \\
\hline 2008-03-22 21:00:00.0 & 24.8 \\
\hline 2008-03-22 18:00:00.0 & 26.6 \\
\hline 2008-03-22 15:00:00.0 & 14.3 \\
\hline 2008-03-22 12:00:00.0 & 10.9 \\
\hline 2008-03-22 09:00:00.0 & 17.3 \\
\hline 2008-03-22 06:00:00.0 & 17.8 \\
\hline 2008-03-22 03:00:00.0 & 9.7 \\
\hline 2008-03-22 00:00:00.0 & 12.0 \\
\hline 2008-03-21 21:00:00.0 & 17.3 \\
\hline 2008-03-21 18:00:00.0 & 13.7 \\
\hline 2008-03-21 15:00:00.0 & \\
\hline 2008-03-21 12:00:00.0 & 11.5 \\
\hline 2008-03-21 09:00:00.0 & 14.2 \\
\hline 2008-03-21 06:00:00.0 & 12.7 \\
\hline 2008-03-21 03:00:00.0 & 12.6 \\
\hline 2008-03-21 00:00:00.0 & 8.6 \\
\hline 2008-03-20 21:00:00.0 & 11.3 \\
\hline 2008-03-20 18:00:00.0 & 13.3 \\
\hline 2008-03-20 15:00:00.0 & 17.8 \\
\hline 2008-03-20 12:00:00.0 & 14.1 \\
\hline 2008-03-20 09:00:00.0 & 13.4 \\
\hline 2008-03-20 06:00:00.0 & 17.6 \\
\hline 2008-03-20 03:00:00.0 & 14.8 \\
\hline 2008-03-20 00:00:00.0 & 13.7 \\
\hline 2008-03-19 21:00:00.0 & 17.1 \\
\hline
\end{tabular}




\section{ANEXO 01 - RAJADAS DE VENTO REGISTRADAS EM SÃO MARTINHO DA SERRA / RS}

\begin{tabular}{|c|c|}
\hline DataHora & VelVentoMax \\
\hline 2008-03-19 18:00:00.0 & 24.8 \\
\hline 2008-03-19 15:00:00.0 & 22.2 \\
\hline 2008-03-19 12:00:00.0 & 23.4 \\
\hline 2008-03-19 09:00:00.0 & 18.6 \\
\hline 2008-03-19 06:00:00.0 & 14.3 \\
\hline 2008-03-19 03:00:00.0 & 13.8 \\
\hline 2008-03-19 00:00:00.0 & 14.6 \\
\hline 2008-03-18 21:00:00.0 & 21.2 \\
\hline 2008-03-18 18:00:00.0 & 18.6 \\
\hline 2008-03-18 15:00:00.0 & 14.8 \\
\hline 2008-03-18 12:00:00.0 & 15.7 \\
\hline 2008-03-18 09:00:00.0 & 16.0 \\
\hline 2008-03-18 06:00:00.0 & 21.9 \\
\hline 2008-03-18 03:00:00.0 & 11.8 \\
\hline 2008-03-18 00:00:00.0 & 12.0 \\
\hline 2008-03-17 21:00:00.0 & 13.6 \\
\hline 2008-03-17 18:00:00.0 & 13.0 \\
\hline 2008-03-17 15:00:00.0 & 18.8 \\
\hline 2008-03-17 12:00:00.0 & 19.1 \\
\hline 2008-03-17 09:00:00.0 & 18.2 \\
\hline 2008-03-17 06:00:00.0 & \\
\hline 2008-03-17 03:00:00.0 & 14.4 \\
\hline 2008-03-17 00:00:00.0 & 12.1 \\
\hline 2008-03-16 21:00:00.0 & 16.3 \\
\hline 2008-03-16 18:00:00.0 & 22.7 \\
\hline 2008-03-16 15:00:00.0 & 21.7 \\
\hline 2008-03-16 12:00:00.0 & 20.3 \\
\hline 2008-03-16 09:00:00.0 & 16.9 \\
\hline 2008-03-16 06:00:00.0 & 15.3 \\
\hline 2008-03-16 03:00:00.0 & 11.6 \\
\hline 2008-03-16 00:00:00.0 & 15.7 \\
\hline 2008-03-15 21:00:00.0 & 19.1 \\
\hline 2008-03-15 18:00:00.0 & \\
\hline 2008-03-15 15:00:00.0 & 28.5 \\
\hline 2008-03-15 12:00:00.0 & 26.6 \\
\hline 2008-03-15 09:00:00.0 & 14.2 \\
\hline 2008-03-15 06:00:00.0 & 11.5 \\
\hline 2008-03-15 03:00:00.0 & 12.3 \\
\hline 2008-03-15 00:00:00.0 & 16.5 \\
\hline 2008-03-14 21:00:00.0 & 19.7 \\
\hline 2008-03-14 18:00:00.0 & 21.4 \\
\hline 2008-03-14 15:00:00.0 & 19.8 \\
\hline 2008-03-14 12:00:00.0 & 43.2 \\
\hline 2008-03-14 09:00:00.0 & 12.6 \\
\hline 2008-03-14 06:00:00.0 & 14.2 \\
\hline 2008-03-14 03:00:00.0 & 16.1 \\
\hline 2008-03-14 00:00:00.0 & 11.7 \\
\hline 2008-03-13 21:00:00.0 & 20.5 \\
\hline 2008-03-13 18:00:00.0 & 21.5 \\
\hline 2008-03-13 15:00:00.0 & 18.1 \\
\hline 2008-03-13 12:00:00.0 & 15.8 \\
\hline 2008-03-13 09:00:00.0 & 9.9 \\
\hline 2008-03-13 06:00:00.0 & 8.6 \\
\hline 2008-03-13 03:00:00.0 & 8.4 \\
\hline 2008-03-13 00:00:00.0 & 12.2 \\
\hline 2008-03-12 21:00:00.0 & 30.8 \\
\hline 2008-03-12 18:00:00.0 & 16.7 \\
\hline 2008-03-12 15:00:00.0 & 18.3 \\
\hline 2008-03-12 12:00:00.0 & 19.0 \\
\hline 2008-03-12 09:00:00.0 & 16.2 \\
\hline 2008-03-12 06:00:00.0 & 17.9 \\
\hline 2008-03-12 03:00:00.0 & 15.5 \\
\hline 2008-03-12 00:00:00.0 & 17.2 \\
\hline 2008-03-11 21:00:00.0 & 18.8 \\
\hline 2008-03-11 18:00:00.0 & 18.3 \\
\hline 2008-03-11 15:00:00.0 & 17.6 \\
\hline 2008-03-11 12:00:00.0 & 18.1 \\
\hline 2008-03-11 09:00:00.0 & 18.8 \\
\hline 2008-03-11 06:00:00.0 & 22.2 \\
\hline
\end{tabular}

\begin{tabular}{|c|c|}
\hline DataHora & VelVentoMax \\
\hline 2008-03-11 03:00:00.0 & 20.8 \\
\hline 2008-03-11 00:00:00.0 & 12.9 \\
\hline 2008-03-10 21:00:00.0 & \\
\hline 2008-03-10 18:00:00.0 & \\
\hline 2008-03-10 15:00:00.0 & 27.1 \\
\hline 2008-03-10 12:00:00.0 & 10.2 \\
\hline 2008-03-10 09:00:00.0 & \\
\hline 2008-03-10 06:00:00.0 & 8.4 \\
\hline 2008-03-10 03:00:00.0 & 15.6 \\
\hline 2008-03-10 00:00:00.0 & 19.6 \\
\hline 2008-03-09 21:00:00.0 & 15.3 \\
\hline 2008-03-09 18:00:00.0 & 11.5 \\
\hline 2008-03-09 15:00:00.0 & 13.3 \\
\hline 2008-03-09 12:00:00.0 & 20.0 \\
\hline 2008-03-09 09:00:00.0 & 10.9 \\
\hline 2008-03-09 06:00:00.0 & 13.1 \\
\hline 2008-03-09 03:00:00.0 & 13.5 \\
\hline 2008-03-09 00:00:00.0 & 14.1 \\
\hline 2008-03-08 21:00:00.0 & 16.9 \\
\hline 2008-03-08 18:00:00.0 & 12.7 \\
\hline 2008-03-08 15:00:00.0 & 14.7 \\
\hline 2008-03-08 12:00:00.0 & 13.4 \\
\hline 2008-03-08 09:00:00.0 & 11.5 \\
\hline 2008-03-08 06:00:00.0 & 10.3 \\
\hline 2008-03-08 03:00:00.0 & 10.0 \\
\hline 2008-03-08 00:00:00.0 & 9.0 \\
\hline 2008-03-07 21:00:00.0 & \\
\hline 2008-03-07 18:00:00.0 & 12.5 \\
\hline 2008-03-07 15:00:00.0 & 9.4 \\
\hline 2008-03-07 12:00:00.0 & 6.4 \\
\hline 2008-03-07 09:00:00.0 & 5.5 \\
\hline 2008-03-07 06:00:00.0 & 5.6 \\
\hline 2008-03-07 03:00:00.0 & 4.9 \\
\hline 2008-03-07 00:00:00.0 & 7.4 \\
\hline 2008-03-06 21:00:00.0 & 20.4 \\
\hline 2008-03-06 18:00:00.0 & 23.3 \\
\hline 2008-03-06 15:00:00.0 & 18.8 \\
\hline 2008-03-06 12:00:00.0 & 10.7 \\
\hline 2008-03-06 09:00:00.0 & \\
\hline 2008-03-06 06:00:00.0 & 7.0 \\
\hline 2008-03-06 03:00:00.0 & 6.1 \\
\hline 2008-03-06 00:00:00.0 & 9.6 \\
\hline 2008-03-05 21:00:00.0 & 19.0 \\
\hline 2008-03-05 18:00:00.0 & 23.8 \\
\hline 2008-03-05 15:00:00.0 & 19.2 \\
\hline 2008-03-05 12:00:00.0 & 14.3 \\
\hline 2008-03-05 09:00:00.0 & 8.2 \\
\hline 2008-03-05 06:00:00.0 & 8.8 \\
\hline 2008-03-05 03:00:00.0 & 8.0 \\
\hline 2008-03-05 00:00:00.0 & 16.2 \\
\hline 2008-03-04 21:00:00.0 & 22.4 \\
\hline 2008-03-04 18:00:00.0 & 23.4 \\
\hline 2008-03-04 15:00:00.0 & 16.2 \\
\hline 2008-03-04 12:00:00.0 & 32.0 \\
\hline 2008-03-04 09:00:00.0 & 15.1 \\
\hline 2008-03-04 06:00:00.0 & 11.6 \\
\hline 2008-03-04 03:00:00.0 & 13.6 \\
\hline 2008-03-04 00:00:00.0 & 16.1 \\
\hline 2008-03-03 21:00:00.0 & 25.0 \\
\hline 2008-03-03 18:00:00.0 & 27.2 \\
\hline 2008-03-03 15:00:00.0 & 27.8 \\
\hline 2008-03-03 12:00:00.0 & 14.9 \\
\hline 2008-03-03 09:00:00.0 & 14.6 \\
\hline 2008-03-03 06:00:00.0 & 16.5 \\
\hline 2008-03-03 03:00:00.0 & 10.5 \\
\hline 2008-03-03 00:00:00.0 & 10.5 \\
\hline 2008-03-02 21:00:00.0 & 17.8 \\
\hline 2008-03-02 18:00:00.0 & 20.6 \\
\hline 2008-03-02 15:00:00.0 & 24.8 \\
\hline
\end{tabular}

\begin{tabular}{|c|c|}
\hline DataHora & VelVentoMax \\
\hline 2008-03-02 12:00:00.0 & 14.8 \\
\hline 2008-03-02 09:00:00.0 & 15.9 \\
\hline 2008-03-02 06:00:00.0 & 13.4 \\
\hline 2008-03-02 03:00:00.0 & 12.8 \\
\hline 2008-03-02 00:00:00.0 & 11.1 \\
\hline 2008-03-01 21:00:00.0 & 30.7 \\
\hline 2008-03-01 18:00:00.0 & 25.2 \\
\hline 2008-03-01 15:00:00.0 & 19.1 \\
\hline 2008-03-01 12:00:00.0 & 13.4 \\
\hline 2008-03-01 09:00:00.0 & 8.1 \\
\hline 2008-03-01 06:00:00.0 & 11.8 \\
\hline 2008-03-01 03:00:00.0 & 26.4 \\
\hline 2008-03-01 00:00:00.0 & 16.3 \\
\hline 2008-02-29 21:00:00.0 & 12.0 \\
\hline 2008-02-29 18:00:00.0 & 18.5 \\
\hline 2008-02-29 15:00:00.0 & 18.5 \\
\hline 2008-02-29 12:00:00.0 & 22.8 \\
\hline 2008-02-29 09:00:00.0 & 22.6 \\
\hline 2008-02-29 06:00:00.0 & 27.0 \\
\hline 2008-02-29 03:00:00.0 & 19.5 \\
\hline 2008-02-29 00:00:00.0 & 20.6 \\
\hline 2008-02-28 21:00:00.0 & 17.0 \\
\hline $2008-02-28$ 18:00:00.0 & 17.1 \\
\hline 2008-02-28 15:00:00.0 & 26.4 \\
\hline 2008-02-28 12:00:00.0 & 25.3 \\
\hline 2008-02-28 09:00:00.0 & 20.8 \\
\hline 2008-02-28 06:00:00.0 & 19.0 \\
\hline 2008-02-28 03:00:00.0 & 13.0 \\
\hline 2008-02-28 00:00:00.0 & 21.8 \\
\hline 2008-02-27 21:00:00.0 & 20.5 \\
\hline 2008-02-27 18:00:00.0 & 24.0 \\
\hline 2008-02-27 15:00:00.0 & 27.2 \\
\hline 2008-02-27 12:00:00.0 & 24.1 \\
\hline 2008-02-27 09:00:00.0 & 20.2 \\
\hline 2008-02-27 06:00:00.0 & 16.9 \\
\hline 2008-02-27 03:00:00.0 & 15.9 \\
\hline 2008-02-27 00:00:00.0 & 13.3 \\
\hline 2008-02-26 21:00:00.0 & 18.4 \\
\hline 2008-02-26 18:00:00.0 & 35.6 \\
\hline 2008-02-26 15:00:00.0 & 21.6 \\
\hline 2008-02-26 12:00:00.0 & 23.5 \\
\hline 2008-02-26 09:00:00.0 & 18.9 \\
\hline 2008-02-26 06:00:00.0 & 20.4 \\
\hline 2008-02-26 03:00:00.0 & 17.2 \\
\hline 2008-02-26 00:00:00.0 & 20.8 \\
\hline 2008-02-25 21:00:00.0 & 24.2 \\
\hline 2008-02-25 18:00:00.0 & 15.2 \\
\hline 2008-02-25 15:00:00.0 & 20.0 \\
\hline 2008-02-25 12:00:00.0 & 19.2 \\
\hline 2008-02-25 09:00:00.0 & 19.0 \\
\hline 2008-02-25 06:00:00.0 & 17.6 \\
\hline 2008-02-25 03:00:00.0 & 15.1 \\
\hline 2008-02-25 00:00:00.0 & 19.8 \\
\hline 2008-02-24 21:00:00.0 & 20.1 \\
\hline 2008-02-24 18:00:00.0 & 15.5 \\
\hline 2008-02-24 15:00:00.0 & 17.4 \\
\hline 2008-02-24 12:00:00.0 & 17.2 \\
\hline 2008-02-24 09:00:00.0 & 14.6 \\
\hline 2008-02-24 06:00:00.0 & 14.2 \\
\hline 2008-02-24 03:00:00.0 & 13.5 \\
\hline 2008-02-24 00:00:00.0 & 21.2 \\
\hline 2008-02-23 21:00:00.0 & 24.9 \\
\hline 2008-02-23 18:00:00.0 & 25.2 \\
\hline 2008-02-23 15:00:00.0 & 20.5 \\
\hline 2008-02-23 12:00:00.0 & 18.3 \\
\hline 2008-02-23 09:00:00.0 & 17.2 \\
\hline 2008-02-23 06:00:00.0 & 18.7 \\
\hline 2008-02-23 03:00:00.0 & 12.4 \\
\hline 2008-02-23 00:00:00.0 & 13.8 \\
\hline
\end{tabular}




\section{ANEXO 01 - RAJADAS DE VENTO REGISTRADAS EM SÃO MARTINHO DA SERRA / RS}

\begin{tabular}{|c|c|}
\hline DataHora & VelVentoMax \\
\hline 2008-02-22 21:00:00.0 & 16.2 \\
\hline 2008-02-22 18:00:00.0 & 18.8 \\
\hline 2008-02-22 15:00:00.0 & 13.0 \\
\hline 2008-02-22 12:00:00.0 & 12.8 \\
\hline 2008-02-22 09:00:00.0 & 13.3 \\
\hline 2008-02-22 06:00:00.0 & 13.9 \\
\hline 2008-02-22 03:00:00.0 & 13.4 \\
\hline 2008-02-22 00:00:00.0 & 10.8 \\
\hline 2008-02-21 21:00:00.0 & 13.5 \\
\hline 2008-02-21 18:00:00.0 & 17.1 \\
\hline 2008-02-21 15:00:00.0 & 15.7 \\
\hline 2008-02-21 12:00:00.0 & 11.0 \\
\hline 2008-02-21 09:00:00.0 & 12.3 \\
\hline 2008-02-21 06:00:00.0 & 11.7 \\
\hline 2008-02-21 03:00:00.0 & 14.7 \\
\hline 2008-02-21 00:00:00.0 & 10.8 \\
\hline 2008-02-20 21:00:00.0 & 16.7 \\
\hline 2008-02-20 18:00:00.0 & 18.4 \\
\hline 2008-02-20 15:00:00.0 & \\
\hline 2008-02-20 12:00:00.0 & 12.5 \\
\hline 2008-02-20 09:00:00.0 & 13.5 \\
\hline 2008-02-20 06:00:00.0 & 9.1 \\
\hline 2008-02-20 03:00:00.0 & 12.5 \\
\hline 2008-02-20 00:00:00.0 & 21.5 \\
\hline 2008-02-19 21:00:00.0 & 20.5 \\
\hline 2008-02-19 18:00:00.0 & \\
\hline 2008-02-19 15:00:00.0 & 21.2 \\
\hline 2008-02-19 12:00:00.0 & 18.7 \\
\hline 2008-02-19 09:00:00.0 & 40.3 \\
\hline 2008-02-19 06:00:00.0 & \\
\hline 2008-02-19 03:00:00.0 & 16.9 \\
\hline 2008-02-19 00:00:00.0 & 15.7 \\
\hline 2008-02-18 21:00:00.0 & 16.8 \\
\hline 2008-02-18 18:00:00.0 & 12.9 \\
\hline 2008-02-18 15:00:00.0 & 16.9 \\
\hline 2008-02-18 12:00:00.0 & 17.0 \\
\hline 2008-02-18 09:00:00.0 & 12.5 \\
\hline 2008-02-18 06:00:00.0 & 12.3 \\
\hline 2008-02-18 03:00:00.0 & 13.1 \\
\hline 2008-02-18 00:00:00.0 & 16.8 \\
\hline 2008-02-17 21:00:00.0 & 17.2 \\
\hline 2008-02-17 18:00:00.0 & 50.1 \\
\hline 2008-02-17 15:00:00.0 & 9.8 \\
\hline 2008-02-17 12:00:00.0 & 9.5 \\
\hline 2008-02-17 09:00:00.0 & 13.3 \\
\hline 2008-02-17 06:00:00.0 & 14.6 \\
\hline 2008-02-17 03:00:00.0 & 15.1 \\
\hline 2008-02-17 00:00:00.0 & 12.1 \\
\hline 2008-02-16 21:00:00.0 & 13.7 \\
\hline 2008-02-16 18:00:00.0 & \\
\hline 2008-02-16 15:00:00.0 & 14.2 \\
\hline 2008-02-16 12:00:00.0 & 14.7 \\
\hline 2008-02-16 09:00:00.0 & \\
\hline 2008-02-16 06:00:00.0 & 15.5 \\
\hline 2008-02-16 03:00:00.0 & 17.4 \\
\hline 2008-02-16 00:00:00.0 & 15.3 \\
\hline 2008-02-15 21:00:00.0 & \\
\hline 2008-02-15 18:00:00.0 & 14.4 \\
\hline 2008-02-15 15:00:00.0 & 16.5 \\
\hline 2008-02-15 12:00:00.0 & 18.3 \\
\hline 2008-02-15 09:00:00.0 & 18.4 \\
\hline 2008-02-15 06:00:00.0 & 15.7 \\
\hline 2008-02-15 03:00:00.0 & 14.5 \\
\hline 2008-02-15 00:00:00.0 & 14.0 \\
\hline 2008-02-14 21:00:00.0 & 13.8 \\
\hline 2008-02-14 18:00:00.0 & 12.2 \\
\hline 2008-02-14 15:00:00.0 & 11.5 \\
\hline 2008-02-14 12:00:00.0 & 15.2 \\
\hline 2008-02-14 09:00:00.0 & \\
\hline
\end{tabular}

\begin{tabular}{|c|c|}
\hline DataHora & VelVentoMax \\
\hline 2008-02-14 06:00:00.0 & 13.0 \\
\hline 2008-02-14 03:00:00.0 & 8.9 \\
\hline 2008-02-14 00:00:00.0 & 9.2 \\
\hline 2008-02-13 21:00:00.0 & \\
\hline 2008-02-13 18:00:00.0 & 32.5 \\
\hline 2008-02-13 15:00:00.0 & 17.2 \\
\hline 2008-02-13 12:00:00.0 & 8.2 \\
\hline 2008-02-13 09:00:00.0 & 9.2 \\
\hline 2008-02-13 06:00:00.0 & 11.0 \\
\hline 2008-02-13 03:00:00.0 & 13.9 \\
\hline 2008-02-13 00:00:00.0 & 12.6 \\
\hline 2008-02-12 21:00:00.0 & 15.3 \\
\hline 2008-02-12 18:00:00.0 & 16.4 \\
\hline 2008-02-12 15:00:00.0 & 16.6 \\
\hline 2008-02-12 12:00:00.0 & 11.7 \\
\hline 2008-02-12 09:00:00.0 & \\
\hline 2008-02-12 06:00:00.0 & 9.3 \\
\hline 2008-02-12 03:00:00.0 & 13.2 \\
\hline 2008-02-12 00:00:00.0 & 14.5 \\
\hline 2008-02-11 21:00:00.0 & \\
\hline 2008-02-11 18:00:00.0 & 22.4 \\
\hline 2008-02-11 15:00:00.0 & 21.9 \\
\hline 2008-02-11 12:00:00.0 & 21.6 \\
\hline 2008-02-11 09:00:00.0 & 17.5 \\
\hline 2008-02-11 06:00:00.0 & 21.9 \\
\hline 2008-02-11 03:00:00.0 & 23.2 \\
\hline 2008-02-11 00:00:00.0 & 17.5 \\
\hline 2008-02-10 21:00:00.0 & 21.8 \\
\hline 2008-02-10 18:00:00.0 & 17.5 \\
\hline 2008-02-10 15:00:00.0 & 16.6 \\
\hline 2008-02-10 12:00:00.0 & 9.4 \\
\hline 2008-02-10 09:00:00.0 & \\
\hline 2008-02-10 06:00:00.0 & 13.2 \\
\hline 2008-02-10 03:00:00.0 & 12.6 \\
\hline 2008-02-10 00:00:00.0 & 14.5 \\
\hline 2008-02-09 21:00:00.0 & 18.9 \\
\hline 2008-02-09 18:00:00.0 & 33.5 \\
\hline 2008-02-09 15:00:00.0 & 15.0 \\
\hline 2008-02-09 12:00:00.0 & 18.3 \\
\hline 2008-02-09 09:00:00.0 & 23.4 \\
\hline 2008-02-09 06:00:00.0 & 20.0 \\
\hline 2008-02-09 03:00:00.0 & 16.8 \\
\hline 2008-02-09 00:00:00.0 & 15.2 \\
\hline 2008-02-08 21:00:00.0 & 13.5 \\
\hline 2008-02-08 18:00:00.0 & 15.6 \\
\hline 2008-02-08 15:00:00.0 & 17.4 \\
\hline 2008-02-08 12:00:00.0 & 17.2 \\
\hline 2008-02-08 09:00:00.0 & 15.0 \\
\hline 2008-02-08 06:00:00.0 & 11.5 \\
\hline 2008-02-08 03:00:00.0 & 11.3 \\
\hline 2008-02-08 00:00:00.0 & 10.7 \\
\hline 2008-02-07 21:00:00.0 & 12.4 \\
\hline 2008-02-07 18:00:00.0 & 19.3 \\
\hline 2008-02-07 15:00:00.0 & 20.9 \\
\hline 2008-02-07 12:00:00.0 & 21.3 \\
\hline 2008-02-07 09:00:00.0 & 19.9 \\
\hline 2008-02-07 06:00:00.0 & 20.7 \\
\hline 2008-02-07 03:00:00.0 & 15.5 \\
\hline 2008-02-07 00:00:00.0 & 12.6 \\
\hline 2008-02-06 21:00:00.0 & 19.7 \\
\hline 2008-02-06 18:00:00.0 & 20.8 \\
\hline 2008-02-06 15:00:00.0 & 20.4 \\
\hline 2008-02-06 12:00:00.0 & 23.1 \\
\hline 2008-02-06 09:00:00.0 & 20.7 \\
\hline 2008-02-06 06:00:00.0 & 19.7 \\
\hline 2008-02-06 03:00:00.0 & 15.0 \\
\hline 2008-02-06 00:00:00.0 & 17.5 \\
\hline 2008-02-05 21:00:00.0 & 17.1 \\
\hline 2008-02-05 18:00:00.0 & 17.4 \\
\hline
\end{tabular}

\begin{tabular}{|c|c|}
\hline DataHora & VelVentoMax \\
\hline 2008-02-05 15:00:00.0 & 14.5 \\
\hline 2008-02-05 12:00:00.0 & 16.4 \\
\hline 2008-02-05 09:00:00.0 & 15.3 \\
\hline 2008-02-05 06:00:00.0 & 10.6 \\
\hline 2008-02-05 03:00:00.0 & 10.8 \\
\hline 2008-02-05 00:00:00.0 & 9.8 \\
\hline 2008-02-04 21:00:00.0 & 12.9 \\
\hline 2008-02-04 18:00:00.0 & 13.5 \\
\hline 2008-02-04 15:00:00.0 & 14.7 \\
\hline $2008-02-04$ 12:00:00.0 & 12.3 \\
\hline 2008-02-04 09:00:00.0 & 11.9 \\
\hline 2008-02-04 06:00:00.0 & 9.3 \\
\hline 2008-02-04 03:00:00.0 & 9.8 \\
\hline 2008-02-04 00:00:00.0 & 18.3 \\
\hline 2008-02-03 21:00:00.0 & 23.7 \\
\hline $2008-02-03$ 18:00:00.0 & 23.9 \\
\hline $2008-02-03$ 15:00:00.0 & 22.1 \\
\hline 2008-02-03 12:00:00.0 & 22.2 \\
\hline 2008-02-03 09:00:00.0 & 7.5 \\
\hline 2008-02-03 06:00:00.0 & 7.7 \\
\hline 2008-02-03 03:00:00.0 & 10.6 \\
\hline 2008-02-03 00:00:00.0 & 23.1 \\
\hline 2008-02-02 21:00:00.0 & 28.4 \\
\hline 2008-02-02 18:00:00.0 & 26.5 \\
\hline $2008-02-02$ 15:00:00.0 & 16.3 \\
\hline 2008-02-02 12:00:00.0 & 7.5 \\
\hline 2008-02-02 09:00:00.0 & 12.5 \\
\hline 2008-02-02 06:00:00.0 & 10.3 \\
\hline 2008-02-02 03:00:00.0 & 10.0 \\
\hline 2008-02-02 00:00:00.0 & 16.1 \\
\hline 2008-02-01 21:00:00.0 & 16.3 \\
\hline 2008-02-01 18:00:00.0 & 15.0 \\
\hline 2008-02-01 15:00:00.0 & 18.5 \\
\hline 2008-02-01 12:00:00.0 & 18.2 \\
\hline 2008-02-01 09:00:00.0 & 15.5 \\
\hline 2008-02-01 06:00:00.0 & 13.7 \\
\hline 2008-02-01 03:00:00.0 & 15.4 \\
\hline 2008-02-01 00:00:00.0 & 20.5 \\
\hline 2008-01-31 21:00:00.0 & 22.9 \\
\hline 2008-01-31 18:00:00.0 & 17.5 \\
\hline 2008-01-31 15:00:00.0 & 18.8 \\
\hline 2008-01-31 12:00:00.0 & 20.5 \\
\hline 2008-01-31 09:00:00.0 & 14.9 \\
\hline 2008-01-31 06:00:00.0 & 15.7 \\
\hline 2008-01-31 03:00:00.0 & 15.0 \\
\hline 2008-01-31 00:00:00.0 & 13.6 \\
\hline 2008-01-30 21:00:00.0 & 21.4 \\
\hline 2008-01-30 18:00:00.0 & 18.1 \\
\hline $2008-01-3015: 00: 00.0$ & 13.2 \\
\hline 2008-01-30 12:00:00.0 & 13.9 \\
\hline $2008-01-30$ 09:00:00.0 & 11.2 \\
\hline 2008-01-30 06:00:00.0 & 17.4 \\
\hline 2008-01-30 03:00:00.0 & 17.6 \\
\hline $2008-01-3000: 00: 00.0$ & 10.4 \\
\hline 2008-01-29 21:00:00.0 & 15.5 \\
\hline 2008-01-29 18:00:00.0 & 14.2 \\
\hline 2008-01-29 15:00:00.0 & 17.3 \\
\hline 2008-01-29 12:00:00.0 & 21.2 \\
\hline 2008-01-29 09:00:00.0 & 21.5 \\
\hline 2008-01-29 06:00:00.0 & 18.1 \\
\hline 2008-01-29 03:00:00.0 & 11.9 \\
\hline $2008-01-2900: 00: 00.0$ & 14.7 \\
\hline 2008-01-28 21:00:00.0 & 15.6 \\
\hline $2008-01-28$ 18:00:00.0 & 14.7 \\
\hline 2008-01-28 15:00:00.0 & 25.9 \\
\hline 2008-01-28 12:00:00.0 & 24.7 \\
\hline 2008-01-28 09:00:00.0 & 23.2 \\
\hline 2008-01-28 06:00:00.0 & 24.9 \\
\hline 2008-01-28 03:00:00.0 & 13.2 \\
\hline
\end{tabular}




\section{ANEXO 01 - RAJADAS DE VENTO REGISTRADAS EM SÃO MARTINHO DA SERRA / RS}

\begin{tabular}{|c|c|}
\hline DataHora & VelVentoMax \\
\hline 2008-01-28 00:00:00.0 & 22.3 \\
\hline 2008-01-27 21:00:00.0 & 22.5 \\
\hline 2008-01-27 18:00:00.0 & 24.3 \\
\hline 2008-01-27 15:00:00.0 & 25.4 \\
\hline 2008-01-27 12:00:00.0 & 28.0 \\
\hline 2008-01-27 09:00:00.0 & 17.5 \\
\hline 2008-01-27 06:00:00.0 & 17.5 \\
\hline 2008-01-27 03:00:00.0 & 21.7 \\
\hline 2008-01-27 00:00:00.0 & 24.9 \\
\hline 2008-01-26 21:00:00.0 & 25.0 \\
\hline 2008-01-26 18:00:00.0 & 20.2 \\
\hline 2008-01-26 15:00:00.0 & 26.6 \\
\hline 2008-01-26 12:00:00.0 & 19.7 \\
\hline 2008-01-26 09:00:00.0 & 19.3 \\
\hline 2008-01-26 06:00:00.0 & 18.7 \\
\hline 2008-01-26 03:00:00.0 & 22.1 \\
\hline 2008-01-26 00:00:00.0 & 21.3 \\
\hline 2008-01-25 21:00:00.0 & 21.8 \\
\hline 2008-01-25 18:00:00.0 & 15.8 \\
\hline 2008-01-25 15:00:00.0 & 20.0 \\
\hline 2008-01-25 12:00:00.0 & 21.1 \\
\hline 2008-01-25 09:00:00.0 & 18.0 \\
\hline 2008-01-25 06:00:00.0 & 12.9 \\
\hline 2008-01-25 03:00:00.0 & 14.9 \\
\hline 2008-01-25 00:00:00.0 & 19.2 \\
\hline 2008-01-24 21:00:00.0 & 19.7 \\
\hline 2008-01-24 18:00:00.0 & 16.5 \\
\hline 2008-01-24 15:00:00.0 & 21.7 \\
\hline 2008-01-24 12:00:00.0 & 31.1 \\
\hline 2008-01-24 09:00:00.0 & 21.8 \\
\hline 2008-01-24 06:00:00.0 & 20.9 \\
\hline 2008-01-24 03:00:00.0 & 20.3 \\
\hline 2008-01-24 00:00:00.0 & 21.5 \\
\hline 2008-01-23 21:00:00.0 & 20.2 \\
\hline 2008-01-23 18:00:00.0 & 26.9 \\
\hline 2008-01-23 15:00:00.0 & 27.7 \\
\hline 2008-01-23 12:00:00.0 & 24.6 \\
\hline 2008-01-23 09:00:00.0 & 22.1 \\
\hline 2008-01-23 06:00:00.0 & 17.1 \\
\hline 2008-01-23 03:00:00.0 & 17.4 \\
\hline 2008-01-23 00:00:00.0 & 15.4 \\
\hline 2008-01-22 21:00:00.0 & 48.2 \\
\hline 2008-01-22 18:00:00.0 & 23.8 \\
\hline 2008-01-22 15:00:00.0 & 27.5 \\
\hline 2008-01-22 12:00:00.0 & 28.7 \\
\hline 2008-01-22 09:00:00.0 & 25.8 \\
\hline 2008-01-22 06:00:00.0 & 26.6 \\
\hline 2008-01-22 03:00:00.0 & 17.4 \\
\hline 2008-01-22 00:00:00.0 & 25.3 \\
\hline 2008-01-21 21:00:00.0 & 23.1 \\
\hline 2008-01-21 18:00:00.0 & 22.8 \\
\hline 2008-01-21 15:00:00.0 & 25.1 \\
\hline 2008-01-21 12:00:00.0 & 22.0 \\
\hline 2008-01-21 09:00:00.0 & \\
\hline 2008-01-21 06:00:00.0 & 18.5 \\
\hline 2008-01-21 03:00:00.0 & 17.6 \\
\hline 2008-01-21 00:00:00.0 & 20.1 \\
\hline 2008-01-20 21:00:00.0 & 24.2 \\
\hline 2008-01-20 18:00:00.0 & 8.9 \\
\hline 2008-01-20 15:00:00.0 & 22.9 \\
\hline 2008-01-20 12:00:00.0 & 21.6 \\
\hline 2008-01-20 09:00:00.0 & 14.1 \\
\hline 2008-01-20 06:00:00.0 & 24.6 \\
\hline 2008-01-20 03:00:00.0 & 22.9 \\
\hline 2008-01-20 00:00:00.0 & 15.4 \\
\hline 2008-01-19 21:00:00.0 & 32.6 \\
\hline 2008-01-19 18:00:00.0 & 22.6 \\
\hline 2008-01-19 15:00:00.0 & 24.7 \\
\hline 2008-01-19 12:00:00.0 & 19.9 \\
\hline
\end{tabular}

\begin{tabular}{|c|c|}
\hline DataHora & VelVentoMax \\
\hline 2008-01-19 09:00:00.0 & 13.8 \\
\hline 2008-01-19 06:00:00.0 & 15.4 \\
\hline 2008-01-19 03:00:00.0 & 28.4 \\
\hline 2008-01-19 00:00:00.0 & 26.9 \\
\hline 2008-01-18 21:00:00.0 & 41.7 \\
\hline 2008-01-18 18:00:00.0 & 39.3 \\
\hline 2008-01-18 15:00:00.0 & 13.8 \\
\hline 2008-01-18 12:00:00.0 & 14.0 \\
\hline 2008-01-18 09:00:00.0 & 15.3 \\
\hline 2008-01-18 06:00:00.0 & 19.2 \\
\hline 2008-01-18 03:00:00.0 & 14.9 \\
\hline 2008-01-18 00:00:00.0 & 17.0 \\
\hline 2008-01-17 21:00:00.0 & \\
\hline 2008-01-17 18:00:00.0 & 14.8 \\
\hline 2008-01-17 15:00:00.0 & 4.1 \\
\hline 2008-01-17 12:00:00.0 & 15.0 \\
\hline 2008-01-17 09:00:00.0 & 14.2 \\
\hline 2008-01-17 06:00:00.0 & 7.1 \\
\hline 2008-01-17 03:00:00.0 & 7.1 \\
\hline 2008-01-17 00:00:00.0 & 32.2 \\
\hline 2008-01-16 21:00:00.0 & \\
\hline 2008-01-16 18:00:00.0 & 24.9 \\
\hline 2008-01-16 15:00:00.0 & 28.6 \\
\hline 2008-01-16 12:00:00.0 & 17.6 \\
\hline 2008-01-16 09:00:00.0 & \\
\hline 2008-01-16 06:00:00.0 & 15.5 \\
\hline 2008-01-16 03:00:00.0 & 13.1 \\
\hline 2008-01-16 00:00:00.0 & 13.0 \\
\hline 2008-01-15 21:00:00.0 & 12.7 \\
\hline 2008-01-15 18:00:00.0 & 13.9 \\
\hline 2008-01-15 15:00:00.0 & 17.0 \\
\hline 2008-01-15 12:00:00.0 & 19.3 \\
\hline 2008-01-15 09:00:00.0 & 14.5 \\
\hline 2008-01-15 06:00:00.0 & 16.6 \\
\hline 2008-01-15 03:00:00.0 & 44.1 \\
\hline 2008-01-15 00:00:00.0 & 14.5 \\
\hline 2008-01-14 21:00:00.0 & 15.8 \\
\hline 2008-01-14 18:00:00.0 & 15.4 \\
\hline 2008-01-14 15:00:00.0 & 20.0 \\
\hline 2008-01-14 12:00:00.0 & 22.3 \\
\hline 2008-01-14 09:00:00.0 & 18.3 \\
\hline 2008-01-14 06:00:00.0 & 16.9 \\
\hline 2008-01-14 03:00:00.0 & 16.1 \\
\hline 2008-01-14 00:00:00.0 & 23.1 \\
\hline 2008-01-13 21:00:00.0 & 3.9 \\
\hline 2008-01-13 18:00:00.0 & 16.5 \\
\hline 2008-01-13 15:00:00.0 & 25.0 \\
\hline 2008-01-13 12:00:00.0 & 0.6 \\
\hline 2008-01-13 09:00:00.0 & 22.5 \\
\hline 2008-01-13 06:00:00.0 & 3.0 \\
\hline 2008-01-13 03:00:00.0 & 18.3 \\
\hline 2008-01-13 00:00:00.0 & 21.8 \\
\hline 2008-01-12 21:00:00.0 & 21.8 \\
\hline 2008-01-12 18:00:00.0 & 18.9 \\
\hline 2008-01-12 15:00:00.0 & 31.8 \\
\hline 2008-01-12 12:00:00.0 & 25.9 \\
\hline 2008-01-12 09:00:00.0 & 17.6 \\
\hline 2008-01-12 06:00:00.0 & 22.8 \\
\hline 2008-01-12 03:00:00.0 & 27.6 \\
\hline 2008-01-12 00:00:00.0 & 21.6 \\
\hline 2008-01-11 21:00:00.0 & 20.6 \\
\hline 2008-01-11 18:00:00.0 & 14.8 \\
\hline 2008-01-11 15:00:00.0 & 3.8 \\
\hline 2008-01-11 12:00:00.0 & 30.4 \\
\hline 2008-01-11 09:00:00.0 & 34.0 \\
\hline 2008-01-11 06:00:00.0 & 12.2 \\
\hline 2008-01-11 03:00:00.0 & 16.4 \\
\hline 2008-01-11 00:00:00.0 & 10.9 \\
\hline 2008-01-10 21:00:00.0 & 16.8 \\
\hline
\end{tabular}

\begin{tabular}{|c|c|}
\hline DataHora & VelVentoMax \\
\hline 2008-01-10 18:00:00.0 & 18.2 \\
\hline 2008-01-10 15:00:00.0 & 18.0 \\
\hline 2008-01-10 12:00:00.0 & 19.0 \\
\hline 2008-01-10 09:00:00.0 & 14.6 \\
\hline 2008-01-10 06:00:00.0 & 14.2 \\
\hline 2008-01-10 03:00:00.0 & 12.9 \\
\hline 2008-01-10 00:00:00.0 & \\
\hline 2008-01-09 21:00:00.0 & 22.5 \\
\hline 2008-01-09 18:00:00.0 & 15.6 \\
\hline 2008-01-09 15:00:00.0 & 10.6 \\
\hline 2008-01-09 12:00:00.0 & 13.6 \\
\hline 2008-01-09 09:00:00.0 & 11.9 \\
\hline 2008-01-09 06:00:00.0 & 14.3 \\
\hline 2008-01-09 03:00:00.0 & 12.8 \\
\hline 2008-01-09 00:00:00.0 & 14.6 \\
\hline 2008-01-08 21:00:00.0 & 15.9 \\
\hline 2008-01-08 18:00:00.0 & 17.7 \\
\hline 2008-01-08 15:00:00.0 & 14.3 \\
\hline 2008-01-08 12:00:00.0 & 13.2 \\
\hline 2008-01-08 09:00:00.0 & 14.2 \\
\hline 2008-01-08 06:00:00.0 & 10.1 \\
\hline 2008-01-08 03:00:00.0 & 4.8 \\
\hline 2008-01-08 00:00:00.0 & 10.9 \\
\hline 2008-01-07 21:00:00.0 & 15.1 \\
\hline 2008-01-07 18:00:00.0 & 14.2 \\
\hline 2008-01-07 15:00:00.0 & \\
\hline 2008-01-07 12:00:00.0 & \\
\hline 2008-01-07 09:00:00.0 & 17.0 \\
\hline 2008-01-07 06:00:00.0 & 17.0 \\
\hline 2008-01-07 03:00:00.0 & \\
\hline 2008-01-07 00:00:00.0 & 10.9 \\
\hline 2008-01-06 21:00:00.0 & 18.0 \\
\hline 2008-01-06 18:00:00.0 & 16.4 \\
\hline 2008-01-06 15:00:00.0 & 15.7 \\
\hline 2008-01-06 12:00:00.0 & \\
\hline 2008-01-06 09:00:00.0 & 11.8 \\
\hline 2008-01-06 06:00:00.0 & 12.7 \\
\hline 2008-01-06 03:00:00.0 & \\
\hline 2008-01-06 00:00:00.0 & 12.3 \\
\hline 2008-01-05 21:00:00.0 & 13.4 \\
\hline 2008-01-05 18:00:00.0 & 15.5 \\
\hline 2008-01-05 15:00:00.0 & 18.1 \\
\hline 2008-01-05 12:00:00.0 & \\
\hline 2008-01-05 09:00:00.0 & 15.0 \\
\hline 2008-01-05 06:00:00.0 & 9.8 \\
\hline 2008-01-05 03:00:00.0 & \\
\hline 2008-01-05 00:00:00.0 & 21.2 \\
\hline 2008-01-04 21:00:00.0 & 24.1 \\
\hline 2008-01-04 18:00:00.0 & 20.9 \\
\hline 2008-01-04 15:00:00.0 & \\
\hline 2008-01-04 12:00:00.0 & 12.5 \\
\hline 2008-01-04 09:00:00.0 & \\
\hline 2008-01-04 06:00:00.0 & 6.3 \\
\hline 2008-01-04 03:00:00.0 & \\
\hline 2008-01-04 00:00:00.0 & \\
\hline 2008-01-03 21:00:00.0 & 21.9 \\
\hline 2008-01-03 18:00:00.0 & 46.6 \\
\hline 2008-01-03 15:00:00.0 & \\
\hline 2008-01-03 12:00:00.0 & 32.3 \\
\hline 2008-01-03 09:00:00.0 & 15.3 \\
\hline 2008-01-03 06:00:00.0 & 13.8 \\
\hline 2008-01-03 03:00:00.0 & 10.7 \\
\hline 2008-01-03 00:00:00.0 & 10.4 \\
\hline 2008-01-02 21:00:00.0 & 14.5 \\
\hline 2008-01-02 18:00:00.0 & \\
\hline 2008-01-02 15:00:00.0 & 12.5 \\
\hline 2008-01-02 12:00:00.0 & 34.5 \\
\hline 2008-01-02 09:00:00.0 & 18.9 \\
\hline 2008-01-02 06:00:00.0 & 15.5 \\
\hline
\end{tabular}




\section{ANEXO 01 - RAJADAS DE VENTO REGISTRADAS EM SÃO MARTINHO DA SERRA / RS}

\begin{tabular}{|c|c|}
\hline DataHora & VelVentoMax \\
\hline 2008-01-02 03:00:00.0 & 16.5 \\
\hline 2008-01-02 00:00:00.0 & 16.3 \\
\hline 2008-01-01 21:00:00.0 & 27.1 \\
\hline 2008-01-01 18:00:00.0 & 16.2 \\
\hline 2008-01-01 15:00:00.0 & 13.9 \\
\hline 2008-01-01 12:00:00.0 & 12.7 \\
\hline 2008-01-01 09:00:00.0 & 15.1 \\
\hline 2008-01-01 06:00:00.0 & 15.2 \\
\hline 2008-01-01 03:00:00.0 & 14.7 \\
\hline 2008-01-01 00:00:00.0 & 14.9 \\
\hline 2007-12-31 21:00:00.0 & 17.8 \\
\hline 2007-12-31 18:00:00.0 & 15.7 \\
\hline 2007-12-31 15:00:00.0 & 11.4 \\
\hline 2007-12-31 12:00:00.0 & 9.4 \\
\hline 2007-12-31 09:00:00.0 & 10.3 \\
\hline 2007-12-31 06:00:00.0 & 11.6 \\
\hline 2007-12-31 03:00:00.0 & 20.4 \\
\hline 2007-12-31 00:00:00.0 & 20.4 \\
\hline 2007-12-30 21:00:00.0 & 20.7 \\
\hline 2007-12-30 18:00:00.0 & 24.9 \\
\hline 2007-12-30 15:00:00.0 & 19.9 \\
\hline 2007-12-30 12:00:00.0 & 1.6 \\
\hline 2007-12-30 09:00:00.0 & 34.3 \\
\hline 2007-12-30 06:00:00.0 & 15.8 \\
\hline 2007-12-30 03:00:00.0 & 18.0 \\
\hline 2007-12-30 00:00:00.0 & 18.5 \\
\hline 2007-12-29 21:00:00.0 & 13.5 \\
\hline 2007-12-29 18:00:00.0 & 29.4 \\
\hline 2007-12-29 15:00:00.0 & 23.2 \\
\hline 2007-12-29 12:00:00.0 & 20.2 \\
\hline 2007-12-29 09:00:00.0 & 17.1 \\
\hline 2007-12-29 06:00:00.0 & 18.0 \\
\hline 2007-12-29 03:00:00.0 & 32.3 \\
\hline 2007-12-29 00:00:00.0 & 19.4 \\
\hline 2007-12-28 21:00:00.0 & 21.4 \\
\hline 2007-12-28 18:00:00.0 & 26.2 \\
\hline 2007-12-28 15:00:00.0 & 23.4 \\
\hline 2007-12-28 12:00:00.0 & 25.7 \\
\hline 2007-12-28 09:00:00.0 & 17.8 \\
\hline 2007-12-28 06:00:00.0 & 18.4 \\
\hline 2007-12-28 03:00:00.0 & 24.7 \\
\hline 2007-12-28 00:00:00.0 & 24.1 \\
\hline 2007-12-27 21:00:00.0 & 22.6 \\
\hline 2007-12-27 18:00:00.0 & 19.8 \\
\hline 2007-12-27 15:00:00.0 & 17.1 \\
\hline 2007-12-27 12:00:00.0 & 12.1 \\
\hline 2007-12-27 09:00:00.0 & 23.1 \\
\hline 2007-12-27 06:00:00.0 & 28.4 \\
\hline 2007-12-27 03:00:00.0 & 9.4 \\
\hline 2007-12-27 00:00:00.0 & 11.9 \\
\hline 2007-12-26 21:00:00.0 & 18.7 \\
\hline 2007-12-26 18:00:00.0 & 25.7 \\
\hline 2007-12-26 15:00:00.0 & 18.0 \\
\hline 2007-12-26 12:00:00.0 & 13.8 \\
\hline 2007-12-26 09:00:00.0 & \\
\hline 2007-12-26 06:00:00.0 & 12.2 \\
\hline 2007-12-26 03:00:00.0 & 11.3 \\
\hline 2007-12-26 00:00:00.0 & 16.3 \\
\hline 2007-12-25 21:00:00.0 & 20.9 \\
\hline 2007-12-25 18:00:00.0 & 20.7 \\
\hline 2007-12-25 15:00:00.0 & 17.7 \\
\hline 2007-12-25 12:00:00.0 & 16.5 \\
\hline 2007-12-25 09:00:00.0 & 15.9 \\
\hline 2007-12-25 06:00:00.0 & 16.0 \\
\hline 2007-12-25 03:00:00.0 & 19.6 \\
\hline 2007-12-25 00:00:00.0 & 15.5 \\
\hline 2007-12-24 21:00:00.0 & 20.5 \\
\hline 2007-12-24 18:00:00.0 & 34.1 \\
\hline 2007-12-24 15:00:00.0 & 26.1 \\
\hline
\end{tabular}

\begin{tabular}{|c|c|}
\hline DataHora & VelVentoMax \\
\hline 2007-12-24 12:00:00.0 & 22.3 \\
\hline 2007-12-24 09:00:00.0 & 20.9 \\
\hline 2007-12-24 06:00:00.0 & 21.8 \\
\hline 2007-12-24 03:00:00.0 & 19.5 \\
\hline 2007-12-24 00:00:00.0 & 18.6 \\
\hline 2007-12-23 21:00:00.0 & 21.2 \\
\hline 2007-12-23 18:00:00.0 & 25.3 \\
\hline 2007-12-23 15:00:00.0 & 23.4 \\
\hline 2007-12-23 12:00:00.0 & 21.6 \\
\hline 2007-12-23 09:00:00.0 & 17.6 \\
\hline 2007-12-23 06:00:00.0 & 16.1 \\
\hline 2007-12-23 03:00:00.0 & 16.5 \\
\hline 2007-12-23 00:00:00.0 & 28.7 \\
\hline 2007-12-22 21:00:00.0 & 29.0 \\
\hline 2007-12-22 18:00:00.0 & 20.7 \\
\hline 2007-12-22 15:00:00.0 & 19.1 \\
\hline 2007-12-22 12:00:00.0 & 8.9 \\
\hline 2007-12-22 09:00:00.0 & 11.5 \\
\hline 2007-12-22 06:00:00.0 & 14.3 \\
\hline 2007-12-22 03:00:00.0 & 10.9 \\
\hline 2007-12-22 00:00:00.0 & 12.2 \\
\hline 2007-12-21 21:00:00.0 & 15.9 \\
\hline 2007-12-21 18:00:00.0 & 18.6 \\
\hline 2007-12-21 15:00:00.0 & 13.0 \\
\hline 2007-12-21 12:00:00.0 & 13.8 \\
\hline 2007-12-21 09:00:00.0 & 18.7 \\
\hline 2007-12-21 06:00:00.0 & 19.2 \\
\hline 2007-12-21 03:00:00.0 & 17.5 \\
\hline 2007-12-21 00:00:00.0 & 19.3 \\
\hline 2007-12-20 21:00:00.0 & 19.8 \\
\hline 2007-12-20 18:00:00.0 & 17.5 \\
\hline 2007-12-20 15:00:00.0 & 15.8 \\
\hline 2007-12-20 12:00:00.0 & 19.2 \\
\hline 2007-12-20 09:00:00.0 & 18.8 \\
\hline 2007-12-20 06:00:00.0 & 18.7 \\
\hline 2007-12-20 03:00:00.0 & 15.3 \\
\hline 2007-12-20 00:00:00.0 & 15.8 \\
\hline 2007-12-19 21:00:00.0 & 19.2 \\
\hline 2007-12-19 18:00:00.0 & 19.5 \\
\hline 2007-12-19 15:00:00.0 & 21.0 \\
\hline 2007-12-19 12:00:00.0 & 16.7 \\
\hline 2007-12-19 09:00:00.0 & \\
\hline 2007-12-19 06:00:00.0 & 10.2 \\
\hline 2007-12-19 03:00:00.0 & 7.1 \\
\hline 2007-12-19 00:00:00.0 & 7.0 \\
\hline 2007-12-18 21:00:00.0 & 14.8 \\
\hline 2007-12-18 18:00:00.0 & 17.9 \\
\hline 2007-12-18 15:00:00.0 & 15.3 \\
\hline 2007-12-18 12:00:00.0 & 13.8 \\
\hline 2007-12-18 09:00:00.0 & 12.8 \\
\hline 2007-12-18 06:00:00.0 & 16.6 \\
\hline 2007-12-18 03:00:00.0 & 16.8 \\
\hline 2007-12-18 00:00:00.0 & 22.7 \\
\hline 2007-12-17 21:00:00.0 & 22.6 \\
\hline 2007-12-17 18:00:00.0 & 19.6 \\
\hline 2007-12-17 15:00:00.0 & 21.7 \\
\hline 2007-12-17 12:00:00.0 & 26.4 \\
\hline 2007-12-17 09:00:00.0 & 18.2 \\
\hline 2007-12-17 06:00:00.0 & 20.8 \\
\hline 2007-12-17 03:00:00.0 & 18.8 \\
\hline 2007-12-17 00:00:00.0 & \\
\hline 2007-12-16 21:00:00.0 & 25.1 \\
\hline 2007-12-16 18:00:00.0 & 24.7 \\
\hline 2007-12-16 15:00:00.0 & \\
\hline 2007-12-16 12:00:00.0 & 22.1 \\
\hline 2007-12-16 09:00:00.0 & 20.7 \\
\hline 2007-12-16 06:00:00.0 & 25.6 \\
\hline 2007-12-16 03:00:00.0 & 22.9 \\
\hline 2007-12-16 00:00:00.0 & \\
\hline
\end{tabular}

\begin{tabular}{|c|c|}
\hline DataHora & VelVentoMax \\
\hline 2007-12-15 21:00:00.0 & 18.9 \\
\hline 2007-12-15 18:00:00.0 & 19.3 \\
\hline 2007-12-15 15:00:00.0 & 14.5 \\
\hline 2007-12-15 12:00:00.0 & 17.0 \\
\hline 2007-12-15 09:00:00.0 & 14.6 \\
\hline 2007-12-15 06:00:00.0 & 20.1 \\
\hline 2007-12-15 03:00:00.0 & \\
\hline 2007-12-15 00:00:00.0 & 20.2 \\
\hline 2007-12-14 21:00:00.0 & 17.8 \\
\hline 2007-12-14 18:00:00.0 & 14.1 \\
\hline 2007-12-14 15:00:00.0 & 36.2 \\
\hline 2007-12-14 12:00:00.0 & 27.0 \\
\hline 2007-12-14 09:00:00.0 & 22.1 \\
\hline 2007-12-14 06:00:00.0 & 20.2 \\
\hline 2007-12-14 03:00:00.0 & 19.1 \\
\hline 2007-12-14 00:00:00.0 & 21.2 \\
\hline 2007-12-13 21:00:00.0 & 22.7 \\
\hline 2007-12-13 18:00:00.0 & 17.1 \\
\hline $2007-12-13$ 15:00:00.0 & 19.6 \\
\hline 2007-12-13 12:00:00.0 & 25.6 \\
\hline 2007-12-13 09:00:00.0 & 22.0 \\
\hline 2007-12-13 06:00:00.0 & 22.0 \\
\hline 2007-12-13 03:00:00.0 & 26.1 \\
\hline 2007-12-13 00:00:00.0 & 21.3 \\
\hline 2007-12-12 21:00:00.0 & 25.1 \\
\hline 2007-12-12 18:00:00.0 & 22.6 \\
\hline 2007-12-12 15:00:00.0 & 19.8 \\
\hline 2007-12-12 12:00:00.0 & 17.8 \\
\hline 2007-12-12 09:00:00.0 & 15.5 \\
\hline 2007-12-12 06:00:00.0 & 17.0 \\
\hline 2007-12-12 03:00:00.0 & 17.0 \\
\hline 2007-12-12 00:00:00.0 & 16.0 \\
\hline 2007-12-11 21:00:00.0 & 24.5 \\
\hline 2007-12-11 18:00:00.0 & 22.3 \\
\hline 2007-12-11 15:00:00.0 & 23.7 \\
\hline 2007-12-11 12:00:00.0 & 29.0 \\
\hline 2007-12-11 09:00:00.0 & 19.8 \\
\hline 2007-12-11 06:00:00.0 & 32.9 \\
\hline 2007-12-11 03:00:00.0 & 37.7 \\
\hline 2007-12-11 00:00:00.0 & 18.8 \\
\hline 2007-12-10 21:00:00.0 & 26.5 \\
\hline 2007-12-10 18:00:00.0 & 31.1 \\
\hline 2007-12-10 15:00:00.0 & \\
\hline 2007-12-10 12:00:00.0 & 25.4 \\
\hline 2007-12-10 09:00:00.0 & 20.2 \\
\hline 2007-12-10 06:00:00.0 & 19.5 \\
\hline 2007-12-10 03:00:00.0 & 18.2 \\
\hline 2007-12-10 00:00:00.0 & 17.6 \\
\hline 2007-12-09 21:00:00.0 & 20.3 \\
\hline 2007-12-09 18:00:00.0 & 17.7 \\
\hline 2007-12-09 15:00:00.0 & 16.7 \\
\hline 2007-12-09 12:00:00.0 & 19.9 \\
\hline 2007-12-09 09:00:00.0 & 23.6 \\
\hline 2007-12-09 06:00:00.0 & 4.4 \\
\hline 2007-12-09 03:00:00.0 & 5.4 \\
\hline 2007-12-09 00:00:00.0 & 15.3 \\
\hline 2007-12-08 21:00:00.0 & 19.8 \\
\hline 2007-12-08 18:00:00.0 & 23.3 \\
\hline 2007-12-08 15:00:00.0 & 22.6 \\
\hline 2007-12-08 12:00:00.0 & 11.7 \\
\hline 2007-12-08 09:00:00.0 & 13.0 \\
\hline 2007-12-08 06:00:00.0 & 11.9 \\
\hline 2007-12-08 03:00:00.0 & 10.2 \\
\hline 2007-12-08 00:00:00.0 & 14.5 \\
\hline 2007-12-07 21:00:00.0 & 22.6 \\
\hline 2007-12-07 18:00:00.0 & 21.2 \\
\hline 2007-12-07 15:00:00.0 & 29.7 \\
\hline 2007-12-07 12:00:00.0 & 3.0 \\
\hline 2007-12-07 09:00:00.0 & 11.5 \\
\hline
\end{tabular}




\section{ANEXO 01 - RAJADAS DE VENTO REGISTRADAS EM SÃO MARTINHO DA SERRA / RS}

\begin{tabular}{|c|c|}
\hline DataHora & VelVentoMax \\
\hline 2007-12-07 06:00:00.0 & 12.4 \\
\hline 2007-12-07 03:00:00.0 & 12.9 \\
\hline 2007-12-07 00:00:00.0 & 12.8 \\
\hline 2007-12-06 21:00:00.0 & 15.8 \\
\hline 2007-12-06 18:00:00.0 & 18.4 \\
\hline 2007-12-06 15:00:00.0 & 20.4 \\
\hline 2007-12-06 12:00:00.0 & 22.4 \\
\hline 2007-12-06 09:00:00.0 & 19.1 \\
\hline 2007-12-06 06:00:00.0 & 24.0 \\
\hline 2007-12-06 03:00:00.0 & 26.5 \\
\hline 2007-12-06 00:00:00.0 & 27.2 \\
\hline 2007-12-05 21:00:00.0 & 25.3 \\
\hline 2007-12-05 18:00:00.0 & 18.9 \\
\hline 2007-12-05 15:00:00.0 & 11.9 \\
\hline 2007-12-05 12:00:00.0 & 8.9 \\
\hline 2007-12-05 09:00:00.0 & 27.3 \\
\hline 2007-12-05 06:00:00.0 & 26.0 \\
\hline 2007-12-05 03:00:00.0 & 36.0 \\
\hline 2007-12-05 00:00:00.0 & 1.2 \\
\hline 2007-12-04 21:00:00.0 & 18.3 \\
\hline 2007-12-04 18:00:00.0 & \\
\hline 2007-12-04 15:00:00.0 & 15.7 \\
\hline 2007-12-04 12:00:00.0 & 31.5 \\
\hline 2007-12-04 09:00:00.0 & 15.2 \\
\hline 2007-12-04 06:00:00.0 & 17.3 \\
\hline 2007-12-04 03:00:00.0 & 14.8 \\
\hline 2007-12-04 00:00:00.0 & 17.0 \\
\hline 2007-12-03 21:00:00.0 & 19.9 \\
\hline 2007-12-03 18:00:00.0 & 15.8 \\
\hline 2007-12-03 15:00:00.0 & 13.9 \\
\hline 2007-12-03 12:00:00.0 & 16.2 \\
\hline 2007-12-03 09:00:00.0 & 17.5 \\
\hline 2007-12-03 06:00:00.0 & 11.4 \\
\hline 2007-12-03 03:00:00.0 & 12.7 \\
\hline 2007-12-03 00:00:00.0 & 15.4 \\
\hline 2007-12-02 21:00:00.0 & 22.5 \\
\hline 2007-12-02 18:00:00.0 & \\
\hline 2007-12-02 15:00:00.0 & 13.9 \\
\hline 2007-12-02 12:00:00.0 & 12.9 \\
\hline 2007-12-02 09:00:00.0 & 12.0 \\
\hline 2007-12-02 06:00:00.0 & 13.7 \\
\hline 2007-12-02 03:00:00.0 & 14.5 \\
\hline 2007-12-02 00:00:00.0 & 21.2 \\
\hline 2007-12-01 21:00:00.0 & 20.5 \\
\hline 2007-12-01 18:00:00.0 & 19.0 \\
\hline 2007-12-01 15:00:00.0 & 18.1 \\
\hline 2007-12-01 12:00:00.0 & 23.2 \\
\hline 2007-12-01 09:00:00.0 & 19.4 \\
\hline 2007-12-01 06:00:00.0 & 19.6 \\
\hline 2007-12-01 03:00:00.0 & 18.0 \\
\hline 2007-12-01 00:00:00.0 & 23.1 \\
\hline 2007-11-30 21:00:00.0 & 25.3 \\
\hline 2007-11-30 18:00:00.0 & 24.8 \\
\hline 2007-11-30 15:00:00.0 & 18.3 \\
\hline 2007-11-30 12:00:00.0 & 20.2 \\
\hline 2007-11-30 09:00:00.0 & 20.8 \\
\hline 2007-11-30 06:00:00.0 & 14.9 \\
\hline 2007-11-30 03:00:00.0 & 16.6 \\
\hline 2007-11-30 00:00:00.0 & 23.1 \\
\hline 2007-11-29 21:00:00.0 & 22.7 \\
\hline 2007-11-29 18:00:00.0 & 24.2 \\
\hline 2007-11-29 15:00:00.0 & 22.7 \\
\hline 2007-11-29 12:00:00.0 & 24.9 \\
\hline 2007-11-29 09:00:00.0 & 18.0 \\
\hline 2007-11-29 06:00:00.0 & 18.1 \\
\hline 2007-11-29 03:00:00.0 & 17.6 \\
\hline 2007-11-29 00:00:00.0 & 27.0 \\
\hline 2007-11-28 21:00:00.0 & 28.0 \\
\hline 2007-11-28 18:00:00.0 & 25.8 \\
\hline
\end{tabular}

\begin{tabular}{|c|c|}
\hline DataHora & VelVentoMax \\
\hline 2007-11-28 15:00:00.0 & 17.0 \\
\hline 2007-11-28 12:00:00.0 & 15.9 \\
\hline 2007-11-28 09:00:00.0 & 13.0 \\
\hline 2007-11-28 06:00:00.0 & 14.4 \\
\hline 2007-11-28 03:00:00.0 & \\
\hline 2007-11-28 00:00:00.0 & \\
\hline 2007-11-27 21:00:00.0 & 19.2 \\
\hline 2007-11-27 18:00:00.0 & \\
\hline 2007-11-27 15:00:00.0 & 23.3 \\
\hline 2007-11-27 12:00:00.0 & 11.9 \\
\hline 2007-11-27 09:00:00.0 & \\
\hline 2007-11-27 06:00:00.0 & 12.4 \\
\hline 2007-11-27 03:00:00.0 & 11.7 \\
\hline 2007-11-27 00:00:00.0 & 10.6 \\
\hline 2007-11-26 21:00:00.0 & 22.7 \\
\hline 2007-11-26 18:00:00.0 & 26.8 \\
\hline 2007-11-26 15:00:00.0 & 27.9 \\
\hline 2007-11-26 12:00:00.0 & 21.4 \\
\hline 2007-11-26 09:00:00.0 & 8.2 \\
\hline 2007-11-26 06:00:00.0 & 6.0 \\
\hline 2007-11-26 03:00:00.0 & 8.2 \\
\hline 2007-11-26 00:00:00.0 & 14.6 \\
\hline 2007-11-25 21:00:00.0 & 16.1 \\
\hline 2007-11-25 18:00:00.0 & 17.0 \\
\hline 2007-11-25 15:00:00.0 & 17.2 \\
\hline 2007-11-25 12:00:00.0 & 18.0 \\
\hline 2007-11-25 09:00:00.0 & 15.9 \\
\hline 2007-11-25 06:00:00.0 & 12.4 \\
\hline 2007-11-25 03:00:00.0 & 12.5 \\
\hline 2007-11-25 00:00:00.0 & 14.6 \\
\hline 2007-11-24 21:00:00.0 & 12.9 \\
\hline 2007-11-24 18:00:00.0 & 14.1 \\
\hline 2007-11-24 15:00:00.0 & 13.6 \\
\hline 2007-11-24 12:00:00.0 & 14.4 \\
\hline 2007-11-24 09:00:00.0 & 11.5 \\
\hline 2007-11-24 06:00:00.0 & 10.7 \\
\hline 2007-11-24 03:00:00.0 & 18.4 \\
\hline 2007-11-24 00:00:00.0 & 25.3 \\
\hline 2007-11-23 21:00:00.0 & 39.1 \\
\hline 2007-11-23 18:00:00.0 & 18.0 \\
\hline 2007-11-23 15:00:00.0 & 22.4 \\
\hline 2007-11-23 12:00:00.0 & 26.3 \\
\hline 2007-11-23 09:00:00.0 & 22.9 \\
\hline 2007-11-23 06:00:00.0 & 21.2 \\
\hline 2007-11-23 03:00:00.0 & 19.8 \\
\hline 2007-11-23 00:00:00.0 & 17.7 \\
\hline 2007-11-22 21:00:00.0 & 19.1 \\
\hline 2007-11-22 18:00:00.0 & 23.9 \\
\hline 2007-11-22 15:00:00.0 & 30.0 \\
\hline 2007-11-22 12:00:00.0 & 30.0 \\
\hline 2007-11-22 09:00:00.0 & 18.7 \\
\hline 2007-11-22 06:00:00.0 & 13.9 \\
\hline 2007-11-22 03:00:00.0 & 14.9 \\
\hline 2007-11-22 00:00:00.0 & 19.9 \\
\hline 2007-11-21 21:00:00.0 & 23.1 \\
\hline 2007-11-21 18:00:00.0 & 21.1 \\
\hline 2007-11-21 15:00:00.0 & 13.9 \\
\hline 2007-11-21 12:00:00.0 & 11.2 \\
\hline 2007-11-21 09:00:00.0 & \\
\hline 2007-11-21 06:00:00.0 & 13.0 \\
\hline 2007-11-21 03:00:00.0 & 10.7 \\
\hline 2007-11-21 00:00:00.0 & 13.7 \\
\hline 2007-11-20 21:00:00.0 & 16.7 \\
\hline 2007-11-20 18:00:00.0 & 18.3 \\
\hline 2007-11-20 15:00:00.0 & 16.4 \\
\hline 2007-11-20 12:00:00.0 & 12.0 \\
\hline 2007-11-20 09:00:00.0 & 8.9 \\
\hline 2007-11-20 06:00:00.0 & 7.6 \\
\hline 2007-11-20 03:00:00.0 & 9.7 \\
\hline
\end{tabular}

\begin{tabular}{|c|c|}
\hline DataHora & VelVentoMax \\
\hline 2007-11-20 00:00:00.0 & 12.8 \\
\hline 2007-11-19 21:00:00.0 & 13.7 \\
\hline 2007-11-19 18:00:00.0 & 30.1 \\
\hline 2007-11-19 15:00:00.0 & 10.5 \\
\hline 2007-11-19 12:00:00.0 & 12.5 \\
\hline 2007-11-19 09:00:00.0 & 14.6 \\
\hline 2007-11-19 06:00:00.0 & 14.1 \\
\hline 2007-11-19 03:00:00.0 & 10.1 \\
\hline 2007-11-19 00:00:00.0 & 6.6 \\
\hline 2007-11-18 21:00:00.0 & 12.5 \\
\hline 2007-11-18 18:00:00.0 & 14.9 \\
\hline 2007-11-18 15:00:00.0 & 34.8 \\
\hline 2007-11-18 12:00:00.0 & 24.1 \\
\hline 2007-11-18 09:00:00.0 & 36.4 \\
\hline 2007-11-18 06:00:00.0 & 24.5 \\
\hline 2007-11-18 03:00:00.0 & 22.3 \\
\hline 2007-11-18 00:00:00.0 & 19.6 \\
\hline 2007-11-17 21:00:00.0 & 39.7 \\
\hline 2007-11-17 18:00:00.0 & 27.8 \\
\hline 2007-11-17 15:00:00.0 & 24.4 \\
\hline 2007-11-17 12:00:00.0 & 27.0 \\
\hline 2007-11-17 09:00:00.0 & 27.3 \\
\hline 2007-11-17 06:00:00.0 & 27.1 \\
\hline 2007-11-17 03:00:00.0 & 21.9 \\
\hline 2007-11-17 00:00:00.0 & 17.3 \\
\hline 2007-11-16 21:00:00.0 & 20.3 \\
\hline 2007-11-16 18:00:00.0 & 20.8 \\
\hline 2007-11-16 15:00:00.0 & 27.4 \\
\hline 2007-11-16 12:00:00.0 & 30.3 \\
\hline 2007-11-16 09:00:00.0 & 25.4 \\
\hline 2007-11-16 06:00:00.0 & 21.2 \\
\hline 2007-11-16 03:00:00.0 & 18.2 \\
\hline 2007-11-16 00:00:00.0 & 22.2 \\
\hline 2007-11-15 21:00:00.0 & 22.6 \\
\hline 2007-11-15 18:00:00.0 & 21.9 \\
\hline 2007-11-15 15:00:00.0 & 20.6 \\
\hline 2007-11-15 12:00:00.0 & 20.3 \\
\hline 2007-11-15 09:00:00.0 & 13.9 \\
\hline 2007-11-15 06:00:00.0 & 25.2 \\
\hline 2007-11-15 03:00:00.0 & 28.0 \\
\hline 2007-11-15 00:00:00.0 & 32.6 \\
\hline 2007-11-14 21:00:00.0 & 28.1 \\
\hline 2007-11-14 18:00:00.0 & 23.3 \\
\hline 2007-11-14 15:00:00.0 & 17.3 \\
\hline 2007-11-14 12:00:00.0 & 32.0 \\
\hline 2007-11-14 09:00:00.0 & 11.3 \\
\hline 2007-11-14 06:00:00.0 & 23.0 \\
\hline 2007-11-14 03:00:00.0 & 20.0 \\
\hline 2007-11-14 00:00:00.0 & 26.3 \\
\hline 2007-11-13 21:00:00.0 & 20.7 \\
\hline 2007-11-13 18:00:00.0 & 26.4 \\
\hline 2007-11-13 15:00:00.0 & 25.4 \\
\hline 2007-11-13 12:00:00.0 & 27.1 \\
\hline 2007-11-13 09:00:00.0 & 21.2 \\
\hline 2007-11-13 06:00:00.0 & 1.679 .999 \\
\hline 2007-11-13 03:00:00.0 & 8.9 \\
\hline 2007-11-13 00:00:00.0 & 12.3 \\
\hline 2007-11-12 21:00:00.0 & 16.1 \\
\hline 2007-11-12 18:00:00.0 & 14.5 \\
\hline 2007-11-12 15:00:00.0 & 13.4 \\
\hline 2007-11-12 12:00:00.0 & 14.7 \\
\hline 2007-11-12 09:00:00.0 & 9.0 \\
\hline 2007-11-12 06:00:00.0 & 9.6 \\
\hline 2007-11-12 03:00:00.0 & \\
\hline 2007-11-12 00:00:00.0 & 14.8 \\
\hline 2007-11-11 21:00:00.0 & 24.0 \\
\hline 2007-11-11 18:00:00.0 & 26.6 \\
\hline 2007-11-11 15:00:00.0 & 7.5 \\
\hline 2007-11-11 12:00:00.0 & 41.4 \\
\hline
\end{tabular}




\section{ANEXO 01 - RAJADAS DE VENTO REGISTRADAS EM SÃO MARTINHO DA SERRA / RS}

\begin{tabular}{|c|c|}
\hline DataHora & VelVentoMax \\
\hline 2007-11-11 09:00:00.0 & 16.3 \\
\hline 2007-11-11 06:00:00.0 & 13.3 \\
\hline 2007-11-11 03:00:00.0 & 17.6 \\
\hline 2007-11-11 00:00:00.0 & 25.8 \\
\hline 2007-11-10 21:00:00.0 & 22.9 \\
\hline 2007-11-10 18:00:00.0 & 18.6 \\
\hline 2007-11-10 15:00:00.0 & 48.0 \\
\hline 2007-11-10 12:00:00.0 & 14.6 \\
\hline 2007-11-10 09:00:00.0 & 18.0 \\
\hline 2007-11-10 06:00:00.0 & 16.6 \\
\hline 2007-11-10 03:00:00.0 & 13.4 \\
\hline 2007-11-10 00:00:00.0 & 12.3 \\
\hline 2007-11-09 21:00:00.0 & 41.9 \\
\hline 2007-11-09 18:00:00.0 & 15.4 \\
\hline 2007-11-09 15:00:00.0 & 6.1 \\
\hline 2007-11-09 12:00:00.0 & 28.7 \\
\hline 2007-11-09 09:00:00.0 & 24.9 \\
\hline 2007-11-09 06:00:00.0 & 18.0 \\
\hline 2007-11-09 03:00:00.0 & 17.4 \\
\hline 2007-11-09 00:00:00.0 & 13.1 \\
\hline 2007-11-08 21:00:00.0 & 16.2 \\
\hline 2007-11-08 18:00:00.0 & \\
\hline 2007-11-08 15:00:00.0 & 15.8 \\
\hline 2007-11-08 12:00:00.0 & 19.7 \\
\hline 2007-11-08 09:00:00.0 & 16.0 \\
\hline 2007-11-08 06:00:00.0 & 16.5 \\
\hline 2007-11-08 03:00:00.0 & 15.2 \\
\hline 2007-11-08 00:00:00.0 & 14.9 \\
\hline 2007-11-07 21:00:00.0 & 49.5 \\
\hline 2007-11-07 18:00:00.0 & 17.2 \\
\hline 2007-11-07 15:00:00.0 & 17.6 \\
\hline 2007-11-07 12:00:00.0 & 20.7 \\
\hline 2007-11-07 09:00:00.0 & 20.4 \\
\hline 2007-11-07 06:00:00.0 & 15.7 \\
\hline 2007-11-07 03:00:00.0 & 50.4 \\
\hline 2007-11-07 00:00:00.0 & 11.0 \\
\hline 2007-11-06 21:00:00.0 & 13.6 \\
\hline 2007-11-06 18:00:00.0 & 33.4 \\
\hline 2007-11-06 15:00:00.0 & 28.0 \\
\hline 2007-11-06 12:00:00.0 & 9.0 \\
\hline 2007-11-06 09:00:00.0 & 15.0 \\
\hline 2007-11-06 06:00:00.0 & 12.0 \\
\hline 2007-11-06 03:00:00.0 & 12.0 \\
\hline 2007-11-06 00:00:00.0 & 12.0 \\
\hline 2007-11-05 21:00:00.0 & 13.0 \\
\hline 2007-11-05 18:00:00.0 & 32.0 \\
\hline 2007-11-05 15:00:00.0 & 11.0 \\
\hline 2007-11-05 12:00:00.0 & 14.0 \\
\hline 2007-11-05 09:00:00.0 & 9.0 \\
\hline 2007-11-05 06:00:00.0 & 11.0 \\
\hline 2007-11-05 03:00:00.0 & 8.0 \\
\hline 2007-11-05 00:00:00.0 & 16.0 \\
\hline 2007-11-04 21:00:00.0 & 26.0 \\
\hline 2007-11-04 18:00:00.0 & 31.0 \\
\hline 2007-11-04 15:00:00.0 & 25.0 \\
\hline 2007-11-04 12:00:00.0 & 24.0 \\
\hline 2007-11-04 09:00:00.0 & 24.0 \\
\hline 2007-11-04 06:00:00.0 & 30.0 \\
\hline 2007-11-04 03:00:00.0 & 10.0 \\
\hline 2007-11-04 00:00:00.0 & 15.0 \\
\hline 2007-11-03 21:00:00.0 & 24.0 \\
\hline 2007-11-03 18:00:00.0 & 20.0 \\
\hline 2007-11-03 15:00:00.0 & 11.0 \\
\hline 2007-11-03 12:00:00.0 & 9.0 \\
\hline 2007-11-03 09:00:00.0 & 5.0 \\
\hline 2007-11-03 06:00:00.0 & 9.0 \\
\hline 2007-11-03 03:00:00.0 & 10.0 \\
\hline 2007-11-03 00:00:00.0 & 8.0 \\
\hline 2007-11-02 21:00:00.0 & 15.0 \\
\hline
\end{tabular}

\begin{tabular}{|c|c|}
\hline DataHora & VelVentoMax \\
\hline 2007-11-02 18:00:00.0 & 16.0 \\
\hline 2007-11-02 15:00:00.0 & 12.0 \\
\hline 2007-11-02 12:00:00.0 & 11.0 \\
\hline 2007-11-02 09:00:00.0 & \\
\hline 2007-11-02 06:00:00.0 & 11.0 \\
\hline 2007-11-02 03:00:00.0 & 7.0 \\
\hline 2007-11-02 00:00:00.0 & 15.0 \\
\hline 2007-11-01 21:00:00.0 & 19.0 \\
\hline 2007-11-01 18:00:00.0 & 19.3 \\
\hline 2007-11-01 15:00:00.0 & 19.7 \\
\hline 2007-11-01 12:00:00.0 & 19.1 \\
\hline 2007-11-01 09:00:00.0 & \\
\hline 2007-11-01 06:00:00.0 & 21.3 \\
\hline 2007-11-01 03:00:00.0 & 24.6 \\
\hline 2007-11-01 00:00:00.0 & 35.6 \\
\hline 2007-10-31 21:00:00.0 & 29.6 \\
\hline 2007-10-31 18:00:00.0 & 21.8 \\
\hline 2007-10-31 15:00:00.0 & 20.1 \\
\hline 2007-10-31 12:00:00.0 & 18.5 \\
\hline 2007-10-31 09:00:00.0 & 16.4 \\
\hline 2007-10-31 06:00:00.0 & 15.3 \\
\hline 2007-10-31 03:00:00.0 & 9.9 \\
\hline 2007-10-31 00:00:00.0 & 21.5 \\
\hline 2007-10-30 21:00:00.0 & 20.6 \\
\hline 2007-10-30 18:00:00.0 & 13.0 \\
\hline 2007-10-30 15:00:00.0 & 17.2 \\
\hline 2007-10-30 12:00:00.0 & 22.2 \\
\hline 2007-10-30 09:00:00.0 & 24.2 \\
\hline 2007-10-30 06:00:00.0 & 17.8 \\
\hline 2007-10-30 03:00:00.0 & 20.1 \\
\hline 2007-10-30 00:00:00.0 & 21.2 \\
\hline 2007-10-29 21:00:00.0 & \\
\hline 2007-10-29 18:00:00.0 & 33.0 \\
\hline 2007-10-29 15:00:00.0 & 26.1 \\
\hline 2007-10-29 12:00:00.0 & 40.9 \\
\hline 2007-10-29 09:00:00.0 & 31.6 \\
\hline 2007-10-29 06:00:00.0 & 33.0 \\
\hline 2007-10-29 03:00:00.0 & 26.0 \\
\hline 2007-10-29 00:00:00.0 & 31.0 \\
\hline 2007-10-28 21:00:00.0 & 26.0 \\
\hline 2007-10-28 18:00:00.0 & 17.0 \\
\hline 2007-10-28 15:00:00.0 & 25.0 \\
\hline 2007-10-28 12:00:00.0 & 24.0 \\
\hline 2007-10-28 09:00:00.0 & 20.0 \\
\hline 2007-10-28 06:00:00.0 & 15.0 \\
\hline 2007-10-28 03:00:00.0 & 10.0 \\
\hline 2007-10-28 00:00:00.0 & 8.0 \\
\hline 2007-10-27 21:00:00.0 & 22.0 \\
\hline 2007-10-27 18:00:00.0 & 34.0 \\
\hline 2007-10-27 15:00:00.0 & 24.0 \\
\hline 2007-10-27 12:00:00.0 & 25.0 \\
\hline 2007-10-27 09:00:00.0 & 23.0 \\
\hline 2007-10-27 06:00:00.0 & 8.0 \\
\hline 2007-10-27 03:00:00.0 & 7.0 \\
\hline 2007-10-27 00:00:00.0 & 5.0 \\
\hline 2007-10-26 21:00:00.0 & 19.0 \\
\hline 2007-10-26 18:00:00.0 & 20.0 \\
\hline 2007-10-26 15:00:00.0 & 19.0 \\
\hline 2007-10-26 12:00:00.0 & 17.0 \\
\hline 2007-10-26 09:00:00.0 & 13.0 \\
\hline 2007-10-26 06:00:00.0 & 12.0 \\
\hline 2007-10-26 03:00:00.0 & 6.0 \\
\hline 2007-10-26 00:00:00.0 & 6.0 \\
\hline 2007-10-25 21:00:00.0 & 14.0 \\
\hline 2007-10-25 18:00:00.0 & 20.0 \\
\hline 2007-10-25 15:00:00.0 & 23.0 \\
\hline 2007-10-25 12:00:00.0 & 26.0 \\
\hline 2007-10-25 09:00:00.0 & 21.0 \\
\hline 2007-10-25 06:00:00.0 & 25.0 \\
\hline
\end{tabular}

\begin{tabular}{|c|c|}
\hline DataHora & VelVentoMax \\
\hline 2007-10-25 03:00:00.0 & 23.0 \\
\hline 2007-10-25 00:00:00.0 & 18.0 \\
\hline 2007-10-24 21:00:00.0 & 18.0 \\
\hline $2007-10-24$ 18:00:00.0 & 17.0 \\
\hline 2007-10-24 15:00:00.0 & 24.0 \\
\hline 2007-10-24 12:00:00.0 & 23.0 \\
\hline 2007-10-24 09:00:00.0 & 13.0 \\
\hline 2007-10-24 06:00:00.0 & 17.0 \\
\hline 2007-10-24 03:00:00.0 & 16.0 \\
\hline 2007-10-24 00:00:00.0 & 15.0 \\
\hline 2007-10-23 21:00:00.0 & 21.0 \\
\hline $2007-10-23$ 18:00:00.0 & 19.0 \\
\hline 2007-10-23 15:00:00.0 & 4.0 \\
\hline 2007-10-23 12:00:00.0 & 21.0 \\
\hline 2007-10-23 09:00:00.0 & 21.0 \\
\hline 2007-10-23 06:00:00.0 & 22.0 \\
\hline 2007-10-23 03:00:00.0 & 20.0 \\
\hline 2007-10-23 00:00:00.0 & 18.0 \\
\hline 2007-10-22 21:00:00.0 & 19.0 \\
\hline 2007-10-22 18:00:00.0 & 16.0 \\
\hline 2007-10-22 15:00:00.0 & 24.0 \\
\hline $2007-10-22$ 12:00:00.0 & 25.0 \\
\hline $2007-10-22$ 09:00:00.0 & 17.0 \\
\hline 2007-10-22 06:00:00.0 & 18.0 \\
\hline 2007-10-22 03:00:00.0 & 15.0 \\
\hline $2007-10-22$ 00:00:00.0 & 15.0 \\
\hline 2007-10-21 21:00:00.0 & 14.0 \\
\hline 2007-10-21 18:00:00.0 & 10.0 \\
\hline 2007-10-21 15:00:00.0 & 11.0 \\
\hline 2007-10-21 12:00:00.0 & 15.0 \\
\hline 2007-10-21 09:00:00.0 & 20.0 \\
\hline 2007-10-21 06:00:00.0 & 19.0 \\
\hline 2007-10-21 03:00:00.0 & 22.0 \\
\hline 2007-10-21 00:00:00.0 & 20.0 \\
\hline 2007-10-20 21:00:00.0 & 32.0 \\
\hline 2007-10-20 18:00:00.0 & 30.0 \\
\hline $2007-10-20$ 15:00:00.0 & 27.0 \\
\hline 2007-10-20 12:00:00.0 & 7.0 \\
\hline 2007-10-20 09:00:00.0 & 11.0 \\
\hline 2007-10-20 06:00:00.0 & 14.0 \\
\hline 2007-10-20 03:00:00.0 & 14.0 \\
\hline 2007-10-20 00:00:00.0 & 13.0 \\
\hline 2007-10-19 21:00:00.0 & 17.0 \\
\hline 2007-10-19 18:00:00.0 & 11.0 \\
\hline 2007-10-19 15:00:00.0 & \\
\hline 2007-10-19 12:00:00.0 & 16.0 \\
\hline 2007-10-19 09:00:00.0 & 12.0 \\
\hline 2007-10-19 06:00:00.0 & 13.0 \\
\hline 2007-10-19 03:00:00.0 & 12.0 \\
\hline 2007-10-19 00:00:00.0 & 15.0 \\
\hline 2007-10-18 21:00:00.0 & 17.0 \\
\hline 2007-10-18 18:00:00.0 & 15.0 \\
\hline 2007-10-18 15:00:00.0 & 15.7 \\
\hline 2007-10-18 12:00:00.0 & 2.7 \\
\hline 2007-10-18 09:00:00.0 & 16.1 \\
\hline 2007-10-18 06:00:00.0 & 15.5 \\
\hline 2007-10-18 03:00:00.0 & \\
\hline 2007-10-18 00:00:00.0 & 12.7 \\
\hline 2007-10-17 21:00:00.0 & 11.3 \\
\hline 2007-10-17 18:00:00.0 & 12.7 \\
\hline 2007-10-17 15:00:00.0 & 8.7 \\
\hline 2007-10-17 12:00:00.0 & 9.7 \\
\hline 2007-10-17 09:00:00.0 & 7.1 \\
\hline 2007-10-17 06:00:00.0 & 14.3 \\
\hline 2007-10-17 03:00:00.0 & 18.2 \\
\hline 2007-10-17 00:00:00.0 & 14.1 \\
\hline 2007-10-16 21:00:00.0 & 21.9 \\
\hline $2007-10-16$ 18:00:00.0 & 24.5 \\
\hline 2007-10-16 15:00:00.0 & 23.3 \\
\hline
\end{tabular}




\section{ANEXO 01 - RAJADAS DE VENTO REGISTRADAS EM SÃO MARTINHO DA SERRA / RS}

\begin{tabular}{|c|c|}
\hline DataHora & VelVentoMax \\
\hline 2007-10-16 12:00:00.0 & 18.6 \\
\hline 2007-10-16 09:00:00.0 & 13.4 \\
\hline 2007-10-16 06:00:00.0 & 14.0 \\
\hline 2007-10-16 03:00:00.0 & 18.5 \\
\hline 2007-10-16 00:00:00.0 & 18.8 \\
\hline 2007-10-15 21:00:00.0 & 17.6 \\
\hline 2007-10-15 18:00:00.0 & 23.4 \\
\hline 2007-10-15 15:00:00.0 & 29.1 \\
\hline 2007-10-15 12:00:00.0 & 28.3 \\
\hline 2007-10-15 09:00:00.0 & 23.0 \\
\hline 2007-10-15 06:00:00.0 & 22.9 \\
\hline 2007-10-15 03:00:00.0 & 22.3 \\
\hline 2007-10-15 00:00:00.0 & 22.4 \\
\hline 2007-10-14 21:00:00.0 & 20.8 \\
\hline 2007-10-14 18:00:00.0 & 26.9 \\
\hline 2007-10-14 15:00:00.0 & 24.2 \\
\hline 2007-10-14 12:00:00.0 & 22.7 \\
\hline 2007-10-14 09:00:00.0 & 22.1 \\
\hline 2007-10-14 06:00:00.0 & 15.1 \\
\hline 2007-10-14 03:00:00.0 & 11.8 \\
\hline 2007-10-14 00:00:00.0 & 9.2 \\
\hline 2007-10-13 21:00:00.0 & 16.6 \\
\hline 2007-10-13 18:00:00.0 & 22.5 \\
\hline 2007-10-13 15:00:00.0 & 22.3 \\
\hline 2007-10-13 12:00:00.0 & 14.2 \\
\hline 2007-10-13 09:00:00.0 & 14.6 \\
\hline 2007-10-13 06:00:00.0 & 23.4 \\
\hline 2007-10-13 03:00:00.0 & 23.9 \\
\hline 2007-10-13 00:00:00.0 & 29.5 \\
\hline 2007-10-12 21:00:00.0 & 33.4 \\
\hline 2007-10-12 18:00:00.0 & 36.2 \\
\hline 2007-10-12 15:00:00.0 & 32.0 \\
\hline 2007-10-12 12:00:00.0 & 22.2 \\
\hline 2007-10-12 09:00:00.0 & 25.2 \\
\hline 2007-10-12 06:00:00.0 & 22.4 \\
\hline 2007-10-12 03:00:00.0 & 16.0 \\
\hline 2007-10-12 00:00:00.0 & 18.4 \\
\hline 2007-10-11 21:00:00.0 & 19.7 \\
\hline 2007-10-11 18:00:00.0 & 19.2 \\
\hline 2007-10-11 15:00:00.0 & 27.1 \\
\hline 2007-10-11 12:00:00.0 & 20.6 \\
\hline 2007-10-11 09:00:00.0 & 17.5 \\
\hline 2007-10-11 06:00:00.0 & 17.5 \\
\hline 2007-10-11 03:00:00.0 & 21.0 \\
\hline 2007-10-11 00:00:00.0 & 28.6 \\
\hline 2007-10-10 21:00:00.0 & 14.1 \\
\hline 2007-10-10 18:00:00.0 & 16.1 \\
\hline 2007-10-10 15:00:00.0 & 3.6 \\
\hline 2007-10-10 12:00:00.0 & 33.8 \\
\hline 2007-10-10 09:00:00.0 & 35.6 \\
\hline 2007-10-10 06:00:00.0 & 15.4 \\
\hline 2007-10-10 03:00:00.0 & 13.3 \\
\hline 2007-10-10 00:00:00.0 & 14.5 \\
\hline 2007-10-09 21:00:00.0 & 23.2 \\
\hline 2007-10-09 18:00:00.0 & 22.6 \\
\hline 2007-10-09 15:00:00.0 & 25.6 \\
\hline 2007-10-09 12:00:00.0 & 4.5 \\
\hline 2007-10-09 09:00:00.0 & 23.6 \\
\hline 2007-10-09 06:00:00.0 & 18.6 \\
\hline 2007-10-09 03:00:00.0 & 25.8 \\
\hline 2007-10-09 00:00:00.0 & 26.6 \\
\hline 2007-10-08 21:00:00.0 & \\
\hline 2007-10-08 18:00:00.0 & 38.8 \\
\hline 2007-10-08 15:00:00.0 & 26.8 \\
\hline 2007-10-08 12:00:00.0 & 36.1 \\
\hline 2007-10-08 09:00:00.0 & 25.5 \\
\hline 2007-10-08 06:00:00.0 & 24.4 \\
\hline 2007-10-08 03:00:00.0 & 23.2 \\
\hline 2007-10-08 00:00:00.0 & 26.2 \\
\hline
\end{tabular}

\begin{tabular}{|c|c|}
\hline DataHora & VelVentoMax \\
\hline 2007-10-07 21:00:00.0 & 24.4 \\
\hline 2007-10-07 18:00:00.0 & 20.6 \\
\hline 2007-10-07 15:00:00.0 & 24.5 \\
\hline 2007-10-07 12:00:00.0 & 25.4 \\
\hline 2007-10-07 09:00:00.0 & 18.9 \\
\hline 2007-10-07 06:00:00.0 & 12.7 \\
\hline 2007-10-07 03:00:00.0 & 13.6 \\
\hline 2007-10-07 00:00:00.0 & 19.4 \\
\hline 2007-10-06 21:00:00.0 & 22.4 \\
\hline 2007-10-06 18:00:00.0 & 33.3 \\
\hline 2007-10-06 15:00:00.0 & 29.4 \\
\hline 2007-10-06 12:00:00.0 & 25.6 \\
\hline 2007-10-06 09:00:00.0 & 27.6 \\
\hline 2007-10-06 06:00:00.0 & 29.7 \\
\hline 2007-10-06 03:00:00.0 & 24.0 \\
\hline 2007-10-06 00:00:00.0 & 23.3 \\
\hline 2007-10-05 21:00:00.0 & 27.2 \\
\hline 2007-10-05 18:00:00.0 & 31.0 \\
\hline 2007-10-05 15:00:00.0 & 34.0 \\
\hline 2007-10-05 12:00:00.0 & 31.3 \\
\hline 2007-10-05 09:00:00.0 & 21.7 \\
\hline 2007-10-05 06:00:00.0 & 23.4 \\
\hline 2007-10-05 03:00:00.0 & 25.3 \\
\hline 2007-10-05 00:00:00.0 & 28.3 \\
\hline 2007-10-04 21:00:00.0 & 21.5 \\
\hline 2007-10-04 18:00:00.0 & 27.0 \\
\hline 2007-10-04 15:00:00.0 & 29.7 \\
\hline 2007-10-04 12:00:00.0 & 22.7 \\
\hline 2007-10-04 09:00:00.0 & 29.3 \\
\hline 2007-10-04 06:00:00.0 & 24.9 \\
\hline 2007-10-04 03:00:00.0 & 26.0 \\
\hline 2007-10-04 00:00:00.0 & 23.1 \\
\hline 2007-10-03 21:00:00.0 & 23.4 \\
\hline 2007-10-03 18:00:00.0 & 19.4 \\
\hline 2007-10-03 15:00:00.0 & 21.6 \\
\hline 2007-10-03 12:00:00.0 & 23.7 \\
\hline 2007-10-03 09:00:00.0 & 23.7 \\
\hline 2007-10-03 06:00:00.0 & 30.3 \\
\hline 2007-10-03 03:00:00.0 & 22.5 \\
\hline 2007-10-03 00:00:00.0 & 18.2 \\
\hline 2007-10-02 21:00:00.0 & 17.2 \\
\hline 2007-10-02 18:00:00.0 & 22.4 \\
\hline 2007-10-02 15:00:00.0 & 26.7 \\
\hline 2007-10-02 12:00:00.0 & 29.7 \\
\hline 2007-10-02 09:00:00.0 & 31.1 \\
\hline 2007-10-02 06:00:00.0 & 25.5 \\
\hline 2007-10-02 03:00:00.0 & 22.7 \\
\hline 2007-10-02 00:00:00.0 & 18.4 \\
\hline 2007-10-01 21:00:00.0 & 25.2 \\
\hline 2007-10-01 18:00:00.0 & 22.7 \\
\hline 2007-10-01 15:00:00.0 & 27.3 \\
\hline 2007-10-01 12:00:00.0 & 29.4 \\
\hline 2007-10-01 09:00:00.0 & 26.6 \\
\hline 2007-10-01 06:00:00.0 & 24.5 \\
\hline 2007-10-01 03:00:00.0 & 18.1 \\
\hline 2007-10-01 00:00:00.0 & 17.6 \\
\hline 2007-09-30 21:00:00.0 & 19.8 \\
\hline 2007-09-30 18:00:00.0 & 21.1 \\
\hline 2007-09-30 15:00:00.0 & 24.6 \\
\hline 2007-09-30 12:00:00.0 & 24.4 \\
\hline 2007-09-30 09:00:00.0 & 17.9 \\
\hline 2007-09-30 06:00:00.0 & 21.5 \\
\hline 2007-09-30 03:00:00.0 & 23.4 \\
\hline 2007-09-30 00:00:00.0 & 20.5 \\
\hline 2007-09-29 21:00:00.0 & 23.3 \\
\hline 2007-09-29 18:00:00.0 & 21.5 \\
\hline 2007-09-29 15:00:00.0 & 24.1 \\
\hline 2007-09-29 12:00:00.0 & 24.1 \\
\hline 2007-09-29 09:00:00.0 & 21.8 \\
\hline
\end{tabular}

\begin{tabular}{|c|c|}
\hline DataHora & VelVentoMax \\
\hline 2007-09-29 06:00:00.0 & 14.2 \\
\hline 2007-09-29 03:00:00.0 & 12.2 \\
\hline 2007-09-29 00:00:00.0 & 11.6 \\
\hline 2007-09-28 21:00:00.0 & 16.3 \\
\hline 2007-09-28 18:00:00.0 & 15.2 \\
\hline 2007-09-28 15:00:00.0 & 13.9 \\
\hline 2007-09-28 12:00:00.0 & 13.7 \\
\hline 2007-09-28 09:00:00.0 & 9.4 \\
\hline 2007-09-28 06:00:00.0 & 6.7 \\
\hline 2007-09-28 03:00:00.0 & 7.8 \\
\hline 2007-09-28 00:00:00.0 & 10.3 \\
\hline 2007-09-27 21:00:00.0 & 20.7 \\
\hline 2007-09-27 18:00:00.0 & 38.9 \\
\hline 2007-09-27 15:00:00.0 & 24.5 \\
\hline 2007-09-27 12:00:00.0 & 22.4 \\
\hline 2007-09-27 09:00:00.0 & 24.6 \\
\hline 2007-09-27 06:00:00.0 & 23.8 \\
\hline 2007-09-27 03:00:00.0 & 19.1 \\
\hline 2007-09-27 00:00:00.0 & 20.2 \\
\hline 2007-09-26 21:00:00.0 & 18.4 \\
\hline 2007-09-26 18:00:00.0 & 23.5 \\
\hline 2007-09-26 15:00:00.0 & 47.3 \\
\hline 2007-09-26 12:00:00.0 & 28.2 \\
\hline 2007-09-26 09:00:00.0 & 25.8 \\
\hline 2007-09-26 06:00:00.0 & 20.3 \\
\hline 2007-09-26 03:00:00.0 & 18.7 \\
\hline 2007-09-26 00:00:00.0 & 15.4 \\
\hline 2007-09-25 21:00:00.0 & 19.5 \\
\hline $2007-09-25$ 18:00:00.0 & 26.5 \\
\hline 2007-09-25 15:00:00.0 & 5.2 \\
\hline 2007-09-25 12:00:00.0 & 18.3 \\
\hline 2007-09-25 09:00:00.0 & 9.7 \\
\hline 2007-09-25 06:00:00.0 & 15.6 \\
\hline 2007-09-25 03:00:00.0 & 14.7 \\
\hline 2007-09-25 00:00:00.0 & \\
\hline 2007-09-24 21:00:00.0 & 29.5 \\
\hline $2007-09-24$ 18:00:00.0 & 31.6 \\
\hline $2007-09-24$ 15:00:00.0 & 32.5 \\
\hline 2007-09-24 12:00:00.0 & 25.9 \\
\hline 2007-09-24 09:00:00.0 & 25.0 \\
\hline 2007-09-24 06:00:00.0 & 37.1 \\
\hline 2007-09-24 03:00:00.0 & 35.9 \\
\hline 2007-09-24 00:00:00.0 & 33.7 \\
\hline 2007-09-23 21:00:00.0 & 29.5 \\
\hline $2007-09-23$ 18:00:00.0 & 20.6 \\
\hline 2007-09-23 15:00:00.0 & 21.9 \\
\hline 2007-09-23 12:00:00.0 & 20.1 \\
\hline 2007-09-23 09:00:00.0 & 21.9 \\
\hline 2007-09-23 06:00:00.0 & 19.1 \\
\hline 2007-09-23 03:00:00.0 & 27.1 \\
\hline 2007-09-23 00:00:00.0 & 26.5 \\
\hline 2007-09-22 21:00:00.0 & 23.1 \\
\hline 2007-09-22 18:00:00.0 & \\
\hline 2007-09-22 15:00:00.0 & 23.4 \\
\hline 2007-09-22 12:00:00.0 & 20.1 \\
\hline 2007-09-22 09:00:00.0 & 32.8 \\
\hline 2007-09-22 06:00:00.0 & 21.2 \\
\hline 2007-09-22 03:00:00.0 & 17.5 \\
\hline 2007-09-22 00:00:00.0 & 16.4 \\
\hline 2007-09-21 21:00:00.0 & 15.6 \\
\hline 2007-09-21 18:00:00.0 & 25.3 \\
\hline 2007-09-21 15:00:00.0 & 20.8 \\
\hline 2007-09-21 12:00:00.0 & 30.6 \\
\hline 2007-09-21 09:00:00.0 & 15.8 \\
\hline 2007-09-21 06:00:00.0 & 16.0 \\
\hline 2007-09-21 03:00:00.0 & 8.1 \\
\hline 2007-09-21 00:00:00.0 & 17.3 \\
\hline 2007-09-20 21:00:00.0 & 20.4 \\
\hline 2007-09-20 18:00:00.0 & 18.3 \\
\hline
\end{tabular}




\section{ANEXO 01 - RAJADAS DE VENTO REGISTRADAS EM SÃO MARTINHO DA SERRA / RS}

\begin{tabular}{|c|c|}
\hline DataHora & VelVentoMax \\
\hline 2007-09-20 15:00:00.0 & 24.9 \\
\hline 2007-09-20 12:00:00.0 & \\
\hline 2007-09-20 09:00:00.0 & 17.9 \\
\hline 2007-09-20 06:00:00.0 & 16.2 \\
\hline 2007-09-20 03:00:00.0 & 12.9 \\
\hline 2007-09-20 00:00:00.0 & 11.4 \\
\hline 2007-09-19 21:00:00.0 & 15.0 \\
\hline 2007-09-19 18:00:00.0 & 15.1 \\
\hline 2007-09-19 15:00:00.0 & 18.3 \\
\hline 2007-09-19 12:00:00.0 & 20.4 \\
\hline 2007-09-19 09:00:00.0 & 12.0 \\
\hline 2007-09-19 06:00:00.0 & 23.4 \\
\hline 2007-09-19 03:00:00.0 & 18.7 \\
\hline 2007-09-19 00:00:00.0 & 28.7 \\
\hline 2007-09-18 21:00:00.0 & 35.1 \\
\hline 2007-09-18 18:00:00.0 & 29.7 \\
\hline 2007-09-18 15:00:00.0 & 24.6 \\
\hline 2007-09-18 12:00:00.0 & 31.1 \\
\hline 2007-09-18 09:00:00.0 & 32.5 \\
\hline 2007-09-18 06:00:00.0 & 35.7 \\
\hline 2007-09-18 03:00:00.0 & 34.5 \\
\hline 2007-09-18 00:00:00.0 & 33.4 \\
\hline 2007-09-17 21:00:00.0 & 31.9 \\
\hline 2007-09-17 18:00:00.0 & \\
\hline 2007-09-17 15:00:00.0 & 33.7 \\
\hline 2007-09-17 12:00:00.0 & 29.2 \\
\hline 2007-09-17 09:00:00.0 & 29.8 \\
\hline 2007-09-17 06:00:00.0 & 23.1 \\
\hline 2007-09-17 03:00:00.0 & 25.2 \\
\hline 2007-09-17 00:00:00.0 & 22.0 \\
\hline 2007-09-16 21:00:00.0 & 20.4 \\
\hline 2007-09-16 18:00:00.0 & 22.8 \\
\hline 2007-09-16 15:00:00.0 & 17.5 \\
\hline 2007-09-16 12:00:00.0 & 12.9 \\
\hline 2007-09-16 09:00:00.0 & 12.0 \\
\hline 2007-09-16 06:00:00.0 & 9.0 \\
\hline 2007-09-16 03:00:00.0 & 15.4 \\
\hline 2007-09-16 00:00:00.0 & 14.5 \\
\hline 2007-09-15 21:00:00.0 & 14.0 \\
\hline 2007-09-15 18:00:00.0 & 28.9 \\
\hline 2007-09-15 15:00:00.0 & 2.3 \\
\hline 2007-09-15 12:00:00.0 & 34.1 \\
\hline 2007-09-15 09:00:00.0 & 13.7 \\
\hline 2007-09-15 06:00:00.0 & 14.6 \\
\hline 2007-09-15 03:00:00.0 & 18.1 \\
\hline 2007-09-15 00:00:00.0 & 42.8 \\
\hline 2007-09-14 21:00:00.0 & \\
\hline 2007-09-14 18:00:00.0 & 25.1 \\
\hline 2007-09-14 15:00:00.0 & 21.0 \\
\hline 2007-09-14 12:00:00.0 & 23.5 \\
\hline 2007-09-14 09:00:00.0 & 22.2 \\
\hline 2007-09-14 06:00:00.0 & 18.9 \\
\hline 2007-09-14 03:00:00.0 & 16.6 \\
\hline 2007-09-14 00:00:00.0 & 15.4 \\
\hline 2007-09-13 21:00:00.0 & 10.4 \\
\hline 2007-09-13 18:00:00.0 & 13.7 \\
\hline 2007-09-13 15:00:00.0 & 22.1 \\
\hline 2007-09-13 12:00:00.0 & 25.2 \\
\hline 2007-09-13 09:00:00.0 & 21.9 \\
\hline 2007-09-13 06:00:00.0 & 18.1 \\
\hline 2007-09-13 03:00:00.0 & 14.8 \\
\hline 2007-09-13 00:00:00.0 & 11.5 \\
\hline 2007-09-12 21:00:00.0 & \\
\hline 2007-09-12 18:00:00.0 & 12.7 \\
\hline 2007-09-12 15:00:00.0 & 16.5 \\
\hline 2007-09-12 12:00:00.0 & 15.0 \\
\hline 2007-09-12 09:00:00.0 & 21.3 \\
\hline 2007-09-12 06:00:00.0 & 21.5 \\
\hline 2007-09-12 03:00:00.0 & 21.3 \\
\hline
\end{tabular}

\begin{tabular}{|c|c|}
\hline DataHora & VelVentoMax \\
\hline 2007-09-12 00:00:00.0 & 12.8 \\
\hline 2007-09-11 21:00:00.0 & 31.5 \\
\hline 2007-09-11 18:00:00.0 & 23.4 \\
\hline 2007-09-11 15:00:00.0 & 23.6 \\
\hline 2007-09-11 12:00:00.0 & 21.6 \\
\hline 2007-09-11 09:00:00.0 & 20.6 \\
\hline 2007-09-11 06:00:00.0 & 21.1 \\
\hline 2007-09-11 03:00:00.0 & 22.4 \\
\hline 2007-09-11 00:00:00.0 & 18.9 \\
\hline 2007-09-10 21:00:00.0 & 30.5 \\
\hline 2007-09-10 18:00:00.0 & 22.3 \\
\hline 2007-09-10 15:00:00.0 & 22.7 \\
\hline 2007-09-10 12:00:00.0 & 14.8 \\
\hline 2007-09-10 09:00:00.0 & 20.5 \\
\hline 2007-09-10 06:00:00.0 & 19.3 \\
\hline 2007-09-10 03:00:00.0 & 19.6 \\
\hline 2007-09-10 00:00:00.0 & 16.0 \\
\hline 2007-09-09 21:00:00.0 & 19.6 \\
\hline 2007-09-09 18:00:00.0 & 21.2 \\
\hline 2007-09-09 15:00:00.0 & 27.1 \\
\hline 2007-09-09 12:00:00.0 & 20.9 \\
\hline 2007-09-09 09:00:00.0 & 22.3 \\
\hline 2007-09-09 06:00:00.0 & 17.3 \\
\hline 2007-09-09 03:00:00.0 & 14.1 \\
\hline 2007-09-09 00:00:00.0 & 31.7 \\
\hline 2007-09-08 21:00:00.0 & 15.6 \\
\hline 2007-09-08 18:00:00.0 & 18.1 \\
\hline 2007-09-08 15:00:00.0 & 36.1 \\
\hline 2007-09-08 12:00:00.0 & 36.2 \\
\hline 2007-09-08 09:00:00.0 & 16.3 \\
\hline 2007-09-08 06:00:00.0 & 14.8 \\
\hline 2007-09-08 03:00:00.0 & 17.0 \\
\hline 2007-09-08 00:00:00.0 & 11.2 \\
\hline 2007-09-07 21:00:00.0 & 9.6 \\
\hline 2007-09-07 18:00:00.0 & 8.7 \\
\hline 2007-09-07 15:00:00.0 & 27.4 \\
\hline 2007-09-07 12:00:00.0 & 17.3 \\
\hline 2007-09-07 09:00:00.0 & 17.9 \\
\hline 2007-09-07 06:00:00.0 & 16.8 \\
\hline 2007-09-07 03:00:00.0 & 18.2 \\
\hline 2007-09-07 00:00:00.0 & \\
\hline 2007-09-06 21:00:00.0 & 10.4 \\
\hline 2007-09-06 18:00:00.0 & 17.5 \\
\hline 2007-09-06 15:00:00.0 & 34.6 \\
\hline 2007-09-06 12:00:00.0 & 20.7 \\
\hline 2007-09-06 09:00:00.0 & 23.6 \\
\hline 2007-09-06 06:00:00.0 & 26.3 \\
\hline 2007-09-06 03:00:00.0 & 17.9 \\
\hline 2007-09-06 00:00:00.0 & \\
\hline 2007-09-05 21:00:00.0 & 16.0 \\
\hline 2007-09-05 18:00:00.0 & 24.3 \\
\hline 2007-09-05 15:00:00.0 & 25.9 \\
\hline 2007-09-05 12:00:00.0 & 21.3 \\
\hline 2007-09-05 09:00:00.0 & 21.2 \\
\hline 2007-09-05 06:00:00.0 & 19.1 \\
\hline 2007-09-05 03:00:00.0 & 18.0 \\
\hline 2007-09-05 00:00:00.0 & \\
\hline 2007-09-04 21:00:00.0 & 35.4 \\
\hline 2007-09-04 18:00:00.0 & 14.9 \\
\hline 2007-09-04 15:00:00.0 & 27.7 \\
\hline 2007-09-04 12:00:00.0 & 23.1 \\
\hline 2007-09-04 09:00:00.0 & 21.5 \\
\hline 2007-09-04 06:00:00.0 & 18.9 \\
\hline 2007-09-04 03:00:00.0 & 18.1 \\
\hline 2007-09-04 00:00:00.0 & 14.9 \\
\hline 2007-09-03 21:00:00.0 & 16.9 \\
\hline 2007-09-03 18:00:00.0 & 16.3 \\
\hline 2007-09-03 15:00:00.0 & 23.0 \\
\hline 2007-09-03 12:00:00.0 & 22.7 \\
\hline
\end{tabular}

\begin{tabular}{|c|c|}
\hline DataHora & VelVentoMax \\
\hline 2007-09-03 09:00:00.0 & 23.5 \\
\hline 2007-09-03 06:00:00.0 & 23.0 \\
\hline 2007-09-03 03:00:00.0 & 20.1 \\
\hline 2007-09-03 00:00:00.0 & 17.6 \\
\hline 2007-09-02 21:00:00.0 & 19.5 \\
\hline 2007-09-02 18:00:00.0 & 19.0 \\
\hline 2007-09-02 15:00:00.0 & 20.4 \\
\hline 2007-09-02 12:00:00.0 & 17.6 \\
\hline 2007-09-02 09:00:00.0 & 14.5 \\
\hline 2007-09-02 06:00:00.0 & 21.3 \\
\hline 2007-09-02 03:00:00.0 & 27.5 \\
\hline 2007-09-02 00:00:00.0 & 29.4 \\
\hline 2007-09-01 21:00:00.0 & 13.1 \\
\hline 2007-09-01 18:00:00.0 & 13.6 \\
\hline 2007-09-01 15:00:00.0 & 11.9 \\
\hline 2007-09-01 12:00:00.0 & 30.4 \\
\hline 2007-09-01 09:00:00.0 & 13.9 \\
\hline 2007-09-01 06:00:00.0 & 10.4 \\
\hline 2007-09-01 03:00:00.0 & 10.8 \\
\hline 2007-09-01 00:00:00.0 & 10.6 \\
\hline 2007-08-31 21:00:00.0 & 13.0 \\
\hline $2007-08-31$ 18:00:00.0 & 6.6 \\
\hline $2007-08-31$ 15:00:00.0 & 13.1 \\
\hline 2007-08-31 12:00:00.0 & 17.2 \\
\hline 2007-08-31 09:00:00.0 & 21.8 \\
\hline 2007-08-31 06:00:00.0 & 20.9 \\
\hline 2007-08-31 03:00:00.0 & 19.9 \\
\hline 2007-08-31 00:00:00.0 & \\
\hline 2007-08-30 21:00:00.0 & 23.1 \\
\hline 2007-08-30 18:00:00.0 & 22.2 \\
\hline 2007-08-30 15:00:00.0 & 31.5 \\
\hline 2007-08-30 12:00:00.0 & 29.2 \\
\hline 2007-08-30 09:00:00.0 & 23.8 \\
\hline 2007-08-30 06:00:00.0 & 20.5 \\
\hline $2007-08-30$ 03:00:00.0 & 20.0 \\
\hline 2007-08-30 00:00:00.0 & 21.5 \\
\hline 2007-08-29 21:00:00.0 & 28.6 \\
\hline $2007-08-2918: 00: 00.0$ & 27.1 \\
\hline 2007-08-29 15:00:00.0 & 24.7 \\
\hline $2007-08-29$ 12:00:00.0 & 11.7 \\
\hline 2007-08-29 09:00:00.0 & 10.1 \\
\hline 2007-08-29 06:00:00.0 & 10.6 \\
\hline 2007-08-29 03:00:00.0 & 4.9 \\
\hline 2007-08-29 00:00:00.0 & 10.4 \\
\hline 2007-08-28 21:00:00.0 & 12.9 \\
\hline $2007-08-28$ 18:00:00.0 & 11.7 \\
\hline 2007-08-28 15:00:00.0 & 10.8 \\
\hline 2007-08-28 12:00:00.0 & 14.4 \\
\hline $2007-08-28$ 09:00:00.0 & 13.0 \\
\hline 2007-08-28 06:00:00.0 & 8.3 \\
\hline $2007-08-28$ 03:00:00.0 & 4.2 \\
\hline 2007-08-28 00:00:00.0 & 11.2 \\
\hline 2007-08-27 21:00:00.0 & 16.5 \\
\hline $2007-08-27$ 18:00:00.0 & 22.0 \\
\hline 2007-08-27 15:00:00.0 & 26.8 \\
\hline 2007-08-27 12:00:00.0 & 14.7 \\
\hline 2007-08-27 09:00:00.0 & 16.0 \\
\hline 2007-08-27 06:00:00.0 & 23.0 \\
\hline 2007-08-27 03:00:00.0 & 23.0 \\
\hline 2007-08-27 00:00:00.0 & 21.0 \\
\hline 2007-08-26 21:00:00.0 & 27.0 \\
\hline $2007-08-26$ 18:00:00.0 & 22.0 \\
\hline $2007-08-26$ 15:00:00.0 & 22.0 \\
\hline $2007-08-26$ 12:00:00.0 & 16.0 \\
\hline 2007-08-26 09:00:00.0 & 11.0 \\
\hline 2007-08-26 06:00:00.0 & 13.0 \\
\hline 2007-08-26 03:00:00.0 & 19.0 \\
\hline 2007-08-26 00:00:00.0 & 19.0 \\
\hline 2007-08-25 21:00:00.0 & 4.0 \\
\hline
\end{tabular}




\section{ANEXO 01 - RAJADAS DE VENTO REGISTRADAS EM SÃO MARTINHO DA SERRA / RS}

\begin{tabular}{|c|c|}
\hline DataHora & VelVentoMax \\
\hline 2007-08-25 18:00:00.0 & 34.1 \\
\hline 2007-08-25 15:00:00.0 & 36.1 \\
\hline 2007-08-25 12:00:00.0 & 36.3 \\
\hline 2007-08-25 09:00:00.0 & 34.9 \\
\hline 2007-08-25 06:00:00.0 & 35.7 \\
\hline 2007-08-25 03:00:00.0 & 40.8 \\
\hline 2007-08-25 00:00:00.0 & 31.7 \\
\hline 2007-08-24 21:00:00.0 & 31.2 \\
\hline 2007-08-24 18:00:00.0 & 29.9 \\
\hline 2007-08-24 15:00:00.0 & 23.2 \\
\hline 2007-08-24 12:00:00.0 & 21.4 \\
\hline 2007-08-24 09:00:00.0 & 24.6 \\
\hline 2007-08-24 06:00:00.0 & 20.6 \\
\hline 2007-08-24 03:00:00.0 & 15.0 \\
\hline 2007-08-24 00:00:00.0 & 24.8 \\
\hline 2007-08-23 21:00:00.0 & 24.8 \\
\hline 2007-08-23 18:00:00.0 & 31.8 \\
\hline 2007-08-23 15:00:00.0 & 32.3 \\
\hline 2007-08-23 12:00:00.0 & 23.8 \\
\hline 2007-08-23 09:00:00.0 & 19.7 \\
\hline 2007-08-23 06:00:00.0 & 18.8 \\
\hline 2007-08-23 03:00:00.0 & 16.9 \\
\hline 2007-08-23 00:00:00.0 & 19.3 \\
\hline 2007-08-22 21:00:00.0 & 14.3 \\
\hline 2007-08-22 18:00:00.0 & 15.3 \\
\hline 2007-08-22 15:00:00.0 & 20.7 \\
\hline 2007-08-22 12:00:00.0 & 19.8 \\
\hline 2007-08-22 09:00:00.0 & 23.7 \\
\hline 2007-08-22 06:00:00.0 & 24.6 \\
\hline 2007-08-22 03:00:00.0 & 21.3 \\
\hline 2007-08-22 00:00:00.0 & \\
\hline 2007-08-21 21:00:00.0 & 16.0 \\
\hline 2007-08-21 18:00:00.0 & 16.5 \\
\hline 2007-08-21 15:00:00.0 & 17.0 \\
\hline 2007-08-21 12:00:00.0 & 16.5 \\
\hline 2007-08-21 09:00:00.0 & 12.7 \\
\hline 2007-08-21 06:00:00.0 & 15.5 \\
\hline 2007-08-21 03:00:00.0 & 13.1 \\
\hline 2007-08-21 00:00:00.0 & 9.1 \\
\hline 2007-08-20 21:00:00.0 & \\
\hline 2007-08-20 18:00:00.0 & 16.4 \\
\hline 2007-08-20 15:00:00.0 & 33.2 \\
\hline 2007-08-20 12:00:00.0 & 19.7 \\
\hline 2007-08-20 09:00:00.0 & \\
\hline 2007-08-20 06:00:00.0 & 24.0 \\
\hline 2007-08-20 03:00:00.0 & 27.0 \\
\hline 2007-08-20 00:00:00.0 & 24.0 \\
\hline 2007-08-19 21:00:00.0 & \\
\hline 2007-08-19 18:00:00.0 & 18.0 \\
\hline 2007-08-19 15:00:00.0 & 9.0 \\
\hline 2007-08-19 12:00:00.0 & 15.0 \\
\hline 2007-08-19 09:00:00.0 & 18.0 \\
\hline 2007-08-19 06:00:00.0 & 20.0 \\
\hline 2007-08-19 03:00:00.0 & 20.0 \\
\hline 2007-08-19 00:00:00.0 & 20.0 \\
\hline 2007-08-18 21:00:00.0 & 35.0 \\
\hline 2007-08-18 18:00:00.0 & 23.0 \\
\hline 2007-08-18 15:00:00.0 & 27.0 \\
\hline 2007-08-18 12:00:00.0 & 20.0 \\
\hline 2007-08-18 09:00:00.0 & \\
\hline 2007-08-18 06:00:00.0 & 26.0 \\
\hline 2007-08-18 03:00:00.0 & 30.0 \\
\hline 2007-08-18 00:00:00.0 & 25.0 \\
\hline 2007-08-17 21:00:00.0 & 29.8 \\
\hline 2007-08-17 18:00:00.0 & 33.2 \\
\hline 2007-08-17 15:00:00.0 & 31.0 \\
\hline 2007-08-17 12:00:00.0 & 43.2 \\
\hline 2007-08-17 09:00:00.0 & 37.0 \\
\hline 2007-08-17 06:00:00.0 & 33.8 \\
\hline
\end{tabular}

\begin{tabular}{|c|c|}
\hline DataHora & VelVentoMax \\
\hline 2007-08-17 03:00:00.0 & 31.1 \\
\hline 2007-08-17 00:00:00.0 & 28.1 \\
\hline 2007-08-16 21:00:00.0 & 32.0 \\
\hline 2007-08-16 18:00:00.0 & 34.9 \\
\hline 2007-08-16 15:00:00.0 & 31.0 \\
\hline 2007-08-16 12:00:00.0 & 28.6 \\
\hline 2007-08-16 09:00:00.0 & 26.1 \\
\hline 2007-08-16 06:00:00.0 & 22.4 \\
\hline 2007-08-16 03:00:00.0 & 13.3 \\
\hline 2007-08-16 00:00:00.0 & 12.6 \\
\hline 2007-08-15 21:00:00.0 & 22.4 \\
\hline 2007-08-15 18:00:00.0 & 34.3 \\
\hline 2007-08-15 15:00:00.0 & 36.9 \\
\hline 2007-08-15 12:00:00.0 & 33.3 \\
\hline 2007-08-15 09:00:00.0 & 31.1 \\
\hline 2007-08-15 06:00:00.0 & 33.5 \\
\hline 2007-08-15 03:00:00.0 & 33.6 \\
\hline 2007-08-15 00:00:00.0 & 30.8 \\
\hline 2007-08-14 21:00:00.0 & 32.1 \\
\hline 2007-08-14 18:00:00.0 & 35.6 \\
\hline 2007-08-14 15:00:00.0 & 32.7 \\
\hline 2007-08-14 12:00:00.0 & 26.5 \\
\hline 2007-08-14 09:00:00.0 & \\
\hline 2007-08-14 06:00:00.0 & 21.9 \\
\hline 2007-08-14 03:00:00.0 & 20.7 \\
\hline 2007-08-14 00:00:00.0 & 17.3 \\
\hline 2007-08-13 21:00:00.0 & 24.4 \\
\hline 2007-08-13 18:00:00.0 & 25.7 \\
\hline 2007-08-13 15:00:00.0 & 28.5 \\
\hline 2007-08-13 12:00:00.0 & 29.2 \\
\hline 2007-08-13 09:00:00.0 & 26.4 \\
\hline 2007-08-13 06:00:00.0 & 22.4 \\
\hline 2007-08-13 03:00:00.0 & 22.2 \\
\hline 2007-08-13 00:00:00.0 & \\
\hline 2007-08-12 21:00:00.0 & 18.6 \\
\hline 2007-08-12 18:00:00.0 & 21.7 \\
\hline 2007-08-12 15:00:00.0 & 26.9 \\
\hline 2007-08-12 12:00:00.0 & 27.0 \\
\hline 2007-08-12 09:00:00.0 & 23.0 \\
\hline 2007-08-12 06:00:00.0 & 25.3 \\
\hline 2007-08-12 03:00:00.0 & 19.8 \\
\hline 2007-08-12 00:00:00.0 & \\
\hline 2007-08-11 21:00:00.0 & 14.5 \\
\hline 2007-08-11 18:00:00.0 & 10.1 \\
\hline 2007-08-11 15:00:00.0 & 11.7 \\
\hline 2007-08-11 12:00:00.0 & 6.4 \\
\hline 2007-08-11 09:00:00.0 & 9.3 \\
\hline 2007-08-11 06:00:00.0 & 8.7 \\
\hline 2007-08-11 03:00:00.0 & \\
\hline 2007-08-11 00:00:00.0 & 24.1 \\
\hline 2007-08-10 21:00:00.0 & 27.6 \\
\hline 2007-08-10 18:00:00.0 & 27.2 \\
\hline 2007-08-10 15:00:00.0 & 29.0 \\
\hline 2007-08-10 12:00:00.0 & 31.4 \\
\hline 2007-08-10 09:00:00.0 & 34.6 \\
\hline 2007-08-10 06:00:00.0 & 28.8 \\
\hline 2007-08-10 03:00:00.0 & 23.4 \\
\hline 2007-08-10 00:00:00.0 & 21.4 \\
\hline 2007-08-09 21:00:00.0 & 31.4 \\
\hline 2007-08-09 18:00:00.0 & 31.6 \\
\hline 2007-08-09 15:00:00.0 & 24.5 \\
\hline 2007-08-09 12:00:00.0 & 27.0 \\
\hline 2007-08-09 09:00:00.0 & 25.0 \\
\hline 2007-08-09 06:00:00.0 & 26.2 \\
\hline 2007-08-09 03:00:00.0 & 24.6 \\
\hline 2007-08-09 00:00:00.0 & 20.5 \\
\hline 2007-08-08 21:00:00.0 & 19.3 \\
\hline 2007-08-08 18:00:00.0 & 19.4 \\
\hline 2007-08-08 15:00:00.0 & 21.7 \\
\hline
\end{tabular}

\begin{tabular}{|c|c|}
\hline DataHora & VelVentoMax \\
\hline \multicolumn{2}{|l|}{ 2007-08-08 12:00:00.0 } \\
\hline 2007-08-08 09:00:00.0 & 16.7 \\
\hline 2007-08-08 06:00:00.0 & 11.7 \\
\hline 2007-08-08 03:00:00.0 & 9.4 \\
\hline 2007-08-08 00:00:00.0 & 9.8 \\
\hline 2007-08-07 21:00:00.0 & 19.1 \\
\hline 2007-08-07 18:00:00.0 & 23.4 \\
\hline \multicolumn{2}{|l|}{ 2007-08-07 15:00:00.0 } \\
\hline 2007-08-07 12:00:00.0 & 14.7 \\
\hline 2007-08-07 09:00:00.0 & 17.5 \\
\hline 2007-08-07 06:00:00.0 & 21.0 \\
\hline 2007-08-07 03:00:00.0 & 28.2 \\
\hline 2007-08-07 00:00:00.0 & 31.8 \\
\hline 2007-08-06 21:00:00.0 & 35.5 \\
\hline 2007-08-06 18:00:00.0 & 29.3 \\
\hline 2007-08-06 15:00:00.0 & 24.2 \\
\hline 2007-08-06 12:00:00.0 & 29.8 \\
\hline 2007-08-06 09:00:00.0 & 23.8 \\
\hline 2007-08-06 06:00:00.0 & 25.5 \\
\hline 2007-08-06 03:00:00.0 & 23.6 \\
\hline 2007-08-06 00:00:00.0 & 22.9 \\
\hline 2007-08-05 21:00:00.0 & 20.2 \\
\hline 2007-08-05 18:00:00.0 & 19.0 \\
\hline 2007-08-05 15:00:00.0 & 24.4 \\
\hline 2007-08-05 12:00:00.0 & 22.2 \\
\hline 2007-08-05 09:00:00.0 & 20.2 \\
\hline 2007-08-05 06:00:00.0 & 8.4 \\
\hline 2007-08-05 03:00:00.0 & 13.4 \\
\hline 2007-08-05 00:00:00.0 & 14.6 \\
\hline 2007-08-04 21:00:00.0 & 31.7 \\
\hline 2007-08-04 18:00:00.0 & 15.0 \\
\hline \multicolumn{2}{|l|}{ 2007-08-04 15:00:00.0 } \\
\hline 2007-08-04 12:00:00.0 & 17.1 \\
\hline 2007-08-04 09:00:00.0 & 18.9 \\
\hline 2007-08-04 06:00:00.0 & 18.9 \\
\hline 2007-08-04 03:00:00.0 & 19.6 \\
\hline 2007-08-04 00:00:00.0 & 23.0 \\
\hline 2007-08-03 21:00:00.0 & 24.2 \\
\hline 2007-08-03 18:00:00.0 & 23.4 \\
\hline 2007-08-03 15:00:00.0 & 18.0 \\
\hline 2007-08-03 12:00:00.0 & 22.8 \\
\hline 2007-08-03 09:00:00.0 & 22.6 \\
\hline 2007-08-03 06:00:00.0 & 23.5 \\
\hline 2007-08-03 03:00:00.0 & 26.0 \\
\hline 2007-08-03 00:00:00.0 & 27.1 \\
\hline 2007-08-02 21:00:00.0 & 30.1 \\
\hline 2007-08-02 18:00:00.0 & 26.7 \\
\hline 2007-08-02 15:00:00.0 & 25.2 \\
\hline 2007-08-02 12:00:00.0 & 28.1 \\
\hline 2007-08-02 09:00:00.0 & 25.0 \\
\hline 2007-08-02 06:00:00.0 & 21.5 \\
\hline \multicolumn{2}{|l|}{ 2007-08-02 03:00:00.0 } \\
\hline 2007-08-02 00:00:00.0 & 8.1 \\
\hline 2007-08-01 21:00:00.0 & 32.1 \\
\hline \multicolumn{2}{|l|}{ 2007-08-01 18:00:00.0 } \\
\hline 2007-08-01 15:00:00.0 & 20.3 \\
\hline 2007-08-01 12:00:00.0 & 18.7 \\
\hline 2007-08-01 09:00:00.0 & 17.9 \\
\hline \multicolumn{2}{|l|}{ 2007-08-01 06:00:00.0 } \\
\hline 2007-08-01 03:00:00.0 & 16.0 \\
\hline 2007-08-01 00:00:00.0 & 12.6 \\
\hline 2007-07-31 21:00:00.0 & 14.0 \\
\hline \multicolumn{2}{|l|}{$2007-07-31$ 18:00:00.0 } \\
\hline 2007-07-31 15:00:00.0 & 18.4 \\
\hline \multicolumn{2}{|l|}{ 2007-07-31 12:00:00.0 } \\
\hline 2007-07-31 09:00:00.0 & 14.3 \\
\hline \multicolumn{2}{|l|}{ 2007-07-31 06:00:00.0 } \\
\hline 2007-07-31 03:00:00.0 & 10.1 \\
\hline 2007-07-31 00:00:00.0 & 12.7 \\
\hline
\end{tabular}




\section{ANEXO 01 - RAJADAS DE VENTO REGISTRADAS EM SÃO MARTINHO DA SERRA / RS}

\begin{tabular}{|c|c|}
\hline DataHora & VelVentoMax \\
\hline 2007-07-30 21:00:00.0 & 11.9 \\
\hline 2007-07-30 18:00:00.0 & 11.8 \\
\hline 2007-07-30 15:00:00.0 & 23.9 \\
\hline 2007-07-30 12:00:00.0 & 25.0 \\
\hline 2007-07-30 09:00:00.0 & 23.6 \\
\hline 2007-07-30 06:00:00.0 & 22.8 \\
\hline 2007-07-30 03:00:00.0 & 18.7 \\
\hline 2007-07-30 00:00:00.0 & 18.4 \\
\hline 2007-07-29 21:00:00.0 & 17.6 \\
\hline 2007-07-29 18:00:00.0 & 25.2 \\
\hline 2007-07-29 15:00:00.0 & 24.4 \\
\hline 2007-07-29 12:00:00.0 & 17.0 \\
\hline 2007-07-29 09:00:00.0 & 14.4 \\
\hline 2007-07-29 06:00:00.0 & 13.0 \\
\hline 2007-07-29 03:00:00.0 & 13.2 \\
\hline 2007-07-29 00:00:00.0 & 12.7 \\
\hline 2007-07-28 21:00:00.0 & \\
\hline 2007-07-28 18:00:00.0 & 25.5 \\
\hline 2007-07-28 15:00:00.0 & 26.5 \\
\hline 2007-07-28 12:00:00.0 & 23.0 \\
\hline 2007-07-28 09:00:00.0 & 22.9 \\
\hline 2007-07-28 06:00:00.0 & 23.8 \\
\hline 2007-07-28 03:00:00.0 & 20.8 \\
\hline 2007-07-28 00:00:00.0 & 10.8 \\
\hline 2007-07-27 21:00:00.0 & 16.9 \\
\hline 2007-07-27 18:00:00.0 & 17.7 \\
\hline 2007-07-27 15:00:00.0 & 15.0 \\
\hline 2007-07-27 12:00:00.0 & 10.3 \\
\hline 2007-07-27 09:00:00.0 & 10.4 \\
\hline 2007-07-27 06:00:00.0 & 40.0 \\
\hline 2007-07-27 03:00:00.0 & 11.2 \\
\hline 2007-07-27 00:00:00.0 & 11.5 \\
\hline 2007-07-26 21:00:00.0 & 17.9 \\
\hline 2007-07-26 18:00:00.0 & 20.3 \\
\hline 2007-07-26 15:00:00.0 & 18.8 \\
\hline 2007-07-26 12:00:00.0 & 13.5 \\
\hline 2007-07-26 09:00:00.0 & 16.0 \\
\hline 2007-07-26 06:00:00.0 & 17.4 \\
\hline 2007-07-26 03:00:00.0 & 14.8 \\
\hline 2007-07-26 00:00:00.0 & 11.2 \\
\hline 2007-07-25 21:00:00.0 & 28.9 \\
\hline 2007-07-25 18:00:00.0 & 26.1 \\
\hline 2007-07-25 15:00:00.0 & 26.1 \\
\hline 2007-07-25 12:00:00.0 & 17.1 \\
\hline 2007-07-25 09:00:00.0 & 12.9 \\
\hline 2007-07-25 06:00:00.0 & 7.9 \\
\hline 2007-07-25 03:00:00.0 & 13.7 \\
\hline 2007-07-25 00:00:00.0 & \\
\hline 2007-07-24 21:00:00.0 & \\
\hline 2007-07-24 18:00:00.0 & 21.1 \\
\hline 2007-07-24 15:00:00.0 & 24.7 \\
\hline 2007-07-24 12:00:00.0 & 21.0 \\
\hline 2007-07-24 09:00:00.0 & \\
\hline 2007-07-24 06:00:00.0 & 6.0 \\
\hline 2007-07-24 03:00:00.0 & 10.7 \\
\hline 2007-07-24 00:00:00.0 & 11.3 \\
\hline 2007-07-23 21:00:00.0 & \\
\hline 2007-07-23 18:00:00.0 & 24.7 \\
\hline 2007-07-23 15:00:00.0 & 19.4 \\
\hline 2007-07-23 12:00:00.0 & \\
\hline 2007-07-23 09:00:00.0 & \\
\hline 2007-07-23 06:00:00.0 & 11.5 \\
\hline 2007-07-23 03:00:00.0 & 27.3 \\
\hline 2007-07-23 00:00:00.0 & 33.6 \\
\hline 2007-07-22 21:00:00.0 & \\
\hline 2007-07-22 18:00:00.0 & 18.2 \\
\hline 2007-07-22 15:00:00.0 & 21.0 \\
\hline 2007-07-22 12:00:00.0 & \\
\hline 2007-07-22 09:00:00.0 & 23.7 \\
\hline
\end{tabular}

\begin{tabular}{|c|c|}
\hline DataHora & VelVentoMax \\
\hline 2007-07-22 06:00:00.0 & 23.0 \\
\hline 2007-07-22 03:00:00.0 & 23.3 \\
\hline 2007-07-22 00:00:00.0 & \\
\hline 2007-07-21 21:00:00.0 & 24.0 \\
\hline 2007-07-21 18:00:00.0 & 32.5 \\
\hline 2007-07-21 15:00:00.0 & 20.0 \\
\hline 2007-07-21 12:00:00.0 & \\
\hline 2007-07-21 09:00:00.0 & \\
\hline 2007-07-21 06:00:00.0 & 12.5 \\
\hline 2007-07-21 03:00:00.0 & 8.9 \\
\hline 2007-07-21 00:00:00.0 & 6.3 \\
\hline 2007-07-20 21:00:00.0 & 13.8 \\
\hline 2007-07-20 18:00:00.0 & 21.7 \\
\hline 2007-07-20 15:00:00.0 & 18.4 \\
\hline 2007-07-20 12:00:00.0 & \\
\hline 2007-07-20 09:00:00.0 & 8.7 \\
\hline 2007-07-20 06:00:00.0 & 22.0 \\
\hline 2007-07-20 03:00:00.0 & 20.2 \\
\hline 2007-07-20 00:00:00.0 & \\
\hline 2007-07-19 21:00:00.0 & 18.3 \\
\hline 2007-07-19 18:00:00.0 & 18.3 \\
\hline 2007-07-19 15:00:00.0 & 15.0 \\
\hline 2007-07-19 12:00:00.0 & \\
\hline 2007-07-19 09:00:00.0 & 8.9 \\
\hline 2007-07-19 06:00:00.0 & 5.8 \\
\hline 2007-07-19 03:00:00.0 & \\
\hline 2007-07-19 00:00:00.0 & \\
\hline 2007-07-18 21:00:00.0 & 11.2 \\
\hline 2007-07-18 18:00:00.0 & 15.6 \\
\hline 2007-07-18 15:00:00.0 & \\
\hline 2007-07-18 12:00:00.0 & \\
\hline 2007-07-18 09:00:00.0 & \\
\hline 2007-07-18 06:00:00.0 & 17.5 \\
\hline 2007-07-18 03:00:00.0 & 14.7 \\
\hline 2007-07-18 00:00:00.0 & 20.1 \\
\hline 2007-07-17 21:00:00.0 & 32.3 \\
\hline 2007-07-17 18:00:00.0 & 29.7 \\
\hline 2007-07-17 15:00:00.0 & \\
\hline 2007-07-17 12:00:00.0 & \\
\hline 2007-07-17 09:00:00.0 & 20.3 \\
\hline 2007-07-17 06:00:00.0 & 21.9 \\
\hline 2007-07-17 03:00:00.0 & 21.5 \\
\hline 2007-07-17 00:00:00.0 & 16.7 \\
\hline 2007-07-16 21:00:00.0 & 14.4 \\
\hline 2007-07-16 18:00:00.0 & 14.3 \\
\hline 2007-07-16 15:00:00.0 & 15.3 \\
\hline 2007-07-16 12:00:00.0 & 12.1 \\
\hline 2007-07-16 09:00:00.0 & 11.1 \\
\hline 2007-07-16 06:00:00.0 & 10.2 \\
\hline 2007-07-16 03:00:00.0 & 9.9 \\
\hline 2007-07-16 00:00:00.0 & 7.7 \\
\hline 2007-07-15 21:00:00.0 & 9.4 \\
\hline 2007-07-15 18:00:00.0 & 15.0 \\
\hline 2007-07-15 15:00:00.0 & 16.1 \\
\hline 2007-07-15 12:00:00.0 & \\
\hline 2007-07-15 09:00:00.0 & 14.5 \\
\hline 2007-07-15 06:00:00.0 & 14.0 \\
\hline 2007-07-15 03:00:00.0 & 11.9 \\
\hline 2007-07-15 00:00:00.0 & \\
\hline 2007-07-14 21:00:00.0 & 20.4 \\
\hline 2007-07-14 18:00:00.0 & 23.5 \\
\hline 2007-07-14 15:00:00.0 & 22.6 \\
\hline 2007-07-14 12:00:00.0 & \\
\hline 2007-07-14 09:00:00.0 & 12.4 \\
\hline 2007-07-14 06:00:00.0 & 11.2 \\
\hline 2007-07-14 03:00:00.0 & \\
\hline 2007-07-14 00:00:00.0 & 15.7 \\
\hline 2007-07-13 21:00:00.0 & 30.5 \\
\hline 2007-07-13 18:00:00.0 & 10.7 \\
\hline
\end{tabular}

\begin{tabular}{|c|c|}
\hline DataHora & VelVentoMax \\
\hline \multicolumn{2}{|l|}{ 2007-07-13 15:00:00.0 } \\
\hline 2007-07-13 12:00:00.0 & 15.7 \\
\hline 2007-07-13 09:00:00.0 & 18.9 \\
\hline 2007-07-13 06:00:00.0 & 19.2 \\
\hline 2007-07-13 03:00:00.0 & 20.4 \\
\hline \multicolumn{2}{|l|}{ 2007-07-13 00:00:00.0 } \\
\hline 2007-07-12 21:00:00.0 & 17.8 \\
\hline 2007-07-12 18:00:00.0 & 18.6 \\
\hline $2007-07-12$ 15:00:00.0 & 15.2 \\
\hline $2007-07-12$ 12:00:00.0 & 12.7 \\
\hline 2007-07-12 09:00:00.0 & 14.2 \\
\hline $2007-07-12$ 06:00:00.0 & 13.4 \\
\hline \multicolumn{2}{|l|}{ 2007-07-12 03:00:00.0 } \\
\hline 2007-07-12 00:00:00.0 & 8.6 \\
\hline 2007-07-11 21:00:00.0 & 9.1 \\
\hline 2007-07-11 18:00:00.0 & 10.5 \\
\hline 2007-07-11 15:00:00.0 & 11.2 \\
\hline 2007-07-11 12:00:00.0 & 10.3 \\
\hline 2007-07-11 09:00:00.0 & 17.0 \\
\hline 2007-07-11 06:00:00.0 & 15.2 \\
\hline 2007-07-11 03:00:00.0 & 21.5 \\
\hline \multicolumn{2}{|l|}{ 2007-07-11 00:00:00.0 } \\
\hline $2007-07-10$ 21:00:00.0 & 25.4 \\
\hline \multicolumn{2}{|l|}{$2007-07-1018: 00: 00.0$} \\
\hline $2007-07-1015: 00: 00.0$ & 20.3 \\
\hline 2007-07-10 12:00:00.0 & 15.3 \\
\hline 2007-07-10 09:00:00.0 & 8.1 \\
\hline 2007-07-10 06:00:00.0 & 9.8 \\
\hline \multicolumn{2}{|l|}{ 2007-07-10 03:00:00.0 } \\
\hline \multicolumn{2}{|l|}{ 2007-07-10 00:00:00.0 } \\
\hline 2007-07-09 21:00:00.0 & 25.6 \\
\hline 2007-07-09 18:00:00.0 & 23.9 \\
\hline 2007-07-09 15:00:00.0 & 23.8 \\
\hline 2007-07-09 12:00:00.0 & 26.7 \\
\hline 2007-07-09 09:00:00.0 & 28.1 \\
\hline 2007-07-09 06:00:00.0 & 24.9 \\
\hline 2007-07-09 03:00:00.0 & 25.4 \\
\hline \multicolumn{2}{|l|}{ 2007-07-09 00:00:00.0 } \\
\hline 2007-07-08 21:00:00.0 & 29.7 \\
\hline 2007-07-08 18:00:00.0 & 29.8 \\
\hline 2007-07-08 15:00:00.0 & 35.6 \\
\hline 2007-07-08 12:00:00.0 & 35.6 \\
\hline 2007-07-08 09:00:00.0 & 34.6 \\
\hline 2007-07-08 06:00:00.0 & 30.6 \\
\hline 2007-07-08 03:00:00.0 & 28.0 \\
\hline 2007-07-08 00:00:00.0 & 17.7 \\
\hline 2007-07-07 21:00:00.0 & 23.1 \\
\hline \multicolumn{2}{|l|}{ 2007-07-07 18:00:00.0 } \\
\hline $2007-07-07$ 15:00:00.0 & 28.8 \\
\hline 2007-07-07 12:00:00.0 & 21.9 \\
\hline 2007-07-07 09:00:00.0 & 14.3 \\
\hline \multicolumn{2}{|l|}{ 2007-07-07 06:00:00.0 } \\
\hline 2007-07-07 03:00:00.0 & 15.6 \\
\hline 2007-07-07 00:00:00.0 & 12.8 \\
\hline 2007-07-06 21:00:00.0 & 16.1 \\
\hline 2007-07-06 18:00:00.0 & 21.4 \\
\hline \multicolumn{2}{|l|}{$2007-07-06$ 15:00:00.0 } \\
\hline 2007-07-06 12:00:00.0 & 18.9 \\
\hline 2007-07-06 09:00:00.0 & 25.5 \\
\hline 2007-07-06 06:00:00.0 & 28.1 \\
\hline 2007-07-06 03:00:00.0 & 30.2 \\
\hline 2007-07-06 00:00:00.0 & 27.1 \\
\hline 2007-07-05 21:00:00.0 & 26.9 \\
\hline $2007-07-05$ 18:00:00.0 & 32.2 \\
\hline 2007-07-05 15:00:00.0 & \\
\hline 2007-07-05 12:00:00.0 & \\
\hline 2007-07-05 09:00:00.0 & 22.9 \\
\hline 2007-07-05 06:00:00.0 & \\
\hline 2007-07-05 03:00:00.0 & 21.3 \\
\hline
\end{tabular}




\section{ANEXO 01 - RAJADAS DE VENTO REGISTRADAS EM SÃO MARTINHO DA SERRA / RS}

\begin{tabular}{|c|c|}
\hline DataHora & VelVentoMax \\
\hline 2007-07-05 00:00:00.0 & 16.4 \\
\hline 2007-07-04 21:00:00.0 & 25.2 \\
\hline 2007-07-04 18:00:00.0 & 28.5 \\
\hline 2007-07-04 15:00:00.0 & 25.9 \\
\hline 2007-07-04 12:00:00.0 & 19.5 \\
\hline 2007-07-04 09:00:00.0 & 19.2 \\
\hline 2007-07-04 06:00:00.0 & \\
\hline 2007-07-04 03:00:00.0 & \\
\hline 2007-07-04 00:00:00.0 & 16.9 \\
\hline 2007-07-03 21:00:00.0 & 19.5 \\
\hline 2007-07-03 18:00:00.0 & 21.4 \\
\hline 2007-07-03 15:00:00.0 & 18.3 \\
\hline 2007-07-03 12:00:00.0 & 12.6 \\
\hline 2007-07-03 09:00:00.0 & 12.4 \\
\hline 2007-07-03 06:00:00.0 & \\
\hline 2007-07-03 03:00:00.0 & 14.0 \\
\hline 2007-07-03 00:00:00.0 & 11.1 \\
\hline 2007-07-02 21:00:00.0 & 27.1 \\
\hline 2007-07-02 18:00:00.0 & 12.0 \\
\hline 2007-07-02 15:00:00.0 & 11.0 \\
\hline 2007-07-02 12:00:00.0 & 17.8 \\
\hline 2007-07-02 09:00:00.0 & \\
\hline 2007-07-02 06:00:00.0 & 17.3 \\
\hline 2007-07-02 03:00:00.0 & 18.3 \\
\hline 2007-07-02 00:00:00.0 & 10.3 \\
\hline 2007-07-01 21:00:00.0 & 30.9 \\
\hline 2007-07-01 18:00:00.0 & 9.7 \\
\hline 2007-07-01 15:00:00.0 & 9.1 \\
\hline 2007-07-01 12:00:00.0 & 8.5 \\
\hline 2007-07-01 09:00:00.0 & 26.4 \\
\hline 2007-07-01 06:00:00.0 & 31.2 \\
\hline 2007-07-01 03:00:00.0 & 5.6 \\
\hline 2007-07-01 00:00:00.0 & \\
\hline 2007-06-30 21:00:00.0 & 11.8 \\
\hline 2007-06-30 18:00:00.0 & 11.9 \\
\hline 2007-06-30 15:00:00.0 & 31.2 \\
\hline 2007-06-30 12:00:00.0 & 10.6 \\
\hline 2007-06-30 09:00:00.0 & 9.5 \\
\hline 2007-06-30 06:00:00.0 & 6.6 \\
\hline 2007-06-30 03:00:00.0 & 17.8 \\
\hline 2007-06-30 00:00:00.0 & 6.0 \\
\hline 2007-06-29 21:00:00.0 & 12.8 \\
\hline 2007-06-29 18:00:00.0 & 11.6 \\
\hline 2007-06-29 15:00:00.0 & 9.4 \\
\hline 2007-06-29 12:00:00.0 & 6.1 \\
\hline 2007-06-29 09:00:00.0 & 2.5 \\
\hline 2007-06-29 06:00:00.0 & 5.4 \\
\hline 2007-06-29 03:00:00.0 & 6.4 \\
\hline 2007-06-29 00:00:00.0 & 9.1 \\
\hline 2007-06-28 21:00:00.0 & 13.9 \\
\hline 2007-06-28 18:00:00.0 & 9.7 \\
\hline 2007-06-28 15:00:00.0 & 8.9 \\
\hline 2007-06-28 12:00:00.0 & 11.0 \\
\hline 2007-06-28 09:00:00.0 & 12.2 \\
\hline 2007-06-28 06:00:00.0 & 7.2 \\
\hline 2007-06-28 03:00:00.0 & 7.1 \\
\hline 2007-06-28 00:00:00.0 & 8.7 \\
\hline 2007-06-27 21:00:00.0 & 13.9 \\
\hline 2007-06-27 18:00:00.0 & 17.3 \\
\hline 2007-06-27 15:00:00.0 & 18.6 \\
\hline 2007-06-27 12:00:00.0 & 14.4 \\
\hline 2007-06-27 09:00:00.0 & 19.9 \\
\hline 2007-06-27 06:00:00.0 & 16.8 \\
\hline 2007-06-27 03:00:00.0 & 12.3 \\
\hline 2007-06-27 00:00:00.0 & \\
\hline 2007-06-26 21:00:00.0 & 12.3 \\
\hline 2007-06-26 18:00:00.0 & \\
\hline 2007-06-26 15:00:00.0 & 16.8 \\
\hline 2007-06-26 12:00:00.0 & 13.6 \\
\hline
\end{tabular}

\begin{tabular}{|c|c|}
\hline DataHora & VelVentoMax \\
\hline 2007-06-26 09:00:00.0 & 10.9 \\
\hline 2007-06-26 06:00:00.0 & 11.3 \\
\hline 2007-06-26 03:00:00.0 & 18.8 \\
\hline 2007-06-26 00:00:00.0 & \\
\hline 2007-06-25 21:00:00.0 & 16.4 \\
\hline 2007-06-25 18:00:00.0 & 18.7 \\
\hline 2007-06-25 15:00:00.0 & 19.7 \\
\hline 2007-06-25 12:00:00.0 & 15.9 \\
\hline 2007-06-25 09:00:00.0 & \\
\hline 2007-06-25 06:00:00.0 & 8.2 \\
\hline 2007-06-25 03:00:00.0 & 7.3 \\
\hline 2007-06-25 00:00:00.0 & 7.8 \\
\hline 2007-06-24 21:00:00.0 & 2.4 \\
\hline 2007-06-24 18:00:00.0 & 19.3 \\
\hline 2007-06-24 15:00:00.0 & 20.4 \\
\hline 2007-06-24 12:00:00.0 & 19.7 \\
\hline 2007-06-24 09:00:00.0 & 15.5 \\
\hline 2007-06-24 06:00:00.0 & 21.0 \\
\hline 2007-06-24 03:00:00.0 & 19.8 \\
\hline 2007-06-24 00:00:00.0 & 20.4 \\
\hline 2007-06-23 21:00:00.0 & 1.7 \\
\hline 2007-06-23 18:00:00.0 & 9.2 \\
\hline 2007-06-23 15:00:00.0 & 20.2 \\
\hline 2007-06-23 12:00:00.0 & 15.6 \\
\hline 2007-06-23 09:00:00.0 & 29.7 \\
\hline 2007-06-23 06:00:00.0 & 30.7 \\
\hline 2007-06-23 03:00:00.0 & 27.5 \\
\hline 2007-06-23 00:00:00.0 & 27.4 \\
\hline 2007-06-22 21:00:00.0 & 27.3 \\
\hline 2007-06-22 18:00:00.0 & 35.3 \\
\hline 2007-06-22 15:00:00.0 & 35.0 \\
\hline 2007-06-22 12:00:00.0 & 37.1 \\
\hline 2007-06-22 09:00:00.0 & 22.6 \\
\hline 2007-06-22 06:00:00.0 & 18.1 \\
\hline 2007-06-22 03:00:00.0 & 18.1 \\
\hline 2007-06-22 00:00:00.0 & 17.6 \\
\hline 2007-06-21 21:00:00.0 & 16.4 \\
\hline 2007-06-21 18:00:00.0 & 22.8 \\
\hline 2007-06-21 15:00:00.0 & 25.7 \\
\hline 2007-06-21 12:00:00.0 & 30.7 \\
\hline 2007-06-21 09:00:00.0 & 25.5 \\
\hline 2007-06-21 06:00:00.0 & 25.7 \\
\hline 2007-06-21 03:00:00.0 & 25.8 \\
\hline 2007-06-21 00:00:00.0 & \\
\hline 2007-06-20 21:00:00.0 & 29.4 \\
\hline 2007-06-20 18:00:00.0 & 29.9 \\
\hline 2007-06-20 15:00:00.0 & 30.7 \\
\hline 2007-06-20 12:00:00.0 & 22.6 \\
\hline 2007-06-20 09:00:00.0 & 19.5 \\
\hline 2007-06-20 06:00:00.0 & 17.5 \\
\hline 2007-06-20 03:00:00.0 & 19.9 \\
\hline 2007-06-20 00:00:00.0 & 17.9 \\
\hline 2007-06-19 21:00:00.0 & 16.0 \\
\hline 2007-06-19 18:00:00.0 & 17.0 \\
\hline 2007-06-19 15:00:00.0 & 15.1 \\
\hline 2007-06-19 12:00:00.0 & 18.5 \\
\hline 2007-06-19 09:00:00.0 & 20.5 \\
\hline 2007-06-19 06:00:00.0 & \\
\hline 2007-06-19 03:00:00.0 & 18.9 \\
\hline 2007-06-19 00:00:00.0 & 17.0 \\
\hline 2007-06-18 21:00:00.0 & 15.2 \\
\hline 2007-06-18 18:00:00.0 & 35.4 \\
\hline 2007-06-18 15:00:00.0 & 30.0 \\
\hline 2007-06-18 12:00:00.0 & 4.0 \\
\hline 2007-06-18 09:00:00.0 & 6.8 \\
\hline 2007-06-18 06:00:00.0 & 12.7 \\
\hline 2007-06-18 03:00:00.0 & 10.4 \\
\hline 2007-06-18 00:00:00.0 & 9.2 \\
\hline 2007-06-17 21:00:00.0 & 11.3 \\
\hline
\end{tabular}

\begin{tabular}{|c|c|}
\hline DataHora & VelVentoMax \\
\hline 2007-06-17 18:00:00.0 & 16.3 \\
\hline 2007-06-17 15:00:00.0 & 16.2 \\
\hline 2007-06-17 12:00:00.0 & 15.6 \\
\hline 2007-06-17 09:00:00.0 & 15.9 \\
\hline 2007-06-17 06:00:00.0 & 12.8 \\
\hline 2007-06-17 03:00:00.0 & 11.7 \\
\hline 2007-06-17 00:00:00.0 & 10.2 \\
\hline 2007-06-16 21:00:00.0 & 35.9 \\
\hline 2007-06-16 18:00:00.0 & 9.4 \\
\hline 2007-06-16 15:00:00.0 & 11.2 \\
\hline 2007-06-16 12:00:00.0 & 10.5 \\
\hline 2007-06-16 09:00:00.0 & 9.0 \\
\hline 2007-06-16 06:00:00.0 & 15.8 \\
\hline 2007-06-16 03:00:00.0 & 25.3 \\
\hline 2007-06-16 00:00:00.0 & 27.7 \\
\hline 2007-06-15 21:00:00.0 & 24.6 \\
\hline 2007-06-15 18:00:00.0 & 40.8 \\
\hline 2007-06-15 15:00:00.0 & 30.2 \\
\hline 2007-06-15 12:00:00.0 & 30.8 \\
\hline 2007-06-15 09:00:00.0 & 32.1 \\
\hline 2007-06-15 06:00:00.0 & 30.1 \\
\hline 2007-06-15 03:00:00.0 & 19.5 \\
\hline 2007-06-15 00:00:00.0 & 17.3 \\
\hline 2007-06-14 21:00:00.0 & 13.5 \\
\hline 2007-06-14 18:00:00.0 & 13.9 \\
\hline 2007-06-14 15:00:00.0 & 11.2 \\
\hline 2007-06-14 12:00:00.0 & 7.0 \\
\hline 2007-06-14 09:00:00.0 & 9.0 \\
\hline 2007-06-14 06:00:00.0 & 15.0 \\
\hline 2007-06-14 03:00:00.0 & 16.0 \\
\hline 2007-06-14 00:00:00.0 & \\
\hline 2007-06-13 21:00:00.0 & 18.0 \\
\hline 2007-06-13 18:00:00.0 & 26.0 \\
\hline 2007-06-13 15:00:00.0 & 20.0 \\
\hline 2007-06-13 12:00:00.0 & 20.0 \\
\hline 2007-06-13 09:00:00.0 & 22.0 \\
\hline 2007-06-13 06:00:00.0 & 23.5 \\
\hline 2007-06-13 03:00:00.0 & 21.2 \\
\hline 2007-06-13 00:00:00.0 & 15.4 \\
\hline 2007-06-12 21:00:00.0 & 14.2 \\
\hline 2007-06-12 18:00:00.0 & 17.4 \\
\hline 2007-06-12 15:00:00.0 & 15.3 \\
\hline 2007-06-12 12:00:00.0 & 10.4 \\
\hline 2007-06-12 09:00:00.0 & 9.6 \\
\hline 2007-06-12 06:00:00.0 & 12.9 \\
\hline 2007-06-12 03:00:00.0 & 11.2 \\
\hline 2007-06-12 00:00:00.0 & 19.3 \\
\hline 2007-06-11 21:00:00.0 & 18.4 \\
\hline 2007-06-11 18:00:00.0 & 26.6 \\
\hline 2007-06-11 15:00:00.0 & 16.7 \\
\hline 2007-06-11 12:00:00.0 & 18.3 \\
\hline 2007-06-11 09:00:00.0 & 18.9 \\
\hline 2007-06-11 06:00:00.0 & 20.3 \\
\hline 2007-06-11 03:00:00.0 & 17.8 \\
\hline 2007-06-11 00:00:00.0 & 19.7 \\
\hline 2007-06-10 21:00:00.0 & 28.7 \\
\hline 2007-06-10 18:00:00.0 & 18.2 \\
\hline 2007-06-10 15:00:00.0 & 16.6 \\
\hline 2007-06-10 12:00:00.0 & 10.3 \\
\hline 2007-06-10 09:00:00.0 & 11.0 \\
\hline 2007-06-10 06:00:00.0 & 20.1 \\
\hline 2007-06-10 03:00:00.0 & 36.8 \\
\hline 2007-06-10 00:00:00.0 & 8.8 \\
\hline 2007-06-09 21:00:00.0 & 21.3 \\
\hline 2007-06-09 18:00:00.0 & 17.8 \\
\hline 2007-06-09 15:00:00.0 & 20.3 \\
\hline 2007-06-09 12:00:00.0 & 19.4 \\
\hline 2007-06-09 09:00:00.0 & 14.9 \\
\hline 2007-06-09 06:00:00.0 & 19.5 \\
\hline
\end{tabular}




\section{ANEXO 01 - RAJADAS DE VENTO REGISTRADAS EM SÃO MARTINHO DA SERRA / RS}

\begin{tabular}{|c|c|}
\hline DataHora & VelVentoMax \\
\hline 2007-06-09 03:00:00.0 & 19.6 \\
\hline 2007-06-09 00:00:00.0 & 20.8 \\
\hline 2007-06-08 21:00:00.0 & 17.1 \\
\hline 2007-06-08 18:00:00.0 & 18.5 \\
\hline 2007-06-08 15:00:00.0 & 19.3 \\
\hline 2007-06-08 12:00:00.0 & 20.3 \\
\hline 2007-06-08 09:00:00.0 & 18.2 \\
\hline 2007-06-08 06:00:00.0 & \\
\hline 2007-06-08 03:00:00.0 & 10.4 \\
\hline 2007-06-08 00:00:00.0 & 10.0 \\
\hline 2007-06-07 21:00:00.0 & 32.7 \\
\hline 2007-06-07 18:00:00.0 & 13.4 \\
\hline 2007-06-07 15:00:00.0 & 10.7 \\
\hline 2007-06-07 12:00:00.0 & 11.0 \\
\hline 2007-06-07 09:00:00.0 & 9.4 \\
\hline 2007-06-07 06:00:00.0 & \\
\hline 2007-06-07 03:00:00.0 & 12.3 \\
\hline 2007-06-07 00:00:00.0 & 12.4 \\
\hline 2007-06-06 21:00:00.0 & 27.1 \\
\hline 2007-06-06 18:00:00.0 & 15.3 \\
\hline 2007-06-06 15:00:00.0 & 11.1 \\
\hline 2007-06-06 12:00:00.0 & 14.4 \\
\hline 2007-06-06 09:00:00.0 & 14.0 \\
\hline 2007-06-06 06:00:00.0 & 11.6 \\
\hline 2007-06-06 03:00:00.0 & 7.7 \\
\hline 2007-06-06 00:00:00.0 & 9.5 \\
\hline 2007-06-05 21:00:00.0 & 13.0 \\
\hline 2007-06-05 18:00:00.0 & 13.3 \\
\hline 2007-06-05 15:00:00.0 & 10.7 \\
\hline 2007-06-05 12:00:00.0 & 29.4 \\
\hline 2007-06-05 09:00:00.0 & \\
\hline 2007-06-05 06:00:00.0 & 6.9 \\
\hline 2007-06-05 03:00:00.0 & 6.7 \\
\hline 2007-06-05 00:00:00.0 & 6.9 \\
\hline 2007-06-04 21:00:00.0 & \\
\hline 2007-06-04 18:00:00.0 & \\
\hline 2007-06-04 15:00:00.0 & \\
\hline 2007-06-04 12:00:00.0 & 10.0 \\
\hline 2007-06-04 09:00:00.0 & 9.0 \\
\hline 2007-06-04 06:00:00.0 & \\
\hline 2007-06-04 03:00:00.0 & 9.4 \\
\hline 2007-06-04 00:00:00.0 & 12.4 \\
\hline 2007-06-03 21:00:00.0 & \\
\hline 2007-06-03 18:00:00.0 & 26.0 \\
\hline 2007-06-03 15:00:00.0 & 20.9 \\
\hline 2007-06-03 12:00:00.0 & 13.6 \\
\hline 2007-06-03 09:00:00.0 & 14.0 \\
\hline 2007-06-03 06:00:00.0 & 15.2 \\
\hline 2007-06-03 03:00:00.0 & 16.3 \\
\hline 2007-06-03 00:00:00.0 & 14.3 \\
\hline 2007-06-02 21:00:00.0 & 18.9 \\
\hline 2007-06-02 18:00:00.0 & 20.3 \\
\hline 2007-06-02 15:00:00.0 & 15.6 \\
\hline 2007-06-02 12:00:00.0 & 8.3 \\
\hline 2007-06-02 09:00:00.0 & 7.0 \\
\hline 2007-06-02 06:00:00.0 & 8.8 \\
\hline 2007-06-02 03:00:00.0 & 7.8 \\
\hline 2007-06-02 00:00:00.0 & 10.3 \\
\hline 2007-06-01 21:00:00.0 & 18.6 \\
\hline 2007-06-01 18:00:00.0 & 19.6 \\
\hline 2007-06-01 15:00:00.0 & 12.8 \\
\hline 2007-06-01 12:00:00.0 & 11.9 \\
\hline 2007-06-01 09:00:00.0 & 12.1 \\
\hline 2007-06-01 06:00:00.0 & 9.7 \\
\hline 2007-06-01 03:00:00.0 & 7.6 \\
\hline 2007-06-01 00:00:00.0 & 6.9 \\
\hline 2007-05-31 21:00:00.0 & 14.1 \\
\hline 2007-05-31 18:00:00.0 & 24.0 \\
\hline 2007-05-31 15:00:00.0 & 24.8 \\
\hline
\end{tabular}

\begin{tabular}{|c|c|}
\hline DataHora & VelVentoMax \\
\hline 2007-05-31 12:00:00.0 & 24.8 \\
\hline 2007-05-31 09:00:00.0 & 20.9 \\
\hline 2007-05-31 06:00:00.0 & 18.4 \\
\hline 2007-05-31 03:00:00.0 & 16.2 \\
\hline 2007-05-31 00:00:00.0 & \\
\hline 2007-05-30 21:00:00.0 & 13.5 \\
\hline 2007-05-30 18:00:00.0 & 17.1 \\
\hline 2007-05-30 15:00:00.0 & 13.4 \\
\hline 2007-05-30 12:00:00.0 & 13.1 \\
\hline 2007-05-30 09:00:00.0 & 4.7 \\
\hline 2007-05-30 06:00:00.0 & 10.2 \\
\hline 2007-05-30 03:00:00.0 & 10.9 \\
\hline 2007-05-30 00:00:00.0 & 14.5 \\
\hline 2007-05-29 21:00:00.0 & 19.4 \\
\hline 2007-05-29 18:00:00.0 & 22.9 \\
\hline 2007-05-29 15:00:00.0 & 19.5 \\
\hline 2007-05-29 12:00:00.0 & 15.7 \\
\hline 2007-05-29 09:00:00.0 & 15.7 \\
\hline 2007-05-29 06:00:00.0 & 15.1 \\
\hline 2007-05-29 03:00:00.0 & 12.1 \\
\hline 2007-05-29 00:00:00.0 & 12.2 \\
\hline 2007-05-28 21:00:00.0 & 21.3 \\
\hline 2007-05-28 18:00:00.0 & 20.5 \\
\hline 2007-05-28 15:00:00.0 & 20.4 \\
\hline 2007-05-28 12:00:00.0 & 19.0 \\
\hline 2007-05-28 09:00:00.0 & 16.7 \\
\hline 2007-05-28 06:00:00.0 & 14.9 \\
\hline 2007-05-28 03:00:00.0 & 38.4 \\
\hline 2007-05-28 00:00:00.0 & 12.3 \\
\hline 2007-05-27 21:00:00.0 & 32.9 \\
\hline 2007-05-27 18:00:00.0 & 14.1 \\
\hline 2007-05-27 15:00:00.0 & 11.2 \\
\hline 2007-05-27 12:00:00.0 & 13.5 \\
\hline 2007-05-27 09:00:00.0 & 11.5 \\
\hline 2007-05-27 06:00:00.0 & 12.0 \\
\hline 2007-05-27 03:00:00.0 & 10.8 \\
\hline 2007-05-27 00:00:00.0 & 8.2 \\
\hline 2007-05-26 21:00:00.0 & 11.0 \\
\hline 2007-05-26 18:00:00.0 & 15.8 \\
\hline 2007-05-26 15:00:00.0 & 17.9 \\
\hline 2007-05-26 12:00:00.0 & 11.5 \\
\hline 2007-05-26 09:00:00.0 & 11.9 \\
\hline 2007-05-26 06:00:00.0 & 11.8 \\
\hline 2007-05-26 03:00:00.0 & 10.9 \\
\hline 2007-05-26 00:00:00.0 & 6.9 \\
\hline 2007-05-25 21:00:00.0 & 13.4 \\
\hline 2007-05-25 18:00:00.0 & \\
\hline 2007-05-25 15:00:00.0 & \\
\hline 2007-05-25 12:00:00.0 & 10.7 \\
\hline 2007-05-25 09:00:00.0 & 8.8 \\
\hline 2007-05-25 06:00:00.0 & 2.4 \\
\hline 2007-05-25 03:00:00.0 & 11.2 \\
\hline 2007-05-25 00:00:00.0 & 36.0 \\
\hline 2007-05-24 21:00:00.0 & 13.3 \\
\hline $2007-05-24$ 18:00:00.0 & 17.0 \\
\hline 2007-05-24 15:00:00.0 & 16.6 \\
\hline 2007-05-24 12:00:00.0 & 27.1 \\
\hline 2007-05-24 09:00:00.0 & 18.1 \\
\hline 2007-05-24 06:00:00.0 & 18.9 \\
\hline 2007-05-24 03:00:00.0 & 18.0 \\
\hline 2007-05-24 00:00:00.0 & 21.5 \\
\hline 2007-05-23 21:00:00.0 & 25.1 \\
\hline 2007-05-23 18:00:00.0 & 36.6 \\
\hline 2007-05-23 15:00:00.0 & 23.7 \\
\hline 2007-05-23 12:00:00.0 & 21.5 \\
\hline 2007-05-23 09:00:00.0 & 24.7 \\
\hline 2007-05-23 06:00:00.0 & 23.9 \\
\hline 2007-05-23 03:00:00.0 & \\
\hline 2007-05-23 00:00:00.0 & 12.4 \\
\hline
\end{tabular}

\begin{tabular}{|c|c|}
\hline DataHora & VelVentoMax \\
\hline 2007-05-22 21:00:00.0 & 29.4 \\
\hline 2007-05-22 18:00:00.0 & 9.4 \\
\hline 2007-05-22 15:00:00.0 & 11.8 \\
\hline 2007-05-22 12:00:00.0 & \\
\hline 2007-05-22 09:00:00.0 & 8.9 \\
\hline 2007-05-22 06:00:00.0 & 5.8 \\
\hline 2007-05-22 03:00:00.0 & 6.4 \\
\hline 2007-05-22 00:00:00.0 & 6.1 \\
\hline 2007-05-21 21:00:00.0 & 16.3 \\
\hline 2007-05-21 18:00:00.0 & 30.7 \\
\hline 2007-05-21 15:00:00.0 & 19.9 \\
\hline 2007-05-21 12:00:00.0 & 18.6 \\
\hline 2007-05-21 09:00:00.0 & 18.5 \\
\hline 2007-05-21 06:00:00.0 & 27.1 \\
\hline 2007-05-21 03:00:00.0 & 17.7 \\
\hline 2007-05-21 00:00:00.0 & 12.0 \\
\hline 2007-05-20 21:00:00.0 & 12.2 \\
\hline 2007-05-20 18:00:00.0 & 16.7 \\
\hline 2007-05-20 15:00:00.0 & 23.5 \\
\hline 2007-05-20 12:00:00.0 & 20.2 \\
\hline 2007-05-20 09:00:00.0 & \\
\hline 2007-05-20 06:00:00.0 & 18.5 \\
\hline 2007-05-20 03:00:00.0 & 17.4 \\
\hline 2007-05-20 00:00:00.0 & \\
\hline 2007-05-19 21:00:00.0 & 46.6 \\
\hline 2007-05-19 18:00:00.0 & \\
\hline 2007-05-19 15:00:00.0 & 26.0 \\
\hline 2007-05-19 12:00:00.0 & 34.2 \\
\hline 2007-05-19 09:00:00.0 & 12.6 \\
\hline 2007-05-19 06:00:00.0 & 9.4 \\
\hline 2007-05-19 03:00:00.0 & 12.4 \\
\hline 2007-05-19 00:00:00.0 & 14.3 \\
\hline 2007-05-18 21:00:00.0 & 13.8 \\
\hline 2007-05-18 18:00:00.0 & 20.5 \\
\hline 2007-05-18 15:00:00.0 & 22.9 \\
\hline 2007-05-18 12:00:00.0 & 11.9 \\
\hline 2007-05-18 09:00:00.0 & 28.8 \\
\hline 2007-05-18 06:00:00.0 & 30.4 \\
\hline 2007-05-18 03:00:00.0 & 4.8 \\
\hline 2007-05-18 00:00:00.0 & 13.0 \\
\hline 2007-05-17 21:00:00.0 & 17.7 \\
\hline 2007-05-17 18:00:00.0 & 21.5 \\
\hline 2007-05-17 15:00:00.0 & 24.0 \\
\hline 2007-05-17 12:00:00.0 & 15.0 \\
\hline 2007-05-17 09:00:00.0 & 12.1 \\
\hline 2007-05-17 06:00:00.0 & 12.8 \\
\hline 2007-05-17 03:00:00.0 & 10.8 \\
\hline 2007-05-17 00:00:00.0 & 11.5 \\
\hline 2007-05-16 21:00:00.0 & 32.6 \\
\hline 2007-05-16 18:00:00.0 & 14.5 \\
\hline 2007-05-16 15:00:00.0 & 15.6 \\
\hline 2007-05-16 12:00:00.0 & 18.9 \\
\hline 2007-05-16 09:00:00.0 & 21.4 \\
\hline 2007-05-16 06:00:00.0 & 8.2 \\
\hline 2007-05-16 03:00:00.0 & 11.2 \\
\hline 2007-05-16 00:00:00.0 & 10.5 \\
\hline 2007-05-15 21:00:00.0 & 17.7 \\
\hline 2007-05-15 18:00:00.0 & 20.8 \\
\hline 2007-05-15 15:00:00.0 & 25.3 \\
\hline 2007-05-15 12:00:00.0 & 20.8 \\
\hline 2007-05-15 09:00:00.0 & 24.6 \\
\hline 2007-05-15 06:00:00.0 & 24.5 \\
\hline 2007-05-15 03:00:00.0 & 17.0 \\
\hline 2007-05-15 00:00:00.0 & \\
\hline 2007-05-14 21:00:00.0 & 34.4 \\
\hline 2007-05-14 18:00:00.0 & 21.4 \\
\hline 2007-05-14 15:00:00.0 & 19.0 \\
\hline 2007-05-14 12:00:00.0 & 19.3 \\
\hline 2007-05-14 09:00:00.0 & 15.9 \\
\hline
\end{tabular}




\section{ANEXO 01 - RAJADAS DE VENTO REGISTRADAS EM SÃO MARTINHO DA SERRA / RS}

\begin{tabular}{|c|c|}
\hline DataHora & VelVentoMax \\
\hline 2007-05-14 06:00:00.0 & 30.1 \\
\hline 2007-05-14 03:00:00.0 & 16.4 \\
\hline 2007-05-14 00:00:00.0 & 21.4 \\
\hline 2007-05-13 21:00:00.0 & 21.0 \\
\hline 2007-05-13 18:00:00.0 & 23.1 \\
\hline 2007-05-13 15:00:00.0 & 20.2 \\
\hline 2007-05-13 12:00:00.0 & 19.3 \\
\hline 2007-05-13 09:00:00.0 & 22.5 \\
\hline 2007-05-13 06:00:00.0 & 18.9 \\
\hline 2007-05-13 03:00:00.0 & 17.5 \\
\hline 2007-05-13 00:00:00.0 & 12.9 \\
\hline 2007-05-12 21:00:00.0 & 7.0 \\
\hline 2007-05-12 18:00:00.0 & 14.2 \\
\hline 2007-05-12 15:00:00.0 & 14.0 \\
\hline 2007-05-12 12:00:00.0 & 10.4 \\
\hline 2007-05-12 09:00:00.0 & 3.9 \\
\hline 2007-05-12 06:00:00.0 & 6.3 \\
\hline 2007-05-12 03:00:00.0 & 7.4 \\
\hline 2007-05-12 00:00:00.0 & 6.1 \\
\hline 2007-05-11 21:00:00.0 & 8.1 \\
\hline 2007-05-11 18:00:00.0 & 19.5 \\
\hline 2007-05-11 15:00:00.0 & 21.7 \\
\hline 2007-05-11 12:00:00.0 & 19.6 \\
\hline 2007-05-11 09:00:00.0 & 15.4 \\
\hline 2007-05-11 06:00:00.0 & 14.9 \\
\hline 2007-05-11 03:00:00.0 & 17.8 \\
\hline 2007-05-11 00:00:00.0 & \\
\hline 2007-05-10 21:00:00.0 & 4.8 \\
\hline 2007-05-10 18:00:00.0 & 24.7 \\
\hline 2007-05-10 15:00:00.0 & 25.9 \\
\hline 2007-05-10 12:00:00.0 & 18.6 \\
\hline 2007-05-10 09:00:00.0 & 18.6 \\
\hline 2007-05-10 06:00:00.0 & 10.0 \\
\hline 2007-05-10 03:00:00.0 & 9.5 \\
\hline 2007-05-10 00:00:00.0 & 8.2 \\
\hline 2007-05-09 21:00:00.0 & 15.7 \\
\hline 2007-05-09 18:00:00.0 & 20.9 \\
\hline 2007-05-09 15:00:00.0 & 21.7 \\
\hline 2007-05-09 12:00:00.0 & 14.4 \\
\hline 2007-05-09 09:00:00.0 & \\
\hline 2007-05-09 06:00:00.0 & 16.1 \\
\hline 2007-05-09 03:00:00.0 & 23.4 \\
\hline 2007-05-09 00:00:00.0 & 11.1 \\
\hline 2007-05-08 21:00:00.0 & 27.2 \\
\hline 2007-05-08 18:00:00.0 & 25.2 \\
\hline 2007-05-08 15:00:00.0 & 8.0 \\
\hline 2007-05-08 12:00:00.0 & 33.6 \\
\hline 2007-05-08 09:00:00.0 & 2.0 \\
\hline 2007-05-08 06:00:00.0 & 21.3 \\
\hline 2007-05-08 03:00:00.0 & 16.7 \\
\hline 2007-05-08 00:00:00.0 & 17.9 \\
\hline 2007-05-07 21:00:00.0 & 18.0 \\
\hline 2007-05-07 18:00:00.0 & 20.5 \\
\hline 2007-05-07 15:00:00.0 & 22.8 \\
\hline 2007-05-07 12:00:00.0 & 24.6 \\
\hline 2007-05-07 09:00:00.0 & 25.0 \\
\hline 2007-05-07 06:00:00.0 & 24.0 \\
\hline 2007-05-07 03:00:00.0 & 17.0 \\
\hline 2007-05-07 00:00:00.0 & 24.0 \\
\hline 2007-05-06 21:00:00.0 & 32.0 \\
\hline 2007-05-06 18:00:00.0 & 35.3 \\
\hline 2007-05-06 15:00:00.0 & 32.0 \\
\hline 2007-05-06 12:00:00.0 & 34.2 \\
\hline 2007-05-06 09:00:00.0 & \\
\hline 2007-05-06 06:00:00.0 & 10.2 \\
\hline 2007-05-06 03:00:00.0 & 11.3 \\
\hline 2007-05-06 00:00:00.0 & 11.3 \\
\hline 2007-05-05 21:00:00.0 & 21.6 \\
\hline 2007-05-05 18:00:00.0 & 22.3 \\
\hline
\end{tabular}

\begin{tabular}{|c|c|}
\hline DataHora & VelVentoMax \\
\hline 2007-05-05 15:00:00.0 & 18.9 \\
\hline 2007-05-05 12:00:00.0 & 17.0 \\
\hline 2007-05-05 09:00:00.0 & 20.1 \\
\hline 2007-05-05 06:00:00.0 & 21.1 \\
\hline 2007-05-05 03:00:00.0 & 19.2 \\
\hline 2007-05-05 00:00:00.0 & 19.3 \\
\hline 2007-05-04 21:00:00.0 & 27.0 \\
\hline 2007-05-04 18:00:00.0 & 27.7 \\
\hline 2007-05-04 15:00:00.0 & 27.1 \\
\hline 2007-05-04 12:00:00.0 & 26.6 \\
\hline 2007-05-04 09:00:00.0 & 22.4 \\
\hline 2007-05-04 06:00:00.0 & 22.0 \\
\hline 2007-05-04 03:00:00.0 & 21.2 \\
\hline 2007-05-04 00:00:00.0 & 25.2 \\
\hline 2007-05-03 21:00:00.0 & 17.3 \\
\hline 2007-05-03 18:00:00.0 & 26.2 \\
\hline 2007-05-03 15:00:00.0 & 27.3 \\
\hline 2007-05-03 12:00:00.0 & 21.2 \\
\hline 2007-05-03 09:00:00.0 & 16.0 \\
\hline 2007-05-03 06:00:00.0 & 10.2 \\
\hline 2007-05-03 03:00:00.0 & \\
\hline 2007-05-03 00:00:00.0 & 17.7 \\
\hline 2007-05-02 21:00:00.0 & 3.9 \\
\hline 2007-05-02 18:00:00.0 & 22.1 \\
\hline 2007-05-02 15:00:00.0 & 22.0 \\
\hline 2007-05-02 12:00:00.0 & 42.0 \\
\hline 2007-05-02 09:00:00.0 & 12.0 \\
\hline 2007-05-02 06:00:00.0 & 10.0 \\
\hline 2007-05-02 03:00:00.0 & 12.0 \\
\hline 2007-05-02 00:00:00.0 & 12.6 \\
\hline 2007-05-01 21:00:00.0 & 14.4 \\
\hline 2007-05-01 18:00:00.0 & 18.6 \\
\hline 2007-05-01 15:00:00.0 & 21.3 \\
\hline 2007-05-01 12:00:00.0 & 19.0 \\
\hline 2007-05-01 09:00:00.0 & 17.9 \\
\hline 2007-05-01 06:00:00.0 & 20.3 \\
\hline 2007-05-01 03:00:00.0 & 19.5 \\
\hline 2007-05-01 00:00:00.0 & 13.9 \\
\hline 2007-04-30 21:00:00.0 & 12.6 \\
\hline 2007-04-30 18:00:00.0 & 16.5 \\
\hline 2007-04-30 15:00:00.0 & 20.3 \\
\hline 2007-04-30 12:00:00.0 & 18.6 \\
\hline 2007-04-30 09:00:00.0 & 16.5 \\
\hline 2007-04-30 06:00:00.0 & 14.5 \\
\hline 2007-04-30 03:00:00.0 & 12.3 \\
\hline 2007-04-30 00:00:00.0 & 10.6 \\
\hline 2007-04-29 21:00:00.0 & 13.0 \\
\hline 2007-04-29 18:00:00.0 & 33.6 \\
\hline 2007-04-29 15:00:00.0 & \\
\hline 2007-04-29 12:00:00.0 & 18.4 \\
\hline 2007-04-29 09:00:00.0 & 16.2 \\
\hline 2007-04-29 06:00:00.0 & 15.8 \\
\hline 2007-04-29 03:00:00.0 & 12.9 \\
\hline 2007-04-29 00:00:00.0 & 12.4 \\
\hline 2007-04-28 21:00:00.0 & 17.6 \\
\hline 2007-04-28 18:00:00.0 & 23.7 \\
\hline 2007-04-28 15:00:00.0 & 28.9 \\
\hline 2007-04-28 12:00:00.0 & 21.2 \\
\hline 2007-04-28 09:00:00.0 & 14.8 \\
\hline 2007-04-28 06:00:00.0 & 13.2 \\
\hline 2007-04-28 03:00:00.0 & 9.3 \\
\hline 2007-04-28 00:00:00.0 & 10.7 \\
\hline 2007-04-27 21:00:00.0 & 34.3 \\
\hline 2007-04-27 18:00:00.0 & 21.1 \\
\hline 2007-04-27 15:00:00.0 & 21.0 \\
\hline 2007-04-27 12:00:00.0 & 12.2 \\
\hline 2007-04-27 09:00:00.0 & 11.0 \\
\hline 2007-04-27 06:00:00.0 & 18.0 \\
\hline 2007-04-27 03:00:00.0 & 18.0 \\
\hline
\end{tabular}

\begin{tabular}{|c|c|}
\hline DataHora & VelVentoMax \\
\hline 2007-04-27 00:00:00.0 & 16.0 \\
\hline 2007-04-26 21:00:00.0 & 28.0 \\
\hline 2007-04-26 18:00:00.0 & 25.0 \\
\hline 2007-04-26 15:00:00.0 & 23.0 \\
\hline 2007-04-26 12:00:00.0 & 27.0 \\
\hline 2007-04-26 09:00:00.0 & 26.0 \\
\hline 2007-04-26 06:00:00.0 & 24.0 \\
\hline 2007-04-26 03:00:00.0 & 9.0 \\
\hline 2007-04-26 00:00:00.0 & 5.0 \\
\hline 2007-04-25 21:00:00.0 & 2.0 \\
\hline 2007-04-25 18:00:00.0 & \\
\hline 2007-04-25 15:00:00.0 & 27.0 \\
\hline 2007-04-25 12:00:00.0 & 12.0 \\
\hline 2007-04-25 09:00:00.0 & 8.0 \\
\hline 2007-04-25 06:00:00.0 & 14.0 \\
\hline 2007-04-25 03:00:00.0 & 17.0 \\
\hline 2007-04-25 00:00:00.0 & 18.0 \\
\hline 2007-04-24 21:00:00.0 & 15.0 \\
\hline $2007-04-24$ 18:00:00.0 & 21.0 \\
\hline 2007-04-24 15:00:00.0 & 33.0 \\
\hline 2007-04-24 12:00:00.0 & 18.0 \\
\hline 2007-04-24 09:00:00.0 & 21.0 \\
\hline 2007-04-24 06:00:00.0 & 19.0 \\
\hline 2007-04-24 03:00:00.0 & 18.0 \\
\hline 2007-04-24 00:00:00.0 & 13.0 \\
\hline 2007-04-23 21:00:00.0 & 17.0 \\
\hline 2007-04-23 18:00:00.0 & 32.0 \\
\hline $2007-04-23$ 15:00:00.0 & 17.9 \\
\hline 2007-04-23 12:00:00.0 & 16.2 \\
\hline 2007-04-23 09:00:00.0 & 12.8 \\
\hline 2007-04-23 06:00:00.0 & 11.8 \\
\hline 2007-04-23 03:00:00.0 & 15.2 \\
\hline 2007-04-23 00:00:00.0 & 5.4 \\
\hline 2007-04-22 21:00:00.0 & 15.0 \\
\hline 2007-04-22 18:00:00.0 & 34.7 \\
\hline 2007-04-22 15:00:00.0 & 46.8 \\
\hline 2007-04-22 12:00:00.0 & 18.3 \\
\hline 2007-04-22 09:00:00.0 & 15.8 \\
\hline 2007-04-22 06:00:00.0 & 13.6 \\
\hline 2007-04-22 03:00:00.0 & 10.4 \\
\hline 2007-04-22 00:00:00.0 & 8.4 \\
\hline 2007-04-21 21:00:00.0 & 14.5 \\
\hline 2007-04-21 18:00:00.0 & 12.9 \\
\hline 2007-04-21 15:00:00.0 & 33.4 \\
\hline 2007-04-21 12:00:00.0 & 13.6 \\
\hline 2007-04-21 09:00:00.0 & 12.8 \\
\hline 2007-04-21 06:00:00.0 & 12.1 \\
\hline 2007-04-21 03:00:00.0 & 9.6 \\
\hline 2007-04-21 00:00:00.0 & 46.2 \\
\hline 2007-04-20 21:00:00.0 & 15.2 \\
\hline 2007-04-20 18:00:00.0 & 16.8 \\
\hline 2007-04-20 15:00:00.0 & 20.2 \\
\hline 2007-04-20 12:00:00.0 & 21.3 \\
\hline 2007-04-20 09:00:00.0 & 19.0 \\
\hline 2007-04-20 06:00:00.0 & 14.3 \\
\hline 2007-04-20 03:00:00.0 & 13.1 \\
\hline 2007-04-20 00:00:00.0 & 9.7 \\
\hline 2007-04-19 21:00:00.0 & \\
\hline 2007-04-19 18:00:00.0 & 21.2 \\
\hline 2007-04-19 15:00:00.0 & 24.7 \\
\hline 2007-04-19 12:00:00.0 & 20.7 \\
\hline 2007-04-19 09:00:00.0 & 16.3 \\
\hline 2007-04-19 06:00:00.0 & 17.2 \\
\hline 2007-04-19 03:00:00.0 & \\
\hline 2007-04-19 00:00:00.0 & \\
\hline 2007-04-18 21:00:00.0 & \\
\hline 2007-04-18 18:00:00.0 & 12.5 \\
\hline 2007-04-18 15:00:00.0 & 19.3 \\
\hline 2007-04-18 12:00:00.0 & \\
\hline
\end{tabular}




\section{ANEXO 01 - RAJADAS DE VENTO REGISTRADAS EM SÃO MARTINHO DA SERRA / RS}

\begin{tabular}{|c|c|}
\hline \multirow{3}{*}{ DataHora } & VelVentoMax \\
\hline & \\
\hline & 15.3 \\
\hline 2007-04-18 03:00:00.0 & 12.9 \\
\hline 2007-04-18 00:00:00.0 & 11.3 \\
\hline 2007-04-17 21:00:00.0 & 35.3 \\
\hline 2007-04-17 18:00:00.0 & 15.8 \\
\hline 2007-04-17 15:00:00.0 & 16.3 \\
\hline 2007-04-17 12:00:00.0 & 17.0 \\
\hline 2007-04-17 09:00:00.0 & \\
\hline 2007-04-17 06:00:00.0 & 21.3 \\
\hline 2007-04-17 03:00:00.0 & 18.3 \\
\hline 2007-04-17 00:00:00.0 & 13.3 \\
\hline 2007-04-16 21:00:00.0 & 15.7 \\
\hline 2007-04-16 18:00:00.0 & 15.0 \\
\hline 2007-04-16 15:00:00.0 & 13.0 \\
\hline 2007-04-16 12:00:00.0 & 14.4 \\
\hline 2007-04-16 09:00:00.0 & 16.1 \\
\hline 2007-04-16 06:00:00.0 & 13.2 \\
\hline 2007-04-16 03:00:00.0 & 10.5 \\
\hline 2007-04-16 00:00:00.0 & 10.7 \\
\hline 2007-04-15 21:00:00.0 & 4.7 \\
\hline 2007-04-15 18:00:00.0 & 15.9 \\
\hline 2007-04-15 15:00:00.0 & 18.6 \\
\hline 2007-04-15 12:00:00.0 & 13.8 \\
\hline 2007-04-15 09:00:00.0 & 12.1 \\
\hline 2007-04-15 06:00:00.0 & 35.2 \\
\hline 2007-04-15 03:00:00.0 & 26.9 \\
\hline 2007-04-15 00:00:00.0 & 29.4 \\
\hline 2007-04-14 21:00:00.0 & \\
\hline 2007-04-14 18:00:00.0 & 22.9 \\
\hline 2007-04-14 15:00:00.0 & 22.9 \\
\hline 2007-04-14 12:00:00.0 & 26.0 \\
\hline 2007-04-14 09:00:00.0 & 9.6 \\
\hline 2007-04-14 06:00:00.0 & 22.0 \\
\hline 2007-04-14 03:00:00.0 & 21.1 \\
\hline 2007-04-14 00:00:00.0 & 15.8 \\
\hline 2007-04-13 21:00:00.0 & 17.1 \\
\hline 2007-04-13 18:00:00.0 & 19.4 \\
\hline 2007-04-13 15:00:00.0 & 20.9 \\
\hline 2007-04-13 12:00:00.0 & 21.0 \\
\hline 2007-04-13 09:00:00.0 & 15.3 \\
\hline 2007-04-13 06:00:00.0 & 13.6 \\
\hline 2007-04-13 03:00:00.0 & 12.6 \\
\hline 2007-04-13 00:00:00.0 & 9.8 \\
\hline 2007-04-12 21:00:00.0 & 15.4 \\
\hline 2007-04-12 18:00:00.0 & 20.4 \\
\hline 2007-04-12 15:00:00.0 & 18.4 \\
\hline 2007-04-12 12:00:00.0 & 10.8 \\
\hline 2007-04-12 09:00:00.0 & \\
\hline 2007-04-12 06:00:00.0 & 22.3 \\
\hline 2007-04-12 03:00:00.0 & 7.2 \\
\hline 2007-04-12 00:00:00.0 & 10.7 \\
\hline 2007-04-11 21:00:00.0 & 26.8 \\
\hline 2007-04-11 18:00:00.0 & 29.8 \\
\hline 2007-04-11 15:00:00.0 & 23.4 \\
\hline 2007-04-11 12:00:00.0 & 14.2 \\
\hline 2007-04-11 09:00:00.0 & 12.5 \\
\hline 2007-04-11 06:00:00.0 & 10.4 \\
\hline 2007-04-11 03:00:00.0 & 14.4 \\
\hline 2007-04-11 00:00:00.0 & 11.0 \\
\hline 2007-04-10 21:00:00.0 & 12.8 \\
\hline 2007-04-10 18:00:00.0 & 13.5 \\
\hline 2007-04-10 15:00:00.0 & 15.4 \\
\hline 2007-04-10 12:00:00.0 & 17.4 \\
\hline 2007-04-10 09:00:00.0 & 19.5 \\
\hline 2007-04-10 06:00:00.0 & 15.0 \\
\hline 2007-04-10 03:00:00.0 & 12.3 \\
\hline 2007-04-10 00:00:00.0 & 9.9 \\
\hline 2007-04-09 21:00:00.0 & 12.8 \\
\hline
\end{tabular}

\begin{tabular}{|c|c|}
\hline DataHora & VelVentoMax \\
\hline 2007-04-09 18:00:00.0 & 14.5 \\
\hline 2007-04-09 15:00:00.0 & 12.3 \\
\hline 2007-04-09 12:00:00.0 & \\
\hline 2007-04-09 09:00:00.0 & 13.5 \\
\hline 2007-04-09 06:00:00.0 & 14.3 \\
\hline 2007-04-09 03:00:00.0 & 13.2 \\
\hline 2007-04-09 00:00:00.0 & 14.9 \\
\hline 2007-04-08 21:00:00.0 & 21.5 \\
\hline 2007-04-08 18:00:00.0 & 22.5 \\
\hline 2007-04-08 15:00:00.0 & 20.5 \\
\hline 2007-04-08 12:00:00.0 & 13.8 \\
\hline 2007-04-08 09:00:00.0 & 13.7 \\
\hline 2007-04-08 06:00:00.0 & 11.2 \\
\hline 2007-04-08 03:00:00.0 & 9.2 \\
\hline 2007-04-08 00:00:00.0 & 9.1 \\
\hline 2007-04-07 21:00:00.0 & 15.5 \\
\hline 2007-04-07 18:00:00.0 & 18.9 \\
\hline 2007-04-07 15:00:00.0 & 14.4 \\
\hline 2007-04-07 12:00:00.0 & 13.8 \\
\hline 2007-04-07 09:00:00.0 & 22.3 \\
\hline 2007-04-07 06:00:00.0 & 18.4 \\
\hline 2007-04-07 03:00:00.0 & 16.3 \\
\hline 2007-04-07 00:00:00.0 & 22.1 \\
\hline 2007-04-06 21:00:00.0 & 17.5 \\
\hline 2007-04-06 18:00:00.0 & 18.1 \\
\hline 2007-04-06 15:00:00.0 & 43.1 \\
\hline 2007-04-06 12:00:00.0 & 21.9 \\
\hline 2007-04-06 09:00:00.0 & 18.2 \\
\hline 2007-04-06 06:00:00.0 & 16.7 \\
\hline 2007-04-06 03:00:00.0 & 15.0 \\
\hline 2007-04-06 00:00:00.0 & 26.1 \\
\hline 2007-04-05 21:00:00.0 & 25.4 \\
\hline 2007-04-05 18:00:00.0 & 22.4 \\
\hline 2007-04-05 15:00:00.0 & 23.0 \\
\hline 2007-04-05 12:00:00.0 & 19.7 \\
\hline 2007-04-05 09:00:00.0 & 22.2 \\
\hline 2007-04-05 06:00:00.0 & 20.2 \\
\hline 2007-04-05 03:00:00.0 & 16.6 \\
\hline 2007-04-05 00:00:00.0 & 11.2 \\
\hline 2007-04-04 21:00:00.0 & 17.0 \\
\hline 2007-04-04 18:00:00.0 & 17.6 \\
\hline 2007-04-04 15:00:00.0 & 16.9 \\
\hline 2007-04-04 12:00:00.0 & 14.5 \\
\hline 2007-04-04 09:00:00.0 & 8.5 \\
\hline 2007-04-04 06:00:00.0 & 13.4 \\
\hline 2007-04-04 03:00:00.0 & 9.9 \\
\hline 2007-04-04 00:00:00.0 & 12.0 \\
\hline 2007-04-03 21:00:00.0 & 13.4 \\
\hline 2007-04-03 18:00:00.0 & 16.8 \\
\hline 2007-04-03 15:00:00.0 & 24.2 \\
\hline 2007-04-03 12:00:00.0 & 13.8 \\
\hline 2007-04-03 09:00:00.0 & 13.9 \\
\hline 2007-04-03 06:00:00.0 & 15.4 \\
\hline 2007-04-03 03:00:00.0 & 10.3 \\
\hline 2007-04-03 00:00:00.0 & 7.3 \\
\hline 2007-04-02 21:00:00.0 & 22.9 \\
\hline 2007-04-02 18:00:00.0 & 19.0 \\
\hline 2007-04-02 15:00:00.0 & 18.8 \\
\hline 2007-04-02 12:00:00.0 & 16.4 \\
\hline 2007-04-02 09:00:00.0 & 16.2 \\
\hline 2007-04-02 06:00:00.0 & 17.4 \\
\hline 2007-04-02 03:00:00.0 & 20.8 \\
\hline 2007-04-02 00:00:00.0 & 18.6 \\
\hline 2007-04-01 21:00:00.0 & 20.9 \\
\hline 2007-04-01 18:00:00.0 & 19.6 \\
\hline 2007-04-01 15:00:00.0 & \\
\hline 2007-04-01 12:00:00.0 & 17.5 \\
\hline 2007-04-01 09:00:00.0 & 9.6 \\
\hline 2007-04-01 06:00:00.0 & 10.0 \\
\hline
\end{tabular}

\begin{tabular}{|c|c|}
\hline DataHora & VelVentoMax \\
\hline 2007-04-01 03:00:00.0 & 10.6 \\
\hline 2007-04-01 00:00:00.0 & 9.3 \\
\hline 2007-03-31 21:00:00.0 & 13.0 \\
\hline 2007-03-31 18:00:00.0 & 19.4 \\
\hline 2007-03-31 15:00:00.0 & 29.6 \\
\hline 2007-03-31 12:00:00.0 & 21.6 \\
\hline 2007-03-31 09:00:00.0 & 12.3 \\
\hline 2007-03-31 06:00:00.0 & 14.7 \\
\hline 2007-03-31 03:00:00.0 & 22.1 \\
\hline 2007-03-31 00:00:00.0 & 9.9 \\
\hline $2007-03-3021: 00: 00.0$ & 21.9 \\
\hline $2007-03-3018: 00: 00.0$ & 24.6 \\
\hline 2007-03-30 15:00:00.0 & 17.4 \\
\hline 2007-03-30 12:00:00.0 & 18.8 \\
\hline 2007-03-30 09:00:00.0 & 16.3 \\
\hline $2007-03-3006: 00: 00.0$ & 15.5 \\
\hline $2007-03-30$ 03:00:00.0 & \\
\hline 2007-03-30 00:00:00.0 & 10.4 \\
\hline 2007-03-29 21:00:00.0 & 13.1 \\
\hline 2007-03-29 18:00:00.0 & 18.2 \\
\hline $2007-03-29$ 15:00:00.0 & 14.4 \\
\hline $2007-03-2912: 00: 00.0$ & 15.1 \\
\hline $2007-03-29$ 09:00:00.0 & 17.2 \\
\hline 2007-03-29 06:00:00.0 & 15.7 \\
\hline 2007-03-29 03:00:00.0 & 20.2 \\
\hline 2007-03-29 00:00:00.0 & 21.3 \\
\hline 2007-03-28 21:00:00.0 & 23.7 \\
\hline $2007-03-28$ 18:00:00.0 & 23.7 \\
\hline 2007-03-28 15:00:00.0 & 17.1 \\
\hline 2007-03-28 12:00:00.0 & 14.6 \\
\hline 2007-03-28 09:00:00.0 & 14.4 \\
\hline 2007-03-28 06:00:00.0 & 4.3 \\
\hline 2007-03-28 03:00:00.0 & 11.2 \\
\hline 2007-03-28 00:00:00.0 & 6.5 \\
\hline $2007-03-27$ 21:00:00.0 & 21.3 \\
\hline 2007-03-27 18:00:00.0 & 27.2 \\
\hline 2007-03-27 15:00:00.0 & 21.9 \\
\hline $2007-03-27$ 12:00:00.0 & 19.9 \\
\hline 2007-03-27 09:00:00.0 & 22.2 \\
\hline 2007-03-27 06:00:00.0 & \\
\hline 2007-03-27 03:00:00.0 & 25.7 \\
\hline 2007-03-27 00:00:00.0 & 22.8 \\
\hline 2007-03-26 21:00:00.0 & 44.6 \\
\hline 2007-03-26 18:00:00.0 & 13.2 \\
\hline 2007-03-26 15:00:00.0 & 17.9 \\
\hline 2007-03-26 12:00:00.0 & 21.7 \\
\hline 2007-03-26 09:00:00.0 & 19.3 \\
\hline 2007-03-26 06:00:00.0 & 19.3 \\
\hline 2007-03-26 03:00:00.0 & 19.4 \\
\hline 2007-03-26 00:00:00.0 & 11.8 \\
\hline $2007-03-25$ 21:00:00.0 & 12.6 \\
\hline 2007-03-25 18:00:00.0 & 27.2 \\
\hline 2007-03-25 15:00:00.0 & 2.0 \\
\hline $2007-03-25$ 12:00:00.0 & 18.9 \\
\hline 2007-03-25 09:00:00.0 & 18.5 \\
\hline 2007-03-25 06:00:00.0 & 18.0 \\
\hline 2007-03-25 03:00:00.0 & 17.7 \\
\hline 2007-03-25 00:00:00.0 & 18.3 \\
\hline 2007-03-24 21:00:00.0 & 17.2 \\
\hline 2007-03-24 18:00:00.0 & 16.5 \\
\hline 2007-03-24 15:00:00.0 & 34.3 \\
\hline $2007-03-24$ 12:00:00.0 & 17.5 \\
\hline 2007-03-24 09:00:00.0 & \\
\hline $2007-03-24$ 06:00:00.0 & 11.5 \\
\hline 2007-03-24 03:00:00.0 & 8.4 \\
\hline 2007-03-24 00:00:00.0 & 6.3 \\
\hline 2007-03-23 21:00:00.0 & 15.3 \\
\hline $2007-03-23$ 18:00:00.0 & 19.1 \\
\hline 2007-03-23 15:00:00.0 & 3.4 \\
\hline
\end{tabular}




\section{ANEXO 01 - RAJADAS DE VENTO REGISTRADAS EM SÃO MARTINHO DA SERRA / RS}

\begin{tabular}{|c|c|}
\hline DataHora & VelVentoMax \\
\hline 2007-03-23 12:00:00.0 & 13.0 \\
\hline 2007-03-23 09:00:00.0 & 16.5 \\
\hline 2007-03-23 06:00:00.0 & 14.1 \\
\hline 2007-03-23 03:00:00.0 & 13.8 \\
\hline 2007-03-23 00:00:00.0 & 11.2 \\
\hline 2007-03-22 21:00:00.0 & 31.9 \\
\hline 2007-03-22 18:00:00.0 & 11.8 \\
\hline 2007-03-22 15:00:00.0 & 14.1 \\
\hline 2007-03-22 12:00:00.0 & 17.1 \\
\hline 2007-03-22 09:00:00.0 & \\
\hline 2007-03-22 06:00:00.0 & 16.4 \\
\hline 2007-03-22 03:00:00.0 & 12.4 \\
\hline 2007-03-22 00:00:00.0 & \\
\hline 2007-03-21 21:00:00.0 & 12.5 \\
\hline 2007-03-21 18:00:00.0 & 12.2 \\
\hline 2007-03-21 15:00:00.0 & 15.0 \\
\hline 2007-03-21 12:00:00.0 & 13.0 \\
\hline 2007-03-21 09:00:00.0 & 9.0 \\
\hline 2007-03-21 06:00:00.0 & 9.0 \\
\hline 2007-03-21 03:00:00.0 & 8.0 \\
\hline 2007-03-21 00:00:00.0 & 10.0 \\
\hline 2007-03-20 21:00:00.0 & 16.0 \\
\hline 2007-03-20 18:00:00.0 & 16.0 \\
\hline 2007-03-20 15:00:00.0 & 17.0 \\
\hline 2007-03-20 12:00:00.0 & 14.0 \\
\hline 2007-03-20 09:00:00.0 & 15.0 \\
\hline 2007-03-20 06:00:00.0 & 16.0 \\
\hline 2007-03-20 03:00:00.0 & 18.0 \\
\hline 2007-03-20 00:00:00.0 & 14.0 \\
\hline 2007-03-19 21:00:00.0 & 19.0 \\
\hline 2007-03-19 18:00:00.0 & 17.0 \\
\hline 2007-03-19 15:00:00.0 & 15.0 \\
\hline 2007-03-19 12:00:00.0 & 2.0 \\
\hline 2007-03-19 09:00:00.0 & \\
\hline 2007-03-19 06:00:00.0 & 16.0 \\
\hline 2007-03-19 03:00:00.0 & 14.0 \\
\hline 2007-03-19 00:00:00.0 & 16.0 \\
\hline 2007-03-18 21:00:00.0 & 25.0 \\
\hline 2007-03-18 18:00:00.0 & 16.0 \\
\hline 2007-03-18 15:00:00.0 & 13.0 \\
\hline 2007-03-18 12:00:00.0 & 11.0 \\
\hline 2007-03-18 09:00:00.0 & \\
\hline 2007-03-18 06:00:00.0 & 8.0 \\
\hline 2007-03-18 03:00:00.0 & 16.0 \\
\hline 2007-03-18 00:00:00.0 & 16.0 \\
\hline 2007-03-17 21:00:00.0 & 18.0 \\
\hline 2007-03-17 18:00:00.0 & 16.0 \\
\hline 2007-03-17 15:00:00.0 & \\
\hline 2007-03-17 12:00:00.0 & 16.0 \\
\hline 2007-03-17 09:00:00.0 & 13.0 \\
\hline 2007-03-17 06:00:00.0 & 14.0 \\
\hline 2007-03-17 03:00:00.0 & 18.0 \\
\hline 2007-03-17 00:00:00.0 & 19.0 \\
\hline 2007-03-16 21:00:00.0 & 20.0 \\
\hline 2007-03-16 18:00:00.0 & 39.0 \\
\hline 2007-03-16 15:00:00.0 & 4.0 \\
\hline 2007-03-16 12:00:00.0 & 13.0 \\
\hline 2007-03-16 09:00:00.0 & 10.0 \\
\hline 2007-03-16 06:00:00.0 & 8.0 \\
\hline 2007-03-16 03:00:00.0 & 8.0 \\
\hline 2007-03-16 00:00:00.0 & 9.0 \\
\hline 2007-03-15 21:00:00.0 & 20.0 \\
\hline 2007-03-15 18:00:00.0 & 21.0 \\
\hline 2007-03-15 15:00:00.0 & 17.0 \\
\hline 2007-03-15 12:00:00.0 & 18.0 \\
\hline 2007-03-15 09:00:00.0 & 10.0 \\
\hline 2007-03-15 06:00:00.0 & 12.0 \\
\hline 2007-03-15 03:00:00.0 & 13.0 \\
\hline 2007-03-15 00:00:00.0 & 10.0 \\
\hline
\end{tabular}

\begin{tabular}{|c|c|}
\hline DataHora & VelVentoMax \\
\hline 2007-03-14 21:00:00.0 & 30.0 \\
\hline 2007-03-14 18:00:00.0 & 12.0 \\
\hline 2007-03-14 15:00:00.0 & 13.0 \\
\hline 2007-03-14 12:00:00.0 & 16.0 \\
\hline 2007-03-14 09:00:00.0 & 15.0 \\
\hline 2007-03-14 06:00:00.0 & 12.0 \\
\hline 2007-03-14 03:00:00.0 & 12.0 \\
\hline 2007-03-14 00:00:00.0 & \\
\hline 2007-03-13 21:00:00.0 & 16.0 \\
\hline 2007-03-13 18:00:00.0 & 10.0 \\
\hline 2007-03-13 15:00:00.0 & 14.0 \\
\hline 2007-03-13 12:00:00.0 & 16.0 \\
\hline 2007-03-13 09:00:00.0 & 12.0 \\
\hline 2007-03-13 06:00:00.0 & \\
\hline 2007-03-13 03:00:00.0 & 11.0 \\
\hline 2007-03-13 00:00:00.0 & \\
\hline 2007-03-12 21:00:00.0 & 14.0 \\
\hline 2007-03-12 18:00:00.0 & 15.0 \\
\hline 2007-03-12 15:00:00.0 & 9.0 \\
\hline 2007-03-12 12:00:00.0 & 38.0 \\
\hline 2007-03-12 09:00:00.0 & 10.0 \\
\hline 2007-03-12 06:00:00.0 & 11.0 \\
\hline 2007-03-12 03:00:00.0 & 11.0 \\
\hline 2007-03-12 00:00:00.0 & 10.0 \\
\hline 2007-03-11 21:00:00.0 & 12.0 \\
\hline 2007-03-11 18:00:00.0 & 12.0 \\
\hline 2007-03-11 15:00:00.0 & 11.0 \\
\hline 2007-03-11 12:00:00.0 & \\
\hline 2007-03-11 09:00:00.0 & 8.0 \\
\hline 2007-03-11 06:00:00.0 & 5.0 \\
\hline 2007-03-11 03:00:00.0 & 5.0 \\
\hline 2007-03-11 00:00:00.0 & 28.0 \\
\hline 2007-03-10 21:00:00.0 & 12.0 \\
\hline 2007-03-10 18:00:00.0 & 7.0 \\
\hline 2007-03-10 15:00:00.0 & 7.0 \\
\hline 2007-03-10 12:00:00.0 & 9.0 \\
\hline 2007-03-10 09:00:00.0 & 10.0 \\
\hline 2007-03-10 06:00:00.0 & 5.0 \\
\hline 2007-03-10 03:00:00.0 & 12.0 \\
\hline 2007-03-10 00:00:00.0 & 19.0 \\
\hline 2007-03-09 21:00:00.0 & 24.0 \\
\hline 2007-03-09 18:00:00.0 & 9.0 \\
\hline 2007-03-09 15:00:00.0 & 32.0 \\
\hline 2007-03-09 12:00:00.0 & 21.0 \\
\hline 2007-03-09 09:00:00.0 & 17.0 \\
\hline 2007-03-09 06:00:00.0 & 39.0 \\
\hline 2007-03-09 03:00:00.0 & 15.0 \\
\hline 2007-03-09 00:00:00.0 & \\
\hline 2007-03-08 21:00:00.0 & 27.0 \\
\hline 2007-03-08 18:00:00.0 & 28.0 \\
\hline 2007-03-08 15:00:00.0 & 20.0 \\
\hline 2007-03-08 12:00:00.0 & 17.0 \\
\hline 2007-03-08 09:00:00.0 & 21.0 \\
\hline 2007-03-08 06:00:00.0 & 19.0 \\
\hline 2007-03-08 03:00:00.0 & 17.0 \\
\hline 2007-03-08 00:00:00.0 & 21.0 \\
\hline 2007-03-07 21:00:00.0 & 22.0 \\
\hline 2007-03-07 18:00:00.0 & 12.0 \\
\hline 2007-03-07 15:00:00.0 & 17.0 \\
\hline 2007-03-07 12:00:00.0 & 16.0 \\
\hline 2007-03-07 09:00:00.0 & 18.0 \\
\hline 2007-03-07 06:00:00.0 & 18.0 \\
\hline 2007-03-07 03:00:00.0 & \\
\hline 2007-03-07 00:00:00.0 & 20.0 \\
\hline 2007-03-06 21:00:00.0 & 33.0 \\
\hline 2007-03-06 18:00:00.0 & 14.0 \\
\hline 2007-03-06 15:00:00.0 & 14.0 \\
\hline 2007-03-06 12:00:00.0 & 13.0 \\
\hline 2007-03-06 09:00:00.0 & 15.0 \\
\hline
\end{tabular}

\begin{tabular}{|c|c|}
\hline DataHora & VelVentoMax \\
\hline 2007-03-06 06:00:00.0 & 14.0 \\
\hline 2007-03-06 03:00:00.0 & 12.0 \\
\hline 2007-03-06 00:00:00.0 & 18.0 \\
\hline 2007-03-05 21:00:00.0 & 31.0 \\
\hline 2007-03-05 18:00:00.0 & 20.0 \\
\hline 2007-03-05 15:00:00.0 & 20.0 \\
\hline 2007-03-05 12:00:00.0 & 10.0 \\
\hline 2007-03-05 09:00:00.0 & 10.0 \\
\hline 2007-03-05 06:00:00.0 & 8.0 \\
\hline 2007-03-05 03:00:00.0 & 12.0 \\
\hline 2007-03-05 00:00:00.0 & 28.0 \\
\hline 2007-03-04 21:00:00.0 & \\
\hline 2007-03-04 18:00:00.0 & \\
\hline 2007-03-04 15:00:00.0 & 35.0 \\
\hline 2007-03-04 12:00:00.0 & 20.0 \\
\hline 2007-03-04 09:00:00.0 & 19.0 \\
\hline 2007-03-04 06:00:00.0 & 18.0 \\
\hline 2007-03-04 03:00:00.0 & 23.0 \\
\hline 2007-03-04 00:00:00.0 & 29.0 \\
\hline 2007-03-03 21:00:00.0 & 29.0 \\
\hline 2007-03-03 18:00:00.0 & 18.0 \\
\hline 2007-03-03 15:00:00.0 & 24.0 \\
\hline 2007-03-03 12:00:00.0 & 22.0 \\
\hline 2007-03-03 09:00:00.0 & 21.0 \\
\hline 2007-03-03 06:00:00.0 & 18.0 \\
\hline 2007-03-03 03:00:00.0 & 19.0 \\
\hline 2007-03-03 00:00:00.0 & \\
\hline 2007-03-02 21:00:00.0 & \\
\hline 2007-03-02 18:00:00.0 & 17.0 \\
\hline 2007-03-02 15:00:00.0 & 17.0 \\
\hline 2007-03-02 12:00:00.0 & 23.0 \\
\hline 2007-03-02 09:00:00.0 & 28.0 \\
\hline 2007-03-02 06:00:00.0 & 39.0 \\
\hline 2007-03-02 03:00:00.0 & 15.0 \\
\hline 2007-03-02 00:00:00.0 & 21.0 \\
\hline 2007-03-01 21:00:00.0 & 28.0 \\
\hline 2007-03-01 18:00:00.0 & 28.0 \\
\hline 2007-03-01 15:00:00.0 & 31.0 \\
\hline 2007-03-01 12:00:00.0 & 22.6 \\
\hline 2007-03-01 09:00:00.0 & 16.2 \\
\hline 2007-03-01 06:00:00.0 & \\
\hline 2007-03-01 03:00:00.0 & 15.3 \\
\hline 2007-03-01 00:00:00.0 & 19.9 \\
\hline 2007-02-28 21:00:00.0 & 18.6 \\
\hline $2007-02-28$ 18:00:00.0 & 21.3 \\
\hline 2007-02-28 15:00:00.0 & 50.0 \\
\hline 2007-02-28 12:00:00.0 & 25.0 \\
\hline 2007-02-28 09:00:00.0 & 14.7 \\
\hline 2007-02-28 06:00:00.0 & 13.8 \\
\hline 2007-02-28 03:00:00.0 & 14.1 \\
\hline 2007-02-28 00:00:00.0 & 14.5 \\
\hline 2007-02-27 21:00:00.0 & 22.9 \\
\hline 2007-02-27 18:00:00.0 & 24.3 \\
\hline 2007-02-27 15:00:00.0 & 23.4 \\
\hline 2007-02-27 12:00:00.0 & 21.6 \\
\hline 2007-02-27 09:00:00.0 & 18.8 \\
\hline 2007-02-27 06:00:00.0 & 18.3 \\
\hline 2007-02-27 03:00:00.0 & 14.5 \\
\hline 2007-02-27 00:00:00.0 & 11.5 \\
\hline 2007-02-26 21:00:00.0 & 11.9 \\
\hline 2007-02-26 18:00:00.0 & 14.1 \\
\hline 2007-02-26 15:00:00.0 & 16.9 \\
\hline 2007-02-26 12:00:00.0 & 15.7 \\
\hline 2007-02-26 09:00:00.0 & \\
\hline 2007-02-26 06:00:00.0 & 18.0 \\
\hline 2007-02-26 03:00:00.0 & 18.0 \\
\hline 2007-02-26 00:00:00.0 & 11.9 \\
\hline 2007-02-25 21:00:00.0 & 14.9 \\
\hline 2007-02-25 18:00:00.0 & \\
\hline
\end{tabular}




\section{ANEXO 01 - RAJADAS DE VENTO REGISTRADAS EM SÃO MARTINHO DA SERRA / RS}

\begin{tabular}{|c|c|}
\hline DataHora & VelVentoMax \\
\hline 2007-02-25 15:00:00.0 & 12.0 \\
\hline 2007-02-25 12:00:00.0 & 9.2 \\
\hline 2007-02-25 09:00:00.0 & \\
\hline 2007-02-25 06:00:00.0 & 13.7 \\
\hline 2007-02-25 03:00:00.0 & 13.9 \\
\hline 2007-02-25 00:00:00.0 & 12.8 \\
\hline 2007-02-24 21:00:00.0 & \\
\hline 2007-02-24 18:00:00.0 & 24.0 \\
\hline 2007-02-24 15:00:00.0 & 18.0 \\
\hline 2007-02-24 12:00:00.0 & 17.2 \\
\hline 2007-02-24 09:00:00.0 & 16.8 \\
\hline 2007-02-24 06:00:00.0 & 23.7 \\
\hline 2007-02-24 03:00:00.0 & 27.6 \\
\hline 2007-02-24 00:00:00.0 & 16.6 \\
\hline 2007-02-23 21:00:00.0 & 28.7 \\
\hline 2007-02-23 18:00:00.0 & 7.5 \\
\hline 2007-02-23 15:00:00.0 & 28.7 \\
\hline 2007-02-23 12:00:00.0 & 18.7 \\
\hline 2007-02-23 09:00:00.0 & \\
\hline 2007-02-23 06:00:00.0 & 15.9 \\
\hline 2007-02-23 03:00:00.0 & 19.4 \\
\hline 2007-02-23 00:00:00.0 & 14.1 \\
\hline 2007-02-22 21:00:00.0 & 34.5 \\
\hline 2007-02-22 18:00:00.0 & 31.2 \\
\hline 2007-02-22 15:00:00.0 & 29.7 \\
\hline 2007-02-22 12:00:00.0 & 49.2 \\
\hline 2007-02-22 09:00:00.0 & 24.6 \\
\hline 2007-02-22 06:00:00.0 & 19.6 \\
\hline 2007-02-22 03:00:00.0 & 16.0 \\
\hline 2007-02-22 00:00:00.0 & 19.6 \\
\hline 2007-02-21 21:00:00.0 & 21.9 \\
\hline 2007-02-21 18:00:00.0 & 18.7 \\
\hline 2007-02-21 15:00:00.0 & 24.4 \\
\hline 2007-02-21 12:00:00.0 & 21.6 \\
\hline 2007-02-21 09:00:00.0 & 20.5 \\
\hline 2007-02-21 06:00:00.0 & 20.7 \\
\hline 2007-02-21 03:00:00.0 & 22.6 \\
\hline 2007-02-21 00:00:00.0 & 24.9 \\
\hline 2007-02-20 21:00:00.0 & 24.9 \\
\hline 2007-02-20 18:00:00.0 & 21.9 \\
\hline 2007-02-20 15:00:00.0 & 23.0 \\
\hline 2007-02-20 12:00:00.0 & 21.7 \\
\hline 2007-02-20 09:00:00.0 & 16.9 \\
\hline 2007-02-20 06:00:00.0 & 18.4 \\
\hline 2007-02-20 03:00:00.0 & 19.9 \\
\hline 2007-02-20 00:00:00.0 & 22.8 \\
\hline 2007-02-19 21:00:00.0 & 22.5 \\
\hline 2007-02-19 18:00:00.0 & 23.0 \\
\hline 2007-02-19 15:00:00.0 & 27.0 \\
\hline 2007-02-19 12:00:00.0 & 28.1 \\
\hline 2007-02-19 09:00:00.0 & 14.4 \\
\hline 2007-02-19 06:00:00.0 & 11.8 \\
\hline 2007-02-19 03:00:00.0 & 10.2 \\
\hline 2007-02-19 00:00:00.0 & 13.8 \\
\hline 2007-02-18 21:00:00.0 & 17.2 \\
\hline 2007-02-18 18:00:00.0 & 32.4 \\
\hline 2007-02-18 15:00:00.0 & 21.8 \\
\hline 2007-02-18 12:00:00.0 & 22.0 \\
\hline 2007-02-18 09:00:00.0 & 24.7 \\
\hline 2007-02-18 06:00:00.0 & 22.6 \\
\hline 2007-02-18 03:00:00.0 & 22.2 \\
\hline 2007-02-18 00:00:00.0 & \\
\hline 2007-02-17 21:00:00.0 & 19.0 \\
\hline 2007-02-17 18:00:00.0 & 23.2 \\
\hline 2007-02-17 15:00:00.0 & 25.2 \\
\hline 2007-02-17 12:00:00.0 & 51.0 \\
\hline 2007-02-17 09:00:00.0 & 24.3 \\
\hline 2007-02-17 06:00:00.0 & 16.5 \\
\hline 2007-02-17 03:00:00.0 & 13.1 \\
\hline
\end{tabular}

\begin{tabular}{|c|c|}
\hline DataHora & VelVentoMax \\
\hline 2007-02-17 00:00:00.0 & 13.6 \\
\hline 2007-02-16 21:00:00.0 & 22.5 \\
\hline 2007-02-16 18:00:00.0 & 24.5 \\
\hline 2007-02-16 15:00:00.0 & 23.3 \\
\hline 2007-02-16 12:00:00.0 & 19.4 \\
\hline 2007-02-16 09:00:00.0 & 17.6 \\
\hline 2007-02-16 06:00:00.0 & 14.8 \\
\hline 2007-02-16 03:00:00.0 & 14.9 \\
\hline 2007-02-16 00:00:00.0 & 14.9 \\
\hline 2007-02-15 21:00:00.0 & 12.9 \\
\hline 2007-02-15 18:00:00.0 & 13.0 \\
\hline 2007-02-15 15:00:00.0 & 12.1 \\
\hline 2007-02-15 12:00:00.0 & 17.2 \\
\hline 2007-02-15 09:00:00.0 & 17.3 \\
\hline 2007-02-15 06:00:00.0 & 6.5 \\
\hline 2007-02-15 03:00:00.0 & 13.1 \\
\hline 2007-02-15 00:00:00.0 & 12.1 \\
\hline 2007-02-14 21:00:00.0 & 12.9 \\
\hline 2007-02-14 18:00:00.0 & 24.9 \\
\hline 2007-02-14 15:00:00.0 & 13.8 \\
\hline 2007-02-14 12:00:00.0 & 16.5 \\
\hline 2007-02-14 09:00:00.0 & 16.0 \\
\hline 2007-02-14 06:00:00.0 & 16.0 \\
\hline 2007-02-14 03:00:00.0 & 16.0 \\
\hline 2007-02-14 00:00:00.0 & 15.0 \\
\hline 2007-02-13 21:00:00.0 & 20.0 \\
\hline 2007-02-13 18:00:00.0 & 17.0 \\
\hline 2007-02-13 15:00:00.0 & 22.0 \\
\hline 2007-02-13 12:00:00.0 & 26.1 \\
\hline 2007-02-13 09:00:00.0 & 21.8 \\
\hline 2007-02-13 06:00:00.0 & 18.8 \\
\hline 2007-02-13 03:00:00.0 & 16.3 \\
\hline 2007-02-13 00:00:00.0 & 22.5 \\
\hline 2007-02-12 21:00:00.0 & 22.7 \\
\hline 2007-02-12 18:00:00.0 & 20.3 \\
\hline 2007-02-12 15:00:00.0 & 23.0 \\
\hline 2007-02-12 12:00:00.0 & 24.6 \\
\hline 2007-02-12 09:00:00.0 & 13.9 \\
\hline 2007-02-12 06:00:00.0 & 19.7 \\
\hline 2007-02-12 03:00:00.0 & 23.7 \\
\hline 2007-02-12 00:00:00.0 & 27.1 \\
\hline 2007-02-11 21:00:00.0 & 26.3 \\
\hline 2007-02-11 18:00:00.0 & 30.5 \\
\hline 2007-02-11 15:00:00.0 & 28.6 \\
\hline 2007-02-11 12:00:00.0 & 25.5 \\
\hline 2007-02-11 09:00:00.0 & 20.7 \\
\hline 2007-02-11 06:00:00.0 & 21.1 \\
\hline 2007-02-11 03:00:00.0 & \\
\hline 2007-02-11 00:00:00.0 & 17.3 \\
\hline 2007-02-10 21:00:00.0 & 25.9 \\
\hline 2007-02-10 18:00:00.0 & 24.4 \\
\hline 2007-02-10 15:00:00.0 & 17.7 \\
\hline 2007-02-10 12:00:00.0 & 13.7 \\
\hline 2007-02-10 09:00:00.0 & 17.9 \\
\hline 2007-02-10 06:00:00.0 & 14.9 \\
\hline 2007-02-10 03:00:00.0 & \\
\hline 2007-02-10 00:00:00.0 & 17.6 \\
\hline 2007-02-09 21:00:00.0 & 35.0 \\
\hline 2007-02-09 18:00:00.0 & 20.5 \\
\hline 2007-02-09 15:00:00.0 & 23.1 \\
\hline 2007-02-09 12:00:00.0 & 23.5 \\
\hline 2007-02-09 09:00:00.0 & 24.2 \\
\hline 2007-02-09 06:00:00.0 & 20.4 \\
\hline 2007-02-09 03:00:00.0 & 17.8 \\
\hline 2007-02-09 00:00:00.0 & 38.4 \\
\hline 2007-02-08 21:00:00.0 & 23.7 \\
\hline 2007-02-08 18:00:00.0 & 12.3 \\
\hline 2007-02-08 15:00:00.0 & 18.6 \\
\hline 2007-02-08 12:00:00.0 & 19.8 \\
\hline
\end{tabular}

\begin{tabular}{|c|c|}
\hline DataHora & VelVentoMax \\
\hline 2007-02-08 09:00:00.0 & 21.0 \\
\hline 2007-02-08 06:00:00.0 & \\
\hline 2007-02-08 03:00:00.0 & 17.6 \\
\hline 2007-02-08 00:00:00.0 & 15.3 \\
\hline 2007-02-07 21:00:00.0 & 16.9 \\
\hline 2007-02-07 18:00:00.0 & 19.3 \\
\hline 2007-02-07 15:00:00.0 & 18.0 \\
\hline 2007-02-07 12:00:00.0 & 17.2 \\
\hline 2007-02-07 09:00:00.0 & 18.0 \\
\hline 2007-02-07 06:00:00.0 & 24.4 \\
\hline 2007-02-07 03:00:00.0 & 9.2 \\
\hline 2007-02-07 00:00:00.0 & 17.8 \\
\hline 2007-02-06 21:00:00.0 & 27.7 \\
\hline 2007-02-06 18:00:00.0 & 31.5 \\
\hline 2007-02-06 15:00:00.0 & 33.9 \\
\hline 2007-02-06 12:00:00.0 & 19.4 \\
\hline 2007-02-06 09:00:00.0 & 12.1 \\
\hline 2007-02-06 06:00:00.0 & 9.4 \\
\hline 2007-02-06 03:00:00.0 & 15.7 \\
\hline 2007-02-06 00:00:00.0 & \\
\hline 2007-02-05 21:00:00.0 & \\
\hline 2007-02-05 18:00:00.0 & \\
\hline 2007-02-05 15:00:00.0 & 23.6 \\
\hline 2007-02-05 12:00:00.0 & 18.2 \\
\hline 2007-02-05 09:00:00.0 & 16.4 \\
\hline 2007-02-05 06:00:00.0 & 14.7 \\
\hline 2007-02-05 03:00:00.0 & \\
\hline 2007-02-05 00:00:00.0 & 21.8 \\
\hline 2007-02-04 21:00:00.0 & 21.1 \\
\hline 2007-02-04 18:00:00.0 & 39.8 \\
\hline 2007-02-04 15:00:00.0 & 21.6 \\
\hline 2007-02-04 12:00:00.0 & 21.7 \\
\hline 2007-02-04 09:00:00.0 & 20.1 \\
\hline 2007-02-04 06:00:00.0 & 14.9 \\
\hline 2007-02-04 03:00:00.0 & 15.5 \\
\hline 2007-02-04 00:00:00.0 & 17.7 \\
\hline 2007-02-03 21:00:00.0 & 19.9 \\
\hline 2007-02-03 18:00:00.0 & 27.1 \\
\hline 2007-02-03 15:00:00.0 & 14.3 \\
\hline 2007-02-03 12:00:00.0 & 15.9 \\
\hline 2007-02-03 09:00:00.0 & 16.8 \\
\hline 2007-02-03 06:00:00.0 & \\
\hline 2007-02-03 03:00:00.0 & 13.9 \\
\hline 2007-02-03 00:00:00.0 & 12.1 \\
\hline 2007-02-02 21:00:00.0 & 14.8 \\
\hline 2007-02-02 18:00:00.0 & 16.3 \\
\hline 2007-02-02 15:00:00.0 & 15.9 \\
\hline 2007-02-02 12:00:00.0 & 12.2 \\
\hline 2007-02-02 09:00:00.0 & 13.4 \\
\hline 2007-02-02 06:00:00.0 & 10.7 \\
\hline 2007-02-02 03:00:00.0 & 7.6 \\
\hline 2007-02-02 00:00:00.0 & 8.2 \\
\hline 2007-02-01 21:00:00.0 & 13.2 \\
\hline 2007-02-01 18:00:00.0 & 16.7 \\
\hline 2007-02-01 15:00:00.0 & 15.6 \\
\hline 2007-02-01 12:00:00.0 & 10.7 \\
\hline 2007-02-01 09:00:00.0 & 11.0 \\
\hline 2007-02-01 06:00:00.0 & 10.4 \\
\hline 2007-02-01 03:00:00.0 & 13.6 \\
\hline 2007-02-01 00:00:00.0 & 14.0 \\
\hline 2007-01-31 21:00:00.0 & 16.4 \\
\hline 2007-01-31 18:00:00.0 & 17.6 \\
\hline 2007-01-31 15:00:00.0 & 11.5 \\
\hline 2007-01-31 12:00:00.0 & 11.6 \\
\hline 2007-01-31 09:00:00.0 & 13.3 \\
\hline 2007-01-31 06:00:00.0 & 13.0 \\
\hline 2007-01-31 03:00:00.0 & 14.0 \\
\hline 2007-01-31 00:00:00.0 & 14.0 \\
\hline 2007-01-30 21:00:00.0 & 15.0 \\
\hline
\end{tabular}




\section{ANEXO 01 - RAJADAS DE VENTO REGISTRADAS EM SÃO MARTINHO DA SERRA / RS}

\begin{tabular}{|c|c|}
\hline DataHora & VelVentoMax \\
\hline 2007-01-30 18:00:00.0 & 14.0 \\
\hline 2007-01-30 15:00:00.0 & 15.0 \\
\hline 2007-01-30 12:00:00.0 & 14.0 \\
\hline 2007-01-30 09:00:00.0 & 13.0 \\
\hline 2007-01-30 06:00:00.0 & 16.0 \\
\hline 2007-01-30 03:00:00.0 & 17.0 \\
\hline 2007-01-30 00:00:00.0 & 19.0 \\
\hline 2007-01-29 21:00:00.0 & 21.0 \\
\hline 2007-01-29 18:00:00.0 & 19.0 \\
\hline 2007-01-29 15:00:00.0 & 17.0 \\
\hline 2007-01-29 12:00:00.0 & 20.0 \\
\hline 2007-01-29 09:00:00.0 & 18.0 \\
\hline 2007-01-29 06:00:00.0 & 23.0 \\
\hline 2007-01-29 03:00:00.0 & 26.0 \\
\hline 2007-01-29 00:00:00.0 & \\
\hline 2007-01-28 21:00:00.0 & 22.0 \\
\hline 2007-01-28 18:00:00.0 & 15.0 \\
\hline 2007-01-28 15:00:00.0 & 16.0 \\
\hline 2007-01-28 12:00:00.0 & 18.0 \\
\hline 2007-01-28 09:00:00.0 & \\
\hline 2007-01-28 06:00:00.0 & \\
\hline 2007-01-28 03:00:00.0 & 8.0 \\
\hline 2007-01-28 00:00:00.0 & 23.0 \\
\hline 2007-01-27 21:00:00.0 & \\
\hline 2007-01-27 18:00:00.0 & 17.0 \\
\hline 2007-01-27 15:00:00.0 & 14.0 \\
\hline 2007-01-27 12:00:00.0 & 20.0 \\
\hline 2007-01-27 09:00:00.0 & \\
\hline 2007-01-27 06:00:00.0 & 13.0 \\
\hline 2007-01-27 03:00:00.0 & 9.0 \\
\hline 2007-01-27 00:00:00.0 & \\
\hline 2007-01-26 18:00:00.0 & 18.7 \\
\hline 2007-01-26 15:00:00.0 & 18.5 \\
\hline 2007-01-26 12:00:00.0 & \\
\hline 2007-01-26 09:00:00.0 & 15.0 \\
\hline 2007-01-26 06:00:00.0 & 12.0 \\
\hline 2007-01-26 03:00:00.0 & 19.6 \\
\hline 2007-01-26 00:00:00.0 & 19.4 \\
\hline 2007-01-25 21:00:00.0 & 16.8 \\
\hline 2007-01-25 18:00:00.0 & 19.2 \\
\hline 2007-01-25 15:00:00.0 & 19.0 \\
\hline 2007-01-25 12:00:00.0 & 14.9 \\
\hline 2007-01-25 09:00:00.0 & 13.1 \\
\hline 2007-01-25 06:00:00.0 & 9.0 \\
\hline 2007-01-25 03:00:00.0 & 10.4 \\
\hline 2007-01-25 00:00:00.0 & 17.1 \\
\hline 2007-01-24 21:00:00.0 & 16.3 \\
\hline 2007-01-24 18:00:00.0 & 15.7 \\
\hline 2007-01-24 15:00:00.0 & 15.7 \\
\hline 2007-01-24 12:00:00.0 & 22.1 \\
\hline 2007-01-24 09:00:00.0 & \\
\hline 2007-01-24 06:00:00.0 & 13.6 \\
\hline 2007-01-24 03:00:00.0 & 14.6 \\
\hline 2007-01-24 00:00:00.0 & \\
\hline 2007-01-23 21:00:00.0 & 15.4 \\
\hline 2007-01-23 18:00:00.0 & 16.5 \\
\hline 2007-01-23 15:00:00.0 & 22.8 \\
\hline 2007-01-23 12:00:00.0 & 18.0 \\
\hline 2007-01-23 09:00:00.0 & 17.7 \\
\hline 2007-01-23 06:00:00.0 & 17.6 \\
\hline 2007-01-23 03:00:00.0 & \\
\hline 2007-01-23 00:00:00.0 & 15.4 \\
\hline 2007-01-22 21:00:00.0 & 15.5 \\
\hline 2007-01-22 18:00:00.0 & 13.5 \\
\hline 2007-01-22 15:00:00.0 & 19.9 \\
\hline 2007-01-22 12:00:00.0 & 21.4 \\
\hline 2007-01-22 09:00:00.0 & 20.2 \\
\hline 2007-01-22 06:00:00.0 & 21.8 \\
\hline 2007-01-22 03:00:00.0 & 20.0 \\
\hline
\end{tabular}

\begin{tabular}{|c|c|}
\hline DataHora & VelVentoMax \\
\hline 2007-01-22 00:00:00.0 & 21.3 \\
\hline 2007-01-21 21:00:00.0 & 24.6 \\
\hline 2007-01-21 18:00:00.0 & 19.2 \\
\hline 2007-01-21 15:00:00.0 & 26.1 \\
\hline 2007-01-21 12:00:00.0 & 26.7 \\
\hline 2007-01-21 09:00:00.0 & 15.1 \\
\hline 2007-01-21 06:00:00.0 & 16.4 \\
\hline 2007-01-21 03:00:00.0 & 21.1 \\
\hline 2007-01-21 00:00:00.0 & 27.4 \\
\hline 2007-01-20 21:00:00.0 & 33.1 \\
\hline 2007-01-20 18:00:00.0 & 31.5 \\
\hline 2007-01-20 15:00:00.0 & 31.1 \\
\hline 2007-01-20 12:00:00.0 & \\
\hline 2007-01-20 09:00:00.0 & 18.3 \\
\hline 2007-01-20 06:00:00.0 & 17.3 \\
\hline 2007-01-20 03:00:00.0 & 19.7 \\
\hline 2007-01-20 00:00:00.0 & 28.5 \\
\hline 2007-01-19 21:00:00.0 & 29.9 \\
\hline 2007-01-19 18:00:00.0 & 12.9 \\
\hline 2007-01-19 15:00:00.0 & 17.2 \\
\hline 2007-01-19 12:00:00.0 & 18.6 \\
\hline 2007-01-19 09:00:00.0 & 16.9 \\
\hline 2007-01-19 06:00:00.0 & 15.7 \\
\hline 2007-01-19 03:00:00.0 & 7.8 \\
\hline 2007-01-19 00:00:00.0 & 16.2 \\
\hline 2007-01-18 21:00:00.0 & 24.2 \\
\hline 2007-01-18 18:00:00.0 & 19.6 \\
\hline 2007-01-18 15:00:00.0 & \\
\hline 2007-01-18 12:00:00.0 & \\
\hline 2007-01-18 09:00:00.0 & \\
\hline 2007-01-17 12:00:00.0 & 20.5 \\
\hline 2007-01-17 09:00:00.0 & 19.9 \\
\hline 2007-01-17 06:00:00.0 & \\
\hline 2007-01-17 03:00:00.0 & 23.2 \\
\hline 2007-01-17 00:00:00.0 & 16.6 \\
\hline 2007-01-16 21:00:00.0 & 17.9 \\
\hline 2007-01-16 18:00:00.0 & 21.1 \\
\hline 2007-01-16 15:00:00.0 & 24.0 \\
\hline 2007-01-16 12:00:00.0 & 23.7 \\
\hline 2007-01-16 09:00:00.0 & 13.6 \\
\hline 2007-01-16 06:00:00.0 & 16.9 \\
\hline 2007-01-16 03:00:00.0 & \\
\hline 2007-01-16 00:00:00.0 & 21.1 \\
\hline 2007-01-15 21:00:00.0 & 22.4 \\
\hline 2007-01-15 18:00:00.0 & 20.3 \\
\hline 2007-01-15 15:00:00.0 & 18.1 \\
\hline 2007-01-15 12:00:00.0 & \\
\hline 2007-01-15 09:00:00.0 & 13.3 \\
\hline 2007-01-15 06:00:00.0 & 12.6 \\
\hline 2007-01-15 03:00:00.0 & 8.9 \\
\hline 2007-01-15 00:00:00.0 & \\
\hline 2007-01-14 21:00:00.0 & 15.3 \\
\hline 2007-01-14 18:00:00.0 & 18.2 \\
\hline 2007-01-14 15:00:00.0 & \\
\hline 2007-01-14 12:00:00.0 & \\
\hline 2007-01-14 09:00:00.0 & 22.2 \\
\hline 2007-01-14 06:00:00.0 & 29.6 \\
\hline 2007-01-14 03:00:00.0 & 5.0 \\
\hline 2007-01-14 00:00:00.0 & 14.0 \\
\hline 2007-01-13 21:00:00.0 & 15.0 \\
\hline 2007-01-13 18:00:00.0 & 14.0 \\
\hline 2007-01-13 15:00:00.0 & 33.0 \\
\hline 2007-01-13 12:00:00.0 & 13.0 \\
\hline 2007-01-13 09:00:00.0 & 12.0 \\
\hline 2007-01-13 06:00:00.0 & \\
\hline 2007-01-13 03:00:00.0 & 9.0 \\
\hline 2007-01-13 00:00:00.0 & \\
\hline 2007-01-12 21:00:00.0 & 18.0 \\
\hline 2007-01-12 18:00:00.0 & \\
\hline
\end{tabular}

\begin{tabular}{|c|c|}
\hline DataHora & VelVentoMax \\
\hline 2007-01-12 15:00:00.0 & 21.0 \\
\hline 2007-01-12 12:00:00.0 & 20.0 \\
\hline 2007-01-12 09:00:00.0 & 13.0 \\
\hline 2007-01-12 06:00:00.0 & \\
\hline 2007-01-12 03:00:00.0 & 9.0 \\
\hline 2007-01-12 00:00:00.0 & 8.0 \\
\hline 2007-01-11 21:00:00.0 & 15.0 \\
\hline 2007-01-11 18:00:00.0 & 19.0 \\
\hline 2007-01-11 15:00:00.0 & 17.0 \\
\hline 2007-01-11 12:00:00.0 & \\
\hline 2007-01-11 09:00:00.0 & \\
\hline 2007-01-11 06:00:00.0 & 17.0 \\
\hline 2007-01-11 03:00:00.0 & 36.0 \\
\hline 2007-01-11 00:00:00.0 & 36.0 \\
\hline 2007-01-10 21:00:00.0 & 15.0 \\
\hline 2007-01-10 18:00:00.0 & 19.0 \\
\hline 2007-01-10 15:00:00.0 & 17.0 \\
\hline 2007-01-10 12:00:00.0 & 17.0 \\
\hline 2007-01-10 09:00:00.0 & 16.0 \\
\hline 2007-01-10 06:00:00.0 & \\
\hline 2007-01-10 03:00:00.0 & 12.0 \\
\hline 2007-01-10 00:00:00.0 & 15.0 \\
\hline 2007-01-09 21:00:00.0 & 11.0 \\
\hline 2007-01-09 18:00:00.0 & 16.0 \\
\hline 2007-01-09 15:00:00.0 & 14.0 \\
\hline 2007-01-09 12:00:00.0 & 16.0 \\
\hline 2007-01-09 09:00:00.0 & 13.0 \\
\hline 2007-01-09 06:00:00.0 & 15.0 \\
\hline 2007-01-09 03:00:00.0 & 16.0 \\
\hline 2007-01-09 00:00:00.0 & 23.0 \\
\hline 2007-01-08 21:00:00.0 & 22.0 \\
\hline 2007-01-08 18:00:00.0 & 19.0 \\
\hline 2007-01-08 15:00:00.0 & 21.0 \\
\hline 2007-01-08 12:00:00.0 & 22.0 \\
\hline 2007-01-08 09:00:00.0 & \\
\hline 2007-01-08 06:00:00.0 & 25.0 \\
\hline 2007-01-08 03:00:00.0 & 26.0 \\
\hline 2007-01-08 00:00:00.0 & 27.0 \\
\hline 2007-01-07 21:00:00.0 & 25.0 \\
\hline 2007-01-07 18:00:00.0 & 21.0 \\
\hline 2007-01-07 15:00:00.0 & 23.0 \\
\hline 2007-01-07 12:00:00.0 & 26.0 \\
\hline 2007-01-07 09:00:00.0 & 17.0 \\
\hline 2007-01-07 06:00:00.0 & 19.0 \\
\hline 2007-01-07 03:00:00.0 & 21.0 \\
\hline 2007-01-07 00:00:00.0 & 23.0 \\
\hline 2007-01-06 21:00:00.0 & 23.0 \\
\hline 2007-01-06 18:00:00.0 & 22.0 \\
\hline 2007-01-06 15:00:00.0 & 23.0 \\
\hline 2007-01-06 12:00:00.0 & 21.0 \\
\hline 2007-01-06 09:00:00.0 & 15.0 \\
\hline 2007-01-06 06:00:00.0 & 19.0 \\
\hline 2007-01-06 03:00:00.0 & 26.0 \\
\hline 2007-01-06 00:00:00.0 & 24.0 \\
\hline 2007-01-05 21:00:00.0 & 26.0 \\
\hline 2007-01-05 18:00:00.0 & 24.0 \\
\hline 2007-01-05 15:00:00.0 & 4.0 \\
\hline 2007-01-05 12:00:00.0 & \\
\hline 2007-01-05 09:00:00.0 & 5.0 \\
\hline 2007-01-05 06:00:00.0 & 8.0 \\
\hline 2007-01-05 03:00:00.0 & 10.0 \\
\hline 2007-01-05 00:00:00.0 & 13.0 \\
\hline 2007-01-04 21:00:00.0 & 11.0 \\
\hline 2007-01-04 18:00:00.0 & 14.0 \\
\hline 2007-01-04 15:00:00.0 & 10.0 \\
\hline 2007-01-04 12:00:00.0 & 15.0 \\
\hline 2007-01-04 09:00:00.0 & \\
\hline 2007-01-04 06:00:00.0 & 10.0 \\
\hline 2007-01-04 03:00:00.0 & 10.0 \\
\hline
\end{tabular}




\section{ANEXO 01 - RAJADAS DE VENTO REGISTRADAS EM SÃO MARTINHO DA SERRA / RS}

\begin{tabular}{|c|c|}
\hline DataHora & VelVentoMax \\
\hline 2007-01-04 00:00:00.0 & 15.0 \\
\hline 2007-01-03 21:00:00.0 & 22.0 \\
\hline 2007-01-03 18:00:00.0 & 19.0 \\
\hline 2007-01-03 15:00:00.0 & 20.0 \\
\hline 2007-01-03 12:00:00.0 & 17.0 \\
\hline 2007-01-03 09:00:00.0 & 17.0 \\
\hline 2007-01-03 06:00:00.0 & 23.0 \\
\hline 2007-01-03 03:00:00.0 & 17.0 \\
\hline 2007-01-03 00:00:00.0 & 18.0 \\
\hline 2007-01-02 21:00:00.0 & 17.0 \\
\hline 2007-01-02 18:00:00.0 & 14.0 \\
\hline 2007-01-02 15:00:00.0 & 24.0 \\
\hline 2007-01-02 12:00:00.0 & 22.0 \\
\hline 2007-01-02 09:00:00.0 & 21.0 \\
\hline 2007-01-02 06:00:00.0 & 19.0 \\
\hline 2007-01-02 03:00:00.0 & 12.0 \\
\hline 2007-01-02 00:00:00.0 & 15.0 \\
\hline 2007-01-01 21:00:00.0 & 17.0 \\
\hline 2007-01-01 18:00:00.0 & 17.0 \\
\hline 2007-01-01 15:00:00.0 & 22.0 \\
\hline 2007-01-01 12:00:00.0 & 23.0 \\
\hline 2007-01-01 09:00:00.0 & 16.0 \\
\hline 2007-01-01 06:00:00.0 & 15.0 \\
\hline 2007-01-01 03:00:00.0 & 15.0 \\
\hline 2007-01-01 00:00:00.0 & 18.0 \\
\hline 2006-12-31 21:00:00.0 & 19.0 \\
\hline 2006-12-31 18:00:00.0 & 16.8 \\
\hline 2006-12-31 15:00:00.0 & 15.8 \\
\hline 2006-12-31 12:00:00.0 & 15.8 \\
\hline 2006-12-31 09:00:00.0 & 11.5 \\
\hline 2006-12-31 06:00:00.0 & 14.7 \\
\hline 2006-12-31 03:00:00.0 & 16.5 \\
\hline 2006-12-31 00:00:00.0 & \\
\hline 2006-12-30 21:00:00.0 & 14.1 \\
\hline 2006-12-30 18:00:00.0 & 20.1 \\
\hline 2006-12-30 15:00:00.0 & 11.2 \\
\hline 2006-12-30 12:00:00.0 & 14.1 \\
\hline 2006-12-30 09:00:00.0 & 14.6 \\
\hline 2006-12-30 06:00:00.0 & 14.3 \\
\hline 2006-12-30 03:00:00.0 & 16.6 \\
\hline 2006-12-30 00:00:00.0 & 28.5 \\
\hline 2006-12-29 21:00:00.0 & 12.7 \\
\hline 2006-12-29 18:00:00.0 & 18.8 \\
\hline 2006-12-29 15:00:00.0 & 21.8 \\
\hline 2006-12-29 12:00:00.0 & 25.7 \\
\hline 2006-12-29 09:00:00.0 & 19.0 \\
\hline 2006-12-29 06:00:00.0 & 20.0 \\
\hline 2006-12-29 03:00:00.0 & \\
\hline 2006-12-29 00:00:00.0 & 20.5 \\
\hline 2006-12-28 21:00:00.0 & 22.4 \\
\hline 2006-12-28 18:00:00.0 & 16.1 \\
\hline 2006-12-28 15:00:00.0 & 18.6 \\
\hline 2006-12-28 12:00:00.0 & 20.1 \\
\hline 2006-12-28 09:00:00.0 & 35.4 \\
\hline 2006-12-28 06:00:00.0 & 13.2 \\
\hline 2006-12-28 03:00:00.0 & 11.4 \\
\hline 2006-12-28 00:00:00.0 & 14.1 \\
\hline 2006-12-27 21:00:00.0 & 13.0 \\
\hline 2006-12-27 18:00:00.0 & 15.8 \\
\hline 2006-12-27 15:00:00.0 & 15.7 \\
\hline 2006-12-27 12:00:00.0 & 17.6 \\
\hline 2006-12-27 09:00:00.0 & 16.0 \\
\hline 2006-12-27 06:00:00.0 & 9.4 \\
\hline 2006-12-27 03:00:00.0 & 4.9 \\
\hline 2006-12-27 00:00:00.0 & 13.4 \\
\hline 2006-12-26 21:00:00.0 & 22.9 \\
\hline 2006-12-26 18:00:00.0 & 22.5 \\
\hline 2006-12-26 15:00:00.0 & 33.1 \\
\hline 2006-12-26 12:00:00.0 & 16.6 \\
\hline
\end{tabular}

\begin{tabular}{|c|c|}
\hline DataHora & VelVentoMax \\
\hline 2006-12-26 09:00:00.0 & 8.3 \\
\hline 2006-12-26 06:00:00.0 & 9.1 \\
\hline 2006-12-26 03:00:00.0 & 14.1 \\
\hline 2006-12-26 00:00:00.0 & 42.1 \\
\hline 2006-12-25 21:00:00.0 & 22.1 \\
\hline 2006-12-25 18:00:00.0 & 23.9 \\
\hline 2006-12-25 15:00:00.0 & 41.7 \\
\hline 2006-12-25 12:00:00.0 & 21.0 \\
\hline 2006-12-25 09:00:00.0 & 13.7 \\
\hline 2006-12-25 06:00:00.0 & 12.9 \\
\hline 2006-12-25 03:00:00.0 & 16.7 \\
\hline 2006-12-25 00:00:00.0 & 8.2 \\
\hline 2006-12-24 21:00:00.0 & 19.9 \\
\hline 2006-12-24 18:00:00.0 & 30.8 \\
\hline 2006-12-24 15:00:00.0 & 20.8 \\
\hline 2006-12-24 12:00:00.0 & 21.5 \\
\hline 2006-12-24 09:00:00.0 & 18.0 \\
\hline 2006-12-24 06:00:00.0 & 20.9 \\
\hline 2006-12-24 03:00:00.0 & 23.7 \\
\hline 2006-12-24 00:00:00.0 & 35.1 \\
\hline 2006-12-23 21:00:00.0 & 14.1 \\
\hline 2006-12-23 18:00:00.0 & 15.9 \\
\hline 2006-12-23 15:00:00.0 & \\
\hline 2006-12-23 12:00:00.0 & 25.3 \\
\hline 2006-12-23 09:00:00.0 & 16.2 \\
\hline 2006-12-23 06:00:00.0 & 14.0 \\
\hline 2006-12-23 03:00:00.0 & 15.8 \\
\hline 2006-12-23 00:00:00.0 & 21.8 \\
\hline 2006-12-22 21:00:00.0 & 21.6 \\
\hline 2006-12-22 18:00:00.0 & 41.5 \\
\hline 2006-12-22 15:00:00.0 & 16.2 \\
\hline 2006-12-22 12:00:00.0 & 34.5 \\
\hline 2006-12-22 09:00:00.0 & 15.9 \\
\hline 2006-12-22 06:00:00.0 & 12.9 \\
\hline 2006-12-22 03:00:00.0 & 11.7 \\
\hline 2006-12-22 00:00:00.0 & 11.4 \\
\hline 2006-12-21 21:00:00.0 & 13.1 \\
\hline 2006-12-21 18:00:00.0 & 11.8 \\
\hline 2006-12-21 15:00:00.0 & 31.5 \\
\hline 2006-12-21 12:00:00.0 & 32.5 \\
\hline 2006-12-21 09:00:00.0 & 7.9 \\
\hline 2006-12-21 06:00:00.0 & 5.5 \\
\hline 2006-12-21 03:00:00.0 & 9.6 \\
\hline 2006-12-21 00:00:00.0 & 25.5 \\
\hline 2006-12-20 21:00:00.0 & 24.4 \\
\hline 2006-12-20 18:00:00.0 & 37.9 \\
\hline 2006-12-20 15:00:00.0 & \\
\hline 2006-12-20 12:00:00.0 & 32.2 \\
\hline 2006-12-20 09:00:00.0 & 30.0 \\
\hline 2006-12-20 06:00:00.0 & 19.5 \\
\hline 2006-12-20 03:00:00.0 & 18.3 \\
\hline 2006-12-20 00:00:00.0 & 17.2 \\
\hline 2006-12-19 21:00:00.0 & 31.6 \\
\hline 2006-12-19 18:00:00.0 & 21.8 \\
\hline 2006-12-19 15:00:00.0 & 19.1 \\
\hline 2006-12-19 12:00:00.0 & 15.7 \\
\hline 2006-12-19 09:00:00.0 & 15.9 \\
\hline 2006-12-19 06:00:00.0 & 18.3 \\
\hline 2006-12-19 03:00:00.0 & 13.8 \\
\hline 2006-12-19 00:00:00.0 & 15.4 \\
\hline 2006-12-18 21:00:00.0 & 16.3 \\
\hline 2006-12-18 18:00:00.0 & 16.6 \\
\hline 2006-12-18 15:00:00.0 & \\
\hline 2006-12-18 12:00:00.0 & 17.3 \\
\hline 2006-12-18 09:00:00.0 & 20.7 \\
\hline 2006-12-18 06:00:00.0 & 39.8 \\
\hline 2006-12-18 03:00:00.0 & 43.3 \\
\hline 2006-12-18 00:00:00.0 & 11.9 \\
\hline 2006-12-17 21:00:00.0 & 32.9 \\
\hline
\end{tabular}

\begin{tabular}{|c|c|}
\hline DataHora & VelVentoMax \\
\hline 2006-12-17 18:00:00.0 & 23.6 \\
\hline 2006-12-17 15:00:00.0 & 20.8 \\
\hline 2006-12-17 12:00:00.0 & 16.3 \\
\hline 2006-12-17 09:00:00.0 & 17.6 \\
\hline 2006-12-17 06:00:00.0 & 20.4 \\
\hline 2006-12-17 03:00:00.0 & \\
\hline 2006-12-17 00:00:00.0 & 16.3 \\
\hline 2006-12-12 03:00:00.0 & 12.5 \\
\hline 2006-12-12 00:00:00.0 & 20.2 \\
\hline 2006-12-11 21:00:00.0 & 23.5 \\
\hline 2006-12-11 18:00:00.0 & 20.8 \\
\hline 2006-12-11 15:00:00.0 & 16.6 \\
\hline 2006-12-11 12:00:00.0 & 27.1 \\
\hline 2006-12-11 09:00:00.0 & 14.4 \\
\hline 2006-12-11 06:00:00.0 & 15.8 \\
\hline 2006-12-11 03:00:00.0 & 18.3 \\
\hline 2006-12-11 00:00:00.0 & 17.9 \\
\hline 2006-12-10 21:00:00.0 & \\
\hline 2006-12-10 18:00:00.0 & 24.5 \\
\hline 2006-12-10 15:00:00.0 & 23.7 \\
\hline 2006-12-10 12:00:00.0 & 28.4 \\
\hline 2006-12-10 09:00:00.0 & \\
\hline 2006-12-10 06:00:00.0 & 10.6 \\
\hline 2006-12-10 03:00:00.0 & 13.2 \\
\hline 2006-12-10 00:00:00.0 & 15.1 \\
\hline 2006-12-09 21:00:00.0 & \\
\hline 2006-12-09 18:00:00.0 & 33.2 \\
\hline 2006-12-09 15:00:00.0 & 20.5 \\
\hline 2006-12-09 12:00:00.0 & 14.6 \\
\hline 2006-12-09 09:00:00.0 & \\
\hline 2006-12-09 06:00:00.0 & 12.4 \\
\hline 2006-12-09 03:00:00.0 & 14.3 \\
\hline 2006-12-09 00:00:00.0 & \\
\hline 2006-12-08 21:00:00.0 & \\
\hline 2006-12-08 18:00:00.0 & 19.0 \\
\hline 2006-12-08 15:00:00.0 & 43.9 \\
\hline 2006-12-08 12:00:00.0 & 19.9 \\
\hline 2006-12-08 09:00:00.0 & 21.0 \\
\hline 2006-12-08 06:00:00.0 & 20.5 \\
\hline 2006-12-08 03:00:00.0 & 19.1 \\
\hline 2006-12-08 00:00:00.0 & \\
\hline 2006-12-07 21:00:00.0 & \\
\hline 2006-12-07 18:00:00.0 & 24.6 \\
\hline 2006-12-07 15:00:00.0 & 34.5 \\
\hline 2006-12-07 12:00:00.0 & 22.3 \\
\hline 2006-12-07 09:00:00.0 & 18.9 \\
\hline 2006-12-07 06:00:00.0 & 14.2 \\
\hline 2006-12-07 03:00:00.0 & 15.3 \\
\hline 2006-12-07 00:00:00.0 & \\
\hline 2006-12-06 21:00:00.0 & 28.3 \\
\hline 2006-12-06 18:00:00.0 & 28.3 \\
\hline 2006-12-06 15:00:00.0 & 33.3 \\
\hline 2006-12-06 12:00:00.0 & 18.4 \\
\hline 2006-12-06 09:00:00.0 & 13.9 \\
\hline 2006-12-06 06:00:00.0 & 20.4 \\
\hline 2006-12-06 03:00:00.0 & 22.1 \\
\hline 2006-12-06 00:00:00.0 & 26.2 \\
\hline 2006-12-05 21:00:00.0 & 27.2 \\
\hline 2006-12-05 18:00:00.0 & 21.5 \\
\hline 2006-12-05 15:00:00.0 & 20.5 \\
\hline 2006-12-05 12:00:00.0 & 21.5 \\
\hline 2006-12-05 09:00:00.0 & 18.8 \\
\hline 2006-12-05 06:00:00.0 & 20.4 \\
\hline 2006-12-05 03:00:00.0 & 23.3 \\
\hline 2006-12-05 00:00:00.0 & 28.1 \\
\hline 2006-12-04 21:00:00.0 & 26.0 \\
\hline 2006-12-04 18:00:00.0 & 22.9 \\
\hline 2006-12-04 15:00:00.0 & 17.0 \\
\hline 2006-12-04 12:00:00.0 & \\
\hline
\end{tabular}




\section{ANEXO 01 - RAJADAS DE VENTO REGISTRADAS EM SÃO MARTINHO DA SERRA / RS}

\begin{tabular}{|c|c|}
\hline DataHora & VelVentoMax \\
\hline 2006-12-04 09:00:00.0 & 11.3 \\
\hline 2006-12-04 06:00:00.0 & 10.7 \\
\hline 2006-12-04 03:00:00.0 & 12.4 \\
\hline 2006-12-04 00:00:00.0 & 19.4 \\
\hline 2006-12-03 21:00:00.0 & 19.5 \\
\hline 2006-12-03 18:00:00.0 & 20.0 \\
\hline 2006-12-03 15:00:00.0 & 13.6 \\
\hline 2006-12-03 12:00:00.0 & \\
\hline 2006-12-03 09:00:00.0 & 8.9 \\
\hline 2006-12-03 06:00:00.0 & 15.7 \\
\hline 2006-12-03 03:00:00.0 & 17.1 \\
\hline 2006-12-03 00:00:00.0 & 19.6 \\
\hline 2006-12-02 21:00:00.0 & 22.4 \\
\hline 2006-12-02 18:00:00.0 & 13.4 \\
\hline 2006-12-02 15:00:00.0 & 22.2 \\
\hline 2006-12-02 12:00:00.0 & \\
\hline 2006-12-02 09:00:00.0 & 20.9 \\
\hline 2006-12-02 06:00:00.0 & 19.8 \\
\hline 2006-12-02 03:00:00.0 & 17.1 \\
\hline 2006-12-02 00:00:00.0 & 19.3 \\
\hline 2006-12-01 21:00:00.0 & 17.8 \\
\hline 2006-12-01 18:00:00.0 & 26.1 \\
\hline 2006-12-01 15:00:00.0 & 25.0 \\
\hline 2006-12-01 12:00:00.0 & 26.9 \\
\hline 2006-12-01 09:00:00.0 & 23.5 \\
\hline 2006-12-01 06:00:00.0 & 21.3 \\
\hline 2006-12-01 03:00:00.0 & 18.4 \\
\hline 2006-12-01 00:00:00.0 & \\
\hline 2006-11-30 21:00:00.0 & 22.0 \\
\hline 2006-11-30 18:00:00.0 & 19.4 \\
\hline 2006-11-30 15:00:00.0 & 19.4 \\
\hline 2006-11-30 12:00:00.0 & 21.9 \\
\hline 2006-11-30 09:00:00.0 & 21.6 \\
\hline 2006-11-30 06:00:00.0 & 18.0 \\
\hline 2006-11-30 03:00:00.0 & \\
\hline 2006-11-30 00:00:00.0 & 28.5 \\
\hline 2006-11-29 21:00:00.0 & 24.3 \\
\hline 2006-11-29 18:00:00.0 & 19.4 \\
\hline 2006-11-29 15:00:00.0 & 17.0 \\
\hline 2006-11-29 12:00:00.0 & 13.9 \\
\hline 2006-11-29 09:00:00.0 & 13.8 \\
\hline 2006-11-29 06:00:00.0 & 13.7 \\
\hline 2006-11-29 03:00:00.0 & 17.7 \\
\hline 2006-11-29 00:00:00.0 & 19.5 \\
\hline 2006-11-28 21:00:00.0 & 20.6 \\
\hline 2006-11-28 18:00:00.0 & 18.4 \\
\hline 2006-11-28 15:00:00.0 & 14.2 \\
\hline 2006-11-28 12:00:00.0 & 13.3 \\
\hline 2006-11-28 09:00:00.0 & 16.5 \\
\hline 2006-11-28 06:00:00.0 & 15.4 \\
\hline 2006-11-28 03:00:00.0 & 24.4 \\
\hline 2006-11-28 00:00:00.0 & 27.3 \\
\hline 2006-11-27 21:00:00.0 & 22.4 \\
\hline 2006-11-27 18:00:00.0 & 22.6 \\
\hline 2006-11-27 15:00:00.0 & 28.3 \\
\hline 2006-11-27 12:00:00.0 & 31.6 \\
\hline 2006-11-27 09:00:00.0 & 23.3 \\
\hline 2006-11-27 06:00:00.0 & 23.6 \\
\hline 2006-11-27 03:00:00.0 & 33.5 \\
\hline 2006-11-27 00:00:00.0 & 32.0 \\
\hline 2006-11-26 21:00:00.0 & 32.7 \\
\hline 2006-11-26 18:00:00.0 & 25.9 \\
\hline 2006-11-26 15:00:00.0 & \\
\hline 2006-11-26 12:00:00.0 & \\
\hline 2006-11-26 09:00:00.0 & 21.7 \\
\hline 2006-11-26 06:00:00.0 & 11.9 \\
\hline 2006-11-26 03:00:00.0 & \\
\hline 2006-11-26 00:00:00.0 & \\
\hline 2006-11-25 21:00:00.0 & \\
\hline
\end{tabular}

\begin{tabular}{|c|c|}
\hline DataHora & VelVentoMax \\
\hline 2006-11-25 12:00:00.0 & 13.4 \\
\hline 2006-11-25 09:00:00.0 & 19.8 \\
\hline 2006-11-25 06:00:00.0 & 16.3 \\
\hline 2006-11-25 03:00:00.0 & 27.6 \\
\hline 2006-11-25 00:00:00.0 & 20.7 \\
\hline 2006-11-24 21:00:00.0 & 18.0 \\
\hline 2006-11-24 18:00:00.0 & \\
\hline 2006-11-24 15:00:00.0 & 22.1 \\
\hline 2006-11-24 12:00:00.0 & 21.6 \\
\hline 2006-11-24 09:00:00.0 & 20.6 \\
\hline 2006-11-24 06:00:00.0 & \\
\hline 2006-11-24 03:00:00.0 & 11.8 \\
\hline 2006-11-24 00:00:00.0 & 17.5 \\
\hline 2006-11-23 21:00:00.0 & 17.5 \\
\hline 2006-11-23 18:00:00.0 & 27.8 \\
\hline 2006-11-23 15:00:00.0 & 21.3 \\
\hline 2006-11-23 12:00:00.0 & 22.3 \\
\hline 2006-11-23 09:00:00.0 & 17.9 \\
\hline 2006-11-23 06:00:00.0 & \\
\hline 2006-11-23 03:00:00.0 & 11.2 \\
\hline 2006-11-23 00:00:00.0 & 14.7 \\
\hline 2006-11-22 21:00:00.0 & 19.0 \\
\hline 2006-11-22 18:00:00.0 & 17.9 \\
\hline 2006-11-22 15:00:00.0 & \\
\hline 2006-11-22 12:00:00.0 & 13.7 \\
\hline 2006-11-22 09:00:00.0 & 5.9 \\
\hline 2006-11-22 06:00:00.0 & \\
\hline 2006-11-22 03:00:00.0 & \\
\hline 2006-11-22 00:00:00.0 & 8.8 \\
\hline 2006-11-21 21:00:00.0 & 16.4 \\
\hline 2006-11-21 18:00:00.0 & 19.6 \\
\hline 2006-11-21 15:00:00.0 & 20.4 \\
\hline 2006-11-21 12:00:00.0 & 21.9 \\
\hline 2006-11-21 09:00:00.0 & 21.1 \\
\hline 2006-11-21 06:00:00.0 & \\
\hline 2006-11-21 03:00:00.0 & 14.8 \\
\hline 2006-11-21 00:00:00.0 & 15.7 \\
\hline 2006-11-20 21:00:00.0 & 20.7 \\
\hline 2006-11-20 18:00:00.0 & \\
\hline 2006-11-20 15:00:00.0 & 28.1 \\
\hline 2006-11-20 12:00:00.0 & \\
\hline 2006-11-20 09:00:00.0 & 22.9 \\
\hline 2006-11-20 06:00:00.0 & \\
\hline 2006-11-20 03:00:00.0 & \\
\hline 2006-11-20 00:00:00.0 & \\
\hline 2006-11-17 12:00:00.0 & 33.5 \\
\hline 2006-11-17 09:00:00.0 & 25.8 \\
\hline 2006-11-17 06:00:00.0 & \\
\hline 2006-11-17 03:00:00.0 & \\
\hline 2006-11-17 00:00:00.0 & \\
\hline 2006-11-16 21:00:00.0 & 34.1 \\
\hline 2006-11-16 18:00:00.0 & \\
\hline 2006-11-16 15:00:00.0 & 31.0 \\
\hline 2006-11-16 12:00:00.0 & 20.9 \\
\hline 2006-11-16 09:00:00.0 & 14.7 \\
\hline 2006-11-16 06:00:00.0 & \\
\hline 2006-11-16 03:00:00.0 & 14.6 \\
\hline 2006-11-16 00:00:00.0 & \\
\hline 2006-11-15 21:00:00.0 & \\
\hline 2006-11-15 18:00:00.0 & 25.6 \\
\hline 2006-11-15 15:00:00.0 & 27.0 \\
\hline 2006-11-15 12:00:00.0 & 27.9 \\
\hline 2006-11-15 09:00:00.0 & \\
\hline 2006-11-15 06:00:00.0 & 18.5 \\
\hline 2006-11-15 03:00:00.0 & 13.7 \\
\hline 2006-11-15 00:00:00.0 & 13.0 \\
\hline 2006-11-14 21:00:00.0 & 19.3 \\
\hline 2006-11-14 18:00:00.0 & 29.3 \\
\hline 2006-11-14 15:00:00.0 & 24.1 \\
\hline
\end{tabular}

\begin{tabular}{|c|c|}
\hline DataHora & VelVentoMax \\
\hline 2006-11-14 12:00:00.0 & \\
\hline 2006-11-14 09:00:00.0 & 23.5 \\
\hline 2006-11-14 06:00:00.0 & \\
\hline 2006-11-14 03:00:00.0 & 18.0 \\
\hline 2006-11-14 00:00:00.0 & \\
\hline 2006-11-13 21:00:00.0 & 21.2 \\
\hline 2006-11-13 18:00:00.0 & 23.7 \\
\hline 2006-11-13 15:00:00.0 & 29.3 \\
\hline 2006-11-13 12:00:00.0 & \\
\hline 2006-11-13 09:00:00.0 & \\
\hline 2006-11-13 06:00:00.0 & 22.8 \\
\hline 2006-11-13 03:00:00.0 & 22.3 \\
\hline 2006-11-13 00:00:00.0 & 21.6 \\
\hline 2006-11-12 21:00:00.0 & \\
\hline 2006-11-12 18:00:00.0 & 15.6 \\
\hline 2006-11-12 15:00:00.0 & 22.0 \\
\hline 2006-11-12 12:00:00.0 & 23.8 \\
\hline 2006-11-12 09:00:00.0 & 13.3 \\
\hline 2006-11-12 06:00:00.0 & 13.5 \\
\hline 2006-11-12 03:00:00.0 & 15.5 \\
\hline 2006-11-12 00:00:00.0 & 17.3 \\
\hline 2006-11-11 21:00:00.0 & 18.3 \\
\hline 2006-11-11 18:00:00.0 & \\
\hline 2006-11-11 15:00:00.0 & 17.4 \\
\hline 2006-11-11 12:00:00.0 & \\
\hline 2006-11-11 09:00:00.0 & \\
\hline 2006-11-11 06:00:00.0 & \\
\hline 2006-11-11 03:00:00.0 & 9.6 \\
\hline 2006-11-11 00:00:00.0 & 14.5 \\
\hline 2006-11-10 21:00:00.0 & 31.0 \\
\hline 2006-11-10 18:00:00.0 & 25.0 \\
\hline 2006-11-10 15:00:00.0 & 31.9 \\
\hline 2006-11-10 12:00:00.0 & \\
\hline 2006-11-10 09:00:00.0 & 15.3 \\
\hline 2006-11-10 06:00:00.0 & 13.4 \\
\hline 2006-11-10 03:00:00.0 & 6.1 \\
\hline 2006-11-10 00:00:00.0 & \\
\hline 2006-11-09 21:00:00.0 & 24.3 \\
\hline 2006-11-09 18:00:00.0 & 30.9 \\
\hline 2006-11-09 15:00:00.0 & 26.0 \\
\hline 2006-11-09 12:00:00.0 & \\
\hline 2006-11-09 09:00:00.0 & 13.2 \\
\hline 2006-11-09 06:00:00.0 & 21.0 \\
\hline 2006-11-09 03:00:00.0 & 15.4 \\
\hline 2006-11-09 00:00:00.0 & \\
\hline 2006-11-08 21:00:00.0 & 19.8 \\
\hline 2006-11-08 18:00:00.0 & 22.7 \\
\hline 2006-11-08 15:00:00.0 & 20.7 \\
\hline 2006-11-08 12:00:00.0 & 19.5 \\
\hline 2006-11-08 09:00:00.0 & 28.8 \\
\hline 2006-11-08 06:00:00.0 & 16.6 \\
\hline 2006-11-08 03:00:00.0 & 17.0 \\
\hline 2006-11-08 00:00:00.0 & \\
\hline 2006-11-07 21:00:00.0 & 20.0 \\
\hline 2006-11-07 18:00:00.0 & 20.1 \\
\hline 2006-11-07 15:00:00.0 & 26.6 \\
\hline 2006-11-07 12:00:00.0 & 27.9 \\
\hline 2006-11-07 09:00:00.0 & 14.8 \\
\hline 2006-11-07 06:00:00.0 & 21.6 \\
\hline 2006-11-07 03:00:00.0 & 11.3 \\
\hline 2006-11-07 00:00:00.0 & 21.3 \\
\hline 2006-11-06 21:00:00.0 & 26.9 \\
\hline 2006-11-06 18:00:00.0 & 26.5 \\
\hline 2006-11-06 15:00:00.0 & \\
\hline 2006-11-06 12:00:00.0 & 19.8 \\
\hline 2006-11-06 09:00:00.0 & 37.0 \\
\hline 2006-11-06 06:00:00.0 & 34.1 \\
\hline 2006-11-06 03:00:00.0 & 22.6 \\
\hline 2006-11-06 00:00:00.0 & 19.1 \\
\hline
\end{tabular}




\section{ANEXO 01 - RAJADAS DE VENTO REGISTRADAS EM SÃO MARTINHO DA SERRA / RS}

\begin{tabular}{|c|c|}
\hline DataHora & VelVentoMax \\
\hline 2006-11-05 21:00:00.0 & 11.5 \\
\hline 2006-11-05 18:00:00.0 & 26.9 \\
\hline 2006-11-05 15:00:00.0 & 23.5 \\
\hline 2006-11-05 12:00:00.0 & \\
\hline 2006-11-05 09:00:00.0 & 20.8 \\
\hline 2006-11-05 06:00:00.0 & 23.5 \\
\hline 2006-11-05 03:00:00.0 & 24.9 \\
\hline 2006-11-05 00:00:00.0 & \\
\hline 2006-11-04 21:00:00.0 & 27.3 \\
\hline 2006-11-04 18:00:00.0 & 25.1 \\
\hline 2006-11-04 15:00:00.0 & 29.2 \\
\hline 2006-11-04 12:00:00.0 & \\
\hline 2006-11-04 09:00:00.0 & 21.9 \\
\hline 2006-11-04 06:00:00.0 & 20.6 \\
\hline 2006-11-04 03:00:00.0 & 26.2 \\
\hline 2006-11-04 00:00:00.0 & \\
\hline 2006-11-03 21:00:00.0 & 26.3 \\
\hline 2006-11-03 18:00:00.0 & 23.3 \\
\hline 2006-11-03 15:00:00.0 & 19.9 \\
\hline 2006-11-03 12:00:00.0 & 19.8 \\
\hline 2006-11-03 09:00:00.0 & 20.0 \\
\hline 2006-11-03 06:00:00.0 & 16.0 \\
\hline 2006-11-03 03:00:00.0 & 12.2 \\
\hline 2006-11-03 00:00:00.0 & 14.4 \\
\hline 2006-11-02 21:00:00.0 & 15.8 \\
\hline 2006-11-02 18:00:00.0 & 15.4 \\
\hline 2006-11-02 15:00:00.0 & \\
\hline 2006-11-02 12:00:00.0 & 12.5 \\
\hline 2006-11-02 09:00:00.0 & 13.7 \\
\hline 2006-11-02 06:00:00.0 & 16.9 \\
\hline 2006-11-02 03:00:00.0 & \\
\hline 2006-11-02 00:00:00.0 & 21.7 \\
\hline 2006-11-01 21:00:00.0 & 17.4 \\
\hline 2006-11-01 18:00:00.0 & 22.6 \\
\hline 2006-11-01 15:00:00.0 & \\
\hline 2006-11-01 12:00:00.0 & 32.3 \\
\hline 2006-11-01 09:00:00.0 & 14.4 \\
\hline 2006-11-01 06:00:00.0 & 17.0 \\
\hline 2006-11-01 03:00:00.0 & \\
\hline 2006-11-01 00:00:00.0 & 20.4 \\
\hline 2006-10-31 21:00:00.0 & 20.5 \\
\hline 2006-10-31 18:00:00.0 & 26.0 \\
\hline 2006-10-31 15:00:00.0 & 22.1 \\
\hline 2006-10-31 12:00:00.0 & 18.2 \\
\hline 2006-10-31 09:00:00.0 & 17.6 \\
\hline 2006-10-31 06:00:00.0 & 16.5 \\
\hline 2006-10-31 03:00:00.0 & \\
\hline 2006-10-31 00:00:00.0 & 15.3 \\
\hline 2006-10-30 21:00:00.0 & 16.2 \\
\hline 2006-10-30 18:00:00.0 & 15.6 \\
\hline 2006-10-30 15:00:00.0 & 16.9 \\
\hline 2006-10-30 12:00:00.0 & 11.0 \\
\hline 2006-10-30 09:00:00.0 & 14.7 \\
\hline 2006-10-30 06:00:00.0 & 24.2 \\
\hline 2006-10-30 03:00:00.0 & 24.9 \\
\hline 2006-10-30 00:00:00.0 & 41.2 \\
\hline 2006-10-29 21:00:00.0 & 1.6 \\
\hline 2006-10-29 18:00:00.0 & 22.1 \\
\hline 2006-10-29 15:00:00.0 & 17.0 \\
\hline 2006-10-29 12:00:00.0 & 24.5 \\
\hline 2006-10-29 09:00:00.0 & 22.9 \\
\hline 2006-10-29 06:00:00.0 & \\
\hline 2006-10-29 03:00:00.0 & 15.9 \\
\hline 2006-10-29 00:00:00.0 & 17.1 \\
\hline 2006-10-28 21:00:00.0 & 14.2 \\
\hline 2006-10-28 18:00:00.0 & \\
\hline 2006-10-28 15:00:00.0 & 15.9 \\
\hline 2006-10-28 12:00:00.0 & 15.7 \\
\hline 2006-10-28 09:00:00.0 & 23.7 \\
\hline
\end{tabular}

\begin{tabular}{|c|c|}
\hline DataHora & VelVentoMax \\
\hline 2006-10-28 06:00:00.0 & 23.5 \\
\hline \multicolumn{2}{|l|}{ 2006-10-28 03:00:00.0 } \\
\hline 2006-10-28 00:00:00.0 & 21.5 \\
\hline 2006-10-27 21:00:00.0 & 20.5 \\
\hline 2006-10-27 18:00:00.0 & 20.0 \\
\hline 2006-10-27 15:00:00.0 & 29.6 \\
\hline 2006-10-27 12:00:00.0 & 27.3 \\
\hline 2006-10-27 09:00:00.0 & 24.6 \\
\hline \multicolumn{2}{|l|}{ 2006-10-27 06:00:00.0 } \\
\hline 2006-10-27 03:00:00.0 & 21.9 \\
\hline 2006-10-27 00:00:00.0 & 23.3 \\
\hline 2006-10-26 21:00:00.0 & 22.3 \\
\hline 2006-10-26 18:00:00.0 & 23.0 \\
\hline 2006-10-26 15:00:00.0 & 24.5 \\
\hline 2006-10-26 12:00:00.0 & 20.9 \\
\hline 2006-10-26 09:00:00.0 & 18.8 \\
\hline \multicolumn{2}{|l|}{ 2006-10-26 06:00:00.0 } \\
\hline 2006-10-26 03:00:00.0 & 6.6 \\
\hline \multicolumn{2}{|l|}{ 2006-10-26 00:00:00.0 } \\
\hline 2006-10-25 21:00:00.0 & 24.2 \\
\hline 2006-10-25 18:00:00.0 & 23.6 \\
\hline 2006-10-25 15:00:00.0 & 22.1 \\
\hline 2006-10-25 12:00:00.0 & 23.9 \\
\hline 2006-10-25 09:00:00.0 & 21.5 \\
\hline 2006-10-25 06:00:00.0 & 18.7 \\
\hline 2006-10-25 03:00:00.0 & 12.9 \\
\hline 2006-10-25 00:00:00.0 & 12.5 \\
\hline 2006-10-24 21:00:00.0 & 14.7 \\
\hline 2006-10-24 18:00:00.0 & 17.5 \\
\hline 2006-10-24 15:00:00.0 & 24.7 \\
\hline 2006-10-24 12:00:00.0 & 26.9 \\
\hline 2006-10-24 09:00:00.0 & 23.1 \\
\hline 2006-10-24 06:00:00.0 & 20.8 \\
\hline 2006-10-24 03:00:00.0 & 15.4 \\
\hline 2006-10-24 00:00:00.0 & 13.1 \\
\hline 2006-10-23 21:00:00.0 & 14.5 \\
\hline 2006-10-23 18:00:00.0 & 19.3 \\
\hline 2006-10-23 15:00:00.0 & 25.0 \\
\hline 2006-10-23 12:00:00.0 & 27.2 \\
\hline 2006-10-23 09:00:00.0 & 20.7 \\
\hline \multicolumn{2}{|l|}{ 2006-10-23 06:00:00.0 } \\
\hline 2006-10-23 03:00:00.0 & 13.7 \\
\hline 2006-10-23 00:00:00.0 & 16.8 \\
\hline 2006-10-22 21:00:00.0 & 20.0 \\
\hline 2006-10-22 18:00:00.0 & 20.3 \\
\hline 2006-10-22 15:00:00.0 & 20.2 \\
\hline 2006-10-22 12:00:00.0 & 11.5 \\
\hline 2006-10-22 09:00:00.0 & 6.9 \\
\hline 2006-10-22 06:00:00.0 & 10.4 \\
\hline 2006-10-22 03:00:00.0 & 12.2 \\
\hline 2006-10-22 00:00:00.0 & 11.5 \\
\hline \multicolumn{2}{|l|}{ 2006-10-21 21:00:00.0 } \\
\hline 2006-10-21 18:00:00.0 & 29.9 \\
\hline 2006-10-21 15:00:00.0 & 31.0 \\
\hline 2006-10-21 12:00:00.0 & 14.9 \\
\hline \multicolumn{2}{|l|}{ 2006-10-21 09:00:00.0 } \\
\hline 2006-10-21 06:00:00.0 & 20.1 \\
\hline 2006-10-21 03:00:00.0 & 23.6 \\
\hline 2006-10-21 00:00:00.0 & 22.0 \\
\hline 2006-10-20 21:00:00.0 & 27.3 \\
\hline 2006-10-20 18:00:00.0 & 21.9 \\
\hline 2006-10-20 15:00:00.0 & 19.0 \\
\hline 2006-10-20 12:00:00.0 & 24.0 \\
\hline 2006-10-20 09:00:00.0 & 17.0 \\
\hline 2006-10-20 06:00:00.0 & 18.0 \\
\hline 2006-10-20 03:00:00.0 & 23.0 \\
\hline 2006-10-20 00:00:00.0 & 20.0 \\
\hline 2006-10-19 21:00:00.0 & 29.0 \\
\hline 2006-10-19 18:00:00.0 & 25.0 \\
\hline
\end{tabular}

\begin{tabular}{|c|c|}
\hline DataHora & VelVentoMax \\
\hline 2006-10-19 15:00:00.0 & 20.0 \\
\hline 2006-10-19 12:00:00.0 & 35.0 \\
\hline 2006-10-19 09:00:00.0 & 19.0 \\
\hline 2006-10-19 06:00:00.0 & 21.0 \\
\hline 2006-10-19 03:00:00.0 & 17.0 \\
\hline 2006-10-19 00:00:00.0 & 19.0 \\
\hline 2006-10-18 21:00:00.0 & 20.0 \\
\hline 2006-10-18 18:00:00.0 & 18.0 \\
\hline 2006-10-18 15:00:00.0 & 21.0 \\
\hline $2006-10-18$ 12:00:00.0 & 23.0 \\
\hline 2006-10-18 09:00:00.0 & \\
\hline 2006-10-18 06:00:00.0 & 20.0 \\
\hline 2006-10-18 03:00:00.0 & 20.0 \\
\hline 2006-10-18 00:00:00.0 & 22.0 \\
\hline 2006-10-17 21:00:00.0 & \\
\hline 2006-10-17 18:00:00.0 & 27.0 \\
\hline 2006-10-17 15:00:00.0 & 19.0 \\
\hline 2006-10-17 12:00:00.0 & 20.0 \\
\hline 2006-10-17 09:00:00.0 & \\
\hline 2006-10-17 06:00:00.0 & 18.0 \\
\hline 2006-10-17 03:00:00.0 & 21.0 \\
\hline 2006-10-17 00:00:00.0 & \\
\hline 2006-10-16 21:00:00.0 & 25.0 \\
\hline 2006-10-16 18:00:00.0 & 25.0 \\
\hline 2006-10-16 15:00:00.0 & 32.0 \\
\hline 2006-10-16 12:00:00.0 & 29.0 \\
\hline 2006-10-16 09:00:00.0 & 16.0 \\
\hline 2006-10-16 06:00:00.0 & 19.0 \\
\hline 2006-10-16 03:00:00.0 & 26.0 \\
\hline 2006-10-16 00:00:00.0 & \\
\hline 2006-10-15 21:00:00.0 & 17.0 \\
\hline 2006-10-15 18:00:00.0 & 29.0 \\
\hline 2006-10-15 15:00:00.0 & 30.0 \\
\hline 2006-10-15 12:00:00.0 & \\
\hline 2006-10-15 09:00:00.0 & 16.0 \\
\hline 2006-10-15 06:00:00.0 & 35.0 \\
\hline 2006-10-15 03:00:00.0 & 13.0 \\
\hline 2006-10-15 00:00:00.0 & 18.0 \\
\hline 2006-10-14 21:00:00.0 & 29.0 \\
\hline 2006-10-14 18:00:00.0 & 26.0 \\
\hline 2006-10-14 15:00:00.0 & 26.0 \\
\hline 2006-10-14 12:00:00.0 & 26.0 \\
\hline 2006-10-14 09:00:00.0 & 23.0 \\
\hline 2006-10-14 06:00:00.0 & 22.0 \\
\hline 2006-10-14 03:00:00.0 & 18.0 \\
\hline 2006-10-14 00:00:00.0 & 16.0 \\
\hline 2006-10-13 21:00:00.0 & 16.0 \\
\hline 2006-10-13 18:00:00.0 & 3.0 \\
\hline $2006-10-13$ 15:00:00.0 & 19.0 \\
\hline 2006-10-13 12:00:00.0 & \\
\hline 2006-10-13 09:00:00.0 & 15.0 \\
\hline 2006-10-13 06:00:00.0 & 16.0 \\
\hline 2006-10-13 03:00:00.0 & 19.0 \\
\hline $2006-10-13$ 00:00:00.0 & \\
\hline 2006-10-12 21:00:00.0 & \\
\hline 2006-10-12 18:00:00.0 & \\
\hline 2006-10-12 09:00:00.0 & 21.0 \\
\hline 2006-10-12 06:00:00.0 & 20.0 \\
\hline 2006-10-12 03:00:00.0 & \\
\hline 2006-10-12 00:00:00.0 & \\
\hline 2006-10-11 21:00:00.0 & \\
\hline $2006-10-11$ 12:00:00.0 & 32.0 \\
\hline 2006-10-11 09:00:00.0 & 18.0 \\
\hline 2006-10-11 06:00:00.0 & 26.0 \\
\hline 2006-10-11 03:00:00.0 & \\
\hline 2006-10-11 00:00:00.0 & \\
\hline 2006-10-10 21:00:00.0 & \\
\hline 2006-10-10 12:00:00.0 & 35.0 \\
\hline 2006-10-10 09:00:00.0 & 19.0 \\
\hline
\end{tabular}




\section{ANEXO 01 - RAJADAS DE VENTO REGISTRADAS EM SÃO MARTINHO DA SERRA / RS}

\begin{tabular}{|c|c|}
\hline DataHora & VelVentoMax \\
\hline 2006-10-10 06:00:00.0 & 19.0 \\
\hline \multicolumn{2}{|l|}{ 2006-10-10 03:00:00.0 } \\
\hline \multicolumn{2}{|l|}{ 2006-10-10 00:00:00.0 } \\
\hline 2006-10-09 21:00:00.0 & 12.0 \\
\hline 2006-10-09 18:00:00.0 & 13.0 \\
\hline 2006-10-09 15:00:00.0 & 16.0 \\
\hline 2006-10-09 12:00:00.0 & 21.0 \\
\hline 2006-10-09 09:00:00.0 & 25.0 \\
\hline 2006-10-09 06:00:00.0 & 18.0 \\
\hline \multicolumn{2}{|l|}{ 2006-10-09 03:00:00.0 } \\
\hline 2006-10-09 00:00:00.0 & 14.0 \\
\hline 2006-10-08 21:00:00.0 & 17.0 \\
\hline 2006-10-08 18:00:00.0 & 18.0 \\
\hline 2006-10-08 15:00:00.0 & 21.0 \\
\hline 2006-10-08 12:00:00.0 & 21.0 \\
\hline 2006-10-08 09:00:00.0 & 19.0 \\
\hline 2006-10-08 06:00:00.0 & 12.0 \\
\hline \multicolumn{2}{|l|}{ 2006-10-08 03:00:00.0 } \\
\hline 2006-10-08 00:00:00.0 & 11.0 \\
\hline 2006-10-07 21:00:00.0 & 20.0 \\
\hline 2006-10-07 18:00:00.0 & 21.0 \\
\hline \multicolumn{2}{|l|}{ 2006-10-07 15:00:00.0 } \\
\hline 2006-10-07 12:00:00.0 & 10.0 \\
\hline 2006-10-07 09:00:00.0 & 10.0 \\
\hline 2006-10-07 06:00:00.0 & 17.0 \\
\hline 2006-10-07 03:00:00.0 & 17.0 \\
\hline 2006-10-07 00:00:00.0 & 19.0 \\
\hline 2006-10-06 21:00:00.0 & 19.0 \\
\hline 2006-10-06 18:00:00.0 & 27.0 \\
\hline \multicolumn{2}{|l|}{ 2006-10-06 15:00:00.0 } \\
\hline 2006-10-06 12:00:00.0 & 22.0 \\
\hline 2006-10-06 09:00:00.0 & 22.0 \\
\hline 2006-10-06 06:00:00.0 & 41.0 \\
\hline 2006-10-06 03:00:00.0 & 13.0 \\
\hline 2006-10-06 00:00:00.0 & 18.0 \\
\hline 2006-10-05 21:00:00.0 & 25.0 \\
\hline \multicolumn{2}{|l|}{ 2006-10-05 18:00:00.0 } \\
\hline \multicolumn{2}{|l|}{ 2006-10-05 15:00:00.0 } \\
\hline 2006-10-05 12:00:00.0 & 15.0 \\
\hline 2006-10-05 09:00:00.0 & 35.0 \\
\hline \multicolumn{2}{|l|}{ 2006-10-05 06:00:00.0 } \\
\hline 2006-10-05 03:00:00.0 & 15.0 \\
\hline 2006-10-05 00:00:00.0 & 30.0 \\
\hline 2006-10-04 21:00:00.0 & 20.0 \\
\hline 2006-10-04 18:00:00.0 & 13.0 \\
\hline 2006-10-04 15:00:00.0 & 18.0 \\
\hline \multicolumn{2}{|l|}{ 2006-10-04 12:00:00.0 } \\
\hline 2006-10-04 09:00:00.0 & 16.0 \\
\hline 2006-10-04 06:00:00.0 & 14.0 \\
\hline 2006-10-04 03:00:00.0 & 36.0 \\
\hline 2006-10-04 00:00:00.0 & 21.0 \\
\hline 2006-10-03 21:00:00.0 & 12.0 \\
\hline 2006-10-03 18:00:00.0 & 14.0 \\
\hline 2006-10-03 15:00:00.0 & 15.0 \\
\hline 2006-10-03 12:00:00.0 & 21.0 \\
\hline 2006-10-03 09:00:00.0 & 24.0 \\
\hline \multicolumn{2}{|l|}{ 2006-10-03 06:00:00.0 } \\
\hline 2006-10-03 03:00:00.0 & 13.0 \\
\hline 2006-10-03 00:00:00.0 & 13.0 \\
\hline 2006-10-02 21:00:00.0 & 15.0 \\
\hline 2006-10-02 18:00:00.0 & 14.0 \\
\hline 2006-10-02 15:00:00.0 & 32.0 \\
\hline 2006-10-02 12:00:00.0 & 32.0 \\
\hline 2006-10-02 09:00:00.0 & 19.0 \\
\hline \multicolumn{2}{|l|}{ 2006-10-02 06:00:00.0 } \\
\hline 2006-10-02 03:00:00.0 & 16.7 \\
\hline 2006-10-02 00:00:00.0 & 12.5 \\
\hline \multicolumn{2}{|l|}{ 2006-10-01 21:00:00.0 } \\
\hline 2006-10-01 18:00:00.0 & 27.1 \\
\hline
\end{tabular}

\begin{tabular}{|c|c|}
\hline DataHora & VelVentoMax \\
\hline 2006-10-01 15:00:00.0 & 20.8 \\
\hline 2006-10-01 12:00:00.0 & 25.6 \\
\hline 2006-10-01 09:00:00.0 & 24.9 \\
\hline 2006-10-01 06:00:00.0 & \\
\hline 2006-10-01 03:00:00.0 & 21.9 \\
\hline 2006-10-01 00:00:00.0 & \\
\hline 2006-09-30 21:00:00.0 & 16.3 \\
\hline 2006-09-30 18:00:00.0 & 19.8 \\
\hline 2006-09-30 15:00:00.0 & 25.7 \\
\hline 2006-09-30 12:00:00.0 & 26.0 \\
\hline 2006-09-30 09:00:00.0 & 24.6 \\
\hline 2006-09-30 06:00:00.0 & \\
\hline 2006-09-30 03:00:00.0 & 16.7 \\
\hline 2006-09-30 00:00:00.0 & 15.7 \\
\hline 2006-09-29 21:00:00.0 & \\
\hline 2006-09-29 18:00:00.0 & \\
\hline 2006-09-29 15:00:00.0 & 25.5 \\
\hline 2006-09-29 12:00:00.0 & 25.4 \\
\hline 2006-09-29 09:00:00.0 & 22.5 \\
\hline 2006-09-29 06:00:00.0 & 25.1 \\
\hline 2006-09-29 03:00:00.0 & 22.7 \\
\hline 2006-09-29 00:00:00.0 & 19.0 \\
\hline 2006-09-28 21:00:00.0 & 17.6 \\
\hline 2006-09-28 18:00:00.0 & \\
\hline 2006-09-28 15:00:00.0 & 15.7 \\
\hline 2006-09-28 12:00:00.0 & 10.6 \\
\hline 2006-09-28 09:00:00.0 & \\
\hline 2006-09-28 06:00:00.0 & \\
\hline 2006-09-28 03:00:00.0 & 19.9 \\
\hline 2006-09-28 00:00:00.0 & 19.8 \\
\hline 2006-09-27 21:00:00.0 & 13.9 \\
\hline 2006-09-27 18:00:00.0 & \\
\hline 2006-09-27 15:00:00.0 & 27.4 \\
\hline 2006-09-27 12:00:00.0 & 27.6 \\
\hline 2006-09-27 09:00:00.0 & \\
\hline 2006-09-27 06:00:00.0 & \\
\hline 2006-09-27 03:00:00.0 & 26.2 \\
\hline 2006-09-27 00:00:00.0 & 14.9 \\
\hline 2006-09-26 21:00:00.0 & 22.5 \\
\hline 2006-09-26 18:00:00.0 & 36.0 \\
\hline 2006-09-26 15:00:00.0 & 28.6 \\
\hline 2006-09-26 12:00:00.0 & 26.7 \\
\hline 2006-09-26 09:00:00.0 & \\
\hline 2006-09-26 06:00:00.0 & 23.4 \\
\hline 2006-09-26 03:00:00.0 & 18.1 \\
\hline 2006-09-26 00:00:00.0 & 13.1 \\
\hline 2006-09-25 21:00:00.0 & \\
\hline 2006-09-25 18:00:00.0 & 15.9 \\
\hline 2006-09-25 15:00:00.0 & 17.2 \\
\hline 2006-09-25 12:00:00.0 & 13.2 \\
\hline 2006-09-25 09:00:00.0 & 10.6 \\
\hline 2006-09-25 06:00:00.0 & 11.4 \\
\hline 2006-09-25 03:00:00.0 & 10.9 \\
\hline 2006-09-25 00:00:00.0 & 11.5 \\
\hline 2006-09-24 21:00:00.0 & 15.6 \\
\hline 2006-09-24 18:00:00.0 & 16.1 \\
\hline 2006-09-24 15:00:00.0 & 31.2 \\
\hline 2006-09-24 12:00:00.0 & 26.0 \\
\hline 2006-09-24 09:00:00.0 & \\
\hline 2006-09-24 06:00:00.0 & 24.0 \\
\hline 2006-09-24 03:00:00.0 & 19.0 \\
\hline 2006-09-24 00:00:00.0 & 12.0 \\
\hline 2006-09-23 21:00:00.0 & 21.8 \\
\hline 2006-09-23 18:00:00.0 & 23.6 \\
\hline 2006-09-23 15:00:00.0 & 20.5 \\
\hline 2006-09-23 12:00:00.0 & \\
\hline 2006-09-23 09:00:00.0 & 33.8 \\
\hline 2006-09-23 06:00:00.0 & 11.1 \\
\hline 2006-09-23 03:00:00.0 & 12.0 \\
\hline
\end{tabular}

\begin{tabular}{|c|c|}
\hline DataHora & VelVentoMax \\
\hline 2006-09-23 00:00:00.0 & 15.2 \\
\hline 2006-09-22 21:00:00.0 & 18.6 \\
\hline 2006-09-22 18:00:00.0 & 23.7 \\
\hline 2006-09-22 15:00:00.0 & 22.3 \\
\hline 2006-09-22 12:00:00.0 & 17.7 \\
\hline 2006-09-22 09:00:00.0 & 16.8 \\
\hline 2006-09-22 06:00:00.0 & 13.0 \\
\hline 2006-09-22 03:00:00.0 & 14.5 \\
\hline 2006-09-22 00:00:00.0 & 16.6 \\
\hline 2006-09-21 21:00:00.0 & \\
\hline 2006-09-21 18:00:00.0 & 14.9 \\
\hline 2006-09-21 15:00:00.0 & 13.2 \\
\hline 2006-09-21 12:00:00.0 & \\
\hline 2006-09-21 09:00:00.0 & 10.7 \\
\hline 2006-09-21 06:00:00.0 & 10.8 \\
\hline 2006-09-21 03:00:00.0 & 12.3 \\
\hline 2006-09-21 00:00:00.0 & \\
\hline 2006-09-20 21:00:00.0 & 19.4 \\
\hline 2006-09-20 18:00:00.0 & 17.3 \\
\hline 2006-09-20 15:00:00.0 & 18.1 \\
\hline 2006-09-20 12:00:00.0 & \\
\hline 2006-09-20 09:00:00.0 & 17.3 \\
\hline 2006-09-20 06:00:00.0 & 16.7 \\
\hline 2006-09-20 03:00:00.0 & 19.4 \\
\hline 2006-09-20 00:00:00.0 & \\
\hline 2006-09-19 21:00:00.0 & 16.1 \\
\hline 2006-09-19 18:00:00.0 & 17.2 \\
\hline 2006-09-19 15:00:00.0 & 19.4 \\
\hline 2006-09-19 12:00:00.0 & \\
\hline 2006-09-19 09:00:00.0 & 16.9 \\
\hline 2006-09-19 06:00:00.0 & 16.1 \\
\hline 2006-09-19 03:00:00.0 & 14.1 \\
\hline 2006-09-19 00:00:00.0 & 13.8 \\
\hline 2006-09-18 21:00:00.0 & 31.2 \\
\hline 2006-09-18 18:00:00.0 & 14.6 \\
\hline 2006-09-18 15:00:00.0 & 29.0 \\
\hline 2006-09-18 12:00:00.0 & \\
\hline 2006-09-18 09:00:00.0 & 15.2 \\
\hline 2006-09-18 06:00:00.0 & 15.4 \\
\hline 2006-09-18 03:00:00.0 & 10.5 \\
\hline 2006-09-18 00:00:00.0 & 3.9 \\
\hline 2006-09-17 21:00:00.0 & 13.4 \\
\hline 2006-09-17 18:00:00.0 & 14.6 \\
\hline 2006-09-17 15:00:00.0 & 18.0 \\
\hline 2006-09-17 12:00:00.0 & \\
\hline 2006-09-17 09:00:00.0 & 12.4 \\
\hline 2006-09-17 06:00:00.0 & 16.7 \\
\hline 2006-09-17 03:00:00.0 & 14.4 \\
\hline 2006-09-17 00:00:00.0 & \\
\hline 2006-09-16 21:00:00.0 & 27.1 \\
\hline 2006-09-16 18:00:00.0 & 24.9 \\
\hline 2006-09-16 15:00:00.0 & \\
\hline 2006-09-16 12:00:00.0 & 17.7 \\
\hline 2006-09-16 09:00:00.0 & 11.6 \\
\hline 2006-09-16 06:00:00.0 & 11.2 \\
\hline 2006-09-16 03:00:00.0 & \\
\hline 2006-09-16 00:00:00.0 & \\
\hline 2006-09-15 21:00:00.0 & 25.2 \\
\hline 2006-09-15 18:00:00.0 & 22.7 \\
\hline 2006-09-15 15:00:00.0 & \\
\hline 2006-09-15 12:00:00.0 & \\
\hline 2006-09-15 09:00:00.0 & 35.2 \\
\hline 2006-09-15 06:00:00.0 & 43.0 \\
\hline 2006-09-15 03:00:00.0 & \\
\hline 2006-09-15 00:00:00.0 & 19.3 \\
\hline 2006-09-14 21:00:00.0 & 14.6 \\
\hline 2006-09-14 18:00:00.0 & 14.3 \\
\hline 2006-09-14 15:00:00.0 & 11.9 \\
\hline 2006-09-14 12:00:00.0 & 12.2 \\
\hline
\end{tabular}




\section{ANEXO 01 - RAJADAS DE VENTO REGISTRADAS EM SÃO MARTINHO DA SERRA / RS}

\begin{tabular}{|c|c|}
\hline DataHora & VelVentoMax \\
\hline 2006-09-14 09:00:00.0 & 12.9 \\
\hline 2006-09-14 06:00:00.0 & 16.2 \\
\hline 2006-09-14 03:00:00.0 & 10.8 \\
\hline \multicolumn{2}{|l|}{ 2006-09-14 00:00:00.0 } \\
\hline 2006-09-13 21:00:00.0 & 14.0 \\
\hline 2006-09-13 18:00:00.0 & 25.8 \\
\hline \multicolumn{2}{|l|}{ 2006-09-13 15:00:00.0 } \\
\hline 2006-09-13 12:00:00.0 & 30.6 \\
\hline 2006-09-13 09:00:00.0 & 25.9 \\
\hline 2006-09-13 06:00:00.0 & 29.0 \\
\hline 2006-09-13 03:00:00.0 & 30.0 \\
\hline 2006-09-13 00:00:00.0 & 25.5 \\
\hline 2006-09-12 21:00:00.0 & 22.5 \\
\hline 2006-09-12 18:00:00.0 & 28.3 \\
\hline \multicolumn{2}{|l|}{ 2006-09-12 15:00:00.0 } \\
\hline 2006-09-12 12:00:00.0 & 12.2 \\
\hline 2006-09-12 09:00:00.0 & 13.4 \\
\hline 2006-09-12 06:00:00.0 & 12.7 \\
\hline \multicolumn{2}{|l|}{ 2006-09-12 03:00:00.0 } \\
\hline 2006-09-12 00:00:00.0 & 14.3 \\
\hline 2006-09-11 21:00:00.0 & 30.7 \\
\hline 2006-09-11 18:00:00.0 & 34.3 \\
\hline 2006-09-11 15:00:00.0 & 31.4 \\
\hline 2006-09-11 12:00:00.0 & 28.2 \\
\hline 2006-09-11 09:00:00.0 & 27.8 \\
\hline 2006-09-11 06:00:00.0 & 23.8 \\
\hline 2006-09-11 03:00:00.0 & 19.8 \\
\hline 2006-09-11 00:00:00.0 & 15.9 \\
\hline 2006-09-10 21:00:00.0 & 21.7 \\
\hline 2006-09-10 18:00:00.0 & 22.2 \\
\hline \multicolumn{2}{|l|}{ 2006-09-10 15:00:00.0 } \\
\hline 2006-09-10 12:00:00.0 & 14.6 \\
\hline 2006-09-10 09:00:00.0 & 15.0 \\
\hline 2006-09-10 06:00:00.0 & 9.0 \\
\hline \multicolumn{2}{|l|}{ 2006-09-10 03:00:00.0 } \\
\hline 2006-09-10 00:00:00.0 & 8.0 \\
\hline 2006-09-09 21:00:00.0 & 16.5 \\
\hline 2006-09-09 18:00:00.0 & 17.4 \\
\hline 2006-09-09 15:00:00.0 & 18.7 \\
\hline 2006-09-09 12:00:00.0 & 14.8 \\
\hline 2006-09-09 09:00:00.0 & 10.3 \\
\hline \multicolumn{2}{|l|}{ 2006-09-09 06:00:00.0 } \\
\hline 2006-09-09 03:00:00.0 & 11.7 \\
\hline 2006-09-09 00:00:00.0 & 16.7 \\
\hline 2006-09-08 21:00:00.0 & 14.1 \\
\hline 2006-09-08 18:00:00.0 & 32.3 \\
\hline 2006-09-08 15:00:00.0 & 21.2 \\
\hline 2006-09-08 12:00:00.0 & 22.0 \\
\hline 2006-09-08 09:00:00.0 & 22.6 \\
\hline 2006-09-08 06:00:00.0 & 22.1 \\
\hline 2006-09-08 03:00:00.0 & 19.7 \\
\hline 2006-09-08 00:00:00.0 & 14.9 \\
\hline 2006-09-07 21:00:00.0 & 33.3 \\
\hline 2006-09-07 18:00:00.0 & 18.7 \\
\hline 2006-09-07 15:00:00.0 & 23.9 \\
\hline 2006-09-07 12:00:00.0 & 28.3 \\
\hline 2006-09-07 09:00:00.0 & 21.0 \\
\hline \multicolumn{2}{|l|}{ 2006-09-07 06:00:00.0 } \\
\hline 2006-09-07 03:00:00.0 & 19.1 \\
\hline 2006-09-07 00:00:00.0 & 12.0 \\
\hline 2006-09-06 21:00:00.0 & 16.7 \\
\hline 2006-09-06 18:00:00.0 & 22.8 \\
\hline 2006-09-06 15:00:00.0 & 19.3 \\
\hline 2006-09-06 12:00:00.0 & 19.6 \\
\hline 2006-09-06 09:00:00.0 & 12.7 \\
\hline \multicolumn{2}{|l|}{ 2006-09-06 06:00:00.0 } \\
\hline 2006-09-06 03:00:00.0 & 11.5 \\
\hline 2006-09-06 00:00:00.0 & 11.2 \\
\hline 2006-09-05 21:00:00.0 & 11.6 \\
\hline
\end{tabular}

\begin{tabular}{|c|c|}
\hline DataHora & VelVentoMax \\
\hline 2006-09-05 18:00:00.0 & 12.6 \\
\hline 2006-09-05 15:00:00.0 & 14.1 \\
\hline 2006-09-05 12:00:00.0 & 5.2 \\
\hline 2006-09-05 09:00:00.0 & 4.7 \\
\hline 2006-09-05 06:00:00.0 & 5.9 \\
\hline 2006-09-05 03:00:00.0 & 9.0 \\
\hline 2006-09-05 00:00:00.0 & \\
\hline 2006-09-04 21:00:00.0 & 28.7 \\
\hline 2006-09-04 18:00:00.0 & \\
\hline 2006-09-04 15:00:00.0 & 22.5 \\
\hline 2006-09-04 12:00:00.0 & 11.3 \\
\hline 2006-09-04 09:00:00.0 & 7.5 \\
\hline 2006-09-04 06:00:00.0 & \\
\hline 2006-09-04 03:00:00.0 & 16.0 \\
\hline 2006-09-04 00:00:00.0 & \\
\hline 2006-09-03 21:00:00.0 & 28.2 \\
\hline 2006-09-03 18:00:00.0 & \\
\hline 2006-09-03 15:00:00.0 & 27.5 \\
\hline 2006-09-03 12:00:00.0 & 11.4 \\
\hline 2006-09-03 09:00:00.0 & \\
\hline 2006-09-03 06:00:00.0 & 23.6 \\
\hline 2006-09-03 03:00:00.0 & 26.1 \\
\hline 2006-09-03 00:00:00.0 & 28.6 \\
\hline 2006-09-02 21:00:00.0 & 35.0 \\
\hline 2006-09-02 18:00:00.0 & 36.5 \\
\hline 2006-09-02 15:00:00.0 & 34.0 \\
\hline 2006-09-02 12:00:00.0 & 32.1 \\
\hline 2006-09-02 09:00:00.0 & 29.0 \\
\hline 2006-09-02 06:00:00.0 & 9.7 \\
\hline 2006-09-02 03:00:00.0 & 29.9 \\
\hline 2006-09-02 00:00:00.0 & 20.3 \\
\hline 2006-09-01 21:00:00.0 & 15.8 \\
\hline 2006-09-01 18:00:00.0 & 20.5 \\
\hline 2006-09-01 15:00:00.0 & 22.7 \\
\hline 2006-09-01 12:00:00.0 & 17.6 \\
\hline 2006-09-01 09:00:00.0 & 16.1 \\
\hline 2006-09-01 06:00:00.0 & \\
\hline 2006-09-01 03:00:00.0 & 11.3 \\
\hline 2006-09-01 00:00:00.0 & 13.7 \\
\hline 2006-08-31 21:00:00.0 & 12.6 \\
\hline 2006-08-31 18:00:00.0 & 18.6 \\
\hline 2006-08-31 15:00:00.0 & 22.3 \\
\hline 2006-08-31 12:00:00.0 & 23.6 \\
\hline 2006-08-31 06:00:00.0 & 19.7 \\
\hline 2006-08-31 03:00:00.0 & 10.8 \\
\hline 2006-08-31 00:00:00.0 & 17.1 \\
\hline 2006-08-30 21:00:00.0 & 18.8 \\
\hline 2006-08-30 18:00:00.0 & 23.5 \\
\hline 2006-08-30 15:00:00.0 & 29.2 \\
\hline 2006-08-30 12:00:00.0 & 25.6 \\
\hline 2006-08-30 09:00:00.0 & 25.2 \\
\hline 2006-08-30 06:00:00.0 & 24.0 \\
\hline 2006-08-30 03:00:00.0 & 17.9 \\
\hline 2006-08-30 00:00:00.0 & 13.6 \\
\hline 2006-08-29 18:00:00.0 & 22.6 \\
\hline 2006-08-29 15:00:00.0 & 18.5 \\
\hline 2006-08-29 12:00:00.0 & 7.3 \\
\hline 2006-08-29 09:00:00.0 & 8.7 \\
\hline 2006-08-29 06:00:00.0 & 7.7 \\
\hline 2006-08-29 03:00:00.0 & 6.8 \\
\hline 2006-08-29 00:00:00.0 & 9.8 \\
\hline 2006-08-28 21:00:00.0 & 20.1 \\
\hline 2006-08-28 18:00:00.0 & 19.1 \\
\hline 2006-08-28 15:00:00.0 & 17.5 \\
\hline 2006-08-28 09:00:00.0 & 6.9 \\
\hline 2006-08-28 06:00:00.0 & 10.2 \\
\hline 2006-08-28 03:00:00.0 & 10.5 \\
\hline 2006-08-28 00:00:00.0 & 11.2 \\
\hline 2006-08-27 21:00:00.0 & 21.5 \\
\hline
\end{tabular}

\begin{tabular}{|c|c|}
\hline DataHora & VelVentoMax \\
\hline 2006-08-27 18:00:00.0 & 21.0 \\
\hline 2006-08-27 15:00:00.0 & 20.7 \\
\hline 2006-08-27 12:00:00.0 & 17.7 \\
\hline 2006-08-27 09:00:00.0 & 21.1 \\
\hline 2006-08-27 06:00:00.0 & 27.1 \\
\hline 2006-08-27 03:00:00.0 & 28.3 \\
\hline 2006-08-27 00:00:00.0 & 25.1 \\
\hline 2006-08-26 21:00:00.0 & 24.7 \\
\hline 2006-08-26 18:00:00.0 & 28.6 \\
\hline 2006-08-26 15:00:00.0 & 31.6 \\
\hline 2006-08-26 12:00:00.0 & 16.6 \\
\hline 2006-08-26 09:00:00.0 & 37.5 \\
\hline 2006-08-26 06:00:00.0 & 21.8 \\
\hline 2006-08-25 21:00:00.0 & 16.1 \\
\hline 2006-08-25 18:00:00.0 & 4.5 \\
\hline 2006-08-25 15:00:00.0 & 21.9 \\
\hline 2006-08-25 09:00:00.0 & 19.1 \\
\hline 2006-08-25 06:00:00.0 & 16.7 \\
\hline 2006-08-25 03:00:00.0 & 16.4 \\
\hline 2006-08-24 21:00:00.0 & 14.8 \\
\hline 2006-08-24 18:00:00.0 & 15.1 \\
\hline 2006-08-24 15:00:00.0 & 15.8 \\
\hline 2006-08-24 12:00:00.0 & 34.8 \\
\hline 2006-08-24 09:00:00.0 & 16.0 \\
\hline 2006-08-24 06:00:00.0 & 12.9 \\
\hline 2006-08-24 03:00:00.0 & 12.8 \\
\hline 2006-08-24 00:00:00.0 & 11.3 \\
\hline 2006-08-23 21:00:00.0 & 13.7 \\
\hline 2006-08-23 18:00:00.0 & 14.3 \\
\hline 2006-08-23 15:00:00.0 & 12.8 \\
\hline 2006-08-23 09:00:00.0 & 7.6 \\
\hline 2006-08-23 06:00:00.0 & 8.9 \\
\hline 2006-08-23 03:00:00.0 & 9.6 \\
\hline 2006-08-23 00:00:00.0 & 7.4 \\
\hline 2006-08-22 21:00:00.0 & 15.8 \\
\hline 2006-08-22 18:00:00.0 & 14.8 \\
\hline 2006-08-22 12:00:00.0 & 12.5 \\
\hline 2006-08-22 09:00:00.0 & 13.9 \\
\hline 2006-08-22 06:00:00.0 & 10.2 \\
\hline 2006-08-22 03:00:00.0 & 8.8 \\
\hline 2006-08-22 00:00:00.0 & 5.9 \\
\hline 2006-08-21 21:00:00.0 & 10.2 \\
\hline 2006-08-21 18:00:00.0 & 30.3 \\
\hline 2006-08-21 12:00:00.0 & 14.0 \\
\hline 2006-08-21 09:00:00.0 & 11.5 \\
\hline 2006-08-21 06:00:00.0 & 15.3 \\
\hline 2006-08-21 03:00:00.0 & 21.8 \\
\hline 2006-08-21 00:00:00.0 & 18.9 \\
\hline 2006-08-20 21:00:00.0 & 28.6 \\
\hline 2006-08-20 18:00:00.0 & 30.3 \\
\hline 2006-08-20 15:00:00.0 & 26.2 \\
\hline 2006-08-20 09:00:00.0 & 12.0 \\
\hline 2006-08-20 06:00:00.0 & 37.2 \\
\hline 2006-08-20 00:00:00.0 & 6.5 \\
\hline 2006-08-19 21:00:00.0 & 17.0 \\
\hline 2006-08-19 18:00:00.0 & 17.3 \\
\hline 2006-08-19 15:00:00.0 & 9.6 \\
\hline 2006-08-19 12:00:00.0 & 8.8 \\
\hline 2006-08-19 09:00:00.0 & 5.8 \\
\hline 2006-08-19 06:00:00.0 & 5.9 \\
\hline 2006-08-19 03:00:00.0 & 6.7 \\
\hline 2006-08-18 21:00:00.0 & 20.0 \\
\hline 2006-08-18 18:00:00.0 & 21.6 \\
\hline 2006-08-18 15:00:00.0 & 20.3 \\
\hline 2006-08-18 09:00:00.0 & 13.9 \\
\hline 2006-08-18 06:00:00.0 & 15.4 \\
\hline 2006-08-18 03:00:00.0 & 11.6 \\
\hline 2006-08-18 00:00:00.0 & 12.6 \\
\hline 2006-08-17 21:00:00.0 & 17.7 \\
\hline
\end{tabular}




\section{ANEXO 01 - RAJADAS DE VENTO REGISTRADAS EM SÃO MARTINHO DA SERRA / RS}

\begin{tabular}{|c|c|}
\hline DataHora & VelVentoMax \\
\hline 2006-08-17 18:00:00.0 & 16.6 \\
\hline 2006-08-17 12:00:00.0 & 15.2 \\
\hline 2006-08-17 09:00:00.0 & 15.2 \\
\hline 2006-08-17 06:00:00.0 & 11.8 \\
\hline 2006-08-17 03:00:00.0 & 15.0 \\
\hline 2006-08-17 00:00:00.0 & 15.2 \\
\hline 2006-08-16 21:00:00.0 & 23.2 \\
\hline 2006-08-16 18:00:00.0 & 33.0 \\
\hline 2006-08-16 12:00:00.0 & 33.2 \\
\hline 2006-08-16 09:00:00.0 & 32.7 \\
\hline 2006-08-16 03:00:00.0 & 29.0 \\
\hline 2006-08-16 00:00:00.0 & 22.9 \\
\hline 2006-08-15 21:00:00.0 & 26.2 \\
\hline 2006-08-15 18:00:00.0 & 25.2 \\
\hline 2006-08-15 15:00:00.0 & 26.0 \\
\hline 2006-08-15 12:00:00.0 & 25.5 \\
\hline 2006-08-15 09:00:00.0 & 18.3 \\
\hline 2006-08-15 06:00:00.0 & 22.5 \\
\hline 2006-08-15 03:00:00.0 & 20.3 \\
\hline 2006-08-15 00:00:00.0 & 16.4 \\
\hline 2006-08-14 21:00:00.0 & 19.7 \\
\hline 2006-08-14 18:00:00.0 & 26.1 \\
\hline 2006-08-14 15:00:00.0 & 36.0 \\
\hline 2006-08-14 09:00:00.0 & 27.5 \\
\hline 2006-08-14 03:00:00.0 & 15.0 \\
\hline 2006-08-14 00:00:00.0 & 13.7 \\
\hline 2006-08-13 21:00:00.0 & 13.9 \\
\hline 2006-08-13 18:00:00.0 & 20.5 \\
\hline 2006-08-13 15:00:00.0 & 23.5 \\
\hline 2006-08-13 03:00:00.0 & 20.9 \\
\hline 2006-08-13 00:00:00.0 & 16.8 \\
\hline 2006-08-12 21:00:00.0 & 21.0 \\
\hline 2006-08-12 15:00:00.0 & 22.4 \\
\hline 2006-08-12 12:00:00.0 & 21.9 \\
\hline 2006-08-12 09:00:00.0 & 20.0 \\
\hline 2006-08-12 03:00:00.0 & 19.9 \\
\hline 2006-08-12 00:00:00.0 & 14.3 \\
\hline 2006-08-11 21:00:00.0 & 16.4 \\
\hline 2006-08-11 15:00:00.0 & 27.8 \\
\hline 2006-08-11 12:00:00.0 & 39.7 \\
\hline 2006-08-11 09:00:00.0 & 23.9 \\
\hline 2006-08-11 06:00:00.0 & 22.9 \\
\hline 2006-08-11 03:00:00.0 & 22.0 \\
\hline 2006-08-11 00:00:00.0 & 14.0 \\
\hline 2006-08-10 21:00:00.0 & 11.3 \\
\hline 2006-08-10 15:00:00.0 & 14.7 \\
\hline 2006-08-10 12:00:00.0 & 13.2 \\
\hline 2006-08-10 09:00:00.0 & \\
\hline 2006-08-10 06:00:00.0 & \\
\hline 2006-08-10 03:00:00.0 & 22.4 \\
\hline 2006-08-10 00:00:00.0 & 16.2 \\
\hline 2006-08-09 21:00:00.0 & 38.5 \\
\hline 2006-08-09 18:00:00.0 & 41.9 \\
\hline 2006-08-09 15:00:00.0 & 6.1 \\
\hline 2006-08-09 12:00:00.0 & 27.2 \\
\hline 2006-08-09 09:00:00.0 & \\
\hline 2006-08-09 06:00:00.0 & \\
\hline 2006-08-09 03:00:00.0 & 24.0 \\
\hline 2006-08-09 00:00:00.0 & \\
\hline 2006-08-08 21:00:00.0 & 29.0 \\
\hline 2006-08-08 18:00:00.0 & 26.0 \\
\hline 2006-08-08 15:00:00.0 & 27.2 \\
\hline 2006-08-08 12:00:00.0 & 24.5 \\
\hline 2006-08-08 09:00:00.0 & \\
\hline 2006-08-08 06:00:00.0 & \\
\hline 2006-08-08 03:00:00.0 & 17.7 \\
\hline 2006-08-08 00:00:00.0 & 14.4 \\
\hline 2006-08-07 21:00:00.0 & 23.7 \\
\hline 2006-08-07 18:00:00.0 & 25.8 \\
\hline
\end{tabular}

\begin{tabular}{|c|c|}
\hline DataHora & VelVentoMax \\
\hline 2006-08-07 15:00:00.0 & 26.8 \\
\hline 2006-08-07 12:00:00.0 & 20.9 \\
\hline 2006-08-07 09:00:00.0 & \\
\hline 2006-08-07 06:00:00.0 & 20.0 \\
\hline 2006-08-07 03:00:00.0 & 20.0 \\
\hline 2006-08-07 00:00:00.0 & 19.0 \\
\hline 2006-08-06 21:00:00.0 & 15.0 \\
\hline 2006-08-06 18:00:00.0 & \\
\hline 2006-08-06 15:00:00.0 & 19.0 \\
\hline 2006-08-06 12:00:00.0 & \\
\hline 2006-08-06 09:00:00.0 & \\
\hline 2006-08-06 06:00:00.0 & \\
\hline 2006-08-06 03:00:00.0 & 13.0 \\
\hline 2006-08-06 00:00:00.0 & 9.0 \\
\hline 2006-08-05 21:00:00.0 & \\
\hline 2006-08-05 18:00:00.0 & 6.0 \\
\hline 2006-08-05 15:00:00.0 & \\
\hline 2006-08-05 12:00:00.0 & \\
\hline 2006-08-05 09:00:00.0 & \\
\hline 2006-08-05 06:00:00.0 & 16.0 \\
\hline 2006-08-05 03:00:00.0 & 16.0 \\
\hline 2006-08-05 00:00:00.0 & \\
\hline 2006-08-04 21:00:00.0 & \\
\hline 2006-08-04 18:00:00.0 & 14.0 \\
\hline 2006-08-04 15:00:00.0 & 12.0 \\
\hline 2006-08-04 12:00:00.0 & \\
\hline 2006-08-04 09:00:00.0 & \\
\hline 2006-08-04 06:00:00.0 & 18.0 \\
\hline 2006-08-04 03:00:00.0 & 16.0 \\
\hline 2006-08-04 00:00:00.0 & 12.0 \\
\hline 2006-08-03 21:00:00.0 & 14.0 \\
\hline 2006-08-03 18:00:00.0 & 11.0 \\
\hline 2006-08-03 15:00:00.0 & 18.0 \\
\hline 2006-08-03 12:00:00.0 & \\
\hline 2006-08-03 09:00:00.0 & \\
\hline 2006-08-03 06:00:00.0 & 21.0 \\
\hline 2006-08-03 03:00:00.0 & 18.0 \\
\hline 2006-08-03 00:00:00.0 & 14.0 \\
\hline 2006-08-02 21:00:00.0 & 15.0 \\
\hline 2006-08-02 18:00:00.0 & 17.0 \\
\hline 2006-08-02 15:00:00.0 & 21.0 \\
\hline 2006-08-02 12:00:00.0 & \\
\hline 2006-08-02 09:00:00.0 & 15.9 \\
\hline 2006-08-02 06:00:00.0 & 12.1 \\
\hline 2006-08-02 03:00:00.0 & 10.7 \\
\hline 2006-08-02 00:00:00.0 & 11.6 \\
\hline 2006-08-01 21:00:00.0 & 15.3 \\
\hline 2006-08-01 18:00:00.0 & 19.7 \\
\hline 2006-08-01 15:00:00.0 & 20.7 \\
\hline 2006-08-01 12:00:00.0 & 13.3 \\
\hline 2006-08-01 09:00:00.0 & 11.7 \\
\hline 2006-08-01 06:00:00.0 & 9.0 \\
\hline 2006-08-01 03:00:00.0 & 9.0 \\
\hline 2006-08-01 00:00:00.0 & 10.0 \\
\hline 2006-07-31 21:00:00.0 & 15.0 \\
\hline 2006-07-31 18:00:00.0 & 31.0 \\
\hline 2006-07-31 15:00:00.0 & 14.1 \\
\hline 2006-07-31 12:00:00.0 & \\
\hline 2006-07-31 09:00:00.0 & 8.4 \\
\hline 2006-07-31 06:00:00.0 & 8.1 \\
\hline 2006-07-31 03:00:00.0 & 6.0 \\
\hline 2006-07-31 00:00:00.0 & 47.7 \\
\hline 2006-07-30 21:00:00.0 & 17.4 \\
\hline 2006-07-30 18:00:00.0 & 18.0 \\
\hline 2006-07-30 15:00:00.0 & 17.3 \\
\hline 2006-07-30 12:00:00.0 & 8.3 \\
\hline 2006-07-30 09:00:00.0 & 8.8 \\
\hline 2006-07-30 06:00:00.0 & 12.6 \\
\hline 2006-07-30 03:00:00.0 & 18.3 \\
\hline
\end{tabular}

\begin{tabular}{|c|c|}
\hline DataHora & VelVentoMax \\
\hline 2006-07-30 00:00:00.0 & 14.9 \\
\hline 2006-07-29 21:00:00.0 & 24.6 \\
\hline 2006-07-29 18:00:00.0 & 24.2 \\
\hline 2006-07-29 15:00:00.0 & \\
\hline 2006-07-29 12:00:00.0 & \\
\hline 2006-07-29 09:00:00.0 & 23.5 \\
\hline 2006-07-29 06:00:00.0 & 23.7 \\
\hline 2006-07-29 03:00:00.0 & 26.8 \\
\hline 2006-07-29 00:00:00.0 & \\
\hline 2006-07-28 21:00:00.0 & 37.9 \\
\hline $2006-07-28$ 18:00:00.0 & 21.6 \\
\hline $2006-07-28$ 15:00:00.0 & \\
\hline 2006-07-28 12:00:00.0 & 15.8 \\
\hline 2006-07-28 09:00:00.0 & 21.0 \\
\hline 2006-07-28 06:00:00.0 & 20.1 \\
\hline 2006-07-28 03:00:00.0 & 16.5 \\
\hline 2006-07-28 00:00:00.0 & 16.5 \\
\hline 2006-07-27 21:00:00.0 & 22.9 \\
\hline 2006-07-27 18:00:00.0 & 26.2 \\
\hline 2006-07-27 15:00:00.0 & 34.3 \\
\hline 2006-07-27 12:00:00.0 & \\
\hline 2006-07-27 09:00:00.0 & 26.8 \\
\hline 2006-07-27 06:00:00.0 & 24.1 \\
\hline 2006-07-27 03:00:00.0 & 26.1 \\
\hline 2006-07-27 00:00:00.0 & 24.6 \\
\hline 2006-07-26 21:00:00.0 & 22.8 \\
\hline 2006-07-26 18:00:00.0 & 22.4 \\
\hline 2006-07-26 15:00:00.0 & 29.4 \\
\hline 2006-07-26 12:00:00.0 & 26.1 \\
\hline 2006-07-26 09:00:00.0 & 34.6 \\
\hline 2006-07-26 06:00:00.0 & 17.9 \\
\hline 2006-07-26 03:00:00.0 & 19.5 \\
\hline 2006-07-26 00:00:00.0 & 19.2 \\
\hline 2006-07-25 21:00:00.0 & 20.3 \\
\hline 2006-07-25 18:00:00.0 & 18.7 \\
\hline 2006-07-25 15:00:00.0 & 19.3 \\
\hline 2006-07-25 12:00:00.0 & 19.0 \\
\hline 2006-07-25 09:00:00.0 & 16.4 \\
\hline 2006-07-25 06:00:00.0 & 16.0 \\
\hline 2006-07-25 03:00:00.0 & 15.1 \\
\hline 2006-07-25 00:00:00.0 & 16.7 \\
\hline 2006-07-24 21:00:00.0 & 16.7 \\
\hline 2006-07-24 18:00:00.0 & 16.6 \\
\hline 2006-07-24 15:00:00.0 & 20.8 \\
\hline 2006-07-24 12:00:00.0 & 20.5 \\
\hline 2006-07-24 09:00:00.0 & 19.9 \\
\hline 2006-07-24 06:00:00.0 & 19.9 \\
\hline 2006-07-24 03:00:00.0 & \\
\hline 2006-07-24 00:00:00.0 & 17.3 \\
\hline 2006-07-23 21:00:00.0 & 19.6 \\
\hline $2006-07-23$ 18:00:00.0 & 18.6 \\
\hline 2006-07-23 15:00:00.0 & 16.6 \\
\hline 2006-07-23 12:00:00.0 & 7.0 \\
\hline 2006-07-23 09:00:00.0 & 21.5 \\
\hline 2006-07-23 06:00:00.0 & 18.1 \\
\hline 2006-07-23 03:00:00.0 & 19.3 \\
\hline 2006-07-23 00:00:00.0 & 18.3 \\
\hline 2006-07-22 21:00:00.0 & 37.0 \\
\hline 2006-07-22 18:00:00.0 & 39.9 \\
\hline 2006-07-22 15:00:00.0 & 37.4 \\
\hline 2006-07-22 12:00:00.0 & 27.2 \\
\hline 2006-07-22 09:00:00.0 & 24.4 \\
\hline 2006-07-22 06:00:00.0 & 17.8 \\
\hline 2006-07-22 03:00:00.0 & 15.0 \\
\hline 2006-07-22 00:00:00.0 & 13.3 \\
\hline 2006-07-21 21:00:00.0 & 26.8 \\
\hline 2006-07-21 18:00:00.0 & 21.6 \\
\hline 2006-07-21 15:00:00.0 & 21.2 \\
\hline 2006-07-21 12:00:00.0 & 22.2 \\
\hline
\end{tabular}




\section{ANEXO 01 - RAJADAS DE VENTO REGISTRADAS EM SÃO MARTINHO DA SERRA / RS}

\begin{tabular}{|c|c|}
\hline DataHora & VelVentoMax \\
\hline 2006-07-21 09:00:00.0 & 22.9 \\
\hline 2006-07-21 06:00:00.0 & 17.9 \\
\hline 2006-07-21 03:00:00.0 & 17.9 \\
\hline 2006-07-21 00:00:00.0 & 13.1 \\
\hline 2006-07-20 21:00:00.0 & 19.8 \\
\hline 2006-07-20 18:00:00.0 & 18.2 \\
\hline 2006-07-20 15:00:00.0 & 14.9 \\
\hline 2006-07-20 12:00:00.0 & 10.9 \\
\hline 2006-07-20 09:00:00.0 & 6.4 \\
\hline 2006-07-20 06:00:00.0 & \\
\hline 2006-07-20 03:00:00.0 & 12.4 \\
\hline 2006-07-20 00:00:00.0 & 7.4 \\
\hline 2006-07-19 21:00:00.0 & 18.9 \\
\hline 2006-07-19 18:00:00.0 & 19.9 \\
\hline 2006-07-19 15:00:00.0 & 13.6 \\
\hline 2006-07-19 12:00:00.0 & 10.9 \\
\hline 2006-07-19 09:00:00.0 & 9.6 \\
\hline 2006-07-19 06:00:00.0 & 9.0 \\
\hline 2006-07-19 03:00:00.0 & 7.1 \\
\hline 2006-07-19 00:00:00.0 & 8.9 \\
\hline 2006-07-18 21:00:00.0 & 14.4 \\
\hline 2006-07-18 18:00:00.0 & 18.1 \\
\hline 2006-07-18 15:00:00.0 & 14.9 \\
\hline 2006-07-18 12:00:00.0 & 13.7 \\
\hline 2006-07-18 09:00:00.0 & 13.9 \\
\hline 2006-07-18 06:00:00.0 & \\
\hline 2006-07-18 03:00:00.0 & 12.7 \\
\hline 2006-07-18 00:00:00.0 & 12.9 \\
\hline 2006-07-17 21:00:00.0 & 7.1 \\
\hline 2006-07-17 18:00:00.0 & 9.1 \\
\hline 2006-07-17 15:00:00.0 & 9.5 \\
\hline 2006-07-17 12:00:00.0 & \\
\hline 2006-07-17 09:00:00.0 & 11.9 \\
\hline 2006-07-17 06:00:00.0 & 12.4 \\
\hline 2006-07-17 03:00:00.0 & 14.4 \\
\hline 2006-07-17 00:00:00.0 & 14.2 \\
\hline 2006-07-16 21:00:00.0 & 14.4 \\
\hline 2006-07-16 18:00:00.0 & 20.6 \\
\hline 2006-07-16 15:00:00.0 & 20.1 \\
\hline 2006-07-16 12:00:00.0 & \\
\hline 2006-07-16 09:00:00.0 & 19.7 \\
\hline 2006-07-16 06:00:00.0 & 21.6 \\
\hline 2006-07-16 03:00:00.0 & 22.1 \\
\hline 2006-07-16 00:00:00.0 & 22.6 \\
\hline 2006-07-15 21:00:00.0 & 22.1 \\
\hline 2006-07-15 18:00:00.0 & \\
\hline 2006-07-15 15:00:00.0 & 24.1 \\
\hline 2006-07-15 12:00:00.0 & 22.9 \\
\hline 2006-07-15 09:00:00.0 & \\
\hline 2006-07-15 06:00:00.0 & 24.0 \\
\hline 2006-07-15 03:00:00.0 & 25.0 \\
\hline 2006-07-15 00:00:00.0 & \\
\hline 2006-07-14 21:00:00.0 & 22.6 \\
\hline 2006-07-14 18:00:00.0 & 15.8 \\
\hline 2006-07-14 15:00:00.0 & 22.1 \\
\hline 2006-07-14 12:00:00.0 & 25.6 \\
\hline 2006-07-14 09:00:00.0 & \\
\hline 2006-07-14 06:00:00.0 & \\
\hline 2006-07-14 03:00:00.0 & 14.3 \\
\hline 2006-07-14 00:00:00.0 & 13.8 \\
\hline 2006-07-13 21:00:00.0 & 22.4 \\
\hline 2006-07-13 18:00:00.0 & 29.0 \\
\hline 2006-07-13 15:00:00.0 & 28.9 \\
\hline 2006-07-13 12:00:00.0 & 23.8 \\
\hline 2006-07-13 09:00:00.0 & \\
\hline 2006-07-13 06:00:00.0 & 26.5 \\
\hline 2006-07-13 03:00:00.0 & 27.7 \\
\hline 2006-07-13 00:00:00.0 & 21.7 \\
\hline 2006-07-12 21:00:00.0 & \\
\hline
\end{tabular}

\begin{tabular}{|c|c|}
\hline DataHora & VelVentoMax \\
\hline 2006-07-12 18:00:00.0 & 35.4 \\
\hline 2006-07-12 15:00:00.0 & 31.2 \\
\hline 2006-07-12 12:00:00.0 & 25.3 \\
\hline 2006-07-12 09:00:00.0 & 25.6 \\
\hline 2006-07-12 06:00:00.0 & 26.6 \\
\hline 2006-07-12 03:00:00.0 & 26.5 \\
\hline 2006-07-12 00:00:00.0 & 25.8 \\
\hline 2006-07-11 21:00:00.0 & \\
\hline 2006-07-11 18:00:00.0 & 24.9 \\
\hline 2006-07-11 15:00:00.0 & 25.7 \\
\hline 2006-07-11 12:00:00.0 & 25.7 \\
\hline 2006-07-11 09:00:00.0 & \\
\hline 2006-07-11 06:00:00.0 & 22.7 \\
\hline 2006-07-11 03:00:00.0 & 25.5 \\
\hline 2006-07-11 00:00:00.0 & 18.3 \\
\hline 2006-07-10 21:00:00.0 & 11.9 \\
\hline 2006-07-10 18:00:00.0 & 17.4 \\
\hline 2006-07-10 15:00:00.0 & 19.2 \\
\hline 2006-07-10 12:00:00.0 & 14.8 \\
\hline 2006-07-10 09:00:00.0 & \\
\hline 2006-07-10 06:00:00.0 & 9.1 \\
\hline 2006-07-10 03:00:00.0 & 8.4 \\
\hline 2006-07-10 00:00:00.0 & \\
\hline 2006-07-09 21:00:00.0 & 14.1 \\
\hline 2006-07-09 18:00:00.0 & 20.0 \\
\hline 2006-07-09 15:00:00.0 & 30.7 \\
\hline 2006-07-09 12:00:00.0 & 38.1 \\
\hline 2006-07-09 09:00:00.0 & 27.2 \\
\hline 2006-07-09 06:00:00.0 & 33.2 \\
\hline 2006-07-09 03:00:00.0 & 24.4 \\
\hline 2006-07-09 00:00:00.0 & 22.3 \\
\hline 2006-07-08 21:00:00.0 & 22.1 \\
\hline 2006-07-08 18:00:00.0 & 27.4 \\
\hline 2006-07-08 15:00:00.0 & 30.5 \\
\hline 2006-07-08 12:00:00.0 & \\
\hline 2006-07-08 09:00:00.0 & \\
\hline 2006-07-08 06:00:00.0 & 20.8 \\
\hline 2006-07-08 03:00:00.0 & 18.6 \\
\hline 2006-07-08 00:00:00.0 & 16.9 \\
\hline 2006-07-07 21:00:00.0 & 18.0 \\
\hline 2006-07-07 18:00:00.0 & 20.6 \\
\hline 2006-07-07 15:00:00.0 & 20.1 \\
\hline 2006-07-07 12:00:00.0 & 16.8 \\
\hline 2006-07-07 09:00:00.0 & 18.8 \\
\hline 2006-07-07 06:00:00.0 & 19.1 \\
\hline 2006-07-07 03:00:00.0 & 18.5 \\
\hline 2006-07-07 00:00:00.0 & 15.2 \\
\hline 2006-07-06 21:00:00.0 & 11.9 \\
\hline 2006-07-06 18:00:00.0 & 13.5 \\
\hline 2006-07-06 15:00:00.0 & 14.4 \\
\hline 2006-07-06 12:00:00.0 & \\
\hline 2006-07-06 09:00:00.0 & 15.8 \\
\hline 2006-07-06 06:00:00.0 & 16.5 \\
\hline 2006-07-06 03:00:00.0 & 14.4 \\
\hline 2006-07-06 00:00:00.0 & \\
\hline 2006-07-05 21:00:00.0 & 9.0 \\
\hline 2006-07-05 18:00:00.0 & 13.2 \\
\hline 2006-07-05 15:00:00.0 & 11.3 \\
\hline 2006-07-05 12:00:00.0 & 9.2 \\
\hline 2006-07-05 09:00:00.0 & \\
\hline 2006-07-05 06:00:00.0 & 5.3 \\
\hline 2006-07-05 03:00:00.0 & 3.0 \\
\hline 2006-07-05 00:00:00.0 & 5.7 \\
\hline 2006-07-04 21:00:00.0 & 11.6 \\
\hline 2006-07-04 18:00:00.0 & 17.0 \\
\hline 2006-07-04 15:00:00.0 & 13.9 \\
\hline 2006-07-04 12:00:00.0 & \\
\hline 2006-07-04 09:00:00.0 & \\
\hline 2006-07-04 06:00:00.0 & 10.9 \\
\hline
\end{tabular}

\begin{tabular}{|c|c|}
\hline \multirow{2}{*}{$\begin{array}{l}\text { DataHora } \\
\text { 2006-07-04 03:00:00.0 }\end{array}$} & \multirow[t]{2}{*}{ VelVentoMax } \\
\hline & \\
\hline 2006-07-04 00:00:00.0 & 12.3 \\
\hline 2006-07-03 21:00:00.0 & 12.1 \\
\hline 2006-07-03 18:00:00.0 & 15.2 \\
\hline 2006-07-03 15:00:00.0 & 15.7 \\
\hline 2006-07-03 12:00:00.0 & \\
\hline 2006-07-03 09:00:00.0 & 15.1 \\
\hline 2006-07-03 06:00:00.0 & 14.5 \\
\hline 2006-07-03 03:00:00.0 & 15.0 \\
\hline 2006-07-03 00:00:00.0 & 10.5 \\
\hline 2006-07-02 21:00:00.0 & 12.1 \\
\hline 2006-07-02 18:00:00.0 & 11.5 \\
\hline 2006-07-02 15:00:00.0 & 10.6 \\
\hline 2006-07-02 12:00:00.0 & \\
\hline 2006-07-02 09:00:00.0 & 6.1 \\
\hline 2006-07-02 06:00:00.0 & 6.4 \\
\hline 2006-07-02 03:00:00.0 & 5.8 \\
\hline 2006-07-02 00:00:00.0 & \\
\hline 2006-07-01 21:00:00.0 & \\
\hline 2006-07-01 18:00:00.0 & \\
\hline 2006-07-01 06:00:00.0 & 17.2 \\
\hline 2006-07-01 03:00:00.0 & 14.7 \\
\hline 2006-07-01 00:00:00.0 & \\
\hline 2006-06-30 21:00:00.0 & \\
\hline 2006-06-30 18:00:00.0 & \\
\hline 2006-06-30 09:00:00.0 & 16.0 \\
\hline 2006-06-30 06:00:00.0 & 15.1 \\
\hline 2006-06-30 03:00:00.0 & 15.5 \\
\hline 2006-06-30 00:00:00.0 & \\
\hline 2006-06-29 21:00:00.0 & \\
\hline 2006-06-29 18:00:00.0 & \\
\hline 2006-06-29 09:00:00.0 & 17.1 \\
\hline 2006-06-29 06:00:00.0 & 13.0 \\
\hline 2006-06-29 03:00:00.0 & \\
\hline 2006-06-29 00:00:00.0 & 14.0 \\
\hline 2006-06-28 21:00:00.0 & 16.1 \\
\hline $2006-06-28$ 18:00:00.0 & 16.3 \\
\hline $2006-06-28$ 15:00:00.0 & \\
\hline 2006-06-28 12:00:00.0 & 13.5 \\
\hline 2006-06-28 09:00:00.0 & 12.6 \\
\hline 2006-06-28 06:00:00.0 & 10.5 \\
\hline 2006-06-28 03:00:00.0 & 9.2 \\
\hline 2006-06-28 00:00:00.0 & 8.7 \\
\hline 2006-06-27 21:00:00.0 & 11.9 \\
\hline 2006-06-27 18:00:00.0 & 14.2 \\
\hline 2006-06-27 15:00:00.0 & 12.5 \\
\hline 2006-06-27 12:00:00.0 & \\
\hline 2006-06-27 09:00:00.0 & 19.3 \\
\hline 2006-06-27 06:00:00.0 & \\
\hline 2006-06-27 03:00:00.0 & \\
\hline 2006-06-27 00:00:00.0 & 19.3 \\
\hline 2006-06-26 21:00:00.0 & 22.1 \\
\hline 2006-06-26 18:00:00.0 & \\
\hline 2006-06-26 15:00:00.0 & \\
\hline 2006-06-26 12:00:00.0 & \\
\hline 2006-06-26 06:00:00.0 & 38.3 \\
\hline 2006-06-26 03:00:00.0 & \\
\hline 2006-06-26 00:00:00.0 & 20.5 \\
\hline 2006-06-25 21:00:00.0 & 18.0 \\
\hline 2006-06-25 18:00:00.0 & 45.6 \\
\hline 2006-06-25 15:00:00.0 & 36.2 \\
\hline 2006-06-25 12:00:00.0 & 23.7 \\
\hline 2006-06-25 09:00:00.0 & 21.4 \\
\hline 2006-06-25 06:00:00.0 & \\
\hline 2006-06-25 03:00:00.0 & 16.7 \\
\hline 2006-06-25 00:00:00.0 & 16.8 \\
\hline 2006-06-24 21:00:00.0 & 21.1 \\
\hline 2006-06-24 18:00:00.0 & \\
\hline $2006-06-24$ 15:00:00.0 & 22.3 \\
\hline
\end{tabular}




\section{ANEXO 01 - RAJADAS DE VENTO REGISTRADAS EM SÃO MARTINHO DA SERRA / RS}

\begin{tabular}{|c|c|}
\hline DataHora & VelVentoMax \\
\hline 2006-06-24 12:00:00.0 & 20.0 \\
\hline 2006-06-24 09:00:00.0 & 17.5 \\
\hline \multicolumn{2}{|l|}{ 2006-06-24 06:00:00.0 } \\
\hline 2006-06-24 03:00:00.0 & 18.6 \\
\hline 2006-06-24 00:00:00.0 & 14.1 \\
\hline \multicolumn{2}{|l|}{ 2006-06-23 21:00:00.0 } \\
\hline 2006-06-23 18:00:00.0 & 18.7 \\
\hline \multicolumn{2}{|l|}{ 2006-06-23 15:00:00.0 } \\
\hline \multicolumn{2}{|l|}{ 2006-06-23 12:00:00.0 } \\
\hline 2006-06-23 09:00:00.0 & 13.7 \\
\hline 2006-06-23 06:00:00.0 & 10.8 \\
\hline \multicolumn{2}{|l|}{ 2006-06-23 03:00:00.0 } \\
\hline \multicolumn{2}{|l|}{ 2006-06-23 00:00:00.0 } \\
\hline \multicolumn{2}{|l|}{ 2006-06-22 21:00:00.0 } \\
\hline 2006-06-22 15:00:00.0 & 18.3 \\
\hline 2006-06-22 12:00:00.0 & 27.1 \\
\hline 2006-06-22 09:00:00.0 & 14.3 \\
\hline \multicolumn{2}{|l|}{ 2006-06-22 06:00:00.0 } \\
\hline \multicolumn{2}{|l|}{ 2006-06-22 03:00:00.0 } \\
\hline \multicolumn{2}{|l|}{ 2006-06-22 00:00:00.0 } \\
\hline 2006-06-21 18:00:00.0 & 33.7 \\
\hline 2006-06-21 15:00:00.0 & 29.6 \\
\hline 2006-06-21 12:00:00.0 & 33.2 \\
\hline \multicolumn{2}{|l|}{ 2006-06-21 09:00:00.0 } \\
\hline \multicolumn{2}{|l|}{ 2006-06-21 06:00:00.0 } \\
\hline 2006-06-21 03:00:00.0 & 24.7 \\
\hline 2006-06-21 00:00:00.0 & 19.9 \\
\hline 2006-06-20 21:00:00.0 & 29.1 \\
\hline 2006-06-20 18:00:00.0 & 27.5 \\
\hline 2006-06-20 15:00:00.0 & 28.5 \\
\hline 2006-06-20 12:00:00.0 & 23.2 \\
\hline \multicolumn{2}{|l|}{ 2006-06-20 09:00:00.0 } \\
\hline 2006-06-20 06:00:00.0 & \\
\hline 2006-06-20 03:00:00.0 & 19.5 \\
\hline 2006-06-20 00:00:00.0 & 16.6 \\
\hline 2006-06-19 21:00:00.0 & 22.7 \\
\hline 2006-06-19 18:00:00.0 & 25.0 \\
\hline 2006-06-19 15:00:00.0 & 25.9 \\
\hline 2006-06-19 12:00:00.0 & 20.0 \\
\hline 2006-06-19 09:00:00.0 & \\
\hline 2006-06-19 06:00:00.0 & \\
\hline 2006-06-19 03:00:00.0 & 14.1 \\
\hline 2006-06-19 00:00:00.0 & 10.7 \\
\hline 2006-06-18 21:00:00.0 & 13.5 \\
\hline 2006-06-18 18:00:00.0 & 19.2 \\
\hline 2006-06-18 15:00:00.0 & 18.9 \\
\hline 2006-06-18 12:00:00.0 & 16.9 \\
\hline 2006-06-18 09:00:00.0 & \\
\hline 2006-06-18 06:00:00.0 & \\
\hline 2006-06-18 03:00:00.0 & 23.8 \\
\hline 2006-06-18 00:00:00.0 & 21.7 \\
\hline 2006-06-17 21:00:00.0 & 15.1 \\
\hline 2006-06-17 18:00:00.0 & \\
\hline 2006-06-17 15:00:00.0 & 27.1 \\
\hline 2006-06-17 12:00:00.0 & 22.1 \\
\hline 2006-06-17 09:00:00.0 & \\
\hline 2006-06-17 06:00:00.0 & \\
\hline 2006-06-17 03:00:00.0 & 17.3 \\
\hline 2006-06-17 00:00:00.0 & 16.0 \\
\hline 2006-06-16 21:00:00.0 & \\
\hline 2006-06-16 18:00:00.0 & 20.3 \\
\hline 2006-06-16 15:00:00.0 & 6.8 \\
\hline 2006-06-16 12:00:00.0 & \\
\hline 2006-06-16 09:00:00.0 & \\
\hline 2006-06-16 06:00:00.0 & 17.8 \\
\hline 2006-06-16 03:00:00.0 & \\
\hline 2006-06-16 00:00:00.0 & \\
\hline 2006-06-15 21:00:00.0 & 25.2 \\
\hline 2006-06-15 18:00:00.0 & 12.8 \\
\hline
\end{tabular}

\begin{tabular}{|c|c|}
\hline DataHora & VelVentoMax \\
\hline 2006-06-15 15:00:00.0 & 12.6 \\
\hline 2006-06-15 12:00:00.0 & \\
\hline 2006-06-15 09:00:00.0 & \\
\hline 2006-06-15 06:00:00.0 & 10.5 \\
\hline 2006-06-15 03:00:00.0 & 13.1 \\
\hline 2006-06-15 00:00:00.0 & 10.3 \\
\hline 2006-06-14 21:00:00.0 & 44.2 \\
\hline 2006-06-14 18:00:00.0 & 19.6 \\
\hline 2006-06-14 15:00:00.0 & 20.4 \\
\hline 2006-06-14 12:00:00.0 & 14.3 \\
\hline 2006-06-14 09:00:00.0 & \\
\hline 2006-06-14 06:00:00.0 & 18.2 \\
\hline 2006-06-14 03:00:00.0 & 16.1 \\
\hline 2006-06-14 00:00:00.0 & 12.0 \\
\hline 2006-06-13 21:00:00.0 & 16.6 \\
\hline 2006-06-13 18:00:00.0 & 20.7 \\
\hline 2006-06-13 15:00:00.0 & 22.2 \\
\hline 2006-06-13 12:00:00.0 & \\
\hline 2006-06-13 09:00:00.0 & \\
\hline 2006-06-13 06:00:00.0 & 21.1 \\
\hline 2006-06-13 03:00:00.0 & 21.7 \\
\hline 2006-06-13 00:00:00.0 & 13.3 \\
\hline 2006-06-12 21:00:00.0 & \\
\hline 2006-06-12 18:00:00.0 & 15.5 \\
\hline 2006-06-12 15:00:00.0 & 17.5 \\
\hline 2006-06-12 12:00:00.0 & \\
\hline 2006-06-12 09:00:00.0 & 16.0 \\
\hline 2006-06-12 06:00:00.0 & 13.0 \\
\hline 2006-06-12 03:00:00.0 & 9.0 \\
\hline 2006-06-12 00:00:00.0 & 10.0 \\
\hline 2006-06-11 21:00:00.0 & 11.0 \\
\hline 2006-06-11 18:00:00.0 & 13.0 \\
\hline 2006-06-11 15:00:00.0 & 11.0 \\
\hline 2006-06-11 12:00:00.0 & \\
\hline 2006-06-11 09:00:00.0 & 15.0 \\
\hline 2006-06-11 06:00:00.0 & 14.0 \\
\hline 2006-06-11 03:00:00.0 & 20.0 \\
\hline 2006-06-11 00:00:00.0 & \\
\hline 2006-06-10 21:00:00.0 & 15.0 \\
\hline 2006-06-10 18:00:00.0 & 19.0 \\
\hline 2006-06-10 15:00:00.0 & 23.0 \\
\hline 2006-06-10 12:00:00.0 & \\
\hline 2006-06-10 09:00:00.0 & 17.0 \\
\hline 2006-06-10 06:00:00.0 & 16.0 \\
\hline 2006-06-10 03:00:00.0 & 14.0 \\
\hline 2006-06-10 00:00:00.0 & 17.0 \\
\hline 2006-06-09 21:00:00.0 & 28.9 \\
\hline 2006-06-09 18:00:00.0 & 34.7 \\
\hline 2006-06-09 15:00:00.0 & 37.7 \\
\hline 2006-06-09 12:00:00.0 & \\
\hline 2006-06-09 09:00:00.0 & 21.0 \\
\hline 2006-06-09 06:00:00.0 & 22.2 \\
\hline 2006-06-09 03:00:00.0 & \\
\hline 2006-06-09 00:00:00.0 & \\
\hline 2006-06-08 21:00:00.0 & 25.3 \\
\hline 2006-06-08 18:00:00.0 & 29.0 \\
\hline 2006-06-08 15:00:00.0 & \\
\hline 2006-06-08 12:00:00.0 & 21.5 \\
\hline 2006-06-08 09:00:00.0 & 20.1 \\
\hline 2006-06-08 06:00:00.0 & 17.6 \\
\hline 2006-06-08 03:00:00.0 & 19.7 \\
\hline 2006-06-08 00:00:00.0 & 15.7 \\
\hline 2006-06-07 21:00:00.0 & 15.5 \\
\hline 2006-06-07 18:00:00.0 & 23.3 \\
\hline 2006-06-07 15:00:00.0 & 23.7 \\
\hline 2006-06-07 12:00:00.0 & \\
\hline 2006-06-07 09:00:00.0 & 12.9 \\
\hline 2006-06-07 06:00:00.0 & 16.4 \\
\hline 2006-06-07 03:00:00.0 & \\
\hline
\end{tabular}

\begin{tabular}{|c|c|}
\hline DataHora & VelVentoMax \\
\hline 2006-06-07 00:00:00.0 & 11.4 \\
\hline 2006-06-06 21:00:00.0 & 17.0 \\
\hline 2006-06-06 18:00:00.0 & 24.5 \\
\hline 2006-06-06 15:00:00.0 & 17.4 \\
\hline 2006-06-06 12:00:00.0 & 16.8 \\
\hline 2006-06-06 09:00:00.0 & 20.5 \\
\hline 2006-06-06 06:00:00.0 & 20.5 \\
\hline 2006-06-06 03:00:00.0 & 19.7 \\
\hline 2006-06-06 00:00:00.0 & \\
\hline 2006-06-05 21:00:00.0 & 12.7 \\
\hline 2006-06-05 18:00:00.0 & 12.7 \\
\hline 2006-06-05 15:00:00.0 & 12.9 \\
\hline 2006-06-05 12:00:00.0 & \\
\hline 2006-06-05 09:00:00.0 & 6.1 \\
\hline 2006-06-05 06:00:00.0 & 6.7 \\
\hline 2006-06-05 03:00:00.0 & \\
\hline 2006-06-05 00:00:00.0 & 10.6 \\
\hline 2006-06-04 21:00:00.0 & 17.6 \\
\hline 2006-06-04 18:00:00.0 & 21.4 \\
\hline 2006-06-04 15:00:00.0 & 22.0 \\
\hline 2006-06-04 12:00:00.0 & 23.4 \\
\hline 2006-06-04 09:00:00.0 & 20.9 \\
\hline 2006-06-04 06:00:00.0 & 24.7 \\
\hline 2006-06-04 03:00:00.0 & \\
\hline 2006-06-04 00:00:00.0 & 20.3 \\
\hline 2006-06-03 21:00:00.0 & 15.6 \\
\hline 2006-06-03 18:00:00.0 & 22.8 \\
\hline 2006-06-03 15:00:00.0 & \\
\hline 2006-06-03 12:00:00.0 & 26.8 \\
\hline 2006-06-03 09:00:00.0 & 27.7 \\
\hline 2006-06-03 06:00:00.0 & 27.7 \\
\hline 2006-06-03 03:00:00.0 & 25.5 \\
\hline 2006-06-03 00:00:00.0 & 22.1 \\
\hline 2006-06-02 21:00:00.0 & 20.0 \\
\hline $2006-06-02$ 18:00:00.0 & 22.6 \\
\hline 2006-06-02 15:00:00.0 & 25.7 \\
\hline 2006-06-02 12:00:00.0 & 25.7 \\
\hline 2006-06-02 09:00:00.0 & 20.5 \\
\hline 2006-06-02 06:00:00.0 & 20.7 \\
\hline 2006-06-02 03:00:00.0 & \\
\hline 2006-06-02 00:00:00.0 & 20.2 \\
\hline 2006-06-01 21:00:00.0 & 18.0 \\
\hline 2006-06-01 18:00:00.0 & 17.8 \\
\hline 2006-06-01 15:00:00.0 & 25.7 \\
\hline 2006-06-01 12:00:00.0 & 20.8 \\
\hline 2006-06-01 09:00:00.0 & 19.5 \\
\hline 2006-06-01 06:00:00.0 & 19.6 \\
\hline 2006-06-01 03:00:00.0 & 14.3 \\
\hline 2006-06-01 00:00:00.0 & 11.4 \\
\hline 2006-05-31 21:00:00.0 & 15.3 \\
\hline 2006-05-31 18:00:00.0 & 17.9 \\
\hline 2006-05-31 15:00:00.0 & \\
\hline 2006-05-31 12:00:00.0 & 15.1 \\
\hline 2006-05-31 09:00:00.0 & 21.0 \\
\hline 2006-05-31 06:00:00.0 & 15.5 \\
\hline 2006-05-31 03:00:00.0 & \\
\hline 2006-05-31 00:00:00.0 & 16.9 \\
\hline 2006-05-30 21:00:00.0 & 9.8 \\
\hline 2006-05-30 18:00:00.0 & 15.4 \\
\hline 2006-05-30 15:00:00.0 & 13.9 \\
\hline 2006-05-30 12:00:00.0 & 12.7 \\
\hline 2006-05-30 09:00:00.0 & 15.9 \\
\hline 2006-05-30 06:00:00.0 & \\
\hline 2006-05-30 03:00:00.0 & 12.9 \\
\hline 2006-05-30 00:00:00.0 & 9.9 \\
\hline 2006-05-29 21:00:00.0 & 32.5 \\
\hline 2006-05-29 18:00:00.0 & 21.2 \\
\hline $2006-05-29$ 15:00:00.0 & 22.0 \\
\hline 2006-05-29 12:00:00.0 & 22.5 \\
\hline
\end{tabular}




\section{ANEXO 01 - RAJADAS DE VENTO REGISTRADAS EM SÃO MARTINHO DA SERRA / RS}

\begin{tabular}{|c|c|}
\hline DataHora & VelVentoMax \\
\hline 2006-05-29 09:00:00.0 & 20.8 \\
\hline 2006-05-29 06:00:00.0 & 23.4 \\
\hline 2006-05-29 03:00:00.0 & 18.3 \\
\hline 2006-05-29 00:00:00.0 & 15.3 \\
\hline 2006-05-28 21:00:00.0 & 13.8 \\
\hline \multicolumn{2}{|l|}{ 2006-05-28 18:00:00.0 } \\
\hline 2006-05-28 15:00:00.0 & 12.4 \\
\hline 2006-05-28 12:00:00.0 & 12.4 \\
\hline 2006-05-28 09:00:00.0 & 10.7 \\
\hline \multicolumn{2}{|l|}{ 2006-05-28 06:00:00.0 } \\
\hline 2006-05-28 03:00:00.0 & 9.9 \\
\hline 2006-05-28 00:00:00.0 & 17.1 \\
\hline 2006-05-27 21:00:00.0 & 22.6 \\
\hline 2006-05-27 18:00:00.0 & 26.0 \\
\hline 2006-05-27 15:00:00.0 & 14.9 \\
\hline 2006-05-27 12:00:00.0 & 12.5 \\
\hline 2006-05-27 09:00:00.0 & 15.5 \\
\hline \multicolumn{2}{|l|}{ 2006-05-27 06:00:00.0 } \\
\hline 2006-05-27 03:00:00.0 & 11.3 \\
\hline 2006-05-27 00:00:00.0 & 10.5 \\
\hline 2006-05-26 21:00:00.0 & 16.7 \\
\hline 2006-05-26 18:00:00.0 & 19.1 \\
\hline 2006-05-26 15:00:00.0 & 18.5 \\
\hline 2006-05-26 12:00:00.0 & 18.6 \\
\hline \multicolumn{2}{|l|}{ 2006-05-26 09:00:00.0 } \\
\hline \multicolumn{2}{|l|}{ 2006-05-26 06:00:00.0 } \\
\hline 2006-05-26 03:00:00.0 & 14.7 \\
\hline 2006-05-26 00:00:00.0 & 14.4 \\
\hline 2006-05-25 21:00:00.0 & 9.7 \\
\hline 2006-05-25 18:00:00.0 & 12.7 \\
\hline 2006-05-25 15:00:00.0 & 21.6 \\
\hline 2006-05-25 12:00:00.0 & 20.8 \\
\hline 2006-05-25 09:00:00.0 & 19.9 \\
\hline 2006-05-25 06:00:00.0 & 19.4 \\
\hline 2006-05-25 03:00:00.0 & 17.6 \\
\hline 2006-05-25 00:00:00.0 & 15.7 \\
\hline 2006-05-24 21:00:00.0 & 16.7 \\
\hline 2006-05-24 18:00:00.0 & 18.1 \\
\hline 2006-05-24 15:00:00.0 & 23.1 \\
\hline 2006-05-24 12:00:00.0 & 16.5 \\
\hline \multicolumn{2}{|l|}{ 2006-05-24 09:00:00.0 } \\
\hline 2006-05-24 06:00:00.0 & 16.8 \\
\hline 2006-05-24 03:00:00.0 & 16.6 \\
\hline \multicolumn{2}{|l|}{ 2006-05-24 00:00:00.0 } \\
\hline 2006-05-23 21:00:00.0 & 23.0 \\
\hline 2006-05-23 18:00:00.0 & 21.8 \\
\hline 2006-05-23 15:00:00.0 & 27.5 \\
\hline 2006-05-23 12:00:00.0 & 18.8 \\
\hline \multicolumn{2}{|l|}{ 2006-05-23 09:00:00.0 } \\
\hline 2006-05-23 06:00:00.0 & 10.0 \\
\hline 2006-05-23 03:00:00.0 & 8.9 \\
\hline 2006-05-23 00:00:00.0 & 9.9 \\
\hline 2006-05-22 21:00:00.0 & 16.4 \\
\hline 2006-05-22 18:00:00.0 & 20.6 \\
\hline 2006-05-22 15:00:00.0 & 21.0 \\
\hline 2006-05-22 12:00:00.0 & 9.8 \\
\hline \multicolumn{2}{|l|}{ 2006-05-22 09:00:00.0 } \\
\hline 2006-05-22 06:00:00.0 & 8.3 \\
\hline 2006-05-22 03:00:00.0 & 7.4 \\
\hline 2006-05-22 00:00:00.0 & 8.3 \\
\hline 2006-05-21 21:00:00.0 & 16.5 \\
\hline 2006-05-21 18:00:00.0 & 19.0 \\
\hline 2006-05-21 15:00:00.0 & 15.6 \\
\hline 2006-05-21 12:00:00.0 & 12.2 \\
\hline \multicolumn{2}{|l|}{ 2006-05-21 09:00:00.0 } \\
\hline 2006-05-21 06:00:00.0 & 9.4 \\
\hline 2006-05-21 03:00:00.0 & 10.7 \\
\hline 2006-05-21 00:00:00.0 & 11.8 \\
\hline 2006-05-20 21:00:00.0 & 15.0 \\
\hline
\end{tabular}

\begin{tabular}{|c|c|}
\hline DataHora & VelVentoMax \\
\hline 2006-05-20 18:00:00.0 & 16.8 \\
\hline 2006-05-20 15:00:00.0 & 20.5 \\
\hline 2006-05-20 12:00:00.0 & 18.9 \\
\hline 2006-05-20 09:00:00.0 & \\
\hline 2006-05-20 06:00:00.0 & 22.0 \\
\hline 2006-05-20 03:00:00.0 & 24.0 \\
\hline 2006-05-20 00:00:00.0 & 25.9 \\
\hline 2006-05-19 21:00:00.0 & 24.0 \\
\hline 2006-05-19 18:00:00.0 & 19.3 \\
\hline 2006-05-19 15:00:00.0 & 8.6 \\
\hline 2006-05-19 12:00:00.0 & 5.8 \\
\hline 2006-05-19 09:00:00.0 & \\
\hline 2006-05-19 06:00:00.0 & 15.8 \\
\hline 2006-05-19 03:00:00.0 & 7.4 \\
\hline 2006-05-19 00:00:00.0 & \\
\hline 2006-05-18 21:00:00.0 & \\
\hline 2006-05-18 18:00:00.0 & 19.0 \\
\hline 2006-05-18 15:00:00.0 & 17.1 \\
\hline 2006-05-18 12:00:00.0 & \\
\hline 2006-05-18 09:00:00.0 & \\
\hline 2006-05-18 06:00:00.0 & 14.8 \\
\hline 2006-05-18 03:00:00.0 & 13.4 \\
\hline 2006-05-18 00:00:00.0 & 4.8 \\
\hline 2006-05-17 21:00:00.0 & 12.1 \\
\hline 2006-05-17 18:00:00.0 & 13.1 \\
\hline 2006-05-17 15:00:00.0 & 17.0 \\
\hline 2006-05-17 12:00:00.0 & \\
\hline 2006-05-17 09:00:00.0 & \\
\hline 2006-05-17 06:00:00.0 & 11.5 \\
\hline 2006-05-17 03:00:00.0 & 13.7 \\
\hline 2006-05-17 00:00:00.0 & \\
\hline 2006-05-16 21:00:00.0 & 14.7 \\
\hline 2006-05-16 18:00:00.0 & 16.0 \\
\hline 2006-05-16 15:00:00.0 & 22.4 \\
\hline 2006-05-16 12:00:00.0 & \\
\hline 2006-05-16 09:00:00.0 & 17.0 \\
\hline 2006-05-16 06:00:00.0 & 17.0 \\
\hline 2006-05-16 03:00:00.0 & 13.0 \\
\hline 2006-05-16 00:00:00.0 & 14.0 \\
\hline 2006-05-15 21:00:00.0 & 13.0 \\
\hline 2006-05-15 18:00:00.0 & 16.0 \\
\hline 2006-05-15 15:00:00.0 & 15.0 \\
\hline 2006-05-15 12:00:00.0 & \\
\hline 2006-05-15 09:00:00.0 & \\
\hline 2006-05-15 06:00:00.0 & 16.0 \\
\hline 2006-05-15 03:00:00.0 & 14.0 \\
\hline 2006-05-15 00:00:00.0 & 12.0 \\
\hline 2006-05-14 21:00:00.0 & 13.0 \\
\hline 2006-05-14 18:00:00.0 & 14.0 \\
\hline 2006-05-14 15:00:00.0 & 17.0 \\
\hline 2006-05-14 12:00:00.0 & \\
\hline 2006-05-14 09:00:00.0 & 16.0 \\
\hline 2006-05-14 06:00:00.0 & 15.0 \\
\hline 2006-05-14 03:00:00.0 & 10.0 \\
\hline 2006-05-14 00:00:00.0 & 9.0 \\
\hline 2006-05-13 21:00:00.0 & 13.0 \\
\hline 2006-05-13 18:00:00.0 & 13.0 \\
\hline 2006-05-13 15:00:00.0 & 15.0 \\
\hline 2006-05-13 12:00:00.0 & \\
\hline 2006-05-13 09:00:00.0 & 10.0 \\
\hline 2006-05-13 06:00:00.0 & 11.0 \\
\hline 2006-05-13 03:00:00.0 & 10.0 \\
\hline 2006-05-13 00:00:00.0 & 12.0 \\
\hline 2006-05-12 21:00:00.0 & 16.0 \\
\hline 2006-05-12 18:00:00.0 & 19.0 \\
\hline 2006-05-12 15:00:00.0 & 15.0 \\
\hline 2006-05-12 12:00:00.0 & \\
\hline 2006-05-12 09:00:00.0 & 5.0 \\
\hline 2006-05-12 06:00:00.0 & 6.0 \\
\hline
\end{tabular}

\begin{tabular}{|c|c|}
\hline \multirow{3}{*}{$\begin{array}{l}\text { DataHora } \\
2006-05-12 \text { 03:00:00.0 }\end{array}$} & VelVentoMax \\
\hline & \\
\hline & 6.0 \\
\hline 2006-05-11 21:00:00.0 & 16.0 \\
\hline 2006-05-11 18:00:00.0 & 22.0 \\
\hline 2006-05-11 15:00:00.0 & 21.0 \\
\hline 2006-05-11 12:00:00.0 & \\
\hline 2006-05-11 09:00:00.0 & 8.0 \\
\hline 2006-05-11 06:00:00.0 & 11.0 \\
\hline 2006-05-11 03:00:00.0 & \\
\hline 2006-05-11 00:00:00.0 & 11.0 \\
\hline $2006-05-10$ 21:00:00.0 & 16.0 \\
\hline $2006-05-10$ 18:00:00.0 & 22.0 \\
\hline 2006-05-10 15:00:00.0 & \\
\hline 2006-05-10 12:00:00.0 & 18.0 \\
\hline 2006-05-10 09:00:00.0 & 17.0 \\
\hline 2006-05-10 06:00:00.0 & 16.0 \\
\hline 2006-05-10 03:00:00.0 & \\
\hline $2006-05-1000: 00: 00.0$ & 15.0 \\
\hline 2006-05-09 21:00:00.0 & 23.0 \\
\hline 2006-05-09 18:00:00.0 & 21.0 \\
\hline 2006-05-09 15:00:00.0 & \\
\hline 2006-05-09 12:00:00.0 & 19.0 \\
\hline 2006-05-09 09:00:00.0 & 16.0 \\
\hline 2006-05-09 06:00:00.0 & 15.0 \\
\hline 2006-05-09 03:00:00.0 & 15.0 \\
\hline 2006-05-09 00:00:00.0 & 18.0 \\
\hline 2006-05-08 21:00:00.0 & 25.0 \\
\hline 2006-05-08 18:00:00.0 & 26.0 \\
\hline 2006-05-08 15:00:00.0 & \\
\hline 2006-05-08 12:00:00.0 & \\
\hline 2006-05-08 09:00:00.0 & 13.0 \\
\hline 2006-05-08 06:00:00.0 & 20.0 \\
\hline 2006-05-08 03:00:00.0 & 23.0 \\
\hline 2006-05-08 00:00:00.0 & 15.0 \\
\hline $2006-05-07$ 21:00:00.0 & 27.0 \\
\hline 2006-05-07 18:00:00.0 & 16.0 \\
\hline 2006-05-07 15:00:00.0 & \\
\hline $2006-05-07$ 12:00:00.0 & 12.0 \\
\hline 2006-05-07 09:00:00.0 & 10.0 \\
\hline 2006-05-07 06:00:00.0 & \\
\hline 2006-05-07 03:00:00.0 & \\
\hline 2006-05-07 00:00:00.0 & 9.0 \\
\hline 2006-05-06 21:00:00.0 & 14.0 \\
\hline 2006-05-06 18:00:00.0 & 13.0 \\
\hline 2006-05-06 15:00:00.0 & 17.0 \\
\hline 2006-05-06 12:00:00.0 & \\
\hline 2006-05-06 09:00:00.0 & 13.0 \\
\hline 2006-05-06 06:00:00.0 & \\
\hline 2006-05-06 03:00:00.0 & \\
\hline 2006-05-06 00:00:00.0 & 9.0 \\
\hline 2006-05-05 21:00:00.0 & 34.0 \\
\hline 2006-05-05 18:00:00.0 & \\
\hline 2006-05-05 15:00:00.0 & 19.0 \\
\hline $2006-05-05$ 12:00:00.0 & 15.0 \\
\hline 2006-05-05 09:00:00.0 & \\
\hline 2006-05-05 06:00:00.0 & \\
\hline 2006-05-05 03:00:00.0 & 5.0 \\
\hline 2006-05-05 00:00:00.0 & 8.0 \\
\hline 2006-05-04 21:00:00.0 & 23.0 \\
\hline 2006-05-04 18:00:00.0 & 19.0 \\
\hline 2006-05-04 15:00:00.0 & 15.0 \\
\hline $2006-05-04$ 12:00:00.0 & 6.0 \\
\hline 2006-05-04 09:00:00.0 & 9.0 \\
\hline 2006-05-04 06:00:00.0 & 8.0 \\
\hline 2006-05-04 03:00:00.0 & 8.0 \\
\hline $2006-05-04$ 00:00:00.0 & 7.0 \\
\hline 2006-05-03 21:00:00.0 & 15.0 \\
\hline 2006-05-03 18:00:00.0 & 18.0 \\
\hline 2006-05-03 15:00:00.0 & 18.0 \\
\hline
\end{tabular}




\section{ANEXO 01 - RAJADAS DE VENTO REGISTRADAS EM SÃO MARTINHO DA SERRA / RS}

\begin{tabular}{|c|c|}
\hline DataHora & VelVentoMax \\
\hline 2006-05-03 12:00:00.0 & 15.0 \\
\hline \multicolumn{2}{|l|}{ 2006-05-03 09:00:00.0 } \\
\hline \multicolumn{2}{|l|}{ 2006-05-03 06:00:00.0 } \\
\hline 2006-05-03 03:00:00.0 & 16.0 \\
\hline 2006-05-03 00:00:00.0 & 16.0 \\
\hline 2006-05-02 21:00:00.0 & 40.0 \\
\hline 2006-05-02 18:00:00.0 & 24.0 \\
\hline 2006-05-02 15:00:00.0 & 24.0 \\
\hline \multicolumn{2}{|l|}{ 2006-05-02 12:00:00.0 } \\
\hline 2006-05-02 09:00:00.0 & 9.0 \\
\hline \multicolumn{2}{|l|}{ 2006-05-02 06:00:00.0 } \\
\hline 2006-05-02 03:00:00.0 & 12.0 \\
\hline 2006-05-02 00:00:00.0 & 10.0 \\
\hline 2006-05-01 21:00:00.0 & 22.0 \\
\hline \multicolumn{2}{|l|}{ 2006-05-01 18:00:00.0 } \\
\hline 2006-05-01 15:00:00.0 & 20.0 \\
\hline 2006-05-01 12:00:00.0 & 11.0 \\
\hline \multicolumn{2}{|l|}{ 2006-05-01 09:00:00.0 } \\
\hline 2006-05-01 06:00:00.0 & 11.0 \\
\hline 2006-05-01 03:00:00.0 & 12.0 \\
\hline 2006-05-01 00:00:00.0 & 13.0 \\
\hline \multicolumn{2}{|l|}{ 2006-04-30 21:00:00.0 } \\
\hline \multicolumn{2}{|l|}{ 2006-04-30 18:00:00.0 } \\
\hline \multicolumn{2}{|l|}{ 2006-04-30 15:00:00.0 } \\
\hline 2006-04-30 12:00:00.0 & 12.0 \\
\hline \multicolumn{2}{|l|}{ 2006-04-30 09:00:00.0 } \\
\hline \multicolumn{2}{|l|}{ 2006-04-30 06:00:00.0 } \\
\hline 2006-04-30 03:00:00.0 & 7.0 \\
\hline \multirow{2}{*}{\multicolumn{2}{|c|}{$\begin{array}{l}2006-04-30 \text { 00:00:00.0 } \\
2006-04-29 \text { 21:00:00.0 }\end{array}$}} \\
\hline & \\
\hline \multicolumn{2}{|l|}{ 2006-04-29 18:00:00.0 } \\
\hline 2006-04-29 15:00:00.0 & 10.0 \\
\hline 2006-04-29 12:00:00.0 & 13.0 \\
\hline 2006-04-29 09:00:00.0 & \\
\hline 2006-04-29 06:00:00.0 & 19.0 \\
\hline 2006-04-29 03:00:00.0 & 15.0 \\
\hline 2006-04-29 00:00:00.0 & 13.0 \\
\hline 2006-04-28 21:00:00.0 & \\
\hline 2006-04-28 18:00:00.0 & 16.0 \\
\hline 2006-04-28 15:00:00.0 & 21.0 \\
\hline 2006-04-28 12:00:00.0 & 14.0 \\
\hline 2006-04-28 09:00:00.0 & \\
\hline 2006-04-28 06:00:00.0 & 10.0 \\
\hline 2006-04-28 03:00:00.0 & 10.0 \\
\hline 2006-04-28 00:00:00.0 & 6.0 \\
\hline 2006-04-27 21:00:00.0 & 13.0 \\
\hline 2006-04-27 18:00:00.0 & 12.0 \\
\hline 2006-04-27 15:00:00.0 & 10.0 \\
\hline 2006-04-27 12:00:00.0 & 5.0 \\
\hline 2006-04-27 09:00:00.0 & \\
\hline 2006-04-27 06:00:00.0 & \\
\hline 2006-04-27 03:00:00.0 & \\
\hline 2006-04-27 00:00:00.0 & 6.0 \\
\hline 2006-04-26 21:00:00.0 & 10.0 \\
\hline 2006-04-26 18:00:00.0 & 10.0 \\
\hline 2006-04-26 15:00:00.0 & 9.0 \\
\hline 2006-04-26 12:00:00.0 & 11.0 \\
\hline 2006-04-26 09:00:00.0 & \\
\hline 2006-04-26 06:00:00.0 & 19.0 \\
\hline 2006-04-26 03:00:00.0 & \\
\hline 2006-04-26 00:00:00.0 & 11.0 \\
\hline 2006-04-25 21:00:00.0 & \\
\hline 2006-04-25 18:00:00.0 & 43.0 \\
\hline 2006-04-25 15:00:00.0 & 19.0 \\
\hline 2006-04-25 12:00:00.0 & 16.0 \\
\hline 2006-04-25 09:00:00.0 & \\
\hline 2006-04-25 06:00:00.0 & 14.0 \\
\hline 2006-04-25 03:00:00.0 & 14.0 \\
\hline 2006-04-25 00:00:00.0 & 12.0 \\
\hline
\end{tabular}

\begin{tabular}{|c|c|}
\hline DataHora & VelVentoMax \\
\hline 2006-04-24 21:00:00.0 & 8.0 \\
\hline 2006-04-24 18:00:00.0 & 9.0 \\
\hline 2006-04-24 15:00:00.0 & 13.0 \\
\hline 2006-04-24 12:00:00.0 & \\
\hline 2006-04-24 09:00:00.0 & 10.0 \\
\hline 2006-04-24 06:00:00.0 & 10.0 \\
\hline 2006-04-24 03:00:00.0 & 7.0 \\
\hline 2006-04-24 00:00:00.0 & \\
\hline 2006-04-23 21:00:00.0 & \\
\hline 2006-04-23 18:00:00.0 & 19.0 \\
\hline 2006-04-23 15:00:00.0 & 24.0 \\
\hline 2006-04-23 12:00:00.0 & \\
\hline 2006-04-23 09:00:00.0 & \\
\hline 2006-04-23 06:00:00.0 & \\
\hline 2006-04-22 21:00:00.0 & 14.0 \\
\hline 2006-04-22 18:00:00.0 & 14.0 \\
\hline 2006-04-22 15:00:00.0 & 12.0 \\
\hline 2006-04-22 12:00:00.0 & \\
\hline 2006-04-22 09:00:00.0 & 7.0 \\
\hline 2006-04-22 06:00:00.0 & 7.0 \\
\hline 2006-04-22 03:00:00.0 & 9.0 \\
\hline 2006-04-22 00:00:00.0 & \\
\hline 2006-04-21 21:00:00.0 & \\
\hline 2006-04-21 18:00:00.0 & 24.0 \\
\hline 2006-04-21 15:00:00.0 & 20.0 \\
\hline 2006-04-21 12:00:00.0 & \\
\hline 2006-04-21 09:00:00.0 & 27.0 \\
\hline 2006-04-21 06:00:00.0 & 22.0 \\
\hline 2006-04-21 03:00:00.0 & 15.0 \\
\hline 2006-04-21 00:00:00.0 & 15.0 \\
\hline 2006-04-20 21:00:00.0 & 14.0 \\
\hline 2006-04-20 18:00:00.0 & 21.0 \\
\hline 2006-04-20 15:00:00.0 & 27.0 \\
\hline 2006-04-20 12:00:00.0 & \\
\hline 2006-04-20 09:00:00.0 & 22.0 \\
\hline 2006-04-20 06:00:00.0 & 21.0 \\
\hline 2006-04-20 03:00:00.0 & \\
\hline 2006-04-20 00:00:00.0 & 15.0 \\
\hline 2006-04-19 21:00:00.0 & 19.0 \\
\hline 2006-04-19 18:00:00.0 & 27.0 \\
\hline 2006-04-19 15:00:00.0 & 22.0 \\
\hline 2006-04-19 12:00:00.0 & 20.0 \\
\hline 2006-04-19 09:00:00.0 & 20.0 \\
\hline 2006-04-19 06:00:00.0 & 16.0 \\
\hline 2006-04-19 03:00:00.0 & 16.0 \\
\hline 2006-04-19 00:00:00.0 & 13.0 \\
\hline 2006-04-18 21:00:00.0 & 17.0 \\
\hline 2006-04-18 18:00:00.0 & 4.0 \\
\hline 2006-04-18 15:00:00.0 & 25.0 \\
\hline 2006-04-18 12:00:00.0 & 26.0 \\
\hline 2006-04-18 09:00:00.0 & 19.0 \\
\hline 2006-04-18 06:00:00.0 & 13.0 \\
\hline 2006-04-18 03:00:00.0 & 10.0 \\
\hline 2006-04-18 00:00:00.0 & 10.0 \\
\hline 2006-04-17 21:00:00.0 & 18.0 \\
\hline 2006-04-17 18:00:00.0 & 19.0 \\
\hline 2006-04-17 15:00:00.0 & 20.0 \\
\hline 2006-04-17 12:00:00.0 & 18.0 \\
\hline 2006-04-17 09:00:00.0 & 11.0 \\
\hline 2006-04-17 06:00:00.0 & 10.0 \\
\hline 2006-04-17 03:00:00.0 & 9.0 \\
\hline 2006-04-17 00:00:00.0 & 11.0 \\
\hline 2006-04-16 21:00:00.0 & 21.0 \\
\hline 2006-04-16 18:00:00.0 & 22.0 \\
\hline 2006-04-16 15:00:00.0 & \\
\hline 2006-04-16 12:00:00.0 & 27.0 \\
\hline 2006-04-16 09:00:00.0 & 20.0 \\
\hline 2006-04-16 06:00:00.0 & \\
\hline 2006-04-16 03:00:00.0 & \\
\hline
\end{tabular}

\begin{tabular}{|c|c|}
\hline DataHora & VelVentoMax \\
\hline 2006-04-16 00:00:00.0 & \\
\hline 2006-04-15 15:00:00.0 & 20.0 \\
\hline 2006-04-15 12:00:00.0 & 13.0 \\
\hline 2006-04-15 09:00:00.0 & \\
\hline 2006-04-15 06:00:00.0 & 10.0 \\
\hline 2006-04-15 03:00:00.0 & 12.0 \\
\hline 2006-04-15 00:00:00.0 & 24.0 \\
\hline 2006-04-14 21:00:00.0 & 18.0 \\
\hline 2006-04-14 18:00:00.0 & 37.0 \\
\hline $2006-04-14$ 15:00:00.0 & \\
\hline $2006-04-14$ 12:00:00.0 & \\
\hline 2006-04-14 09:00:00.0 & 11.0 \\
\hline 2006-04-14 06:00:00.0 & 14.0 \\
\hline 2006-04-14 03:00:00.0 & \\
\hline 2006-04-14 00:00:00.0 & 10.0 \\
\hline 2006-04-13 21:00:00.0 & 9.0 \\
\hline 2006-04-13 18:00:00.0 & 11.0 \\
\hline 2006-04-13 15:00:00.0 & \\
\hline 2006-04-13 12:00:00.0 & 13.0 \\
\hline 2006-04-13 09:00:00.0 & 12.0 \\
\hline 2006-04-13 06:00:00.0 & 11.0 \\
\hline 2006-04-13 03:00:00.0 & \\
\hline $2006-04-13$ 00:00:00.0 & \\
\hline 2006-04-12 21:00:00.0 & 21.0 \\
\hline 2006-04-12 18:00:00.0 & 25.0 \\
\hline 2006-04-12 15:00:00.0 & 19.0 \\
\hline 2006-04-12 12:00:00.0 & 5.0 \\
\hline 2006-04-12 09:00:00.0 & 6.0 \\
\hline 2006-04-12 06:00:00.0 & 6.0 \\
\hline 2006-04-12 03:00:00.0 & 6.0 \\
\hline 2006-04-12 00:00:00.0 & 9.0 \\
\hline 2006-04-11 21:00:00.0 & \\
\hline 2006-04-11 18:00:00.0 & 18.0 \\
\hline 2006-04-11 15:00:00.0 & 13.0 \\
\hline $2006-04-11$ 12:00:00.0 & \\
\hline 2006-04-11 09:00:00.0 & 5.0 \\
\hline 2006-04-11 06:00:00.0 & \\
\hline 2006-04-11 03:00:00.0 & \\
\hline 2006-04-11 00:00:00.0 & 9.0 \\
\hline 2006-04-10 21:00:00.0 & 18.0 \\
\hline 2006-04-10 18:00:00.0 & 16.0 \\
\hline 2006-04-10 15:00:00.0 & \\
\hline 2006-04-10 12:00:00.0 & \\
\hline 2006-04-10 09:00:00.0 & 12.0 \\
\hline 2006-04-10 06:00:00.0 & 11.0 \\
\hline 2006-04-10 03:00:00.0 & \\
\hline 2006-04-10 00:00:00.0 & 14.0 \\
\hline 2006-04-09 21:00:00.0 & 19.0 \\
\hline 2006-04-09 18:00:00.0 & \\
\hline 2006-04-09 15:00:00.0 & 14.0 \\
\hline 2006-04-09 12:00:00.0 & 19.0 \\
\hline 2006-04-09 09:00:00.0 & 14.0 \\
\hline 2006-04-09 06:00:00.0 & 17.0 \\
\hline 2006-04-09 03:00:00.0 & 15.0 \\
\hline 2006-04-09 00:00:00.0 & 12.0 \\
\hline 2006-04-08 21:00:00.0 & 15.0 \\
\hline 2006-04-08 18:00:00.0 & 14.0 \\
\hline 2006-04-08 15:00:00.0 & 13.0 \\
\hline 2006-04-08 12:00:00.0 & 14.0 \\
\hline 2006-04-08 09:00:00.0 & \\
\hline 2006-04-08 06:00:00.0 & \\
\hline 2006-04-08 03:00:00.0 & 12.0 \\
\hline 2006-04-08 00:00:00.0 & 12.0 \\
\hline 2006-04-07 21:00:00.0 & 15.0 \\
\hline 2006-04-07 18:00:00.0 & \\
\hline $2006-04-07$ 15:00:00.0 & 17.0 \\
\hline 2006-04-07 12:00:00.0 & 18.0 \\
\hline 2006-04-07 09:00:00.0 & 17.0 \\
\hline 2006-04-07 06:00:00.0 & 14.0 \\
\hline
\end{tabular}




\section{ANEXO 01 - RAJADAS DE VENTO REGISTRADAS EM SÃO MARTINHO DA SERRA / RS}

\begin{tabular}{|c|c|}
\hline DataHora & VelVentoMax \\
\hline \multicolumn{2}{|l|}{ 2006-04-07 03:00:00.0 } \\
\hline 2006-04-07 00:00:00.0 & 10.0 \\
\hline 2006-04-06 21:00:00.0 & 15.0 \\
\hline \multicolumn{2}{|l|}{ 2006-04-06 18:00:00.0 } \\
\hline 2006-04-06 15:00:00.0 & 13.0 \\
\hline 2006-04-06 12:00:00.0 & 31.0 \\
\hline \multicolumn{2}{|l|}{ 2006-04-06 09:00:00.0 } \\
\hline 2006-04-06 06:00:00.0 & 13.0 \\
\hline 2006-04-06 03:00:00.0 & 20.0 \\
\hline 2006-04-06 00:00:00.0 & 29.0 \\
\hline 2006-04-05 21:00:00.0 & 10.0 \\
\hline 2006-04-05 18:00:00.0 & 17.0 \\
\hline 2006-04-05 15:00:00.0 & 13.0 \\
\hline 2006-04-05 12:00:00.0 & 14.0 \\
\hline 2006-04-05 09:00:00.0 & 15.0 \\
\hline \multicolumn{2}{|l|}{ 2006-04-05 06:00:00.0 } \\
\hline 2006-04-05 03:00:00.0 & 13.0 \\
\hline 2006-04-05 00:00:00.0 & 12.0 \\
\hline 2006-04-04 21:00:00.0 & 15.0 \\
\hline 2006-04-04 18:00:00.0 & 16.0 \\
\hline 2006-04-04 15:00:00.0 & 23.0 \\
\hline 2006-04-04 12:00:00.0 & 23.0 \\
\hline \multicolumn{2}{|l|}{ 2006-04-04 09:00:00.0 } \\
\hline 2006-04-04 06:00:00.0 & 17.0 \\
\hline 2006-04-04 03:00:00.0 & 15.0 \\
\hline \multicolumn{2}{|l|}{ 2006-04-04 00:00:00.0 } \\
\hline 2006-04-03 21:00:00.0 & 16.0 \\
\hline 2006-04-03 18:00:00.0 & 22.0 \\
\hline 2006-04-03 15:00:00.0 & 22.0 \\
\hline 2006-04-03 12:00:00.0 & 20.0 \\
\hline \multicolumn{2}{|l|}{ 2006-04-03 09:00:00.0 } \\
\hline \multicolumn{2}{|l|}{ 2006-04-03 06:00:00.0 } \\
\hline 2006-04-03 03:00:00.0 & 18.0 \\
\hline 2006-04-03 00:00:00.0 & 16.0 \\
\hline 2006-04-02 21:00:00.0 & 23.0 \\
\hline \multicolumn{2}{|l|}{ 2006-04-02 18:00:00.0 } \\
\hline 2006-04-02 15:00:00.0 & 16.0 \\
\hline 2006-04-02 12:00:00.0 & 16.0 \\
\hline \multicolumn{2}{|l|}{ 2006-04-02 09:00:00.0 } \\
\hline 2006-04-02 06:00:00.0 & 15.0 \\
\hline 2006-04-02 03:00:00.0 & 13.0 \\
\hline 2006-04-02 00:00:00.0 & 10.0 \\
\hline \multicolumn{2}{|l|}{ 2006-04-01 21:00:00.0 } \\
\hline \multicolumn{2}{|l|}{ 2006-04-01 18:00:00.0 } \\
\hline 2006-04-01 15:00:00.0 & 18.0 \\
\hline \multicolumn{2}{|l|}{ 2006-04-01 12:00:00.0 } \\
\hline 2006-04-01 09:00:00.0 & 18.0 \\
\hline 2006-04-01 06:00:00.0 & 13.0 \\
\hline 2006-04-01 03:00:00.0 & 13.0 \\
\hline 2006-04-01 00:00:00.0 & 14.0 \\
\hline 2006-03-31 21:00:00.0 & \\
\hline 2006-03-31 18:00:00.0 & 14.0 \\
\hline 2006-03-31 15:00:00.0 & 34.0 \\
\hline 2006-03-31 12:00:00.0 & \\
\hline 2006-03-31 09:00:00.0 & \\
\hline 2006-03-31 06:00:00.0 & 17.0 \\
\hline 2006-03-31 03:00:00.0 & 12.0 \\
\hline 2006-03-31 00:00:00.0 & 12.0 \\
\hline 2006-03-30 21:00:00.0 & \\
\hline 2006-03-30 18:00:00.0 & 12.0 \\
\hline 2006-03-30 15:00:00.0 & 12.0 \\
\hline 2006-03-30 12:00:00.0 & 14.0 \\
\hline 2006-03-30 09:00:00.0 & \\
\hline 2006-03-30 06:00:00.0 & 11.0 \\
\hline 2006-03-30 03:00:00.0 & 9.0 \\
\hline 2006-03-30 00:00:00.0 & 7.0 \\
\hline 2006-03-29 21:00:00.0 & \\
\hline 2006-03-29 18:00:00.0 & 19.0 \\
\hline 2006-03-29 15:00:00.0 & 35.0 \\
\hline
\end{tabular}

\begin{tabular}{|c|c|}
\hline DataHora & VelVentoMax \\
\hline 2006-03-29 12:00:00.0 & 18.0 \\
\hline 2006-03-29 09:00:00.0 & \\
\hline 2006-03-29 06:00:00.0 & 18.0 \\
\hline 2006-03-29 03:00:00.0 & 14.0 \\
\hline 2006-03-29 00:00:00.0 & 17.0 \\
\hline 2006-03-28 21:00:00.0 & \\
\hline 2006-03-28 18:00:00.0 & 16.0 \\
\hline 2006-03-28 15:00:00.0 & 1.0 \\
\hline 2006-03-28 12:00:00.0 & 9.0 \\
\hline 2006-03-28 09:00:00.0 & \\
\hline 2006-03-28 06:00:00.0 & \\
\hline 2006-03-28 03:00:00.0 & \\
\hline 2006-03-27 21:00:00.0 & 15.0 \\
\hline 2006-03-27 18:00:00.0 & 16.0 \\
\hline 2006-03-27 15:00:00.0 & 20.0 \\
\hline 2006-03-27 12:00:00.0 & \\
\hline 2006-03-27 09:00:00.0 & \\
\hline 2006-03-27 06:00:00.0 & \\
\hline 2006-03-26 21:00:00.0 & 20.0 \\
\hline 2006-03-26 18:00:00.0 & 20.0 \\
\hline 2006-03-26 15:00:00.0 & 27.0 \\
\hline 2006-03-26 12:00:00.0 & 24.0 \\
\hline 2006-03-26 09:00:00.0 & \\
\hline 2006-03-26 06:00:00.0 & 25.0 \\
\hline 2006-03-26 03:00:00.0 & 30.0 \\
\hline 2006-03-26 00:00:00.0 & 28.0 \\
\hline 2006-03-25 21:00:00.0 & 29.0 \\
\hline 2006-03-25 18:00:00.0 & 22.0 \\
\hline 2006-03-25 15:00:00.0 & 25.0 \\
\hline 2006-03-25 12:00:00.0 & 16.0 \\
\hline 2006-03-25 09:00:00.0 & 12.0 \\
\hline 2006-03-25 06:00:00.0 & 11.0 \\
\hline 2006-03-25 03:00:00.0 & 5.0 \\
\hline 2006-03-25 00:00:00.0 & 8.0 \\
\hline 2006-03-24 21:00:00.0 & 18.0 \\
\hline 2006-03-24 18:00:00.0 & 21.0 \\
\hline 2006-03-24 15:00:00.0 & 24.0 \\
\hline 2006-03-24 12:00:00.0 & \\
\hline 2006-03-24 09:00:00.0 & 12.0 \\
\hline 2006-03-24 06:00:00.0 & 10.0 \\
\hline 2006-03-24 03:00:00.0 & \\
\hline 2006-03-24 00:00:00.0 & \\
\hline 2006-03-23 21:00:00.0 & 23.0 \\
\hline 2006-03-23 18:00:00.0 & 28.0 \\
\hline 2006-03-23 15:00:00.0 & 31.0 \\
\hline 2006-03-23 12:00:00.0 & 18.0 \\
\hline 2006-03-23 09:00:00.0 & 18.0 \\
\hline 2006-03-23 06:00:00.0 & 15.0 \\
\hline 2006-03-23 03:00:00.0 & 16.0 \\
\hline 2006-03-23 00:00:00.0 & \\
\hline 2006-03-22 21:00:00.0 & 24.0 \\
\hline 2006-03-22 18:00:00.0 & 14.0 \\
\hline 2006-03-22 15:00:00.0 & \\
\hline 2006-03-22 12:00:00.0 & \\
\hline 2006-03-22 09:00:00.0 & 18.0 \\
\hline 2006-03-22 06:00:00.0 & 20.0 \\
\hline 2006-03-22 03:00:00.0 & \\
\hline 2006-03-22 00:00:00.0 & 22.0 \\
\hline 2006-03-21 21:00:00.0 & 23.0 \\
\hline 2006-03-21 18:00:00.0 & 21.0 \\
\hline 2006-03-21 15:00:00.0 & \\
\hline 2006-03-21 12:00:00.0 & \\
\hline 2006-03-21 09:00:00.0 & 19.0 \\
\hline 2006-03-21 06:00:00.0 & 15.0 \\
\hline 2006-03-21 03:00:00.0 & 20.0 \\
\hline 2006-03-21 00:00:00.0 & \\
\hline 2006-03-20 21:00:00.0 & 11.0 \\
\hline 2006-03-20 18:00:00.0 & 12.0 \\
\hline 2006-03-20 15:00:00.0 & 11.0 \\
\hline
\end{tabular}

\begin{tabular}{|c|c|}
\hline DataHora & VelVentoMax \\
\hline \multicolumn{2}{|l|}{ 2006-03-20 12:00:00.0 } \\
\hline 2006-03-20 09:00:00.0 & 8.0 \\
\hline 2006-03-20 06:00:00.0 & 17.0 \\
\hline \multicolumn{2}{|l|}{ 2006-03-20 03:00:00.0 } \\
\hline 2006-03-20 00:00:00.0 & 17.0 \\
\hline 2006-03-19 21:00:00.0 & 22.0 \\
\hline \multicolumn{2}{|l|}{ 2006-03-19 18:00:00.0 } \\
\hline \multicolumn{2}{|l|}{ 2006-03-19 15:00:00.0 } \\
\hline 2006-03-19 12:00:00.0 & 15.0 \\
\hline 2006-03-19 09:00:00.0 & 17.0 \\
\hline 2006-03-19 06:00:00.0 & 16.0 \\
\hline \multicolumn{2}{|l|}{ 2006-03-19 03:00:00.0 } \\
\hline 2006-03-19 00:00:00.0 & 22.0 \\
\hline 2006-03-18 21:00:00.0 & 22.0 \\
\hline 2006-03-18 18:00:00.0 & 17.0 \\
\hline \multicolumn{2}{|l|}{ 2006-03-18 15:00:00.0 } \\
\hline 2006-03-18 12:00:00.0 & 18.0 \\
\hline 2006-03-18 09:00:00.0 & 25.0 \\
\hline 2006-03-18 06:00:00.0 & 8.0 \\
\hline \multicolumn{2}{|l|}{ 2006-03-18 03:00:00.0 } \\
\hline 2006-03-18 00:00:00.0 & 8.0 \\
\hline 2006-03-17 21:00:00.0 & 19.0 \\
\hline 2006-03-17 18:00:00.0 & 25.0 \\
\hline \multicolumn{2}{|l|}{ 2006-03-17 15:00:00.0 } \\
\hline 2006-03-17 12:00:00.0 & 18.0 \\
\hline 2006-03-17 09:00:00.0 & 19.0 \\
\hline \multicolumn{2}{|l|}{ 2006-03-17 06:00:00.0 } \\
\hline 2006-03-17 03:00:00.0 & 14.0 \\
\hline 2006-03-17 00:00:00.0 & 11.0 \\
\hline 2006-03-16 21:00:00.0 & 15.0 \\
\hline \multicolumn{2}{|l|}{ 2006-03-16 18:00:00.0 } \\
\hline 2006-03-16 15:00:00.0 & 15.0 \\
\hline 2006-03-16 12:00:00.0 & 16.0 \\
\hline 2006-03-16 09:00:00.0 & 17.0 \\
\hline 2006-03-16 06:00:00.0 & 15.0 \\
\hline 2006-03-16 03:00:00.0 & 37.0 \\
\hline 2006-03-16 00:00:00.0 & 11.0 \\
\hline \multicolumn{2}{|l|}{ 2006-03-15 21:00:00.0 } \\
\hline 2006-03-15 18:00:00.0 & 17.0 \\
\hline 2006-03-15 15:00:00.0 & 13.0 \\
\hline 2006-03-15 12:00:00.0 & 17.0 \\
\hline 2006-03-15 09:00:00.0 & 17.0 \\
\hline \multicolumn{2}{|l|}{ 2006-03-15 06:00:00.0 } \\
\hline 2006-03-15 03:00:00.0 & 15.0 \\
\hline 2006-03-15 00:00:00.0 & 15.0 \\
\hline 2006-03-14 21:00:00.0 & 17.0 \\
\hline 2006-03-14 18:00:00.0 & 20.0 \\
\hline 2006-03-14 15:00:00.0 & 19.0 \\
\hline 2006-03-14 12:00:00.0 & 19.0 \\
\hline 2006-03-14 09:00:00.0 & 17.0 \\
\hline \multicolumn{2}{|l|}{ 2006-03-14 06:00:00.0 } \\
\hline 2006-03-14 03:00:00.0 & \\
\hline 2006-03-14 00:00:00.0 & \\
\hline 2006-03-13 21:00:00.0 & 14.0 \\
\hline 2006-03-13 18:00:00.0 & 18.0 \\
\hline 2006-03-13 15:00:00.0 & 19.0 \\
\hline 2006-03-13 12:00:00.0 & 20.0 \\
\hline 2006-03-13 09:00:00.0 & 17.0 \\
\hline 2006-03-13 06:00:00.0 & \\
\hline 2006-03-13 03:00:00.0 & 12.0 \\
\hline 2006-03-13 00:00:00.0 & \\
\hline 2006-03-12 21:00:00.0 & \\
\hline 2006-03-12 18:00:00.0 & \\
\hline 2006-03-12 15:00:00.0 & 20.0 \\
\hline 2006-03-12 12:00:00.0 & 21.0 \\
\hline 2006-03-12 09:00:00.0 & \\
\hline 2006-03-12 06:00:00.0 & \\
\hline 2006-03-12 03:00:00.0 & \\
\hline 2006-03-12 00:00:00.0 & \\
\hline
\end{tabular}




\section{ANEXO 01 - RAJADAS DE VENTO REGISTRADAS EM SÃO MARTINHO DA SERRA / RS}

\begin{tabular}{|c|c|}
\hline DataHora & VelVentoMax \\
\hline 2006-03-11 21:00:00.0 & 18.0 \\
\hline \multicolumn{2}{|l|}{ 2006-03-11 18:00:00.0 } \\
\hline 2006-03-11 15:00:00.0 & 21.0 \\
\hline \multicolumn{2}{|l|}{ 2006-03-11 12:00:00.0 } \\
\hline \multicolumn{2}{|l|}{ 2006-03-11 09:00:00.0 } \\
\hline \multicolumn{2}{|l|}{ 2006-03-11 06:00:00.0 } \\
\hline 2006-03-11 03:00:00.0 & 12.0 \\
\hline \multicolumn{2}{|l|}{ 2006-03-11 00:00:00.0 } \\
\hline \multicolumn{2}{|l|}{ 2006-03-10 21:00:00.0 } \\
\hline \multicolumn{2}{|l|}{ 2006-03-10 18:00:00.0 } \\
\hline 2006-03-10 15:00:00.0 & 19.9 \\
\hline 2006-03-10 12:00:00.0 & 22.0 \\
\hline \multicolumn{2}{|l|}{ 2006-03-10 09:00:00.0 } \\
\hline 2006-03-10 06:00:00.0 & 15.5 \\
\hline 2006-03-10 03:00:00.0 & 11.6 \\
\hline 2006-03-10 00:00:00.0 & 10.3 \\
\hline 2006-03-09 21:00:00.0 & 16.7 \\
\hline \multicolumn{2}{|l|}{ 2006-03-09 18:00:00.0 } \\
\hline \multicolumn{2}{|l|}{ 2006-03-09 15:00:00.0 } \\
\hline 2006-03-09 12:00:00.0 & 28.3 \\
\hline \multicolumn{2}{|l|}{ 2006-03-09 09:00:00.0 } \\
\hline 2006-03-09 06:00:00.0 & 13.5 \\
\hline 2006-03-09 03:00:00.0 & 10.8 \\
\hline 2006-03-09 00:00:00.0 & 11.2 \\
\hline 2006-03-08 21:00:00.0 & 15.8 \\
\hline \multicolumn{2}{|l|}{ 2006-03-08 18:00:00.0 } \\
\hline \multicolumn{2}{|l|}{ 2006-03-08 15:00:00.0 } \\
\hline 2006-03-08 12:00:00.0 & \\
\hline 2006-03-08 09:00:00.0 & \\
\hline 2006-03-08 06:00:00.0 & 15.0 \\
\hline 2006-03-08 03:00:00.0 & 14.0 \\
\hline 2006-03-08 00:00:00.0 & 13.0 \\
\hline 2006-03-07 21:00:00.0 & 12.0 \\
\hline 2006-03-07 18:00:00.0 & 14.0 \\
\hline 2006-03-07 15:00:00.0 & 27.0 \\
\hline 2006-03-07 12:00:00.0 & 12.0 \\
\hline 2006-03-07 09:00:00.0 & \\
\hline 2006-03-07 06:00:00.0 & 39.0 \\
\hline 2006-03-07 03:00:00.0 & 12.0 \\
\hline 2006-03-07 00:00:00.0 & 10.0 \\
\hline 2006-03-06 21:00:00.0 & \\
\hline 2006-03-06 18:00:00.0 & 13.0 \\
\hline 2006-03-06 15:00:00.0 & 14.0 \\
\hline 2006-03-06 12:00:00.0 & 7.0 \\
\hline 2006-03-06 09:00:00.0 & 5.0 \\
\hline 2006-03-06 06:00:00.0 & 8.0 \\
\hline 2006-03-06 03:00:00.0 & 7.0 \\
\hline 2006-03-06 00:00:00.0 & 10.0 \\
\hline 2006-03-05 21:00:00.0 & \\
\hline 2006-03-05 18:00:00.0 & 20.0 \\
\hline 2006-03-05 15:00:00.0 & 18.8 \\
\hline 2006-03-05 12:00:00.0 & \\
\hline 2006-03-05 09:00:00.0 & 10.9 \\
\hline 2006-03-05 06:00:00.0 & 11.1 \\
\hline 2006-03-05 03:00:00.0 & 16.5 \\
\hline 2006-03-05 00:00:00.0 & \\
\hline 2006-03-04 21:00:00.0 & 24.1 \\
\hline 2006-03-04 18:00:00.0 & 23.6 \\
\hline 2006-03-04 15:00:00.0 & 21.9 \\
\hline 2006-03-04 12:00:00.0 & \\
\hline 2006-03-04 09:00:00.0 & 12.2 \\
\hline 2006-03-04 06:00:00.0 & 8.4 \\
\hline 2006-03-04 03:00:00.0 & 9.5 \\
\hline 2006-03-04 00:00:00.0 & \\
\hline 2006-03-03 21:00:00.0 & 28.3 \\
\hline 2006-03-03 18:00:00.0 & 28.3 \\
\hline 2006-03-03 15:00:00.0 & 20.9 \\
\hline 2006-03-03 12:00:00.0 & \\
\hline 2006-03-03 09:00:00.0 & 10.6 \\
\hline
\end{tabular}

\begin{tabular}{|c|c|}
\hline DataHora & VelVentoMax \\
\hline 2006-03-03 06:00:00.0 & 17.6 \\
\hline 2006-03-03 03:00:00.0 & 17.0 \\
\hline \multicolumn{2}{|l|}{ 2006-03-03 00:00:00.0 } \\
\hline 2006-03-02 21:00:00.0 & 16.9 \\
\hline 2006-03-02 18:00:00.0 & 12.6 \\
\hline 2006-03-02 15:00:00.0 & 12.1 \\
\hline \multicolumn{2}{|l|}{ 2006-03-02 12:00:00.0 } \\
\hline 2006-03-02 09:00:00.0 & 14.5 \\
\hline 2006-03-02 06:00:00.0 & 11.8 \\
\hline 2006-03-02 03:00:00.0 & 14.2 \\
\hline \multicolumn{2}{|l|}{ 2006-03-02 00:00:00.0 } \\
\hline 2006-03-01 21:00:00.0 & 23.9 \\
\hline 2006-03-01 18:00:00.0 & 19.2 \\
\hline 2006-03-01 15:00:00.0 & 11.6 \\
\hline \multicolumn{2}{|l|}{ 2006-03-01 12:00:00.0 } \\
\hline 2006-03-01 09:00:00.0 & 9.7 \\
\hline 2006-03-01 06:00:00.0 & 17.1 \\
\hline 2006-03-01 03:00:00.0 & 15.5 \\
\hline \multicolumn{2}{|l|}{ 2006-03-01 00:00:00.0 } \\
\hline 2006-02-28 21:00:00.0 & 15.0 \\
\hline 2006-02-28 18:00:00.0 & 16.1 \\
\hline \multicolumn{2}{|l|}{ 2006-02-28 15:00:00.0 } \\
\hline 2006-02-28 12:00:00.0 & 15.8 \\
\hline 2006-02-28 09:00:00.0 & 15.7 \\
\hline 2006-02-28 06:00:00.0 & 16.6 \\
\hline \multicolumn{2}{|l|}{ 2006-02-28 03:00:00.0 } \\
\hline 2006-02-28 00:00:00.0 & 27.1 \\
\hline 2006-02-27 21:00:00.0 & 15.4 \\
\hline 2006-02-27 18:00:00.0 & 17.8 \\
\hline 2006-02-27 15:00:00.0 & 27.1 \\
\hline \multicolumn{2}{|l|}{ 2006-02-27 12:00:00.0 } \\
\hline 2006-02-27 09:00:00.0 & 16.1 \\
\hline 2006-02-27 06:00:00.0 & 17.2 \\
\hline \multicolumn{2}{|l|}{ 2006-02-27 03:00:00.0 } \\
\hline 2006-02-27 00:00:00.0 & 13.3 \\
\hline 2006-02-26 21:00:00.0 & 13.7 \\
\hline 2006-02-26 18:00:00.0 & 2.1 \\
\hline 2006-02-26 15:00:00.0 & 10.6 \\
\hline 2006-02-26 12:00:00.0 & 9.2 \\
\hline \multicolumn{2}{|l|}{ 2006-02-26 09:00:00.0 } \\
\hline 2006-02-26 06:00:00.0 & 10.8 \\
\hline 2006-02-26 03:00:00.0 & 15.9 \\
\hline 2006-02-26 00:00:00.0 & 22.1 \\
\hline 2006-02-25 21:00:00.0 & 24.8 \\
\hline 2006-02-25 18:00:00.0 & 20.2 \\
\hline \multicolumn{2}{|l|}{ 2006-02-25 15:00:00.0 } \\
\hline \multicolumn{2}{|l|}{ 2006-02-25 12:00:00.0 } \\
\hline 2006-02-25 09:00:00.0 & 21.2 \\
\hline \multicolumn{2}{|l|}{ 2006-02-25 06:00:00.0 } \\
\hline 2006-02-25 03:00:00.0 & 19.5 \\
\hline 2006-02-25 00:00:00.0 & 24.7 \\
\hline 2006-02-24 21:00:00.0 & 28.6 \\
\hline 2006-02-24 18:00:00.0 & 25.7 \\
\hline 2006-02-24 15:00:00.0 & 21.1 \\
\hline 2006-02-24 12:00:00.0 & 19.5 \\
\hline 2006-02-24 09:00:00.0 & 6.2 \\
\hline 2006-02-24 06:00:00.0 & 4.9 \\
\hline 2006-02-24 03:00:00.0 & \\
\hline 2006-02-24 00:00:00.0 & 8.9 \\
\hline 2006-02-23 21:00:00.0 & 27.1 \\
\hline 2006-02-23 18:00:00.0 & 16.8 \\
\hline 2006-02-23 15:00:00.0 & \\
\hline 2006-02-23 12:00:00.0 & 18.9 \\
\hline 2006-02-23 09:00:00.0 & 18.6 \\
\hline 2006-02-23 06:00:00.0 & 14.8 \\
\hline 2006-02-23 03:00:00.0 & \\
\hline 2006-02-23 00:00:00.0 & 9.7 \\
\hline 2006-02-22 21:00:00.0 & 15.8 \\
\hline 2006-02-22 18:00:00.0 & \\
\hline
\end{tabular}

\begin{tabular}{|c|c|}
\hline \multirow{2}{*}{$\begin{array}{l}\text { DataHora } \\
\text { 2006-02-22 15:00:00.0 }\end{array}$} & VelVentoMax \\
\hline & \\
\hline 2006-02-22 12:00:00.0 & 9.8 \\
\hline 2006-02-22 09:00:00.0 & 15.2 \\
\hline 2006-02-22 06:00:00.0 & \\
\hline 2006-02-22 03:00:00.0 & \\
\hline 2006-02-22 00:00:00.0 & 10.2 \\
\hline 2006-02-21 21:00:00.0 & 14.0 \\
\hline 2006-02-21 18:00:00.0 & 14.5 \\
\hline 2006-02-21 15:00:00.0 & \\
\hline 2006-02-21 12:00:00.0 & 27.1 \\
\hline 2006-02-21 09:00:00.0 & 14.4 \\
\hline 2006-02-21 06:00:00.0 & 27.1 \\
\hline 2006-02-21 03:00:00.0 & 15.5 \\
\hline 2006-02-21 00:00:00.0 & 15.5 \\
\hline 2006-02-20 21:00:00.0 & 31.6 \\
\hline 2006-02-20 18:00:00.0 & 38.2 \\
\hline 2006-02-20 15:00:00.0 & 18.9 \\
\hline 2006-02-20 12:00:00.0 & 18.9 \\
\hline 2006-02-20 09:00:00.0 & 18.0 \\
\hline 2006-02-20 06:00:00.0 & 15.7 \\
\hline 2006-02-20 03:00:00.0 & 19.8 \\
\hline 2006-02-20 00:00:00.0 & \\
\hline 2006-02-19 21:00:00.0 & 20.6 \\
\hline 2006-02-19 18:00:00.0 & 13.8 \\
\hline 2006-02-19 15:00:00.0 & 20.6 \\
\hline 2006-02-19 12:00:00.0 & 20.2 \\
\hline 2006-02-19 09:00:00.0 & 16.3 \\
\hline 2006-02-19 06:00:00.0 & 14.2 \\
\hline 2006-02-19 03:00:00.0 & \\
\hline 2006-02-19 00:00:00.0 & 21.1 \\
\hline 2006-02-18 21:00:00.0 & 21.2 \\
\hline 2006-02-18 18:00:00.0 & 27.5 \\
\hline 2006-02-18 15:00:00.0 & \\
\hline 2006-02-18 12:00:00.0 & \\
\hline 2006-02-18 09:00:00.0 & \\
\hline 2006-02-18 03:00:00.0 & 10.0 \\
\hline 2006-02-18 00:00:00.0 & 10.4 \\
\hline 2006-02-17 21:00:00.0 & 16.8 \\
\hline 2006-02-17 18:00:00.0 & \\
\hline 2006-02-17 15:00:00.0 & \\
\hline 2006-02-17 12:00:00.0 & \\
\hline 2006-02-17 09:00:00.0 & \\
\hline 2006-02-17 06:00:00.0 & 13.3 \\
\hline 2006-02-17 03:00:00.0 & 14.4 \\
\hline 2006-02-17 00:00:00.0 & 23.1 \\
\hline 2006-02-16 21:00:00.0 & \\
\hline 2006-02-16 18:00:00.0 & \\
\hline 2006-02-16 15:00:00.0 & 13.2 \\
\hline 2006-02-16 12:00:00.0 & 13.5 \\
\hline 2006-02-16 09:00:00.0 & 14.7 \\
\hline 2006-02-16 06:00:00.0 & 14.5 \\
\hline 2006-02-16 03:00:00.0 & 21.7 \\
\hline 2006-02-16 00:00:00.0 & 28.1 \\
\hline 2006-02-15 21:00:00.0 & 19.5 \\
\hline 2006-02-15 18:00:00.0 & \\
\hline 2006-02-15 15:00:00.0 & \\
\hline 2006-02-15 12:00:00.0 & 17.6 \\
\hline 2006-02-15 09:00:00.0 & 14.2 \\
\hline 2006-02-15 06:00:00.0 & 14.0 \\
\hline 2006-02-15 03:00:00.0 & 17.3 \\
\hline 2006-02-15 00:00:00.0 & 14.5 \\
\hline 2006-02-14 21:00:00.0 & 18.9 \\
\hline 2006-02-14 18:00:00.0 & 18.3 \\
\hline 2006-02-14 15:00:00.0 & 18.0 \\
\hline 2006-02-14 12:00:00.0 & 13.3 \\
\hline 2006-02-14 09:00:00.0 & 12.7 \\
\hline 2006-02-14 06:00:00.0 & \\
\hline 2006-02-14 03:00:00.0 & 13.3 \\
\hline 2006-02-14 00:00:00.0 & 32.3 \\
\hline
\end{tabular}




\section{ANEXO 01 - RAJADAS DE VENTO REGISTRADAS EM SÃO MARTINHO DA SERRA / RS}

\begin{tabular}{|c|c|}
\hline DataHora & VelVentoMax \\
\hline 2006-02-13 21:00:00.0 & 18.5 \\
\hline 2006-02-13 18:00:00.0 & 20.1 \\
\hline 2006-02-13 15:00:00.0 & 22.1 \\
\hline 2006-02-13 12:00:00.0 & 21.7 \\
\hline 2006-02-13 09:00:00.0 & 20.0 \\
\hline \multicolumn{2}{|l|}{ 2006-02-13 06:00:00.0 } \\
\hline 2006-02-13 03:00:00.0 & 21.1 \\
\hline 2006-02-13 00:00:00.0 & 17.3 \\
\hline 2006-02-12 21:00:00.0 & 22.7 \\
\hline 2006-02-12 18:00:00.0 & 23.4 \\
\hline 2006-02-12 15:00:00.0 & 12.9 \\
\hline 2006-02-12 12:00:00.0 & 13.9 \\
\hline \multicolumn{2}{|l|}{ 2006-02-12 09:00:00.0 } \\
\hline 2006-02-12 06:00:00.0 & 16.5 \\
\hline 2006-02-12 03:00:00.0 & 16.5 \\
\hline 2006-02-12 00:00:00.0 & 22.9 \\
\hline \multicolumn{2}{|l|}{ 2006-02-11 21:00:00.0 } \\
\hline 2006-02-11 18:00:00.0 & 20.0 \\
\hline 2006-02-11 15:00:00.0 & 21.9 \\
\hline 2006-02-11 12:00:00.0 & 19.0 \\
\hline \multicolumn{2}{|l|}{ 2006-02-11 09:00:00.0 } \\
\hline \multicolumn{2}{|l|}{ 2006-02-11 06:00:00.0 } \\
\hline 2006-02-11 03:00:00.0 & 12.1 \\
\hline 2006-02-11 00:00:00.0 & 31.4 \\
\hline 2006-02-10 21:00:00.0 & 23.9 \\
\hline 2006-02-10 18:00:00.0 & 23.7 \\
\hline 2006-02-10 15:00:00.0 & 22.4 \\
\hline 2006-02-10 12:00:00.0 & 20.8 \\
\hline 2006-02-10 09:00:00.0 & 12.1 \\
\hline 2006-02-10 06:00:00.0 & 12.2 \\
\hline 2006-02-10 03:00:00.0 & 15.0 \\
\hline 2006-02-10 00:00:00.0 & 13.1 \\
\hline \multicolumn{2}{|l|}{ 2006-02-09 21:00:00.0 } \\
\hline 2006-02-09 18:00:00.0 & 20.4 \\
\hline 2006-02-09 15:00:00.0 & 19.9 \\
\hline 2006-02-09 12:00:00.0 & 15.2 \\
\hline \multicolumn{2}{|l|}{ 2006-02-09 09:00:00.0 } \\
\hline 2006-02-09 06:00:00.0 & 11.5 \\
\hline 2006-02-09 03:00:00.0 & 12.6 \\
\hline 2006-02-09 00:00:00.0 & 14.9 \\
\hline 2006-02-08 21:00:00.0 & 19.6 \\
\hline 2006-02-08 18:00:00.0 & 12.3 \\
\hline 2006-02-08 15:00:00.0 & 13.8 \\
\hline 2006-02-08 12:00:00.0 & 16.7 \\
\hline \multicolumn{2}{|l|}{ 2006-02-08 09:00:00.0 } \\
\hline \multicolumn{2}{|l|}{ 2006-02-08 06:00:00.0 } \\
\hline 2006-02-08 03:00:00.0 & 10.1 \\
\hline 2006-02-08 00:00:00.0 & 11.8 \\
\hline 2006-02-07 21:00:00.0 & 14.4 \\
\hline 2006-02-07 18:00:00.0 & 16.0 \\
\hline 2006-02-07 15:00:00.0 & 15.3 \\
\hline 2006-02-07 12:00:00.0 & 13.9 \\
\hline \multicolumn{2}{|l|}{ 2006-02-07 09:00:00.0 } \\
\hline 2006-02-07 06:00:00.0 & 46.0 \\
\hline 2006-02-07 03:00:00.0 & 11.7 \\
\hline 2006-02-07 00:00:00.0 & 12.2 \\
\hline 2006-02-06 21:00:00.0 & 18.5 \\
\hline 2006-02-06 18:00:00.0 & 25.8 \\
\hline 2006-02-06 15:00:00.0 & 33.1 \\
\hline \multicolumn{2}{|l|}{ 2006-02-06 12:00:00.0 } \\
\hline \multicolumn{2}{|l|}{ 2006-02-06 09:00:00.0 } \\
\hline 2006-02-06 06:00:00.0 & 12.4 \\
\hline 2006-02-06 03:00:00.0 & 18.3 \\
\hline \multicolumn{2}{|l|}{ 2006-02-06 00:00:00.0 } \\
\hline 2006-02-05 21:00:00.0 & \\
\hline 2006-02-05 18:00:00.0 & \\
\hline 2006-02-05 06:00:00.0 & 17.0 \\
\hline 2006-02-05 03:00:00.0 & 15.2 \\
\hline 2006-02-05 00:00:00.0 & \\
\hline
\end{tabular}

\begin{tabular}{|c|c|}
\hline DataHora & VelVentoMax \\
\hline 2006-02-04 21:00:00.0 & 15.5 \\
\hline 2006-02-04 18:00:00.0 & 22.6 \\
\hline 2006-02-04 15:00:00.0 & 20.0 \\
\hline 2006-02-04 12:00:00.0 & 19.7 \\
\hline 2006-02-04 09:00:00.0 & 19.0 \\
\hline 2006-02-04 06:00:00.0 & 17.2 \\
\hline 2006-02-04 03:00:00.0 & \\
\hline 2006-02-04 00:00:00.0 & \\
\hline 2006-02-03 21:00:00.0 & 29.0 \\
\hline 2006-02-03 18:00:00.0 & 16.4 \\
\hline 2006-02-03 15:00:00.0 & 16.0 \\
\hline 2006-02-03 12:00:00.0 & 15.9 \\
\hline 2006-02-03 09:00:00.0 & \\
\hline 2006-02-03 06:00:00.0 & 18.5 \\
\hline 2006-02-03 03:00:00.0 & 14.2 \\
\hline 2006-02-03 00:00:00.0 & 31.3 \\
\hline 2006-02-02 21:00:00.0 & 11.6 \\
\hline 2006-02-02 18:00:00.0 & 11.9 \\
\hline 2006-02-02 15:00:00.0 & 19.8 \\
\hline 2006-02-02 12:00:00.0 & \\
\hline 2006-02-02 09:00:00.0 & \\
\hline 2006-02-02 06:00:00.0 & 16.5 \\
\hline 2006-02-02 03:00:00.0 & 13.0 \\
\hline 2006-02-02 00:00:00.0 & \\
\hline 2006-02-01 21:00:00.0 & \\
\hline 2006-02-01 18:00:00.0 & \\
\hline 2006-02-01 09:00:00.0 & 16.2 \\
\hline 2006-02-01 06:00:00.0 & 17.3 \\
\hline 2006-02-01 03:00:00.0 & \\
\hline 2006-02-01 00:00:00.0 & \\
\hline 2006-01-31 21:00:00.0 & \\
\hline 2006-01-31 09:00:00.0 & 13.6 \\
\hline 2006-01-31 06:00:00.0 & \\
\hline 2006-01-31 03:00:00.0 & \\
\hline 2006-01-31 00:00:00.0 & \\
\hline 2006-01-30 21:00:00.0 & 20.3 \\
\hline 2006-01-30 18:00:00.0 & 17.0 \\
\hline 2006-01-30 15:00:00.0 & 12.8 \\
\hline 2006-01-30 12:00:00.0 & \\
\hline 2006-01-30 09:00:00.0 & 12.1 \\
\hline 2006-01-30 06:00:00.0 & \\
\hline 2006-01-30 03:00:00.0 & \\
\hline 2006-01-30 00:00:00.0 & \\
\hline 2006-01-29 21:00:00.0 & 15.0 \\
\hline 2006-01-29 18:00:00.0 & 12.6 \\
\hline 2006-01-29 15:00:00.0 & 16.8 \\
\hline 2006-01-29 12:00:00.0 & \\
\hline 2006-01-29 09:00:00.0 & 18.3 \\
\hline 2006-01-29 06:00:00.0 & 13.9 \\
\hline 2006-01-29 03:00:00.0 & \\
\hline 2006-01-29 00:00:00.0 & 16.5 \\
\hline 2006-01-28 21:00:00.0 & 17.8 \\
\hline 2006-01-28 18:00:00.0 & 16.2 \\
\hline 2006-01-28 15:00:00.0 & 15.1 \\
\hline 2006-01-28 12:00:00.0 & \\
\hline 2006-01-28 09:00:00.0 & 14.5 \\
\hline 2006-01-28 06:00:00.0 & 17.2 \\
\hline 2006-01-28 03:00:00.0 & \\
\hline 2006-01-28 00:00:00.0 & 15.3 \\
\hline 2006-01-27 21:00:00.0 & 17.6 \\
\hline 2006-01-27 18:00:00.0 & 14.2 \\
\hline 2006-01-27 15:00:00.0 & \\
\hline 2006-01-27 12:00:00.0 & \\
\hline 2006-01-27 09:00:00.0 & 16.8 \\
\hline 2006-01-27 06:00:00.0 & \\
\hline 2006-01-27 03:00:00.0 & \\
\hline 2006-01-27 00:00:00.0 & 17.5 \\
\hline 2006-01-26 21:00:00.0 & 19.4 \\
\hline 2006-01-26 18:00:00.0 & 17.9 \\
\hline
\end{tabular}

\begin{tabular}{|c|c|}
\hline DataHora & VelVentoMax \\
\hline 2006-01-26 15:00:00.0 & 20.8 \\
\hline 2006-01-26 12:00:00.0 & 21.2 \\
\hline 2006-01-26 09:00:00.0 & 14.5 \\
\hline 2006-01-26 06:00:00.0 & \\
\hline 2006-01-26 03:00:00.0 & \\
\hline 2006-01-26 00:00:00.0 & 11.8 \\
\hline 2006-01-25 21:00:00.0 & 38.2 \\
\hline $2006-01-25$ 18:00:00.0 & \\
\hline $2006-01-25$ 15:00:00.0 & 22.7 \\
\hline $2006-01-25$ 12:00:00.0 & 17.4 \\
\hline 2006-01-25 09:00:00.0 & 12.1 \\
\hline $2006-01-25$ 06:00:00.0 & \\
\hline 2006-01-25 03:00:00.0 & 20.0 \\
\hline 2006-01-25 00:00:00.0 & 13.3 \\
\hline 2006-01-24 21:00:00.0 & 21.0 \\
\hline $2006-01-24$ 18:00:00.0 & 23.5 \\
\hline 2006-01-24 15:00:00.0 & 30.7 \\
\hline 2006-01-24 12:00:00.0 & 9.9 \\
\hline 2006-01-24 09:00:00.0 & \\
\hline 2006-01-24 06:00:00.0 & 12.6 \\
\hline 2006-01-24 03:00:00.0 & 16.4 \\
\hline $2006-01-2400: 00: 00.0$ & 15.3 \\
\hline $2006-01-23$ 21:00:00.0 & 19.8 \\
\hline 2006-01-23 18:00:00.0 & 24.1 \\
\hline $2006-01-23$ 15:00:00.0 & \\
\hline 2006-01-23 12:00:00.0 & 12.9 \\
\hline 2006-01-23 09:00:00.0 & 12.7 \\
\hline 2006-01-23 06:00:00.0 & \\
\hline 2006-01-23 03:00:00.0 & 18.6 \\
\hline 2006-01-23 00:00:00.0 & 31.3 \\
\hline 2006-01-22 21:00:00.0 & 15.7 \\
\hline 2006-01-22 18:00:00.0 & 15.6 \\
\hline 2006-01-22 15:00:00.0 & 12.1 \\
\hline 2006-01-22 12:00:00.0 & 13.5 \\
\hline 2006-01-22 09:00:00.0 & 9.7 \\
\hline 2006-01-22 06:00:00.0 & 6.8 \\
\hline 2006-01-22 03:00:00.0 & 6.9 \\
\hline 2006-01-22 00:00:00.0 & 18.7 \\
\hline 2006-01-21 21:00:00.0 & 18.6 \\
\hline 2006-01-21 18:00:00.0 & 20.7 \\
\hline 2006-01-21 15:00:00.0 & 18.7 \\
\hline 2006-01-21 12:00:00.0 & 28.3 \\
\hline 2006-01-21 09:00:00.0 & \\
\hline 2006-01-21 06:00:00.0 & 14.0 \\
\hline 2006-01-21 03:00:00.0 & 14.8 \\
\hline 2006-01-21 00:00:00.0 & 11.0 \\
\hline 2006-01-20 21:00:00.0 & 10.1 \\
\hline 2006-01-20 18:00:00.0 & 11.9 \\
\hline $2006-01-20$ 15:00:00.0 & 12.4 \\
\hline 2006-01-20 12:00:00.0 & 8.8 \\
\hline 2006-01-20 09:00:00.0 & 10.7 \\
\hline 2006-01-20 06:00:00.0 & 22.1 \\
\hline 2006-01-20 03:00:00.0 & 14.6 \\
\hline $2006-01-2000: 00: 00.0$ & 20.9 \\
\hline 2006-01-19 21:00:00.0 & 21.0 \\
\hline 2006-01-19 18:00:00.0 & 18.8 \\
\hline 2006-01-19 15:00:00.0 & 15.1 \\
\hline 2006-01-19 12:00:00.0 & 15.9 \\
\hline 2006-01-19 09:00:00.0 & 16.3 \\
\hline 2006-01-19 06:00:00.0 & \\
\hline 2006-01-19 03:00:00.0 & 24.1 \\
\hline 2006-01-19 00:00:00.0 & 23.4 \\
\hline 2006-01-18 21:00:00.0 & 24.4 \\
\hline 2006-01-18 18:00:00.0 & 37.2 \\
\hline 2006-01-18 15:00:00.0 & 30.0 \\
\hline 2006-01-18 12:00:00.0 & \\
\hline 2006-01-18 09:00:00.0 & \\
\hline 2006-01-18 06:00:00.0 & 25.1 \\
\hline 2006-01-18 03:00:00.0 & 27.7 \\
\hline
\end{tabular}




\section{ANEXO 01 - RAJADAS DE VENTO REGISTRADAS EM SÃO MARTINHO DA SERRA / RS}

\begin{tabular}{|c|c|}
\hline DataHora & VelVentoMax \\
\hline 2006-01-18 00:00:00.0 & 26.2 \\
\hline 2006-01-17 21:00:00.0 & 27.4 \\
\hline 2006-01-17 18:00:00.0 & 28.8 \\
\hline 2006-01-17 15:00:00.0 & 25.8 \\
\hline 2006-01-17 12:00:00.0 & 31.2 \\
\hline 2006-01-17 09:00:00.0 & \\
\hline 2006-01-17 06:00:00.0 & 29.8 \\
\hline 2006-01-17 03:00:00.0 & 27.9 \\
\hline 2006-01-17 00:00:00.0 & \\
\hline 2006-01-16 21:00:00.0 & \\
\hline 2006-01-16 18:00:00.0 & 38.7 \\
\hline 2006-01-16 15:00:00.0 & 23.9 \\
\hline 2006-01-16 12:00:00.0 & 17.4 \\
\hline 2006-01-16 09:00:00.0 & \\
\hline 2006-01-16 06:00:00.0 & 13.4 \\
\hline 2006-01-16 03:00:00.0 & 11.3 \\
\hline 2006-01-16 00:00:00.0 & \\
\hline 2006-01-15 21:00:00.0 & \\
\hline 2006-01-15 18:00:00.0 & 20.8 \\
\hline 2006-01-15 15:00:00.0 & 18.4 \\
\hline 2006-01-15 12:00:00.0 & \\
\hline 2006-01-15 09:00:00.0 & 18.4 \\
\hline 2006-01-15 06:00:00.0 & 16.7 \\
\hline 2006-01-15 03:00:00.0 & 16.4 \\
\hline 2006-01-15 00:00:00.0 & 15.0 \\
\hline 2006-01-14 21:00:00.0 & 13.9 \\
\hline 2006-01-14 18:00:00.0 & 12.6 \\
\hline 2006-01-14 15:00:00.0 & 16.3 \\
\hline 2006-01-14 12:00:00.0 & \\
\hline 2006-01-14 09:00:00.0 & 15.1 \\
\hline 2006-01-14 06:00:00.0 & 18.0 \\
\hline 2006-01-14 03:00:00.0 & 22.6 \\
\hline 2006-01-14 00:00:00.0 & \\
\hline 2006-01-13 21:00:00.0 & 20.7 \\
\hline 2006-01-13 18:00:00.0 & 14.7 \\
\hline 2006-01-13 15:00:00.0 & 16.0 \\
\hline 2006-01-13 12:00:00.0 & \\
\hline 2006-01-13 09:00:00.0 & 11.5 \\
\hline 2006-01-13 06:00:00.0 & 13.8 \\
\hline 2006-01-13 03:00:00.0 & 22.6 \\
\hline 2006-01-13 00:00:00.0 & 19.2 \\
\hline 2006-01-12 21:00:00.0 & \\
\hline 2006-01-12 18:00:00.0 & 23.9 \\
\hline 2006-01-12 15:00:00.0 & 26.5 \\
\hline 2006-01-12 12:00:00.0 & \\
\hline 2006-01-12 09:00:00.0 & 20.4 \\
\hline 2006-01-12 06:00:00.0 & 18.0 \\
\hline 2006-01-12 03:00:00.0 & 25.3 \\
\hline 2006-01-12 00:00:00.0 & \\
\hline 2006-01-11 21:00:00.0 & 15.0 \\
\hline 2006-01-11 18:00:00.0 & \\
\hline 2006-01-11 15:00:00.0 & 23.7 \\
\hline 2006-01-11 12:00:00.0 & \\
\hline 2006-01-11 09:00:00.0 & 26.9 \\
\hline 2006-01-11 06:00:00.0 & 19.6 \\
\hline 2006-01-11 03:00:00.0 & 21.2 \\
\hline 2006-01-11 00:00:00.0 & 31.3 \\
\hline 2006-01-10 21:00:00.0 & 15.3 \\
\hline 2006-01-10 18:00:00.0 & 18.9 \\
\hline 2006-01-10 15:00:00.0 & 21.7 \\
\hline 2006-01-10 12:00:00.0 & \\
\hline 2006-01-10 09:00:00.0 & 17.3 \\
\hline 2006-01-10 06:00:00.0 & 17.8 \\
\hline 2006-01-10 03:00:00.0 & 20.7 \\
\hline 2006-01-10 00:00:00.0 & \\
\hline 2006-01-09 21:00:00.0 & 4.1 \\
\hline 2006-01-09 18:00:00.0 & 16.1 \\
\hline 2006-01-09 15:00:00.0 & 19.8 \\
\hline 2006-01-09 12:00:00.0 & 20.6 \\
\hline
\end{tabular}

\begin{tabular}{|c|c|}
\hline DataHora & VelVentoMax \\
\hline 2006-01-09 09:00:00.0 & 20.1 \\
\hline 2006-01-09 06:00:00.0 & 23.1 \\
\hline 2006-01-09 03:00:00.0 & 26.8 \\
\hline \multicolumn{2}{|l|}{ 2006-01-09 00:00:00.0 } \\
\hline 2006-01-08 21:00:00.0 & 29.1 \\
\hline 2006-01-08 18:00:00.0 & 23.7 \\
\hline 2006-01-08 15:00:00.0 & 26.3 \\
\hline \multicolumn{2}{|l|}{ 2006-01-08 12:00:00.0 } \\
\hline 2006-01-08 09:00:00.0 & 18.6 \\
\hline 2006-01-08 06:00:00.0 & 16.4 \\
\hline \multicolumn{2}{|l|}{ 2006-01-08 03:00:00.0 } \\
\hline 2006-01-08 00:00:00.0 & 13.8 \\
\hline 2006-01-07 21:00:00.0 & 15.7 \\
\hline 2006-01-07 18:00:00.0 & 15.0 \\
\hline 2006-01-07 15:00:00.0 & 18.0 \\
\hline 2006-01-07 12:00:00.0 & 16.6 \\
\hline 2006-01-07 09:00:00.0 & 17.1 \\
\hline 2006-01-07 06:00:00.0 & 16.5 \\
\hline 2006-01-07 03:00:00.0 & 14.9 \\
\hline 2006-01-07 00:00:00.0 & 12.0 \\
\hline 2006-01-06 21:00:00.0 & 13.5 \\
\hline 2006-01-06 18:00:00.0 & 15.3 \\
\hline 2006-01-06 15:00:00.0 & 12.3 \\
\hline 2006-01-06 12:00:00.0 & 33.8 \\
\hline 2006-01-06 09:00:00.0 & 18.3 \\
\hline 2006-01-06 06:00:00.0 & 15.9 \\
\hline \multicolumn{2}{|l|}{ 2006-01-06 03:00:00.0 } \\
\hline \multicolumn{2}{|l|}{ 2006-01-06 00:00:00.0 } \\
\hline 2006-01-05 21:00:00.0 & 18.4 \\
\hline 2006-01-05 18:00:00.0 & 20.1 \\
\hline \multicolumn{2}{|l|}{ 2006-01-05 15:00:00.0 } \\
\hline 2006-01-05 12:00:00.0 & 5.2 \\
\hline 2006-01-05 09:00:00.0 & 14.7 \\
\hline 2006-01-05 06:00:00.0 & 16.2 \\
\hline 2006-01-05 03:00:00.0 & 15.5 \\
\hline 2006-01-05 00:00:00.0 & 31.7 \\
\hline 2006-01-04 21:00:00.0 & 19.3 \\
\hline 2006-01-04 18:00:00.0 & 19.1 \\
\hline \multicolumn{2}{|l|}{ 2006-01-04 15:00:00.0 } \\
\hline 2006-01-04 12:00:00.0 & 30.0 \\
\hline 2006-01-04 09:00:00.0 & 12.5 \\
\hline 2006-01-04 06:00:00.0 & 12.0 \\
\hline \multicolumn{2}{|l|}{ 2006-01-04 03:00:00.0 } \\
\hline 2006-01-04 00:00:00.0 & 6.3 \\
\hline 2006-01-03 21:00:00.0 & 14.7 \\
\hline 2006-01-03 18:00:00.0 & 18.4 \\
\hline \multicolumn{2}{|l|}{ 2006-01-03 15:00:00.0 } \\
\hline 2006-01-03 12:00:00.0 & 11.0 \\
\hline 2006-01-03 09:00:00.0 & 11.1 \\
\hline 2006-01-03 06:00:00.0 & 11.5 \\
\hline \multicolumn{2}{|l|}{ 2006-01-03 03:00:00.0 } \\
\hline 2006-01-03 00:00:00.0 & 30.7 \\
\hline 2006-01-02 21:00:00.0 & 16.7 \\
\hline 2006-01-02 18:00:00.0 & 17.7 \\
\hline \multicolumn{2}{|l|}{ 2006-01-02 15:00:00.0 } \\
\hline 2006-01-02 12:00:00.0 & 12.1 \\
\hline 2006-01-02 09:00:00.0 & 10.5 \\
\hline \multicolumn{2}{|l|}{ 2006-01-02 06:00:00.0 } \\
\hline 2006-01-02 03:00:00.0 & 21.0 \\
\hline 2006-01-02 00:00:00.0 & 13.8 \\
\hline 2006-01-01 21:00:00.0 & 14.9 \\
\hline 2006-01-01 18:00:00.0 & 13.8 \\
\hline 2006-01-01 15:00:00.0 & 11.4 \\
\hline 2006-01-01 12:00:00.0 & 12.8 \\
\hline 2006-01-01 09:00:00.0 & 14.3 \\
\hline 2006-01-01 06:00:00.0 & 13.8 \\
\hline 2006-01-01 03:00:00.0 & \\
\hline 2006-01-01 00:00:00.0 & 16.0 \\
\hline 2005-12-29 12:00:00.0 & 21.5 \\
\hline
\end{tabular}

\begin{tabular}{|c|c|}
\hline DataHora & VelVentoMax \\
\hline 2005-12-29 09:00:00.0 & 22.3 \\
\hline 2005-12-29 06:00:00.0 & 19.8 \\
\hline 2005-12-29 03:00:00.0 & 16.2 \\
\hline 2005-12-29 00:00:00.0 & 12.1 \\
\hline 2005-12-28 21:00:00.0 & 16.6 \\
\hline 2005-12-28 18:00:00.0 & 17.4 \\
\hline 2005-12-28 15:00:00.0 & 22.6 \\
\hline 2005-12-28 12:00:00.0 & 26.2 \\
\hline 2005-12-28 09:00:00.0 & 24.3 \\
\hline 2005-12-28 06:00:00.0 & 20.2 \\
\hline 2005-12-28 03:00:00.0 & 18.3 \\
\hline 2005-12-28 00:00:00.0 & 36.1 \\
\hline 2005-12-27 21:00:00.0 & 4.6 \\
\hline 2005-12-27 18:00:00.0 & \\
\hline 2005-12-27 15:00:00.0 & 16.6 \\
\hline 2005-12-27 12:00:00.0 & 16.9 \\
\hline 2005-12-27 09:00:00.0 & 14.3 \\
\hline 2005-12-27 06:00:00.0 & 14.6 \\
\hline 2005-12-27 03:00:00.0 & 18.8 \\
\hline 2005-12-27 00:00:00.0 & 19.4 \\
\hline 2005-12-26 21:00:00.0 & 19.0 \\
\hline 2005-12-26 18:00:00.0 & 20.6 \\
\hline 2005-12-26 15:00:00.0 & 25.0 \\
\hline 2005-12-26 12:00:00.0 & 20.3 \\
\hline 2005-12-26 09:00:00.0 & 31.8 \\
\hline 2005-12-26 06:00:00.0 & \\
\hline 2005-12-26 03:00:00.0 & 20.7 \\
\hline 2005-12-26 00:00:00.0 & 19.3 \\
\hline 2005-12-25 21:00:00.0 & 21.1 \\
\hline 2005-12-25 18:00:00.0 & 22.3 \\
\hline 2005-12-25 15:00:00.0 & 18.7 \\
\hline 2005-12-25 12:00:00.0 & \\
\hline 2005-12-25 09:00:00.0 & \\
\hline 2005-12-25 06:00:00.0 & 13.2 \\
\hline 2005-12-25 03:00:00.0 & 16.7 \\
\hline 2005-12-25 00:00:00.0 & 18.9 \\
\hline 2005-12-24 21:00:00.0 & 24.3 \\
\hline 2005-12-24 18:00:00.0 & 24.0 \\
\hline 2005-12-24 15:00:00.0 & 16.1 \\
\hline 2005-12-24 12:00:00.0 & 11.0 \\
\hline 2005-12-24 09:00:00.0 & 5.6 \\
\hline 2005-12-24 06:00:00.0 & 7.5 \\
\hline 2005-12-24 03:00:00.0 & 11.5 \\
\hline 2005-12-24 00:00:00.0 & 11.2 \\
\hline 2005-12-23 21:00:00.0 & 16.1 \\
\hline 2005-12-23 18:00:00.0 & 23.1 \\
\hline 2005-12-23 15:00:00.0 & 26.8 \\
\hline 2005-12-23 12:00:00.0 & 47.7 \\
\hline 2005-12-23 09:00:00.0 & 18.8 \\
\hline 2005-12-23 06:00:00.0 & \\
\hline 2005-12-23 03:00:00.0 & 14.8 \\
\hline 2005-12-23 00:00:00.0 & \\
\hline 2005-12-22 21:00:00.0 & 19.2 \\
\hline 2005-12-22 18:00:00.0 & 22.5 \\
\hline $2005-12-22$ 15:00:00.0 & 14.1 \\
\hline 2005-12-22 12:00:00.0 & 17.6 \\
\hline 2005-12-22 09:00:00.0 & 17.5 \\
\hline 2005-12-22 06:00:00.0 & 20.5 \\
\hline 2005-12-22 03:00:00.0 & 18.3 \\
\hline 2005-12-22 00:00:00.0 & 20.4 \\
\hline 2005-12-21 21:00:00.0 & 25.0 \\
\hline 2005-12-21 18:00:00.0 & 2.7 \\
\hline 2005-12-21 15:00:00.0 & 21.7 \\
\hline 2005-12-21 12:00:00.0 & 19.6 \\
\hline 2005-12-21 09:00:00.0 & \\
\hline 2005-12-21 06:00:00.0 & 19.8 \\
\hline 2005-12-21 03:00:00.0 & 18.2 \\
\hline 2005-12-21 00:00:00.0 & 4.8 \\
\hline $2005-12-20$ 21:00:00.0 & 18.3 \\
\hline
\end{tabular}




\section{ANEXO 01 - RAJADAS DE VENTO REGISTRADAS EM SÃO MARTINHO DA SERRA / RS}

\begin{tabular}{|c|c|}
\hline DataHora & VelVentoMax \\
\hline 2005-12-20 18:00:00.0 & 17.3 \\
\hline 2005-12-20 15:00:00.0 & 21.2 \\
\hline 2005-12-20 12:00:00.0 & 20.1 \\
\hline 2005-12-20 09:00:00.0 & \\
\hline 2005-12-20 06:00:00.0 & \\
\hline 2005-12-20 03:00:00.0 & 24.0 \\
\hline 2005-12-20 00:00:00.0 & \\
\hline 2005-12-19 21:00:00.0 & 23.9 \\
\hline 2005-12-19 18:00:00.0 & 32.5 \\
\hline 2005-12-19 15:00:00.0 & 19.0 \\
\hline 2005-12-19 12:00:00.0 & 31.4 \\
\hline 2005-12-19 09:00:00.0 & \\
\hline 2005-12-19 06:00:00.0 & 6.3 \\
\hline 2005-12-19 03:00:00.0 & 7.5 \\
\hline 2005-12-19 00:00:00.0 & \\
\hline 2005-12-18 21:00:00.0 & 23.9 \\
\hline 2005-12-18 18:00:00.0 & 22.6 \\
\hline 2005-12-18 15:00:00.0 & 20.5 \\
\hline 2005-12-18 12:00:00.0 & 15.4 \\
\hline 2005-12-18 09:00:00.0 & \\
\hline 2005-12-18 06:00:00.0 & 16.3 \\
\hline 2005-12-18 03:00:00.0 & 14.9 \\
\hline 2005-12-18 00:00:00.0 & 20.4 \\
\hline 2005-12-17 21:00:00.0 & 24.7 \\
\hline 2005-12-17 18:00:00.0 & 23.1 \\
\hline 2005-12-17 15:00:00.0 & 19.3 \\
\hline 2005-12-17 12:00:00.0 & 13.8 \\
\hline 2005-12-17 09:00:00.0 & \\
\hline 2005-12-17 06:00:00.0 & 12.1 \\
\hline 2005-12-17 03:00:00.0 & 25.6 \\
\hline 2005-12-17 00:00:00.0 & 13.3 \\
\hline 2005-12-16 21:00:00.0 & 20.3 \\
\hline 2005-12-16 18:00:00.0 & 22.3 \\
\hline 2005-12-16 15:00:00.0 & 15.9 \\
\hline 2005-12-16 12:00:00.0 & 12.3 \\
\hline 2005-12-16 09:00:00.0 & 14.1 \\
\hline 2005-12-16 06:00:00.0 & 12.4 \\
\hline 2005-12-16 03:00:00.0 & \\
\hline 2005-12-16 00:00:00.0 & \\
\hline 2005-12-15 21:00:00.0 & 18.8 \\
\hline 2005-12-15 18:00:00.0 & 17.7 \\
\hline 2005-12-15 15:00:00.0 & 14.8 \\
\hline 2005-12-15 12:00:00.0 & \\
\hline 2005-12-15 09:00:00.0 & \\
\hline 2005-12-15 06:00:00.0 & 18.8 \\
\hline 2005-12-15 03:00:00.0 & 19.0 \\
\hline 2005-12-15 00:00:00.0 & \\
\hline 2005-12-14 21:00:00.0 & 26.2 \\
\hline 2005-12-14 18:00:00.0 & 17.5 \\
\hline 2005-12-14 15:00:00.0 & 20.0 \\
\hline 2005-12-14 12:00:00.0 & \\
\hline 2005-12-14 09:00:00.0 & \\
\hline 2005-12-14 06:00:00.0 & 16.9 \\
\hline 2005-12-14 03:00:00.0 & 23.3 \\
\hline 2005-12-14 00:00:00.0 & \\
\hline 2005-12-13 21:00:00.0 & 23.2 \\
\hline 2005-12-13 18:00:00.0 & \\
\hline 2005-12-13 15:00:00.0 & 17.8 \\
\hline 2005-12-13 12:00:00.0 & \\
\hline 2005-12-13 09:00:00.0 & 14.5 \\
\hline 2005-12-13 06:00:00.0 & 14.9 \\
\hline 2005-12-13 03:00:00.0 & \\
\hline 2005-12-13 00:00:00.0 & 17.9 \\
\hline 2005-12-12 21:00:00.0 & 19.8 \\
\hline 2005-12-12 18:00:00.0 & 20.1 \\
\hline 2005-12-12 15:00:00.0 & 18.7 \\
\hline 2005-12-12 12:00:00.0 & \\
\hline 2005-12-12 09:00:00.0 & 21.3 \\
\hline 2005-12-12 06:00:00.0 & 19.5 \\
\hline
\end{tabular}

\begin{tabular}{|c|c|}
\hline DataHora & VelVentoMax \\
\hline 2005-12-12 03:00:00.0 & 19.5 \\
\hline 2005-12-12 00:00:00.0 & 20.1 \\
\hline 2005-12-11 21:00:00.0 & 20.3 \\
\hline 2005-12-11 18:00:00.0 & 20.3 \\
\hline 2005-12-11 15:00:00.0 & 21.4 \\
\hline \multicolumn{2}{|l|}{ 2005-12-11 12:00:00.0 } \\
\hline 2005-12-11 09:00:00.0 & 16.0 \\
\hline 2005-12-11 06:00:00.0 & 22.4 \\
\hline 2005-12-11 03:00:00.0 & 25.3 \\
\hline 2005-12-11 00:00:00.0 & 15.4 \\
\hline 2005-12-10 21:00:00.0 & 21.3 \\
\hline 2005-12-10 18:00:00.0 & 22.2 \\
\hline 2005-12-10 15:00:00.0 & 21.7 \\
\hline \multicolumn{2}{|l|}{ 2005-12-10 12:00:00.0 } \\
\hline 2005-12-10 09:00:00.0 & 12.8 \\
\hline 2005-12-10 06:00:00.0 & 16.5 \\
\hline \multicolumn{2}{|l|}{$2005-12-1003: 00: 00.0$} \\
\hline 2005-12-10 00:00:00.0 & 21.2 \\
\hline 2005-12-09 09:00:00.0 & 15.7 \\
\hline 2005-12-09 06:00:00.0 & 7.7 \\
\hline 2005-12-09 03:00:00.0 & 15.9 \\
\hline \multicolumn{2}{|l|}{ 2005-12-09 00:00:00.0 } \\
\hline 2005-12-08 21:00:00.0 & 16.5 \\
\hline 2005-12-08 18:00:00.0 & 19.4 \\
\hline \multicolumn{2}{|l|}{ 2005-12-08 15:00:00.0 } \\
\hline \multicolumn{2}{|l|}{ 2005-12-08 12:00:00.0 } \\
\hline 2005-12-08 09:00:00.0 & 17.2 \\
\hline \multicolumn{2}{|l|}{ 2005-12-08 06:00:00.0 } \\
\hline \multicolumn{2}{|l|}{ 2005-12-08 03:00:00.0 } \\
\hline 2005-12-08 00:00:00.0 & 13.4 \\
\hline 2005-12-07 21:00:00.0 & 13.0 \\
\hline 2005-12-07 18:00:00.0 & 15.4 \\
\hline 2005-12-07 15:00:00.0 & 18.8 \\
\hline 2005-12-07 12:00:00.0 & 20.9 \\
\hline 2005-12-07 09:00:00.0 & 12.6 \\
\hline 2005-12-07 06:00:00.0 & 14.1 \\
\hline \multicolumn{2}{|l|}{ 2005-12-07 03:00:00.0 } \\
\hline 2005-12-07 00:00:00.0 & 21.2 \\
\hline 2005-12-06 21:00:00.0 & 21.9 \\
\hline 2005-12-06 18:00:00.0 & 20.8 \\
\hline \multicolumn{2}{|l|}{ 2005-12-06 15:00:00.0 } \\
\hline \multicolumn{2}{|l|}{ 2005-12-06 12:00:00.0 } \\
\hline 2005-12-06 09:00:00.0 & 14.9 \\
\hline 2005-12-06 06:00:00.0 & 6.9 \\
\hline 2005-12-06 03:00:00.0 & 8.2 \\
\hline 2005-12-06 00:00:00.0 & 18.1 \\
\hline 2005-12-05 21:00:00.0 & 23.7 \\
\hline 2005-12-05 18:00:00.0 & 23.8 \\
\hline 2005-12-05 15:00:00.0 & 2.3 \\
\hline 2005-12-05 12:00:00.0 & 34.4 \\
\hline 2005-12-05 09:00:00.0 & 21.5 \\
\hline 2005-12-05 06:00:00.0 & 19.6 \\
\hline 2005-12-05 03:00:00.0 & 16.1 \\
\hline 2005-12-05 00:00:00.0 & 15.0 \\
\hline 2005-12-04 21:00:00.0 & 12.8 \\
\hline \multicolumn{2}{|l|}{ 2005-12-04 18:00:00.0 } \\
\hline 2005-12-04 15:00:00.0 & 18.2 \\
\hline 2005-12-04 12:00:00.0 & 22.0 \\
\hline 2005-12-04 09:00:00.0 & 20.6 \\
\hline \multicolumn{2}{|l|}{ 2005-12-04 06:00:00.0 } \\
\hline 2005-12-04 03:00:00.0 & 17.9 \\
\hline 2005-12-04 00:00:00.0 & \\
\hline 2005-12-03 21:00:00.0 & 18.7 \\
\hline 2005-12-03 18:00:00.0 & \\
\hline 2005-12-03 15:00:00.0 & 26.7 \\
\hline 2005-12-03 12:00:00.0 & 28.4 \\
\hline 2005-12-03 09:00:00.0 & 24.8 \\
\hline 2005-12-03 06:00:00.0 & \\
\hline 2005-12-03 03:00:00.0 & 25.3 \\
\hline
\end{tabular}

\begin{tabular}{|c|c|}
\hline DataHora & VelVentoMax \\
\hline 2005-12-03 00:00:00.0 & 27.0 \\
\hline 2005-12-02 21:00:00.0 & \\
\hline 2005-12-02 18:00:00.0 & \\
\hline 2005-12-02 15:00:00.0 & 26.5 \\
\hline 2005-12-02 12:00:00.0 & 26.0 \\
\hline 2005-12-02 09:00:00.0 & 23.2 \\
\hline 2005-12-02 06:00:00.0 & \\
\hline 2005-12-02 03:00:00.0 & 30.5 \\
\hline 2005-12-02 00:00:00.0 & 25.7 \\
\hline 2005-12-01 21:00:00.0 & \\
\hline 2005-12-01 18:00:00.0 & 29.5 \\
\hline 2005-12-01 15:00:00.0 & 33.5 \\
\hline 2005-12-01 12:00:00.0 & 34.5 \\
\hline 2005-12-01 09:00:00.0 & 30.4 \\
\hline 2005-12-01 06:00:00.0 & \\
\hline 2005-12-01 03:00:00.0 & \\
\hline 2005-12-01 00:00:00.0 & \\
\hline 2005-11-30 18:00:00.0 & 18.6 \\
\hline 2005-11-30 15:00:00.0 & 19.6 \\
\hline 2005-11-30 12:00:00.0 & 11.5 \\
\hline 2005-11-30 09:00:00.0 & 11.3 \\
\hline 2005-11-30 06:00:00.0 & \\
\hline 2005-11-30 03:00:00.0 & \\
\hline 2005-11-30 00:00:00.0 & \\
\hline 2005-11-29 18:00:00.0 & 13.1 \\
\hline 2005-11-29 15:00:00.0 & 15.8 \\
\hline 2005-11-29 12:00:00.0 & 34.2 \\
\hline 2005-11-29 09:00:00.0 & 17.2 \\
\hline 2005-11-29 06:00:00.0 & \\
\hline 2005-11-29 03:00:00.0 & 12.0 \\
\hline 2005-11-29 00:00:00.0 & 14.8 \\
\hline 2005-11-28 21:00:00.0 & \\
\hline 2005-11-28 18:00:00.0 & 27.1 \\
\hline 2005-11-28 15:00:00.0 & 26.4 \\
\hline 2005-11-28 12:00:00.0 & 32.1 \\
\hline 2005-11-28 09:00:00.0 & \\
\hline 2005-11-28 06:00:00.0 & 25.6 \\
\hline 2005-11-28 03:00:00.0 & 15.7 \\
\hline 2005-11-28 00:00:00.0 & 13.9 \\
\hline 2005-11-27 21:00:00.0 & 19.7 \\
\hline 2005-11-27 18:00:00.0 & 24.3 \\
\hline 2005-11-27 15:00:00.0 & 23.8 \\
\hline 2005-11-27 12:00:00.0 & \\
\hline 2005-11-27 09:00:00.0 & \\
\hline 2005-11-27 06:00:00.0 & 19.5 \\
\hline 2005-11-27 03:00:00.0 & 15.2 \\
\hline 2005-11-27 00:00:00.0 & 19.3 \\
\hline 2005-11-26 21:00:00.0 & 21.8 \\
\hline 2005-11-26 18:00:00.0 & \\
\hline 2005-11-26 15:00:00.0 & 16.1 \\
\hline 2005-11-26 12:00:00.0 & 6.9 \\
\hline 2005-11-26 09:00:00.0 & 10.3 \\
\hline 2005-11-26 06:00:00.0 & 9.2 \\
\hline 2005-11-26 03:00:00.0 & 8.1 \\
\hline 2005-11-26 00:00:00.0 & 28.8 \\
\hline 2005-11-25 21:00:00.0 & \\
\hline 2005-11-25 18:00:00.0 & 20.5 \\
\hline 2005-11-25 15:00:00.0 & 16.1 \\
\hline 2005-11-25 12:00:00.0 & 18.2 \\
\hline 2005-11-25 09:00:00.0 & \\
\hline 2005-11-25 06:00:00.0 & 10.8 \\
\hline 2005-11-25 03:00:00.0 & 11.1 \\
\hline 2005-11-25 00:00:00.0 & \\
\hline 2005-11-24 21:00:00.0 & 24.1 \\
\hline 2005-11-24 18:00:00.0 & \\
\hline 2005-11-24 15:00:00.0 & 23.4 \\
\hline 2005-11-24 12:00:00.0 & 12.2 \\
\hline 2005-11-24 09:00:00.0 & 4.9 \\
\hline 2005-11-24 06:00:00.0 & 41.4 \\
\hline
\end{tabular}




\section{ANEXO 01 - RAJADAS DE VENTO REGISTRADAS EM SÃO MARTINHO DA SERRA / RS}

\begin{tabular}{|c|c|}
\hline DataHora & VelVentoMax \\
\hline 2005-11-24 03:00:00.0 & 25.9 \\
\hline 2005-11-24 00:00:00.0 & \\
\hline 2005-11-23 21:00:00.0 & \\
\hline 2005-11-23 18:00:00.0 & 25.9 \\
\hline 2005-11-23 15:00:00.0 & 22.8 \\
\hline 2005-11-23 12:00:00.0 & \\
\hline 2005-11-23 09:00:00.0 & 16.3 \\
\hline 2005-11-23 06:00:00.0 & 13.3 \\
\hline 2005-11-23 03:00:00.0 & 22.3 \\
\hline 2005-11-23 00:00:00.0 & \\
\hline 2005-11-22 21:00:00.0 & \\
\hline 2005-11-22 18:00:00.0 & 11.3 \\
\hline 2005-11-22 15:00:00.0 & 22.6 \\
\hline 2005-11-22 12:00:00.0 & \\
\hline 2005-11-22 09:00:00.0 & 10.1 \\
\hline 2005-11-22 06:00:00.0 & 11.2 \\
\hline 2005-11-22 03:00:00.0 & 10.5 \\
\hline 2005-11-22 00:00:00.0 & \\
\hline 2005-11-21 21:00:00.0 & 30.0 \\
\hline 2005-11-21 18:00:00.0 & 25.2 \\
\hline 2005-11-21 15:00:00.0 & 22.0 \\
\hline 2005-11-21 12:00:00.0 & \\
\hline 2005-11-21 09:00:00.0 & 22.1 \\
\hline 2005-11-21 06:00:00.0 & 18.1 \\
\hline 2005-11-21 03:00:00.0 & \\
\hline 2005-11-21 00:00:00.0 & \\
\hline 2005-11-20 21:00:00.0 & 17.9 \\
\hline 2005-11-20 18:00:00.0 & 23.7 \\
\hline 2005-11-20 15:00:00.0 & 21.8 \\
\hline 2005-11-20 12:00:00.0 & 22.7 \\
\hline 2005-11-20 09:00:00.0 & 12.5 \\
\hline 2005-11-20 06:00:00.0 & 14.4 \\
\hline 2005-11-20 03:00:00.0 & 11.8 \\
\hline 2005-11-20 00:00:00.0 & \\
\hline 2005-11-19 21:00:00.0 & 18.7 \\
\hline 2005-11-19 18:00:00.0 & 19.8 \\
\hline 2005-11-19 15:00:00.0 & 19.8 \\
\hline 2005-11-19 12:00:00.0 & \\
\hline 2005-11-19 09:00:00.0 & 8.1 \\
\hline 2005-11-19 06:00:00.0 & 12.0 \\
\hline 2005-11-19 03:00:00.0 & 12.2 \\
\hline 2005-11-19 00:00:00.0 & 13.6 \\
\hline 2005-11-18 12:00:00.0 & 29.4 \\
\hline 2005-11-18 09:00:00.0 & 10.2 \\
\hline 2005-11-18 06:00:00.0 & 17.1 \\
\hline 2005-11-18 03:00:00.0 & 17.9 \\
\hline 2005-11-18 00:00:00.0 & \\
\hline 2005-11-17 21:00:00.0 & 24.9 \\
\hline 2005-11-17 18:00:00.0 & 20.4 \\
\hline 2005-11-17 15:00:00.0 & 27.2 \\
\hline 2005-11-17 12:00:00.0 & \\
\hline 2005-11-17 09:00:00.0 & 22.3 \\
\hline 2005-11-17 06:00:00.0 & 22.4 \\
\hline 2005-11-17 03:00:00.0 & 19.3 \\
\hline 2005-11-17 00:00:00.0 & 19.1 \\
\hline 2005-11-16 21:00:00.0 & 20.3 \\
\hline 2005-11-16 18:00:00.0 & 21.2 \\
\hline 2005-11-16 15:00:00.0 & 33.7 \\
\hline 2005-11-16 12:00:00.0 & 16.4 \\
\hline 2005-11-16 09:00:00.0 & 10.4 \\
\hline 2005-11-16 06:00:00.0 & 10.0 \\
\hline 2005-11-16 03:00:00.0 & \\
\hline 2005-11-16 00:00:00.0 & 11.1 \\
\hline 2005-11-15 21:00:00.0 & 20.4 \\
\hline 2005-11-15 18:00:00.0 & 20.7 \\
\hline 2005-11-15 15:00:00.0 & \\
\hline 2005-11-15 12:00:00.0 & 20.1 \\
\hline 2005-11-15 09:00:00.0 & 18.2 \\
\hline 2005-11-15 06:00:00.0 & 18.9 \\
\hline
\end{tabular}

\begin{tabular}{|c|c|}
\hline DataHora & VelVentoMax \\
\hline 2005-11-15 03:00:00.0 & 15.3 \\
\hline 2005-11-15 00:00:00.0 & 15.2 \\
\hline 2005-11-14 21:00:00.0 & 20.4 \\
\hline 2005-11-14 18:00:00.0 & 15.8 \\
\hline 2005-11-14 15:00:00.0 & \\
\hline 2005-11-14 12:00:00.0 & 15.1 \\
\hline 2005-11-14 09:00:00.0 & 14.1 \\
\hline 2005-11-14 06:00:00.0 & 11.1 \\
\hline 2005-11-14 03:00:00.0 & \\
\hline 2005-11-14 00:00:00.0 & 14.1 \\
\hline 2005-11-13 21:00:00.0 & 18.1 \\
\hline 2005-11-13 18:00:00.0 & 20.6 \\
\hline 2005-11-13 15:00:00.0 & 20.6 \\
\hline 2005-11-13 12:00:00.0 & 14.5 \\
\hline 2005-11-13 09:00:00.0 & 15.1 \\
\hline 2005-11-13 06:00:00.0 & 15.1 \\
\hline 2005-11-13 03:00:00.0 & 11.7 \\
\hline 2005-11-13 00:00:00.0 & 11.3 \\
\hline 2005-11-12 21:00:00.0 & 16.2 \\
\hline 2005-11-12 18:00:00.0 & 15.0 \\
\hline 2005-11-12 15:00:00.0 & 8.7 \\
\hline 2005-11-12 12:00:00.0 & \\
\hline 2005-11-12 09:00:00.0 & 14.8 \\
\hline 2005-11-12 06:00:00.0 & 14.5 \\
\hline 2005-11-12 03:00:00.0 & 11.4 \\
\hline 2005-11-12 00:00:00.0 & 17.0 \\
\hline 2005-11-11 21:00:00.0 & 19.5 \\
\hline 2005-11-11 18:00:00.0 & 15.7 \\
\hline 2005-11-11 15:00:00.0 & 16.3 \\
\hline 2005-11-11 12:00:00.0 & \\
\hline 2005-11-11 09:00:00.0 & 19.0 \\
\hline 2005-11-11 06:00:00.0 & 17.2 \\
\hline 2005-11-11 03:00:00.0 & 17.3 \\
\hline 2005-11-11 00:00:00.0 & 10.1 \\
\hline 2005-11-10 21:00:00.0 & 22.5 \\
\hline 2005-11-10 18:00:00.0 & 18.9 \\
\hline 2005-11-10 15:00:00.0 & 23.1 \\
\hline 2005-11-10 12:00:00.0 & 22.5 \\
\hline 2005-11-10 09:00:00.0 & 18.8 \\
\hline 2005-11-10 06:00:00.0 & 18.7 \\
\hline 2005-11-10 03:00:00.0 & 21.3 \\
\hline 2005-11-10 00:00:00.0 & 28.3 \\
\hline 2005-11-09 21:00:00.0 & 26.4 \\
\hline 2005-11-09 18:00:00.0 & 24.7 \\
\hline 2005-11-09 15:00:00.0 & \\
\hline 2005-11-09 12:00:00.0 & 22.5 \\
\hline 2005-11-09 09:00:00.0 & 18.2 \\
\hline 2005-11-09 06:00:00.0 & 16.8 \\
\hline 2005-11-09 03:00:00.0 & 21.3 \\
\hline 2005-11-09 00:00:00.0 & 24.6 \\
\hline 2005-11-08 21:00:00.0 & 27.6 \\
\hline 2005-11-08 18:00:00.0 & 29.0 \\
\hline 2005-11-08 15:00:00.0 & 25.7 \\
\hline 2005-11-08 12:00:00.0 & 27.8 \\
\hline 2005-11-08 09:00:00.0 & 17.2 \\
\hline 2005-11-08 06:00:00.0 & \\
\hline 2005-11-08 03:00:00.0 & 17.3 \\
\hline 2005-11-08 00:00:00.0 & \\
\hline 2005-11-07 21:00:00.0 & 29.4 \\
\hline 2005-11-07 18:00:00.0 & 28.3 \\
\hline 2005-11-07 15:00:00.0 & 29.0 \\
\hline 2005-11-07 12:00:00.0 & 29.3 \\
\hline 2005-11-07 09:00:00.0 & 23.2 \\
\hline 2005-11-07 06:00:00.0 & 25.9 \\
\hline 2005-11-07 03:00:00.0 & 27.5 \\
\hline 2005-11-07 00:00:00.0 & 31.2 \\
\hline 2005-11-06 21:00:00.0 & 27.3 \\
\hline 2005-11-06 18:00:00.0 & \\
\hline 2005-11-06 15:00:00.0 & 24.8 \\
\hline
\end{tabular}

\begin{tabular}{|c|c|}
\hline DataHora & VelVentoMax \\
\hline 2005-11-06 12:00:00.0 & 29.4 \\
\hline 2005-11-06 09:00:00.0 & 17.7 \\
\hline 2005-11-06 06:00:00.0 & 28.3 \\
\hline 2005-11-06 03:00:00.0 & 19.4 \\
\hline 2005-11-06 00:00:00.0 & 7.1 \\
\hline 2005-11-05 21:00:00.0 & 24.1 \\
\hline 2005-11-05 18:00:00.0 & 25.2 \\
\hline 2005-11-05 15:00:00.0 & 19.9 \\
\hline 2005-11-05 12:00:00.0 & 19.4 \\
\hline 2005-11-05 09:00:00.0 & 19.8 \\
\hline 2005-11-05 06:00:00.0 & \\
\hline 2005-11-05 03:00:00.0 & 11.9 \\
\hline 2005-11-05 00:00:00.0 & 15.9 \\
\hline 2005-11-04 21:00:00.0 & 5.6 \\
\hline 2005-11-04 18:00:00.0 & 24.7 \\
\hline 2005-11-04 15:00:00.0 & 26.7 \\
\hline 2005-11-04 12:00:00.0 & 26.4 \\
\hline 2005-11-04 09:00:00.0 & 21.0 \\
\hline 2005-11-04 06:00:00.0 & 18.6 \\
\hline 2005-11-04 03:00:00.0 & 19.5 \\
\hline 2005-11-04 00:00:00.0 & 14.3 \\
\hline 2005-11-03 21:00:00.0 & 27.1 \\
\hline 2005-11-03 18:00:00.0 & 15.2 \\
\hline 2005-11-03 15:00:00.0 & 20.7 \\
\hline 2005-11-03 12:00:00.0 & 22.7 \\
\hline 2005-11-03 09:00:00.0 & 13.7 \\
\hline 2005-11-03 06:00:00.0 & 16.7 \\
\hline 2005-11-03 03:00:00.0 & 17.6 \\
\hline 2005-11-03 00:00:00.0 & 18.0 \\
\hline 2005-11-02 21:00:00.0 & \\
\hline 2005-11-02 18:00:00.0 & 19.5 \\
\hline 2005-11-02 15:00:00.0 & 20.6 \\
\hline 2005-11-02 12:00:00.0 & 20.4 \\
\hline 2005-11-02 09:00:00.0 & 15.5 \\
\hline 2005-11-02 06:00:00.0 & 15.0 \\
\hline 2005-11-02 03:00:00.0 & 13.4 \\
\hline 2005-11-02 00:00:00.0 & 14.1 \\
\hline 2005-11-01 21:00:00.0 & 17.3 \\
\hline 2005-11-01 18:00:00.0 & 11.8 \\
\hline 2005-11-01 15:00:00.0 & 15.7 \\
\hline 2005-11-01 12:00:00.0 & 18.8 \\
\hline 2005-11-01 09:00:00.0 & \\
\hline 2005-11-01 06:00:00.0 & 14.9 \\
\hline 2005-11-01 03:00:00.0 & 8.6 \\
\hline 2005-11-01 00:00:00.0 & 16.3 \\
\hline 2005-10-31 21:00:00.0 & 18.2 \\
\hline 2005-10-31 18:00:00.0 & \\
\hline 2005-10-31 15:00:00.0 & 18.6 \\
\hline 2005-10-31 12:00:00.0 & 12.7 \\
\hline 2005-10-31 09:00:00.0 & \\
\hline 2005-10-31 06:00:00.0 & 9.4 \\
\hline 2005-10-31 03:00:00.0 & 8.6 \\
\hline 2005-10-31 00:00:00.0 & 17.9 \\
\hline 2005-10-30 21:00:00.0 & \\
\hline 2005-10-30 18:00:00.0 & 37.6 \\
\hline 2005-10-30 15:00:00.0 & 19.6 \\
\hline 2005-10-30 12:00:00.0 & 20.5 \\
\hline 2005-10-30 09:00:00.0 & \\
\hline 2005-10-30 06:00:00.0 & 15.9 \\
\hline 2005-10-30 03:00:00.0 & \\
\hline 2005-10-30 00:00:00.0 & \\
\hline 2005-10-29 21:00:00.0 & 22.2 \\
\hline 2005-10-29 18:00:00.0 & 18.5 \\
\hline 2005-10-29 15:00:00.0 & 14.2 \\
\hline 2005-10-29 12:00:00.0 & 20.1 \\
\hline 2005-10-29 09:00:00.0 & 17.6 \\
\hline 2005-10-29 06:00:00.0 & 23.7 \\
\hline 2005-10-29 03:00:00.0 & 28.0 \\
\hline 2005-10-29 00:00:00.0 & \\
\hline
\end{tabular}




\section{ANEXO 01 - RAJADAS DE VENTO REGISTRADAS EM SÃO MARTINHO DA SERRA / RS}

\begin{tabular}{|c|c|}
\hline DataHora & VelVentoMax \\
\hline 2005-10-27 09:00:00.0 & 17.3 \\
\hline 2005-10-27 06:00:00.0 & 20.5 \\
\hline 2005-10-27 03:00:00.0 & 21.9 \\
\hline 2005-10-27 00:00:00.0 & \\
\hline 2005-10-26 21:00:00.0 & 20.4 \\
\hline 2005-10-26 18:00:00.0 & 21.6 \\
\hline 2005-10-26 15:00:00.0 & 27.1 \\
\hline 2005-10-26 12:00:00.0 & 21.2 \\
\hline 2005-10-26 09:00:00.0 & 17.6 \\
\hline 2005-10-26 06:00:00.0 & 15.1 \\
\hline 2005-10-26 03:00:00.0 & 22.6 \\
\hline 2005-10-26 00:00:00.0 & \\
\hline 2005-10-25 21:00:00.0 & \\
\hline 2005-10-25 18:00:00.0 & \\
\hline 2005-10-25 09:00:00.0 & 12.9 \\
\hline 2005-10-25 06:00:00.0 & 16.1 \\
\hline 2005-10-25 03:00:00.0 & 25.5 \\
\hline $2005-10-25$ 00:00:00.0 & \\
\hline 2005-10-24 21:00:00.0 & \\
\hline 2005-10-24 18:00:00.0 & \\
\hline 2005-10-24 09:00:00.0 & 25.8 \\
\hline 2005-10-24 06:00:00.0 & 27.2 \\
\hline 2005-10-24 03:00:00.0 & 29.0 \\
\hline 2005-10-24 00:00:00.0 & \\
\hline 2005-10-23 21:00:00.0 & 19.7 \\
\hline 2005-10-23 18:00:00.0 & 16.1 \\
\hline 2005-10-23 15:00:00.0 & 14.7 \\
\hline 2005-10-23 12:00:00.0 & \\
\hline 2005-10-23 09:00:00.0 & 23.6 \\
\hline 2005-10-23 06:00:00.0 & 17.0 \\
\hline 2005-10-23 03:00:00.0 & \\
\hline $2005-10-23$ 00:00:00.0 & 10.5 \\
\hline 2005-10-22 21:00:00.0 & 17.7 \\
\hline 2005-10-22 18:00:00.0 & 19.5 \\
\hline 2005-10-22 15:00:00.0 & 19.5 \\
\hline 2005-10-22 12:00:00.0 & \\
\hline 2005-10-22 09:00:00.0 & 13.4 \\
\hline 2005-10-22 06:00:00.0 & 14.2 \\
\hline 2005-10-22 03:00:00.0 & \\
\hline 2005-10-22 00:00:00.0 & \\
\hline 2005-10-21 21:00:00.0 & 27.6 \\
\hline 2005-10-21 18:00:00.0 & 13.3 \\
\hline 2005-10-21 15:00:00.0 & \\
\hline 2005-10-21 12:00:00.0 & 7.4 \\
\hline 2005-10-21 09:00:00.0 & 17.1 \\
\hline 2005-10-21 06:00:00.0 & 15.5 \\
\hline 2005-10-21 03:00:00.0 & 14.3 \\
\hline 2005-10-21 00:00:00.0 & 10.6 \\
\hline 2005-10-20 21:00:00.0 & 10.4 \\
\hline 2005-10-20 18:00:00.0 & 13.6 \\
\hline 2005-10-20 15:00:00.0 & 16.5 \\
\hline 2005-10-20 12:00:00.0 & 19.9 \\
\hline 2005-10-20 09:00:00.0 & 18.8 \\
\hline 2005-10-20 06:00:00.0 & 18.5 \\
\hline 2005-10-20 03:00:00.0 & 14.6 \\
\hline $2005-10-20$ 00:00:00.0 & 11.5 \\
\hline 2005-10-19 21:00:00.0 & 13.5 \\
\hline 2005-10-19 18:00:00.0 & 15.3 \\
\hline 2005-10-19 15:00:00.0 & \\
\hline 2005-10-19 12:00:00.0 & 13.6 \\
\hline 2005-10-19 09:00:00.0 & 9.7 \\
\hline 2005-10-19 06:00:00.0 & 10.4 \\
\hline 2005-10-19 03:00:00.0 & 5.6 \\
\hline 2005-10-19 00:00:00.0 & 9.9 \\
\hline 2005-10-18 21:00:00.0 & 33.9 \\
\hline 2005-10-18 18:00:00.0 & 33.9 \\
\hline 2005-10-18 15:00:00.0 & \\
\hline 2005-10-18 12:00:00.0 & 12.6 \\
\hline 2005-10-18 09:00:00.0 & 17.6 \\
\hline
\end{tabular}

\begin{tabular}{|c|c|}
\hline DataHora & VelVentoMax \\
\hline 2005-10-18 06:00:00.0 & \\
\hline 2005-10-18 03:00:00.0 & \\
\hline 2005-10-18 00:00:00.0 & 21.5 \\
\hline 2005-10-17 21:00:00.0 & 19.6 \\
\hline 2005-10-17 18:00:00.0 & \\
\hline 2005-10-17 15:00:00.0 & 16.6 \\
\hline 2005-10-17 12:00:00.0 & \\
\hline 2005-10-17 09:00:00.0 & 12.7 \\
\hline 2005-10-17 06:00:00.0 & 24.3 \\
\hline 2005-10-17 03:00:00.0 & 23.2 \\
\hline 2005-10-17 00:00:00.0 & 21.5 \\
\hline 2005-10-16 21:00:00.0 & 30.1 \\
\hline 2005-10-16 18:00:00.0 & 26.1 \\
\hline 2005-10-16 15:00:00.0 & \\
\hline 2005-10-16 12:00:00.0 & 32.6 \\
\hline 2005-10-16 09:00:00.0 & 26.4 \\
\hline 2005-10-16 06:00:00.0 & \\
\hline 2005-10-16 03:00:00.0 & 25.2 \\
\hline 2005-10-16 00:00:00.0 & 26.0 \\
\hline 2005-10-15 21:00:00.0 & 26.1 \\
\hline 2005-10-15 18:00:00.0 & \\
\hline 2005-10-15 15:00:00.0 & 27.3 \\
\hline 2005-10-15 12:00:00.0 & 35.1 \\
\hline 2005-10-15 09:00:00.0 & 24.7 \\
\hline 2005-10-15 06:00:00.0 & \\
\hline 2005-10-15 03:00:00.0 & 24.4 \\
\hline 2005-10-15 00:00:00.0 & 27.7 \\
\hline 2005-10-14 21:00:00.0 & 24.6 \\
\hline 2005-10-14 18:00:00.0 & \\
\hline 2005-10-14 15:00:00.0 & 15.3 \\
\hline 2005-10-14 12:00:00.0 & 21.1 \\
\hline 2005-10-14 09:00:00.0 & 38.1 \\
\hline 2005-10-14 06:00:00.0 & \\
\hline 2005-10-14 03:00:00.0 & 23.0 \\
\hline 2005-10-14 00:00:00.0 & 38.7 \\
\hline 2005-10-13 21:00:00.0 & 43.6 \\
\hline 2005-10-13 18:00:00.0 & \\
\hline 2005-10-13 15:00:00.0 & \\
\hline 2005-10-13 12:00:00.0 & 28.0 \\
\hline 2005-10-13 09:00:00.0 & 8.8 \\
\hline 2005-10-13 06:00:00.0 & 8.5 \\
\hline 2005-10-13 03:00:00.0 & 8.3 \\
\hline 2005-10-13 00:00:00.0 & 7.7 \\
\hline 2005-10-12 21:00:00.0 & 21.0 \\
\hline 2005-10-12 18:00:00.0 & \\
\hline 2005-10-12 15:00:00.0 & 23.3 \\
\hline 2005-10-12 12:00:00.0 & 18.8 \\
\hline 2005-10-12 09:00:00.0 & 15.0 \\
\hline 2005-10-12 06:00:00.0 & \\
\hline 2005-10-12 03:00:00.0 & 16.5 \\
\hline 2005-10-12 00:00:00.0 & 10.5 \\
\hline 2005-10-11 21:00:00.0 & \\
\hline 2005-10-11 18:00:00.0 & \\
\hline 2005-10-11 15:00:00.0 & 11.6 \\
\hline 2005-10-11 12:00:00.0 & 12.7 \\
\hline 2005-10-11 09:00:00.0 & \\
\hline 2005-10-11 06:00:00.0 & 15.6 \\
\hline 2005-10-11 03:00:00.0 & 17.3 \\
\hline 2005-10-11 00:00:00.0 & 16.8 \\
\hline 2005-10-10 21:00:00.0 & 16.6 \\
\hline 2005-10-10 18:00:00.0 & 17.6 \\
\hline 2005-10-10 15:00:00.0 & 18.4 \\
\hline 2005-10-10 12:00:00.0 & 21.2 \\
\hline 2005-10-10 09:00:00.0 & \\
\hline 2005-10-10 06:00:00.0 & 19.6 \\
\hline 2005-10-10 03:00:00.0 & 14.6 \\
\hline 2005-10-10 00:00:00.0 & 11.1 \\
\hline 2005-10-09 21:00:00.0 & \\
\hline 2005-10-09 18:00:00.0 & 22.6 \\
\hline
\end{tabular}

\begin{tabular}{|c|c|}
\hline DataHora & VelVentoMax \\
\hline 2005-10-09 15:00:00.0 & 19.4 \\
\hline 2005-10-09 12:00:00.0 & 17.9 \\
\hline 2005-10-09 09:00:00.0 & \\
\hline 2005-10-09 06:00:00.0 & 16.7 \\
\hline 2005-10-09 03:00:00.0 & 20.2 \\
\hline 2005-10-09 00:00:00.0 & 8.2 \\
\hline 2005-10-08 21:00:00.0 & \\
\hline 2005-10-08 18:00:00.0 & 16.2 \\
\hline 2005-10-08 15:00:00.0 & 15.5 \\
\hline 2005-10-08 12:00:00.0 & 14.5 \\
\hline 2005-10-08 09:00:00.0 & 9.5 \\
\hline 2005-10-08 06:00:00.0 & 9.2 \\
\hline 2005-10-08 03:00:00.0 & 14.7 \\
\hline 2005-10-08 00:00:00.0 & 10.6 \\
\hline 2005-10-07 18:00:00.0 & 10.9 \\
\hline 2005-10-07 15:00:00.0 & 21.2 \\
\hline 2005-10-07 12:00:00.0 & \\
\hline 2005-10-07 09:00:00.0 & \\
\hline 2005-10-07 06:00:00.0 & 29.1 \\
\hline 2005-10-07 03:00:00.0 & 23.3 \\
\hline 2005-10-07 00:00:00.0 & 19.1 \\
\hline 2005-10-06 21:00:00.0 & 17.1 \\
\hline 2005-10-06 18:00:00.0 & 16.0 \\
\hline 2005-10-06 15:00:00.0 & 25.3 \\
\hline 2005-10-06 12:00:00.0 & 20.3 \\
\hline 2005-10-06 09:00:00.0 & \\
\hline 2005-10-06 06:00:00.0 & \\
\hline 2005-10-06 03:00:00.0 & 10.1 \\
\hline 2005-10-06 00:00:00.0 & \\
\hline 2005-10-05 21:00:00.0 & 27.1 \\
\hline 2005-10-05 18:00:00.0 & 18.6 \\
\hline 2005-10-05 15:00:00.0 & 19.7 \\
\hline 2005-10-05 12:00:00.0 & 23.4 \\
\hline 2005-10-05 09:00:00.0 & \\
\hline 2005-10-05 06:00:00.0 & 25.1 \\
\hline 2005-10-05 03:00:00.0 & 22.5 \\
\hline 2005-10-05 00:00:00.0 & 16.4 \\
\hline 2005-10-04 21:00:00.0 & \\
\hline 2005-10-04 18:00:00.0 & 33.9 \\
\hline 2005-10-04 15:00:00.0 & 28.8 \\
\hline 2005-10-04 12:00:00.0 & \\
\hline 2005-10-04 09:00:00.0 & 32.1 \\
\hline 2005-10-04 06:00:00.0 & 42.3 \\
\hline 2005-10-04 03:00:00.0 & 37.2 \\
\hline 2005-10-04 00:00:00.0 & 13.8 \\
\hline 2005-10-03 21:00:00.0 & \\
\hline 2005-10-03 18:00:00.0 & 26.3 \\
\hline 2005-10-03 15:00:00.0 & 25.2 \\
\hline 2005-10-03 12:00:00.0 & \\
\hline 2005-10-03 09:00:00.0 & 23.6 \\
\hline 2005-10-03 06:00:00.0 & 19.9 \\
\hline 2005-10-03 03:00:00.0 & 20.2 \\
\hline 2005-10-03 00:00:00.0 & \\
\hline 2005-10-02 21:00:00.0 & 19.8 \\
\hline 2005-10-02 18:00:00.0 & 17.5 \\
\hline 2005-10-02 15:00:00.0 & 23.1 \\
\hline 2005-10-02 12:00:00.0 & \\
\hline 2005-10-02 09:00:00.0 & 20.3 \\
\hline 2005-10-02 06:00:00.0 & 21.9 \\
\hline 2005-10-02 03:00:00.0 & \\
\hline 2005-10-02 00:00:00.0 & \\
\hline 2005-10-01 21:00:00.0 & 21.8 \\
\hline 2005-10-01 18:00:00.0 & 18.3 \\
\hline 2005-10-01 15:00:00.0 & 23.1 \\
\hline 2005-10-01 12:00:00.0 & \\
\hline 2005-10-01 09:00:00.0 & \\
\hline 2005-10-01 06:00:00.0 & 21.4 \\
\hline 2005-10-01 03:00:00.0 & 18.4 \\
\hline 2005-10-01 00:00:00.0 & 19.4 \\
\hline
\end{tabular}




\section{ANEXO 01 - RAJADAS DE VENTO REGISTRADAS EM SÃO MARTINHO DA SERRA / RS}

\begin{tabular}{|c|c|}
\hline DataHora & VelVentoMax \\
\hline 2005-09-30 21:00:00.0 & 24.9 \\
\hline 2005-09-30 18:00:00.0 & 8.4 \\
\hline 2005-09-30 15:00:00.0 & 16.3 \\
\hline 2005-09-30 12:00:00.0 & \\
\hline 2005-09-30 09:00:00.0 & 25.3 \\
\hline 2005-09-30 06:00:00.0 & 30.3 \\
\hline 2005-09-30 03:00:00.0 & \\
\hline 2005-09-30 00:00:00.0 & \\
\hline 2005-09-29 21:00:00.0 & 27.4 \\
\hline 2005-09-29 18:00:00.0 & 31.3 \\
\hline 2005-09-29 15:00:00.0 & 33.1 \\
\hline 2005-09-29 12:00:00.0 & 31.6 \\
\hline 2005-09-29 09:00:00.0 & 27.1 \\
\hline 2005-09-29 06:00:00.0 & 26.3 \\
\hline 2005-09-29 03:00:00.0 & 25.4 \\
\hline 2005-09-29 00:00:00.0 & 14.6 \\
\hline 2005-09-28 21:00:00.0 & 15.5 \\
\hline 2005-09-28 18:00:00.0 & 24.4 \\
\hline 2005-09-28 15:00:00.0 & 26.8 \\
\hline 2005-09-28 12:00:00.0 & 28.7 \\
\hline 2005-09-28 09:00:00.0 & 21.5 \\
\hline 2005-09-28 06:00:00.0 & 20.9 \\
\hline 2005-09-28 03:00:00.0 & 14.2 \\
\hline 2005-09-28 00:00:00.0 & \\
\hline 2005-09-27 21:00:00.0 & 20.0 \\
\hline 2005-09-27 18:00:00.0 & 18.7 \\
\hline 2005-09-27 15:00:00.0 & 22.3 \\
\hline 2005-09-27 12:00:00.0 & 23.0 \\
\hline 2005-09-27 09:00:00.0 & 17.7 \\
\hline 2005-09-27 06:00:00.0 & 17.9 \\
\hline 2005-09-27 03:00:00.0 & 15.0 \\
\hline 2005-09-27 00:00:00.0 & \\
\hline 2005-09-26 21:00:00.0 & 18.3 \\
\hline 2005-09-26 18:00:00.0 & 17.9 \\
\hline 2005-09-26 15:00:00.0 & 18.1 \\
\hline 2005-09-26 12:00:00.0 & 17.8 \\
\hline 2005-09-26 09:00:00.0 & 11.8 \\
\hline 2005-09-26 06:00:00.0 & 10.9 \\
\hline 2005-09-26 03:00:00.0 & 11.8 \\
\hline 2005-09-26 00:00:00.0 & \\
\hline 2005-09-25 21:00:00.0 & 26.3 \\
\hline 2005-09-25 18:00:00.0 & 28.8 \\
\hline 2005-09-25 15:00:00.0 & \\
\hline 2005-09-25 12:00:00.0 & 24.9 \\
\hline 2005-09-25 09:00:00.0 & 25.6 \\
\hline 2005-09-25 06:00:00.0 & 22.0 \\
\hline 2005-09-25 03:00:00.0 & \\
\hline 2005-09-25 00:00:00.0 & 20.3 \\
\hline 2005-09-24 21:00:00.0 & 30.3 \\
\hline 2005-09-24 18:00:00.0 & \\
\hline 2005-09-24 15:00:00.0 & \\
\hline 2005-09-24 12:00:00.0 & 22.8 \\
\hline 2005-09-24 09:00:00.0 & 14.0 \\
\hline 2005-09-24 06:00:00.0 & 23.9 \\
\hline 2005-09-24 03:00:00.0 & \\
\hline 2005-09-24 00:00:00.0 & 24.9 \\
\hline 2005-09-23 21:00:00.0 & 20.4 \\
\hline 2005-09-23 18:00:00.0 & 20.0 \\
\hline 2005-09-23 15:00:00.0 & 21.5 \\
\hline 2005-09-23 12:00:00.0 & 24.4 \\
\hline 2005-09-23 09:00:00.0 & 21.6 \\
\hline 2005-09-23 06:00:00.0 & 18.9 \\
\hline 2005-09-23 03:00:00.0 & \\
\hline 2005-09-23 00:00:00.0 & 27.1 \\
\hline 2005-09-22 21:00:00.0 & 34.2 \\
\hline 2005-09-22 18:00:00.0 & 14.6 \\
\hline 2005-09-22 15:00:00.0 & 21.0 \\
\hline 2005-09-22 12:00:00.0 & 20.0 \\
\hline 2005-09-22 09:00:00.0 & 22.0 \\
\hline
\end{tabular}

\begin{tabular}{|c|c|}
\hline DataHora & VelVentoMax \\
\hline 2005-09-22 06:00:00.0 & 20.9 \\
\hline 2005-09-22 03:00:00.0 & 20.1 \\
\hline 2005-09-22 00:00:00.0 & 16.8 \\
\hline 2005-09-21 21:00:00.0 & 33.1 \\
\hline 2005-09-21 18:00:00.0 & 19.1 \\
\hline 2005-09-21 15:00:00.0 & 27.0 \\
\hline 2005-09-21 12:00:00.0 & 26.4 \\
\hline 2005-09-21 09:00:00.0 & 22.4 \\
\hline 2005-09-21 06:00:00.0 & \\
\hline 2005-09-21 03:00:00.0 & 17.2 \\
\hline 2005-09-21 00:00:00.0 & 15.7 \\
\hline 2005-09-20 21:00:00.0 & 20.4 \\
\hline 2005-09-20 18:00:00.0 & 20.1 \\
\hline 2005-09-20 15:00:00.0 & 19.8 \\
\hline 2005-09-20 12:00:00.0 & 15.3 \\
\hline 2005-09-20 09:00:00.0 & 9.5 \\
\hline 2005-09-20 06:00:00.0 & 8.9 \\
\hline 2005-09-20 03:00:00.0 & 6.1 \\
\hline 2005-09-20 00:00:00.0 & 27.1 \\
\hline 2005-09-19 21:00:00.0 & 11.7 \\
\hline 2005-09-19 18:00:00.0 & 15.5 \\
\hline 2005-09-19 15:00:00.0 & 32.6 \\
\hline 2005-09-19 12:00:00.0 & 12.7 \\
\hline 2005-09-19 09:00:00.0 & 12.5 \\
\hline 2005-09-19 06:00:00.0 & \\
\hline 2005-09-19 03:00:00.0 & 14.0 \\
\hline 2005-09-19 00:00:00.0 & 14.5 \\
\hline 2005-09-18 21:00:00.0 & 18.5 \\
\hline 2005-09-18 18:00:00.0 & 15.3 \\
\hline 2005-09-18 15:00:00.0 & 17.0 \\
\hline 2005-09-18 12:00:00.0 & 15.2 \\
\hline 2005-09-18 09:00:00.0 & 19.8 \\
\hline 2005-09-18 06:00:00.0 & \\
\hline 2005-09-18 03:00:00.0 & 25.1 \\
\hline 2005-09-18 00:00:00.0 & 26.3 \\
\hline 2005-09-17 21:00:00.0 & 23.5 \\
\hline 2005-09-17 18:00:00.0 & 26.6 \\
\hline 2005-09-17 15:00:00.0 & 29.2 \\
\hline 2005-09-17 12:00:00.0 & 25.4 \\
\hline 2005-09-17 09:00:00.0 & 18.7 \\
\hline 2005-09-17 06:00:00.0 & \\
\hline 2005-09-17 03:00:00.0 & 17.8 \\
\hline 2005-09-17 00:00:00.0 & \\
\hline 2005-09-16 21:00:00.0 & 15.5 \\
\hline 2005-09-16 18:00:00.0 & \\
\hline 2005-09-16 15:00:00.0 & 21.3 \\
\hline 2005-09-16 12:00:00.0 & 18.3 \\
\hline 2005-09-16 09:00:00.0 & 14.5 \\
\hline 2005-09-16 06:00:00.0 & \\
\hline 2005-09-16 03:00:00.0 & 10.6 \\
\hline 2005-09-16 00:00:00.0 & 9.6 \\
\hline 2005-09-15 21:00:00.0 & 17.7 \\
\hline 2005-09-15 18:00:00.0 & 19.8 \\
\hline 2005-09-15 15:00:00.0 & 20.1 \\
\hline 2005-09-15 12:00:00.0 & 17.3 \\
\hline 2005-09-15 09:00:00.0 & 18.2 \\
\hline 2005-09-15 06:00:00.0 & 18.2 \\
\hline 2005-09-15 03:00:00.0 & 14.4 \\
\hline 2005-09-15 00:00:00.0 & 16.7 \\
\hline 2005-09-14 21:00:00.0 & 16.6 \\
\hline 2005-09-14 18:00:00.0 & \\
\hline 2005-09-14 15:00:00.0 & 26.8 \\
\hline 2005-09-14 12:00:00.0 & 23.5 \\
\hline 2005-09-14 09:00:00.0 & \\
\hline 2005-09-14 06:00:00.0 & \\
\hline 2005-09-14 03:00:00.0 & 17.8 \\
\hline 2005-09-14 00:00:00.0 & 14.6 \\
\hline 2005-09-13 21:00:00.0 & 21.9 \\
\hline 2005-09-13 18:00:00.0 & 25.1 \\
\hline
\end{tabular}

\begin{tabular}{|c|c|}
\hline DataHora & VelVentoMax \\
\hline 2005-09-13 15:00:00.0 & 25.9 \\
\hline 2005-09-13 12:00:00.0 & 17.9 \\
\hline 2005-09-13 09:00:00.0 & 11.1 \\
\hline 2005-09-13 06:00:00.0 & 11.1 \\
\hline 2005-09-13 03:00:00.0 & 11.0 \\
\hline 2005-09-13 00:00:00.0 & 8.8 \\
\hline 2005-09-12 21:00:00.0 & \\
\hline 2005-09-12 18:00:00.0 & 22.7 \\
\hline 2005-09-12 15:00:00.0 & 22.7 \\
\hline $2005-09-12$ 12:00:00.0 & 19.4 \\
\hline 2005-09-12 09:00:00.0 & 22.7 \\
\hline 2005-09-12 06:00:00.0 & 17.4 \\
\hline 2005-09-12 03:00:00.0 & 15.2 \\
\hline 2005-09-12 00:00:00.0 & 20.6 \\
\hline 2005-09-11 21:00:00.0 & 40.2 \\
\hline $2005-09-11$ 18:00:00.0 & 22.8 \\
\hline 2005-09-11 15:00:00.0 & 29.9 \\
\hline 2005-09-11 12:00:00.0 & 29.6 \\
\hline 2005-09-11 09:00:00.0 & \\
\hline 2005-09-11 06:00:00.0 & 34.4 \\
\hline 2005-09-11 03:00:00.0 & 23.0 \\
\hline 2005-09-11 00:00:00.0 & 19.4 \\
\hline $2005-09-10$ 21:00:00.0 & 38.5 \\
\hline 2005-09-10 18:00:00.0 & 22.3 \\
\hline $2005-09-10$ 15:00:00.0 & 27.2 \\
\hline 2005-09-10 12:00:00.0 & 29.3 \\
\hline 2005-09-10 09:00:00.0 & 34.0 \\
\hline 2005-09-10 06:00:00.0 & 31.3 \\
\hline 2005-09-10 03:00:00.0 & 32.9 \\
\hline 2005-09-10 00:00:00.0 & 31.3 \\
\hline 2005-09-09 21:00:00.0 & \\
\hline 2005-09-09 18:00:00.0 & \\
\hline 2005-09-09 15:00:00.0 & 25.6 \\
\hline 2005-09-09 12:00:00.0 & 24.6 \\
\hline 2005-09-09 09:00:00.0 & \\
\hline 2005-09-09 06:00:00.0 & 25.1 \\
\hline 2005-09-09 03:00:00.0 & 22.3 \\
\hline 2005-09-09 00:00:00.0 & 16.2 \\
\hline 2005-09-08 21:00:00.0 & \\
\hline 2005-09-08 18:00:00.0 & \\
\hline 2005-09-08 15:00:00.0 & \\
\hline 2005-09-08 06:00:00.0 & 21.3 \\
\hline 2005-09-08 03:00:00.0 & 17.6 \\
\hline 2005-09-08 00:00:00.0 & 15.6 \\
\hline 2005-09-07 21:00:00.0 & 15.5 \\
\hline 2005-09-07 18:00:00.0 & 15.8 \\
\hline 2005-09-07 15:00:00.0 & 21.3 \\
\hline 2005-09-07 12:00:00.0 & 10.9 \\
\hline 2005-09-07 09:00:00.0 & \\
\hline 2005-09-07 06:00:00.0 & 7.9 \\
\hline 2005-09-07 03:00:00.0 & 7.4 \\
\hline 2005-09-07 00:00:00.0 & \\
\hline 2005-09-06 21:00:00.0 & 14.7 \\
\hline $2005-09-06$ 18:00:00.0 & 15.4 \\
\hline 2005-09-06 15:00:00.0 & 11.8 \\
\hline 2005-09-06 12:00:00.0 & \\
\hline 2005-09-06 09:00:00.0 & \\
\hline 2005-09-06 06:00:00.0 & 8.6 \\
\hline 2005-09-06 03:00:00.0 & 8.8 \\
\hline 2005-09-06 00:00:00.0 & \\
\hline 2005-09-05 21:00:00.0 & 18.1 \\
\hline $2005-09-05$ 18:00:00.0 & 14.4 \\
\hline 2005-09-05 15:00:00.0 & 14.8 \\
\hline $2005-09-05$ 12:00:00.0 & \\
\hline 2005-09-05 09:00:00.0 & 14.8 \\
\hline 2005-09-05 06:00:00.0 & 12.3 \\
\hline 2005-09-05 03:00:00.0 & 10.5 \\
\hline 2005-09-05 00:00:00.0 & 13.6 \\
\hline 2005-09-04 21:00:00.0 & 17.2 \\
\hline
\end{tabular}




\section{ANEXO 01 - RAJADAS DE VENTO REGISTRADAS EM SÃO MARTINHO DA SERRA / RS}

\begin{tabular}{|c|c|}
\hline DataHora & VelVentoMax \\
\hline 2005-09-04 18:00:00.0 & 27.2 \\
\hline 2005-09-04 15:00:00.0 & 15.8 \\
\hline 2005-09-04 12:00:00.0 & 16.9 \\
\hline 2005-09-04 09:00:00.0 & 14.3 \\
\hline 2005-09-04 06:00:00.0 & 19.4 \\
\hline 2005-09-04 03:00:00.0 & 18.1 \\
\hline 2005-09-04 00:00:00.0 & 15.8 \\
\hline 2005-09-03 21:00:00.0 & 17.0 \\
\hline 2005-09-03 18:00:00.0 & 15.7 \\
\hline 2005-09-03 15:00:00.0 & 15.7 \\
\hline 2005-09-03 12:00:00.0 & 6.9 \\
\hline 2005-09-03 09:00:00.0 & 8.1 \\
\hline 2005-09-03 06:00:00.0 & 8.9 \\
\hline 2005-09-03 03:00:00.0 & \\
\hline 2005-09-03 00:00:00.0 & 14.3 \\
\hline 2005-09-02 21:00:00.0 & 19.4 \\
\hline 2005-09-02 18:00:00.0 & 26.4 \\
\hline 2005-09-02 15:00:00.0 & 27.3 \\
\hline 2005-09-02 12:00:00.0 & \\
\hline 2005-09-02 09:00:00.0 & 20.2 \\
\hline 2005-09-02 06:00:00.0 & 18.6 \\
\hline 2005-09-02 03:00:00.0 & 27.4 \\
\hline 2005-09-02 00:00:00.0 & 22.2 \\
\hline 2005-09-01 21:00:00.0 & 19.0 \\
\hline 2005-09-01 18:00:00.0 & 22.8 \\
\hline 2005-09-01 15:00:00.0 & 22.2 \\
\hline 2005-09-01 12:00:00.0 & \\
\hline 2005-09-01 09:00:00.0 & 33.0 \\
\hline 2005-09-01 06:00:00.0 & 26.1 \\
\hline 2005-09-01 03:00:00.0 & 34.1 \\
\hline 2005-09-01 00:00:00.0 & 31.5 \\
\hline 2005-08-31 21:00:00.0 & 28.8 \\
\hline 2005-08-31 18:00:00.0 & 33.8 \\
\hline 2005-08-31 15:00:00.0 & 29.7 \\
\hline 2005-08-31 12:00:00.0 & \\
\hline 2005-08-31 09:00:00.0 & 27.0 \\
\hline 2005-08-31 06:00:00.0 & 30.5 \\
\hline 2005-08-31 03:00:00.0 & \\
\hline 2005-08-31 00:00:00.0 & 13.0 \\
\hline 2005-08-30 21:00:00.0 & 20.0 \\
\hline 2005-08-30 18:00:00.0 & 17.6 \\
\hline 2005-08-30 15:00:00.0 & \\
\hline 2005-08-30 12:00:00.0 & 24.7 \\
\hline 2005-08-30 09:00:00.0 & 27.2 \\
\hline 2005-08-30 06:00:00.0 & 24.3 \\
\hline 2005-08-30 03:00:00.0 & \\
\hline 2005-08-30 00:00:00.0 & 22.0 \\
\hline 2005-08-29 21:00:00.0 & 16.5 \\
\hline 2005-08-29 18:00:00.0 & 18.3 \\
\hline 2005-08-29 15:00:00.0 & 41.2 \\
\hline 2005-08-29 12:00:00.0 & \\
\hline 2005-08-29 09:00:00.0 & 21.8 \\
\hline 2005-08-29 06:00:00.0 & \\
\hline 2005-08-29 03:00:00.0 & \\
\hline 2005-08-29 00:00:00.0 & 19.9 \\
\hline 2005-08-28 21:00:00.0 & 21.1 \\
\hline 2005-08-28 18:00:00.0 & 17.5 \\
\hline 2005-08-28 15:00:00.0 & 12.1 \\
\hline 2005-08-28 12:00:00.0 & 23.6 \\
\hline 2005-08-28 09:00:00.0 & 18.5 \\
\hline 2005-08-28 06:00:00.0 & 21.7 \\
\hline 2005-08-28 03:00:00.0 & 22.7 \\
\hline 2005-08-28 00:00:00.0 & 18.4 \\
\hline 2005-08-27 21:00:00.0 & 22.8 \\
\hline 2005-08-27 18:00:00.0 & 20.1 \\
\hline 2005-08-27 15:00:00.0 & \\
\hline 2005-08-27 12:00:00.0 & 17.3 \\
\hline 2005-08-27 09:00:00.0 & 16.7 \\
\hline 2005-08-27 06:00:00.0 & \\
\hline
\end{tabular}

\begin{tabular}{|c|c|}
\hline DataHora & VelVentoMax \\
\hline 2005-08-27 03:00:00.0 & 16.9 \\
\hline 2005-08-27 00:00:00.0 & 12.7 \\
\hline 2005-08-26 21:00:00.0 & 9.5 \\
\hline 2005-08-26 18:00:00.0 & 10.5 \\
\hline 2005-08-26 15:00:00.0 & 13.8 \\
\hline 2005-08-26 12:00:00.0 & \\
\hline 2005-08-26 09:00:00.0 & 14.9 \\
\hline 2005-08-26 06:00:00.0 & \\
\hline 2005-08-26 03:00:00.0 & 14.0 \\
\hline 2005-08-26 00:00:00.0 & 10.7 \\
\hline 2005-08-25 21:00:00.0 & 16.7 \\
\hline 2005-08-25 18:00:00.0 & 12.8 \\
\hline 2005-08-25 15:00:00.0 & 13.1 \\
\hline 2005-08-25 12:00:00.0 & 5.5 \\
\hline 2005-08-25 09:00:00.0 & 6.9 \\
\hline 2005-08-25 06:00:00.0 & \\
\hline 2005-08-25 03:00:00.0 & 18.2 \\
\hline 2005-08-25 00:00:00.0 & 15.4 \\
\hline 2005-08-24 21:00:00.0 & 30.6 \\
\hline 2005-08-24 18:00:00.0 & 33.0 \\
\hline 2005-08-24 15:00:00.0 & 31.4 \\
\hline 2005-08-24 12:00:00.0 & 33.0 \\
\hline 2005-08-24 09:00:00.0 & 22.8 \\
\hline 2005-08-24 06:00:00.0 & 26.2 \\
\hline 2005-08-24 03:00:00.0 & 38.0 \\
\hline 2005-08-24 00:00:00.0 & 26.0 \\
\hline 2005-08-23 21:00:00.0 & 30.0 \\
\hline 2005-08-23 18:00:00.0 & \\
\hline 2005-08-23 15:00:00.0 & 43.0 \\
\hline 2005-08-23 12:00:00.0 & 26.0 \\
\hline 2005-08-23 09:00:00.0 & \\
\hline 2005-08-23 06:00:00.0 & 26.8 \\
\hline 2005-08-23 03:00:00.0 & 16.8 \\
\hline 2005-08-23 00:00:00.0 & 14.9 \\
\hline 2005-08-22 21:00:00.0 & 17.4 \\
\hline 2005-08-22 18:00:00.0 & 22.3 \\
\hline 2005-08-22 15:00:00.0 & 27.0 \\
\hline $2005-08-22$ 12:00:00.0 & 26.8 \\
\hline 2005-08-22 09:00:00.0 & \\
\hline 2005-08-22 06:00:00.0 & \\
\hline 2005-08-22 03:00:00.0 & 28.1 \\
\hline 2005-08-22 00:00:00.0 & 22.5 \\
\hline 2005-08-21 21:00:00.0 & 20.8 \\
\hline 2005-08-21 18:00:00.0 & 13.2 \\
\hline 2005-08-21 15:00:00.0 & 16.8 \\
\hline 2005-08-21 12:00:00.0 & 17.8 \\
\hline 2005-08-21 09:00:00.0 & \\
\hline 2005-08-21 06:00:00.0 & \\
\hline 2005-08-21 03:00:00.0 & 32.5 \\
\hline 2005-08-21 00:00:00.0 & 20.2 \\
\hline 2005-08-20 21:00:00.0 & \\
\hline 2005-08-20 18:00:00.0 & 16.5 \\
\hline 2005-08-20 15:00:00.0 & 20.8 \\
\hline 2005-08-20 12:00:00.0 & 19.7 \\
\hline 2005-08-20 09:00:00.0 & \\
\hline 2005-08-20 06:00:00.0 & \\
\hline 2005-08-20 03:00:00.0 & 20.2 \\
\hline 2005-08-20 00:00:00.0 & 17.8 \\
\hline 2005-08-19 21:00:00.0 & \\
\hline 2005-08-19 18:00:00.0 & 12.3 \\
\hline 2005-08-19 15:00:00.0 & 13.4 \\
\hline 2005-08-19 12:00:00.0 & 12.8 \\
\hline 2005-08-19 09:00:00.0 & \\
\hline 2005-08-19 06:00:00.0 & \\
\hline 2005-08-19 03:00:00.0 & 22.2 \\
\hline 2005-08-19 00:00:00.0 & \\
\hline 2005-08-18 21:00:00.0 & 20.7 \\
\hline 2005-08-18 18:00:00.0 & 21.2 \\
\hline 2005-08-18 15:00:00.0 & 17.4 \\
\hline
\end{tabular}

\begin{tabular}{|c|c|}
\hline DataHora & VelVentoMax \\
\hline $2005-08-18$ 12:00:00.0 & 17.3 \\
\hline 2005-08-18 09:00:00.0 & \\
\hline 2005-08-18 06:00:00.0 & 16.4 \\
\hline 2005-08-18 03:00:00.0 & 10.7 \\
\hline 2005-08-18 00:00:00.0 & 24.8 \\
\hline 2005-08-17 21:00:00.0 & \\
\hline 2005-08-17 18:00:00.0 & 43.4 \\
\hline 2005-08-17 15:00:00.0 & 33.4 \\
\hline 2005-08-17 12:00:00.0 & \\
\hline 2005-08-17 09:00:00.0 & \\
\hline $2005-08-17$ 06:00:00.0 & 18.0 \\
\hline 2005-08-17 03:00:00.0 & 19.7 \\
\hline $2005-08-17$ 00:00:00.0 & 19.1 \\
\hline 2005-08-16 21:00:00.0 & 22.2 \\
\hline 2005-08-16 18:00:00.0 & 26.1 \\
\hline $2005-08-16$ 15:00:00.0 & 23.3 \\
\hline 2005-08-16 12:00:00.0 & \\
\hline 2005-08-16 09:00:00.0 & \\
\hline 2005-08-16 06:00:00.0 & 23.4 \\
\hline 2005-08-16 03:00:00.0 & 19.0 \\
\hline 2005-08-16 00:00:00.0 & 17.5 \\
\hline $2005-08-15$ 21:00:00.0 & 13.4 \\
\hline $2005-08-15$ 18:00:00.0 & 13.1 \\
\hline 2005-08-15 15:00:00.0 & 11.4 \\
\hline 2005-08-15 12:00:00.0 & \\
\hline 2005-08-15 09:00:00.0 & \\
\hline 2005-08-15 06:00:00.0 & 14.2 \\
\hline 2005-08-15 03:00:00.0 & 11.6 \\
\hline 2005-08-15 00:00:00.0 & \\
\hline 2005-08-14 21:00:00.0 & 14.2 \\
\hline 2005-08-14 18:00:00.0 & 20.2 \\
\hline 2005-08-14 15:00:00.0 & 17.1 \\
\hline 2005-08-14 12:00:00.0 & \\
\hline 2005-08-14 09:00:00.0 & 16.0 \\
\hline $2005-08-14$ 06:00:00.0 & 15.0 \\
\hline 2005-08-14 03:00:00.0 & 16.5 \\
\hline 2005-08-14 00:00:00.0 & \\
\hline $2005-08-13$ 21:00:00.0 & 14.9 \\
\hline 2005-08-13 18:00:00.0 & 22.7 \\
\hline 2005-08-13 15:00:00.0 & 18.8 \\
\hline 2005-08-13 12:00:00.0 & \\
\hline 2005-08-13 09:00:00.0 & 15.5 \\
\hline 2005-08-13 06:00:00.0 & 17.6 \\
\hline 2005-08-13 03:00:00.0 & 14.9 \\
\hline 2005-08-13 00:00:00.0 & 15.0 \\
\hline 2005-08-12 21:00:00.0 & 28.5 \\
\hline 2005-08-12 18:00:00.0 & 13.6 \\
\hline 2005-08-12 15:00:00.0 & 14.5 \\
\hline $2005-08-12$ 12:00:00.0 & \\
\hline 2005-08-12 09:00:00.0 & 12.6 \\
\hline 2005-08-12 06:00:00.0 & 11.7 \\
\hline 2005-08-12 03:00:00.0 & 9.6 \\
\hline 2005-08-12 00:00:00.0 & 8.6 \\
\hline $2005-08-11$ 21:00:00.0 & 12.4 \\
\hline 2005-08-11 18:00:00.0 & \\
\hline 2005-08-11 15:00:00.0 & \\
\hline 2005-08-11 12:00:00.0 & \\
\hline 2005-08-11 09:00:00.0 & 14.9 \\
\hline 2005-08-11 06:00:00.0 & 15.3 \\
\hline 2005-08-11 03:00:00.0 & 13.1 \\
\hline 2005-08-11 00:00:00.0 & 9.6 \\
\hline $2005-08-10$ 21:00:00.0 & 20.8 \\
\hline 2005-08-10 18:00:00.0 & 25.4 \\
\hline $2005-08-10$ 15:00:00.0 & 27.1 \\
\hline 2005-08-10 12:00:00.0 & 25.0 \\
\hline 2005-08-10 09:00:00.0 & \\
\hline 2005-08-10 06:00:00.0 & 19.3 \\
\hline 2005-08-10 03:00:00.0 & 21.0 \\
\hline 2005-08-10 00:00:00.0 & 25.8 \\
\hline
\end{tabular}




\section{ANEXO 01 - RAJADAS DE VENTO REGISTRADAS EM SÃO MARTINHO DA SERRA / RS}

\begin{tabular}{|c|c|}
\hline DataHora & VelVentoMax \\
\hline 2005-08-09 21:00:00.0 & 32.2 \\
\hline 2005-08-09 18:00:00.0 & 35.5 \\
\hline 2005-08-09 15:00:00.0 & 38.4 \\
\hline 2005-08-09 12:00:00.0 & 33.4 \\
\hline 2005-08-09 09:00:00.0 & 31.3 \\
\hline 2005-08-09 06:00:00.0 & 26.7 \\
\hline 2005-08-09 03:00:00.0 & \\
\hline 2005-08-09 00:00:00.0 & \\
\hline 2005-08-08 21:00:00.0 & 21.3 \\
\hline 2005-08-08 18:00:00.0 & 20.9 \\
\hline 2005-08-08 15:00:00.0 & 19.0 \\
\hline 2005-08-08 12:00:00.0 & 14.6 \\
\hline 2005-08-08 09:00:00.0 & 20.8 \\
\hline 2005-08-08 06:00:00.0 & 19.4 \\
\hline 2005-08-08 03:00:00.0 & 14.6 \\
\hline 2005-08-08 00:00:00.0 & 17.2 \\
\hline 2005-08-07 21:00:00.0 & 19.1 \\
\hline 2005-08-07 18:00:00.0 & 28.2 \\
\hline 2005-08-07 15:00:00.0 & \\
\hline 2005-08-07 12:00:00.0 & 27.4 \\
\hline 2005-08-07 09:00:00.0 & 24.0 \\
\hline 2005-08-07 06:00:00.0 & 24.8 \\
\hline 2005-08-07 03:00:00.0 & 27.6 \\
\hline 2005-08-07 00:00:00.0 & 26.2 \\
\hline 2005-08-06 21:00:00.0 & 25.0 \\
\hline 2005-08-06 18:00:00.0 & 19.7 \\
\hline 2005-08-06 15:00:00.0 & \\
\hline 2005-08-06 12:00:00.0 & 20.7 \\
\hline 2005-08-06 09:00:00.0 & 15.7 \\
\hline 2005-08-06 06:00:00.0 & 15.9 \\
\hline 2005-08-06 03:00:00.0 & \\
\hline 2005-08-06 00:00:00.0 & \\
\hline 2005-08-05 21:00:00.0 & 29.6 \\
\hline 2005-08-05 18:00:00.0 & 33.6 \\
\hline 2005-08-05 15:00:00.0 & 27.2 \\
\hline 2005-08-05 12:00:00.0 & 22.1 \\
\hline 2005-08-05 09:00:00.0 & 19.2 \\
\hline 2005-08-05 06:00:00.0 & 19.2 \\
\hline 2005-08-05 03:00:00.0 & 17.5 \\
\hline 2005-08-05 00:00:00.0 & 16.6 \\
\hline 2005-08-04 21:00:00.0 & 21.8 \\
\hline 2005-08-04 18:00:00.0 & 26.3 \\
\hline 2005-08-04 15:00:00.0 & 24.6 \\
\hline 2005-08-04 12:00:00.0 & 20.5 \\
\hline 2005-08-04 09:00:00.0 & 17.7 \\
\hline 2005-08-04 06:00:00.0 & 16.7 \\
\hline 2005-08-04 03:00:00.0 & \\
\hline 2005-08-04 00:00:00.0 & 13.3 \\
\hline 2005-08-03 21:00:00.0 & 20.4 \\
\hline 2005-08-03 18:00:00.0 & 22.6 \\
\hline 2005-08-03 15:00:00.0 & 21.5 \\
\hline 2005-08-03 12:00:00.0 & 18.0 \\
\hline 2005-08-03 09:00:00.0 & 19.2 \\
\hline 2005-08-03 06:00:00.0 & 18.9 \\
\hline 2005-08-03 03:00:00.0 & \\
\hline 2005-08-03 00:00:00.0 & \\
\hline 2005-08-02 21:00:00.0 & 20.7 \\
\hline 2005-08-02 18:00:00.0 & 22.8 \\
\hline 2005-08-02 15:00:00.0 & \\
\hline 2005-08-02 12:00:00.0 & 16.0 \\
\hline 2005-08-02 09:00:00.0 & 18.3 \\
\hline 2005-08-02 06:00:00.0 & 16.2 \\
\hline 2005-08-02 03:00:00.0 & \\
\hline 2005-08-02 00:00:00.0 & 13.6 \\
\hline 2005-08-01 21:00:00.0 & 19.3 \\
\hline 2005-08-01 18:00:00.0 & 23.4 \\
\hline 2005-08-01 15:00:00.0 & 20.3 \\
\hline 2005-08-01 12:00:00.0 & 19.4 \\
\hline 2005-08-01 09:00:00.0 & 17.1 \\
\hline
\end{tabular}

\begin{tabular}{|c|c|}
\hline DataHora & VelVentoMax \\
\hline 2005-08-01 06:00:00.0 & 16.5 \\
\hline 2005-08-01 03:00:00.0 & 16.2 \\
\hline 2005-08-01 00:00:00.0 & 15.5 \\
\hline 2005-07-31 21:00:00.0 & 24.7 \\
\hline 2005-07-31 18:00:00.0 & \\
\hline 2005-07-31 15:00:00.0 & 27.2 \\
\hline 2005-07-31 12:00:00.0 & 25.5 \\
\hline 2005-07-31 09:00:00.0 & 30.4 \\
\hline 2005-07-31 06:00:00.0 & \\
\hline 2005-07-31 03:00:00.0 & 24.5 \\
\hline 2005-07-31 00:00:00.0 & 18.4 \\
\hline 2005-07-30 21:00:00.0 & 27.2 \\
\hline 2005-07-30 18:00:00.0 & 32.1 \\
\hline 2005-07-30 15:00:00.0 & 33.3 \\
\hline 2005-07-30 12:00:00.0 & 23.8 \\
\hline 2005-07-30 09:00:00.0 & 24.5 \\
\hline 2005-07-30 06:00:00.0 & 21.8 \\
\hline 2005-07-30 03:00:00.0 & \\
\hline 2005-07-30 00:00:00.0 & \\
\hline 2005-07-29 21:00:00.0 & 20.3 \\
\hline 2005-07-29 18:00:00.0 & 19.6 \\
\hline 2005-07-29 15:00:00.0 & 21.0 \\
\hline 2005-07-29 12:00:00.0 & 17.9 \\
\hline 2005-07-29 09:00:00.0 & 16.8 \\
\hline 2005-07-29 06:00:00.0 & \\
\hline 2005-07-29 03:00:00.0 & 17.9 \\
\hline 2005-07-29 00:00:00.0 & 17.1 \\
\hline 2005-07-28 21:00:00.0 & 7.8 \\
\hline 2005-07-28 18:00:00.0 & \\
\hline 2005-07-28 15:00:00.0 & 9.7 \\
\hline 2005-07-28 12:00:00.0 & \\
\hline 2005-07-28 09:00:00.0 & 17.2 \\
\hline 2005-07-28 06:00:00.0 & 14.4 \\
\hline 2005-07-28 03:00:00.0 & 16.6 \\
\hline 2005-07-28 00:00:00.0 & 14.5 \\
\hline 2005-07-27 21:00:00.0 & 11.1 \\
\hline 2005-07-27 18:00:00.0 & \\
\hline 2005-07-27 15:00:00.0 & 21.4 \\
\hline 2005-07-27 12:00:00.0 & 20.4 \\
\hline 2005-07-27 09:00:00.0 & \\
\hline 2005-07-27 06:00:00.0 & \\
\hline 2005-07-27 03:00:00.0 & 10.3 \\
\hline 2005-07-27 00:00:00.0 & \\
\hline 2005-07-26 21:00:00.0 & 7.7 \\
\hline 2005-07-26 18:00:00.0 & 8.8 \\
\hline 2005-07-26 15:00:00.0 & 8.8 \\
\hline 2005-07-26 12:00:00.0 & 5.7 \\
\hline 2005-07-26 09:00:00.0 & \\
\hline 2005-07-26 06:00:00.0 & 12.5 \\
\hline 2005-07-26 03:00:00.0 & 15.7 \\
\hline 2005-07-26 00:00:00.0 & 18.3 \\
\hline 2005-07-25 21:00:00.0 & \\
\hline 2005-07-25 18:00:00.0 & 26.2 \\
\hline 2005-07-25 15:00:00.0 & 25.5 \\
\hline 2005-07-25 12:00:00.0 & 16.2 \\
\hline 2005-07-25 09:00:00.0 & \\
\hline 2005-07-25 06:00:00.0 & 16.6 \\
\hline 2005-07-25 03:00:00.0 & 16.8 \\
\hline 2005-07-25 00:00:00.0 & \\
\hline 2005-07-24 21:00:00.0 & 24.2 \\
\hline 2005-07-24 18:00:00.0 & 27.2 \\
\hline 2005-07-24 15:00:00.0 & 22.0 \\
\hline 2005-07-24 12:00:00.0 & 9.9 \\
\hline 2005-07-24 09:00:00.0 & \\
\hline 2005-07-24 06:00:00.0 & 9.2 \\
\hline 2005-07-24 03:00:00.0 & 10.6 \\
\hline 2005-07-24 00:00:00.0 & \\
\hline 2005-07-23 21:00:00.0 & 11.7 \\
\hline 2005-07-23 18:00:00.0 & 27.1 \\
\hline
\end{tabular}

\begin{tabular}{|c|c|}
\hline DataHora & VelVentoMax \\
\hline 2005-07-23 15:00:00.0 & \\
\hline 2005-07-23 12:00:00.0 & \\
\hline 2005-07-23 09:00:00.0 & \\
\hline 2005-07-23 03:00:00.0 & 9.4 \\
\hline 2005-07-23 00:00:00.0 & 14.2 \\
\hline 2005-07-22 21:00:00.0 & \\
\hline 2005-07-22 18:00:00.0 & 17.6 \\
\hline $2005-07-22$ 15:00:00.0 & 19.4 \\
\hline 2005-07-22 12:00:00.0 & 15.9 \\
\hline 2005-07-22 09:00:00.0 & \\
\hline 2005-07-22 06:00:00.0 & 10.5 \\
\hline 2005-07-22 03:00:00.0 & 10.4 \\
\hline 2005-07-22 00:00:00.0 & \\
\hline 2005-07-21 21:00:00.0 & \\
\hline 2005-07-21 18:00:00.0 & 24.3 \\
\hline 2005-07-21 15:00:00.0 & 23.4 \\
\hline 2005-07-21 12:00:00.0 & 21.6 \\
\hline 2005-07-21 09:00:00.0 & 21.5 \\
\hline 2005-07-21 06:00:00.0 & 22.5 \\
\hline 2005-07-21 03:00:00.0 & \\
\hline 2005-07-21 00:00:00.0 & \\
\hline 2005-07-20 21:00:00.0 & 11.9 \\
\hline 2005-07-20 18:00:00.0 & 16.9 \\
\hline 2005-07-20 15:00:00.0 & 21.6 \\
\hline 2005-07-20 12:00:00.0 & 21.6 \\
\hline 2005-07-20 09:00:00.0 & \\
\hline 2005-07-20 06:00:00.0 & 18.6 \\
\hline 2005-07-20 03:00:00.0 & 12.3 \\
\hline 2005-07-20 00:00:00.0 & 9.4 \\
\hline 2005-07-19 21:00:00.0 & 8.4 \\
\hline 2005-07-19 18:00:00.0 & 8.4 \\
\hline 2005-07-19 15:00:00.0 & 12.7 \\
\hline 2005-07-19 12:00:00.0 & \\
\hline 2005-07-19 09:00:00.0 & 3.3 \\
\hline 2005-07-19 06:00:00.0 & 7.0 \\
\hline 2005-07-19 03:00:00.0 & 8.6 \\
\hline 2005-07-19 00:00:00.0 & 13.5 \\
\hline 2005-07-18 21:00:00.0 & 21.9 \\
\hline 2005-07-18 18:00:00.0 & 24.6 \\
\hline 2005-07-18 15:00:00.0 & 17.0 \\
\hline 2005-07-18 12:00:00.0 & \\
\hline 2005-07-18 09:00:00.0 & 9.1 \\
\hline 2005-07-18 06:00:00.0 & 9.7 \\
\hline 2005-07-18 03:00:00.0 & 12.0 \\
\hline 2005-07-18 00:00:00.0 & \\
\hline 2005-07-17 21:00:00.0 & 19.7 \\
\hline 2005-07-17 18:00:00.0 & 19.0 \\
\hline 2005-07-17 15:00:00.0 & 19.2 \\
\hline 2005-07-17 12:00:00.0 & \\
\hline 2005-07-17 09:00:00.0 & 29.3 \\
\hline 2005-07-17 06:00:00.0 & 23.1 \\
\hline 2005-07-17 03:00:00.0 & 21.5 \\
\hline 2005-07-17 00:00:00.0 & 24.2 \\
\hline 2005-07-16 21:00:00.0 & 24.8 \\
\hline 2005-07-16 18:00:00.0 & 28.8 \\
\hline 2005-07-16 15:00:00.0 & 25.4 \\
\hline 2005-07-16 12:00:00.0 & 23.3 \\
\hline 2005-07-16 09:00:00.0 & 19.7 \\
\hline 2005-07-16 06:00:00.0 & 15.1 \\
\hline 2005-07-16 03:00:00.0 & 21.5 \\
\hline 2005-07-16 00:00:00.0 & \\
\hline 2005-07-15 21:00:00.0 & 23.4 \\
\hline 2005-07-15 18:00:00.0 & 20.5 \\
\hline 2005-07-15 15:00:00.0 & 25.3 \\
\hline 2005-07-15 12:00:00.0 & 23.3 \\
\hline 2005-07-15 09:00:00.0 & 25.1 \\
\hline 2005-07-15 06:00:00.0 & 21.2 \\
\hline 2005-07-15 03:00:00.0 & 20.4 \\
\hline 2005-07-15 00:00:00.0 & \\
\hline
\end{tabular}




\section{ANEXO 01 - RAJADAS DE VENTO REGISTRADAS EM SÃO MARTINHO DA SERRA / RS}

\begin{tabular}{|c|c|}
\hline DataHora & VelVentoMax \\
\hline 2005-07-14 21:00:00.0 & 22.9 \\
\hline 2005-07-14 18:00:00.0 & 23.4 \\
\hline 2005-07-14 15:00:00.0 & 22.7 \\
\hline 2005-07-14 12:00:00.0 & \\
\hline 2005-07-14 09:00:00.0 & \\
\hline 2005-07-14 06:00:00.0 & 21.7 \\
\hline 2005-07-14 03:00:00.0 & \\
\hline 2005-07-14 00:00:00.0 & 16.2 \\
\hline 2005-07-13 21:00:00.0 & 20.6 \\
\hline 2005-07-13 18:00:00.0 & 21.2 \\
\hline 2005-07-13 15:00:00.0 & \\
\hline 2005-07-13 12:00:00.0 & \\
\hline 2005-07-13 09:00:00.0 & 21.7 \\
\hline 2005-07-13 06:00:00.0 & 20.3 \\
\hline 2005-07-13 03:00:00.0 & 16.2 \\
\hline 2005-07-13 00:00:00.0 & \\
\hline 2005-07-12 21:00:00.0 & \\
\hline 2005-07-12 18:00:00.0 & \\
\hline 2005-07-12 09:00:00.0 & 19.1 \\
\hline 2005-07-12 06:00:00.0 & 14.6 \\
\hline 2005-07-12 03:00:00.0 & \\
\hline 2005-07-12 00:00:00.0 & \\
\hline 2005-07-11 21:00:00.0 & \\
\hline 2005-07-11 12:00:00.0 & 15.1 \\
\hline 2005-07-11 09:00:00.0 & 13.5 \\
\hline 2005-07-11 06:00:00.0 & 13.7 \\
\hline 2005-07-11 03:00:00.0 & 14.1 \\
\hline 2005-07-11 00:00:00.0 & 13.4 \\
\hline 2005-07-10 21:00:00.0 & 15.7 \\
\hline 2005-07-10 18:00:00.0 & 22.0 \\
\hline 2005-07-10 15:00:00.0 & 24.3 \\
\hline 2005-07-10 12:00:00.0 & \\
\hline 2005-07-10 09:00:00.0 & 18.3 \\
\hline 2005-07-10 06:00:00.0 & 18.3 \\
\hline 2005-07-10 03:00:00.0 & \\
\hline 2005-07-10 00:00:00.0 & 8.8 \\
\hline 2005-07-09 21:00:00.0 & 19.5 \\
\hline 2005-07-09 18:00:00.0 & \\
\hline 2005-07-09 15:00:00.0 & \\
\hline 2005-07-09 12:00:00.0 & 17.2 \\
\hline 2005-07-09 09:00:00.0 & 17.8 \\
\hline 2005-07-09 06:00:00.0 & 18.7 \\
\hline 2005-07-09 03:00:00.0 & 13.6 \\
\hline 2005-07-09 00:00:00.0 & 11.4 \\
\hline 2005-07-08 21:00:00.0 & 22.3 \\
\hline 2005-07-08 18:00:00.0 & 33.7 \\
\hline 2005-07-08 15:00:00.0 & \\
\hline 2005-07-08 12:00:00.0 & 20.6 \\
\hline 2005-07-08 09:00:00.0 & 19.0 \\
\hline 2005-07-08 06:00:00.0 & 18.8 \\
\hline 2005-07-08 03:00:00.0 & \\
\hline 2005-07-08 00:00:00.0 & 19.1 \\
\hline 2005-07-07 21:00:00.0 & 22.1 \\
\hline 2005-07-07 18:00:00.0 & 27.6 \\
\hline 2005-07-07 15:00:00.0 & \\
\hline 2005-07-07 12:00:00.0 & 21.3 \\
\hline 2005-07-07 09:00:00.0 & 18.9 \\
\hline 2005-07-07 06:00:00.0 & \\
\hline 2005-07-07 03:00:00.0 & 10.6 \\
\hline 2005-07-07 00:00:00.0 & \\
\hline 2005-07-06 21:00:00.0 & 17.0 \\
\hline 2005-07-06 18:00:00.0 & 18.0 \\
\hline 2005-07-06 15:00:00.0 & \\
\hline 2005-07-06 12:00:00.0 & \\
\hline 2005-07-06 09:00:00.0 & 13.3 \\
\hline 2005-07-06 06:00:00.0 & \\
\hline 2005-07-06 03:00:00.0 & 15.3 \\
\hline 2005-07-06 00:00:00.0 & 15.4 \\
\hline 2005-07-05 21:00:00.0 & 22.2 \\
\hline
\end{tabular}

\begin{tabular}{|c|c|}
\hline DataHora & VelVentoMax \\
\hline 2005-07-05 18:00:00.0 & \\
\hline 2005-07-05 15:00:00.0 & 18.0 \\
\hline 2005-07-05 12:00:00.0 & \\
\hline 2005-07-05 09:00:00.0 & 20.8 \\
\hline 2005-07-05 06:00:00.0 & \\
\hline 2005-07-05 03:00:00.0 & 23.6 \\
\hline 2005-07-05 00:00:00.0 & 25.5 \\
\hline 2005-07-04 21:00:00.0 & 29.2 \\
\hline 2005-07-04 18:00:00.0 & 33.0 \\
\hline 2005-07-04 15:00:00.0 & 31.7 \\
\hline 2005-07-04 12:00:00.0 & 25.7 \\
\hline 2005-07-04 09:00:00.0 & 18.9 \\
\hline 2005-07-04 06:00:00.0 & \\
\hline 2005-07-04 03:00:00.0 & \\
\hline 2005-07-04 00:00:00.0 & \\
\hline 2005-07-03 15:00:00.0 & 24.0 \\
\hline 2005-07-03 12:00:00.0 & 21.2 \\
\hline 2005-07-03 09:00:00.0 & 18.7 \\
\hline 2005-07-03 06:00:00.0 & \\
\hline 2005-07-03 03:00:00.0 & \\
\hline 2005-07-03 00:00:00.0 & \\
\hline 2005-07-02 15:00:00.0 & 30.0 \\
\hline 2005-07-02 12:00:00.0 & 25.3 \\
\hline 2005-07-02 09:00:00.0 & 17.5 \\
\hline 2005-07-02 06:00:00.0 & \\
\hline 2005-07-02 03:00:00.0 & 14.8 \\
\hline 2005-07-02 00:00:00.0 & 14.1 \\
\hline 2005-07-01 21:00:00.0 & \\
\hline 2005-07-01 18:00:00.0 & \\
\hline 2005-07-01 15:00:00.0 & 19.0 \\
\hline 2005-07-01 12:00:00.0 & 20.3 \\
\hline 2005-07-01 09:00:00.0 & \\
\hline 2005-07-01 06:00:00.0 & \\
\hline 2005-07-01 03:00:00.0 & 18.6 \\
\hline 2005-07-01 00:00:00.0 & 13.6 \\
\hline 2005-06-30 21:00:00.0 & 10.3 \\
\hline 2005-06-30 18:00:00.0 & \\
\hline 2005-06-30 15:00:00.0 & 9.6 \\
\hline 2005-06-30 12:00:00.0 & 9.4 \\
\hline 2005-06-30 09:00:00.0 & \\
\hline 2005-06-30 06:00:00.0 & \\
\hline 2005-06-30 03:00:00.0 & 5.1 \\
\hline 2005-06-30 00:00:00.0 & 6.7 \\
\hline 2005-06-29 21:00:00.0 & 10.2 \\
\hline 2005-06-29 18:00:00.0 & 11.1 \\
\hline 2005-06-29 15:00:00.0 & 18.4 \\
\hline 2005-06-29 12:00:00.0 & 17.6 \\
\hline 2005-06-29 09:00:00.0 & \\
\hline 2005-06-29 06:00:00.0 & \\
\hline 2005-06-29 03:00:00.0 & 13.7 \\
\hline 2005-06-29 00:00:00.0 & 15.6 \\
\hline 2005-06-28 21:00:00.0 & 11.0 \\
\hline 2005-06-28 18:00:00.0 & 15.9 \\
\hline 2005-06-28 15:00:00.0 & 17.2 \\
\hline 2005-06-28 12:00:00.0 & 17.6 \\
\hline 2005-06-28 09:00:00.0 & \\
\hline 2005-06-28 06:00:00.0 & 22.5 \\
\hline 2005-06-28 03:00:00.0 & 26.8 \\
\hline 2005-06-28 00:00:00.0 & \\
\hline 2005-06-27 21:00:00.0 & \\
\hline 2005-06-27 18:00:00.0 & 39.1 \\
\hline 2005-06-27 15:00:00.0 & 24.1 \\
\hline 2005-06-27 12:00:00.0 & 16.4 \\
\hline 2005-06-27 09:00:00.0 & \\
\hline 2005-06-27 06:00:00.0 & \\
\hline 2005-06-27 03:00:00.0 & 12.4 \\
\hline 2005-06-27 00:00:00.0 & \\
\hline 2005-06-26 21:00:00.0 & \\
\hline 2005-06-26 18:00:00.0 & 16.5 \\
\hline
\end{tabular}

\begin{tabular}{|c|c|}
\hline DataHora & VelVentoMax \\
\hline $2005-06-26$ 15:00:00.0 & 17.0 \\
\hline 2005-06-26 12:00:00.0 & \\
\hline 2005-06-26 09:00:00.0 & \\
\hline 2005-06-26 06:00:00.0 & 22.4 \\
\hline 2005-06-26 03:00:00.0 & 18.3 \\
\hline 2005-06-26 00:00:00.0 & 14.4 \\
\hline 2005-06-25 21:00:00.0 & \\
\hline 2005-06-25 18:00:00.0 & 8.1 \\
\hline 2005-06-25 15:00:00.0 & 14.7 \\
\hline $2005-06-25$ 12:00:00.0 & \\
\hline $2005-06-25$ 09:00:00.0 & \\
\hline $2005-06-25$ 06:00:00.0 & \\
\hline 2005-06-24 18:00:00.0 & 14.5 \\
\hline 2005-06-24 15:00:00.0 & 14.6 \\
\hline 2005-06-24 12:00:00.0 & \\
\hline $2005-06-24$ 09:00:00.0 & \\
\hline 2005-06-24 06:00:00.0 & 12.2 \\
\hline 2005-06-24 03:00:00.0 & 11.7 \\
\hline 2005-06-24 00:00:00.0 & 8.9 \\
\hline 2005-06-23 21:00:00.0 & 10.9 \\
\hline 2005-06-23 18:00:00.0 & 18.6 \\
\hline $2005-06-23$ 15:00:00.0 & 10.9 \\
\hline $2005-06-23$ 12:00:00.0 & \\
\hline 2005-06-23 09:00:00.0 & \\
\hline $2005-06-23$ 06:00:00.0 & 12.2 \\
\hline 2005-06-23 03:00:00.0 & 10.5 \\
\hline 2005-06-23 00:00:00.0 & 12.2 \\
\hline 2005-06-22 21:00:00.0 & 9.5 \\
\hline 2005-06-22 18:00:00.0 & 14.3 \\
\hline 2005-06-22 15:00:00.0 & 21.5 \\
\hline 2005-06-22 12:00:00.0 & \\
\hline 2005-06-22 09:00:00.0 & \\
\hline 2005-06-22 06:00:00.0 & 16.1 \\
\hline 2005-06-22 03:00:00.0 & \\
\hline $2005-06-22$ 00:00:00.0 & 18.6 \\
\hline 2005-06-21 21:00:00.0 & 15.9 \\
\hline 2005-06-21 18:00:00.0 & 17.5 \\
\hline $2005-06-21$ 15:00:00.0 & 18.5 \\
\hline 2005-06-21 12:00:00.0 & 16.6 \\
\hline 2005-06-21 09:00:00.0 & 12.7 \\
\hline 2005-06-21 06:00:00.0 & 9.2 \\
\hline 2005-06-21 03:00:00.0 & 9.6 \\
\hline 2005-06-21 00:00:00.0 & \\
\hline 2005-06-20 21:00:00.0 & 19.1 \\
\hline 2005-06-20 18:00:00.0 & 17.0 \\
\hline 2005-06-20 15:00:00.0 & 19.2 \\
\hline 2005-06-20 12:00:00.0 & 6.8 \\
\hline 2005-06-20 09:00:00.0 & \\
\hline $2005-06-20$ 06:00:00.0 & 10.1 \\
\hline 2005-06-20 03:00:00.0 & 23.2 \\
\hline $2005-06-2000: 00: 00.0$ & 17.1 \\
\hline 2005-06-19 21:00:00.0 & 15.1 \\
\hline 2005-06-19 18:00:00.0 & 18.3 \\
\hline $2005-06-19$ 15:00:00.0 & 14.5 \\
\hline 2005-06-19 12:00:00.0 & \\
\hline 2005-06-19 09:00:00.0 & 10.1 \\
\hline 2005-06-19 06:00:00.0 & \\
\hline 2005-06-19 03:00:00.0 & \\
\hline 2005-06-19 00:00:00.0 & 14.7 \\
\hline 2005-06-18 21:00:00.0 & 12.7 \\
\hline 2005-06-18 18:00:00.0 & 11.7 \\
\hline $2005-06-18$ 15:00:00.0 & \\
\hline 2005-06-18 12:00:00.0 & 10.8 \\
\hline 2005-06-18 09:00:00.0 & 10.0 \\
\hline 2005-06-18 06:00:00.0 & 10.9 \\
\hline 2005-06-18 03:00:00.0 & 8.7 \\
\hline 2005-06-18 00:00:00.0 & 11.2 \\
\hline 2005-06-17 21:00:00.0 & \\
\hline 2005-06-17 18:00:00.0 & 14.2 \\
\hline
\end{tabular}




\section{ANEXO 01 - RAJADAS DE VENTO REGISTRADAS EM SÃO MARTINHO DA SERRA / RS}

\begin{tabular}{|c|c|}
\hline \multirow{2}{*}{$\begin{array}{l}\text { DataHora } \\
\text { 2005-06-17 15:00:00.0 }\end{array}$} & VelVentoMax \\
\hline & \\
\hline 2005-06-17 12:00:00.0 & 13.6 \\
\hline 2005-06-17 09:00:00.0 & 13.8 \\
\hline 2005-06-17 06:00:00.0 & 13.3 \\
\hline 2005-06-17 03:00:00.0 & 11.5 \\
\hline 2005-06-17 00:00:00.0 & 7.5 \\
\hline 2005-06-16 21:00:00.0 & 6.3 \\
\hline 2005-06-16 18:00:00.0 & 27.1 \\
\hline 2005-06-16 15:00:00.0 & \\
\hline 2005-06-16 12:00:00.0 & \\
\hline 2005-06-16 09:00:00.0 & 18.2 \\
\hline 2005-06-16 06:00:00.0 & \\
\hline 2005-06-16 03:00:00.0 & 24.1 \\
\hline 2005-06-16 00:00:00.0 & 30.1 \\
\hline 2005-06-15 21:00:00.0 & 36.7 \\
\hline 2005-06-15 18:00:00.0 & 27.0 \\
\hline 2005-06-15 15:00:00.0 & \\
\hline 2005-06-15 12:00:00.0 & \\
\hline 2005-06-15 09:00:00.0 & 22.6 \\
\hline 2005-06-15 06:00:00.0 & 24.4 \\
\hline 2005-06-15 03:00:00.0 & \\
\hline 2005-06-15 00:00:00.0 & 19.5 \\
\hline 2005-06-14 21:00:00.0 & 20.3 \\
\hline 2005-06-14 18:00:00.0 & \\
\hline 2005-06-14 15:00:00.0 & 22.3 \\
\hline 2005-06-14 12:00:00.0 & 22.2 \\
\hline 2005-06-14 09:00:00.0 & 17.6 \\
\hline 2005-06-14 06:00:00.0 & 14.3 \\
\hline 2005-06-14 03:00:00.0 & 15.4 \\
\hline 2005-06-14 00:00:00.0 & 38.9 \\
\hline 2005-06-13 21:00:00.0 & 28.2 \\
\hline 2005-06-13 18:00:00.0 & 19.1 \\
\hline 2005-06-13 15:00:00.0 & \\
\hline 2005-06-13 12:00:00.0 & 14.1 \\
\hline 2005-06-13 09:00:00.0 & 12.3 \\
\hline 2005-06-13 06:00:00.0 & 34.6 \\
\hline 2005-06-13 03:00:00.0 & \\
\hline 2005-06-13 00:00:00.0 & 19.0 \\
\hline 2005-06-12 21:00:00.0 & 13.3 \\
\hline 2005-06-12 18:00:00.0 & 19.2 \\
\hline 2005-06-12 15:00:00.0 & \\
\hline 2005-06-12 12:00:00.0 & 19.6 \\
\hline 2005-06-12 09:00:00.0 & 17.6 \\
\hline 2005-06-12 06:00:00.0 & 18.9 \\
\hline 2005-06-12 03:00:00.0 & 17.3 \\
\hline 2005-06-12 00:00:00.0 & 22.7 \\
\hline 2005-06-11 21:00:00.0 & 32.0 \\
\hline 2005-06-11 18:00:00.0 & 25.8 \\
\hline 2005-06-11 15:00:00.0 & \\
\hline 2005-06-11 12:00:00.0 & 20.4 \\
\hline 2005-06-11 09:00:00.0 & 20.0 \\
\hline 2005-06-11 06:00:00.0 & \\
\hline 2005-06-11 03:00:00.0 & 20.5 \\
\hline 2005-06-11 00:00:00.0 & 18.6 \\
\hline 2005-06-10 21:00:00.0 & 17.0 \\
\hline 2005-06-10 18:00:00.0 & 22.4 \\
\hline 2005-06-10 15:00:00.0 & 19.0 \\
\hline 2005-06-10 12:00:00.0 & 15.5 \\
\hline 2005-06-10 09:00:00.0 & 19.3 \\
\hline 2005-06-10 06:00:00.0 & 15.9 \\
\hline 2005-06-10 03:00:00.0 & 16.2 \\
\hline 2005-06-10 00:00:00.0 & 13.5 \\
\hline 2005-06-09 21:00:00.0 & 16.1 \\
\hline 2005-06-09 18:00:00.0 & 21.3 \\
\hline 2005-06-09 15:00:00.0 & 18.0 \\
\hline 2005-06-09 12:00:00.0 & 17.4 \\
\hline 2005-06-09 09:00:00.0 & 12.7 \\
\hline 2005-06-09 06:00:00.0 & \\
\hline 2005-06-09 03:00:00.0 & 10.9 \\
\hline
\end{tabular}

\begin{tabular}{|c|c|}
\hline DataHora & VelVentoMax \\
\hline 2005-06-09 00:00:00.0 & 6.4 \\
\hline \multicolumn{2}{|l|}{ 2005-06-08 21:00:00.0 } \\
\hline 2005-06-08 18:00:00.0 & 19.0 \\
\hline 2005-06-08 15:00:00.0 & 25.1 \\
\hline 2005-06-08 12:00:00.0 & 20.6 \\
\hline 2005-06-08 09:00:00.0 & 18.0 \\
\hline 2005-06-08 06:00:00.0 & 17.7 \\
\hline 2005-06-08 03:00:00.0 & 17.0 \\
\hline 2005-06-08 00:00:00.0 & 16.0 \\
\hline 2005-06-07 21:00:00.0 & 20.1 \\
\hline \multicolumn{2}{|l|}{ 2005-06-07 18:00:00.0 } \\
\hline 2005-06-07 15:00:00.0 & 20.8 \\
\hline 2005-06-07 12:00:00.0 & 17.8 \\
\hline 2005-06-07 09:00:00.0 & 16.2 \\
\hline \multicolumn{2}{|l|}{ 2005-06-07 06:00:00.0 } \\
\hline 2005-06-07 03:00:00.0 & 12.7 \\
\hline 2005-06-07 00:00:00.0 & 10.5 \\
\hline 2005-06-06 21:00:00.0 & 16.8 \\
\hline 2005-06-06 18:00:00.0 & 18.6 \\
\hline 2005-06-06 15:00:00.0 & 18.5 \\
\hline 2005-06-06 12:00:00.0 & 16.4 \\
\hline 2005-06-06 09:00:00.0 & 10.9 \\
\hline 2005-06-06 06:00:00.0 & 12.5 \\
\hline 2005-06-06 03:00:00.0 & 8.9 \\
\hline 2005-06-06 00:00:00.0 & 6.0 \\
\hline 2005-06-05 21:00:00.0 & 22.2 \\
\hline 2005-06-05 18:00:00.0 & 29.8 \\
\hline 2005-06-05 15:00:00.0 & 28.1 \\
\hline 2005-06-05 12:00:00.0 & 18.4 \\
\hline \multicolumn{2}{|l|}{ 2005-06-05 09:00:00.0 } \\
\hline \multicolumn{2}{|l|}{ 2005-06-05 06:00:00.0 } \\
\hline 2005-06-05 03:00:00.0 & 18.5 \\
\hline 2005-06-05 00:00:00.0 & 16.7 \\
\hline 2005-06-04 21:00:00.0 & 19.3 \\
\hline 2005-06-04 18:00:00.0 & 23.9 \\
\hline 2005-06-04 15:00:00.0 & 24.5 \\
\hline 2005-06-04 12:00:00.0 & 19.5 \\
\hline 2005-06-04 09:00:00.0 & 17.9 \\
\hline \multicolumn{2}{|l|}{ 2005-06-04 06:00:00.0 } \\
\hline \multicolumn{2}{|l|}{ 2005-06-04 03:00:00.0 } \\
\hline 2005-06-04 00:00:00.0 & 13.8 \\
\hline 2005-06-03 21:00:00.0 & 18.3 \\
\hline 2005-06-03 18:00:00.0 & 21.6 \\
\hline 2005-06-03 15:00:00.0 & 18.6 \\
\hline 2005-06-03 12:00:00.0 & 20.4 \\
\hline \multicolumn{2}{|l|}{ 2005-06-03 09:00:00.0 } \\
\hline 2005-06-03 06:00:00.0 & 19.1 \\
\hline 2005-06-03 03:00:00.0 & 16.5 \\
\hline 2005-06-03 00:00:00.0 & 16.4 \\
\hline \multicolumn{2}{|l|}{ 2005-06-02 21:00:00.0 } \\
\hline 2005-06-02 18:00:00.0 & 28.2 \\
\hline 2005-06-02 15:00:00.0 & 26.0 \\
\hline 2005-06-02 12:00:00.0 & 20.1 \\
\hline 2005-06-02 09:00:00.0 & 18.8 \\
\hline \multicolumn{2}{|l|}{ 2005-06-02 06:00:00.0 } \\
\hline 2005-06-02 03:00:00.0 & 15.7 \\
\hline 2005-06-02 00:00:00.0 & 13.8 \\
\hline \multicolumn{2}{|l|}{ 2005-06-01 21:00:00.0 } \\
\hline 2005-06-01 18:00:00.0 & 22.8 \\
\hline 2005-06-01 15:00:00.0 & 23.6 \\
\hline 2005-06-01 12:00:00.0 & 16.5 \\
\hline \multicolumn{2}{|l|}{ 2005-06-01 09:00:00.0 } \\
\hline 2005-06-01 06:00:00.0 & 15.1 \\
\hline 2005-06-01 03:00:00.0 & 15.9 \\
\hline 2005-05-31 18:00:00.0 & 23.9 \\
\hline 2005-05-31 15:00:00.0 & 21.0 \\
\hline 2005-05-31 12:00:00.0 & 17.7 \\
\hline 2005-05-31 09:00:00.0 & 22.7 \\
\hline 2005-05-31 06:00:00.0 & 24.4 \\
\hline
\end{tabular}

\begin{tabular}{|c|c|}
\hline DataHora & VelVentoMax \\
\hline 2005-05-31 03:00:00.0 & 21.2 \\
\hline 2005-05-31 00:00:00.0 & 20.5 \\
\hline \multicolumn{2}{|l|}{ 2005-05-30 21:00:00.0 } \\
\hline 2005-05-30 18:00:00.0 & 20.7 \\
\hline 2005-05-30 15:00:00.0 & 20.5 \\
\hline 2005-05-30 12:00:00.0 & 32.2 \\
\hline \multicolumn{2}{|l|}{ 2005-05-30 09:00:00.0 } \\
\hline 2005-05-30 06:00:00.0 & 21.2 \\
\hline 2005-05-30 03:00:00.0 & 23.8 \\
\hline 2005-05-30 00:00:00.0 & 16.7 \\
\hline 2005-05-29 21:00:00.0 & 24.9 \\
\hline 2005-05-29 18:00:00.0 & 26.4 \\
\hline 2005-05-29 15:00:00.0 & 27.7 \\
\hline 2005-05-29 12:00:00.0 & 23.5 \\
\hline \multicolumn{2}{|l|}{ 2005-05-29 09:00:00.0 } \\
\hline 2005-05-29 06:00:00.0 & 20.8 \\
\hline 2005-05-29 03:00:00.0 & 19.6 \\
\hline 2005-05-29 00:00:00.0 & 18.6 \\
\hline 2005-05-28 21:00:00.0 & 15.9 \\
\hline 2005-05-28 18:00:00.0 & 24.0 \\
\hline 2005-05-28 15:00:00.0 & 23.5 \\
\hline 2005-05-28 12:00:00.0 & 20.5 \\
\hline 2005-05-28 09:00:00.0 & 19.9 \\
\hline 2005-05-28 06:00:00.0 & 20.6 \\
\hline 2005-05-28 03:00:00.0 & 22.3 \\
\hline 2005-05-28 00:00:00.0 & 16.4 \\
\hline 2005-05-27 21:00:00.0 & 14.7 \\
\hline 2005-05-27 18:00:00.0 & 19.1 \\
\hline 2005-05-27 15:00:00.0 & 17.9 \\
\hline 2005-05-27 12:00:00.0 & 19.6 \\
\hline 2005-05-27 09:00:00.0 & 19.0 \\
\hline 2005-05-27 06:00:00.0 & 12.5 \\
\hline 2005-05-27 03:00:00.0 & 13.0 \\
\hline \multicolumn{2}{|l|}{ 2005-05-27 00:00:00.0 } \\
\hline 2005-05-26 21:00:00.0 & 27.1 \\
\hline 2005-05-26 18:00:00.0 & 11.0 \\
\hline 2005-05-26 15:00:00.0 & 11.1 \\
\hline \multicolumn{2}{|l|}{ 2005-05-26 12:00:00.0 } \\
\hline 2005-05-26 09:00:00.0 & 7.8 \\
\hline 2005-05-26 06:00:00.0 & 5.8 \\
\hline \multicolumn{2}{|l|}{ 2005-05-26 03:00:00.0 } \\
\hline 2005-05-26 00:00:00.0 & 9.1 \\
\hline 2005-05-25 21:00:00.0 & 21.7 \\
\hline 2005-05-25 18:00:00.0 & 19.7 \\
\hline 2005-05-25 15:00:00.0 & 27.1 \\
\hline \multicolumn{2}{|l|}{ 2005-05-25 12:00:00.0 } \\
\hline 2005-05-25 09:00:00.0 & 11.7 \\
\hline 2005-05-25 06:00:00.0 & 10.4 \\
\hline 2005-05-25 03:00:00.0 & 12.0 \\
\hline \multicolumn{2}{|l|}{ 2005-05-25 00:00:00.0 } \\
\hline 2005-05-24 21:00:00.0 & 14.2 \\
\hline 2005-05-24 18:00:00.0 & 14.0 \\
\hline \multicolumn{2}{|l|}{ 2005-05-24 15:00:00.0 } \\
\hline \multicolumn{2}{|l|}{ 2005-05-24 12:00:00.0 } \\
\hline 2005-05-24 09:00:00.0 & 16.7 \\
\hline 2005-05-24 06:00:00.0 & 20.2 \\
\hline 2005-05-24 03:00:00.0 & 20.1 \\
\hline 2005-05-24 00:00:00.0 & 21.8 \\
\hline 2005-05-23 21:00:00.0 & 14.7 \\
\hline 2005-05-23 18:00:00.0 & 19.5 \\
\hline 2005-05-23 15:00:00.0 & 23.1 \\
\hline \multicolumn{2}{|l|}{ 2005-05-23 12:00:00.0 } \\
\hline 2005-05-23 09:00:00.0 & 21.2 \\
\hline 2005-05-23 06:00:00.0 & 14.9 \\
\hline 2005-05-23 03:00:00.0 & \\
\hline 2005-05-23 00:00:00.0 & 5.5 \\
\hline 2005-05-22 21:00:00.0 & 10.7 \\
\hline 2005-05-22 18:00:00.0 & 15.3 \\
\hline 2005-05-22 15:00:00.0 & \\
\hline
\end{tabular}




\section{ANEXO 01 - RAJADAS DE VENTO REGISTRADAS EM SÃO MARTINHO DA SERRA / RS}

\begin{tabular}{|c|c|}
\hline \multirow{2}{*}{$\begin{array}{l}\text { DataHora } \\
\text { 2005-05-22 12:00:00.0 }\end{array}$} & VelVentoMax \\
\hline & \\
\hline 2005-05-22 09:00:00.0 & 8.7 \\
\hline 2005-05-22 06:00:00.0 & 7.4 \\
\hline 2005-05-22 03:00:00.0 & 9.3 \\
\hline 2005-05-22 00:00:00.0 & 11.4 \\
\hline 2005-05-21 21:00:00.0 & 23.7 \\
\hline 2005-05-21 18:00:00.0 & 25.4 \\
\hline 2005-05-21 15:00:00.0 & 18.3 \\
\hline 2005-05-21 12:00:00.0 & 7.4 \\
\hline 2005-05-21 09:00:00.0 & 22.8 \\
\hline 2005-05-21 06:00:00.0 & 28.7 \\
\hline 2005-05-21 03:00:00.0 & \\
\hline 2005-05-21 00:00:00.0 & 25.1 \\
\hline 2005-05-20 21:00:00.0 & 18.5 \\
\hline 2005-05-20 18:00:00.0 & 20.7 \\
\hline 2005-05-20 15:00:00.0 & \\
\hline 2005-05-20 12:00:00.0 & 20.2 \\
\hline 2005-05-20 09:00:00.0 & 15.3 \\
\hline 2005-05-20 06:00:00.0 & \\
\hline 2005-05-20 03:00:00.0 & 31.7 \\
\hline 2005-05-20 00:00:00.0 & 8.4 \\
\hline 2005-05-19 21:00:00.0 & 17.7 \\
\hline 2005-05-19 18:00:00.0 & 9.4 \\
\hline 2005-05-19 15:00:00.0 & 14.2 \\
\hline 2005-05-19 12:00:00.0 & 15.5 \\
\hline 2005-05-19 09:00:00.0 & 11.2 \\
\hline 2005-05-19 06:00:00.0 & \\
\hline 2005-05-19 03:00:00.0 & 13.2 \\
\hline 2005-05-19 00:00:00.0 & 13.7 \\
\hline 2005-05-18 21:00:00.0 & 16.2 \\
\hline 2005-05-18 18:00:00.0 & \\
\hline 2005-05-18 15:00:00.0 & \\
\hline 2005-05-18 12:00:00.0 & 8.5 \\
\hline 2005-05-18 09:00:00.0 & 11.3 \\
\hline 2005-05-18 06:00:00.0 & 15.6 \\
\hline 2005-05-18 03:00:00.0 & 21.7 \\
\hline 2005-05-18 00:00:00.0 & 22.8 \\
\hline 2005-05-17 21:00:00.0 & 13.5 \\
\hline 2005-05-17 18:00:00.0 & 14.5 \\
\hline 2005-05-17 15:00:00.0 & \\
\hline 2005-05-17 12:00:00.0 & 10.4 \\
\hline 2005-05-17 09:00:00.0 & 10.8 \\
\hline 2005-05-17 06:00:00.0 & \\
\hline 2005-05-17 03:00:00.0 & 14.5 \\
\hline 2005-05-17 00:00:00.0 & 16.5 \\
\hline 2005-05-16 21:00:00.0 & 16.1 \\
\hline 2005-05-16 18:00:00.0 & 18.0 \\
\hline 2005-05-16 15:00:00.0 & 16.1 \\
\hline 2005-05-16 12:00:00.0 & 16.9 \\
\hline 2005-05-16 09:00:00.0 & 15.6 \\
\hline 2005-05-16 06:00:00.0 & \\
\hline 2005-05-16 03:00:00.0 & 9.8 \\
\hline 2005-05-16 00:00:00.0 & 15.9 \\
\hline 2005-05-15 21:00:00.0 & 16.1 \\
\hline 2005-05-15 18:00:00.0 & 19.9 \\
\hline 2005-05-15 15:00:00.0 & \\
\hline 2005-05-15 12:00:00.0 & 23.0 \\
\hline 2005-05-15 09:00:00.0 & 22.5 \\
\hline 2005-05-15 06:00:00.0 & \\
\hline 2005-05-15 03:00:00.0 & 17.3 \\
\hline 2005-05-15 00:00:00.0 & 11.9 \\
\hline 2005-05-14 21:00:00.0 & \\
\hline 2005-05-14 18:00:00.0 & \\
\hline 2005-05-14 15:00:00.0 & 31.2 \\
\hline 2005-05-14 12:00:00.0 & \\
\hline 2005-05-14 09:00:00.0 & 18.8 \\
\hline 2005-05-14 06:00:00.0 & 21.4 \\
\hline 2005-05-14 03:00:00.0 & 23.8 \\
\hline 2005-05-14 00:00:00.0 & 18.8 \\
\hline
\end{tabular}

\begin{tabular}{|c|c|}
\hline DataHora & VelVentoMax \\
\hline 2005-05-13 21:00:00.0 & 24.7 \\
\hline \multicolumn{2}{|l|}{ 2005-05-13 18:00:00.0 } \\
\hline 2005-05-13 15:00:00.0 & 26.0 \\
\hline 2005-05-13 12:00:00.0 & 23.2 \\
\hline \multicolumn{2}{|l|}{ 2005-05-13 09:00:00.0 } \\
\hline 2005-05-13 06:00:00.0 & 20.9 \\
\hline 2005-05-13 03:00:00.0 & 18.7 \\
\hline 2005-05-13 00:00:00.0 & 19.3 \\
\hline 2005-05-12 21:00:00.0 & 19.5 \\
\hline \multicolumn{2}{|l|}{ 2005-05-12 18:00:00.0 } \\
\hline 2005-05-12 15:00:00.0 & 45.7 \\
\hline \multicolumn{2}{|l|}{ 2005-05-12 12:00:00.0 } \\
\hline \multicolumn{2}{|l|}{ 2005-05-12 09:00:00.0 } \\
\hline 2005-05-12 06:00:00.0 & 22.0 \\
\hline 2005-05-12 03:00:00.0 & 20.9 \\
\hline 2005-05-12 00:00:00.0 & 18.6 \\
\hline 2005-05-11 21:00:00.0 & 27.1 \\
\hline \multicolumn{2}{|l|}{ 2005-05-11 18:00:00.0 } \\
\hline 2005-05-11 15:00:00.0 & 22.2 \\
\hline 2005-05-11 12:00:00.0 & 25.3 \\
\hline \multicolumn{2}{|l|}{ 2005-05-11 09:00:00.0 } \\
\hline 2005-05-11 06:00:00.0 & 24.5 \\
\hline 2005-05-11 03:00:00.0 & 18.1 \\
\hline 2005-05-11 00:00:00.0 & 14.2 \\
\hline 2005-05-10 21:00:00.0 & 16.1 \\
\hline 2005-05-10 18:00:00.0 & 18.9 \\
\hline 2005-05-10 15:00:00.0 & 22.1 \\
\hline 2005-05-10 12:00:00.0 & 19.0 \\
\hline 2005-05-10 09:00:00.0 & 18.8 \\
\hline 2005-05-10 06:00:00.0 & 14.8 \\
\hline 2005-05-10 03:00:00.0 & 12.7 \\
\hline 2005-05-10 00:00:00.0 & 16.2 \\
\hline 2005-05-09 21:00:00.0 & 22.3 \\
\hline 2005-05-09 18:00:00.0 & 19.3 \\
\hline 2005-05-09 15:00:00.0 & 22.2 \\
\hline 2005-05-09 12:00:00.0 & 23.5 \\
\hline \multicolumn{2}{|l|}{ 2005-05-09 09:00:00.0 } \\
\hline 2005-05-09 06:00:00.0 & 22.3 \\
\hline 2005-05-09 03:00:00.0 & 22.7 \\
\hline 2005-05-09 00:00:00.0 & 21.7 \\
\hline 2005-05-08 21:00:00.0 & 24.3 \\
\hline 2005-05-08 18:00:00.0 & 25.5 \\
\hline 2005-05-08 15:00:00.0 & 28.6 \\
\hline 2005-05-08 12:00:00.0 & 21.5 \\
\hline 2005-05-08 09:00:00.0 & 23.1 \\
\hline 2005-05-08 06:00:00.0 & 19.9 \\
\hline 2005-05-08 03:00:00.0 & 23.8 \\
\hline 2005-05-08 00:00:00.0 & 22.5 \\
\hline 2005-05-07 21:00:00.0 & 20.1 \\
\hline \multicolumn{2}{|l|}{ 2005-05-07 18:00:00.0 } \\
\hline 2005-05-07 15:00:00.0 & 21.8 \\
\hline 2005-05-07 12:00:00.0 & 19.4 \\
\hline \multicolumn{2}{|l|}{ 2005-05-07 09:00:00.0 } \\
\hline 2005-05-07 06:00:00.0 & 35.1 \\
\hline 2005-05-07 03:00:00.0 & 16.8 \\
\hline 2005-05-07 00:00:00.0 & 14.6 \\
\hline \multicolumn{2}{|l|}{ 2005-05-06 21:00:00.0 } \\
\hline 2005-05-06 18:00:00.0 & 9.9 \\
\hline \multicolumn{2}{|l|}{ 2005-05-06 15:00:00.0 } \\
\hline \multicolumn{2}{|l|}{ 2005-05-06 12:00:00.0 } \\
\hline 2005-05-06 09:00:00.0 & \\
\hline 2005-05-05 09:00:00.0 & 18.2 \\
\hline 2005-05-05 06:00:00.0 & 15.9 \\
\hline 2005-05-05 03:00:00.0 & 17.1 \\
\hline 2005-05-05 00:00:00.0 & \\
\hline 2005-05-04 21:00:00.0 & 22.7 \\
\hline 2005-05-04 18:00:00.0 & 25.2 \\
\hline 2005-05-04 15:00:00.0 & \\
\hline 2005-05-04 12:00:00.0 & \\
\hline
\end{tabular}

\begin{tabular}{|c|c|}
\hline DataHora & VelVentoMax \\
\hline \multicolumn{2}{|l|}{ 2005-05-04 09:00:00.0 } \\
\hline 2005-05-04 06:00:00.0 & 22.3 \\
\hline 2005-05-04 03:00:00.0 & 16.9 \\
\hline \multicolumn{2}{|l|}{ 2005-05-04 00:00:00.0 } \\
\hline 2005-05-03 21:00:00.0 & 15.0 \\
\hline 2005-05-03 18:00:00.0 & 18.4 \\
\hline 2005-05-03 15:00:00.0 & 24.0 \\
\hline \multicolumn{2}{|l|}{ 2005-05-03 12:00:00.0 } \\
\hline \multicolumn{2}{|l|}{ 2005-05-03 09:00:00.0 } \\
\hline 2005-05-03 06:00:00.0 & 16.9 \\
\hline 2005-05-03 03:00:00.0 & 14.8 \\
\hline \multicolumn{2}{|l|}{ 2005-05-03 00:00:00.0 } \\
\hline 2005-05-02 21:00:00.0 & 22.5 \\
\hline 2005-05-02 18:00:00.0 & 17.9 \\
\hline $2005-05-02$ 15:00:00.0 & 17.7 \\
\hline $2005-05-02$ 12:00:00.0 & 18.8 \\
\hline 2005-05-02 09:00:00.0 & 18.1 \\
\hline 2005-05-02 06:00:00.0 & 18.1 \\
\hline 2005-05-02 03:00:00.0 & 15.0 \\
\hline 2005-05-02 00:00:00.0 & 11.7 \\
\hline 2005-05-01 21:00:00.0 & 13.6 \\
\hline 2005-05-01 18:00:00.0 & 14.6 \\
\hline 2005-05-01 15:00:00.0 & 15.4 \\
\hline 2005-05-01 12:00:00.0 & 14.1 \\
\hline 2005-05-01 09:00:00.0 & 13.0 \\
\hline 2005-05-01 06:00:00.0 & 12.3 \\
\hline 2005-05-01 03:00:00.0 & 12.2 \\
\hline 2005-05-01 00:00:00.0 & 11.4 \\
\hline 2005-04-30 21:00:00.0 & 14.0 \\
\hline $2005-04-30$ 18:00:00.0 & 13.2 \\
\hline 2005-04-30 15:00:00.0 & 16.5 \\
\hline 2005-04-30 12:00:00.0 & 12.1 \\
\hline 2005-04-30 09:00:00.0 & 10.7 \\
\hline 2005-04-30 06:00:00.0 & 10.7 \\
\hline 2005-04-30 03:00:00.0 & 8.7 \\
\hline \multicolumn{2}{|l|}{ 2005-04-30 00:00:00.0 } \\
\hline 2005-04-29 21:00:00.0 & 13.5 \\
\hline $2005-04-29$ 18:00:00.0 & 14.4 \\
\hline \multicolumn{2}{|l|}{ 2005-04-29 15:00:00.0 } \\
\hline 2005-04-29 12:00:00.0 & 10.5 \\
\hline 2005-04-29 09:00:00.0 & 12.5 \\
\hline 2005-04-29 06:00:00.0 & 15.4 \\
\hline 2005-04-29 03:00:00.0 & 11.6 \\
\hline 2005-04-29 00:00:00.0 & 10.9 \\
\hline 2005-04-28 21:00:00.0 & 13.7 \\
\hline 2005-04-28 18:00:00.0 & 14.3 \\
\hline $2005-04-28$ 15:00:00.0 & 13.0 \\
\hline $2005-04-28$ 12:00:00.0 & 13.7 \\
\hline 2005-04-28 09:00:00.0 & 13.2 \\
\hline 2005-04-28 06:00:00.0 & 12.1 \\
\hline 2005-04-28 03:00:00.0 & 12.9 \\
\hline 2005-04-28 00:00:00.0 & 10.4 \\
\hline 2005-04-27 21:00:00.0 & 16.0 \\
\hline $2005-04-27$ 18:00:00.0 & 19.8 \\
\hline \multicolumn{2}{|l|}{ 2005-04-27 15:00:00.0 } \\
\hline 2005-04-27 12:00:00.0 & 13.7 \\
\hline 2005-04-27 09:00:00.0 & 15.7 \\
\hline 2005-04-27 06:00:00.0 & 22.9 \\
\hline \multicolumn{2}{|l|}{ 2005-04-27 03:00:00.0 } \\
\hline 2005-04-27 00:00:00.0 & 10.7 \\
\hline 2005-04-26 21:00:00.0 & 22.7 \\
\hline $2005-04-26$ 18:00:00.0 & 23.7 \\
\hline $2005-04-26$ 15:00:00.0 & 23.2 \\
\hline 2005-04-26 12:00:00.0 & 21.4 \\
\hline 2005-04-26 09:00:00.0 & 16.3 \\
\hline 2005-04-26 06:00:00.0 & 12.2 \\
\hline 2005-04-26 03:00:00.0 & \\
\hline 2005-04-26 00:00:00.0 & \\
\hline 2005-04-25 21:00:00.0 & 22.5 \\
\hline
\end{tabular}




\section{ANEXO 01 - RAJADAS DE VENTO REGISTRADAS EM SÃO MARTINHO DA SERRA / RS}

\begin{tabular}{|c|c|}
\hline \multirow{2}{*}{ DataHora } & VelVentoMax \\
\hline & \\
\hline 2005-04-25 15:00:00.0 & \\
\hline 2005-04-25 12:00:00.0 & 29.8 \\
\hline 2005-04-25 09:00:00.0 & 30.2 \\
\hline 2005-04-25 06:00:00.0 & 34.4 \\
\hline 2005-04-25 03:00:00.0 & 30.1 \\
\hline 2005-04-25 00:00:00.0 & 14.9 \\
\hline 2005-04-24 21:00:00.0 & 17.0 \\
\hline 2005-04-24 18:00:00.0 & 20.7 \\
\hline 2005-04-24 15:00:00.0 & \\
\hline 2005-04-24 12:00:00.0 & 21.2 \\
\hline 2005-04-24 09:00:00.0 & 21.5 \\
\hline 2005-04-24 06:00:00.0 & 20.3 \\
\hline 2005-04-24 03:00:00.0 & 17.0 \\
\hline $2005-04-24$ 00:00:00.0 & \\
\hline 2005-04-23 21:00:00.0 & 15.2 \\
\hline 2005-04-23 18:00:00.0 & \\
\hline 2005-04-23 15:00:00.0 & 19.0 \\
\hline 2005-04-23 12:00:00.0 & \\
\hline 2005-04-23 09:00:00.0 & 16.0 \\
\hline 2005-04-23 06:00:00.0 & \\
\hline 2005-04-23 03:00:00.0 & 11.0 \\
\hline 2005-04-23 00:00:00.0 & 11.2 \\
\hline 2005-04-22 21:00:00.0 & 11.1 \\
\hline 2005-04-22 18:00:00.0 & 11.5 \\
\hline 2005-04-22 15:00:00.0 & 14.3 \\
\hline 2005-04-22 12:00:00.0 & \\
\hline 2005-04-22 09:00:00.0 & 26.1 \\
\hline 2005-04-22 06:00:00.0 & 10.6 \\
\hline 2005-04-22 03:00:00.0 & 9.8 \\
\hline 2005-04-22 00:00:00.0 & 8.7 \\
\hline 2005-04-21 21:00:00.0 & \\
\hline 2005-04-21 18:00:00.0 & 11.4 \\
\hline 2005-04-21 15:00:00.0 & 9.5 \\
\hline 2005-04-21 12:00:00.0 & 12.4 \\
\hline 2005-04-21 09:00:00.0 & 14.6 \\
\hline 2005-04-21 06:00:00.0 & 12.3 \\
\hline 2005-04-21 03:00:00.0 & 13.8 \\
\hline 2005-04-21 00:00:00.0 & 10.0 \\
\hline 2005-04-20 21:00:00.0 & 9.3 \\
\hline 2005-04-20 18:00:00.0 & 15.0 \\
\hline 2005-04-20 15:00:00.0 & 13.4 \\
\hline 2005-04-20 12:00:00.0 & 11.5 \\
\hline 2005-04-20 09:00:00.0 & \\
\hline 2005-04-20 06:00:00.0 & 7.8 \\
\hline 2005-04-20 03:00:00.0 & 15.6 \\
\hline 2005-04-20 00:00:00.0 & 20.0 \\
\hline 2005-04-19 21:00:00.0 & 34.7 \\
\hline 2005-04-19 18:00:00.0 & \\
\hline 2005-04-19 15:00:00.0 & 21.8 \\
\hline 2005-04-19 12:00:00.0 & 15.5 \\
\hline 2005-04-19 09:00:00.0 & 19.0 \\
\hline 2005-04-19 06:00:00.0 & 8.1 \\
\hline 2005-04-19 03:00:00.0 & 8.4 \\
\hline 2005-04-19 00:00:00.0 & 7.7 \\
\hline 2005-04-18 21:00:00.0 & 15.4 \\
\hline 2005-04-18 18:00:00.0 & 14.3 \\
\hline 2005-04-18 15:00:00.0 & 16.3 \\
\hline 2005-04-18 12:00:00.0 & 18.3 \\
\hline 2005-04-18 09:00:00.0 & 17.9 \\
\hline 2005-04-18 06:00:00.0 & 34.3 \\
\hline 2005-04-18 03:00:00.0 & 13.0 \\
\hline 2005-04-18 00:00:00.0 & 9.3 \\
\hline 2005-04-17 21:00:00.0 & 13.7 \\
\hline 2005-04-17 18:00:00.0 & 14.6 \\
\hline 2005-04-17 15:00:00.0 & 26.3 \\
\hline 2005-04-17 12:00:00.0 & 18.9 \\
\hline 2005-04-17 09:00:00.0 & 12.6 \\
\hline 2005-04-17 06:00:00.0 & 37.6 \\
\hline
\end{tabular}

\begin{tabular}{|c|c|}
\hline DataHora & VelVentoMax \\
\hline 2005-04-17 03:00:00.0 & 11.9 \\
\hline 2005-04-17 00:00:00.0 & 10.7 \\
\hline 2005-04-16 21:00:00.0 & 12.7 \\
\hline 2005-04-16 18:00:00.0 & 13.3 \\
\hline 2005-04-16 15:00:00.0 & 12.8 \\
\hline 2005-04-16 12:00:00.0 & 8.5 \\
\hline 2005-04-16 09:00:00.0 & 11.7 \\
\hline 2005-04-16 06:00:00.0 & 9.9 \\
\hline 2005-04-16 03:00:00.0 & 11.6 \\
\hline 2005-04-16 00:00:00.0 & 14.6 \\
\hline 2005-04-15 21:00:00.0 & 23.4 \\
\hline 2005-04-15 18:00:00.0 & 20.1 \\
\hline 2005-04-15 15:00:00.0 & 24.8 \\
\hline 2005-04-15 12:00:00.0 & 21.7 \\
\hline 2005-04-15 09:00:00.0 & 38.0 \\
\hline 2005-04-15 06:00:00.0 & \\
\hline 2005-04-15 03:00:00.0 & \\
\hline 2005-04-15 00:00:00.0 & 21.3 \\
\hline 2005-04-14 21:00:00.0 & \\
\hline 2005-04-14 18:00:00.0 & 38.2 \\
\hline 2005-04-14 15:00:00.0 & 30.0 \\
\hline 2005-04-14 12:00:00.0 & 20.5 \\
\hline 2005-04-14 09:00:00.0 & 21.4 \\
\hline 2005-04-14 06:00:00.0 & 20.0 \\
\hline 2005-04-14 03:00:00.0 & 20.5 \\
\hline 2005-04-14 00:00:00.0 & 21.6 \\
\hline 2005-04-13 21:00:00.0 & 20.3 \\
\hline 2005-04-13 18:00:00.0 & 22.1 \\
\hline 2005-04-13 15:00:00.0 & 26.8 \\
\hline 2005-04-13 12:00:00.0 & 23.6 \\
\hline 2005-04-13 09:00:00.0 & \\
\hline 2005-04-13 06:00:00.0 & 22.8 \\
\hline 2005-04-13 03:00:00.0 & 26.8 \\
\hline 2005-04-13 00:00:00.0 & 21.9 \\
\hline 2005-04-12 21:00:00.0 & 19.2 \\
\hline 2005-04-12 18:00:00.0 & 11.3 \\
\hline 2005-04-12 15:00:00.0 & 11.2 \\
\hline 2005-04-12 12:00:00.0 & 27.0 \\
\hline 2005-04-12 09:00:00.0 & \\
\hline 2005-04-12 06:00:00.0 & 20.0 \\
\hline 2005-04-12 03:00:00.0 & 18.9 \\
\hline 2005-04-12 00:00:00.0 & 26.1 \\
\hline 2005-04-11 21:00:00.0 & 38.0 \\
\hline 2005-04-11 18:00:00.0 & 38.0 \\
\hline 2005-04-11 15:00:00.0 & 35.7 \\
\hline 2005-04-11 12:00:00.0 & 24.5 \\
\hline 2005-04-11 09:00:00.0 & 17.6 \\
\hline 2005-04-11 06:00:00.0 & \\
\hline 2005-04-11 03:00:00.0 & 13.0 \\
\hline 2005-04-11 00:00:00.0 & 12.5 \\
\hline 2005-04-10 21:00:00.0 & 21.9 \\
\hline 2005-04-10 18:00:00.0 & 27.0 \\
\hline 2005-04-10 15:00:00.0 & 21.8 \\
\hline 2005-04-10 12:00:00.0 & 31.8 \\
\hline 2005-04-10 09:00:00.0 & \\
\hline 2005-04-10 06:00:00.0 & 16.1 \\
\hline 2005-04-10 03:00:00.0 & 15.1 \\
\hline 2005-04-10 00:00:00.0 & 15.0 \\
\hline 2005-04-09 21:00:00.0 & 15.2 \\
\hline 2005-04-09 18:00:00.0 & 19.7 \\
\hline 2005-04-09 15:00:00.0 & 26.8 \\
\hline 2005-04-09 12:00:00.0 & 20.8 \\
\hline 2005-04-09 09:00:00.0 & \\
\hline 2005-04-09 06:00:00.0 & 14.6 \\
\hline 2005-04-09 03:00:00.0 & 12.4 \\
\hline 2005-04-09 00:00:00.0 & 5.9 \\
\hline 2005-04-08 21:00:00.0 & 21.1 \\
\hline 2005-04-08 18:00:00.0 & 29.6 \\
\hline 2005-04-08 15:00:00.0 & \\
\hline
\end{tabular}

\begin{tabular}{|c|c|}
\hline DataHora & VelVentoMax \\
\hline 2005-04-08 12:00:00.0 & 18.2 \\
\hline \multicolumn{2}{|l|}{ 2005-04-08 09:00:00.0 } \\
\hline 2005-04-08 06:00:00.0 & 29.6 \\
\hline 2005-04-08 03:00:00.0 & 30.7 \\
\hline 2005-04-08 00:00:00.0 & 24.5 \\
\hline 2005-04-07 21:00:00.0 & 35.4 \\
\hline 2005-04-07 18:00:00.0 & 43.9 \\
\hline 2005-04-07 15:00:00.0 & 40.6 \\
\hline 2005-04-07 12:00:00.0 & 30.5 \\
\hline \multicolumn{2}{|l|}{ 2005-04-07 09:00:00.0 } \\
\hline 2005-04-07 06:00:00.0 & 16.3 \\
\hline \multicolumn{2}{|l|}{ 2005-04-07 03:00:00.0 } \\
\hline 2005-04-07 00:00:00.0 & 14.9 \\
\hline 2005-04-06 21:00:00.0 & 20.7 \\
\hline 2005-04-06 18:00:00.0 & 27.3 \\
\hline 2005-04-06 15:00:00.0 & 20.0 \\
\hline 2005-04-06 12:00:00.0 & 21.1 \\
\hline \multicolumn{2}{|l|}{ 2005-04-06 09:00:00.0 } \\
\hline 2005-04-06 06:00:00.0 & 20.6 \\
\hline 2005-04-06 03:00:00.0 & 19.3 \\
\hline 2005-04-06 00:00:00.0 & 14.7 \\
\hline 2005-04-05 21:00:00.0 & 17.6 \\
\hline $2005-04-05$ 18:00:00.0 & 17.4 \\
\hline \multicolumn{2}{|l|}{$2005-04-05$ 15:00:00.0 } \\
\hline 2005-04-05 12:00:00.0 & 23.2 \\
\hline \multicolumn{2}{|l|}{ 2005-04-05 09:00:00.0 } \\
\hline 2005-04-05 06:00:00.0 & 22.0 \\
\hline 2005-04-05 03:00:00.0 & 20.3 \\
\hline \multicolumn{2}{|l|}{ 2005-04-05 00:00:00.0 } \\
\hline \multicolumn{2}{|l|}{ 2005-04-04 21:00:00.0 } \\
\hline \multicolumn{2}{|l|}{ 2005-04-04 18:00:00.0 } \\
\hline $2005-04-04$ 15:00:00.0 & 19.1 \\
\hline 2005-04-04 12:00:00.0 & 18.6 \\
\hline 2005-04-04 09:00:00.0 & 13.6 \\
\hline \multicolumn{2}{|l|}{ 2005-04-04 06:00:00.0 } \\
\hline 2005-04-04 03:00:00.0 & 11.4 \\
\hline 2005-04-04 00:00:00.0 & 8.2 \\
\hline 2005-04-03 21:00:00.0 & 16.5 \\
\hline 2005-04-03 18:00:00.0 & 20.3 \\
\hline 2005-04-03 15:00:00.0 & 18.9 \\
\hline 2005-04-03 12:00:00.0 & 19.7 \\
\hline 2005-04-03 09:00:00.0 & 22.9 \\
\hline 2005-04-03 06:00:00.0 & 22.2 \\
\hline \multicolumn{2}{|l|}{ 2005-04-03 03:00:00.0 } \\
\hline 2005-04-03 00:00:00.0 & 12.6 \\
\hline 2005-04-01 15:00:00.0 & 32.0 \\
\hline 2005-04-01 12:00:00.0 & 28.7 \\
\hline 2005-04-01 09:00:00.0 & 22.7 \\
\hline 2005-04-01 06:00:00.0 & 21.8 \\
\hline \multicolumn{2}{|l|}{ 2005-04-01 03:00:00.0 } \\
\hline 2005-04-01 00:00:00.0 & 26.3 \\
\hline \multicolumn{2}{|l|}{ 2005-03-31 21:00:00.0 } \\
\hline 2005-03-31 18:00:00.0 & 16.8 \\
\hline $2005-03-31$ 15:00:00.0 & 23.9 \\
\hline 2005-03-31 12:00:00.0 & 22.8 \\
\hline 2005-03-31 09:00:00.0 & 20.6 \\
\hline 2005-03-31 06:00:00.0 & \\
\hline 2005-03-31 03:00:00.0 & 22.3 \\
\hline 2005-03-31 00:00:00.0 & 25.6 \\
\hline 2005-03-30 21:00:00.0 & 26.2 \\
\hline 2005-03-30 18:00:00.0 & 24.4 \\
\hline $2005-03-3015: 00: 00.0$ & \\
\hline 2005-03-30 12:00:00.0 & 20.2 \\
\hline 2005-03-30 09:00:00.0 & 13.3 \\
\hline 2005-03-30 06:00:00.0 & \\
\hline 2005-03-30 03:00:00.0 & 17.9 \\
\hline 2005-03-30 00:00:00.0 & 15.8 \\
\hline 2005-03-29 21:00:00.0 & 29.9 \\
\hline 2005-03-29 18:00:00.0 & 30.7 \\
\hline
\end{tabular}




\section{ANEXO 01 - RAJADAS DE VENTO REGISTRADAS EM SÃO MARTINHO DA SERRA / RS}

\begin{tabular}{|c|c|}
\hline DataHora & VelVentoMax \\
\hline 2005-03-29 15:00:00.0 & 22.3 \\
\hline 2005-03-29 12:00:00.0 & 21.6 \\
\hline 2005-03-29 09:00:00.0 & 19.7 \\
\hline 2005-03-29 06:00:00.0 & \\
\hline 2005-03-29 03:00:00.0 & 15.4 \\
\hline 2005-03-29 00:00:00.0 & 11.7 \\
\hline 2005-03-28 21:00:00.0 & 11.4 \\
\hline 2005-03-28 18:00:00.0 & 15.7 \\
\hline 2005-03-28 15:00:00.0 & \\
\hline 2005-03-28 12:00:00.0 & 19.4 \\
\hline 2005-03-28 09:00:00.0 & 17.7 \\
\hline 2005-03-28 06:00:00.0 & \\
\hline 2005-03-28 03:00:00.0 & 14.2 \\
\hline 2005-03-28 00:00:00.0 & 11.8 \\
\hline 2005-03-27 21:00:00.0 & 11.7 \\
\hline 2005-03-27 18:00:00.0 & 18.2 \\
\hline 2005-03-27 15:00:00.0 & \\
\hline 2005-03-27 12:00:00.0 & 23.2 \\
\hline 2005-03-27 09:00:00.0 & 19.3 \\
\hline 2005-03-27 06:00:00.0 & \\
\hline 2005-03-27 03:00:00.0 & 19.2 \\
\hline 2005-03-27 00:00:00.0 & 16.6 \\
\hline 2005-03-26 21:00:00.0 & 17.5 \\
\hline 2005-03-26 18:00:00.0 & \\
\hline 2005-03-26 15:00:00.0 & 25.3 \\
\hline 2005-03-26 12:00:00.0 & 23.1 \\
\hline 2005-03-26 09:00:00.0 & 22.2 \\
\hline 2005-03-26 06:00:00.0 & \\
\hline 2005-03-26 03:00:00.0 & \\
\hline 2005-03-26 00:00:00.0 & 20.8 \\
\hline 2005-03-25 21:00:00.0 & \\
\hline 2005-03-25 18:00:00.0 & \\
\hline 2005-03-25 15:00:00.0 & 18.0 \\
\hline 2005-03-25 12:00:00.0 & 20.6 \\
\hline 2005-03-25 09:00:00.0 & \\
\hline 2005-03-25 06:00:00.0 & 16.9 \\
\hline 2005-03-25 03:00:00.0 & \\
\hline 2005-03-25 00:00:00.0 & \\
\hline 2005-03-24 21:00:00.0 & \\
\hline 2005-03-24 15:00:00.0 & 28.6 \\
\hline 2005-03-24 12:00:00.0 & 20.8 \\
\hline 2005-03-24 09:00:00.0 & 17.6 \\
\hline 2005-03-24 06:00:00.0 & \\
\hline 2005-03-24 03:00:00.0 & 21.3 \\
\hline 2005-03-24 00:00:00.0 & 22.8 \\
\hline 2005-03-23 21:00:00.0 & 24.2 \\
\hline 2005-03-23 18:00:00.0 & 25.0 \\
\hline 2005-03-23 15:00:00.0 & 23.8 \\
\hline 2005-03-23 12:00:00.0 & 16.4 \\
\hline 2005-03-23 09:00:00.0 & \\
\hline 2005-03-23 06:00:00.0 & \\
\hline 2005-03-23 03:00:00.0 & 28.9 \\
\hline 2005-03-23 00:00:00.0 & 30.8 \\
\hline 2005-03-22 21:00:00.0 & \\
\hline 2005-03-22 18:00:00.0 & \\
\hline 2005-03-22 15:00:00.0 & \\
\hline 2005-03-22 12:00:00.0 & 21.0 \\
\hline 2005-03-22 09:00:00.0 & \\
\hline 2005-03-22 06:00:00.0 & 18.9 \\
\hline 2005-03-22 03:00:00.0 & 15.0 \\
\hline 2005-03-22 00:00:00.0 & 18.8 \\
\hline 2005-03-21 21:00:00.0 & 27.2 \\
\hline 2005-03-21 18:00:00.0 & \\
\hline 2005-03-21 15:00:00.0 & 26.1 \\
\hline 2005-03-21 12:00:00.0 & 19.4 \\
\hline 2005-03-21 09:00:00.0 & \\
\hline 2005-03-21 06:00:00.0 & \\
\hline 2005-03-21 03:00:00.0 & 15.1 \\
\hline 2005-03-21 00:00:00.0 & 13.4 \\
\hline
\end{tabular}

\begin{tabular}{|c|c|}
\hline DataHora & VelVentoMax \\
\hline 2005-03-20 21:00:00.0 & 15.1 \\
\hline 2005-03-20 18:00:00.0 & \\
\hline 2005-03-20 15:00:00.0 & 15.1 \\
\hline 2005-03-20 12:00:00.0 & 17.0 \\
\hline 2005-03-20 09:00:00.0 & \\
\hline 2005-03-20 06:00:00.0 & 13.1 \\
\hline 2005-03-20 03:00:00.0 & 10.6 \\
\hline 2005-03-20 00:00:00.0 & 11.8 \\
\hline 2005-03-19 21:00:00.0 & 14.6 \\
\hline 2005-03-19 18:00:00.0 & \\
\hline 2005-03-19 15:00:00.0 & 13.0 \\
\hline 2005-03-19 12:00:00.0 & 15.4 \\
\hline 2005-03-19 09:00:00.0 & 10.3 \\
\hline 2005-03-19 06:00:00.0 & 13.2 \\
\hline 2005-03-19 03:00:00.0 & 20.1 \\
\hline 2005-03-19 00:00:00.0 & 23.6 \\
\hline 2005-03-18 21:00:00.0 & \\
\hline 2005-03-18 18:00:00.0 & \\
\hline 2005-03-18 15:00:00.0 & 20.7 \\
\hline 2005-03-18 12:00:00.0 & 9.2 \\
\hline 2005-03-18 09:00:00.0 & \\
\hline 2005-03-18 06:00:00.0 & 14.5 \\
\hline 2005-03-18 03:00:00.0 & 10.0 \\
\hline 2005-03-18 00:00:00.0 & 13.2 \\
\hline 2005-03-17 21:00:00.0 & 15.6 \\
\hline 2005-03-17 18:00:00.0 & 18.1 \\
\hline 2005-03-17 15:00:00.0 & 13.8 \\
\hline 2005-03-17 12:00:00.0 & 15.3 \\
\hline 2005-03-17 09:00:00.0 & \\
\hline 2005-03-17 06:00:00.0 & 10.6 \\
\hline 2005-03-17 03:00:00.0 & 9.9 \\
\hline 2005-03-17 00:00:00.0 & 10.2 \\
\hline 2005-03-16 21:00:00.0 & 18.2 \\
\hline 2005-03-16 18:00:00.0 & 18.7 \\
\hline 2005-03-16 15:00:00.0 & 15.2 \\
\hline 2005-03-16 12:00:00.0 & 6.3 \\
\hline 2005-03-16 09:00:00.0 & \\
\hline 2005-03-16 06:00:00.0 & 7.3 \\
\hline 2005-03-16 03:00:00.0 & 10.2 \\
\hline 2005-03-16 00:00:00.0 & 18.2 \\
\hline 2005-03-15 21:00:00.0 & \\
\hline 2005-03-15 18:00:00.0 & 17.4 \\
\hline 2005-03-15 15:00:00.0 & 18.0 \\
\hline 2005-03-15 12:00:00.0 & 14.5 \\
\hline 2005-03-15 09:00:00.0 & 13.8 \\
\hline 2005-03-15 06:00:00.0 & 12.1 \\
\hline 2005-03-15 03:00:00.0 & 12.3 \\
\hline 2005-03-15 00:00:00.0 & 15.6 \\
\hline 2005-03-14 21:00:00.0 & \\
\hline 2005-03-14 18:00:00.0 & 19.1 \\
\hline 2005-03-14 15:00:00.0 & \\
\hline 2005-03-14 12:00:00.0 & 15.7 \\
\hline 2005-03-14 09:00:00.0 & 15.6 \\
\hline 2005-03-14 06:00:00.0 & 22.7 \\
\hline 2005-03-14 03:00:00.0 & 19.8 \\
\hline 2005-03-14 00:00:00.0 & 18.0 \\
\hline 2005-03-13 21:00:00.0 & 16.1 \\
\hline 2005-03-13 18:00:00.0 & 20.4 \\
\hline 2005-03-13 15:00:00.0 & \\
\hline 2005-03-13 12:00:00.0 & 28.9 \\
\hline 2005-03-13 09:00:00.0 & 37.9 \\
\hline 2005-03-13 06:00:00.0 & 43.6 \\
\hline 2005-03-13 03:00:00.0 & 36.9 \\
\hline 2005-03-13 00:00:00.0 & 37.0 \\
\hline 2005-03-12 21:00:00.0 & 29.0 \\
\hline 2005-03-12 18:00:00.0 & 25.3 \\
\hline 2005-03-12 15:00:00.0 & 21.4 \\
\hline 2005-03-12 12:00:00.0 & 19.9 \\
\hline 2005-03-12 09:00:00.0 & 6.5 \\
\hline
\end{tabular}

\begin{tabular}{|c|c|}
\hline DataHora & VelVentoMax \\
\hline 2005-03-12 06:00:00.0 & 5.3 \\
\hline 2005-03-12 03:00:00.0 & 4.0 \\
\hline 2005-03-12 00:00:00.0 & 8.4 \\
\hline 2005-03-11 21:00:00.0 & 18.8 \\
\hline 2005-03-11 18:00:00.0 & 20.5 \\
\hline 2005-03-11 15:00:00.0 & 15.3 \\
\hline 2005-03-11 12:00:00.0 & 9.3 \\
\hline 2005-03-11 09:00:00.0 & 5.7 \\
\hline 2005-03-11 06:00:00.0 & 6.5 \\
\hline 2005-03-11 03:00:00.0 & 5.0 \\
\hline $2005-03-1100: 00: 00.0$ & 12.3 \\
\hline 2005-03-10 21:00:00.0 & 16.2 \\
\hline 2005-03-10 18:00:00.0 & 18.7 \\
\hline 2005-03-10 15:00:00.0 & \\
\hline 2005-03-10 12:00:00.0 & 10.2 \\
\hline 2005-03-10 09:00:00.0 & 11.4 \\
\hline 2005-03-10 06:00:00.0 & 12.2 \\
\hline 2005-03-10 03:00:00.0 & 9.9 \\
\hline 2005-03-10 00:00:00.0 & 11.2 \\
\hline 2005-03-09 21:00:00.0 & 15.8 \\
\hline 2005-03-09 18:00:00.0 & 16.3 \\
\hline 2005-03-09 15:00:00.0 & 23.5 \\
\hline 2005-03-09 12:00:00.0 & 18.5 \\
\hline 2005-03-09 09:00:00.0 & 12.2 \\
\hline 2005-03-09 06:00:00.0 & 21.0 \\
\hline 2005-03-09 03:00:00.0 & \\
\hline 2005-03-09 00:00:00.0 & 20.8 \\
\hline 2005-03-08 21:00:00.0 & 20.3 \\
\hline 2005-03-08 18:00:00.0 & 33.6 \\
\hline 2005-03-08 15:00:00.0 & \\
\hline 2005-03-08 12:00:00.0 & 16.6 \\
\hline 2005-03-08 09:00:00.0 & 14.6 \\
\hline 2005-03-08 06:00:00.0 & \\
\hline 2005-03-08 03:00:00.0 & \\
\hline 2005-03-08 00:00:00.0 & 10.7 \\
\hline 2005-03-07 21:00:00.0 & 20.1 \\
\hline 2005-03-07 18:00:00.0 & 23.4 \\
\hline 2005-03-07 15:00:00.0 & 23.7 \\
\hline 2005-03-07 12:00:00.0 & 17.2 \\
\hline 2005-03-07 09:00:00.0 & \\
\hline 2005-03-07 06:00:00.0 & \\
\hline 2005-03-07 03:00:00.0 & 15.4 \\
\hline 2005-03-07 00:00:00.0 & 13.9 \\
\hline 2005-03-06 21:00:00.0 & 14.7 \\
\hline 2005-03-06 18:00:00.0 & 27.8 \\
\hline 2005-03-06 15:00:00.0 & 21.8 \\
\hline 2005-03-06 12:00:00.0 & 20.2 \\
\hline 2005-03-06 09:00:00.0 & 19.8 \\
\hline 2005-03-06 06:00:00.0 & 19.6 \\
\hline 2005-03-06 03:00:00.0 & \\
\hline 2005-03-06 00:00:00.0 & 15.6 \\
\hline 2005-03-05 21:00:00.0 & 12.1 \\
\hline 2005-03-05 18:00:00.0 & 14.7 \\
\hline 2005-03-05 15:00:00.0 & 19.1 \\
\hline 2005-03-05 12:00:00.0 & 23.4 \\
\hline 2005-03-05 09:00:00.0 & 22.2 \\
\hline 2005-03-05 06:00:00.0 & \\
\hline 2005-03-05 03:00:00.0 & 16.7 \\
\hline 2005-03-05 00:00:00.0 & 15.0 \\
\hline 2005-03-04 21:00:00.0 & 16.3 \\
\hline 2005-03-04 18:00:00.0 & 21.9 \\
\hline 2005-03-04 15:00:00.0 & 23.7 \\
\hline 2005-03-04 12:00:00.0 & 25.9 \\
\hline 2005-03-04 09:00:00.0 & 23.5 \\
\hline 2005-03-04 06:00:00.0 & \\
\hline 2005-03-04 03:00:00.0 & 16.9 \\
\hline 2005-03-04 00:00:00.0 & 19.6 \\
\hline 2005-03-03 21:00:00.0 & 19.9 \\
\hline 2005-03-03 18:00:00.0 & 21.0 \\
\hline
\end{tabular}




\section{ANEXO 01 - RAJADAS DE VENTO REGISTRADAS EM SÃO MARTINHO DA SERRA / RS}

\begin{tabular}{|c|c|}
\hline DataHora & VelVentoMax \\
\hline \multicolumn{2}{|l|}{ 2005-03-03 15:00:00.0 } \\
\hline 2005-03-03 12:00:00.0 & 22.6 \\
\hline 2005-03-03 09:00:00.0 & 15.3 \\
\hline \multicolumn{2}{|l|}{ 2005-03-03 06:00:00.0 } \\
\hline 2005-03-03 03:00:00.0 & 19.3 \\
\hline 2005-03-03 00:00:00.0 & 20.4 \\
\hline 2005-03-02 21:00:00.0 & 21.7 \\
\hline 2005-03-02 18:00:00.0 & 21.9 \\
\hline 2005-03-02 15:00:00.0 & 22.1 \\
\hline 2005-03-02 12:00:00.0 & 21.1 \\
\hline 2005-03-02 09:00:00.0 & 15.6 \\
\hline 2005-03-02 06:00:00.0 & 17.1 \\
\hline 2005-03-02 03:00:00.0 & 17.1 \\
\hline 2005-03-02 00:00:00.0 & 16.0 \\
\hline 2005-03-01 21:00:00.0 & 20.0 \\
\hline \multicolumn{2}{|l|}{ 2005-03-01 18:00:00.0 } \\
\hline 2005-03-01 15:00:00.0 & 19.4 \\
\hline 2005-03-01 12:00:00.0 & 20.9 \\
\hline 2005-03-01 09:00:00.0 & 22.0 \\
\hline 2005-03-01 06:00:00.0 & 28.3 \\
\hline 2005-03-01 03:00:00.0 & 22.8 \\
\hline 2005-03-01 00:00:00.0 & 20.1 \\
\hline $2005-02-28$ 21:00:00.0 & 21.9 \\
\hline 2005-02-28 18:00:00.0 & 19.7 \\
\hline \multicolumn{2}{|l|}{ 2005-02-28 15:00:00.0 } \\
\hline 2005-02-28 12:00:00.0 & 7.7 \\
\hline 2005-02-28 09:00:00.0 & 9.5 \\
\hline \multicolumn{2}{|l|}{$2005-02-28$ 06:00:00.0 } \\
\hline 2005-02-28 03:00:00.0 & 11.3 \\
\hline 2005-02-28 00:00:00.0 & 11.4 \\
\hline 2005-02-27 21:00:00.0 & 22.2 \\
\hline 2005-02-27 18:00:00.0 & 27.0 \\
\hline 2005-02-27 15:00:00.0 & 26.1 \\
\hline 2005-02-27 12:00:00.0 & 12.7 \\
\hline 2005-02-27 09:00:00.0 & 13.8 \\
\hline \multicolumn{2}{|l|}{ 2005-02-27 06:00:00.0 } \\
\hline 2005-02-27 03:00:00.0 & 16.3 \\
\hline 2005-02-27 00:00:00.0 & 13.2 \\
\hline 2005-02-26 21:00:00.0 & 17.8 \\
\hline 2005-02-26 18:00:00.0 & 20.4 \\
\hline 2005-02-26 15:00:00.0 & 17.9 \\
\hline 2005-02-26 12:00:00.0 & 9.2 \\
\hline 2005-02-26 09:00:00.0 & 12.3 \\
\hline \multicolumn{2}{|l|}{ 2005-02-26 06:00:00.0 } \\
\hline 2005-02-26 03:00:00.0 & 10.1 \\
\hline 2005-02-26 00:00:00.0 & 9.1 \\
\hline 2005-02-25 21:00:00.0 & 11.5 \\
\hline \multicolumn{2}{|l|}{ 2005-02-25 18:00:00.0 } \\
\hline 2005-02-25 15:00:00.0 & 14.5 \\
\hline 2005-02-25 12:00:00.0 & 11.6 \\
\hline \multicolumn{2}{|l|}{ 2005-02-25 09:00:00.0 } \\
\hline \multicolumn{2}{|l|}{ 2005-02-25 06:00:00.0 } \\
\hline 2005-02-25 03:00:00.0 & 9.9 \\
\hline 2005-02-25 00:00:00.0 & 10.4 \\
\hline \multicolumn{2}{|l|}{ 2005-02-24 21:00:00.0 } \\
\hline 2005-02-24 18:00:00.0 & 14.3 \\
\hline 2005-02-24 15:00:00.0 & 12.3 \\
\hline 2005-02-24 12:00:00.0 & 11.1 \\
\hline \multicolumn{2}{|l|}{ 2005-02-24 09:00:00.0 } \\
\hline \multicolumn{2}{|l|}{ 2005-02-24 06:00:00.0 } \\
\hline 2005-02-24 03:00:00.0 & 14.3 \\
\hline 2005-02-24 00:00:00.0 & 13.7 \\
\hline 2005-02-23 21:00:00.0 & \\
\hline 2005-02-23 18:00:00.0 & \\
\hline 2005-02-23 15:00:00.0 & 15.9 \\
\hline 2005-02-23 12:00:00.0 & 17.4 \\
\hline 2005-02-23 09:00:00.0 & \\
\hline 2005-02-23 06:00:00.0 & 14.4 \\
\hline 2005-02-23 03:00:00.0 & 13.5 \\
\hline
\end{tabular}

\begin{tabular}{|c|c|}
\hline DataHora & VelVentoMax \\
\hline 2005-02-23 00:00:00.0 & 11.7 \\
\hline \multicolumn{2}{|l|}{ 2005-02-22 21:00:00.0 } \\
\hline 2005-02-22 18:00:00.0 & 20.2 \\
\hline 2005-02-22 15:00:00.0 & 16.8 \\
\hline 2005-02-22 12:00:00.0 & 10.8 \\
\hline \multicolumn{2}{|l|}{ 2005-02-22 09:00:00.0 } \\
\hline \multicolumn{2}{|l|}{ 2005-02-22 06:00:00.0 } \\
\hline 2005-02-22 03:00:00.0 & 17.2 \\
\hline 2005-02-22 00:00:00.0 & 11.6 \\
\hline 2005-02-21 21:00:00.0 & 19.9 \\
\hline 2005-02-21 18:00:00.0 & 19.7 \\
\hline 2005-02-21 15:00:00.0 & 16.5 \\
\hline 2005-02-21 12:00:00.0 & 15.2 \\
\hline \multicolumn{2}{|l|}{ 2005-02-21 09:00:00.0 } \\
\hline 2005-02-21 06:00:00.0 & 15.9 \\
\hline 2005-02-21 03:00:00.0 & 18.6 \\
\hline 2005-02-21 00:00:00.0 & 14.7 \\
\hline 2005-02-20 21:00:00.0 & 19.3 \\
\hline 2005-02-20 18:00:00.0 & 21.2 \\
\hline 2005-02-20 15:00:00.0 & 11.8 \\
\hline 2005-02-20 12:00:00.0 & 14.7 \\
\hline \multicolumn{2}{|l|}{ 2005-02-20 09:00:00.0 } \\
\hline \multicolumn{2}{|l|}{ 2005-02-20 06:00:00.0 } \\
\hline 2005-02-20 03:00:00.0 & 12.5 \\
\hline 2005-02-20 00:00:00.0 & 14.8 \\
\hline \multicolumn{2}{|l|}{ 2005-02-19 21:00:00.0 } \\
\hline 2005-02-19 18:00:00.0 & 16.2 \\
\hline 2005-02-19 15:00:00.0 & 15.2 \\
\hline 2005-02-19 12:00:00.0 & 15.3 \\
\hline \multicolumn{2}{|l|}{ 2005-02-19 09:00:00.0 } \\
\hline 2005-02-19 06:00:00.0 & 11.8 \\
\hline 2005-02-19 03:00:00.0 & 19.1 \\
\hline 2005-02-19 00:00:00.0 & 13.5 \\
\hline \multicolumn{2}{|l|}{ 2005-02-18 21:00:00.0 } \\
\hline 2005-02-18 18:00:00.0 & 20.2 \\
\hline 2005-02-18 15:00:00.0 & 14.4 \\
\hline 2005-02-18 12:00:00.0 & 16.7 \\
\hline \multicolumn{2}{|l|}{ 2005-02-18 09:00:00.0 } \\
\hline \multicolumn{2}{|l|}{ 2005-02-18 06:00:00.0 } \\
\hline 2005-02-18 03:00:00.0 & 16.4 \\
\hline 2005-02-18 00:00:00.0 & 13.1 \\
\hline \multicolumn{2}{|l|}{ 2005-02-17 21:00:00.0 } \\
\hline 2005-02-17 18:00:00.0 & 16.9 \\
\hline 2005-02-17 15:00:00.0 & 16.7 \\
\hline 2005-02-17 12:00:00.0 & 17.0 \\
\hline \multicolumn{2}{|l|}{ 2005-02-17 09:00:00.0 } \\
\hline 2005-02-17 06:00:00.0 & 11.9 \\
\hline 2005-02-17 03:00:00.0 & 17.3 \\
\hline 2005-02-17 00:00:00.0 & 17.1 \\
\hline 2005-02-16 21:00:00.0 & \\
\hline 2005-02-16 18:00:00.0 & 19.0 \\
\hline 2005-02-16 15:00:00.0 & 18.3 \\
\hline 2005-02-16 12:00:00.0 & 18.2 \\
\hline 2005-02-16 09:00:00.0 & \\
\hline 2005-02-16 06:00:00.0 & 15.9 \\
\hline 2005-02-16 03:00:00.0 & \\
\hline 2005-02-16 00:00:00.0 & 17.6 \\
\hline 2005-02-15 21:00:00.0 & 20.2 \\
\hline 2005-02-15 18:00:00.0 & 19.8 \\
\hline 2005-02-15 15:00:00.0 & \\
\hline 2005-02-15 12:00:00.0 & 22.5 \\
\hline 2005-02-15 09:00:00.0 & \\
\hline 2005-02-15 06:00:00.0 & 16.9 \\
\hline 2005-02-15 03:00:00.0 & 24.7 \\
\hline 2005-02-15 00:00:00.0 & 20.7 \\
\hline 2005-02-14 21:00:00.0 & 24.3 \\
\hline 2005-02-14 18:00:00.0 & 24.5 \\
\hline 2005-02-14 15:00:00.0 & 20.8 \\
\hline 2005-02-14 12:00:00.0 & 19.8 \\
\hline
\end{tabular}

\begin{tabular}{|c|c|}
\hline DataHora & VelVentoMax \\
\hline \multicolumn{2}{|l|}{ 2005-02-14 09:00:00.0 } \\
\hline 2005-02-14 06:00:00.0 & 14.7 \\
\hline 2005-02-14 03:00:00.0 & 19.4 \\
\hline 2005-02-14 00:00:00.0 & 20.3 \\
\hline 2005-02-13 21:00:00.0 & 21.1 \\
\hline 2005-02-13 18:00:00.0 & 20.5 \\
\hline 2005-02-13 15:00:00.0 & 20.9 \\
\hline 2005-02-13 12:00:00.0 & 21.2 \\
\hline 2005-02-13 09:00:00.0 & 15.4 \\
\hline 2005-02-13 06:00:00.0 & 9.9 \\
\hline \multicolumn{2}{|l|}{ 2005-02-13 03:00:00.0 } \\
\hline 2005-02-13 00:00:00.0 & 6.7 \\
\hline 2005-02-12 21:00:00.0 & 18.4 \\
\hline 2005-02-12 18:00:00.0 & 22.4 \\
\hline 2005-02-12 15:00:00.0 & 23.9 \\
\hline 2005-02-12 12:00:00.0 & 13.0 \\
\hline 2005-02-12 09:00:00.0 & 9.9 \\
\hline 2005-02-12 06:00:00.0 & 7.8 \\
\hline \multicolumn{2}{|l|}{ 2005-02-12 03:00:00.0 } \\
\hline 2005-02-12 00:00:00.0 & 28.1 \\
\hline 2005-02-11 21:00:00.0 & 25.4 \\
\hline 2005-02-11 18:00:00.0 & 22.5 \\
\hline 2005-02-11 15:00:00.0 & 19.3 \\
\hline 2005-02-11 12:00:00.0 & 14.0 \\
\hline 2005-02-11 09:00:00.0 & 13.7 \\
\hline 2005-02-11 06:00:00.0 & 21.5 \\
\hline \multicolumn{2}{|l|}{ 2005-02-11 03:00:00.0 } \\
\hline \multicolumn{2}{|l|}{ 2005-02-11 00:00:00.0 } \\
\hline 2005-02-10 21:00:00.0 & 23.6 \\
\hline 2005-02-10 18:00:00.0 & 22.1 \\
\hline \multicolumn{2}{|l|}{ 2005-02-10 15:00:00.0 } \\
\hline 2005-02-10 12:00:00.0 & 10.4 \\
\hline 2005-02-10 09:00:00.0 & 5.9 \\
\hline 2005-02-10 06:00:00.0 & 6.0 \\
\hline \multicolumn{2}{|l|}{$2005-02-10$ 03:00:00.0 } \\
\hline 2005-02-10 00:00:00.0 & 17.2 \\
\hline 2005-02-09 21:00:00.0 & 25.1 \\
\hline 2005-02-09 18:00:00.0 & 27.2 \\
\hline 2005-02-09 15:00:00.0 & 25.0 \\
\hline 2005-02-09 12:00:00.0 & 23.5 \\
\hline 2005-02-09 09:00:00.0 & 20.6 \\
\hline \multicolumn{2}{|l|}{ 2005-02-09 06:00:00.0 } \\
\hline 2005-02-09 03:00:00.0 & 11.5 \\
\hline 2005-02-09 00:00:00.0 & 9.4 \\
\hline 2005-02-08 21:00:00.0 & 11.5 \\
\hline 2005-02-08 18:00:00.0 & 19.7 \\
\hline \multicolumn{2}{|l|}{ 2005-02-08 15:00:00.0 } \\
\hline 2005-02-08 12:00:00.0 & 26.1 \\
\hline 2005-02-08 09:00:00.0 & 26.3 \\
\hline 2005-02-08 06:00:00.0 & 23.4 \\
\hline 2005-02-08 03:00:00.0 & 17.3 \\
\hline 2005-02-08 00:00:00.0 & 12.5 \\
\hline 2005-02-07 21:00:00.0 & 13.4 \\
\hline 2005-02-07 18:00:00.0 & 20.9 \\
\hline 2005-02-07 15:00:00.0 & 27.7 \\
\hline 2005-02-07 12:00:00.0 & 29.4 \\
\hline 2005-02-07 09:00:00.0 & 24.9 \\
\hline 2005-02-07 06:00:00.0 & 23.3 \\
\hline \multicolumn{2}{|l|}{ 2005-02-07 03:00:00.0 } \\
\hline 2005-02-07 00:00:00.0 & 13.4 \\
\hline 2005-02-06 21:00:00.0 & 21.6 \\
\hline \multicolumn{2}{|l|}{ 2005-02-06 18:00:00.0 } \\
\hline 2005-02-06 15:00:00.0 & 22.6 \\
\hline 2005-02-06 12:00:00.0 & 25.6 \\
\hline 2005-02-06 09:00:00.0 & 25.3 \\
\hline \multicolumn{2}{|l|}{ 2005-02-06 06:00:00.0 } \\
\hline 2005-02-06 03:00:00.0 & 16.7 \\
\hline 2005-02-06 00:00:00.0 & 15.5 \\
\hline 2005-02-05 21:00:00.0 & 18.6 \\
\hline
\end{tabular}




\section{ANEXO 01 - RAJADAS DE VENTO REGISTRADAS EM SÃO MARTINHO DA SERRA / RS}

\begin{tabular}{|c|c|}
\hline DataHora & VelVentoMax \\
\hline 2005-02-05 18:00:00.0 & 12.7 \\
\hline 2005-02-05 15:00:00.0 & 17.1 \\
\hline 2005-02-05 12:00:00.0 & 18.2 \\
\hline 2005-02-05 09:00:00.0 & 13.0 \\
\hline 2005-02-05 06:00:00.0 & \\
\hline 2005-02-05 03:00:00.0 & 14.1 \\
\hline 2005-02-05 00:00:00.0 & 17.7 \\
\hline 2005-02-04 21:00:00.0 & 17.4 \\
\hline 2005-02-04 18:00:00.0 & 15.0 \\
\hline 2005-02-04 15:00:00.0 & 15.0 \\
\hline 2005-02-04 12:00:00.0 & 16.2 \\
\hline 2005-02-04 09:00:00.0 & 15.4 \\
\hline 2005-02-04 06:00:00.0 & \\
\hline 2005-02-04 03:00:00.0 & 19.0 \\
\hline 2005-02-04 00:00:00.0 & 22.4 \\
\hline 2005-02-03 21:00:00.0 & 23.3 \\
\hline 2005-02-03 18:00:00.0 & \\
\hline 2005-02-03 15:00:00.0 & 25.5 \\
\hline 2005-02-03 12:00:00.0 & 17.8 \\
\hline 2005-02-03 09:00:00.0 & \\
\hline 2005-02-03 06:00:00.0 & \\
\hline 2005-02-03 03:00:00.0 & 23.8 \\
\hline 2005-02-03 00:00:00.0 & 27.3 \\
\hline 2005-02-02 21:00:00.0 & 26.0 \\
\hline 2005-02-02 18:00:00.0 & \\
\hline 2005-02-02 15:00:00.0 & 27.5 \\
\hline 2005-02-02 12:00:00.0 & 28.6 \\
\hline 2005-02-02 09:00:00.0 & \\
\hline 2005-02-02 06:00:00.0 & 26.9 \\
\hline 2005-02-02 03:00:00.0 & 27.2 \\
\hline 2005-02-02 00:00:00.0 & 27.8 \\
\hline 2005-02-01 21:00:00.0 & 23.7 \\
\hline 2005-02-01 18:00:00.0 & 24.8 \\
\hline 2005-02-01 15:00:00.0 & 23.0 \\
\hline 2005-02-01 12:00:00.0 & 25.6 \\
\hline 2005-02-01 09:00:00.0 & \\
\hline 2005-02-01 06:00:00.0 & 22.1 \\
\hline 2005-02-01 03:00:00.0 & 21.5 \\
\hline 2005-02-01 00:00:00.0 & 8.6 \\
\hline 2005-01-31 21:00:00.0 & 31.1 \\
\hline 2005-01-31 18:00:00.0 & \\
\hline 2005-01-31 15:00:00.0 & 27.6 \\
\hline 2005-01-31 12:00:00.0 & 25.3 \\
\hline 2005-01-31 09:00:00.0 & \\
\hline 2005-01-31 06:00:00.0 & 23.9 \\
\hline 2005-01-31 03:00:00.0 & 21.5 \\
\hline 2005-01-31 00:00:00.0 & 16.1 \\
\hline 2005-01-30 21:00:00.0 & \\
\hline 2005-01-30 18:00:00.0 & 26.1 \\
\hline 2005-01-30 15:00:00.0 & 27.8 \\
\hline 2005-01-30 12:00:00.0 & 27.2 \\
\hline 2005-01-30 09:00:00.0 & \\
\hline 2005-01-30 06:00:00.0 & \\
\hline 2005-01-30 03:00:00.0 & 22.2 \\
\hline 2005-01-30 00:00:00.0 & 23.6 \\
\hline 2005-01-29 21:00:00.0 & \\
\hline 2005-01-29 18:00:00.0 & 14.9 \\
\hline 2005-01-29 15:00:00.0 & 22.2 \\
\hline 2005-01-29 12:00:00.0 & 22.5 \\
\hline 2005-01-29 09:00:00.0 & \\
\hline 2005-01-29 06:00:00.0 & 18.7 \\
\hline 2005-01-29 03:00:00.0 & 15.9 \\
\hline 2005-01-29 00:00:00.0 & \\
\hline 2005-01-28 21:00:00.0 & \\
\hline 2005-01-28 18:00:00.0 & 14.4 \\
\hline 2005-01-28 15:00:00.0 & 22.0 \\
\hline 2005-01-28 12:00:00.0 & 20.5 \\
\hline 2005-01-28 09:00:00.0 & 20.0 \\
\hline 2005-01-28 06:00:00.0 & 19.2 \\
\hline
\end{tabular}

\begin{tabular}{|c|c|}
\hline DataHora & VelVentoMax \\
\hline 2005-01-28 03:00:00.0 & 15.6 \\
\hline 2005-01-28 00:00:00.0 & 17.3 \\
\hline 2005-01-27 21:00:00.0 & \\
\hline 2005-01-27 18:00:00.0 & 20.9 \\
\hline 2005-01-27 15:00:00.0 & 22.9 \\
\hline 2005-01-27 12:00:00.0 & 23.2 \\
\hline 2005-01-27 09:00:00.0 & 17.3 \\
\hline 2005-01-27 06:00:00.0 & 17.6 \\
\hline 2005-01-27 03:00:00.0 & 16.0 \\
\hline 2005-01-27 00:00:00.0 & 18.9 \\
\hline 2005-01-26 21:00:00.0 & 24.5 \\
\hline 2005-01-26 18:00:00.0 & \\
\hline 2005-01-26 15:00:00.0 & 26.4 \\
\hline 2005-01-26 12:00:00.0 & 16.5 \\
\hline 2005-01-26 09:00:00.0 & \\
\hline 2005-01-26 06:00:00.0 & 21.7 \\
\hline 2005-01-26 03:00:00.0 & 20.4 \\
\hline 2005-01-26 00:00:00.0 & \\
\hline 2005-01-25 21:00:00.0 & 26.4 \\
\hline 2005-01-25 18:00:00.0 & 25.6 \\
\hline 2005-01-25 15:00:00.0 & 30.7 \\
\hline 2005-01-25 12:00:00.0 & 27.3 \\
\hline 2005-01-25 09:00:00.0 & \\
\hline 2005-01-25 06:00:00.0 & 15.7 \\
\hline 2005-01-25 03:00:00.0 & 20.2 \\
\hline 2005-01-25 00:00:00.0 & 23.8 \\
\hline 2005-01-24 21:00:00.0 & 12.4 \\
\hline 2005-01-24 18:00:00.0 & 16.8 \\
\hline 2005-01-24 15:00:00.0 & 33.3 \\
\hline 2005-01-24 12:00:00.0 & 29.1 \\
\hline 2005-01-24 09:00:00.0 & \\
\hline 2005-01-24 06:00:00.0 & 13.8 \\
\hline 2005-01-24 03:00:00.0 & \\
\hline 2005-01-24 00:00:00.0 & 12.9 \\
\hline 2005-01-23 21:00:00.0 & 15.0 \\
\hline 2005-01-23 18:00:00.0 & 16.3 \\
\hline 2005-01-23 15:00:00.0 & 14.2 \\
\hline 2005-01-23 12:00:00.0 & 17.4 \\
\hline 2005-01-23 09:00:00.0 & 16.9 \\
\hline 2005-01-23 06:00:00.0 & 16.5 \\
\hline 2005-01-23 03:00:00.0 & 10.6 \\
\hline 2005-01-23 00:00:00.0 & \\
\hline 2005-01-22 21:00:00.0 & 15.8 \\
\hline 2005-01-22 18:00:00.0 & 17.0 \\
\hline 2005-01-22 15:00:00.0 & 16.9 \\
\hline 2005-01-22 12:00:00.0 & \\
\hline 2005-01-22 09:00:00.0 & \\
\hline 2005-01-22 06:00:00.0 & \\
\hline 2005-01-22 00:00:00.0 & 13.1 \\
\hline 2005-01-21 12:00:00.0 & 21.2 \\
\hline 2005-01-21 09:00:00.0 & 13.5 \\
\hline 2005-01-21 06:00:00.0 & 11.9 \\
\hline 2005-01-21 03:00:00.0 & 10.6 \\
\hline 2005-01-21 00:00:00.0 & \\
\hline 2005-01-20 21:00:00.0 & 25.8 \\
\hline 2005-01-20 18:00:00.0 & 26.7 \\
\hline 2005-01-20 15:00:00.0 & 23.8 \\
\hline 2005-01-20 12:00:00.0 & 14.4 \\
\hline 2005-01-20 09:00:00.0 & 13.8 \\
\hline 2005-01-20 06:00:00.0 & 12.4 \\
\hline 2005-01-20 03:00:00.0 & 6.8 \\
\hline 2005-01-20 00:00:00.0 & 15.5 \\
\hline 2005-01-19 21:00:00.0 & 17.2 \\
\hline 2005-01-19 18:00:00.0 & 17.7 \\
\hline 2005-01-19 15:00:00.0 & 13.2 \\
\hline 2005-01-19 12:00:00.0 & 33.0 \\
\hline 2005-01-19 09:00:00.0 & 15.3 \\
\hline 2005-01-19 06:00:00.0 & 19.7 \\
\hline 2005-01-19 03:00:00.0 & \\
\hline
\end{tabular}

\begin{tabular}{|c|c|}
\hline DataHora & VelVentoMax \\
\hline 2005-01-19 00:00:00.0 & 13.6 \\
\hline 2005-01-18 21:00:00.0 & 21.4 \\
\hline 2005-01-18 18:00:00.0 & 21.2 \\
\hline 2005-01-18 15:00:00.0 & 17.2 \\
\hline 2005-01-18 12:00:00.0 & 11.1 \\
\hline 2005-01-18 09:00:00.0 & 11.0 \\
\hline 2005-01-18 06:00:00.0 & \\
\hline 2005-01-18 03:00:00.0 & \\
\hline 2005-01-18 00:00:00.0 & 14.2 \\
\hline 2005-01-17 21:00:00.0 & 21.0 \\
\hline 2005-01-17 18:00:00.0 & 25.2 \\
\hline 2005-01-17 15:00:00.0 & 23.2 \\
\hline 2005-01-17 12:00:00.0 & 18.0 \\
\hline 2005-01-17 09:00:00.0 & 17.3 \\
\hline 2005-01-17 06:00:00.0 & 17.2 \\
\hline 2005-01-17 03:00:00.0 & 16.9 \\
\hline 2005-01-17 00:00:00.0 & 21.7 \\
\hline 2005-01-16 21:00:00.0 & 25.9 \\
\hline 2005-01-16 18:00:00.0 & 29.2 \\
\hline 2005-01-16 15:00:00.0 & 27.3 \\
\hline 2005-01-16 12:00:00.0 & 24.6 \\
\hline 2005-01-16 09:00:00.0 & 18.4 \\
\hline 2005-01-16 06:00:00.0 & 27.1 \\
\hline 2005-01-16 03:00:00.0 & 7.5 \\
\hline 2005-01-16 00:00:00.0 & 11.0 \\
\hline 2005-01-15 21:00:00.0 & 12.8 \\
\hline 2005-01-15 18:00:00.0 & 16.7 \\
\hline 2005-01-15 15:00:00.0 & \\
\hline 2005-01-15 12:00:00.0 & 22.9 \\
\hline 2005-01-15 09:00:00.0 & 29.7 \\
\hline 2005-01-15 06:00:00.0 & 13.5 \\
\hline 2005-01-15 03:00:00.0 & \\
\hline 2005-01-15 00:00:00.0 & 13.7 \\
\hline 2005-01-14 21:00:00.0 & 14.9 \\
\hline 2005-01-14 18:00:00.0 & 24.1 \\
\hline 2005-01-14 15:00:00.0 & \\
\hline 2005-01-14 12:00:00.0 & 21.8 \\
\hline 2005-01-14 09:00:00.0 & 20.8 \\
\hline 2005-01-14 06:00:00.0 & 14.4 \\
\hline 2005-01-14 03:00:00.0 & 19.7 \\
\hline 2005-01-14 00:00:00.0 & 16.4 \\
\hline 2005-01-13 21:00:00.0 & 18.2 \\
\hline 2005-01-13 18:00:00.0 & 15.3 \\
\hline 2005-01-13 15:00:00.0 & 11.7 \\
\hline 2005-01-13 12:00:00.0 & 15.1 \\
\hline 2005-01-13 09:00:00.0 & 16.6 \\
\hline 2005-01-13 06:00:00.0 & 16.3 \\
\hline 2005-01-13 03:00:00.0 & \\
\hline 2005-01-13 00:00:00.0 & 14.4 \\
\hline 2005-01-12 21:00:00.0 & 14.1 \\
\hline 2005-01-12 18:00:00.0 & 13.9 \\
\hline 2005-01-12 15:00:00.0 & \\
\hline 2005-01-12 12:00:00.0 & 23.5 \\
\hline 2005-01-12 09:00:00.0 & 18.7 \\
\hline 2005-01-12 06:00:00.0 & \\
\hline 2005-01-12 03:00:00.0 & 16.9 \\
\hline 2005-01-12 00:00:00.0 & 21.3 \\
\hline 2005-01-11 21:00:00.0 & 24.0 \\
\hline 2005-01-11 18:00:00.0 & \\
\hline 2005-01-11 15:00:00.0 & 22.7 \\
\hline 2005-01-11 12:00:00.0 & 20.8 \\
\hline 2005-01-11 09:00:00.0 & 17.7 \\
\hline 2005-01-11 06:00:00.0 & 21.0 \\
\hline 2005-01-11 03:00:00.0 & 20.6 \\
\hline 2005-01-11 00:00:00.0 & 30.6 \\
\hline 2005-01-10 21:00:00.0 & 20.2 \\
\hline 2005-01-10 18:00:00.0 & 18.3 \\
\hline 2005-01-10 15:00:00.0 & 17.6 \\
\hline 2005-01-10 12:00:00.0 & 21.0 \\
\hline
\end{tabular}




\section{ANEXO 01 - RAJADAS DE VENTO REGISTRADAS \\ EM SÃO MARTINHO DA SERRA / RS}

\begin{tabular}{|c|c|}
\hline DataHora & VelVentoMax \\
\hline 2005-01-10 09:00:00.0 & 16.8 \\
\hline 2005-01-10 06:00:00.0 & 8.2 \\
\hline 2005-01-10 03:00:00.0 & 33.3 \\
\hline 2005-01-10 00:00:00.0 & 36.7 \\
\hline 2005-01-09 21:00:00.0 & 19.5 \\
\hline 2005-01-09 18:00:00.0 & 23.6 \\
\hline 2005-01-09 15:00:00.0 & 20.5 \\
\hline 2005-01-09 12:00:00.0 & 14.3 \\
\hline 2005-01-09 09:00:00.0 & \\
\hline 2005-01-09 06:00:00.0 & 10.9 \\
\hline 2005-01-09 03:00:00.0 & 15.5 \\
\hline 2005-01-09 00:00:00.0 & 20.6 \\
\hline 2005-01-08 21:00:00.0 & 23.3 \\
\hline 2005-01-08 18:00:00.0 & \\
\hline 2005-01-08 15:00:00.0 & 16.4 \\
\hline 2005-01-08 12:00:00.0 & 15.7 \\
\hline 2005-01-08 09:00:00.0 & \\
\hline 2005-01-08 06:00:00.0 & 17.2 \\
\hline 2005-01-08 03:00:00.0 & \\
\hline 2005-01-08 00:00:00.0 & 17.1 \\
\hline 2005-01-07 21:00:00.0 & 19.3 \\
\hline 2005-01-07 18:00:00.0 & \\
\hline 2005-01-07 15:00:00.0 & \\
\hline 2005-01-07 12:00:00.0 & 17.1 \\
\hline 2005-01-07 09:00:00.0 & 14.7 \\
\hline 2005-01-07 06:00:00.0 & 14.5 \\
\hline 2005-01-07 03:00:00.0 & 9.6 \\
\hline 2005-01-07 00:00:00.0 & 13.2 \\
\hline 2005-01-06 21:00:00.0 & \\
\hline 2005-01-06 18:00:00.0 & \\
\hline 2005-01-06 15:00:00.0 & 18.1 \\
\hline 2005-01-06 12:00:00.0 & 9.0 \\
\hline 2005-01-06 09:00:00.0 & 11.9 \\
\hline 2005-01-06 06:00:00.0 & \\
\hline 2005-01-06 03:00:00.0 & 12.6 \\
\hline 2005-01-06 00:00:00.0 & 17.8 \\
\hline 2005-01-05 21:00:00.0 & 16.9 \\
\hline 2005-01-05 18:00:00.0 & 16.4 \\
\hline 2005-01-05 15:00:00.0 & 16.0 \\
\hline 2005-01-05 12:00:00.0 & 9.8 \\
\hline 2005-01-05 09:00:00.0 & 10.9 \\
\hline 2005-01-05 06:00:00.0 & \\
\hline 2005-01-05 03:00:00.0 & 12.9 \\
\hline 2005-01-05 00:00:00.0 & 13.1 \\
\hline 2005-01-04 21:00:00.0 & 17.9 \\
\hline 2005-01-04 18:00:00.0 & \\
\hline 2005-01-04 15:00:00.0 & 9.9 \\
\hline 2005-01-04 12:00:00.0 & 8.9 \\
\hline 2005-01-04 09:00:00.0 & 14.7 \\
\hline 2005-01-04 06:00:00.0 & 12.0 \\
\hline 2005-01-04 03:00:00.0 & 11.5 \\
\hline 2005-01-04 00:00:00.0 & 21.1 \\
\hline 2005-01-03 21:00:00.0 & \\
\hline 2005-01-03 18:00:00.0 & 29.0 \\
\hline 2005-01-03 15:00:00.0 & 21.5 \\
\hline 2005-01-03 12:00:00.0 & 18.4 \\
\hline 2005-01-03 09:00:00.0 & 16.2 \\
\hline 2005-01-03 06:00:00.0 & \\
\hline 2005-01-03 03:00:00.0 & 18.3 \\
\hline 2005-01-03 00:00:00.0 & 28.7 \\
\hline 2005-01-02 21:00:00.0 & \\
\hline 2005-01-02 18:00:00.0 & 17.8 \\
\hline 2005-01-02 15:00:00.0 & 20.1 \\
\hline 2005-01-02 12:00:00.0 & 20.5 \\
\hline 2005-01-02 09:00:00.0 & \\
\hline 2005-01-02 06:00:00.0 & 18.9 \\
\hline 2005-01-02 03:00:00.0 & 20.9 \\
\hline 2005-01-02 00:00:00.0 & 22.3 \\
\hline 2005-01-01 21:00:00.0 & 20.4 \\
\hline
\end{tabular}

\begin{tabular}{|l|c|}
\hline DataHora & VelVentoMax \\
\hline 2005-01-01 18:00:00.0 & 17.4 \\
\hline 2005-01-01 15:00:00.0 & 17.8 \\
\hline 2005-01-01 12:00:00.0 & 19.0 \\
\hline 2005-01-01 09:00:00.0 \\
\hline 2005-01-01 06:00:00.0 & 13.3 \\
\hline 2005-01-01 03:00:00.0 & 18.5 \\
\hline 2005-01-01 00:00:00.0 & 17.6 \\
\hline
\end{tabular}

